Joanna Wlochal

A Thesis Submitted for the Degree of PhD at the

University of St Andrews

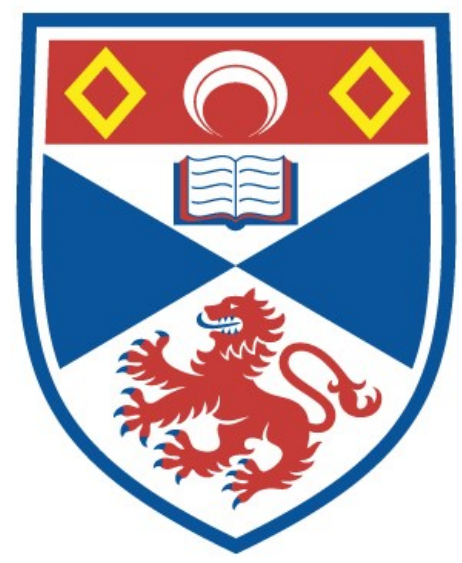

2012

Full metadata for this item is available in

St Andrews Research Repository

at:

http://research-repository.st-andrews.ac.uk/

Please use this identifier to cite or link to this item:

http://hdl.handle.net/10023/3663

This item is protected by original copyright 


\title{
Synthesis of the Originally Proposed Structure of Palmerolide C
}

\author{
Joanna Wlochal \\ School of Chemistry
}

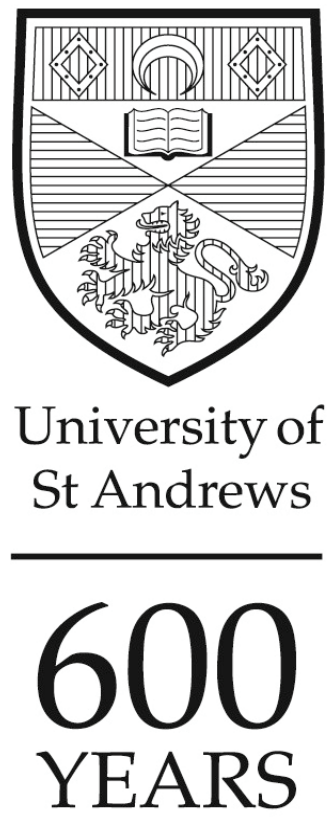

June 2012

Thesis submitted to the University of St Andrews for the degree of Doctor of Philosophy

Supervisor: Dr. Gordon J. Florence 


\section{Declarations}

I, Joanna Wlochal, hereby certify that this thesis, which is approximately 45000 words in length, has been written by me, that it is the record of work carried out by me and that it has not been submitted in any previous application for a higher degree.

I was admitted as a research student in May, 2008 and as a candidate for the degree of $\mathrm{PhD}$ in April 2012; the higher study for which this is a record was carried out in the University of St Andrews between 2008 and 2012

Date Signature of Candidate

\section{Supervisor's declaration}

I hereby certify that the candidate has fulfilled the conditions of the Resolution and Regulations appropriate for the degree of Doctor of Philosophy in the University of St Andrews and that the candidate is qualified to submit this thesis in application for that degree.

Date

Signature of Supervisor 


\title{
Copyright Declarations
}

\author{
Restricted Access
}

In submitting this thesis to the University of St Andrews I understand that I am giving permission for it to be made available for use in accordance with the regulations of the University Library for the time being in force, subject to any copyright vested in the work not being affected thereby. I also understand that the title and the abstract will be published, and that a copy of the work may be made and supplied to any bona fide library or research worker, that my thesis will be electronically accessible for personal or research use unless exempt by award of an embargo as requested below, and that the library has the right to migrate my thesis into new electronic forms as required to ensure continued access to the thesis. I have obtained any third-party copyright permissions that may be required in order to allow such access and migration, or have requested the appropriate embargo below.

The following is an agreed request by candidate and supervisor regarding the electronic publication of this thesis: publication would preclude future publication.

Embargo on both all of printed copy and electronic copy for the same fixed period of two years on the following ground: publication would preclude future publication.

Date

Signature of Candidate

Date

Signature of Supervisor 
"Believe that you can do it, under any circumstances"

- Maria Skłodowska-Curie 
Dedicated to my mother and father 


\section{Preface}

This thesis describes the work carried out during my three and a half years as a Ph.D. student at the School of Chemistry, University of St Andrews under the supervision of Dr. Gordon J. Florence. There are numerous people that have contributed and been involved in the projects I have worked on in the last few years and I would like to thank the following people for their help and collaboration.

I am deeply grateful to Dr. Gordon J. Florence for giving me the confidence to explore my research interests and the guidance to avoid getting lost in my exploration through sharing with me his vast knowledge of chemistry. Gordon was a fabulous advisor: sharp, cheery, perceptive, and mindful of the things that truly matter. He has always motivated and inspired me and during the hard times, remained optimistic and led me in the right direction. Thanks for believing in me.

I would also like to thank my assessor Prof. David O'Hagan for continuous interest in my progress, encouragement and support. I owe many thanks to Dr. Andrew Smith for providing me with scientific insight on numerous occasions, particularly during joint group meeting sessions. Dr. Iain Smellie also deserves a special thanks; he was always full of sound advice when proof-reading this thesis, and was always able to foster a great learning environment for undergraduate students during the time I assisted in his lab-courses.

During my graduate studies I have had the privilege of working with a number of interesting and exceptional people and I would like to take the opportunity to thank them individually. When I first came to the BMS $4^{\text {th }}$ floor, the older students and staff at the School of Chemistry helped to shape me as a scientist. In particularly Dr. Ross G. Murray provided a great deal of guidance during the early years; his work ethic, expertise and music taste kept me coming back every morning, believing my chemistry would start working... and it eventually did! Agnieszka Kosinska took great care of me in and out of the lab, ever since I came for an interview and then through out the first year. I would like to collectively thank the GJF group for making our laboratory a great working environment. There are also a number of people whom I would like to acknowledge individually: the witches from Florence/Florence witches as you like (yes that's about you 
Jo and Katy) thanks for all the sabbatical sessions helping to keep in balance with work, all the support, encouragement, and friendship. Katy deserves all the credit for any coherence in the following pages; Jo, I bet... she would have proof-read your thesis too, if she wouldn't be finishing sooner. The later group members, especially Greg for sharing my 'joy' when I saw the spectra for my final product; to both you and Lloyd, thanks for being understanding lab- and office-mates. Dr. Lik Ren Tai thanks for possibly the most easy going post-doc attitude I have ever come across. I had the good fortune to work with one outstanding undergraduate; Euan, despite sharing my fume-hood and not working on my project, you were by far the best and most cheerful student I have worked with. Guys, having supportive bay mates, and walking this rocky way with me, made a significant difference in my postgraduate experience.

The list of people I would like to thank goes on and on. The Botting, O'Hagan and Smith labs have all been extremely helpful in sharing their lab resources and knowledge. Thank God I had Su one step ahead of me, reminding of any upcoming deadlines for reports and PG bureaucracy to be dealt with; Nikos, for cheering me up when it was needed. I have many fond memories, especially from time spent with Jason, thanks mate. Thanks also to the Polish crew, whom I was fortunate to meet in St Andrews especially Ola, Lila, Artur, Piotr, Ilonka, Gosia and Kris; not a single one of them has ever refused to lend a hand my way. I would also like to thank my friend far away from Scotland, Alma, studies at Lund was the first period in my life where I was living away from my entire family. Fortunately, I met you and you made me feel at home. Your friendship is one of a kind, forgive my negligence over the past years. Thanks for helping me get through the difficult times, and for all the emotional support, camaraderie, entertainment, and care you provided.

For facilities, I would like to thank Dr Tomas Lebl and Melanja Smith in the NMR lab. Caroline Horsburgh and the National Mass Spectrometry Centre in Swansea for mass spectrometry analyses. This project would not be realised without the generous financial support of the University of St Andrews and the EPSRC research council.

At this point, I would like to thank the most important people in my life, my family. I am nothing without them. As much as my time at St Andrews has shaped me as a scientist over the past three-plus years, the continuous support of my parents and sisters 
have helped define me over more than a quarter of a century. My mother, Danuta, is the backbone of our family. She has sacrificed everything to ensure that my sisters and I would always be able to strive for our goals. If there would be a Ph.D. in life in general she would definitely earn summa cum laude. My father, Adam, who I want to thank for showing me the big world, for telling me that nothing is impossible, just further away to reach. He has also taught me the value of hard work. My older sister, Marlena-Zocha, as the eldest paved the way, making my life easier on many occasions, thanks for keeping me up-to-date with everything else beside chemistry, I wish you all the joy and happiness, and remember you are always welcome wherever I go. My younger sister, Alicja, for always bringing good news, tak for al jeres støtte og opmuntring, hvad der går rundt kommer tilbage omkring ;-)

Finally, I want to thank Josep for never giving up on me. It happened to be a distant journey for both of us, the journey that I learnt to cherish, more than a destination...

What else could I write? I don't have the right

Joanna Wlochal

St Andrews, 15 ${ }^{\text {th }}$ June 2012 


\section{Abstract}

The palmerolides are an emerging class of polyketide natural products isolated from marine organisms inhabiting the Antarctic Sea. Although there has been a substantial effort to prepare the most active family member palmerolide A, no synthetic work has been performed in the area of the other members of the family; palmerolides $\mathrm{B}$ and $\mathrm{C}$ of different ring structure. These compounds are particularly interesting due to their complex structures with undefined stereochemistry and both display high selectivity towards human melanoma cancer cell lines.

A highly convergent strategy to access palmerolide $\mathrm{C}$ has been developed, relying on the assembly of three fragments using key bond couplings at C6-C7 (boron-mediated aldol reaction/dehydration) and C14-C15 (Julia-Kocienski olefination), followed by a suitable macrolactonisation step and Buchwald enamide formation. Prior to subunit synthesis, the relative configuration of the $\mathrm{C} 8-\mathrm{C} 10$ stereotriad was resolved via the synthesis of diastereomeric degradation fragments by the application of organocatalytic cross aldol reactions and a suitable 1,3-selective reduction.

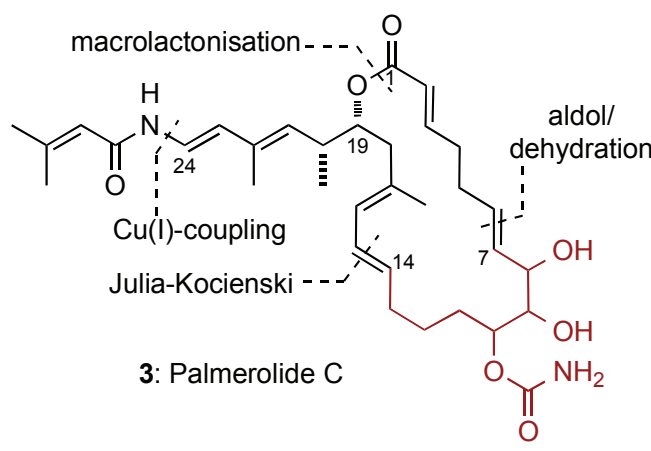




\section{Compound Numbering}

All compounds intended toward the structure elucidation or total synthesis of palmerolide $\mathrm{C}$ will be numbered according to the carbon chain of the natural product. This numbering is given on the structure and is used in the ${ }^{1} \mathrm{H}$ NMR assignment.

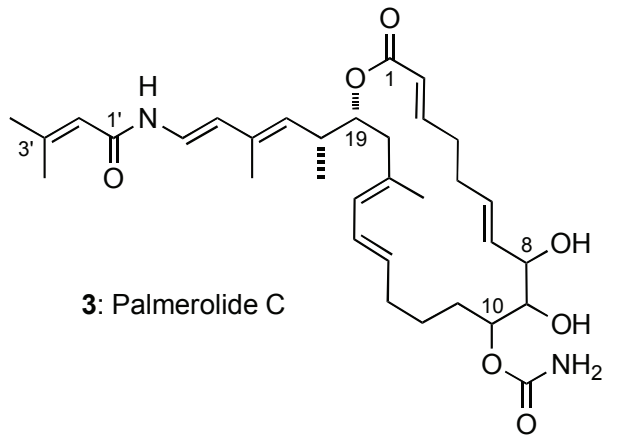

The naming of the compounds in the experimental section uses the IUPAC convention. 


\section{List of Abbreviations}

aq

ASAP MS

$[\alpha]$

Ac

$\mathrm{AcOH}$

$\mathrm{Ar}$

ATPH

BT

br

Burgess reagent

calcd

d

c

CD

CM

COSY

d

$d_{6}$-DMSO

DBU

DCC

dd

de

decomp.

DIAD

DIBAL-H

DMAP

DME

DMF

DMP

DMSO

dr aqueous

atmospheric solids probe analysis mass spectrometry

specific rotation

acetyl

acetic acid

argon

Aluminium tris-(2,6-diphenyl)-phenoxide

benzothiazol-2-yl

broad (NMR)

methyl N-(triethylammoniumsulfonyl)carbamate

calculated

doublet

concentration

circular dichroism

cross-metathesis

correlation spectroscopy

doublet

deuterated dimethyl sulfoxide

1,8-diazabicycloundec-7-ene

dicyclohexylcarbodiimide

doublet of doublets

diastereomeric excess

decomposition

diisopropyl azodicarboxylate

Diisobutylaluminium hydride

4-(N,N-dimethylamino)pyridine

1,2-dimethoxyethane

$\mathrm{N}, \mathrm{N}$-dimethylformamide

Dess-Martin Periodinane

dimethyl sulfoxide

diastereomeric ratio 
$\delta$

ee

EI

eq

ES

Et

EtOAc

g

h

HMBC

HMPA

HRMS

HSQC

HWE

$\mathrm{Hz}$

$\mathrm{IC}_{50}$

Im

IR

$J$

$\mathrm{L}$

LDA

L-Selectride

$\mathrm{m}$

M

Martin sulfurane

$\mathrm{m} / \mathrm{z}$

$\mathrm{Me}$

$\mathrm{MeOH}$

$\mathrm{MHz}$

$\min$

mol

MOM

MS nuclear magnetic resonance chemical shift [ppm]

enantiomeric excess

electron ionisation

equivalent

electrospray

ethyl

ethyl acetate

$\operatorname{gram}(\mathrm{s})$

hour(s)

heteronuclear multiple-bond correlation spectroscopy

hexamethylphosphoramide

high-resolution mass spectrometry

heteronuclear single-quantum correlation spectroscopy

Horner-Wodsworth-Emmons reaction

Hertz

concentration required for $50 \%$ inhibition (of biological effect)

imidazole

infrared

coupling constant

litre

lithium diisopropylamide

lithium tri-sec-butylborohydride

multiplet (NMR)

molarity

bis $[\alpha, \alpha$-bis(trifluoromethyl)benzenemethanolato]diphenylsulfur mass to charge ratio

methyl

methanol

megahertz

minutes

mole

methoxymethyl ether

mass spectroscopy 


\begin{tabular}{|c|c|}
\hline MTPAA & $\alpha$-methoxy- $\alpha$-(trifluoromethyl)phenyl acetic acid \\
\hline MW & microwave \\
\hline$\mu$ & micro \\
\hline NaHMDS & sodium hexamethyldisilazide \\
\hline NMP & $N$-methylpyrrolidone \\
\hline NMR & nuclear magnetic resonance \\
\hline nOe & nuclear Overhauser effect \\
\hline $\mathrm{Nu}$ & nucleophile \\
\hline${ }^{\circ} \mathrm{C}$ & degrees Celsius \\
\hline Obs & obscured \\
\hline $\mathrm{p}$ & para \\
\hline $\mathrm{pH}$ & $-\log [\mathrm{H}+]$ \\
\hline $\mathrm{Ph}$ & phenyl \\
\hline PMB & para-methoxybenzyl \\
\hline ppm & parts per million \\
\hline PT & phenyl tetrazole \\
\hline py & pyridine \\
\hline $\mathrm{q}$ & quartet (NMR) \\
\hline $\mathrm{rt}$ & room temperature \\
\hline $\mathrm{Rf}$ & thin-layer chromatography retention factor \\
\hline s & singlet (NMR) \\
\hline $\mathrm{t}$ & triplet (NMR) \\
\hline$t$ & tertiary $($ tert $)$ \\
\hline TBAF & tetrabutylammonium fluoride \\
\hline TBS & $t$-butyldimethylsilyl \\
\hline TEA & triethylamine \\
\hline TEMPO & 2,2,6,6-tetramethyl-piperidin-1-oxyl \\
\hline TES & triethylsilyl \\
\hline THF & tetrahydrofuran \\
\hline TLC & thin layer chromatography \\
\hline TMS & trimethylsilyl \\
\hline
\end{tabular}




\section{Contents}

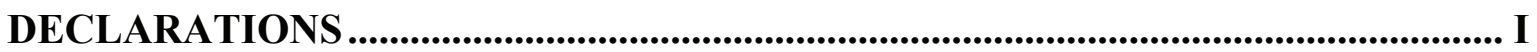

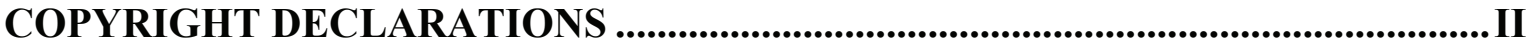

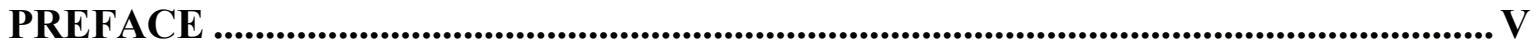

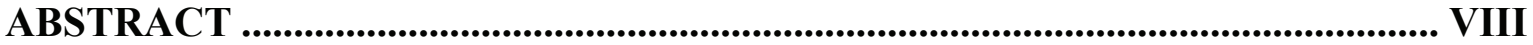

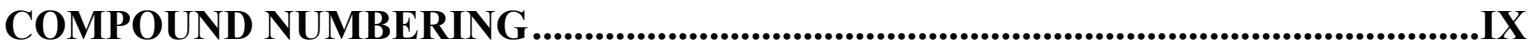

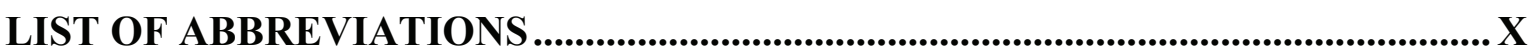

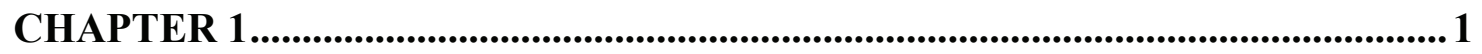

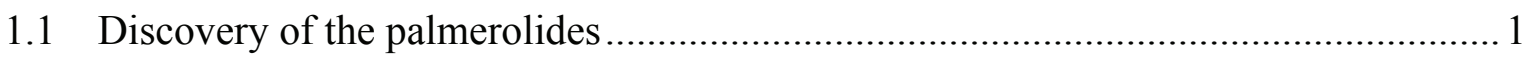

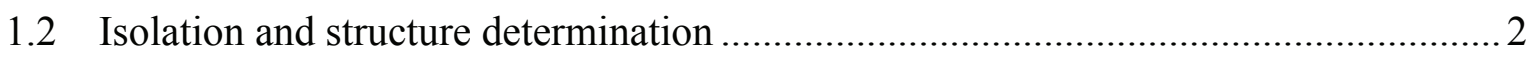

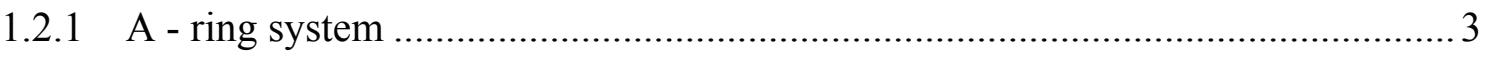

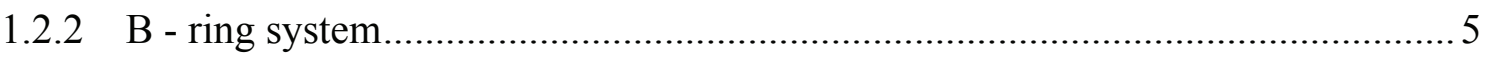

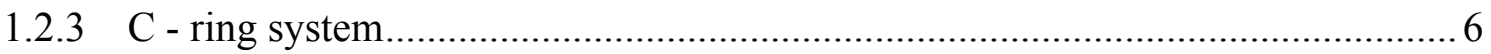

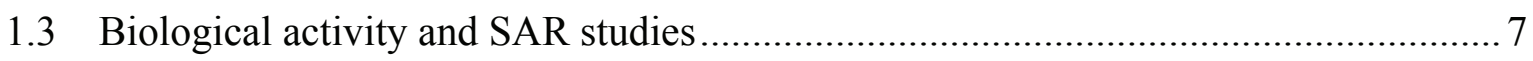

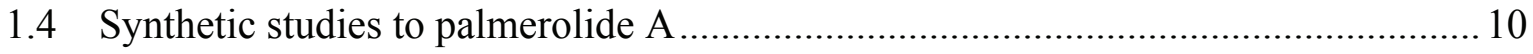

1.4.1 The first synthesis of palmerolide A by De Brabander ..................................... 10

1.4.2 Nicolaou and Chen synthesis of palmerolide A …............................................. 11

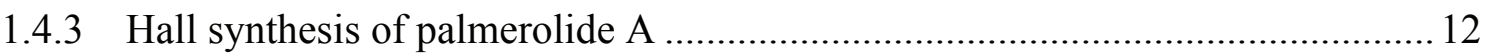

1.5 Methods for the determination of the absolute stereochemistry in complex molecules 14

1.5.1 Degradation studies to confirm the absolute stereochemistry of palmerolide A. 14

1.5.2 Degradation studies in determining the absolute stereochemistry of complex

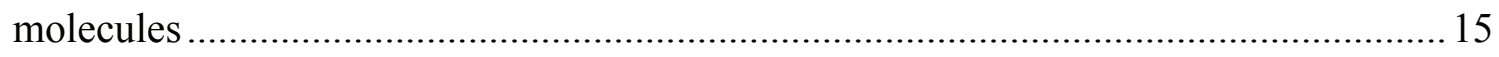

1.5.3 J-based configurational analysis developed by Murata...................................... 19 
CHAPTER 2

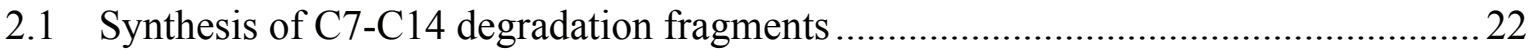

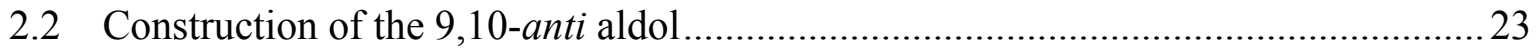

2.2.1 Determination of the absolute configuration at $\mathrm{C} 10$ stereocentre .......................2 27

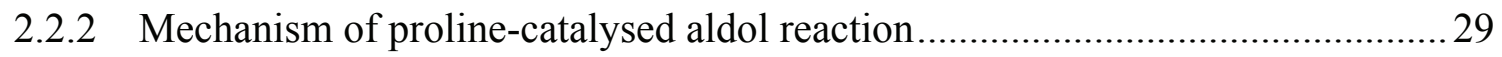

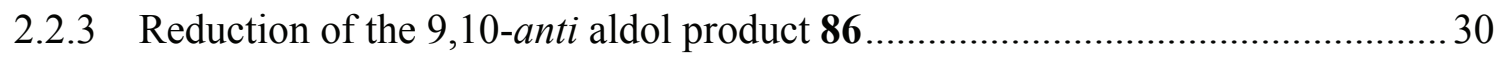

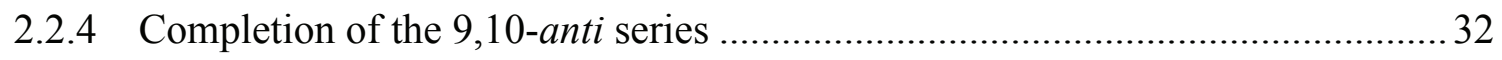

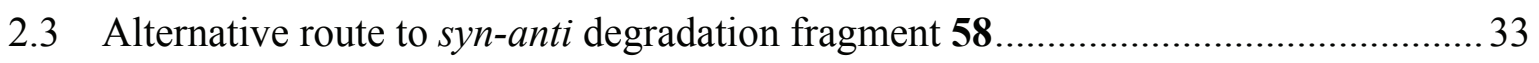

2.4 Construction of the 9,10-syn aldol via an organocatalytic approach.......................... 34

2.4.1 Determination of the absolute configuration at the $\mathrm{C} 10$ stereocentre ................ 35

2.4.2 Mechanism of $\mathrm{O}-\mathrm{t} \mathrm{Bu}$-threonine-catalysed aldol reaction.................................... 36

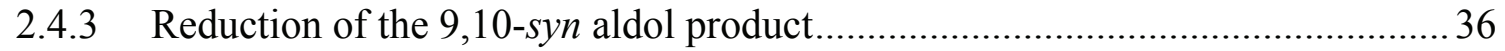

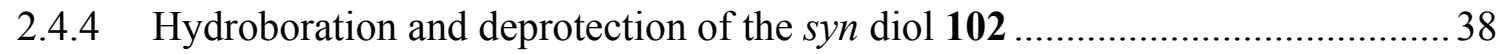

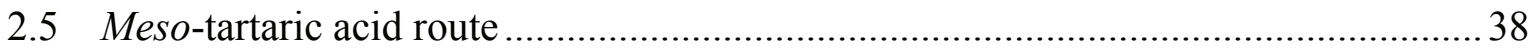

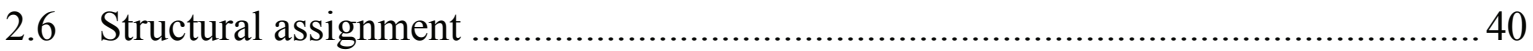

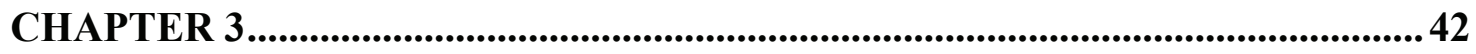

3.1 Retrosynthetic strategy for the synthesis of C1-C14 fragment............................... 42

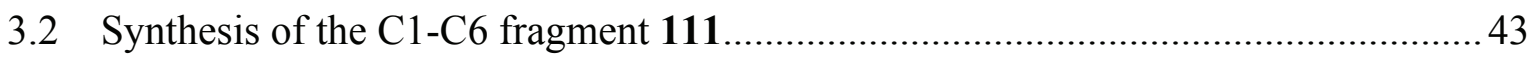

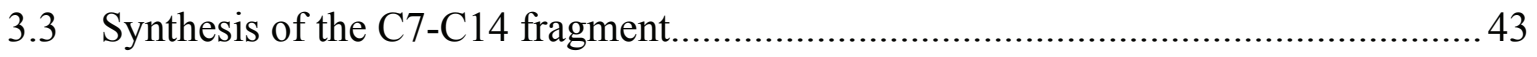

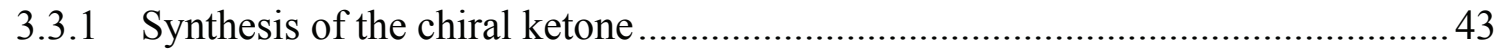

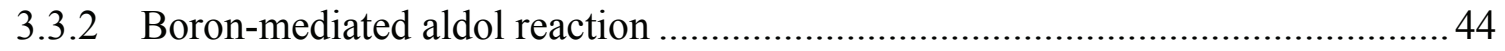

3.3.2.1 Stereocontrol in boron aldol reactions......................................................... 44

3.3.2.2 Boron-mediated aldol reaction using ketone 118....................................... 46

3.3.2.3 Completion of the synthesis of C7-C14 fragment ........................................ 48

3.4 Installation of the C6-C7 junction via cross-metathesis ............................................ 51 


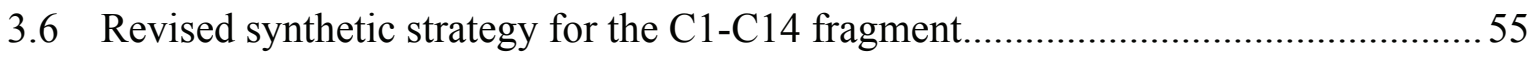

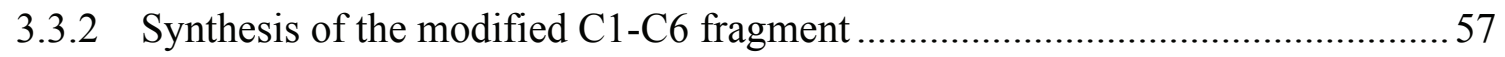

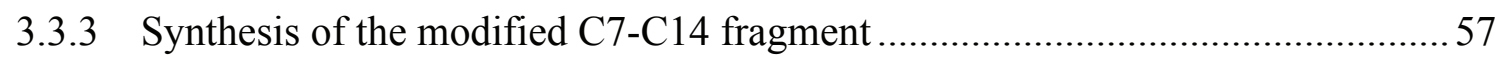

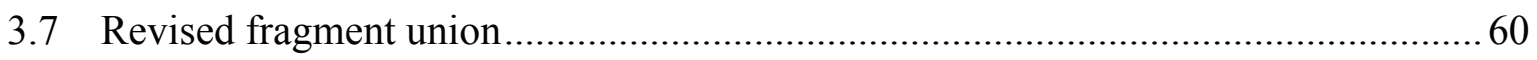

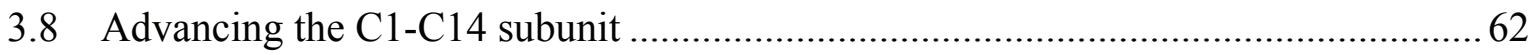

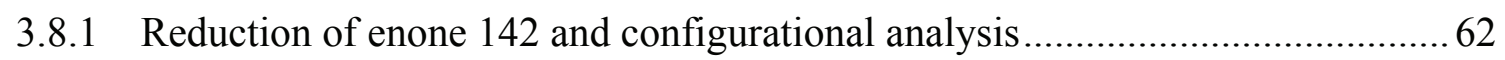

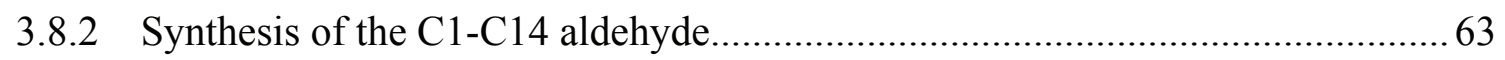

3.9 Summary

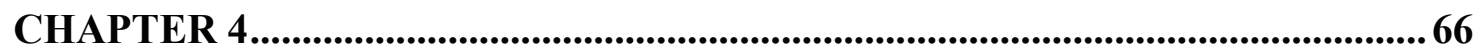

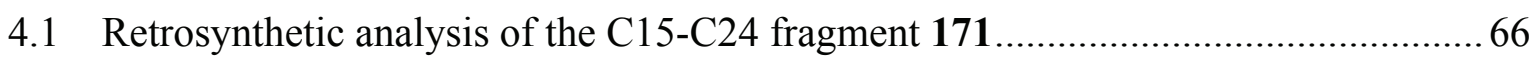

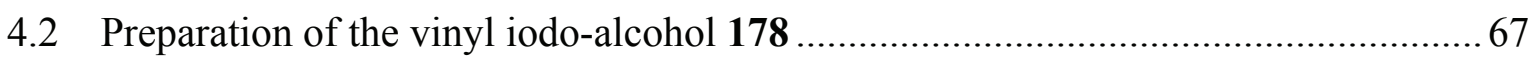

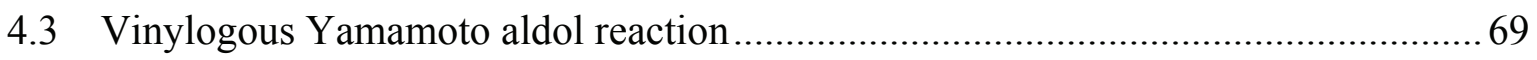

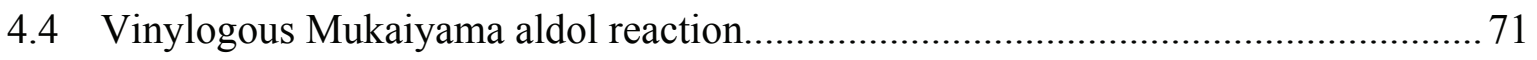

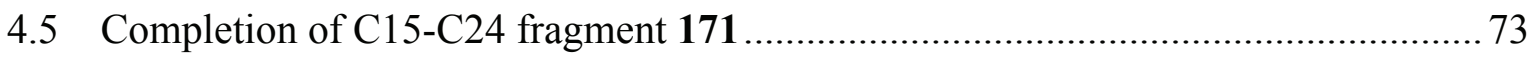

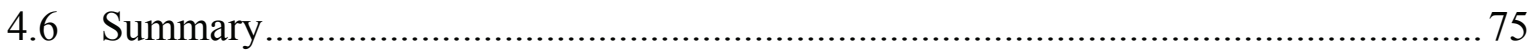

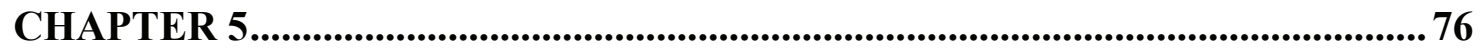

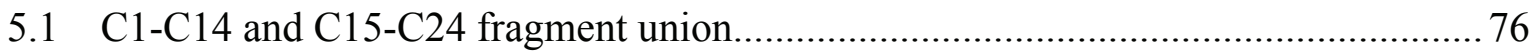

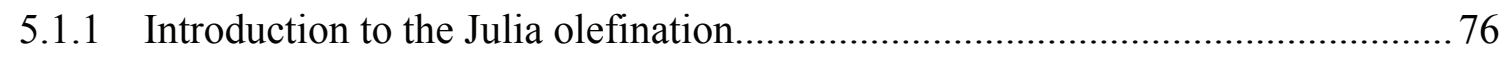

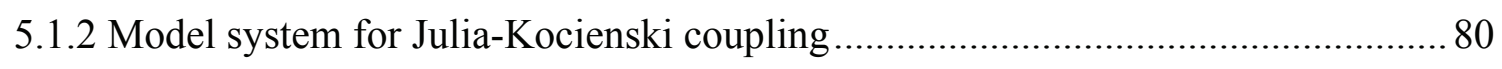

5.1.3 C14-C15 bond formation via Julia-Kocienski coupling ...................................... 81

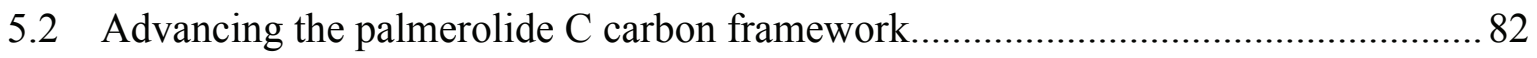

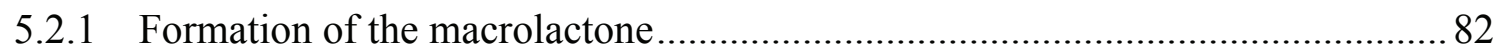

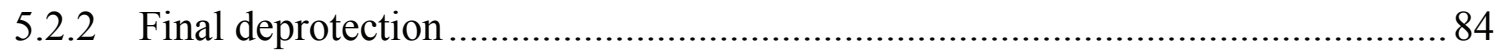


5.3 Introduction of the enamide side chain to complete palmerolide $\mathrm{C}$

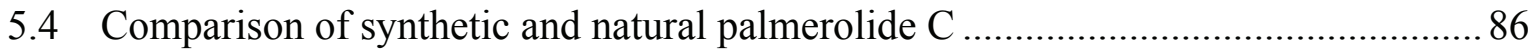

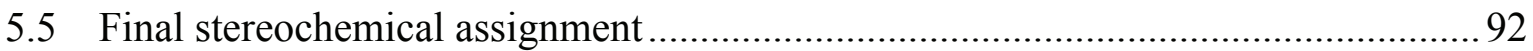

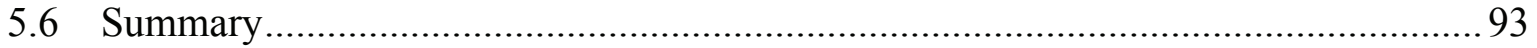

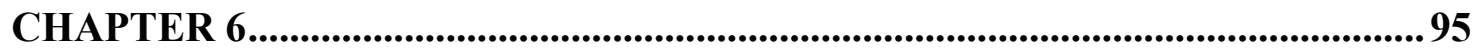

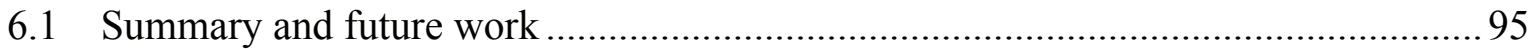

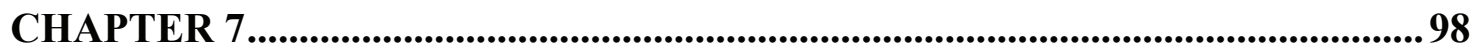

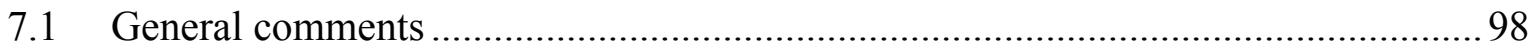

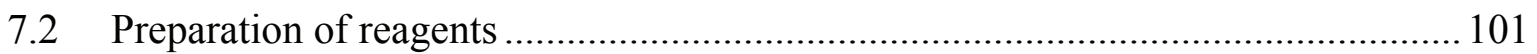

7.3 Experimental procedures for Chapter 2 …....................................................... 104

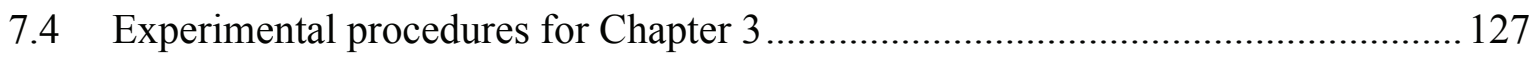

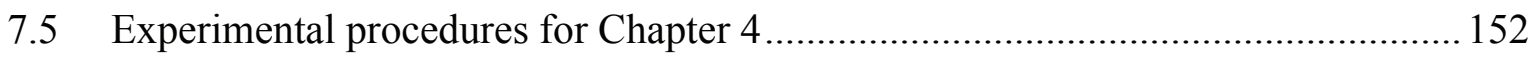

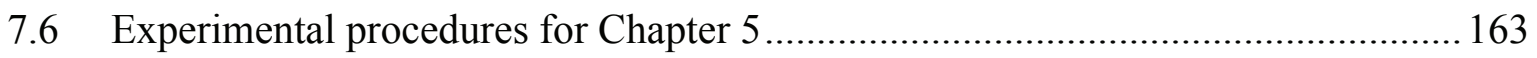

APPENDIX: SELECTED ${ }^{1} \mathrm{H}$ AND ${ }^{13} \mathrm{C}$ NMR SPECTRA ....................................... 172

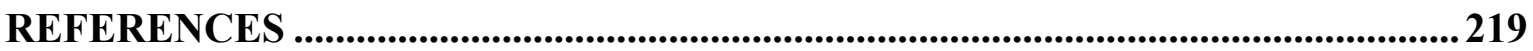




\section{Chapter 1}

\subsection{Discovery of the palmerolides}

The Antarctic polar region is one of the least explored places on our planet due to its inaccessibility. Nevertheless, what is known about its fauna and flora has significantly contributed to our general knowledge of life and uncovered amazing examples of evolutionary adaptation for survival in extreme environmental conditions. ${ }^{1}$ Importantly, this ecosystem has remained undisturbed for millions of years. As a result, the unique phylogenesis of the marine fauna of the Antarctic seas offers distinct structural and functional properties in the secondary metabolites produced by the indigenous species. ${ }^{2}$

In 2006 Baker and co-workers reported the discovery of a family of marine macrolides, which were isolated from the tunicate Synoicum adareanum (Figure 1.1.1) inhabiting the shallow waters in the vicinity of Palmer Station, the only US station on Antarctica north of the Polar Circle. ${ }^{3}$ The isolated compounds were named the palmerolides in recognition of Palmer Station, where they were found. This particular tunicate, found in abundance on the benthos of the Antarctic Sea grows from a common base to multiple fist-sized groups of colonies providing sufficient biomass for collection without harm to the ecosystem. The fact that even a single colony could deliver hundreds of milligrams of palmerolides after processing limited the harvest to a minimum. However, the yields of isolates were variable between colonies, as was the distribution of over a dozen different palmerolides identified. ${ }^{4}$ With the discovery of the palmerolides, Baker has drawn more attention to the potential of Antarctica's remote and unexplored resources. 


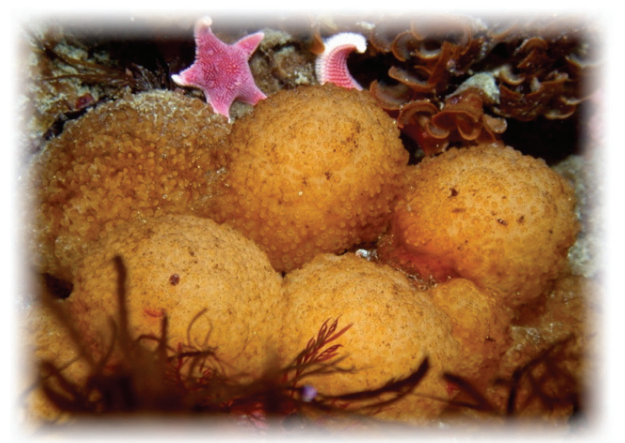

Figure 1.1.1 Synoicum adarenum collected at Palmer Station, Antarctica (Photograph supplied by Bill J. Baker, University of South Florida, USA)

\subsection{Isolation and structure determination}

The collected material was phytochemically processed by first freeze-drying the harvested tunicates to provide $520 \mathrm{~g}$ of dry biomass. The initial extraction with dichloromethane and methanol took three days and resulted in a reddish-brown semi-solid upon evaporation. The crude extracts were then partitioned between ethyl acetate and water. The organic layer was washed, dried and evaporated to provide material for flash column chromatography. The initial isolation gave several separate fractions, which were then purified by reversed-phase HPLC to deliver pure compounds for analysis.

Assessment of the biological activity of the palmerolides began with a 60-cell line panel screen at the National Cancer Institute which revealed unique biological profiles in targeting human cancers. Selectivities towards UACC-62 and M14 melanoma cancer were determined with $\mathrm{IC}_{50}$ of 18 and $76 \mathrm{nM}$ for palmerolide $\mathrm{A}(\mathbf{1})$, rendering the isolated compounds potential candidates for future drug development. In this light the structures of isolated natural products had to be determined.

Investigation of the structural composition of palmerolides by Baker and coworkers culminated in detailed elucidation of the planar structure of twelve family members along with the proposed configurational assignment of palmerolide A (1). The structural characteristics were determined by NMR spectroscopic and HRMS spectrometric experiments. ${ }^{4}$

Structurally, the isolated compounds comprise a 20-membered macrolactone ring bearing a carbamate group at one of the oxygenated positions and four E-configured double bonds including an $(E, E)$-diene motif at $\mathrm{C} 14$ within the macrolide. In addition, a single side chain of varied length and functionality has been identified attached to the C19 
carbon. The palmerolides were then grouped according to the macrolide core structure (Figure 1.2.1). The three different ring systems were distinguished by the structural variation spanning the $\mathrm{C} 6-\mathrm{C} 11$ region of the macrolactone with altering hydroxylation/unsaturation patterns. All the elucidated compounds within these groups bear one of the five different side chains and will be presented in the following sections (1.2.1-1.2.3). It was found that the first compounds isolated, named palmerolide A (1), B (2) and C (3) each represented one of the three unique ring systems (Figure 1.2.1). ${ }^{4}$
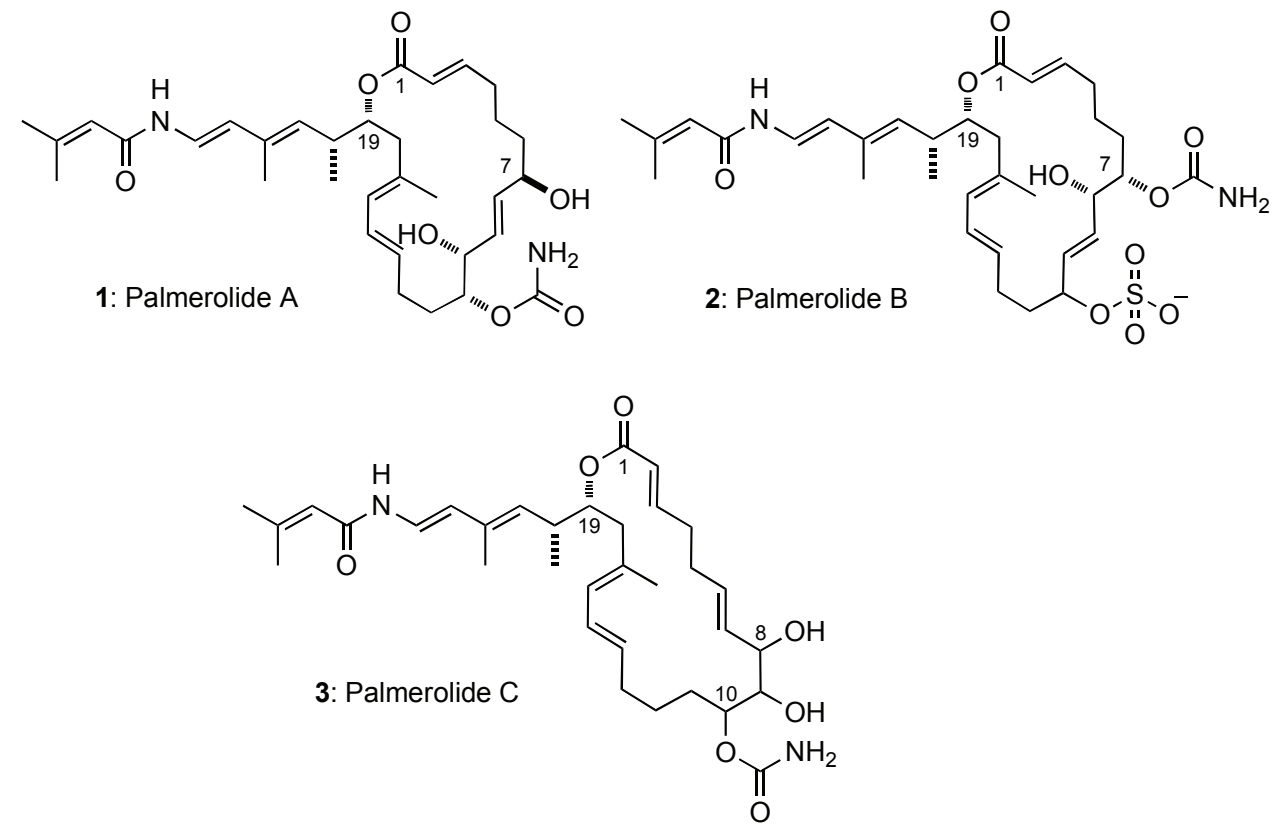

Figure 1.2.1 Three unique ring systems found in the palmerolide family with preliminary stereochemical assignment.

\subsubsection{A - ring system}

Palmerolide A was found to be the primary isolate both in quantity $(200 \mathrm{mg}, 0.02 \%$ dry wt.) and biological activity (melanoma UACC-62, $\mathrm{IC}_{50}=18 \mathrm{nM}$ ). As a result it has received the majority of the attention from the synthetic organic community. ${ }^{5-8}$ The first total synthesis of palmerolide A, achieved by De Brabander within a year from the isolation report, reassigned the proposed relative configuration of $\mathbf{1}$ and established the absolute stereochemistry to be as shown in $\mathbf{4}$ in Table 1.2.1. Elucidation of the planar structure by Baker and co-workers revealed a distinct macrolide with a hydroxyl at $\mathrm{C} 7$ separated by a di-substituted olefin from the dihydroxyl motif at $\mathrm{C} 10$ and $\mathrm{C} 11$. The relative configuration of palmerolide A (1) was established by chemical derivatisation with chiral reagents (MTPA esters of $\mathrm{C} 7, \mathrm{C} 10$ hydroxyl-bearing stereocentres analysed using the 
Mosher method, as discussed further in section 2.2.1) and NMR experiments ( $J$-based configurational analysis of $\mathrm{C} 11$ using the Murata method). ${ }^{3}$ While the Murata analysis ${ }^{9}$ was conducted correctly, it was realised after the first synthesis of 1 by De Brabander that the Mosher ester derivatives were incorrectly assigned. The incorrect interpretation of the C7 and C10 configuration arose as a direct consequence of the applied derivatisation method with $(R)$-MTPA acid chloride, where change in priority of substituents relative to $(R)$-MTPA acid went unnoticed and resulted in the formation of the $(S)$-MTPA ester, not as assigned $(R)$-MTPA ester. ${ }^{3}$

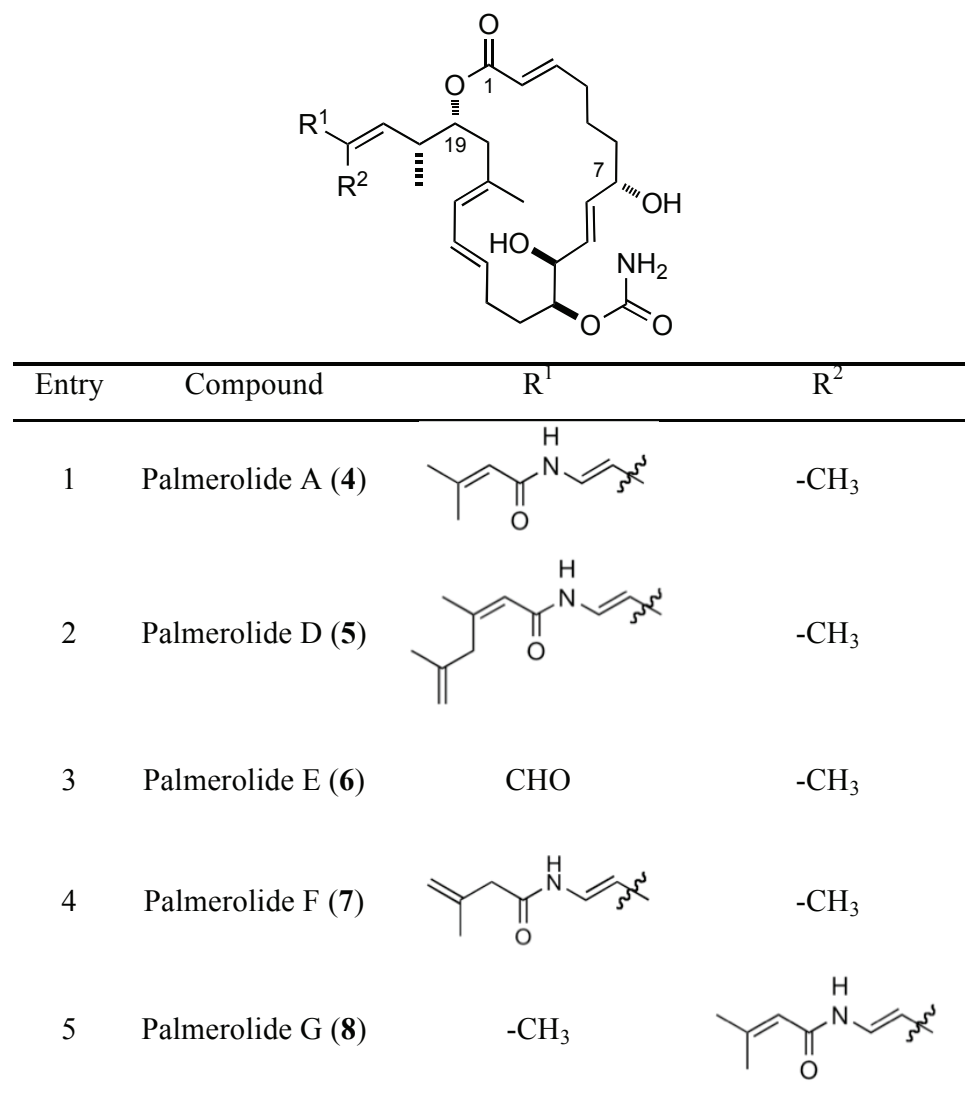

Table 1.2.1 The palmerolides with ring system A with corrected stereochemical assignment.

Structural analogues of palmerolide A (4) have been identified based on the comparison of the collected NMR data. Four palmerolides were found to have the same ring type but varied in the composition of the side chain (Table 1.2.1). The primary isolate (4) comprised of conjugated enamide. Further palmerolides of this ring type presented isomerisation, extension or disruption in conjugation in their side chains (Table 1.2.1). ${ }^{10}$ Palmerolide D (5) was found to contain an elongated side chain with one (Z)- and one terminal double bond, in contrast palmerolide E (6) bears a side chain truncated by one carbon at C-23 terminating in an aldehyde. Shift from conjugation in the enamide to a 
terminal double bond was identified in palmerolide $\mathrm{F}(7)$ and the isomeric $(Z)$-geometry of the $\mathrm{C} 21 / \mathrm{C} 22$ olefin distinguished palmerolide $\mathrm{G}(\mathbf{8})$.

\subsubsection{B - ring system}

A distinctive pattern of hydroxyls at $\mathrm{C} 7$ and $\mathrm{C} 8$ separated by an olefin from the C11 hydroxyl constitute the B-ring macrolide system. The presence of a sulfate group, not found in the other palmerolides substantially affects the properties of the compounds with this core structure, making them very polar and prone to hydrolysis (Table 1.2.2). Determination of the planar structure of palmerolides B (2) and H (9) by NMR spectroscopy proved difficult due to their instability in dimethylsulfoxide. Although, the first attempt to obtain spectroscopic data for $\mathbf{2}$ and $\mathbf{9}$ ended with decomposition of the isolates, further NMR experiments carried out in methanol allowed their structure elucidation. It was found that the side chains of palmerolides B (2) and $\mathrm{H}(9)$ correspond to the previously described structural motifs found in palmerolide A (4) and D (5).

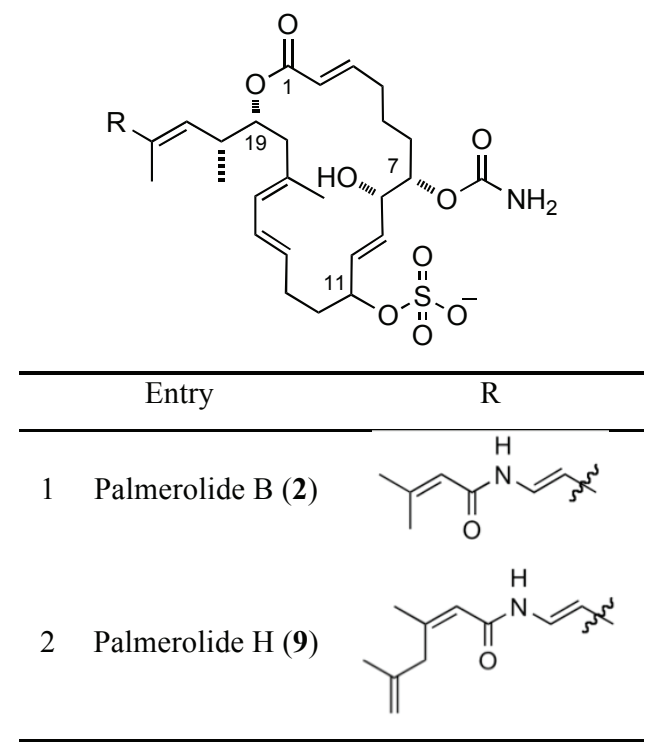

Table 1.2.2 The palmerolides with ring system B with preliminary stereochemical assignments.

Determination of the relative stereochemistry of the C7-C11 stereocentres proved challenging. Chemical derivatisation resulted in the elimination of the sulfate group and hydrolysis of the carbamate. Nevertheless, the C8-Mosher esters were formed and the absolute configuration of the C8 hydroxyl was established. Advanced spectroscopic experiments (gHSQMBC) provided information on the homo- and hetero-nuclear $J$ coupling constants and allowed the determination of the syn arrangement of the C7-C8 
stereocentres. Unfortunately, based on the available data, the configuration of the isolated C11 stereocentre could not be deduced. ${ }^{10}$

\subsubsection{C - ring system}

The final two palmerolides representing the C-ring system, were identified as regioisomers of palmerolide A (4) and E (6). A motif of three contiguous stereocentres at C8-C10 distinguishes palmerolide C (3) and K (10) (Table 1.2.3) from the other family members. Spectroscopic parameters for the C19-C20 positions were found to closely match data reported for the analogous centres in palmerolide A (4). Consistent with a common biogenesis and supported by NMR comparison, the C19-C20 stereogenic centres were determined to have the same configuration within the entire family. ${ }^{11}$ The side chain identified in palmerolide $\mathrm{C}$ (3) corresponds to the $(E, E)$-1,3-dienamide found in palmerolide A (4). Palmerolide K (10) is truncated at C23 with an aldehyde similar to palmerolide E (6). The assignment of the relative and absolute stereochemistry of the remaining centres was not available until recently. ${ }^{4}$ The stereochemical assignment of the C8-C10 stereotriad became one of our major objectives and will be discussed further in Chapter 2.

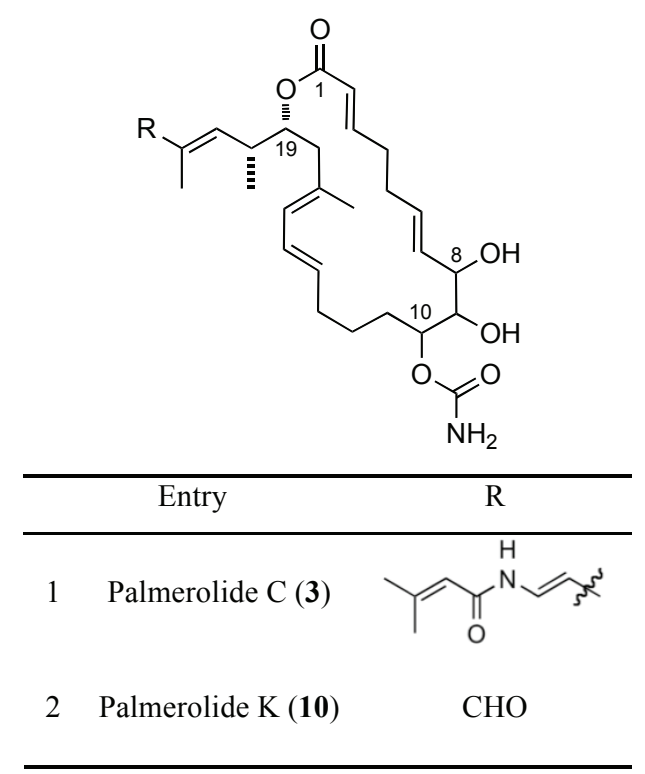

Table 1.2.3 The palmerolides with ring system $\mathrm{C}$.

Characteristic structural features of palmerolides, namely carbamate and enamide side chain suggest involvement of, as yet unknown, bacterial organism in production of 
these natural products in host tunicates. It has been hypothesised that a biosynthesis occurs by hybrid polyketide synthase (PKS)/nonribosomal peptide synthetase (NRPS) pathway. ${ }^{12}$ The macrocyclic part of the molecule could be composed by modular Type I polyketide synthase operative e.g. in the biosynthesis of epothilones (Figure 1.2.2). ${ }^{13}$ Formation of the side chain present in palmerolides may be initiated by NRPS-catalysed incorporation of an amino acid such as glycine (Gly) ${ }^{14}$ and/or valinylglycine (Val-Gly). ${ }^{15}$ Amide portion of the side chain could originate from acetate via an HMG-CoA (3-hydroxy-3-methylglutarylCoA) synthase-type methyltransferase. ${ }^{16,17}$

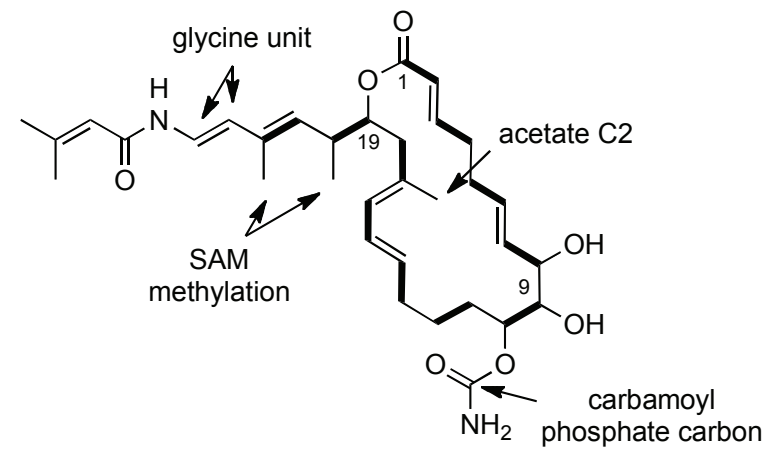

Figure 1.2.2 Hypothesised biosynthetic origin of palmerolide C.

\subsection{Biological activity and SAR studies}

Baker reported that the toxicity profile of the palmerolides correlates with those of several known vacuolar-ATPase inhibitors (salicylhalamide A (11), ${ }^{18,19}$ bafilomycin A (12), ${ }^{20}$ Figure 1.3.1). ${ }^{10}$ Therefore, it was suggested that palmerolides mode of action might be related to an already known inhibition mechanism and undertaking further biological studies would provide relevant information supporting this hypothesis.

Vacuolar-ATPases (v-ATPases) operate as $\mathrm{pH}$ modulators and are widely expressed in eukaryotic cells, as well as metastatic cancer cells. The regulation of the interand intra-organellar acidity within various mammalian cell types is required for a variety of physiological functions including membrane and organellar protein sorting, neurotransmitter uptake, cellular degradative processes, and receptor recycling. ${ }^{18}$ Being responsible for vast molecular regulatory functions, this proton pump system is an interesting target for pharmaceutical research and development. New biological agents, such as the palmerolides, aiming at the control or inhibition of v-ATPase enzymes are of great interest and might serve as potential cancer therapeutics. 


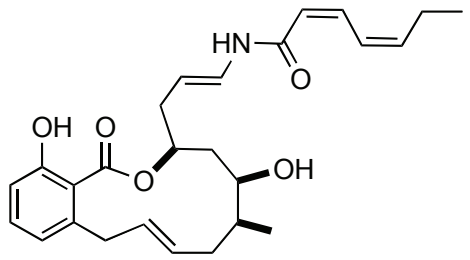

11: (-)-Salicylhalamide A

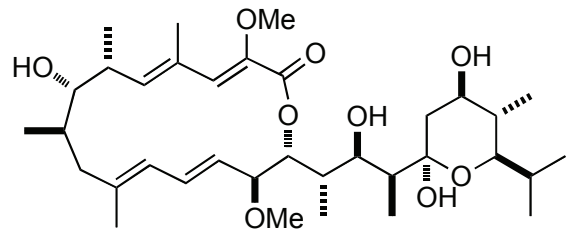

12: Bafilomycin A

Figure 1.3.1 Inhibitors of the v-ATPase enzyme.

Studies undertaken by Baker on the molecular mode of action revealed that palmerolide A (4) affects genes regulating the cholesterol and fatty acid biosynthetic pathway in malignant cells. As this was consistent with published changes in gene expression for bafilomycin A (12), they measured the transcriptional consequences of bafilomycin treatment on the melanoma cell lines (UACC-62 and LOX), and found correlations between the 900 genes dysregulated by palmerolide A (4) and bafilomycin A (12). Bafilomycin has been reported to inhibit cholesteryl ester synthesis through sequestration of free cholesterol in the endosomal/lysosomal compartment as a consequence of v-ATPase inhibition. The high degree of coherence between their gene profiles supports the premise that palmerolide A is a v-ATPase inhibitor, and consequently has an effect on cholesterol sequestration. ${ }^{10}$

On the other hand, De Brabander performed studies on the in vitro inhibition mechanism of v-ATPase by salicylhalamide A (11), ${ }^{19}$ which contains a conjugated enamide, a structural motif shared with palmerolides. This research established several important features regarding interactions of $\mathbf{1 1}$ with v-ATPase enzymes. Salicylchalamide A follows a distinctive mechanism of action from bafilomycin $\mathrm{A}^{20}$ in that it does not compete with it for binding to v-ATPase. In addition, the recognition and binding site for salicylhalamide $A$ was identified to be within the membrane embedded $V_{0}$ domain of $\mathrm{v}$ ATPase enzyme. Interestingly, without the enamide side chain salicylhalamide A loses activity. Further biological assays provided evidence that $\mathbf{1 1}$ does not inhibit the ATP hydrolysis activity of the dissociated $\mathrm{V}_{1}$ domain of the v-ATPase but instead affects the ATPase activity of the holoenzyme by inhibiting the $\mathrm{V}_{0}$ domain. Salicylihalamide A causes a dramatic redistribution of cytosolic $\mathrm{V}_{1}$ from a soluble to a membrane-associated form, a change not observed in cells treated with either bafilomycin A (12) or $\mathrm{NH}_{4} \mathrm{Cl}$. 
Further studies are underway to fully establish the mode of action displayed by the palmerolides, as investigations to date provided some insight but do not answer all the questions in this aspect. In addition, the structure-activity relationship of the palmerolides clearly indicates the importance of the carboxamide side chain for biological activity (Table 1.3.1). Palmerolide A (4) displayed remarkable activity in both targeting v-ATPase enzyme and three orders of magnitude greater selectivity towards human melanoma cancer UACC-62 than other cancer cell lines. In contrast, palmerolide E (6), with a terminal aldehyde at $\mathrm{C} 23$, showed a significant loss in specific enzyme activity but relative cytotoxity towards melanoma cells was retained at reduced levels (Table 1.3.1). Following the structural analysis, palmerolide D (5) with an extended side chain and ring system A proved to be more cytotoxic in comparison to (4), although slightly less selective towards v-ATPase. Terminal double bond shifted from conjugation in palmerolide F (7), consequently reduces the cytotoxicity and diminished enzyme activity. Palmerolide B (2) displayed potent enzyme selectivity, but cytotoxicity was compromised. Palmerolide C (3) showed reduced selectivity and cytotoxicity, however remaining in the nanomolar range (Table 1.3.1). Overall, structure-activity investigation might be inconclusive as even within the same ring type selectivity and cytotoxicity significantly fluctuates. ${ }^{4,21}$

\begin{tabular}{ccc}
\hline Compound & V-ATPase $(\mu \mathrm{M})$ & Cytotoxicity $\mathrm{IC}_{50}(\mu \mathrm{M})$ \\
\cline { 2 - 3 } & Mammalian & UACC-62 melanoma \\
\hline Palmerolide A (4) & 0.002 & 0.024 \\
Palmerolide B (2) & 0.023 & 0.250 \\
Palmerolide C (3) & 0.150 & 0.062 \\
Palmerolide D (5) & 0.025 & 0.002 \\
Palmerolide E (6) & $>10.000$ & 5.000 \\
Palmerolide F (7) & 0.063 & 0.758 \\
\hline
\end{tabular}

Table 1.3.1 Bioactivity data for palmerolides.

Since its initial disclosure palmerolide A (4) was identified as a very potent melanoma cancer inhibitor, it received considerable synthetic attention with several total syntheses and formal approaches published to date ${ }^{5-8}$ and selected syntheses will be presented in the following section (1.4). Palmerolide C (3) and palmerolide B (2), on the other hand, have not been investigated synthetically as their relative stereochemistry remained unknown and selective cytotoxicity was moderately reduced compared to palmerolide A. 


\subsection{Synthetic studies on palmerolide A}

In the six years since the discovery of the palmerolides, a number of approaches to the synthesis of palmerolide A (4), have been reported. ${ }^{5-8}$ The first synthesis of 1 with the incorrect structure was accomplished by De Brabander's group. ${ }^{5}$ They then revised the stereochemical assignment proposed by Baker in the isolation paper to $\mathbf{4}$ and validated this by total synthesis. Shortly after the first synthesis, Nicolaou and Chen reported their approach towards the natural palmerolide A 4 and its analogues. ${ }^{6}$ Hall accomplished the next total synthesis of $4{ }^{8}$ Following these investigations several formal synthetic efforts have been published. ${ }^{22-29}$ The successful approaches are presented herein.

\subsubsection{The first synthesis of palmerolide A by De Brabander}

In 2007 De Brabander and co-workers synthesised the proposed structure of palmerolide A (1). Their strategy relied on a Suzuki coupling ${ }^{30}$ of vinyl iodide $\mathbf{1 3}$ and vinylboronate 14 to construct the advanced diene 15 (Scheme 1.4.1). Yamaguchi esterification with fragment $\mathbf{1 6}^{31}$ and subsequent silyl deprotection gave diol 17. Selective oxidation $^{32}$ of the primary alcohol, followed by an intramolecular Horner-WadsworthEmmons (HWE) macrocyclisation ${ }^{33}$ and subsequent silyl protection gave macrolactone 18. Corey-Bakshi-Shibata reduction of the enone 18 introduced the $\mathrm{C} 7$ stereocentre, ${ }^{34}$ which was subsequently protected as the TBS ether. Ester hydrolysis ${ }^{35}$ and formation of azide 19 set the stage to introduce the enamide side chain. Curtius rearrangement of $\mathbf{1 9}$ and trapping of the intermediate isocyanate with 2-methyl propenylmagnesium bromide completed the enamide side chain. Selective TMS deprotection at C11 allowed for the introduction of the carbamate. Global deprotection furnished the target molecule 1. Unfortunately, the NMR data was inconsistent with the reported ${ }^{1} \mathrm{H}$ and ${ }^{13} \mathrm{C}$ spectra of palmerolide $\mathrm{A}$, indicating that the initial stereochemical assignment was incorrect. After thorough investigation, De Brabander chose to invert the configuration of the C19-C20 centres of the initially synthesised isomer $\mathbf{1 3}$ and subject it to the developed synthetic strategy. Gratifingly, the NMR spectra and remaining analyses matched the data reported for palmerolide A. However, the CD spectra of the synthetic and natural compounds were mirror images. Ultimately, the synthesised structure proved to be the ent-palmerolide A (4) and allowed the definition of the absolute stereochemistry of the natural product. The total synthesis of 
4 was completed in thirty-three steps, with a longest linear sequence of 23 steps and $2.9 \%$ overall yield.

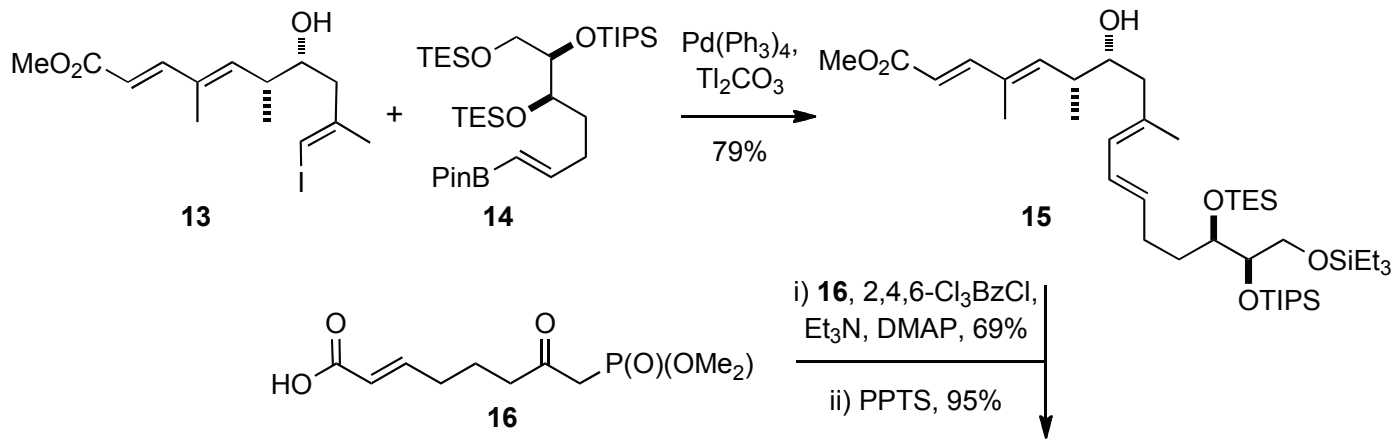

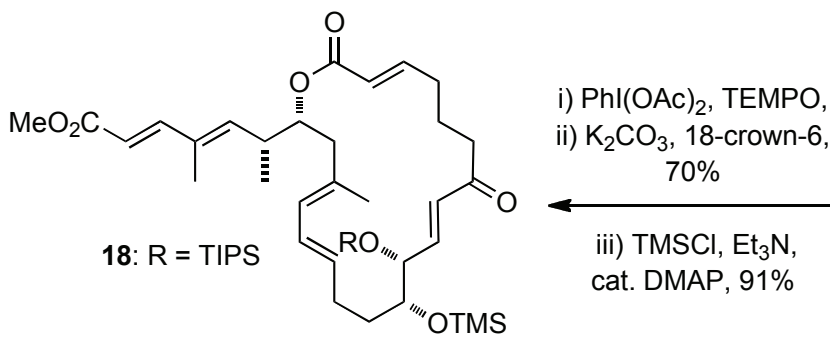

i) (S)-CBS, $\mathrm{BH}_{3}, 99 \%$

ii) TBSOTf, 2,6-lut, 94\%

iii) $\left(\mathrm{Bu}_{3} \mathrm{Sn}\right)_{2} \mathrm{O}, 81 \%$

iv) $(\mathrm{PhO})_{2} \mathrm{P}(\mathrm{O}) \mathrm{N}_{3}, \mathrm{NEt}_{3}, 92 \%$<smiles>COO[PH2]CC(=O)CCC/C=C/C(=O)O[C@H](C/C(C)=C/C=C\CCC(O)C([O-])CO)[C@H](C)/C=C(C)/C=C/C(C)=O</smiles>

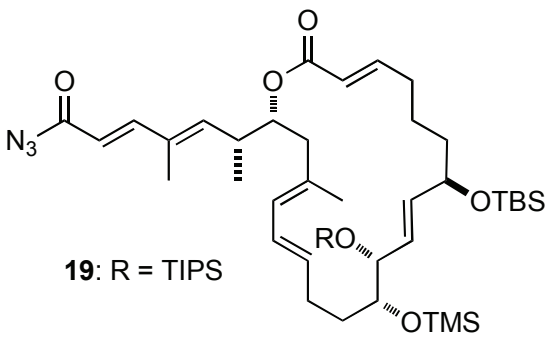

i) $\mathrm{C}_{6} \mathrm{H}_{6}$, reflux

ii) $\mathrm{MgBr}$

iii) HF•py, py, 95\%

iv) $\mathrm{Cl}_{3} \mathrm{CC}(\mathrm{O}) \mathrm{NCO}, 91 \%$

v) TBAF, $41 \%$.

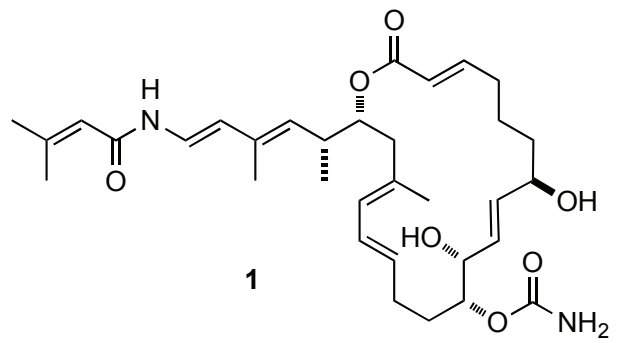

Scheme 1.4.1 Synthesis of the initially proposed structure of palmerolide A by De Brabander.

\subsubsection{Nicolaou and Chen synthesis of palmerolide A}

Shortly after De Brabander published the synthesis of ent-palmerolide A, Nicolaou and Chen presented their approach towards palmerolide A (4). ${ }^{6}$ Their strategy for the assembly of the key subunits began with Stille coupling between vinyl iodide $\mathbf{2 0}$ and vinyl stannane 21 providing tetraene 22 (Scheme 1.4.2). ${ }^{36}$ Carboxylic acid 23 was esterified with alcohol 22 under Yamaguchi conditions to give ester 24. ${ }^{31}$ Subsequent desilylation and oxidation set the stage for Takai olefination to introduce the vinyl iodide. ${ }^{37}$ Removal of the two MOM groups from 25 gave the intermediate diol, which underwent ring-closing metathesis to form a macrocycle 26. The installation of the enamide moiety was achieved through the application of a Buchwald $\mathrm{Cu}(\mathrm{I})$-mediated coupling, ${ }^{38}$ completing the synthesis 
of 4. The synthesis of the naturally occurring palmerolide A 4 was achieved in 28 steps, with a longest linear sequence of 15 steps and $1.7 \%$ overall yield. Thereafter, Chen published the complete synthetic efforts towards the palmerolide $\mathrm{A},{ }^{7}$ followed by the synthesis of analogues, which were subsequently subjected to further SAR studies. ${ }^{39,40}$

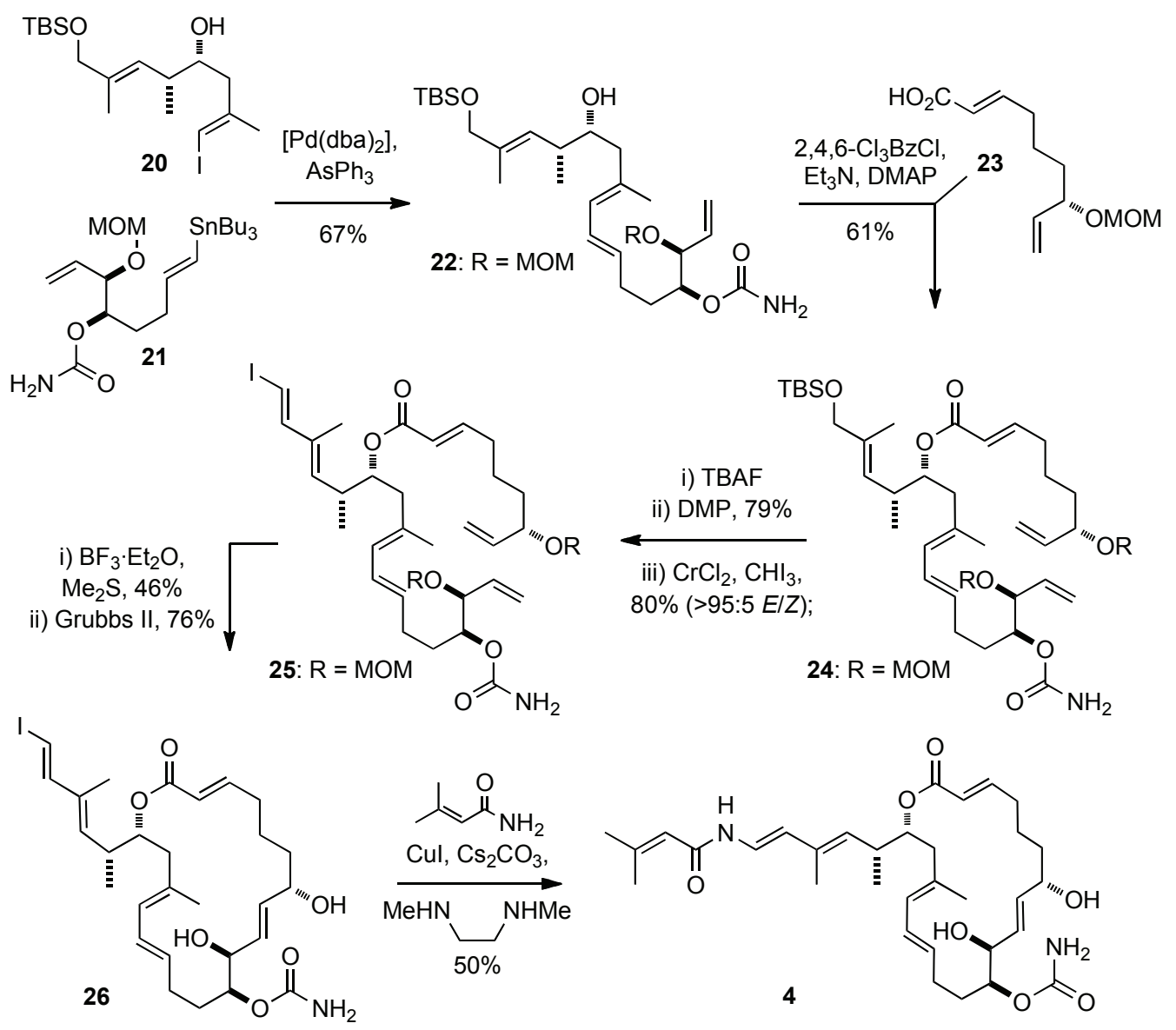

Scheme 1.4.2 Synthesis of natural palmerolide A by Nicolaou and Chen.

\subsubsection{Hall synthesis of palmerolide A}

The Hall approach utilised his organoboron methodology to construct the advanced intermediates (Scheme 1.4.3). At first, fragment 27 was formed using a [4+2] hetero-DielsAlder reaction of $\mathbf{2 8}$ and $\mathbf{2 9},{ }^{41}$ followed by allylboration and subsequent [3,3] IrelandClaisen rearrangement of the alkenylboronate $\mathbf{3 0}$ to form $31 .{ }^{42}$ The left side of the molecule, C14-C25 subunit 32 was accessed by applying enantioselective crotylboration methodology where a tin tetrachloride-based catalyst, developed within the Hall group, was used to install the $\mathrm{C} 19 / \mathrm{C} 20$ stereocentres. ${ }^{43}$ Coupling of the fragments $\mathbf{3 2}$ and $\mathbf{3 3}$ was accomplished via a boron-alkyl Suzuki reaction providing access to the advance intermediate 34. Hydrolysis of the methyl ester with a trimethyltin hydroxide and 
subsequent macrolactonisation under Yamaguchi conditions delivered macrolactone 35 . Introduction of the enamide side chain providing 36 was accomplished following De Brabander's approach via Curtius rearrangement of the azide, which was installed after tert-butyl ester hydrolysis. Cleavage of the PMB group and carbamate formation at C11 preceded final deprotection with TBAF, which completed the natural palmerolide A (4).
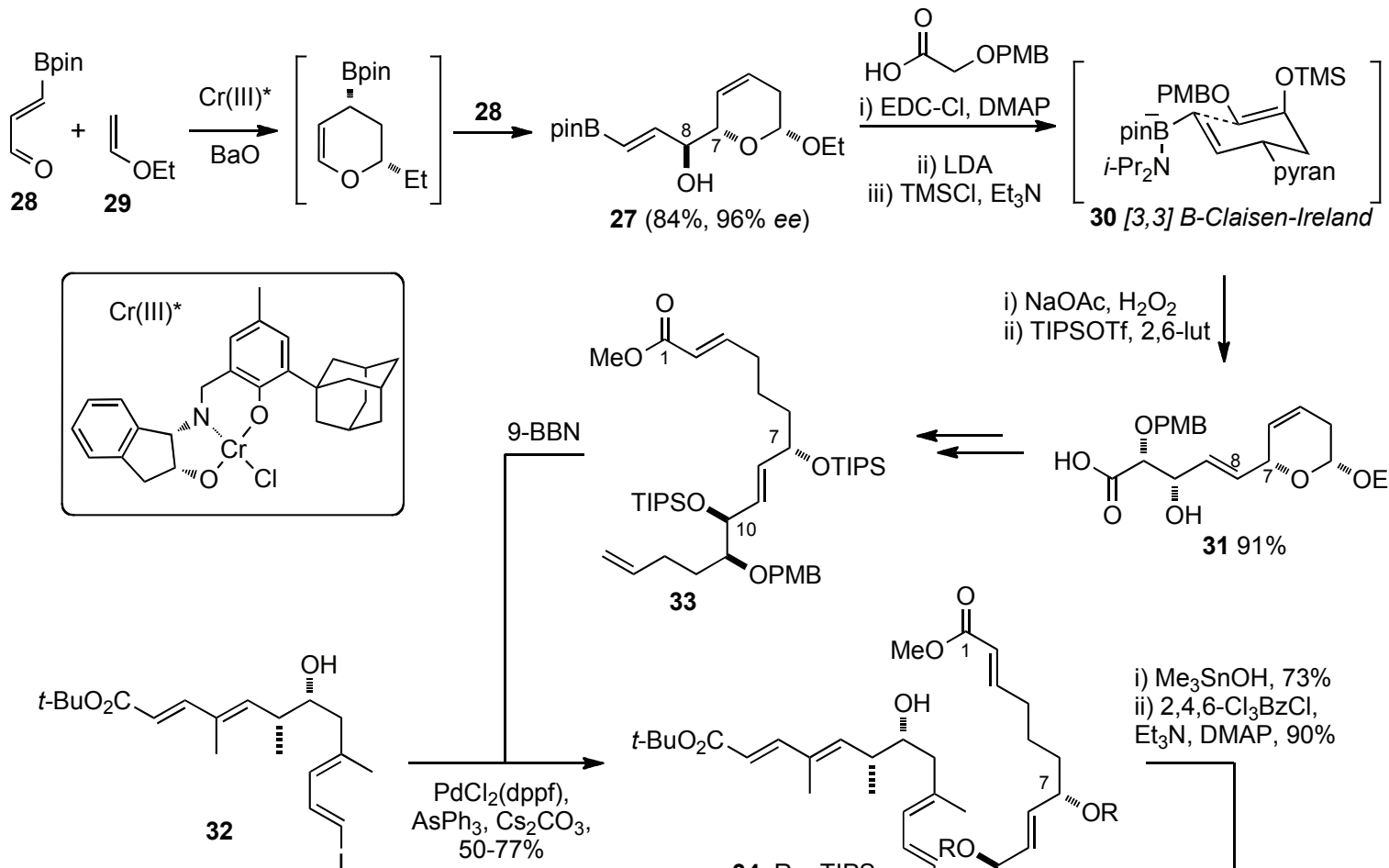

i) $\mathrm{NaOAc}, \mathrm{H}_{2} \mathrm{O}_{2}$
ii) TIPSOTf, 2,6 -lut
$\downarrow$

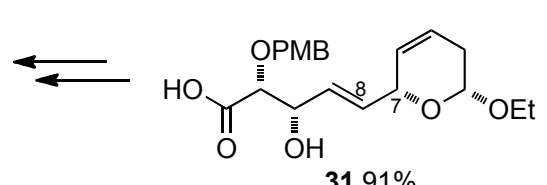<smiles>CC(=C/[C@@H](O)[C@@H](C)C/C(C)=C/C=C\I)/C=C/C(=O)OC(C)(C)C</smiles>

33

$3191 \%$

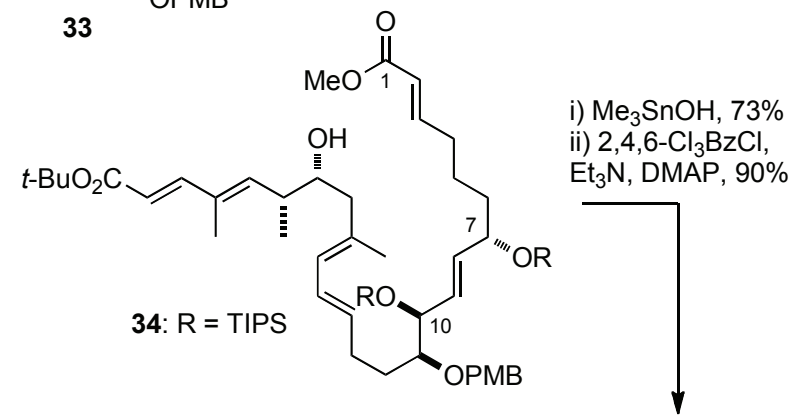

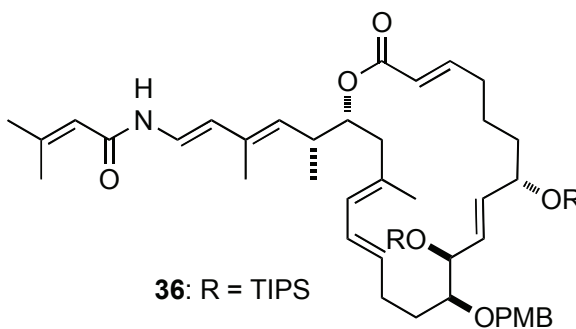

i) TMSOTf, $\mathrm{Et}_{3} \mathrm{~N}, 95 \%$ ii) $\mathrm{N}_{3} \mathrm{P}(\mathrm{O})(\mathrm{OPh})_{2}$, $\mathrm{Et}_{3} \mathrm{~N}, 75 \%$

iii) $\mathrm{C}_{6} \mathrm{H}_{6}$, reflux<smiles>CC(C)=CC(=O)OCl</smiles>

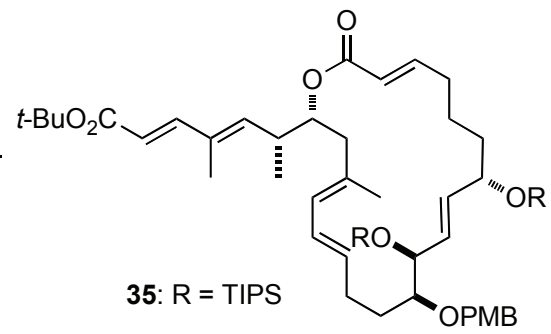

i) $\mathrm{Et}_{2} \mathrm{O}-\mathrm{MgBr}_{2}, \mathrm{Me}_{2} \mathrm{~S}$

ii) $\mathrm{Cl}_{3} \mathrm{CC}(\mathrm{O}) \mathrm{NCO}, \mathrm{Al}_{2} \mathrm{O}_{3}$

iii) TBAF,

4: Palmerolide A

$15-20 \%$ over 3 steps

Scheme 1.4.3 Synthesis of natural palmerolide A by Hall. 


\subsection{Methods for the determination of the absolute stereochemistry in complex molecules}

\subsubsection{Degradation studies to confirm the absolute stereochemistry of palmerolide A}

Following the stereochemical re-assignment of palmerolide A by the first synthesis, Baker and co-workers re-investigated their data for the initially proposed structure 1. In order to clarify all uncertainty, they employed another powerful method used in determination of the absolute stereochemistry of complex molecules. Degradation studies were undertaken to access fragments of the natural palmerolide A with questionable stereochemistry. This approach was found to be a viable method for solving the stereochemical errors in $1 .{ }^{44}$ Their strategy used reductive ozonolysis to access the C3-C8 fragment 37 of palmerolide A, with the ambiguous C7 stereocentre (Scheme 1.5.1). The obtained data for the 1,2,6-hexanetriol 37 was then compared to the literature records for $(R)-1,2,6$-hexanetriol ${ }^{45}$ revealing an inconsistency in the optical rotation values, both in magnitude and sign.

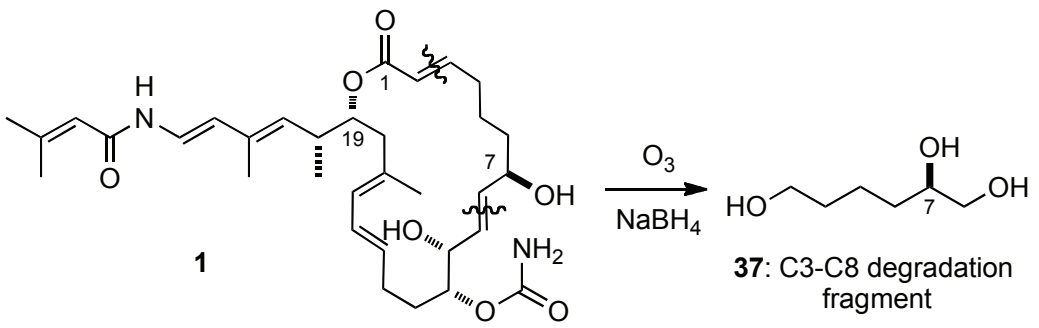

Scheme 1.5.1 Degradation studies of the palmerolide A.

Therefore, they decided to prepare synthetic $(R)$-1,2,6-hexanetriol 37 starting from the $(R)$-acetonide of 1,2,4-trihydroxybutane 38. Following a sequence of Wittig reaction, reduction of enoate 39 and subsequent deprotection gave $(R)$-1,2,6-hexanetriol (Scheme 1.5.2), which identity was confirmed by spectroscopic and spectrometric analysis. However, comparison of the optical rotation in methanol for synthetic $(R)-1,2,6$ hexanetriol $(+11.1)$ with the optical rotation of the natural C3-C8 degradation fragment 37 (-9.0) revealed they were opposite in sign, providing evidence for the $\mathrm{C} 7$ centre being $(S)$ configured. 


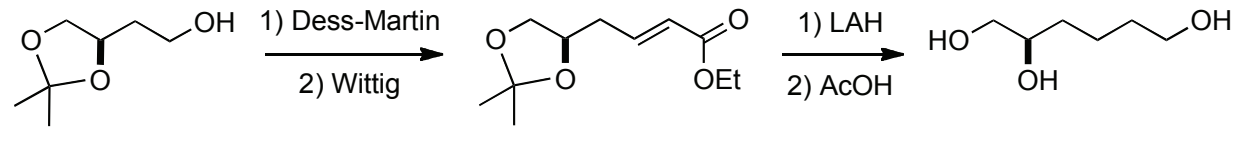

38

39

37

Scheme 1.5.2 Synthesis of the $(R)$-degradation fragment 37 .

To provide a final proof, the synthesis of the ent-degradation fragment $\mathbf{3 7}$ starting from the $(S)$-acetonide of 1,2,4-trihydroxybutane was carried out. In this case, the optical rotation of the $(S)$-triol (-11.6) matched the optical rotation of the degradation product of the natural sample (-9.0) confirming the absolute stereochemistry of the $\mathrm{C} 7$ centre of the palmerolide A bearing $(S)$-configuration.

\subsubsection{Degradation studies in determining the absolute stereochemistry of complex molecules}

The synthesis of degradation fragments of complex molecules is a well-established technique enabling the elucidation of relative and absolute stereochemistry. It is often applied when spectroscopic experiments fail to provide unambiguous information, crystallographic data is not accessible and chemical derivatisation is difficult. However, the presence of functionalities susceptible to controlled fragmentation is essential for making this approach viable.

This technique is well documented in the literature and provides a practical handle for addressing stereochemical issues prior to embarking on the total synthesis. ${ }^{46}$ Rychnovsky employed this approach to determine the relative and absolute stereochemistry of roflamycoin 40 (Scheme 1.5.3), a polyketide macrolide antibiotic consisting of repeating 1,3-diol motifs with 2048 possible stereoisomers. ${ }^{46}$ This remarkable work comprises most of the techniques available for a synthetic chemist to establish the stereo-structure of a complex molecule. The relative stereochemistry of $\mathbf{4 0}$ was determined based on the combination of the chemical derivatisation studies and spectroscopic techniques (Scheme 1.5.3). The arrangement of C13/C15 and C19/C21 was found to be anti upon the inspection of the ${ }^{13} \mathrm{C}$ NMR data obtained for the derivative 41. Based on these findings, next derivative $\mathbf{4 2}$ was studied and provided evidence by ${ }^{1} \mathrm{H}$ NMR and nOe experiments of the syn relation of $\mathrm{C} 15 / \mathrm{C} 19$ stereocentres. The ${ }^{13} \mathrm{C}$-enriched compound $\mathbf{4 3}$ showed ${ }^{13} \mathrm{C}$ NMR signals consistent with the syn relative configuration of the $\mathrm{C} 23 / \mathrm{C} 25$ and C29/C31 stereocentres in the acetonides. 


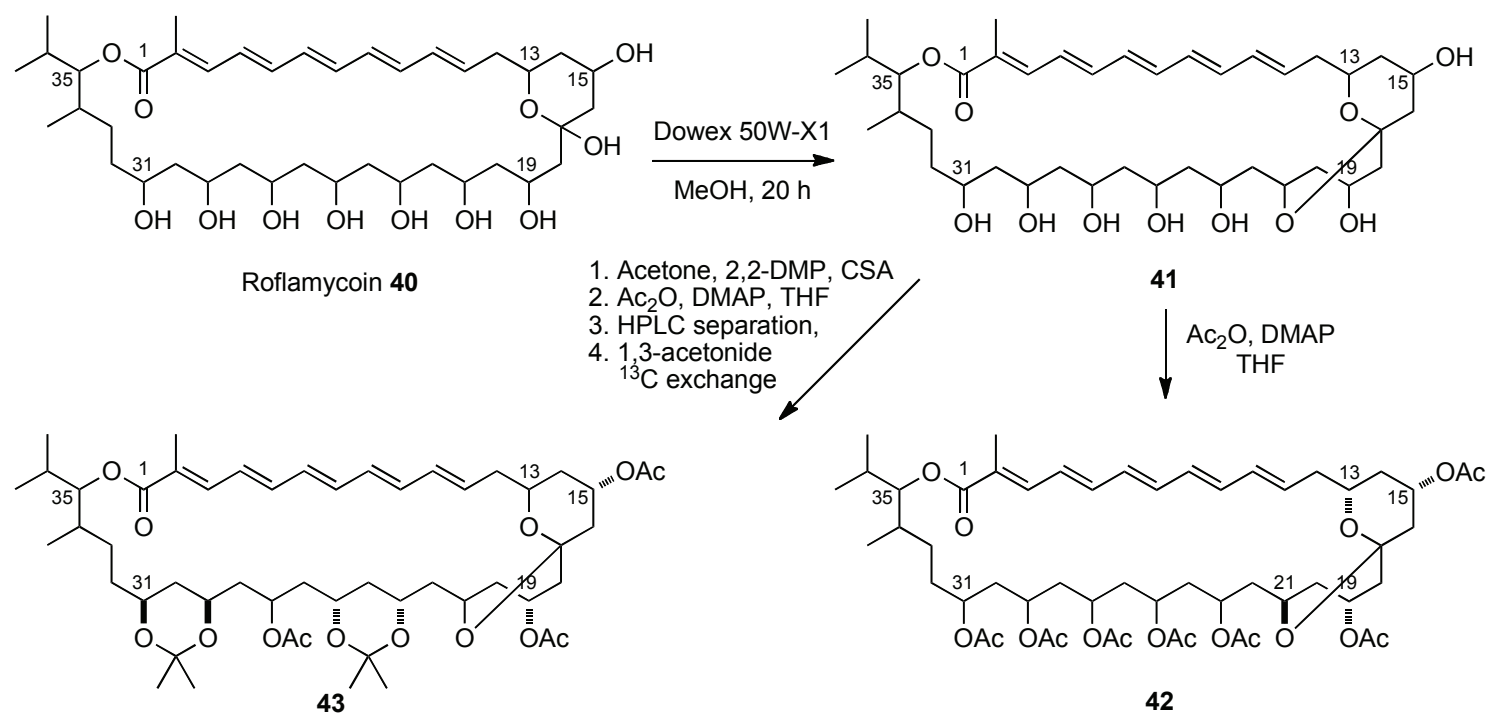

Scheme 1.5.3 Assignment of the relative configuration of the fungal metabolite roflamycoin 40 by chemical derivatisation and ${ }^{13} \mathrm{C}$ NMR spectroscopic analysis.

This observation was supported by the earlier studies within the Rychnovsky group, ${ }^{47,48}$ where ${ }^{13} \mathrm{C}$-NMR spectroscopic data for a wide range of 1,3-diol acetonides were investigated (Figure 1.5.1). Due to the conformational preferences of syn and anti diol acetonides, their methyl and acetal carbons appear at particular chemical shifts in their respective ${ }^{13} \mathrm{C}$ NMR spectra. Their analysis revealed, that 1,3-syn acetonides prefer to adopt chair like conformation, resulting in a specific ${ }^{13} \mathrm{C}$ resonances at 19 and $30 \mathrm{ppm}$ for methyl groups and $98 \mathrm{ppm}$ for acetonide. In comparison, the 1,3-anti acetonides were found to favour a twist-boat conformation with ${ }^{13} \mathrm{C}$ resonances at 23, 25 and $100 \mathrm{ppm}$ for respective carbons.

(H) $\mathrm{Me} \underbrace{\mathrm{R}^{1} \overbrace{0}^{\mathrm{O}}}_{\mathrm{R}^{2}}$ (Me) $\mathrm{H}$ 1,3-syn

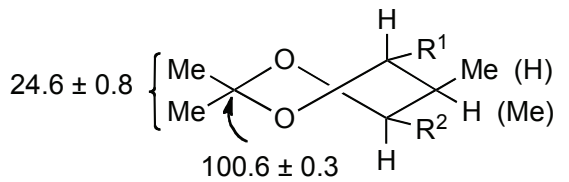

1,3-anti

Figure 1.5.1 ${ }^{13} \mathrm{C}$ shifts in 1,3-syn/anti acetonides.

Reduction of the hemiacetal of $\mathbf{4 0}$ gave a mixture of $\mathrm{C}-17$ epimers (Scheme 1.5.4), which were separated by chiral HPLC and further protected to furnish the acetonides $\mathbf{4 4}$ and 45. Spectroscopic analysis of $\mathbf{4 4}$ and $\mathbf{4 5}$ established the anti relationships at the C13/C15, C21/C23, and C25/C27 stereocentres. 


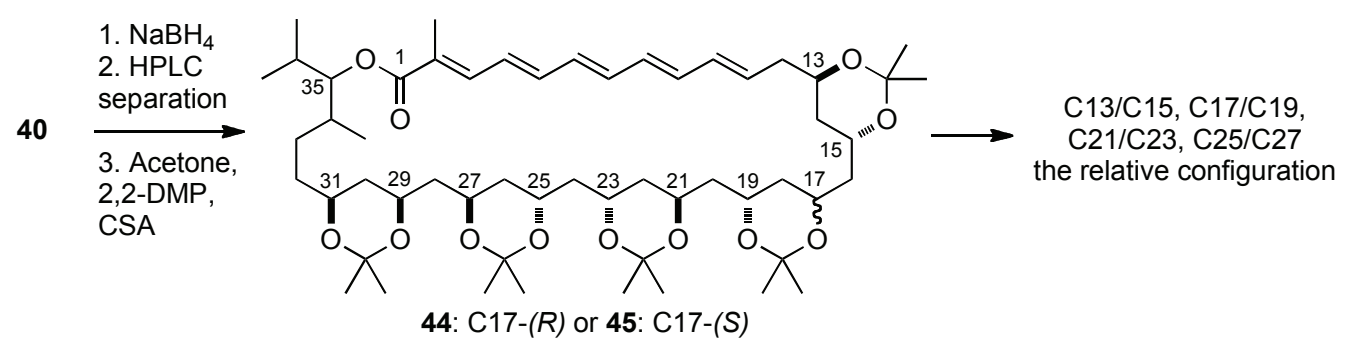

Scheme 1.5.4 Derivatisation of roflamycoin 40.

To determine the relative stereochemistry of the C27/C29, C31/C34, and C34/C35 centres the parent molecule 40 was subjected to degradation studies (Scheme 1.5.5). The polyene fragment was cleaved by ozonolysis and subsequent sodium borohydride reduction provided access to the advanced intermediate. Following sequence of synthetic operations resulted in the formation of peracetylated spiro-acetal 46 enabling the assignment of the pyran C31, C34 and C35 stereocentres. In conjunction with spectroscopic data, computer modeling was employed to elucidate the relative syn stereochemistry at $\mathrm{C} 31 / \mathrm{C} 34$ and $\mathrm{C} 34 / \mathrm{C} 35$ centres. In order to find the final relative relationship of $\mathrm{C} 27 / \mathrm{C} 29$, degradation fragment 47 was prepared. The analysis of the NMR data proved the relative stereochemistry of $\mathrm{C} 27 / \mathrm{C} 29$ to be $s y n$.

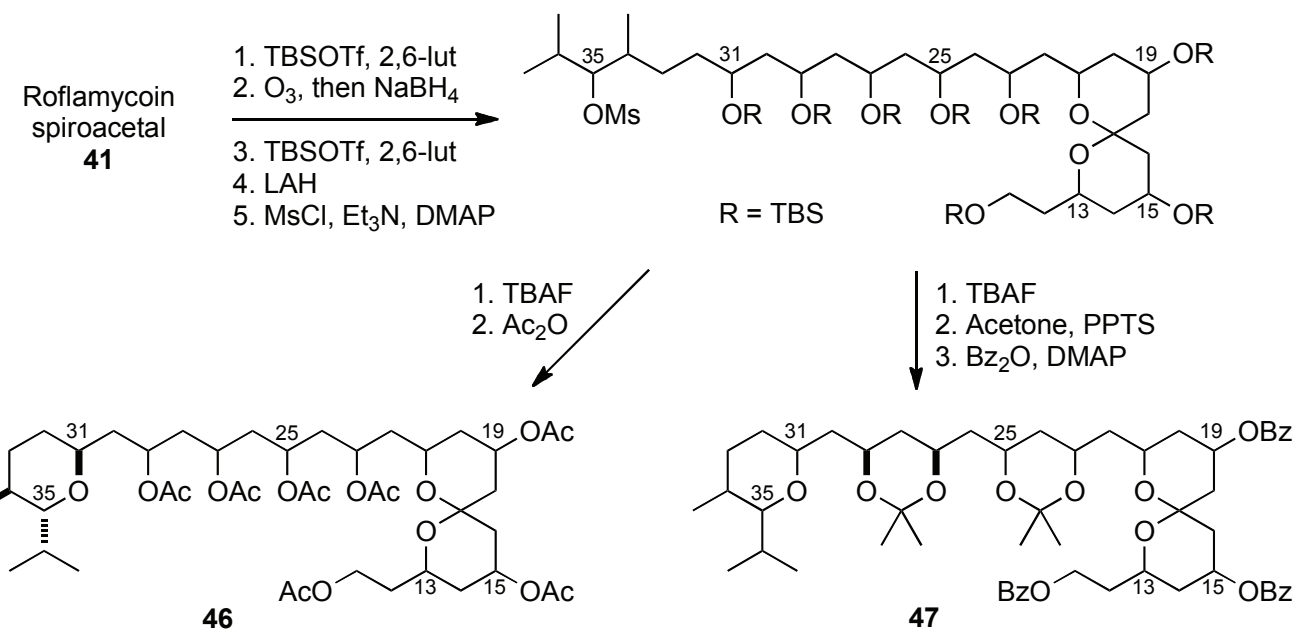

Scheme 1.5.5 Assignment of the relative configuration by degradation fragment studies.

The relative stereochemistry assigned so far was confirmed by analysis of the ${ }^{13} \mathrm{C}$-enriched compound 48, which NMR showed presence of the one anti and four syn acetonides as established previously (Scheme 1.5.6). Determination of the absolute 
stereochemistry of roflamycoin $\mathbf{4 0}$ was achieved by preparation of the Mosher ester derivatives of the C35 hydroxyl of the advanced degradation fragment 48 derived from $\mathbf{4 4}$ (Scheme 1.5.6). Detailed analysis according to the Mosher method confirmed the $S$ configuration of the $\mathrm{C} 35$ stereocentre. Rychnovsky decided then to synthesise degradation fragment 49 instead of the entire molecule $\mathbf{4 0}$, as $\mathbf{4 9}$ contains the entire stereostructure.

The synthetic approach to $\mathbf{4 9}$ was based on the cyanohydrin and bromo-acetonide building blocks $(\mathbf{5 0}, \mathbf{5 1}, \mathbf{5 2}, \mathbf{5 3})$ developed within the Rychnovsky group (Scheme 1.5.6). Their strategy involved the coupling of these pieces using standard alkylation chemistry followed by a stereoselective reductive de-cyanation to give pentaacetonide $49{ }^{46}$ Final comparison of the synthetic and natural material by ${ }^{1} \mathrm{H}$ NMR, ${ }^{13} \mathrm{C}$ NMR, HRMS, and TLC showed identical parameters, verifying the relative and absolute stereochemistry of roflamycoin $\mathbf{4 0}$ as in the fully protected fragment $\mathbf{4 9}$.

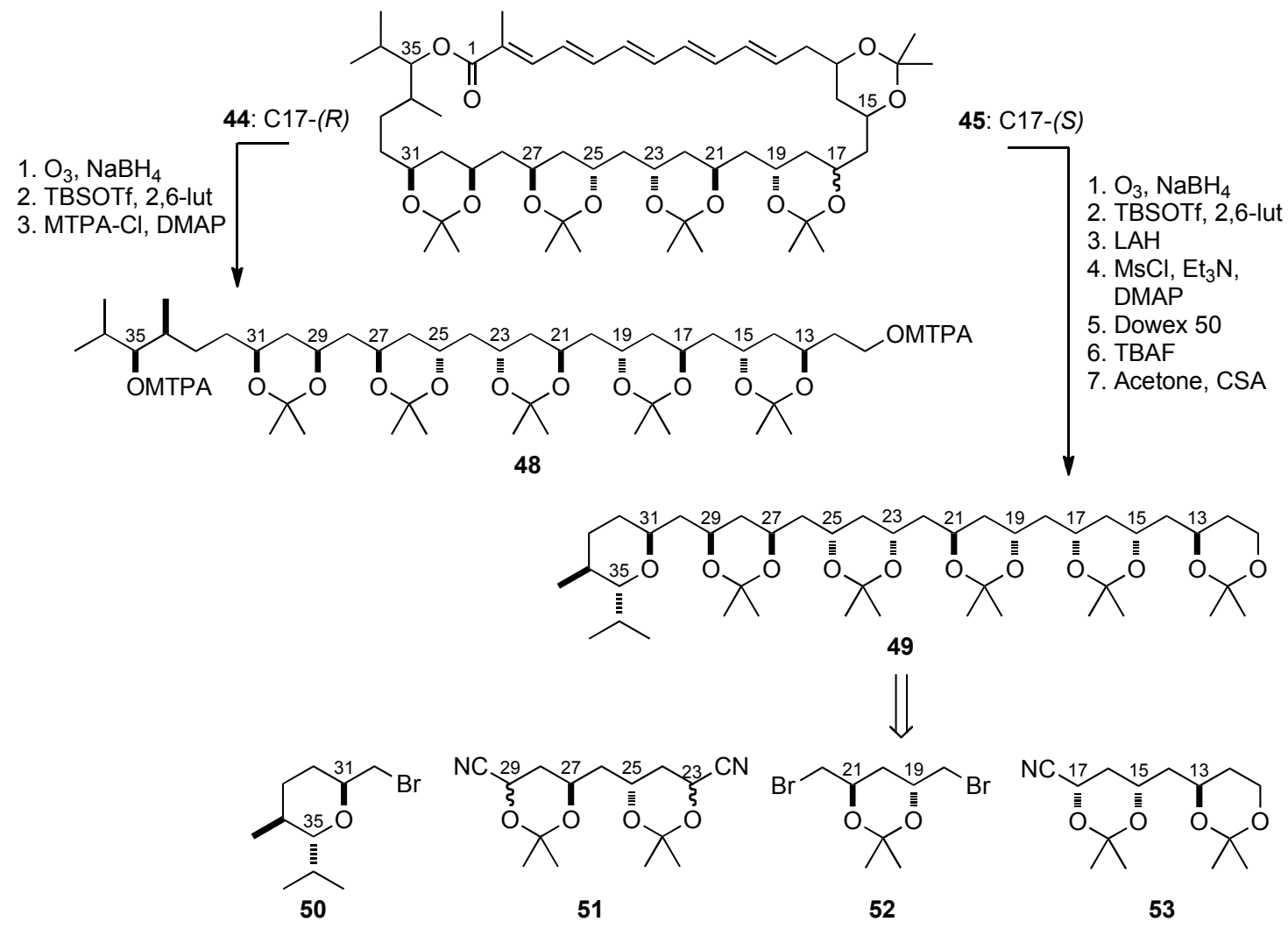

Scheme 1.5.6 Assignment of the absolute stereochemistry by Mosher ester analysis and a synthesis of the degradation fragment 49. 


\subsubsection{J-based configurational analysis developed by Murata}

Traditional NMR methods provide very reliable information for the elucidation of the planar structure. However, configurational analyses often require a combination of various techniques. A method for the determination of the relative configuration of adjacent stereocentres was recently developed by Murata $^{49}$ and instantly has found wide interest and application in establishing the stereochemistry of complex natural products. This method is based on a detailed investigation of hetero- and homo-nuclear spincoupling constants $\left({ }^{2,3} J_{\mathrm{C}, \mathrm{H}}\right.$ and $\left.{ }^{3} J_{\mathrm{H}, \mathrm{H}}\right)$. Commonly employed nOe-based techniques are not reliable in assigning the stereochemistry of highly flexible carbon chains. The observed signal intensities are affected by the presence of multiple conformers, where even minor populations could have a disproportionately large effect, leading to incorrect interpretations. In these systems with conformational flexibilities, the observed coupling constants exist as a weighted average of those associated with each conformer, consequently the most populated one will dominate. Correlation of the vicinal protons spin-couplings $\left({ }^{3} J_{\mathrm{H}, \mathrm{H}}\right)$ depends on the dihedral torsion angle and is described using the Karplus equation. Vicinal carbon-proton spin-coupling constants $\left({ }^{3} J_{\mathrm{C}, \mathrm{H}}\right)$ also follow a Karplus-type relationship and complements the stereochemical information obtained from ${ }^{3} J_{\mathrm{H}, \mathrm{H}}$. Additional information from ${ }^{2} J_{\mathrm{C}, \mathrm{H}}$ further expands the utility of the coupling constants in the configurational analysis (Figure 1.5.2). Advances in the instrumentation and spectroscopic techniques facilitated the calculation of more accurate values of the coupling constants in complex molecules, making the method highly reliable. ${ }^{50}$

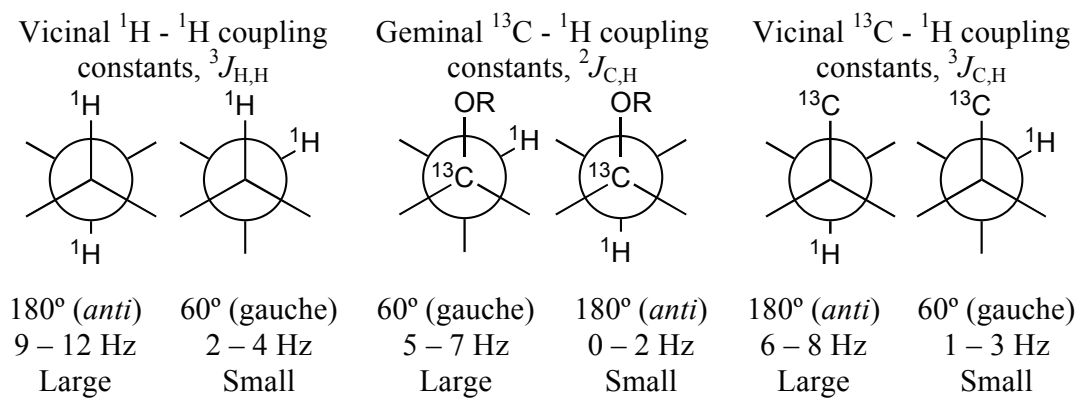

Figure 1.5.2 Coupling constant size dependence on dihedral angle.

This method was applied to establish the configuration of palmerolide A $\mathbf{1}^{3,4,21}$ Although it provided a great amount of detailed conformational information the absolute configuration was affected by the incorrect interpretation of the MTPA-esters. 


\subsection{Retrosynthetic strategy for palmerolide C}

As part of our programme to develop efficient synthetic strategies towards potential drug candidates we embarked on a journey into the total synthesis of palmerolides. This family of marine polyketides with unique chemical and biological properties have already entered the drug discovery arena, however still remaining relatively unexplored. Our contribution towards the studies on palmerolides unveils a detailed investigation of the stereostructure of palmerolide C (3) together with a convergent synthetic approach culminating in the total synthesis of the proposed structure.

The focus was initially set on the elucidation of the relative and absolute stereochemistry of palmerolide C (3). Due to the stereochemical uncertainty of the contiguous $\mathrm{C} 8-\mathrm{C} 10$ stereocentres, this would impose the potantial synthesis of up to sixteen different isomers of the natural product. Drawing from the previous studies on palmerolide A (4), where the absolute stereochemistry was verified by the synthesis of one of the degradation fragments, ${ }^{44}$ we decided to follow a similar approach. Using olefins as the functional handles, 3 would be cleaved by ozonolysis and subsequent reduction to provide poly-hydroxylated fragment 54 (Scheme 1.6.1). The synthesis of diastereomeric degradation fragments (55-58) would then become the primary goal of this project. The relative and absolute stereochemistry would then be defined in collaboration with Professor Bill Baker (University of South Florida, USA) by detailed comparison of the spectroscopic and specific rotation data of the synthetic and natural fragments.
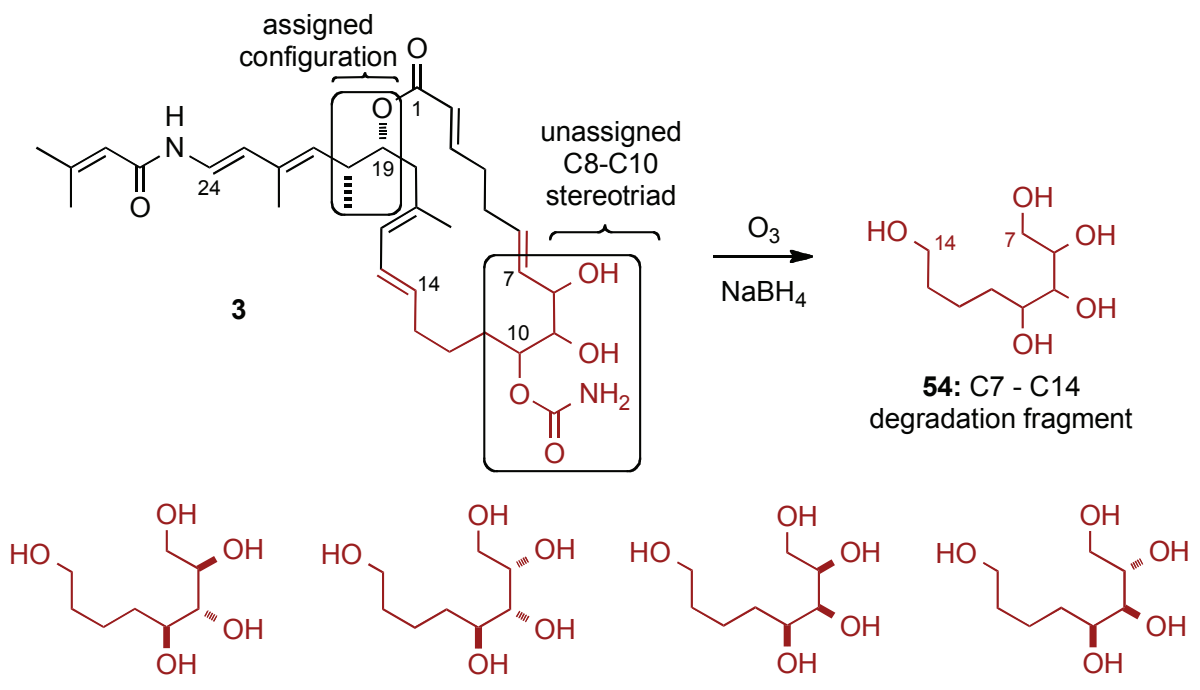

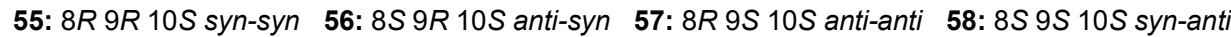

Scheme 1.6.1 Strategy towards stereochemical assignment of the relative and absolute configuration of palmerolide C (3). 
Following this phase, our focus would be the synthesis of the diastereoisomer identified in the degradation fragment studies and the incorporation of the required subunit into a convergent synthetic strategy outlined in Scheme 1.6.2. Initial retrosynthetic disconnection of the $\mathrm{C} 24$ and enamide functionality revealed the carbon framework of palmerolide C (3), which was divided into three key segments after the opening of the macrolactone. The assembly of the $\mathrm{C} 6-\mathrm{C} 7$ and $\mathrm{C} 14-\mathrm{C} 15$ olefins was envisaged to arise from cross-metathesis and Julia-Kocienski olefination, respectively.
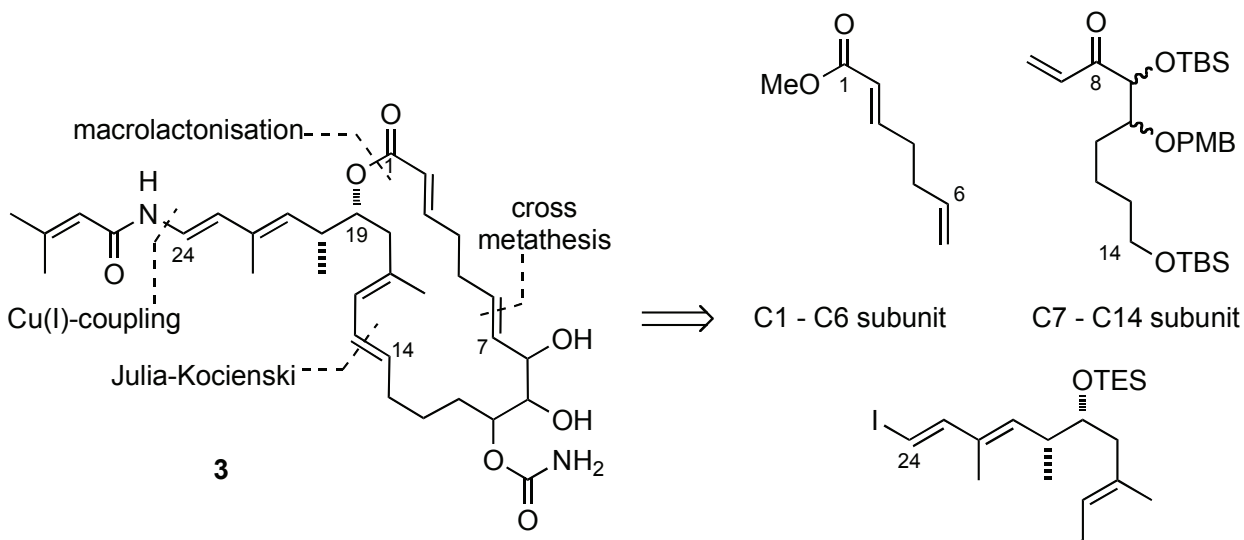

C1 - C6 subunit

C7 - C14 subunit

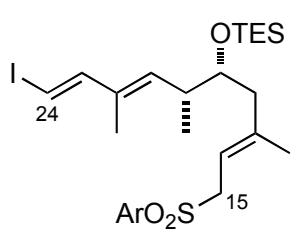

C15 - C24 subunit

Scheme 1.6.2 Retrosynthesis and degradation fragment of palmerolide C (3). 


\section{Chapter 2}

\subsection{Synthesis of C7-C14 degradation fragments}

The determination of the relative and absolute stereochemistry of the natural C7-C14 degradation fragment $\mathbf{5 4}$ would require the synthesis of a minimum of four diastereomeric poly-hydroxylated compounds for spectroscopic comparison. We reasoned that such hydroxylated compounds could be obtained from the 1,3-stereocontrolled reduction of aldol products of type 59, which in turn could arise from dihydroxyacetonederived building block 60 and a suitable aliphatic aldehyde 61 via organocatalysis (Scheme 2.1.1).

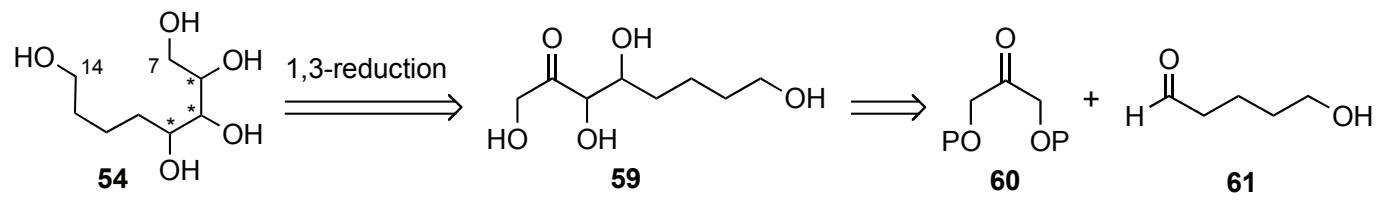

Scheme 2.1.1 Retrosynthesis of the degradation fragment 54 .

In construction of the 9,10-anti aldol product $\mathbf{6 2}$ methodology developed by Enders $^{51}$ would be explored where $(S)$-proline is used in aldol reaction between 2,2-dimethyl-1,3-dioxane-5-one (dioxanone) $\mathbf{6 3}$ and an aldehyde. Following substrate directed 1,3-syn and 1,3-anti reduction, this would provide access to the two degradation fragments $\mathbf{5 7}$ and $\mathbf{5 8}$ (Scheme 2.1.2).<smiles>CC1(C)OCC(=O)CO1</smiles><smiles>[R]C=CCCC(=O)O</smiles><smiles>[R]C(O)C1OC(C)(C)OCC1=O</smiles>

62

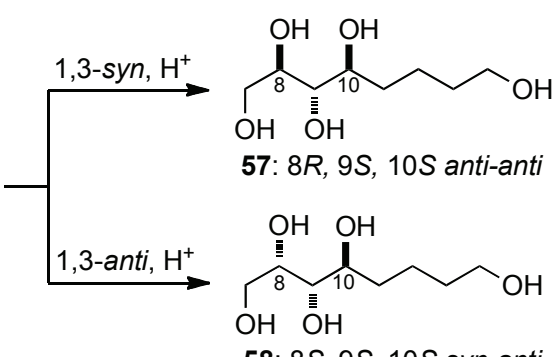

58: 8S, 9S, 10S syn-anti

Scheme 2.1.2 Synthetic strategy to construct anti-anti and syn-anti products. 
An organocatalytic approach would also be explored in the corresponding 9,10-syn series towards 64, where O-t-Bu-threonine-catalysed aldol reaction developed by Barbas would serve as a primary event involving dihydroxyacetone derivative 65. Remaining degradation fragments 55 and 56 (Scheme 2.1.3) would be completed applying the same approach developed in the 9,10-anti series.

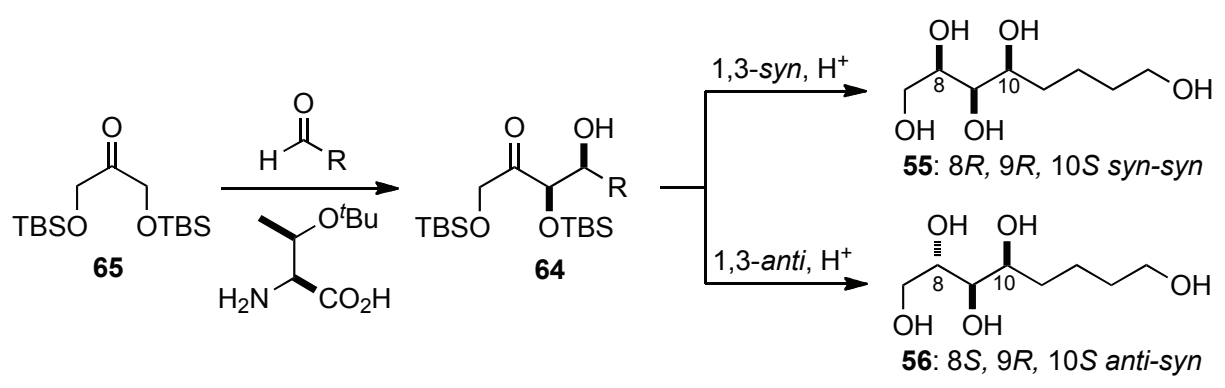

Scheme 2.1.3 Synthetic strategy to construct syn-syn and anti-syn products.

\subsection{Construction of the 9,10-anti aldol}

To test the viability of our strategy for the synthesis of fragment $\mathbf{5 4}$, work commenced with the construction of a system for the cross-aldol condensation. One-step syntheses of poly-oxygenated motifs utilising organocatalytic transformations have been extensively studied over the past decade. ${ }^{51-53}$ Enders and Grondal utilised the prolinecatalysed aldol reaction to effectively synthesise D-psicose (66) with excellent diastereoand enantio-control over the newly formed stereogenic centres (Scheme 2.2.1). Their optimised conditions employed $30 \mathrm{~mol} \%$ of $(R)$-proline, equimolar amount of substrates in dimethylformamide. The reaction mixture was then stored at $2{ }^{\circ} \mathrm{C}$ for a period of six days. Upon cleavage of the acetonides in $\mathbf{6 7}$ cyclisation was induced to furnish a mixture of $\alpha, \beta-$ D-psicofuranose 66 and $\alpha, \beta$-D-psicopyranose $68 .^{51}$

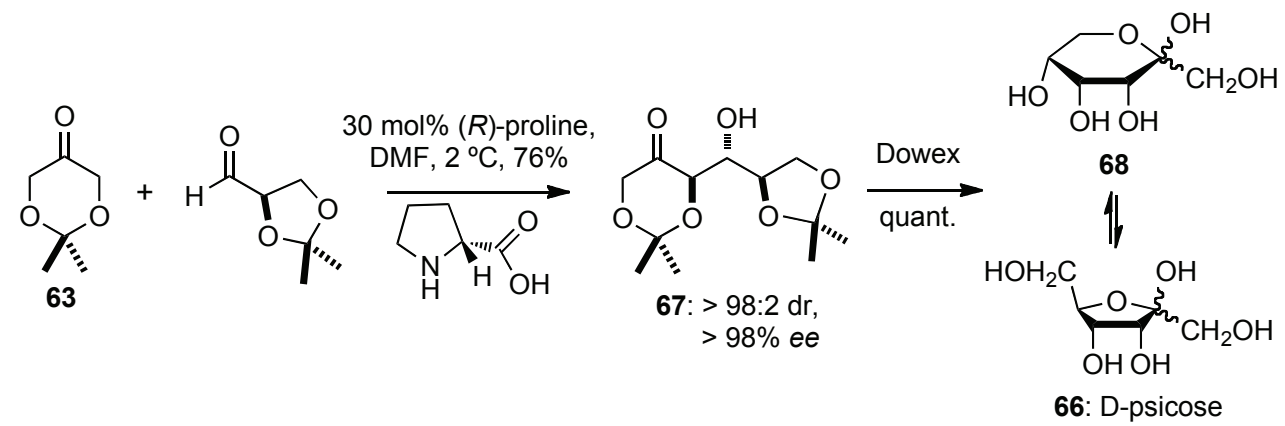

Scheme 2.2.1 Organocatalytic formation of D-psicose. 
Guided by the reported examples, we embarked on the investigation of the $(S)$-proline-catalysed cross aldol reaction between the dioxanone 63 and aldehyde 69. Ketone 63 was readily prepared in a two-step procedure from commercially available Trizma $^{\circledR}$ salt 70. Formation of the 1,3-acetonide $\mathbf{7 1}$ followed by oxidative cleavage with sodium periodate furnished ketone $\mathbf{6 3}$ in good overall yield (Scheme 2.2.2).

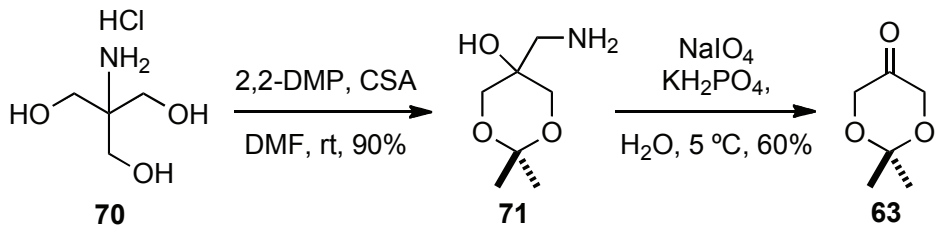

Scheme 2.2.2 Ketone 63 preparation for proline catalysed aldol reaction.

Preparation of aldehyde 69 started with the mono-silyl protection of 1,5-pentanediol 72. Subsequent oxidation of $\mathbf{7 3}$ under Swern conditions afforded aldehyde 69 in $68 \%$ yield over two steps (Scheme 2.2.3).

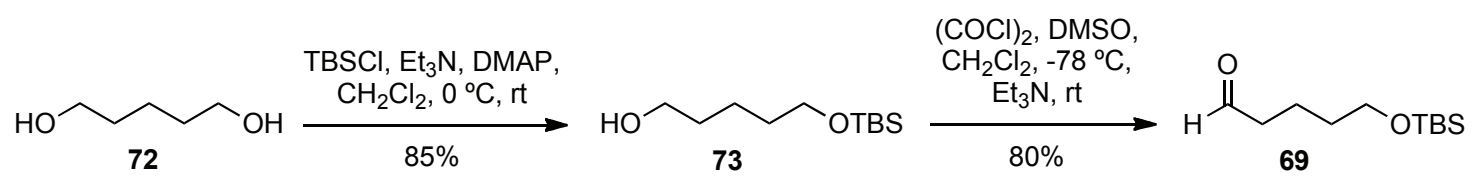

Scheme 2.2.3 Preparation of the aldehyde 69.

Under Enders' conditions (dioxanone 631 eq, 30 mol\% $(S)$-proline, aldehyde 69 1 eq, DMF $1.9 \mathrm{M}, 6$ days at $2{ }^{\circ} \mathrm{C}$ ) formation of the aldol product 74 was not detected, ${ }^{51}$ but instead self-condensation of ketone $\mathbf{6 3}$ was observed (Table 2.2.1, entry 1). Enders also reported this side reaction, where in absence of the aldehyde dioxanone 63 reacts with itself to form protected (S)-dendroketose $\mathbf{7 5}$ in 57\% yield (Scheme 2.2.4).

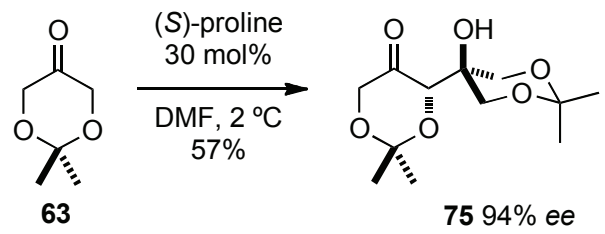

Scheme 2.2.4 Formation of protected $(S)$-dendroketose. 
Further investigations into catalyst loading and substrate equivalents did not deliver the desired product 74. In addition, solvent and temperature were screened. Unfortunately none of the performed operations led to the formation of the cross-coupled product $\mathbf{7 4}$. However, upon changing the protecting group of the aldehyde 69 to $\mathbf{7 6}$, which was readily prepared in two steps from $\mathbf{7 2}$ via mono protection with $\mathrm{BnBr}$, followed by Swern oxidation of 77 in $43 \%$ overall yield (Scheme 2.2.5), the desired aldol product 78 was isolated, albeit in a low 19\% yield (Table 2.2.1, entry 2).
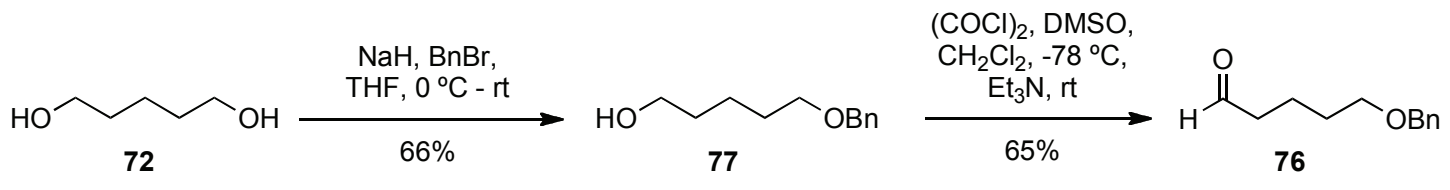

Scheme 2.2.5 Formation of the aldehyde 76 .

Upon attempts to optimise the reaction conditions $\mathbf{7 8}$ was formed in only $23 \%$ yield after carrying out the reaction at room temperature. However, long reaction times and a range of side products in the attempted cross-aldol reactions prompted a search for a suitable aldehyde compatible with an organocatalytic approach.

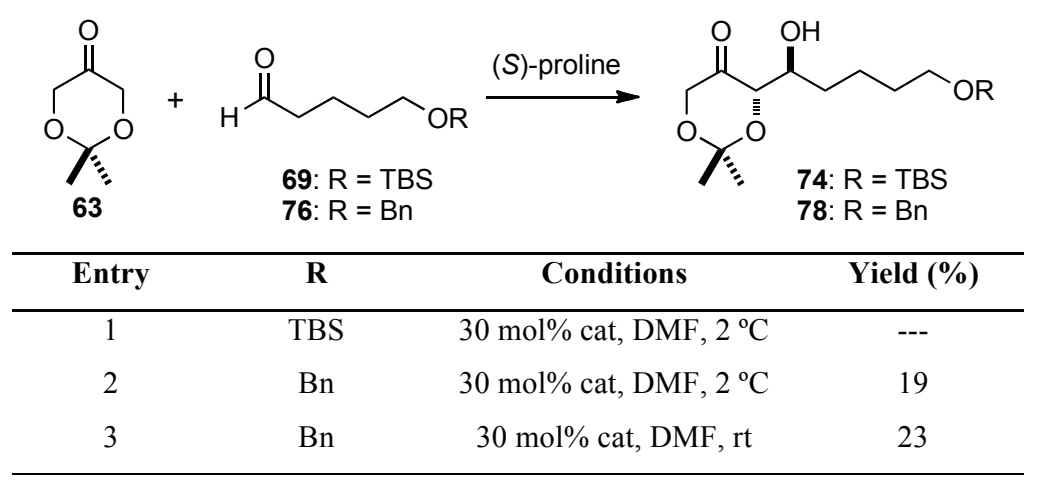

Table 2.2.1 (S)-proline-mediated aldol reaction.

Encouragingly, Grondal and Enders have provided an example of proline catalysed aldol reaction where the pentadecanal 79 was employed in the synthesis of sphingoids $\mathbf{8 0}$ and $\mathbf{8 1}$ (Scheme 2.2.6). ${ }^{54}$ They found that critical to that system was the nature of solvent and temperature. The best results were obtained when the reaction was performed in chloroform at room temperature for four days (60\% isolated yield, $>99: 1 \mathrm{dr}, 95 \%$ ee). 


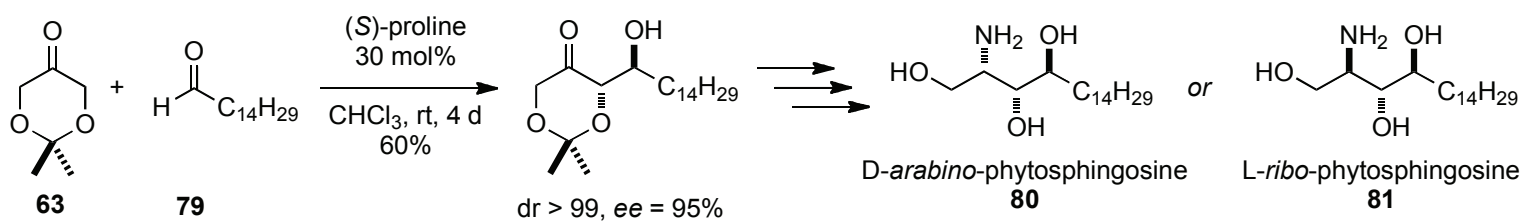

Scheme 2.2.6 Synthesis of sphingoids using proline aldol.

With their findings in mind, unbranched heptanal 82 was used in the reaction with dioxanone 63 and $(S)$-proline $(30 \mathrm{~mol} \%)$ in chloroform. Gratifyingly, the cross-aldol product 83 was isolated as a single diastereoisomer in $45 \%$ yield after storing the reaction for five days at room temperature (Scheme 2.2.7). The apparent difference between the initially examined system and the revised reaction with heptanal 82, was not only the solvent, but also the unexplained influence of the terminal oxygenation of the aldehydes 69 and 76 in the cross-aldol reaction.

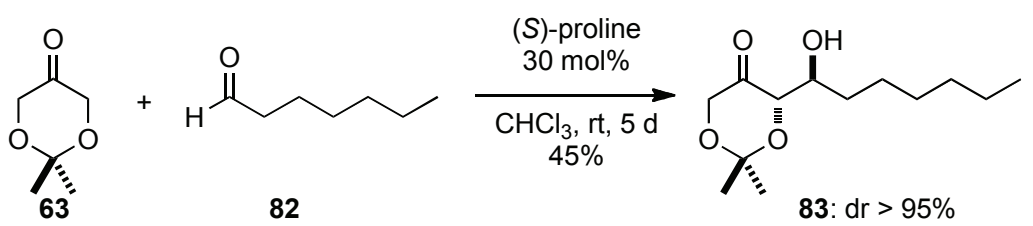

Scheme 2.2.7 Formation of the aldol product 83 catalysed by (S)-proline.

Having established the viability of the proline-catalysed system, 4-pentenal $\mathbf{8 4}$ was deemed a suitable precursor for the introduction of the terminal C14 hydroxyl functionality at a later stage of the synthesis. 4-Pentenal $\mathbf{8 4}$ was readily accessed via Claisen rearrangement of allyl vinyl ether $\mathbf{8 5}$ under microwave irradiation (Scheme 2.2.8) ${ }^{55}$

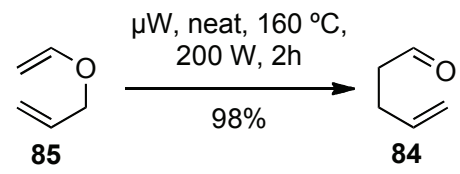

Scheme 2.2.8 Claisen rearrangement of the allyl vinyl ether 85 .

Aldehyde 84 was then added at room temperature to a mixture of ketone $\mathbf{6 3}$ and $30 \mathrm{~mol} \%(S)$-proline in chloroform (Scheme 2.2.9). After five days the desired aldol adduct $\mathbf{8 6}$ was isolated in $44 \%$ yield with excellent diastereoselectivity ( $>95: 5 \mathrm{dr}$ ). 


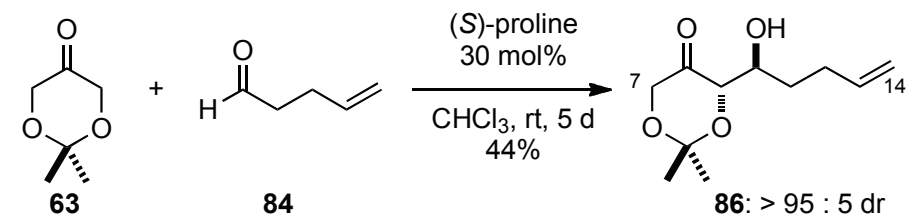

Scheme 2.2.9 Formation of the aldol product 86 .

\subsubsection{Determination of the absolute configuration at $\mathrm{C} 10$ stereocentre}

To establish the absolute configuration at the newly formed $\mathrm{C} 10$ stereocentre and determine enantiomeric purity of $\mathbf{8 6}$, the respective $(R)$ - and $(S)$-Mosher esters $\mathbf{8 7}$ and $\mathbf{8 8}$ were prepared. The derivatisation with chiral reagents such as $\alpha$-methoxy- $\alpha$-trifluoromethylphenyl acetic acid (MTPAA or Mosher's acid) is an established method for the assignment of the absolute configuration of secondary alcohols and amines by ${ }^{1} \mathrm{H}$ NMR spectroscopy. It was introduced in 1973 by Mosher et al. ${ }^{56}$ The presence of a defined chiral group (i.e. aromatic) in the derivatising reagent induces an anisotropic effect on the covalently bound secondary alcohol. Depending on the absolute configuration of the reagent, the planar arrangement of the trifluoromethyl and carbonyl groups with the hydrogen of the carbinol centre, the substituents are shielded/deshielded accordingly to the aromatic group present (Figure 2.2.1). The shielding/deshielding can be measured by ${ }^{1} \mathrm{H}$ NMR spectroscopy. In addition, ${ }^{19} \mathrm{~F}$ NMR can be used to determine the enantiomeric excess as the $(S)$ - or $(R)$-MTPA esters give rise to two distinct signals for derivatised enantiomers.
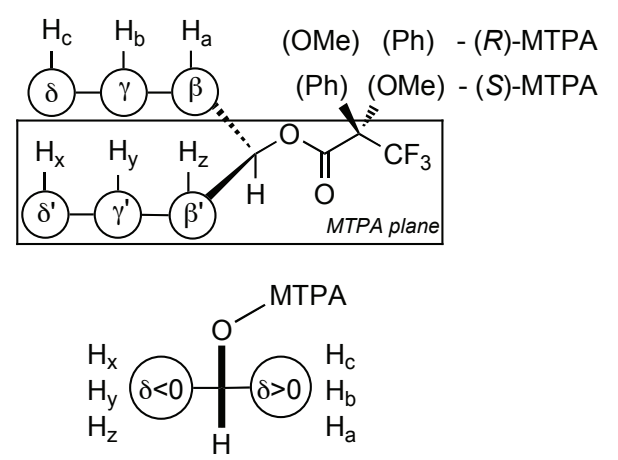

Figure 2.2.1 Preferred conformations of the $(S)$-MTPA and $(R)$-MTPA esters of 86 in $\mathrm{CDCl}_{3}$.

Analysis of the ${ }^{1} \mathrm{H}$ NMR and ${ }^{19}$ F NMR spectra of the $(R)$ - and $(S)$-MTPA esters 87 and $\mathbf{8 8}$ were conducted according to the advanced Mosher method. ${ }^{57}$ The experimental 
data correlated with the expected $10 S$ configuration shown in $\mathbf{8 6}$, the MTPA-aromatic group shielded protons placed on the same face $\mathrm{H} 10-\mathrm{H} 14\left(\mathrm{H}_{\mathrm{x}}, \mathrm{H}_{\mathrm{y}}, \mathrm{H}_{\mathrm{z}}\right.$ in Figure 2.2.1) in the (S)-MTPA ester 88 as evident by ${ }^{1} \mathrm{H}$ NMR spectroscopy. Consequently, the phenyl group in (R)-MTPA ester 87 shielded the H7-H9 protons $\left(\mathrm{H}_{\mathrm{a}}, \mathrm{H}_{\mathrm{b}}, \mathrm{H}_{\mathrm{c}}\right.$ in Figure 2.2.1) situated on the same face. This shielding moves affected protons upfield in the ${ }^{1} \mathrm{H}$ NMR spectrum. The subtraction of the chemical shift values for the particular protons $\left(\delta_{S}-\delta_{R}\right)$ gave negative values in the $\mathrm{H} 10-\mathrm{H} 14$ region and positive in the $\mathrm{H} 7-\mathrm{H} 9$ (Table 2.2.2). Analysis of the data obtained for the $(S)$ - and $(R)$-MTPA esters 87 and 88 confirmed the predicted $10 S$ configuration and also ${ }^{19} \mathrm{~F}$ NMR of 87 (Figure 2.2.2) provided evidence for $97 \%$ enantiopurity of $\mathbf{8 6 .}$

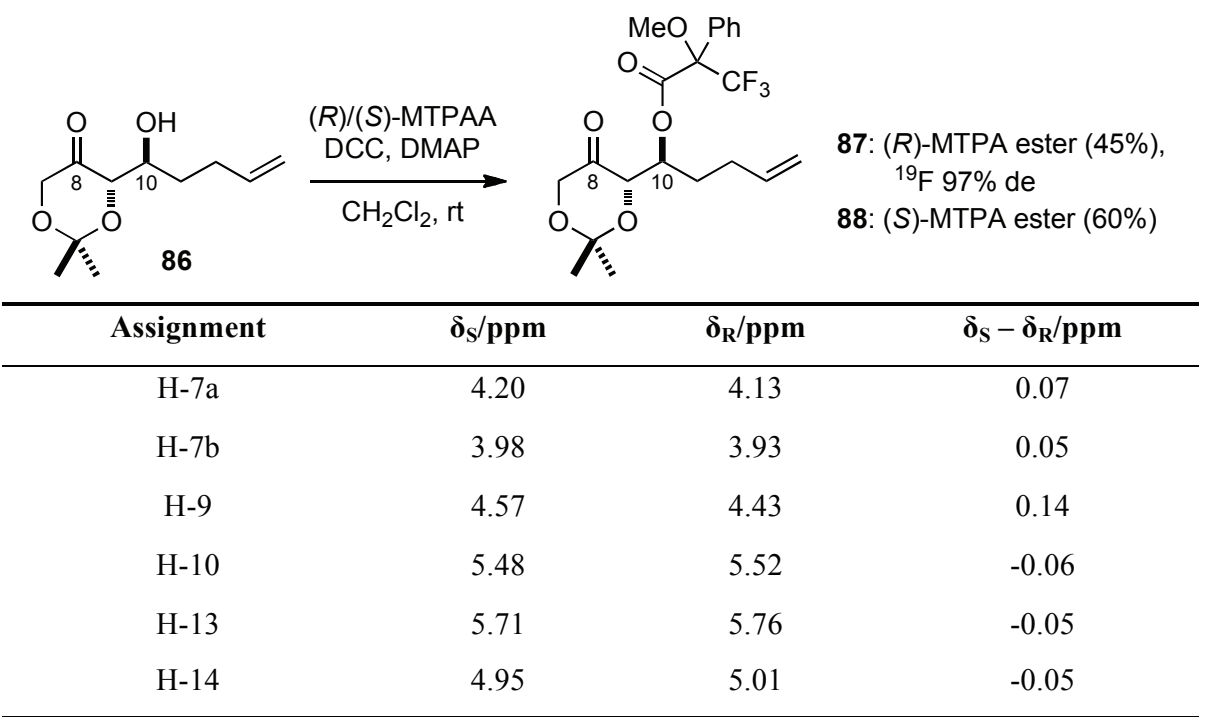

Table 2.2.2 ${ }^{1} \mathrm{H}$ NMR analysis of the $(R)$ - and $(S)$-MTPA esters of $\beta$-hydroxy ketone 86 .

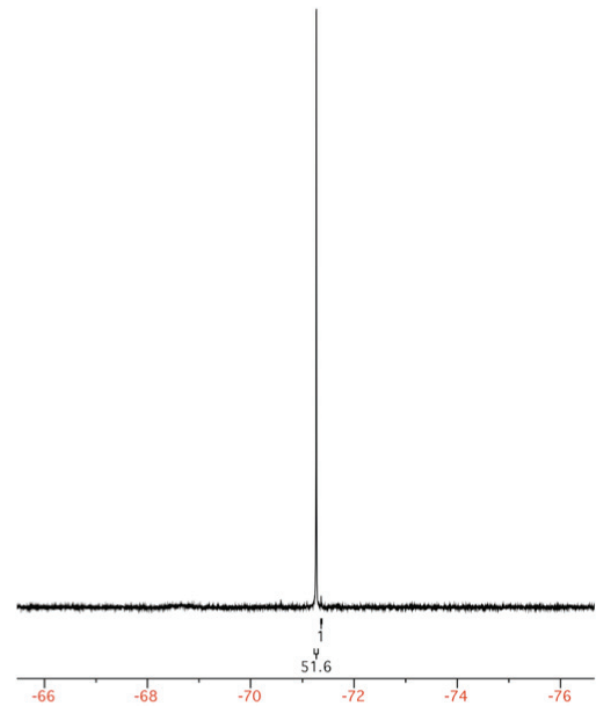

Figure 2.2.2 ${ }^{19}$ F NMR of the (R)-MTPA ester 87 . 


\subsubsection{Mechanism of proline-catalysed aldol reaction}

The mechanistic aspects of the proline-catalysed transformations have been widely studied. ${ }^{58-60}$ The high levels of enantioselectivity obtained in aldol reactions with this amino acid is attributed to its pyrrolidine ring, which upon treatment with a carbonyl compound forms the enamine 89. Iminium ion 90, formed by attack of the enamine on the $r e$-face of the aldehyde is subsequently hydrolysed to afford chiral $\beta$-hydroxyketone $91 .{ }^{60}$ The proposed reaction mechanism (Scheme 2.2.10) was initially based on the established mechanism of class-I aldolases, ${ }^{61}$ which proceed via similar intermediates. In addition, density functional theory (DFT) calculations ${ }^{62}$ supported the hypothesis of this analogy.

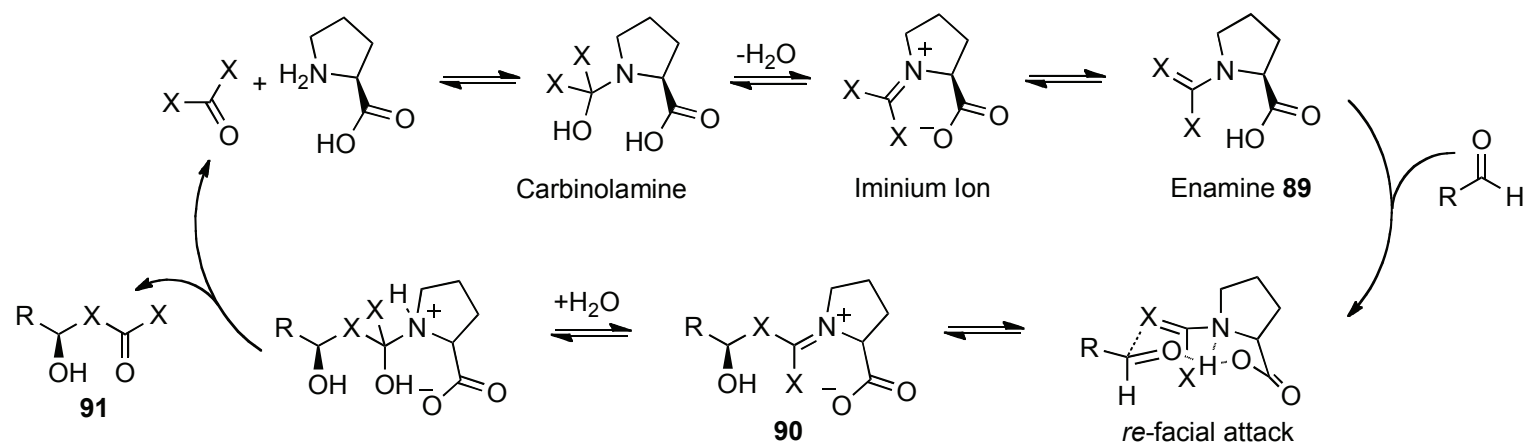

Scheme 2.2.10 Mechanism of $(S)$-proline-catalysed aldol reaction.

The proposed transition state TS I illustrates (Figure 2.2.3) that in the aldol reaction, the enamine attack occurs on the re-face of the aldehyde. This facial selectivity is dictated by minimising steric interactions between the substituents on the aldehyde and enamine (Figure 2.2.3). Moreover, hydrogen transfer between the carboxylate on proline and the oxygen of the aldehyde controls the enantioselectivity by directing which face of the enamine attacks the aldehyde. One of the most attractive features of proline-catalysed aldol reactions is that both $\mathrm{D}$ - and L-proline are readily available, so both enantiomeric products can be accessed. DFT calculations supported the proposed transition state shown in Figure 2.2.3, TS I. Based on these calculations it was postulated that an $\mathrm{N}-\mathrm{H}$ hydrogen bond does not lower the energy of transition state $\mathbf{T S} \mathbf{I} .^{58}$ 


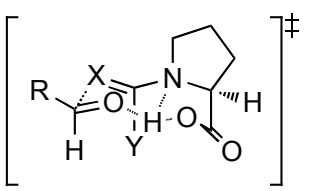

TS I

Figure 2.2.3 Houk's proposed transition state for aldol reaction.

\subsubsection{Reduction of the 9,10-anti aldol product 86}

With the 9,10-anti aldol product 86 in hand, the investigation of the substratedirected 1,3-reduction to install the C8 stereocentre commenced with a screening of borohydride reducing agents, which are summarised in Table 2.2.3. Initial reduction of $\mathbf{8 6}$ was performed with sodium borohydride and delivered mixture of diols 92 and 93 in good yield with moderate diastereoselectivity (3:1), in favour of the 1,3-syn product. Considering this result, standard reduction protocols were employed to explore the possibility of directing selectivity. Reduction with zinc borohydride proceeded in $54 \%$ yield to give 1,3-syn diol 92 in good selectivity (Table 2.2.3, entry 2).

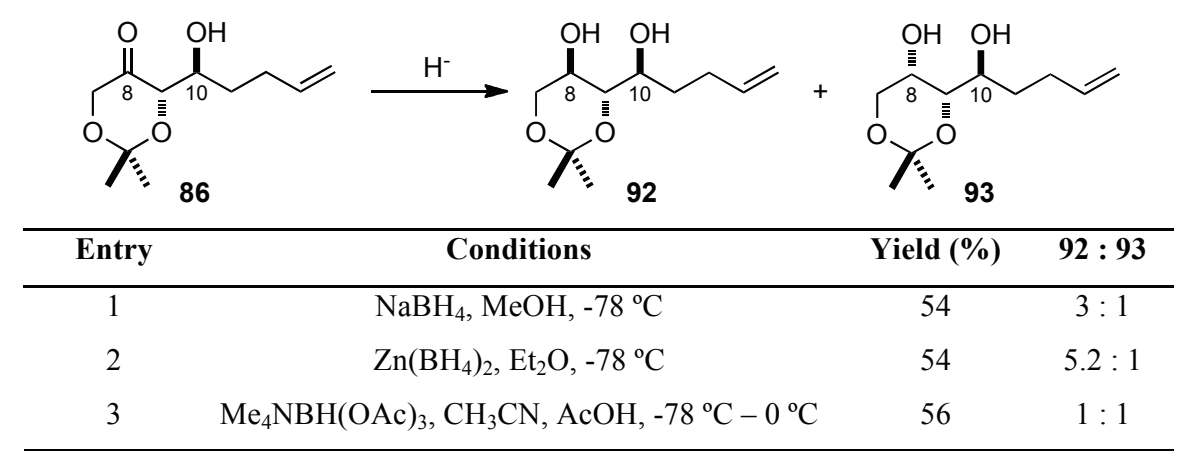

Table 2.2.3 Studies of the reduction of $\beta$-hydroxy ketone 86 .

The predominant formation of the 1,3-syn product 92 can be explained by the proposed transition state TS II shown in Figure 2.2.4. The metal centre coordinates to the free hydroxyl and carbonyl groups placing the molecule in a locked conformation, with hydride attack from a bottom face, giving rise to the formation of 1,3-syn diol. 


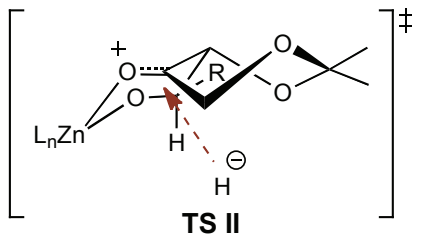

Figure 2.2.4 Transition state for $\mathrm{Zn}\left(\mathrm{BH}_{4}\right)_{2}$ reduction.

We then examined Evans ${ }^{63}$ procedure employing $\mathrm{Me}_{4} \mathrm{NBH}(\mathrm{OAc})_{3}$ to exert 1,3-anti selective reduction, as extensively documented for acyclic substrates. To our surprise, we did not observe good selectivity (Table 2.2.3, entry 3). We reasoned, supported by earlier studies by Enders ${ }^{64}$ that due to the dioxanone unit adopting a twisted boat conformation with the side-chain located in a pseudo-equatorial position, the internal-hydride delivery with preferred axial attack, imposes $s y n$-selectivity, as shown in TS III in Figure 2.2.5.

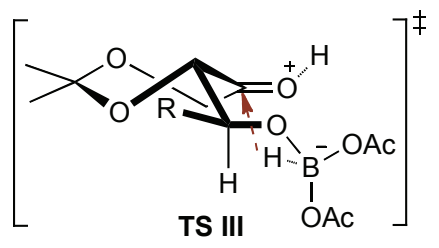

Figure 2.2.5 Enders transition states for 1,3-reduction with $\mathrm{Me}_{4} \mathrm{NBH}(\mathrm{OAc})_{3}$.

In all of the presented reductions, column chromatography on silica gel enabled separation of the diastereomeric diols 92 and $\mathbf{9 3}$. The 1,3-anti diol 93 was isolated as a white solid and recrystallisation provided material for a single crystal X-ray analysis (Figure 2.2.6). The acquired data provided evidence for the 1,3-anti relation of the diol 93. In conjunction with Mosher ester analysis of the C10 hydroxyl, the relative and absolute stereochemistry of $\mathbf{9 3}$ was established to be $8 S, 9 S$ and $10 S$. Consequently, the corresponding major diastereoisomer 92 had the anti-anti arrangement with the $8 R, 9 S$ and $10 S$ configuration.
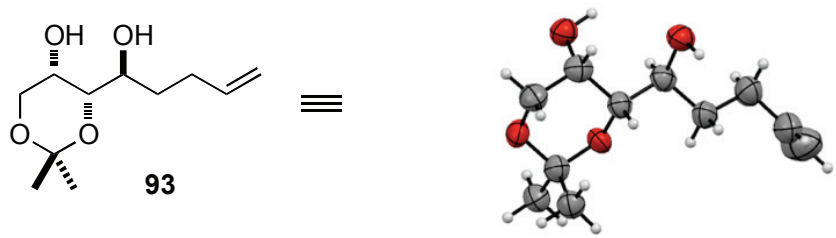

Figure 2.2.6 The 1,3-anti diol 93 and ORTEP representation of its solid-state structure. 


\subsubsection{Completion of the 9,10-anti series}

Having established access to the two required diastereomeric diols 92 and $\mathbf{9 3}$, the introduction of terminal hydroxyl functionality was addressed. The anti-anti diol 92 was examined first to find the optimal conditions. Several reagents to introduce the terminal hydroxyl including dimethylsulfide borane $\left(\mathrm{BH}_{3} \bullet \mathrm{SMe}_{2}\right),{ }^{43}$ 9-borabicyclo-[3.3.1]nonane (9$\mathrm{BBN})^{65}$ and thexyl-borane (thexylBH$\left.{ }_{2}\right)^{66}$ were tested and it was found that the latter reagent provided the best results, delivering the desired product 94 after oxidative work-up in $85 \%$ yield (Scheme 2.2.11).

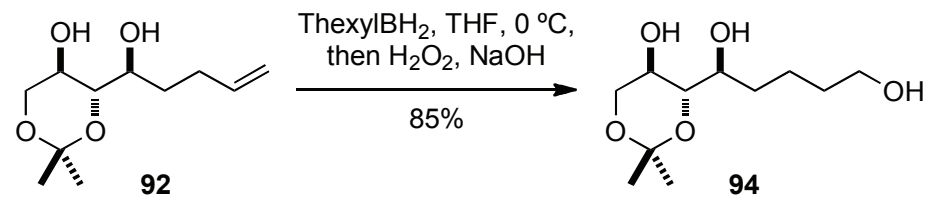

Scheme 2.2.11 Hydroboration to introduce a terminal hydroxyl.

At this point acetonide cleavage would complete the $(8 R, 9 S, 10 S)$-polyol 57. Various conditions were investigated to cleave the isopropylidene group including a screen of organic acids such as camphorsulfonic acid, pyridinium- $p$-toluenesulfonate and $p$-toluenesulfonic acid, which all turned out to be ineffective. The isolation of the highly polar product from aqueous work-up was troublesome. Gratifyingly, using acidic Dowex-50 resin, aqueous work-up was eliminated and simple filtration delivered pure product. The resin was first washed with water, $3 \mathrm{M} \mathrm{HCl}$ and excess methanol before adding to the solution of triol 94 in methanol. When starting material 94 was fully consumed, as indicated by TLC, the suspension was filtered and evaporated to deliver $8 R, 9 S, 10 S$ anti-anti degradation fragment 57 in excellent yield (98\%), without the requirement for further purification (Scheme 2.2.12).

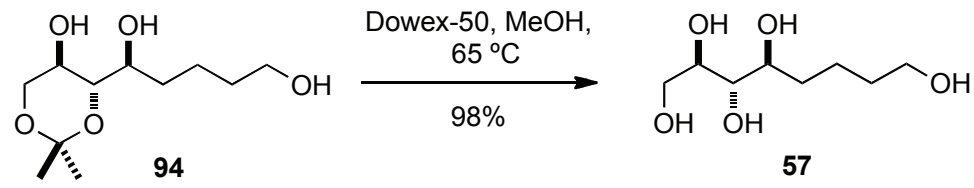

Scheme 2.2.12 Dowex-50 resin mediated deprotection. 
The conditions established for $\mathbf{5 7}$ were then applied to the diastereomeric diol $\mathbf{9 3}$. Accordingly, hydroboration with thexylBH $\mathrm{BH}_{2}$ provided product 93 in $77 \%$ yield, following removal of the acetonide protecting group to deliver the diastereoisomeric polyol $\mathbf{5 8}$ with the $(8 S, 9 S, 10 S)$ configuration and syn-anti arrangement (Scheme 2.2.13).<smiles>C/C=C/CCC(O)[C@@H]1OC(C)(C)OC[C@H]1O</smiles>

Scheme 2.2.13 Hydroboration and deprotection providing degradation fragment 58 .

\subsection{Alternative route to syn-anti degradation fragment 58}

The difficulties in obtaining the 1,3-anti diol 93 were overcome by introducing a bulky TBS protecting group on the free hydroxyl of 86 to form 96 (Scheme 2.3.1). Subsequent reduction with L-Selectride (lithium tri-sec-butylborohydride) afforded the 1,3-anti product 97.

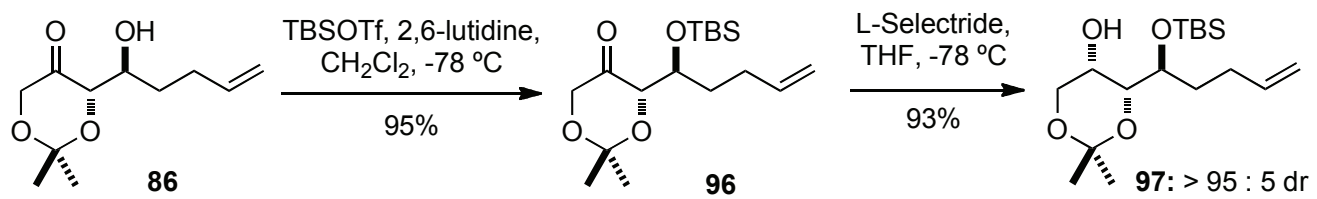

Scheme 2.3.1 Direct reduction of the TBS-protected aldol product 96.

The observed selectivity can be rationalised upon consideration of the transition state TS IV shown in Figure 2.3.1. The $\beta$-OTBS orientating perpendicular to the $\sigma$-framework of the carbonyl moiety and minimisation of steric interaction between the $\mathrm{R}$-substituent and the cyclic dioxanone leading to preferential addition of the hydride from the re-face of the carbonyl. ${ }^{67}$

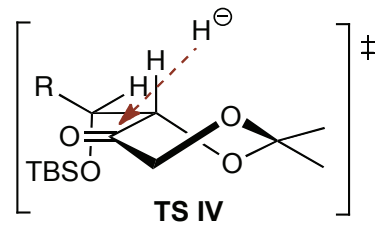

Figure 2.3.1 Enders transition state for a direct hydride delivery in reduction of 96. 
The strategy developed for the synthesis of degradation fragments $\mathbf{5 7}$ and $\mathbf{5 8}$ allowed for further advancement of the compound 97. Hydroboration with thexylBH $\mathrm{BH}_{2}$ delivered intermediate triol in $88 \%$ yield (Scheme 2.3.2). The silyl ether and acetonide cleavage was efficiently accomplished using Dowex-50 resin in methanol to provide the syn-anti degradation fragment $\mathbf{5 8}$ identical to material prepared from $\mathbf{9 3}$.

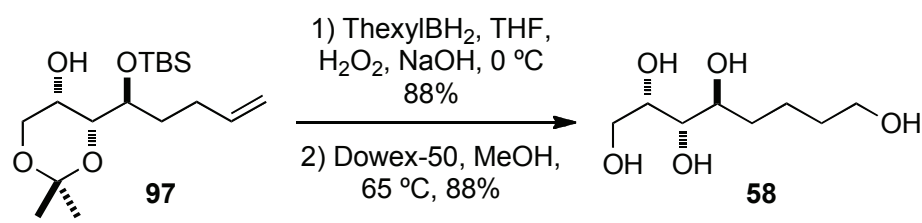

Scheme 2.3.2 Hydroboration and deprotection providing degradation fragment 58.

\subsection{Construction of the 9,10-syn aldol via an organocatalytic approach}

Attention was now directed towards the construction of the second set of diastereomeric degradation fragments $\mathbf{5 5}$ and $\mathbf{5 6}$ with the 9,10-syn configuration. An organocatalytic aldol reaction utilising C3 dihydroxyacetone building block 65 was developed by Barbas. ${ }^{68,69}$ Application of O- $t \mathrm{Bu}$-threonine as a catalyst in the cross-aldol reaction leads to the stereoselective formation of the 1,2-syn aldol product. This methodology was developed primarily for aromatic aldehydes. ${ }^{70}$ However, our system requires the use of an aliphatic aldehyde. Building on previous experience with the prolinecatalysed aldol reactions, 4-pentenal 84 was consequently chosen for investigation with $\mathrm{O}-\mathrm{t} \mathrm{Bu}$-threonine. Ketone $\mathbf{6 5}$ was prepared by reacting dihydroxyacetone dimer $\mathbf{9 8}$ with an excess of TBSCl and imidazole to produce 65 in 95\% yield (Scheme 2.4.1).

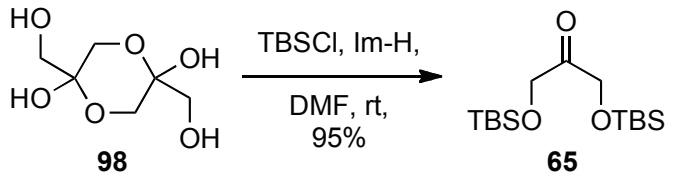

Scheme 2.4.1 Formation of the bis-TBS ketone 65.

With the starting materials in hand, investigation of the $\mathrm{O}-t \mathrm{Bu}$-threonine-catalysed aldol reaction began. The initial reaction was performed under conditions developed by Barbas, where ketone 65 was dissolved in $N$-methylpyrrolidone (NMP), and 4-pentenal 84 was added, followed by water and $O-t \mathrm{Bu}$-threonine. The resulting mixture was stirred for three days (Table 2.4.1, entry 1). After work-up the crude mixture was analysed and traces 
of the corresponding aldol product 99 were detected. Upon changing the solvent to dimethylformamide the aldol product 99 was formed in good selectivity, albeit in low yield (Table 2.4.1, entry 2). However, when the reaction was carried out in chloroform an acceptable, for the purpose of this study, 28\% yield of 99 and good levels of diastereoselectivity $(9: 1)$ were obtained (Table 2.4.1, entry 3$)$.

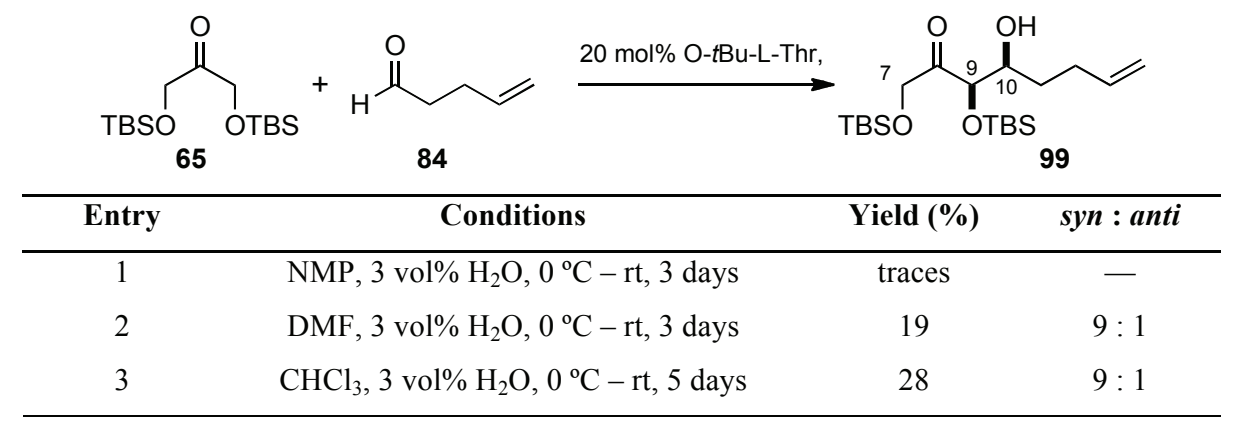

Table 2.4.1 Synthesis of the aldol product 99 and optimisation of the reaction.

\subsubsection{Determination of the absolute configuration at the $\mathrm{C} 10$ stereocentre}

The absolute configuration of $\mathrm{C} 10$ stereocentre was confirmed by the preparation of the corresponding $(R)$ - and $(S)$-MTPA esters 100 and 101, and subsequent analysis by advanced Mosher method as described in section 2.2.1. Investigation of the ${ }^{1} \mathrm{H}$ NMR shifts of the Mosher ester $\mathbf{1 0 0}$ and $\mathbf{1 0 1}$ (Table 2.4.2) confirmed the expected $(S)$-configuration at the C10 hydroxyl-bearing centre. From the ${ }^{19} \mathrm{~F}$ NMR (Error! Reference source not found.) enantiomeric excess of the major 9,10-syn diastereoisomers of 99 was calculated to be $90 \%$ ee.

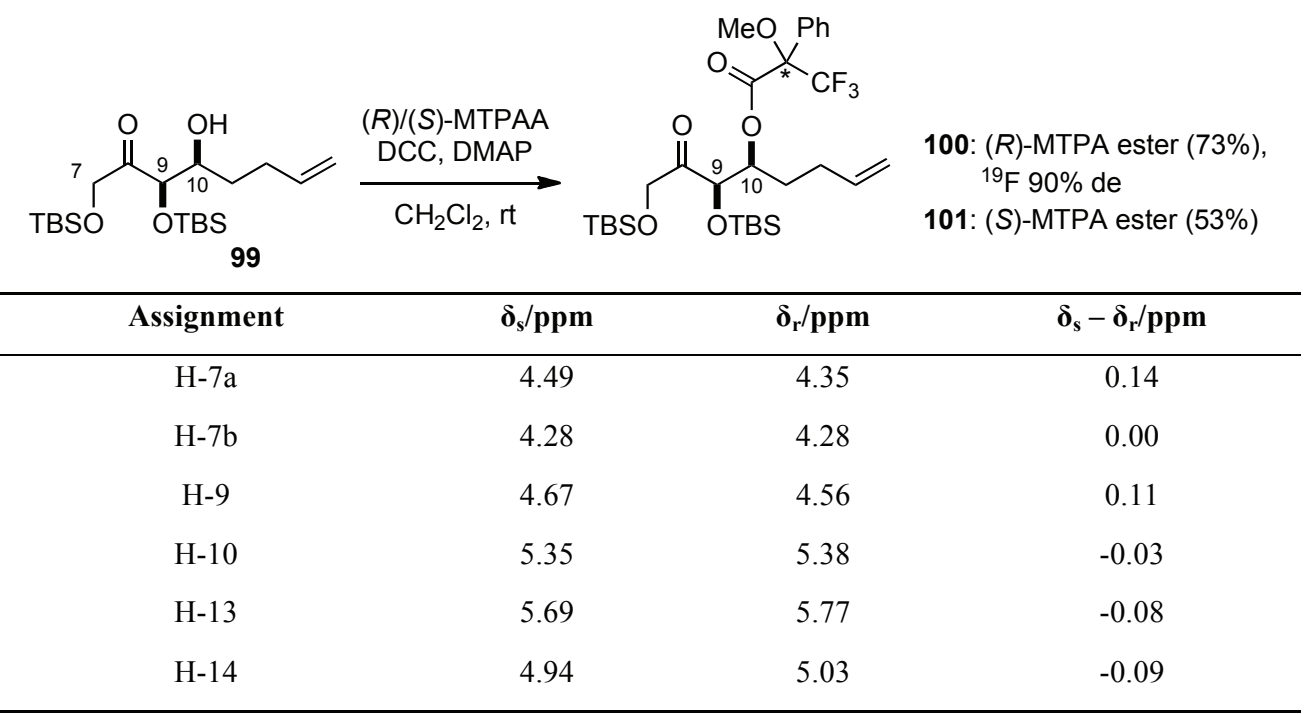

Table 2.4.2 Synthesis of the $(R)$ - and $(S)$-MTPA esters of $\beta$-hydroxy ketone 99 and ${ }^{1}$ H NMR analysis. 

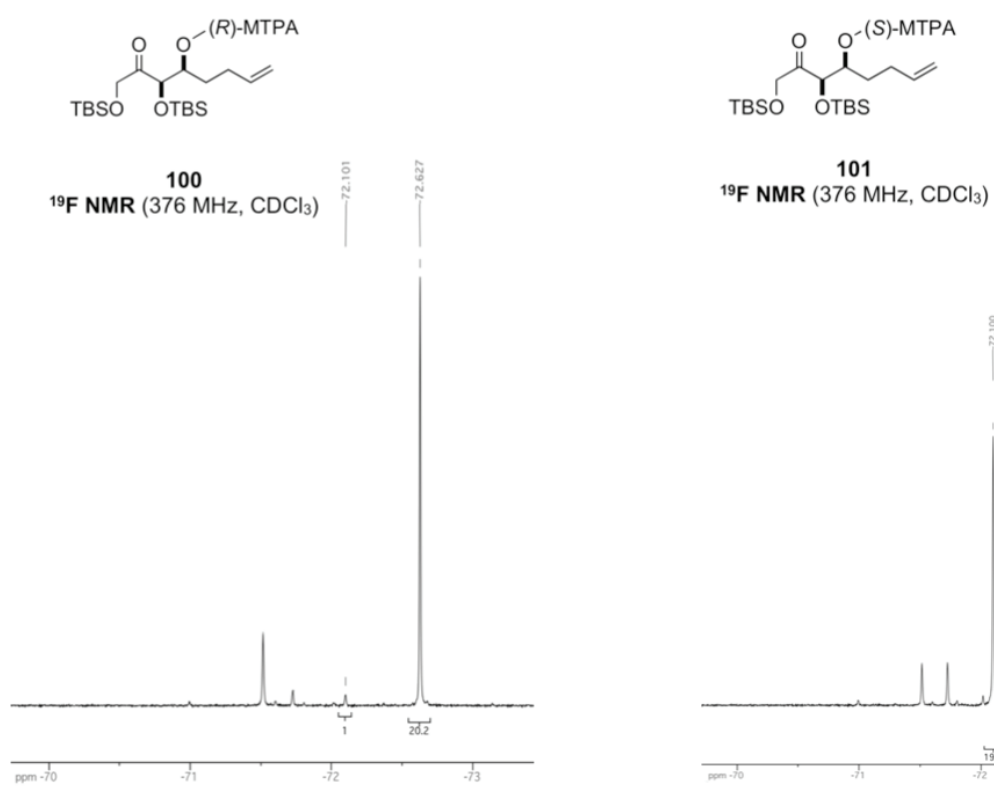

Figure 2.4.1 ${ }^{19} \mathrm{~F}$ NMR of the $(R)$ - and $(S)$-MTPA esters.

\subsubsection{Mechanism of $\mathrm{O}-\mathrm{t} \mathrm{Bu}$-threonine-catalysed aldol reaction}

When $\mathrm{O}-\mathrm{t} \mathrm{Bu}$-threonine is employed as a catalyst, the formation of a $(Z)$-enamine is preferred (Figure 2.4.2, TS V), which is dictated by hydrogen bonding between the amine hydrogen and oxygen of the alkoxy group. Facial selectivity of the approaching acceptor is controlled by the steric hindrance of the aldehyde and enamine substituents as well as proton transfer from the carboxylate onto the aldehyde oxygen (Figure 2.4.2, TS VI). ${ }^{68}$

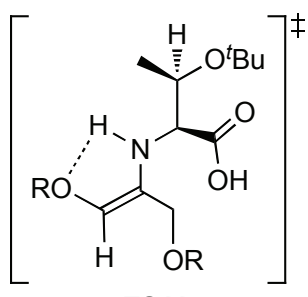

TS V

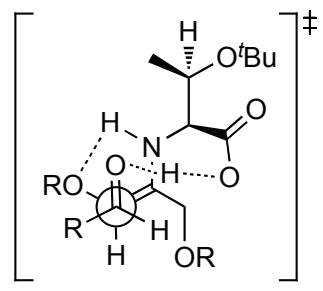

TS VI

Figure 2.4.2 Barbas transition states for $\mathrm{O}-\mathrm{t} \mathrm{Bu}$-threonine-catalysed aldol reaction.

\subsubsection{Reduction of the 9,10-syn aldol product}

With ample quantities of the 9,10-syn configured aldol product 99 in hand, the investigation of the 1,3-reduction commenced. Initial reduction with sodium borohydride gave 1,3-diol 102 in good yield (80\%). However it was observed, that the silyl ether was labile under the reaction conditions returning a range of side products which were difficult to identify (Table 2.4 .3 , entry 1 ). 


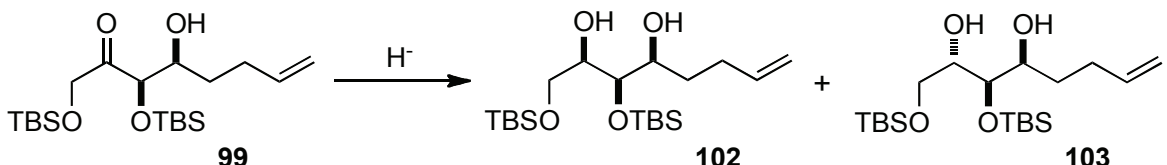

\begin{tabular}{cccc}
\hline Entry & Conditions & Yield (\%) & syn : anti \\
\hline 1 & $\mathrm{NaBH}_{4}, \mathrm{MeOH},-78^{\circ} \mathrm{C}$ & 80 & $n d$ \\
2 & $\left.i) \mathrm{cHex}_{2} \mathrm{BCl} \cdot \mathrm{NEt}_{3}, \mathrm{LiBH}_{3} \mathrm{OMe}, \mathrm{THF},-78{ }^{\circ} \mathrm{C}, i i\right) \mathrm{H}_{2} \mathrm{O}_{2}, \mathrm{MeOH}, \mathrm{pH} 7$ buffer $0{ }^{\circ} \mathrm{C}$ & 84 & $10: 1$ \\
3 & $\mathrm{Me}_{4} \mathrm{NBH}(\mathrm{OAc})_{3}, \mathrm{CH}_{3} \mathrm{CN}, \mathrm{AcOH},-78$ to $-30{ }^{\circ} \mathrm{C}$ & - & - \\
4 & $\mathrm{NaBH}_{4}, \mathrm{CeCl}_{3} \cdot 7 \mathrm{H}_{2} \mathrm{O}, \mathrm{MeOH},-78^{\circ} \mathrm{C}$ & 61 & $10: 1$ \\
\hline
\end{tabular}

Table 2.4.3 Studies of the reduction of $\beta$-hydroxy ketone 99.

Next, modification of the Narasaka-Prasad ${ }^{71,72}$ protocol was investigated where $\beta$-hydroxyketone borinate chelate was reduced with $\mathrm{LiBH}_{3} \mathrm{OMe}^{73}$ (Table 2.4.3, entry 2). The reaction is believed to proceed via a chair-like transition state (Figure 2.4.3) in which a silyl substituent on the $\alpha$-carbon prevents the approach of a reducing agent from the bottom side. Hence, hydride attacks preferentially from the $s i$-face giving rise to the 1,3syn diol 102 in excellent yield (84\%) with good diastereoselectivity (10:1).

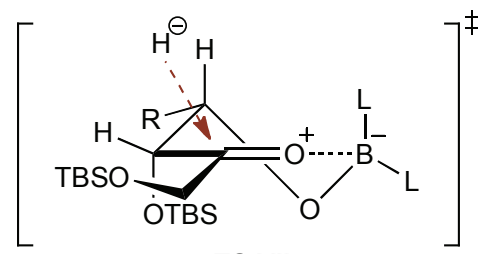

TS VII

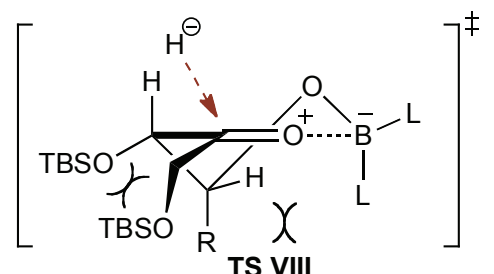

TS VIII

Figure 2.4.3 Proposed transition states for the reduction of the $\beta$-hydroxy ketone 99.

The 1,3-anti reduction of $\beta$-hydroxy ketone 99 was then required to access 103 . It was decided to apply the Evans protocol employing $\mathrm{Me}_{4} \mathrm{NBH}(\mathrm{OAc}) 3 .{ }^{63}$ However, the acidsensitive nature of compound 99 and the instability of the silyl groups in consequence led to degradation of the starting material (Table 2.4.3, entry 3).

The next possibility explored was the Luche reduction (Table 2.4.3, entry 4), which was a cleaner reaction compared to the one with sodium borohydride, and upon inspection of the ${ }^{1} \mathrm{H}$ NMR, the observed selectivity correlated to the results of reaction with $\mathrm{LiBH}_{3} \mathrm{OMe}$. At this point one of the side products, also observed in the reaction with sodium borohydride was isolated and analysed. It was assigned by $\operatorname{COSY}{ }^{1} \mathrm{H}^{-1} \mathrm{H}$ NMR to be compound $\mathbf{1 0 4}$ in which the secondary silyl group had migrated from the C9 position to the neighbouring $\mathrm{C} 8$ hydroxyl (Scheme 2.4.2). Faced with these results, it was necessary to search for an alternative plan to reach the 1,3-anti configured diol 103. 


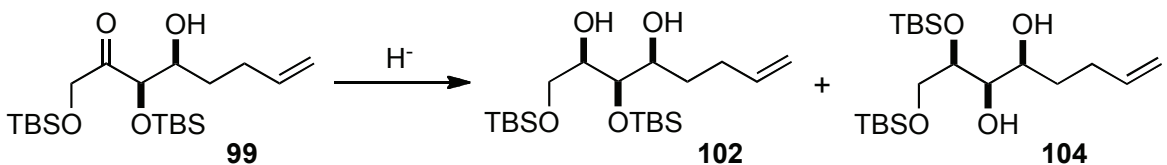

Scheme 2.4.2 Reduction of the $\beta$-hydroxy ketone 99 .

Progress towards the degradation fragment $\mathbf{5 6}$ with the anti-syn arrangement and $8 S, 9 R, 10 S$ configuration was now hampered, nonetheless the syn-syn $8 R, 9 R, 10 S$ configured degradation fragment $\mathbf{5 5}$ was within reach.

\subsubsection{Hydroboration and deprotection of the syn diol 102}

The hydroboration protocol employing thexylBH $\mathrm{H}_{2}$ successfully installed the $\mathrm{C} 14$ hydroxyl in 102. However, it was observed by ${ }^{1} \mathrm{H}$ NMR that the silyl groups were displaced during the reaction. Therefore, Dowex-50 deprotection was instantly carried out to provide the syn-syn $8 R, 9 R, 10 S$ configured degradation fragment $\mathbf{5 5}$ in excellent yield (80\%) over two steps (Scheme 2.4.3).

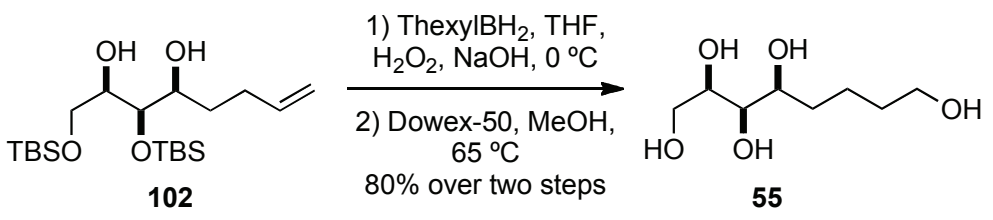

Scheme 2.4.3 Formation of C8R C9R C10S degradation fragment 55.

\subsection{Meso-tartaric acid route}

Due to the difficulties encountered in obtaining the 1,3-anti reduction product 103, a synthesis starting from meso-tartaric acid was designed to access the fourth degradation fragment 56 as a racemate. Employing the protocols developed by Napoli et al, ${ }^{74}$ aldehyde 105 was synthesised in four steps via a series of simple transformations in $66 \%$ yield (Scheme 2.5.1). The methyl ester of meso-tartaric acid 106 was first protected with dimethoxypropane and catalytic $p$-toluenesulfonic acid, followed by lithium-aluminium hydride reduction to afford diol 107. Mono-TBS protection of 107 and oxidation under Swern conditions furnished aldehyde $\mathbf{1 0 5}$. 


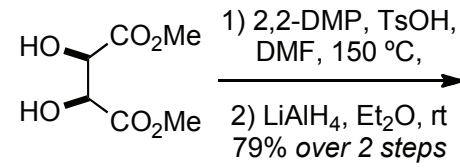

106

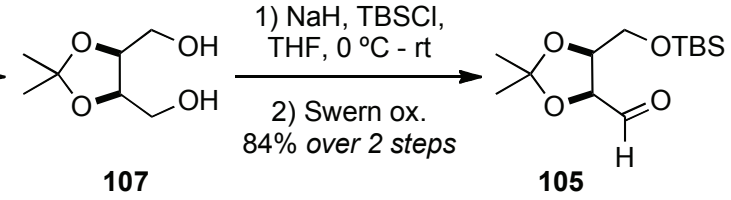

107

Scheme 2.5.1 Synthesis of the aldehyde 105 from meso-tartaric acid.

Addition of the freshly prepared homoallyl magnesium bromide to aldehyde $\mathbf{1 0 5}$ provided a separable mixture of C10-alcohols 108 and 109 (Scheme 2.5.2), which corresponded to the data reported by Napoli for saturated analogues. The isolated alcohol 108 was next subjected to hydroboration with thexylBH $\mathrm{BH}_{2}$ to provide the intermediate diol, which was deprotected with Dowex-50 resin to furnish the racemic anti-syn degradation fragment 56.

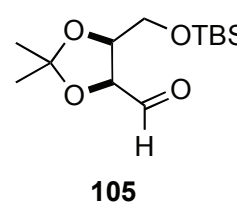

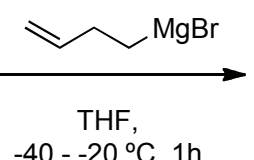

$0{ }^{\circ} \mathrm{C}, 2 \mathrm{~h}, 98 \%$

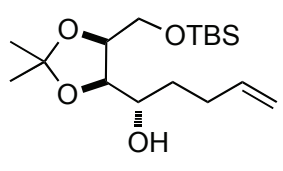

$1082.5: 1 \mathrm{dr}$
1) $\mathrm{ThexylBH}_{2}$, THF, $0^{\circ} \mathrm{C}$, $\mathrm{H}_{2} \mathrm{O}_{2}, \mathrm{NaOH}, 95 \%$

2) Dowex-50, $\mathrm{MeOH}$, $65^{\circ} \mathrm{C}, 98 \%$<smiles>OCCCCC(O)C(O)[C@H](O)CO</smiles>

56 racemic

Scheme 2.5.2 Formation of the racemic degradation fragment 56 via Grignard reaction.

The diastereomeric alcohol $\mathbf{1 0 9}$ was also subjected to hydroboration with thexylBH${ }_{2}$ and Dowex-50 deprotection to yield the racemic form of the previously reported anti-anti degradation fragment 57 (Scheme 2.5.3).

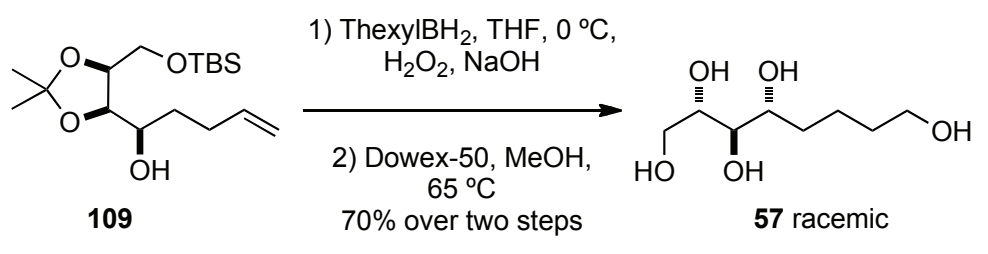

Scheme 2.5.3 Synthesis of the racemic degradation fragment 57 .

As a result, investigation based on the meso-tartaric acid route provided additional justification for the assignment of the relative configuration of the degradation fragments 56 and 57. Assignment of the anti-anti racemic degradation fragment 57 obtained in this way was supported by the corresponding ${ }^{1} \mathrm{H}$ and ${ }^{13} \mathrm{C}$ NMR data of the enantiopure product 57 synthesised in 9,10-anti series. Consequently, product 56 derived from (S)-alcohol 108 would bear anti-syn configuration. 


\subsection{Structural assignment}

At this stage, the first phase of the project was concluded with the synthesis of four diasteroisomeric C7-C14 polyols. The synthetic strategy employed highlights the effectiveness of organocatalysis for the construction of contiguous hydroxylated stereocentres from simple achiral building blocks. The collected ${ }^{1} \mathrm{H}$ and ${ }^{13} \mathrm{C}$ NMR and optical rotation data (Table 2.6.1) were sent to Prof. Bill Baker for comparison with the natural degradation fragment 54. In due course we received confirmation of the relative and absolute stereochemistry of the $\mathrm{C} 7-\mathrm{C} 14$ fragment. Our collaborator identified the arrangement of the triad present in the macrocycle as the syn-anti $8 R, 9 R, 10 R$ isomer, which was the enantiomer of the degradation fragment 58 (Figure 2.6.1). At this point, attention was directed towards the synthesis of the C7-C14 fragment with the proposed stereochemistry and its incorporation into the total synthesis of palmerolide C (3a).

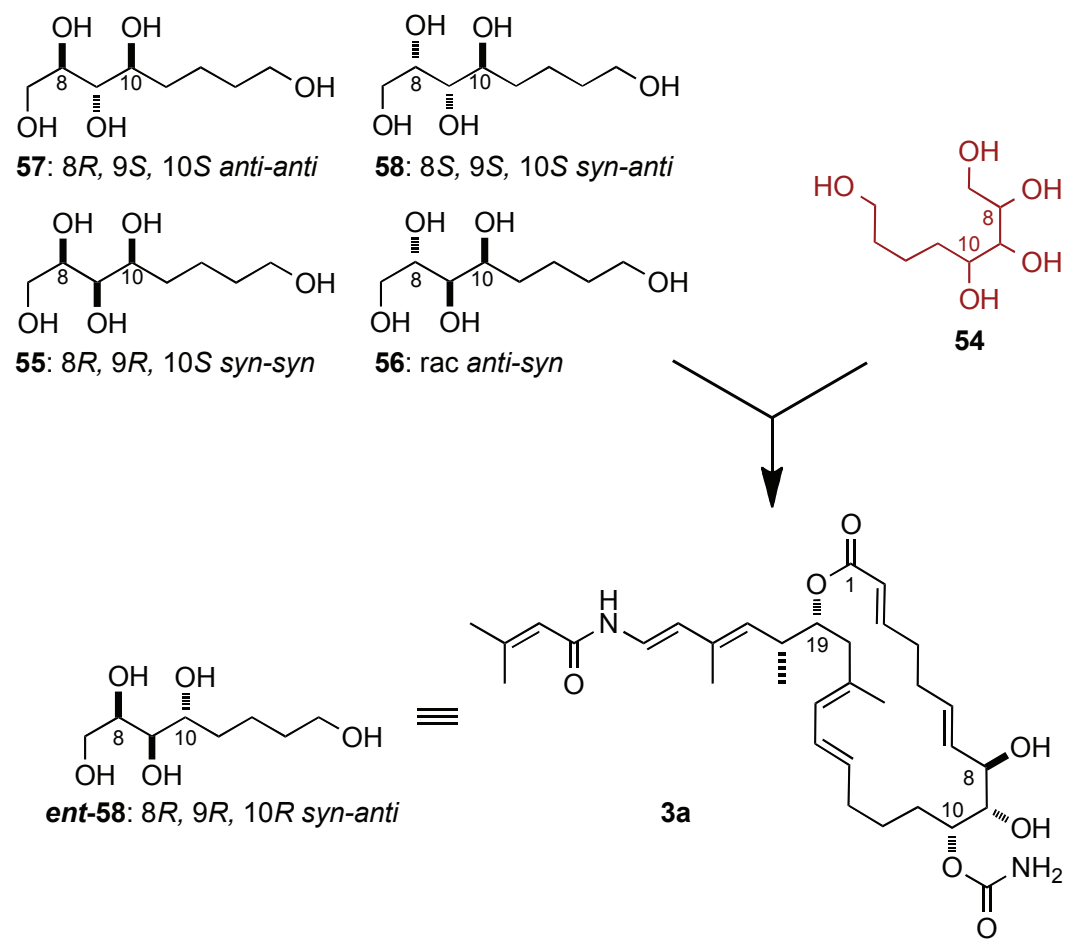

Figure 2.6.1 Comparison studies, performed by Prof Bill Baker, University of South Florida. 


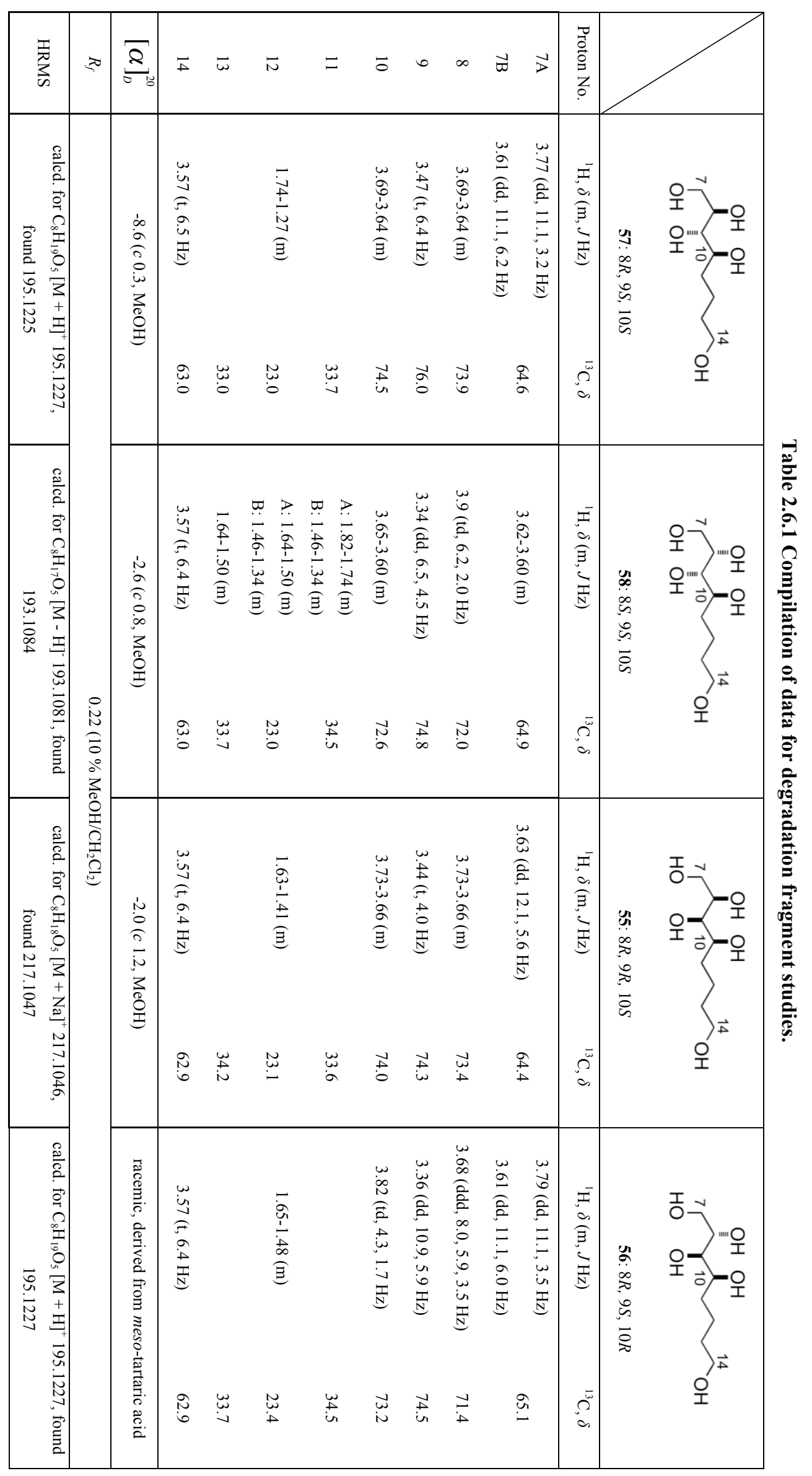




\section{Chapter 3}

\subsection{Retrosynthetic strategy for the synthesis of C1-C14 fragment}

The retrosynthetic strategy for the $\mathrm{C} 1-\mathrm{C} 14$ fragment $\mathbf{1 1 0}$ of palmerolide $\mathrm{C}$ is outlined in Figure 3.1.1. Based on the stereochemical information obtained from the degradation studies a highly convergent synthetic strategy was envisaged with disconnections of the $\mathrm{C} 6-\mathrm{C} 7$ alkene revealing two key fragments. The $\mathrm{C} 1-\mathrm{C} 6$ subunit 111, could be derived from Horner-Wadsworth-Emmons reaction (HWE) of 4-pentenal 84 and triethyl phosphonoacetate 112. The C7-C14 fragment 113 would be assembled via a boron aldol reaction between chiral ketone $\mathbf{1 1 4}$, derived from the L-ascorbic acid and aldehyde 69.
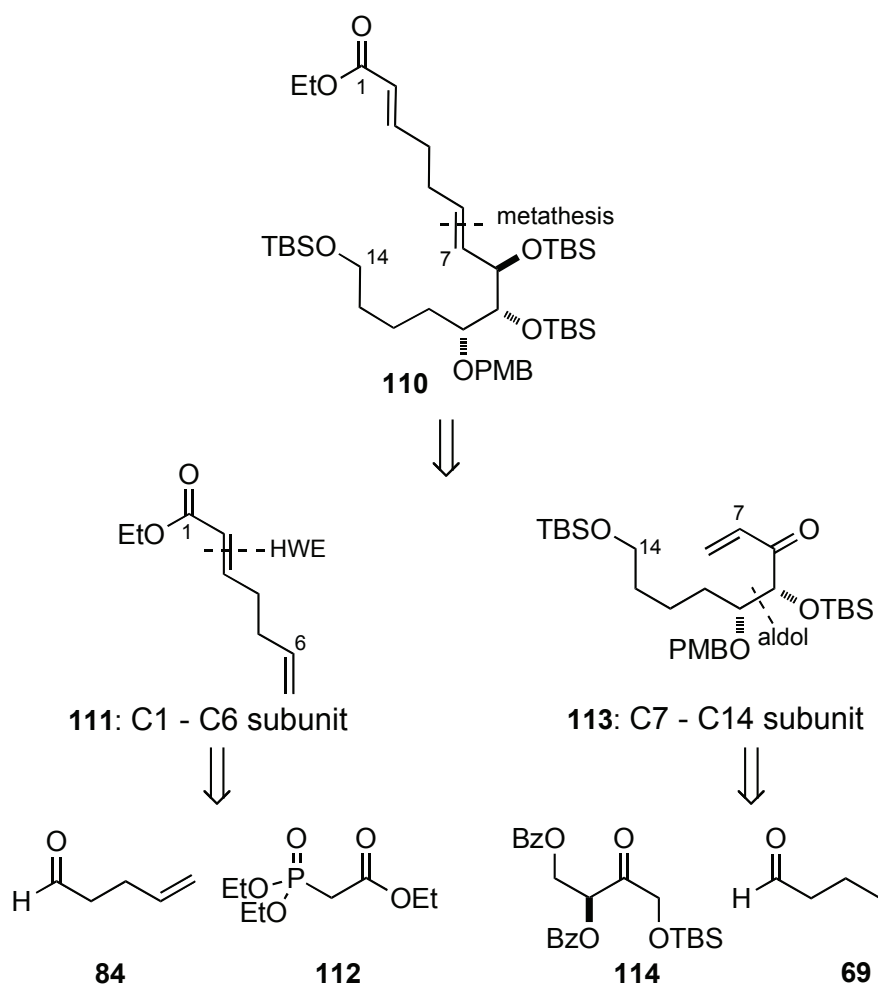

113: $C 7$ - C14 subunit
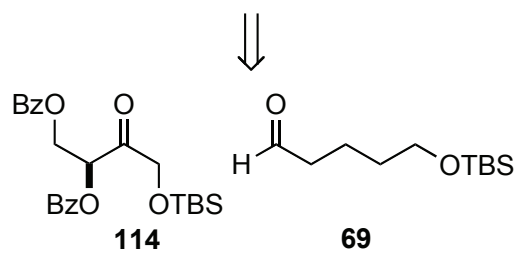

Figure 3.1.1 Retrosynthesis of the C1-C14 fragment 110. 


\subsection{Synthesis of the C1-C6 fragment 111}

Preparation of the C1-C6 fragment 111 began with HWE olefination. Under Masamune-Roush conditions, treatment of 4-pentenal 84 with triethyl phosphonoacetate ester 112, lithium chloride and Hunig's base, provided the $E$-enoate 111 exclusively in $81 \%$ yield (Scheme 3.2 .1$)^{75}$

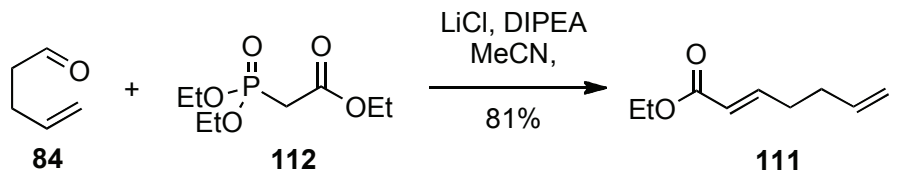

Scheme 3.2.1 Synthesis of the C1-C6 subunit 111 via HWE olefination.

\subsection{Synthesis of the C7-C14 fragment}

\subsubsection{Synthesis of the chiral ketone}

The construction of the key bond in the $\mathrm{C} 7-\mathrm{C} 14$ fragment relies on the boronmediated aldol reaction of $\alpha$-chiral ketone $114 .^{76,77}$ The synthesis of $\mathbf{1 1 4}$ began with the introduction of the isopropylidene acetal on L-ascorbic acid 115 (Scheme 3.3.1). Oxidative cleavage of the resulting intermediate and subsequent esterification provided hydroxy-ester 116. Reduction of the ester and protection of the resulting diol with benzoyl chloride was followed by the deprotection of the acetal, which provided 117. Selective silyl protection of the primary hydroxyl and oxidation under Swern conditions provided access to the chiral ketone 114. A total of eight steps from L-ascorbic acid was required to complete the synthesis of $\mathbf{1 1 4}$ in $23 \%$ overall yield.

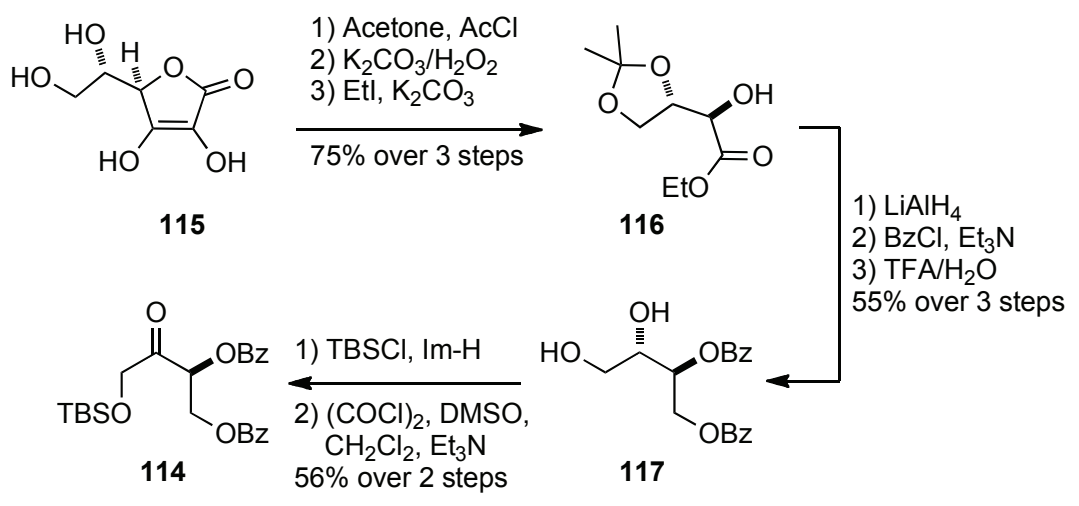

Scheme 3.3.1 Preparation of chiral ketone 114 from L-ascorbic acid. 
Given the lengthy synthetic sequence required to prepare 114 which ultimately only provides two carbons, an alternative $\alpha$-oxygenated chiral ketone was sought. Carda and Marco $^{78}$ demonstrated the potential of the L-erythrulose-derived ketone 118 in the formation of the poly-oxygenated chiral motifs of type 119, which then were used in the synthesis of the bioactive metabolite (+)-goniothalesdiol 120 (Scheme 3.3.2). ${ }^{78}$ Good levels of selectivity ${ }^{79}$ and high yields of product 119 prompted exploration of the Lerythrulose derived ketone 118 in our approach towards C7-C14 subunit 113.

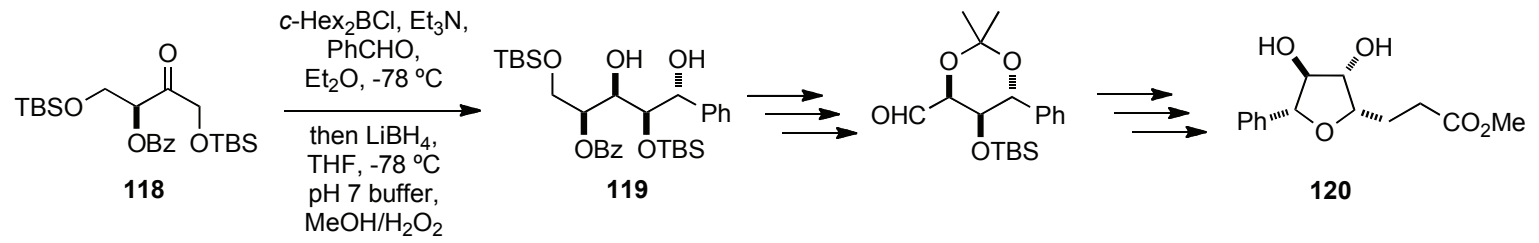

Scheme 3.3.2 Synthesis of (+)-goniothalesdiol 120 from ketone 118.

Marco developed a two-step protocol utilising L-erythrulose $\mathbf{1 2 1}$ as a starting chiral ketone. The synthesis began with TBS-protection of the primary hydroxyl groups in 121, further esterification of the secondary hydroxyl with benzoyl chloride completed 118 in good yield (Scheme 3.3.3).

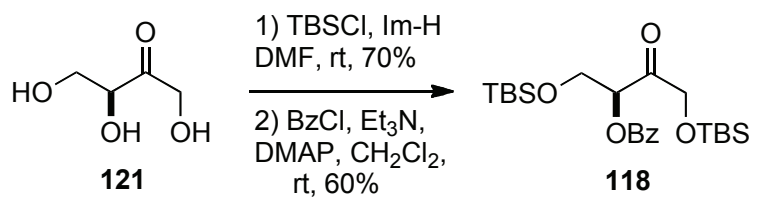

Scheme 3.3.3 Preparation of the chiral ketone 118 from L-erythrulose.

\subsubsection{Boron-mediated aldol reaction}

\subsubsection{Stereocontrol in boron aldol reactions}

The aldol motif is one of the most commonly occurring patterns within molecules derived from natural sources, particularly those of polyketide origin. The construction of the 1,3-hydroxy-ketone pattern presents chemists with a challenge that has been successfully tackled for over thirty years. As a result, examples of well-defined enolisation 
protocols ${ }^{80}$ chiral auxiliaries, ${ }^{81}$ and also ligands and catalysts ${ }^{82}$ for use as stereochemical determinants have been developed.

One of the key factors in predicting the relative configuration of an aldol product (syn or anti) is the geometry of the enolate component; syn-aldol product being generated from $(Z)$-enolates, and anti products arising from $(E)$-enolates. Enolisation protocols using boron as a metal centre give access to defined enolate geometries, where under specific circumstances, factors such as electronic effects and steric effects govern the selectivity. The generation of the $(E)$-boron enolate under kinetic conditions can be achieved employing dicyclohexylboron chloride, which preferentially complexes the ketone $\mathrm{C}=\mathrm{O}$ lone pair cis to the alkyl group allowing stabilisation of a negative charge (Me $\left.>\mathrm{Et}>{ }^{i} \mathrm{Pr}\right)$ (Scheme 3.3.4). As a result of this complexation, a proton on the activated centre can be abstracted by a relatively small amine base such as triethylamine, which consequently gives rise to the $(E)$-boron enolate. Kinetic conditions promoting the formation of the $(Z)$-enolate typically involves the use of small ligands (e.g. $n$-butyl) on boron and a good leaving group such as triflate, in combination with a bulky amine (e.g. diisopropylethylamine), leading to deprotonation of the most accessible proton which provides (Z)-enolate (Scheme 3.3.4). ${ }^{80}$
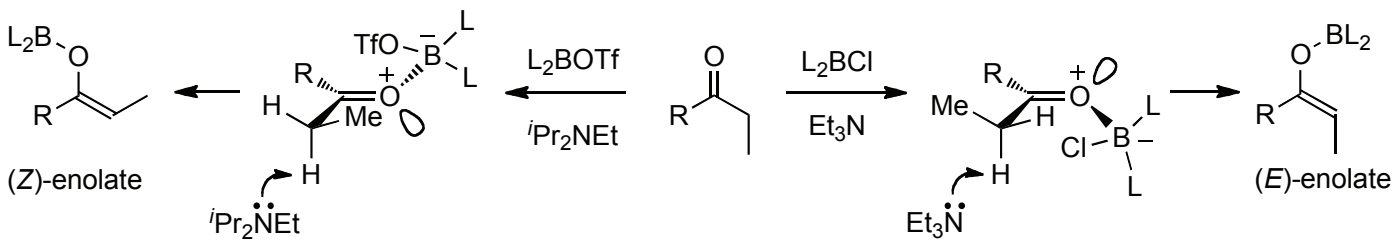

Scheme 3.3.4 Enolisation methods to form $(Z)$ - and $(E)$-enolates.

When the $(Z)$-enolate reacts with the aldehyde, two Zimmerman-Traxler transition states TS I and TS II shown below can be considered (Figure 3.3.1). Due to 1,3-diaxial interactions between the boron-ligand $(\mathrm{L})$, the enolate side chain $\left(\mathrm{R}^{1}\right)$ and the aldehyde substituents $\left(\mathrm{R}^{3}\right)$ transition state TS II is disfavoured, hence the preferred transition state TS I, which is lower in energy, would give rise to the 1,2-syn aldol product. 


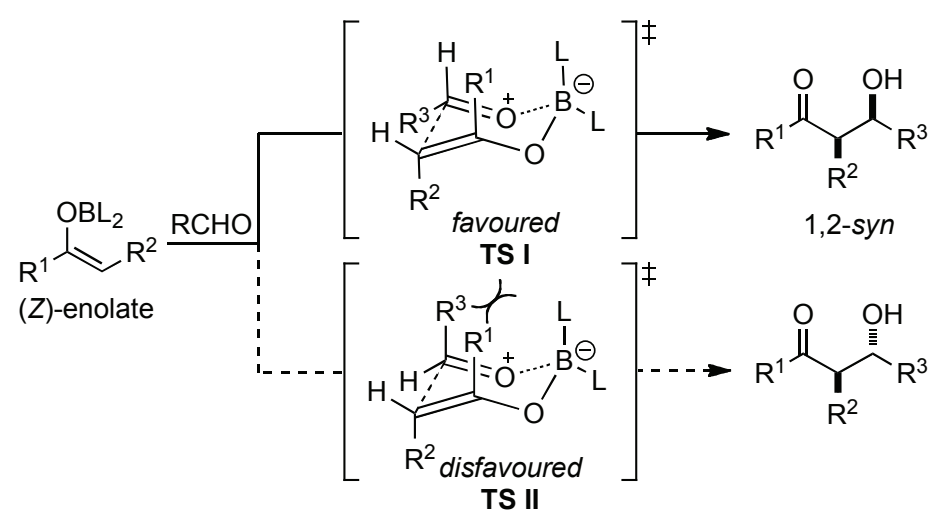

Figure 3.3.1 Zimmerman-Traxler transition states for $(Z)$-enolates.

Recent DFT calculations showed that boat-like transition states predominate when (E)-boron enolates are used in the aldol reaction (Figure 3.3.2). However, the relatively small energy barrier between TS III and TS IV lowers the level of enantiocontrol. ${ }^{83}$ The steric interactions between the metal ligand and enolate side chain $\left(\mathrm{R}^{1}\right)$ are minimised in the aldol reaction proceeding via a boat-like transition state.

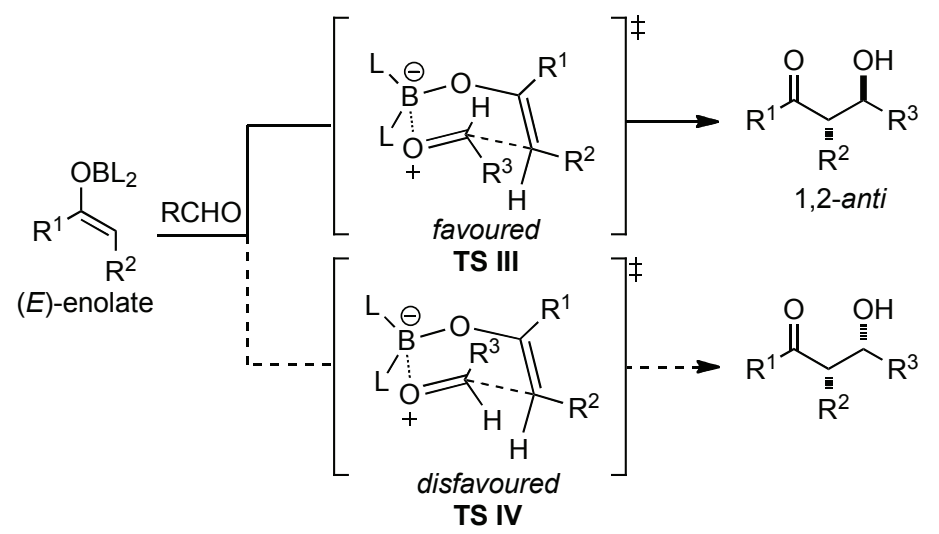

Figure 3.3.2 Boat-like transition states for $(E)$-enolates.

\subsubsection{Boron-mediated aldol reaction using ketone 118}

Investigation of the anti-selective aldol reaction using chiral ketone 118 and aldehyde 69, commenced with the selective enolisation of 118 using $c-\mathrm{Hex}_{2} \mathrm{BCl}$ and $\mathrm{Et}_{3} \mathrm{~N}$ to provide the intermediate $(E)$-boron enolate $\mathbf{1 2 2}$. Followed by the addition of aldehyde 69 and oxidative work-up upon completion of the reaction, the corresponding aldol product 123 (Scheme 3.3.5) was delivered in good yield (81\%) with moderate diastereoselectivity $(4: 1)$. 


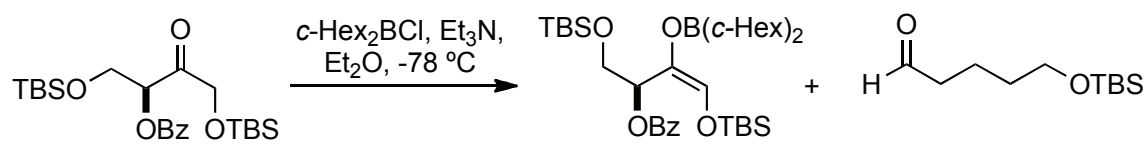

119

122

69

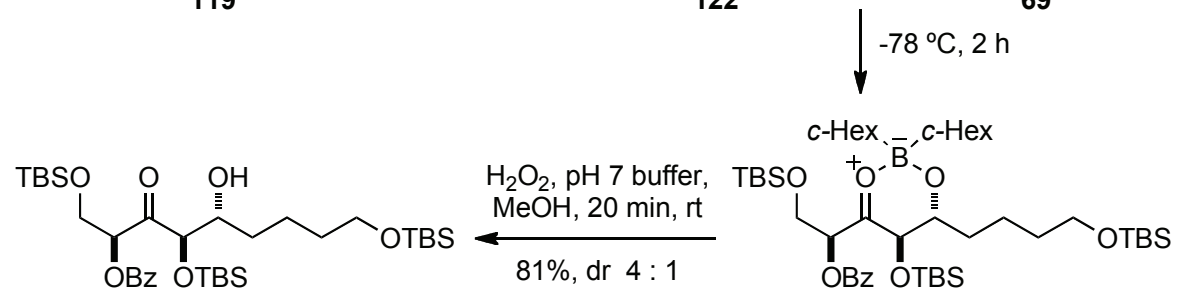

123

Scheme 3.3.5 Boron mediated anti-aldol reaction to form $\beta$-hydroxyketone 123 .

The stereochemical outcome of the aldol reaction of chiral $(E)$-enol borinate $\mathbf{1 2 2}$ is determined by a number of competing effects. A plausible explanation for product distribution in the first instance depends upon enolate geometry and its $\pi$-facial selectivity. Favoured transition state TS V shown in Figure 3.3.3, corresponding to the enolate re-face attack on the aldehyde, would account for the preferred 7,9-syn-9,10-anti (natural product numbering) arrangement in the product $\mathbf{1 2 3}$. The 1,3-allylic strain minimisation between $\alpha$-carbon substituents and the enolate OTBS group overrides the non-reinforcing electronic effect of the enol oxygen and $\alpha$-benzoyl oxygen. The benzoyl substituent is directed in contrasteric fashion, nonetheless stabilising transition state TS $\mathbf{V}$ by hydrogen-bonding to the formyl hydrogen (Figure 3.3.3). ${ }^{79,84}$ To restrain the steric hindrance between the $\alpha-\mathrm{OBz}$ moiety and one of the boron cyclohexyl ligands, the boat-like transition state TS V would predominate over a chair-like transition state. ${ }^{85}$

The product 124 corresponding to the enolate $s i$-face attack on the aldehyde, would be formed as a consequence of a much less stable transition state TS VI. The $\alpha-\mathrm{OBz}$ substituent is now located away from the pseudo-cycle, pushing the sterically more demanding OTBS group inwards, however this interaction can be relieved through the rotation of the $\mathrm{B}-\mathrm{O}$ bonds resulting in a boat-like conformation TS VI, though lacking the formyl hydrogen-bond stabilisation. 


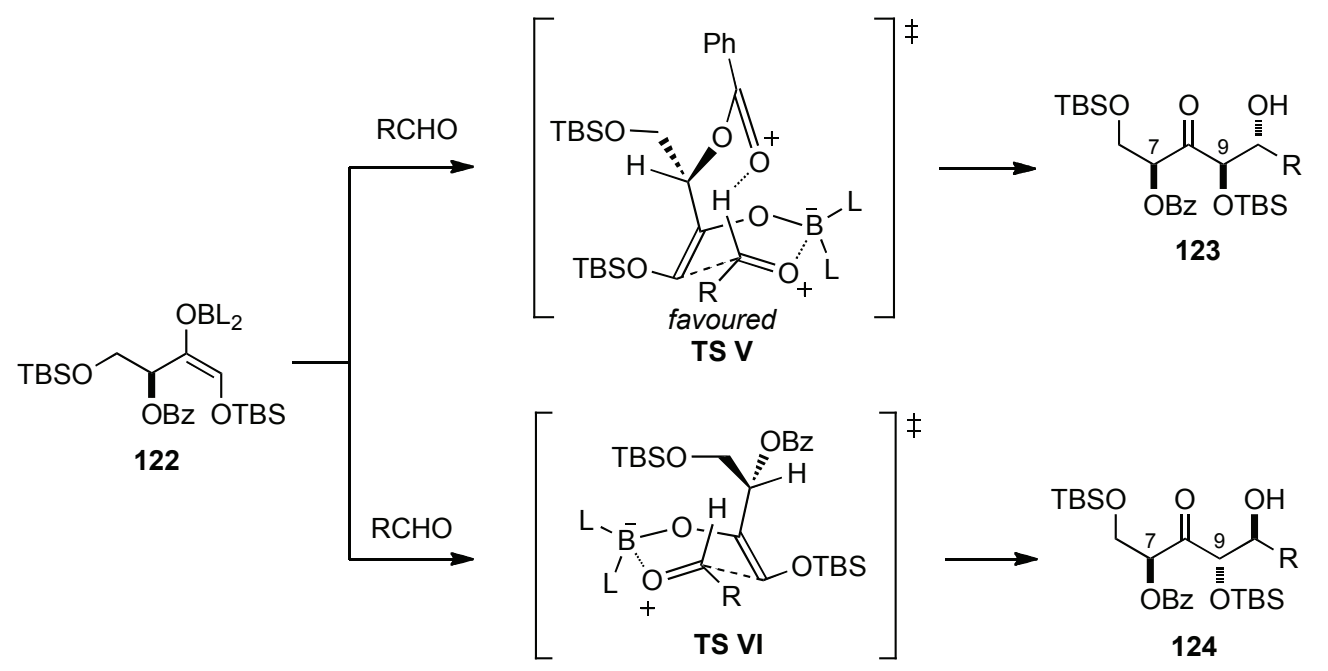

Figure 3.3.3 Rational for observed diastereoselectivity in boron-aldol reaction of chiral $(E)$-borinate 122.

\subsubsection{Completion of the synthesis of C7-C14 fragment}

Having formed the aldol product 123, it was observed that it is prone to rapid decomposition. Therefore, immediate protection of the $\mathrm{C} 10$ hydroxyl was required. However, the introduction of most protecting groups requires strong base or acid to effectively induce the desired transformation. In 2003, Basu and Rai reported a mild procedure to protect a hydroxyl group as its $p$-methoxybenzyl ether. ${ }^{86}$ The protocol employs trichloroacetimidate of $p$-methoxybenzyl alcohol and a catalytic amount of mild Lewis acids, such as $\mathrm{La}(\mathrm{OTf})_{3}$ or $\mathrm{Sc}(\mathrm{OTf})_{3}$ to efficiently form benzyl ethers with primary and secondary alcohols. With the awareness that this procedure has been widely employed in the synthesis of complex molecules, ${ }^{87,88}$ it was attempted first. Aldol product $\mathbf{1 2 3}$ was treated with PMBTCA and $\mathrm{La}(\mathrm{OTf})_{3}$ (Scheme 3.3.6), and the reaction was completed after six hours. Filtration, followed by column chromatography provided 125 in 65\% yield.

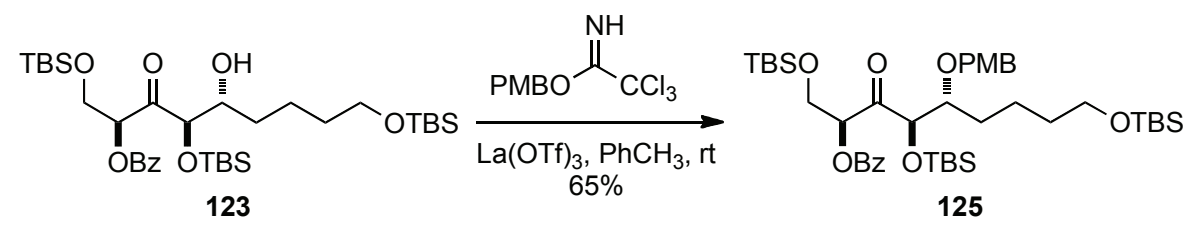

Scheme 3.3.6 Introduction of the PMB group to form 125 . 
Reduction of keto-ester 125 using lithium-aluminium hydride provided poor results, however using lithium borohydride a mixture of diols 126 was produced in 66\% yield. Separation of the diastereoisomers at this point was not required as in the next step the newly formed stereocentre would undergo oxidative cleavage to form the aldehyde 127.

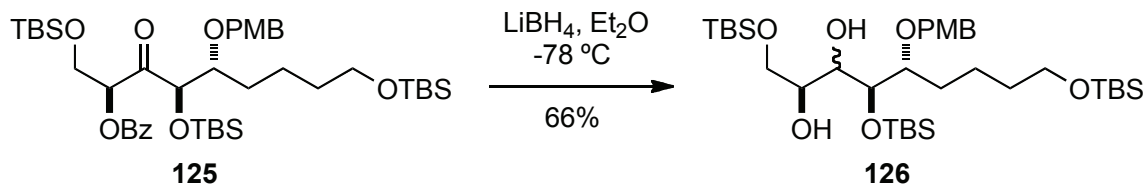

Scheme 3.3.7 Formation of diol 126.

Oxidative cleavage of 1,2-diol 126 with sodium meta-periodate was explored initially, however unsatisfactory results were obtained. Alternative cleavage with lead tetracetate provided the $\alpha, \beta$-bisalkoxy aldehyde 127 almost quantitatively (Scheme 3.3.8). Quick filtration and removal of solvent gave access to crude 127, which was used without further purification in the next step.

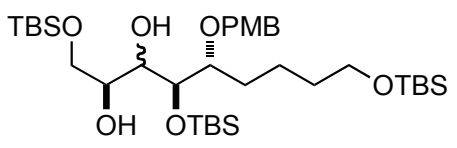

126

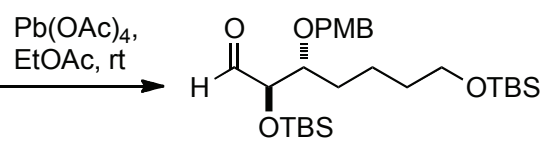

127

Scheme 3.3.8 Synthesis of aldehyde 127.

The synthesis of C7-C14 allylic alcohol 128 was completed with Grignard addition of vinyl magnesium bromide to the aldehyde 127 (Scheme 3.3.9). The allylic alcohol 128 was formed in $60 \%$ yield over two steps and with excellent $>95: 5$ diastereoselectivity.

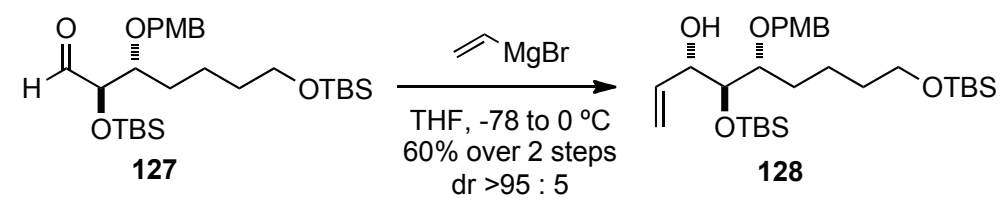

Scheme 3.3.9 Formation of C7-C14 allylic alcohol 128 
In 2006 Evans and co-workers investigated the asymmetric induction in methyl ketone aldol additions to $\alpha, \beta$-bisalkoxy aldehydes and based on the experimental data, reported that the selectivity in acyclic substrates can be predicted upon consideration of the polar Felkin-Anh or Cornforth models. ${ }^{89}$ In the reactions of 4,5-anti-bis-alkoxy aldehyde 129 preference for the formation of 3,4-anti products 130 rather than 131 was observed (Table 3.3.1), resulting from a $\pi$-facial selectivity of the aldehyde, antiparallel arrangement between the $\mathrm{C}=\mathrm{O}$ and $\alpha-\mathrm{C}-\mathrm{O}$ groups, and minimisation of the syn-pentane interactions. Extrapolating these results to our system, the proposed transition state TS VII (Figure 3.3.4) derived from a minimisation of the syn-pentane interactions, accounts for the observed selectivity in the 8,9-anti allylic alcohol 128.

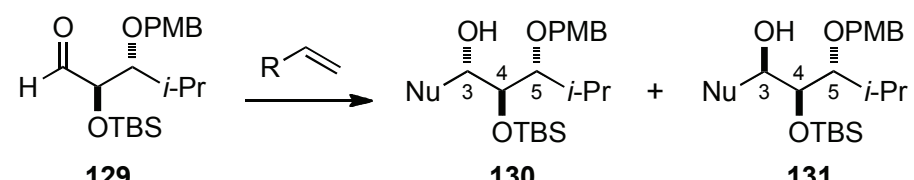

\begin{tabular}{cccc}
\hline \multicolumn{4}{c}{$3,4-$ anti $: 3,4-\operatorname{syn}($ yield \%) } \\
\hline $\mathrm{M}$ & $65: 35(83)$ & $91: 09(88)$ & $\mathrm{M}=\mathrm{Li}$ \\
$\mathrm{Me}$ & $41: 59(95)$ & $86: 14(92)$ & $>99: 01(95)$ \\
$i-\mathrm{Pr}$ & $09: 91(89)$ & $81: 19(90)$ & $>99: 01(98)$ \\
$t-\mathrm{Bu}$ &
\end{tabular}

Table 3.3.1 Aldol addition to $\alpha, \beta$-bisalkoxy aldehyde 129

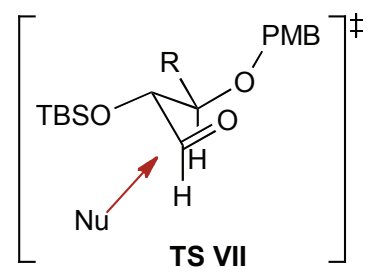

Figure 3.3.4 Evans-Cornforth model for addition to $\alpha, \beta$-bisalkoxy aldehyde.

Comparison with similar allylic alcohols 132 and 133 studied by Pikho during the synthesis of pectenotoxin- $2^{90}$ supported our stereochemical assignment. The reported $J$-coupling values for 3,4-syn arrangement in the allylic alcohol 133 are around $2.0 \mathrm{~Hz}$ and in the 3,4-anti configured product 132 over $6.0 \mathrm{~Hz}$ (Figure 3.3.5). Inspection of the ${ }^{1} \mathrm{H}$ NMR spectra of 128 provided results consistent with 8,9-anti configuration where $J_{\mathrm{H} 8 \text {-H9 }}$ was $5.0 \mathrm{~Hz}$. 


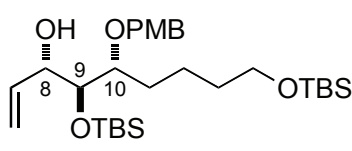

128

${ }^{3} J_{\mathrm{H} 8 \mathrm{H} 9}=5.0 \mathrm{~Hz}$

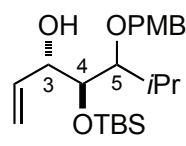

132

${ }^{3} J_{\mathrm{H} 3-\mathrm{H} 4}=6.4 \mathrm{~Hz}$

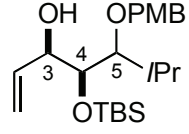

133

${ }^{3} J_{\mathrm{H} 3-\mathrm{H} 4}=2.0 \mathrm{~Hz}$

Figure 3.3.5 Comparison of the $J$ values for 1,2-syn and 1,2-anti arrangement.

After completing the analysis of the obtained data it became apparent that the allylic alcohol 128 possessed the undesired $(S)$ stereochemistry at the newly formed C8 stereocentre. Nevertheless, it was decided to explore the feasibility of a coupling strategy between C1-C6 subunit 111 and C7-C14 fragment 128, while revising the synthetic strategy to install the correct configuration at C8 in allylic alcohol 128.

\subsection{Installation of the $\mathrm{C6}-\mathrm{C} 7$ junction via cross-metathesis}

Synthetic efforts turned towards the investigation of cross-metathesis (CM) between the C1-C6 111 and C7-C14 128 fragments. According to the definition, olefin metathesis is a unique metal-catalysed carbon skeleton redistribution in which a mutual exchange of unsaturated carbon-carbon bonds takes place. ${ }^{91} \mathrm{CM}$ is one variant of that transformation, where two different olefins are cleaved, aided by a catalyst, and recombined in such a way to form the new alkene and liberate ethene. This process is subjected to the thermodynamic rules (Scheme 3.4.1) in which case the external delivery of ethene would reverse the reaction.

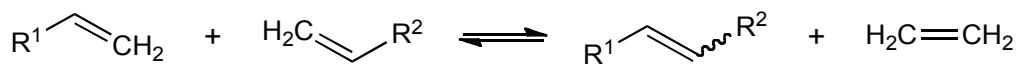

Scheme 3.4.1 Cross-metathesis reaction.

Despite broad interest and vast synthetic applications, the $\mathrm{CM}$ reaction presents certain limitations, with alkene selectivity being the major issue. In addition, complex product mixtures containing homodimers, heterodimers and side products are often obtained (complicating the purification and resulting in low yields).

To systematise the utility of the particular olefins in the process, categorisation and rules for selective CM have been established (Figure 3.4.1) after thorough investigation of literature examples. ${ }^{91}$ 
Unfortunately, under the investigated conditions formation of the heterodimer 134 was not observed, only the homodimer of the C1-C6 fragment 135 was isolated in 30\% yield together with unreacted 128 (40\%). Upon evaluation of this experiment it was hypothesised that the C1-C6 fragment 111 behaves like a Type I olefin whereas the allylic alcohol 128, despite being inactive in the cross metathesis, was involved in undetermined side reactions and so its classification remains ambiguous. The complex ${ }^{1} \mathrm{H}$ NMR spectra and unequal mass balance for compound $\mathbf{1 2 8}$ after the reaction suggested it was decomposing under the reaction conditions. Guided by these findings, considerable efforts were expended to achieve the cross coupled product, whilst employing different reaction conditions, which are summarised in Table 3.4.1. However, none of the catalysts screened in the different combinations of reaction parameters delivered the heterodimeric product 134, promoting, at best the formation of the homodimer 135.
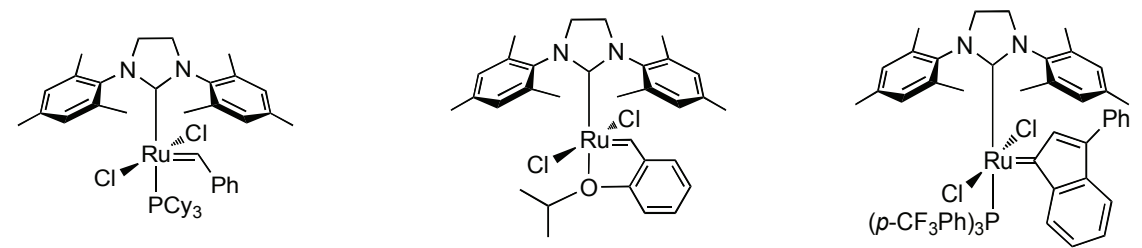

\begin{tabular}{lccc}
\hline Catalyst & Grubbs 2nd & Grubbs-Hoveyda & Nolan-type \\
\hline Cat. loading & $5-50 \mathrm{~mol} \%$ cat & $10 \mathrm{~mol} \%$ cat & $5-50 \mathrm{~mol} \%$ cat \\
Concen. & $0.001-0.1 \mathrm{M} / \mathrm{CH}_{2} \mathrm{Cl}_{2}$ & $0.001 \mathrm{M} / \mathrm{CH}_{2} \mathrm{Cl}_{2}$ & $0.001-0.1 \mathrm{M} / \mathrm{CH}_{2} \mathrm{Cl}_{2}$ \\
Time & $12-\mathbf{4 8} \mathrm{h}$ at rt & $24 \mathrm{~h}$ at rt & $12-48 \mathrm{~h}$ at rt \\
$\mathbf{1 1 1 : 1 2 8}(\mathrm{eq})$ & 1 to $10: 1$ to 2 & $5: 1$ & 1 to $10: 1$ to 2 \\
Result & dimer $\mathbf{1 3 5}(30 \%)$, unreacted $\mathbf{1 2 8}(40 \%)$ & dimer $\mathbf{1 3 5}(25 \%)$, complex mix \\
\hline
\end{tabular}

Table 3.4.1 Investigation of different conditions for $\mathrm{CM}$ reaction.

\subsection{C7-C14 fragment - structural reassignment}

While revising the $\mathrm{C} 7-\mathrm{C} 14$ synthesis and seeking an alternative coupling strategy further information pertaining to the stereochemistry of palmerolide $\mathrm{C}$ was provided. The initial assignment of the $\mathrm{C} 8-\mathrm{C} 10$ stereotriad was incorrect and revised to syn-anti $8 \mathrm{~S}, 9 \mathrm{~S}$, $10 S$, while the $19 R, 20 S$ were the same (Figure 3.5.1). This was due to an error in the Mosher ester analysis arising from the use of MTPA-acid chlorides where, according to Cahn-Ingold-Prelog rules, $(R)$-MTPA acid gives the $(R)$-ester but $(R)$-MTPA acid chloride 
provides the $(S)$-ester. The spectra for the $(R)$ - and $(S)$-esters were transposed resulting in the opposite assignment (Section 2.6, Chapter 2).
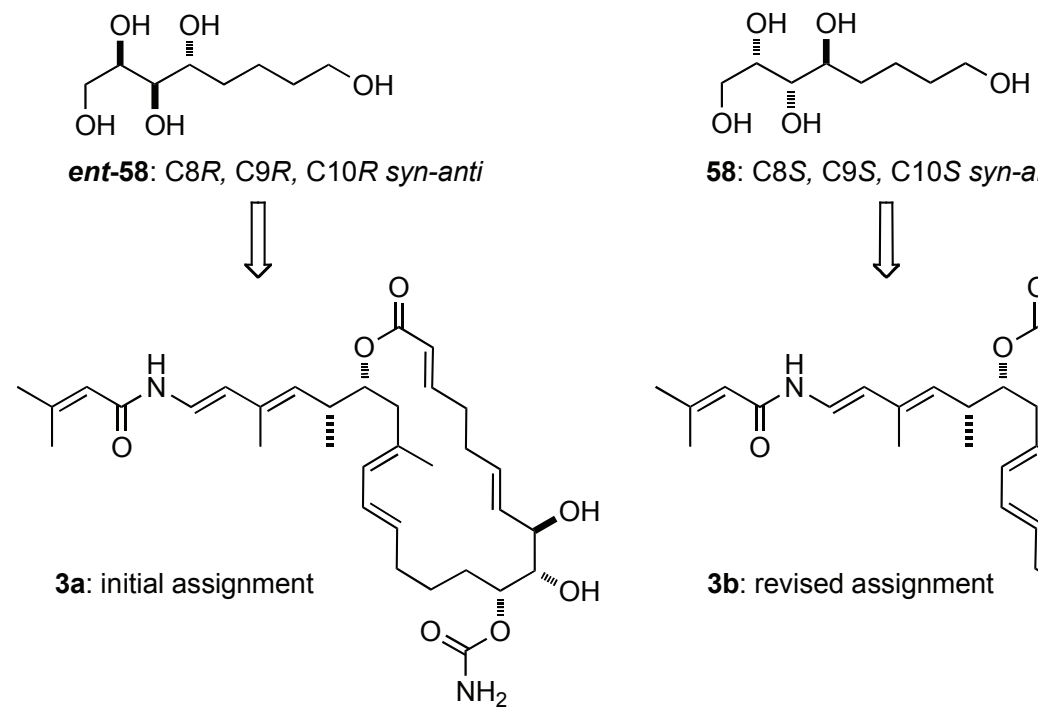

58: C8S, C9S, C10S syn-anti

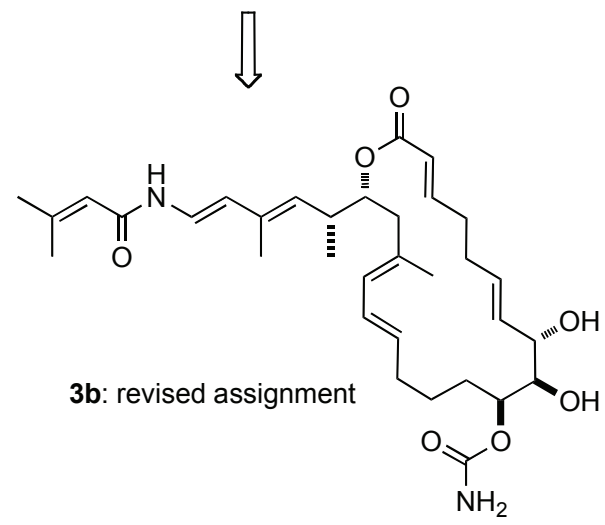

Figure 3.5.1 Revision of the configuration of palmerolide $C$.

In light of this revision, a synthesis reflecting the new assignment had to be devised. The immediate solution would be to change the configuration of the chiral ketone 119 to allow formation of the opposite enantiomer of the aldol product 123. However, D-erythrulose at the cost of $\$ 700$ USD for $100 \mathrm{mg}$ was not seen as viable. Attempts to invert the stereocentre in the TBS-protected L-erythrulose 136 using a Mitsunobu reaction were unsuccessful (Scheme 3.5.1).

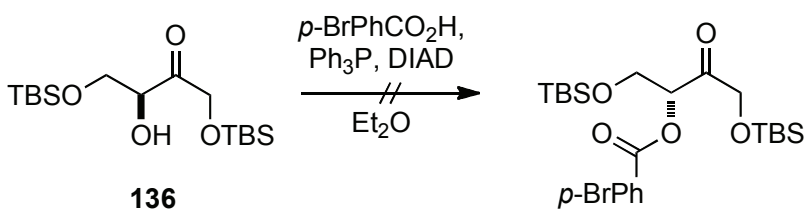

Scheme 3.5.1 Attempted Mitsunobu reaction to form D-erythrulose derivative.

The only possibility left within the approach of changing the stereochemistry on the chiral ketone 114 was to elaborate the route starting from L-ascorbic acid 115. This unfortunately resulted in a nine-step synthesis for one of the starting materials $\mathbf{1 3 7}$ which was produced in $10 \%$ overall yield (Scheme 3.5.2). 
<smiles>O=C1OC([C@@H](O)CO)C(O)=C1O</smiles>

115<smiles>CC(C)(C)C</smiles>

$11675 \%$ over three steps

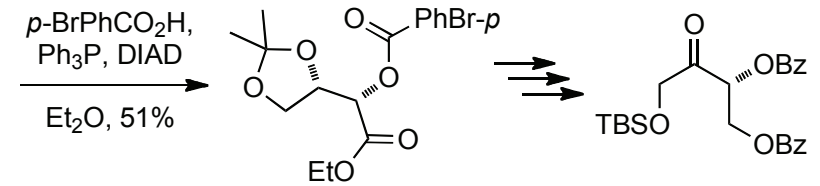

$13727 \%$ over five steps

Scheme 3.5.2 Synthesis of the $(R)$-configured ketone 137.

Undaunted, alternatives were sought and soon a revised plan was developed wherein the Crimmins ${ }^{93}$ thioxazoline 138 was employed in the aldol transformation to control the installation of the required stereocentres (Scheme 3.5.3). A revised strategy would considerably reduce the number of synthetic operations towards C7-C14 fragment, with the $\mathrm{N}$-glycolyloxazolidinethione auxiliary $\mathbf{1 3 8}$ prepared in three steps from (S)-phenylalanine.

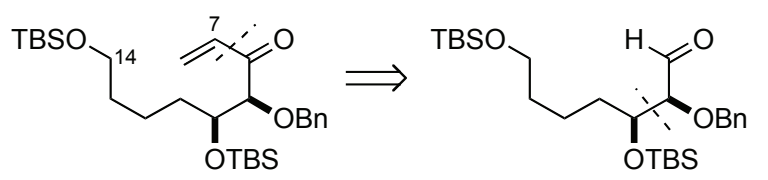

C7 - C14 subunit<smiles>C=COC(=O)N1C(=S)OCC1Br</smiles>

138<smiles>COCCCCC=O</smiles>

69

Scheme 3.5.3 Revised retrosynthesis of the C7-C14 fragment.

\subsection{Revised synthetic strategy for the C1-C14 fragment}

Several steps into our new synthetic approach towards Crimmins' thioauxiliary it was realised that the commercial supply of sparteine, a key component in this aldol reaction, was no longer available. Since our reserve of sparteine were quite limited, revision of the proposed strategy was inevitable. Gratifyingly, it was found that the aldol product $\mathbf{8 6}$ synthesised previously could be converted to $\alpha$-hydroxy ester using a reaction developed by Stoltz. ${ }^{94}$ This study showed application of dioxanone-derived products in a stereoselective alkylation reaction and further elaboration of the product 139 to the corresponding $\alpha$-hydroxyesters $\mathbf{1 4 0}$ as shown in Scheme 3.6.1. 


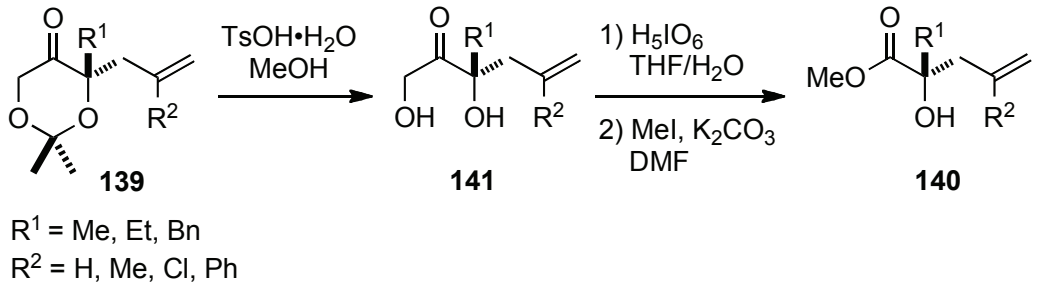

Scheme 3.6.1 Formation of the $\alpha$-hydroxy-ester 140 by Stoltz.

One drawback of this approach would be regioselectivity for oxidative cleavage in the absence of a quaternary $\alpha$-centre as in $\mathbf{1 4 1}$ (Scheme 3.6.1). However, only a synthetic investigation could provide valuable information. Therefore, it was decided to explore the acetonide deprotection, periodic acid-mediated cleavage and esterification on the suitably protected aldol product $\mathbf{8 6}$.

Bearing in mind the unsuccessful cross-metathesis studies, different coupling strategies were sought. As outlined in Scheme 3.6.2, the revised strategy towards $\mathbf{1 4 2}$ would rely on a C6-C7 aldol/dehydration disconnection revealing C1-C6 aldehyde 143 and C7-C14 ketone 144. The synthesis of ketone 144 with C9-C10 stereocentres installed by the proline-catalysed aldol reaction would incorporate the acetonide deprotection and oxidative cleavage to form carboxylic acid. The modified C1-C6 subunit 143 would be accessed using a HWE reaction of aldehyde 145 and triethyl phosphonoacetate 112.

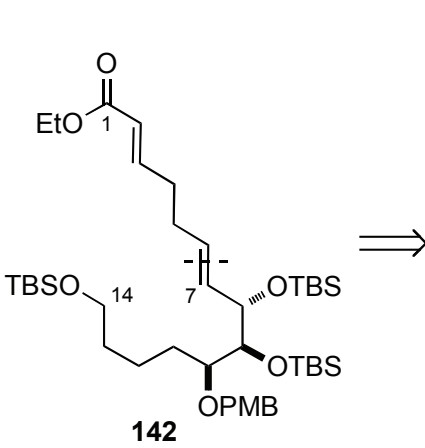

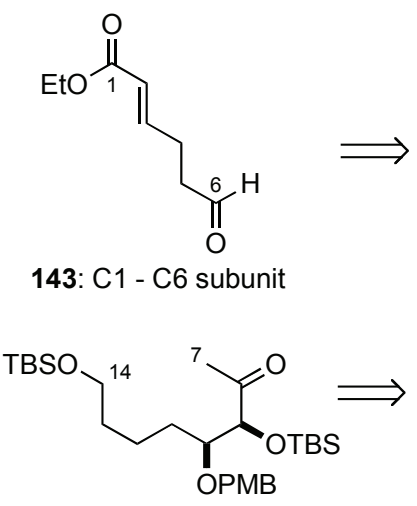

144: C7 - C14 subunit<smiles>CCOC(=O)CP(=O)(OCC)OCC</smiles>

112<smiles>C=CCCC(O)[C@H]1OC(C)(C)OCC1=O</smiles>

86

Scheme 3.6.2 Retrosynthesis of the C1-C14 fragment 142 . 


\subsubsection{Synthesis of the modified C1-C6 fragment}

The construction of the C1-C6 subunit 143 began with the preparation of the aldehyde 145. 1,4-Butanediol 146 was monoprotected with $\mathrm{TBSCl}$, subsequent oxidation under Swern conditions delivered 145 in 68\% over two steps (Scheme 3.6.3). HWE olefination of the aldehyde 145 with triethyl phosphonoacetate 112 was performed under Masamune-Roush conditions. ${ }^{75}$ The enoate $\mathbf{1 4 7}$ was produced in $77 \%$ yield as a single (E)-isomer (Scheme 3.6.3). Following TBS-ether cleavage, Swern oxidation completed the synthesis of C1-C6 fragment $\mathbf{1 4 3}^{95}$ in overall $41 \%$ yield over five steps.

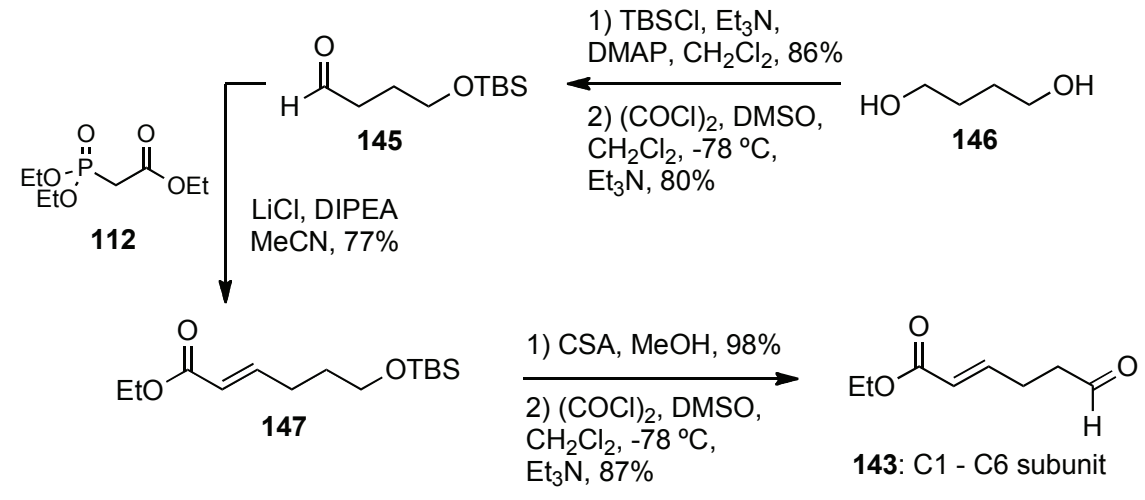

Scheme 3.6.3 Synthesis of the modified C1-C6 subunit 143.

\subsubsection{Synthesis of the modified C7-C14 fragment}

Attention was then turned to the synthesis of the aldol product $\mathbf{8 6}$ under prolinecatalysis. The work commenced with the preparation of bulk quantities of the starting materials applying the same methods as for the small-scale synthesis. The aldehyde $\mathbf{8 4}$ was readily accessed in more than $20 \mathrm{~g}$ scale. Synthesis of dioxanone 63 on $>50 \mathrm{~g}$ scale was more laborious; the initial acetonide $\mathbf{7 1}$ formation was carried out in DMF (Section 2.2), following a long extraction process producing high volume of solvents, which have to be removed. Removal of all traces of DMF before periodate cleavage was essential, as its similar boiling point with the final product made distillation troublesome. Consequently, dioxanone 63 was produced in $>30$ g quantity after distillation. The proline-catalysed reaction was then attempted on $>10 \mathrm{~g}$ (76.9 mmol) of ketone 63 and surprisingly the crossaldol product was produced in very low yield together with a range of side products making the column chromatography purification difficult. Therefore, several reactions (510 ) in parallel on a gram scale of ketone $\mathbf{6 3}$ were carried out and gratifyingly, 86 was 
isolated typically in 40-50\% yield per batch (Scheme 3.6.4).
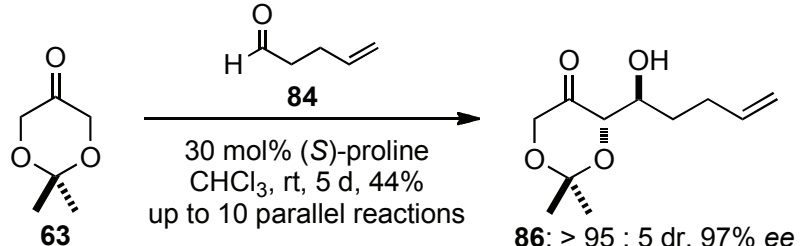

Scheme 3.6.4 Synthesis of the protected $(S)$-proline aldol product 86 .

Following the isolation of $\mathbf{8 6}$, installation of the protecting group required application of a mild protocol (Scheme 3.6.5). Therefore, p-methoxybenzyl trichloroacetimidate was employed in combination with catalytic lanthanide triflate to form C10 PMB-ether 148 in 77\% yield.<smiles>C/C=C\CCC(O)[C@H]1OC(C)(C)OCC1=O</smiles>

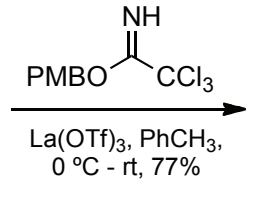<smiles>C=CCCC(O[Na])[C@H]1OC(C)(C)OCC1=O</smiles>

Scheme 3.6.5 Introduction of the PMB group to form 148.

With 148 in hand, attention turned towards formation of the $\alpha$-hydroxy ester 149. The acetonide 148 was subjected to deprotection with $p$-toluenesulfonic acid to access keto-diol 150 in 92\% yield (Scheme 3.6.6). Subsequent periodic acid oxidative cleavage afforded the intermediate $\alpha$-hydroxy carboxylic acid 151 together with aldehyde $\mathbf{1 5 2}$, which were taken as a crude mixture for alkylation with methyl iodide. Upon completion of the reaction, column chromatography afforded $\alpha$-hydroxy ester 149 in $64 \%$ yield and aldehyde 152 in $31 \%$.
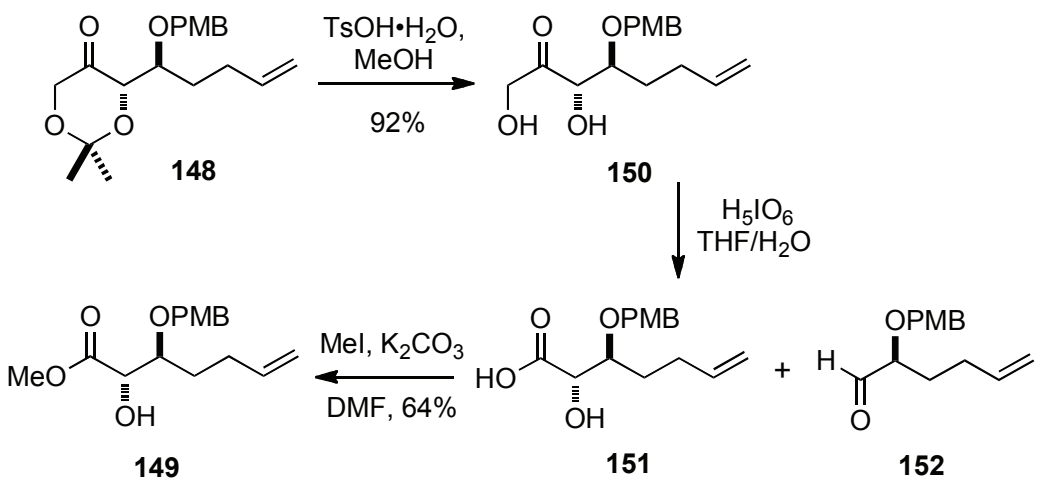

Scheme 3.6.6 Formation of the $\alpha$-hydroxy ester 149 . 
With $\alpha$-hydroxy ester 149 in hand, protection with TBSOTf and 2,6-lutidine gave 153 in $98 \%$ yield (Scheme 3.6.7). Installation of a terminal hydroxyl functionality was then required, and having previously used thexylBH $\mathrm{B}_{2}$ we decided to explore the optimised conditions. Therefore, compound $\mathbf{1 5 3}$ was added to a freshly prepared solution of thexylBH$H_{2}$ in tetrahydrofuran to furnish alcohol 154 after oxidative work-up in excellent 96\% yield (Scheme 3.6.7). Subsequent protection using $\mathrm{TBSCl}$ and imidazole afforded the intermediate 155 in $92 \%$ yield.

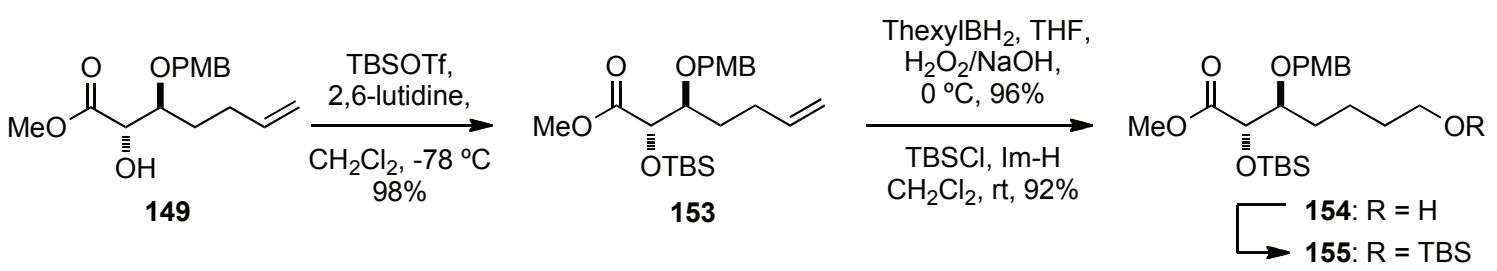

Scheme 3.6.7 Synthetic elaboration of $\alpha$-hydroxy ester 149 .

Having prepared compound $\mathbf{1 5 5}$, all that remained was to form methyl ketone 144 (Scheme 3.6.8). We chose to first introduce a Weinreb amide functionality to allow controlled alkylation with methyl Grignard. To ester 155 and $N, O$-dimethylhydroxylamine hydrochloride was added isopropylmagnesium bromide and intermediate Weinreb amide ${ }^{96}$ 156 was isolated in $97 \%$ yield. Subsequent addition of methylmagnesium bromide afforded methyl ketone $\mathbf{1 4 4}$ in $96 \%$ yield.

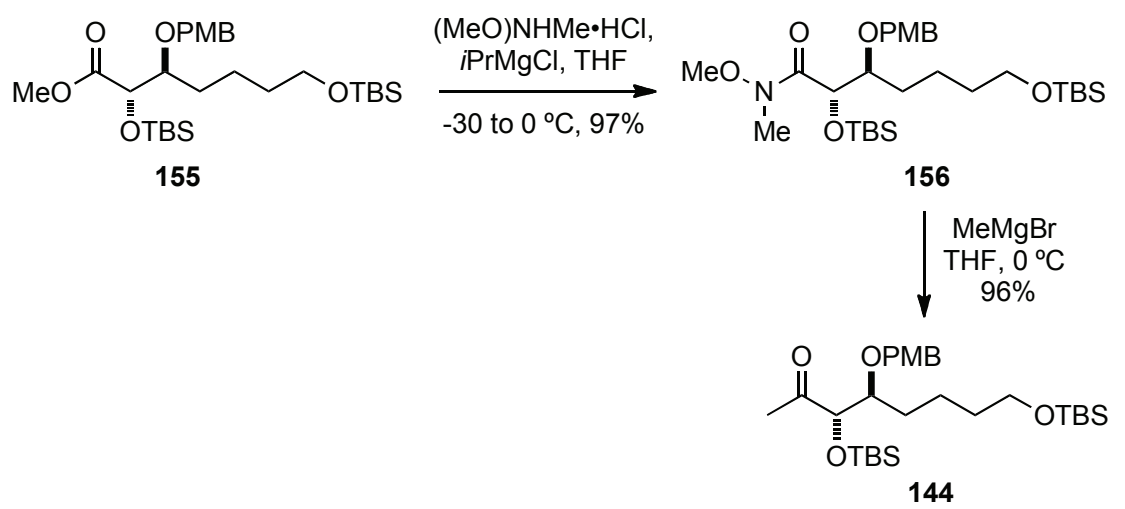

Scheme 3.6.8 Completion of the synthesis of the C7-C14 subunit 144 .

The C7-C14 methyl ketone $\mathbf{1 4 4}$ was synthesised in nine steps including the prolinecatalysed aldol reaction, which installed the desired stereochemistry with an excellent diastereo- and enantiocontrol. However, the isolated yield of the aldol product $\mathbf{8 6}$ is the 
only disadvantageous feature of this organocatalytic approach. Nonetheless, the remaining synthetic operations produced C7-C14 subunit 144 in 36\% yield over eight steps.

\subsection{Revised fragment union}

Having synthesised C1-C6 143 and C7-C14 144 fragments, attention was turned towards their coupling using an aldol/dehydration sequence. Building upon previous experience it was decided to utilise the boron aldol reaction to unite the two carbonyl components. $^{97}$ Selectivity was not an issue, since elimination of the newly formed stereocentre would immediately follows the $\mathrm{C}-\mathrm{C}$ bond forming step. Thus, enolisation of C7-C14 ketone 144 with $c-\mathrm{Hex}_{2} \mathrm{BCl}$ and triethylamine in diethyl ether at $-78{ }^{\circ} \mathrm{C}$, followed by addition of aldehyde 143, provided the corresponding aldol adducts 157 as an inconsequential mixture of C6 diastereoisomers $(90 \%, 1.6: 1 \mathrm{dr}$, Scheme 3.7.1)

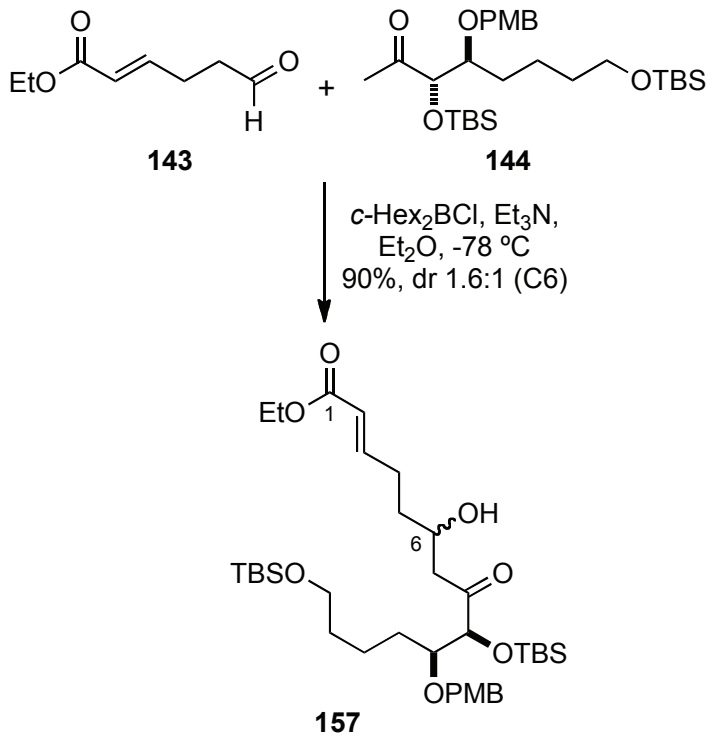

Scheme 3.7.1 C1-C6 and C7-C14 fragment union via aldol coupling.

Next, the one-pot mesylation/elimination procedure ${ }^{98}$ was explored. Even though it provided desired product $\mathbf{1 4 2}$, the obtained yield was unsatisfactory and extended exposure to DBU did not show any improvement in yield (Scheme 3.7.2). An alternative method utilising dehydrating agents such as the Burgess or Martin sulfurane reagents were considered next. Both procedures are reported to efficiently eliminate a molecule of water from secondary and tertiary alcohols. The first reagent methyl $N$-(triethylammoniumsulfonyl)carbamate (Burgess reagent), was found to proceed through E1 
elimination pathway, abstracting hydrogen in syn relation to the leaving group due to the geometrical constraints on the reagent. ${ }^{99}$ Inspection of the literature showed Martin sulfurane reacts with secondary alcohols via E2 elimination. The antiperiplanar geometry in this step enables stereospecific alkene generation from chiral secondary alcohols. Hence, we chose to investigate dehydration with Martin sulfurane reagent. Treatment of $\mathbf{1 5 7}$ with a solution of the Martin sulfurane ${ }^{100}$ reagent at $-10^{\circ} \mathrm{C}$ provided enone 142 in less than one hour in excellent $95 \%$ yield and exclusive $(E)$-selectivity.
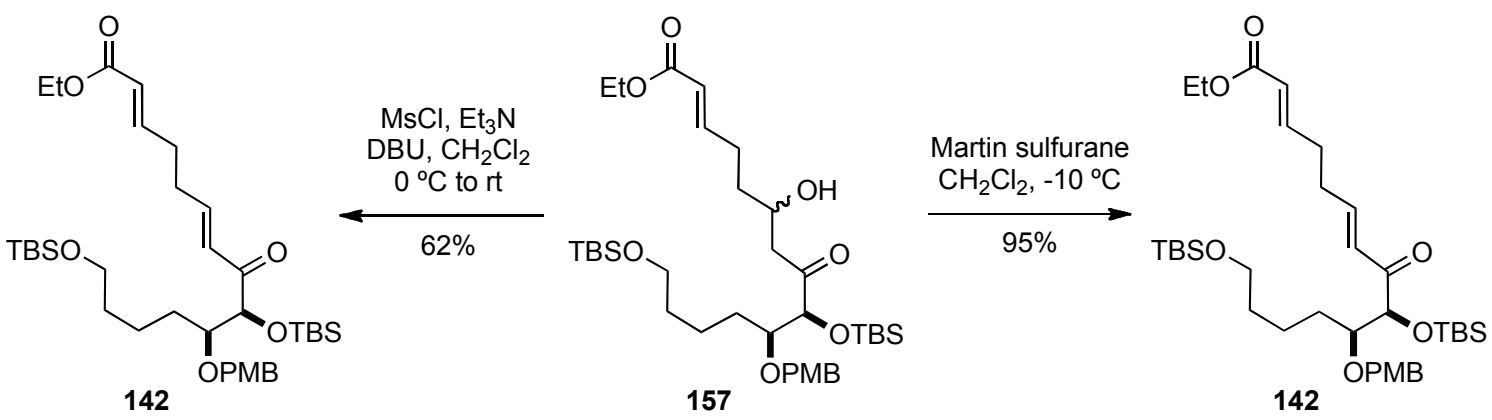

Scheme 3.7.2 Dehydration of the aldol product 157 to form enone 142 .

Martin sulfurane reagent $\mathbf{1 5 8}$ (Scheme 3.7.3), is a ketal analogue of bi-phenylsulfide, which reacts by rapid exchange of one of the alkoxy ligands with the reacting alcohol to yield sulfurane $\mathbf{1 5 9}$ and fluoro-alcohol. Key intermediate alkoxysulfonium ion $\mathbf{1 6 0}$ is formed upon extrusion of the other fluoro-alkoxy ligand, which comes back to attack the $\alpha$-hydrogen and release alkene $\mathbf{1 6 1}$ and bi-phenyl sulfoxide 162 (Scheme 3.7.3).
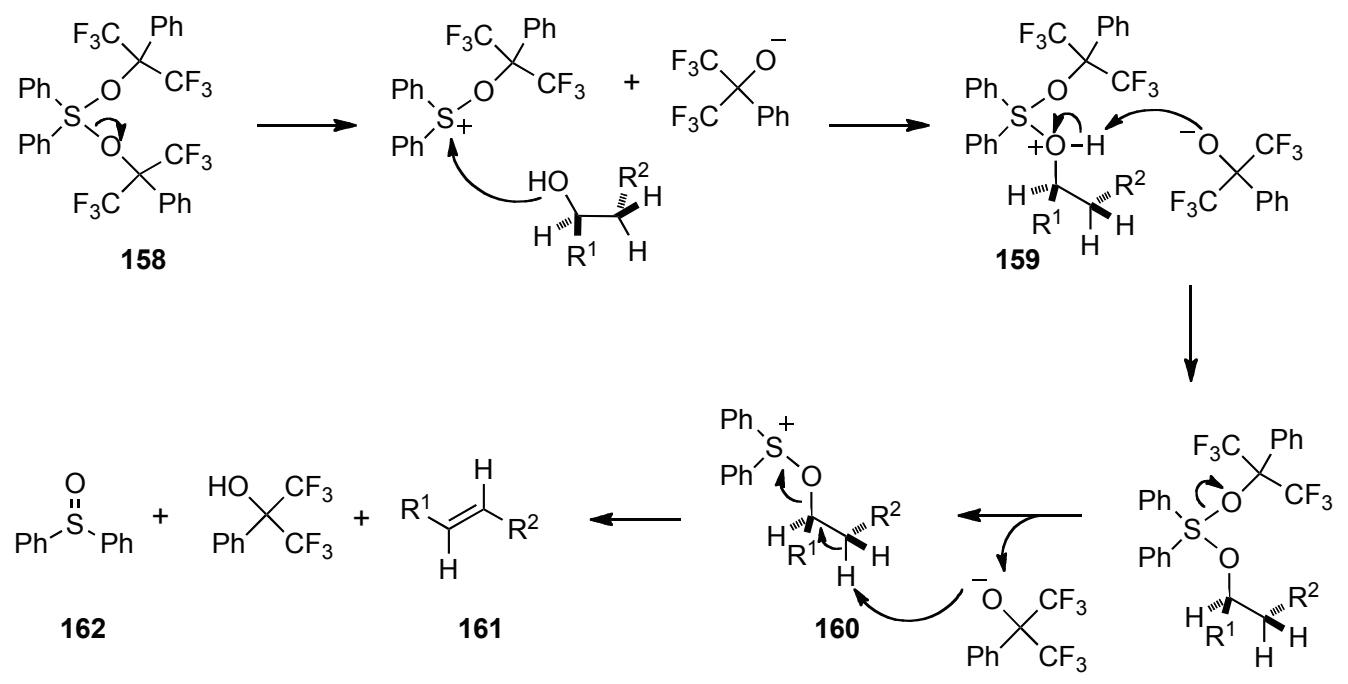

Scheme 3.7.3 Proposed mechanism of Martin sulfurane dehydration. 


\subsection{Advancing the C1-C14 subunit}

\subsubsection{Reduction of enone 142 and configurational analysis}

With the $\mathrm{C} 1-\mathrm{C} 14$ fragment 142 in hand, investigation of the enone reduction began. Drawing upon previous experience with addition to $\alpha, \beta$-bisalkoxyaldehyde $\mathbf{1 2 7}$ high levels of control in the reduction of $\mathbf{1 4 2}$ were expected (see section 3.3.2.3). The selective conversion of enone 142 to the allylic alcohol 163 was achieved under Luche conditions employing cerium chloride and sodium borohydride. The reduction product 163 was formed in excellent 90\% yield and $92: 8$ diastereoselectivity, as established by ${ }^{1} \mathrm{H}$ NMR (Scheme 3.8.1).

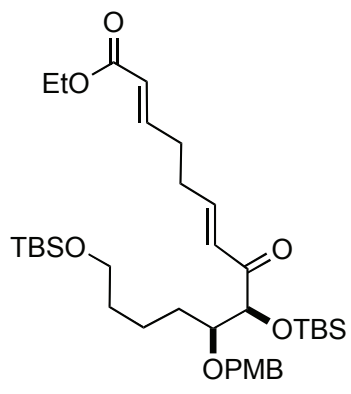

142

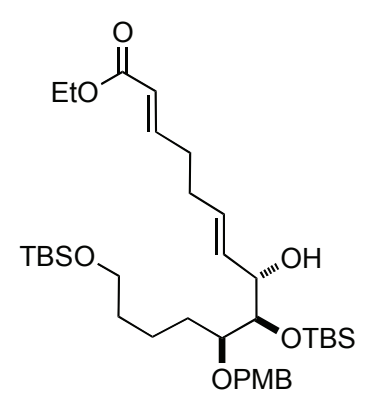

163

Scheme 3.8.1 Reduction of enone 142 introducing C8 stereocentre.

The diastereoselection can be explained by consideration of the Evans-Cornforth model discussed in section 3.3.2.3. ${ }^{89}$ However, in this instance direct minimisation of the syn-pentane interactions (TS VIII) promotes formation of the C8S configured product $\mathbf{1 6 3}$ (Figure 3.8.1).

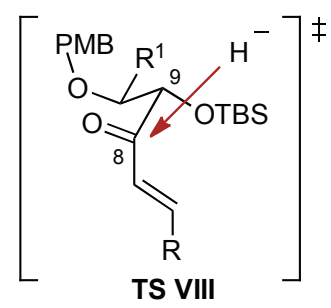

Figure 3.8.1 Evans-Cornforth model for diastereoselective reduction.

The stereochemistry of the C8 hydroxyl was confirmed after the synthesis of $(R) /(S)$-MTPA ester derivatives 164 and 165 (Table 3.8.1). Analysis of the $(R) /(S)$-MTPA 
${ }^{1} \mathrm{H}$ NMR spectra according to the advanced Mosher method, revealed the H1-H7 protons shielded in the (S)-MTPA ester 165 thus giving negative values after deduction, the H8H14 protons returned positive values due to being moved upfield in the (R)-MTPA ester 164, providing proof for the $\mathrm{C} 8 S$ configuration. With the successful installation of the $\mathrm{C} 8$ stereocentre and confirmation of its configuration, the $\mathrm{C} 8-\mathrm{C} 10$ stereotriad has been completed with high levels of diastereo- and enantiocontrol.

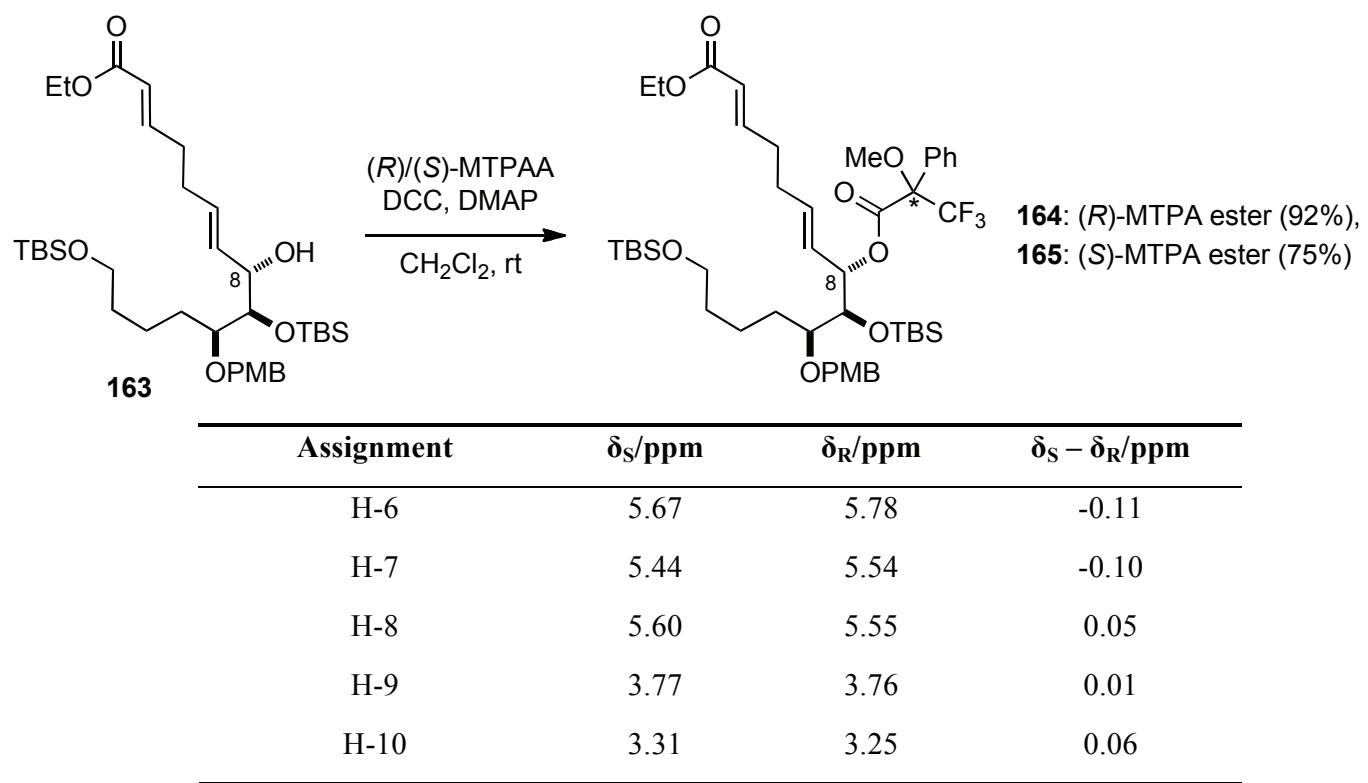

Table 3.8.1 Formation of the (R)- and (S)-MTPA esters 164 and 165, and ${ }^{1} \mathrm{H}$ NMR analysis.

\subsubsection{Synthesis of the C1-C14 aldehyde}

Following the enone reduction, the newly formed C8 hydroxyl in 163 was protected as its TBS ether employing TBSOTf and 2,6-lutidine to provide 166 in 87\% yield (Scheme 3.8.2). In order to minimise the number of synthetic operations on the advanced material and taking into consideration the challenges in removing the PMB ether in the presence of the $\mathrm{C} 14-\mathrm{C} 17$ diene encountered by Hall et al. during the synthesis of palmerolide $\mathrm{A} \mathrm{4},{ }^{8}$ it was decided to introduce the $\mathrm{C} 10$ carbamate at this stage. Therefore, DDQ deprotection of the PMB-ether of 166 revealed the C10 hydroxyl, providing 167 in $88 \%$ yield (Scheme 3.8.2). 


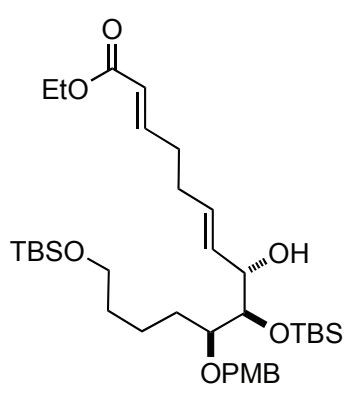

163

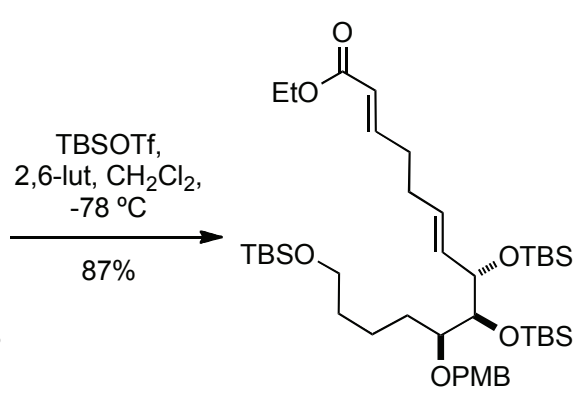

166

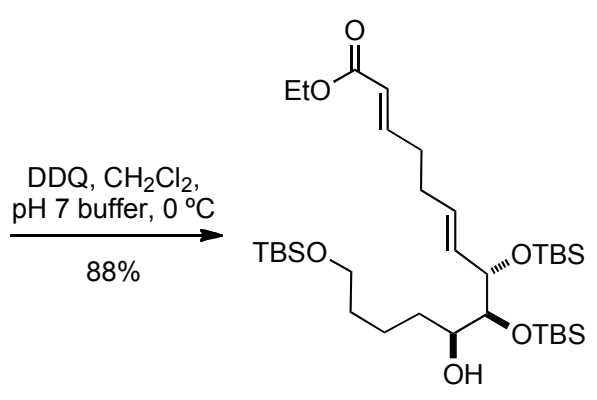

167

Scheme 3.8.2 Protection/deprotection to form C10 alcohol 167.

With compound $\mathbf{1 6 7}$ in hand, installation of the carbamate at the C10 position was required. The employed procedure utilised trichloroacetyl isocyanate ${ }^{101}$ to form the corresponding trichloroacetyl carbamate, subsequent hydrolysis on neutral alumina followed by filtration provided unsubstituted carbamate 168 in excellent $72 \%$ yield (Scheme 3.8.3).

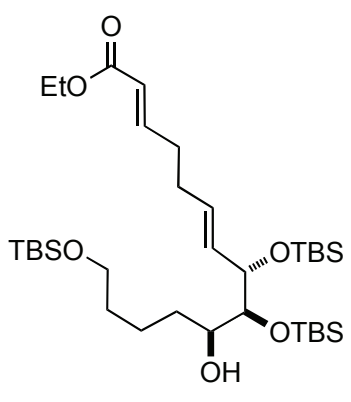

167

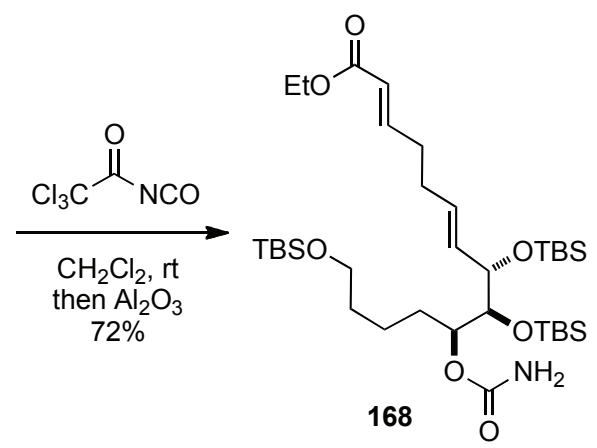

168

Scheme 3.8.3 Formation of the carbamate 168 .

To complete the synthesis of C1-C14 fragment 169 (Scheme 3.8.4), the primary TBS ether in 168 was cleaved under acidic conditions in a $6: 1$ mixture of $\mathrm{CH}_{2} \mathrm{Cl}_{2} / \mathrm{MeOH}$, which proved optimal for selective deprotection. The alcohol 170 was obtained in excellent 93\% yield, and subsequent oxidation with Dess-Martin reagent afforded the C1-C14 aldehyde 169 in 80\% yield. 

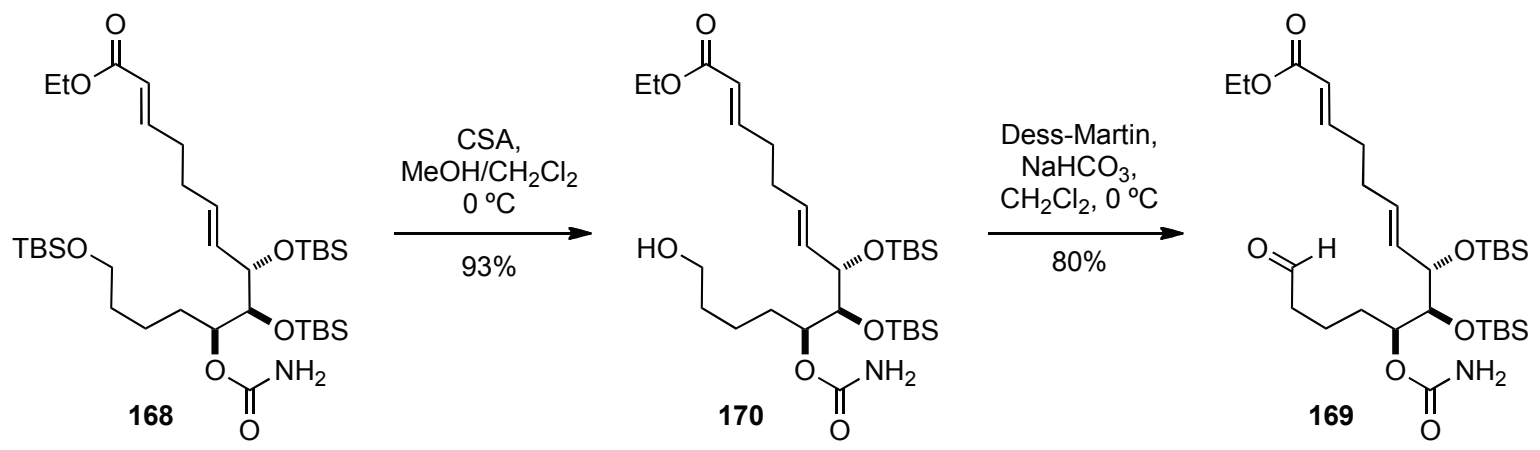

Scheme 3.8.4 Completion of the C1-C14 aldehyde 169.

\subsection{Summary}

The C1-C14 subunit 169 was completed in seventeen steps from the proline-aldol reaction of ketone 63 and aldehyde 84 in $6 \%$ overall yield. Although, the first organocatalytic transformation provided product $\mathbf{8 6}$ in moderate yield, the remaining synthetic operations proved to be very efficient and reliable. Further complexity elements such as the installation of the C8 stereocentre with required $(S)$ stereochemistry and introduction of the C10 carbamate functionality were successfully achieved. 


\section{Chapter 4}

\subsection{Retrosynthetic analysis of the C15-C24 fragment 171}

In planning the synthesis of the C15-C24 fragment 171 (Scheme 4.1.1), a vinylogous aldol disconnection was viewed as the most efficient approach. This required an $\alpha$-chiral aldehyde 172 bearing the sensitive dienyl-iodide functionality and the corresponding silyl ketene acetal 173 or enolate of acrylate 174. By choosing this disconnection, selective installation of the C19 stereocentre could be fully addressed. Either employing Yamamoto's vinylogous aldol reaction between 172 and 174 where bulky Lewis acid aluminum tris-(2,6-diphenyl)-phenoxide (ATPH) would control regioselectivity, or a vinylogous Mukaiyama aldol reaction of $\mathbf{1 7 2}$ and 173, the stereoselectivity would depend on Felkin-Anh control. ${ }^{102,103}$ The synthetic design based on this disconnection would reveal divinyl iodo-aldehyde 172, which would be accessed via Takai olefination of aldehyde 175. Enal 175, would arise from a HWE reaction of aldehyde 176, which could be prepared from methyl (2S)-3-hydroxy-2-methylpropionate 177, commonly known as Roche ester.
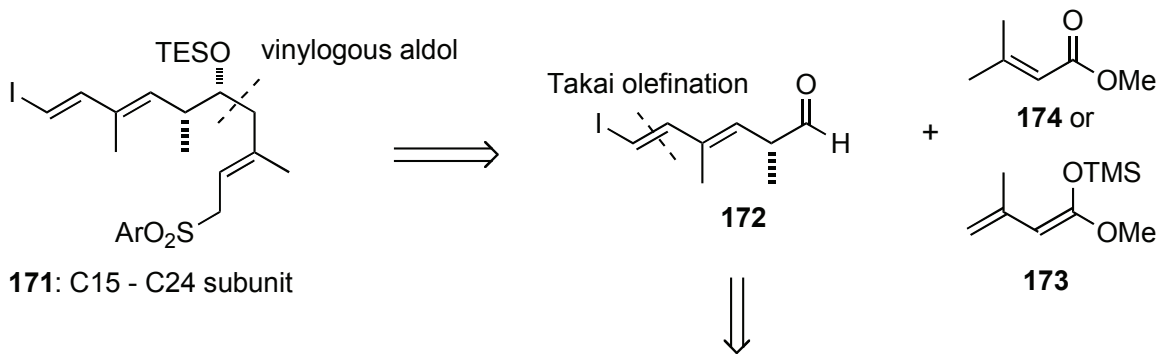

171: C15 - C24 subunit

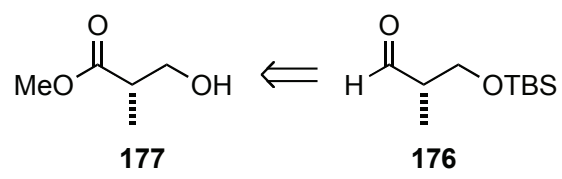<smiles>C=CC(=O)C(C)(C)C(C)(C)C[C@H](C)CO[SbH3]</smiles>

Scheme 4.1.1 Retrosynthetic analysis of C15-C24 fragment 171. 


\subsection{Preparation of the vinyl iodo-alcohol 178}

The synthesis of the C15-C24 subunit 171 began with the three-step synthesis of aldehyde 176. Treatment of $(S)$-Roche ester 177 with TBSCl and imidazole in dichloromethane provided derivative of $\mathbf{1 7 7}$ (Scheme 4.2.1). Subsequent formation of the Weinreb amide 179 via reaction with Weinreb salt and $i$-propylmagnesium chloride in tetrahydrofuran, followed by DIBAL-H reduction of 179 provided aldehyde $176,{ }^{73}$ which was used immediately in the HWE reaction (Scheme 4.2.1).

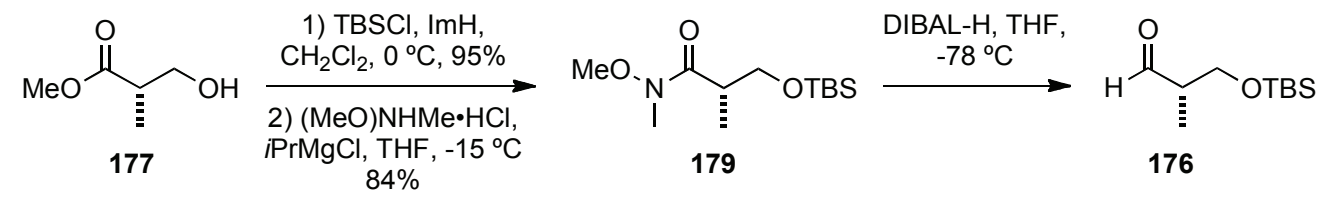

Scheme 4.2.1 Preparation of TBS-protected aldehyde 176.

The freshly prepared aldehyde $\mathbf{1 7 6}$ was used in a HWE reaction with triethyl 2-phosphonopropionate $\mathbf{1 8 0}$ and barium hydroxide (Scheme 4.2.2). ${ }^{104}$ The formation of trisubstituted enoate 181 was achieved in $76 \%$ yield from intermediate 179 with good $E: Z$ selectivity $(7: 1)$. Studies performed by Sinisterra provided some insight into the mechanism and selectivity of the reaction. He found that the interfacial solid-liquid interactions between the substrate and microcrystalline structure of the barium hydroxide were critical to that system. ${ }^{105}$

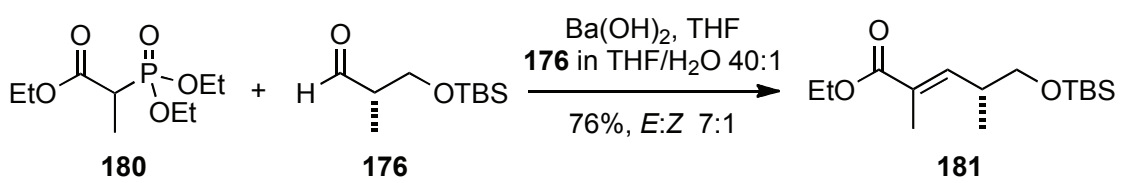

Scheme 4.2.2 Horner-Wadsworth-Emmons olefination to form enoate 181.

Treatment of the enoate 181 with DIBAL-H provided alcohol 182 in $81 \%$ yield, which was readily separated from the remaining $Z$-isomer. Subsequent oxidation using Dess-Martin periodinane afforded aldehyde $\mathbf{1 7 5}$ in 90\% yield (Scheme 4.2.3).<smiles>CCOC(=O)/C=C(\C)C[C@H](C)C[Sb]</smiles>

181

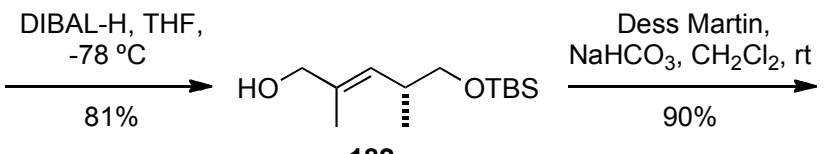

182<smiles>CC(C=O)=C[C@@H](C)CO[Sb](=O)=O</smiles>

175

Scheme 4.2.3 Formation of the aldehyde 175. 
The stage was now set for the introduction of the vinyl iodide moiety. Takai olefination would provide direct access to the vinyl iodide and it generally proceeds in high yield and with good (E)-selectivity. ${ }^{37}$ In principle, this process involves the chromium(II)-mediated one-carbon homologation of an aldehyde with haloform to provide the corresponding vinyl halide. Takai and Utimoto suggested that the haloform first reacts with chromium to form a nucleophilic geminal-dichromium species 183 which attacks the aldehyde (Scheme 4.2.4) $)^{37}$ and the $(E)$-alkene is formed from the $\beta$-oxychromium intermediate 184. The rate of the reaction and selectivity are dependant on the haloform employed and increases in the order of $\mathrm{Cl}<\mathrm{Br}<\mathrm{I}$.

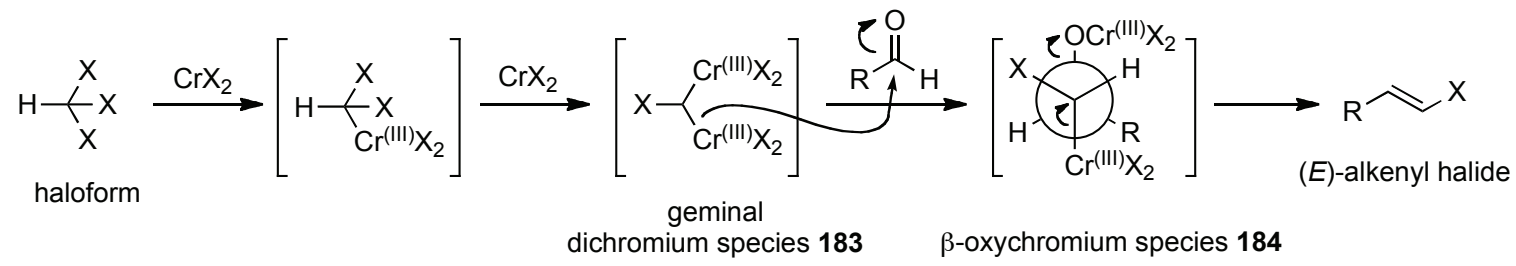

Scheme 4.2.4 Proposed mechanism of Takai olefination.

Thoroughly flame-dried chromium chloride was suspended in tetrahydrofuran (care was taken to mechanically agitate the suspension to ensure that it had not fused) and the mixture of aldehyde 175 and iodoform in 1,4-dioxane was added dropwise over one hour. Although the corresponding product $\mathbf{1 8 5}$ was formed within an hour, as indicated by TLC, the purification by column chromatography significantly reduced the yield of isolated $\mathbf{1 8 5}$ (Scheme 4.2.5). In order to overcome this problem, direct deprotection with TBAF was performed to provide iodo-alcohol $\mathbf{1 7 8}$ in $90 \%$ yield. Upon comparison of different trials of a Takai olefination, it was noticed that the concentration and ratio of 1,4-dioxane : THF was critical to reaction yield and selectivity. The best yield with exclusive $(E)$ product 178 was obtained when $7: 1$ mixture of 1,4-dioxane : THF was used, under strictly anhydrous conditions. It is worth noting that the obtained product $\mathbf{1 7 8}$ could only be stored for short period of time at low temperatures, with the exclusion of light. 


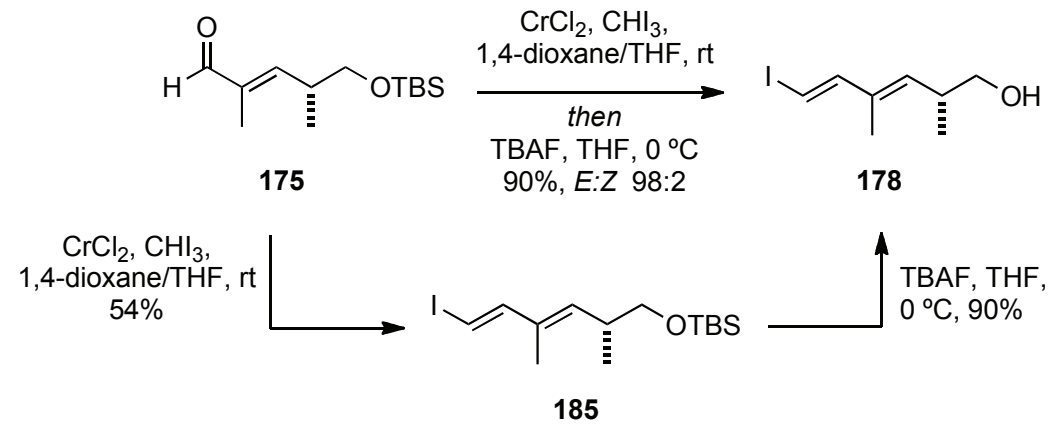

Scheme 4.2.5 Formation of the iodo-alcohol 178 .

\subsection{Vinylogous Yamamoto aldol reaction}

With iodo-alcohol $\mathbf{1 7 8}$ in hand attention turned towards the construction of the C18-C19 bond. The initial plan envisioned a Yamamoto vinylogous aldol reaction using aluminum tris-(2,6-diphenyl)-phenoxide (Figure 4.3.1) as a Lewis acid to build up the required $\mathrm{C} 18-\mathrm{C} 19$ connection.

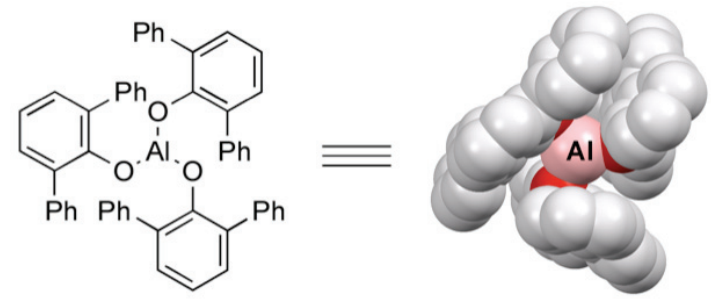

Figure 4.3.1 Yamamoto bulky Lewis acid ATPH.

This transformation is dependant on the host properties of the bulky Lewis acid ATPH for carbonyl guests. The ATPH and carbonyl compounds self-assemble to form complexes where the carbonyl can either be protected towards nucleophilic addition or activated towards selective transformations unattainable under normal conditions. Complexation of an equimolecular mixture of an unsaturated enolisable enoate $\mathbf{1 8 6}$ and an aldehyde 187 with ATPH encapsulates the carbonyl substrates in the cavity of the Lewis acid, shielding their $\alpha$-carbons (Scheme 4.3.1). The sterically accessible $\gamma$-proton can then be deprotonated selectively by a bulky base such as lithium diisopropylamide (LDA), affording an extended enolate which then adds in a $\gamma$-1,2-fashion to the aldehyde $\mathbf{1 8 7}$ giving product 188 in $97 \%$ yield. ${ }^{106-109}$ 


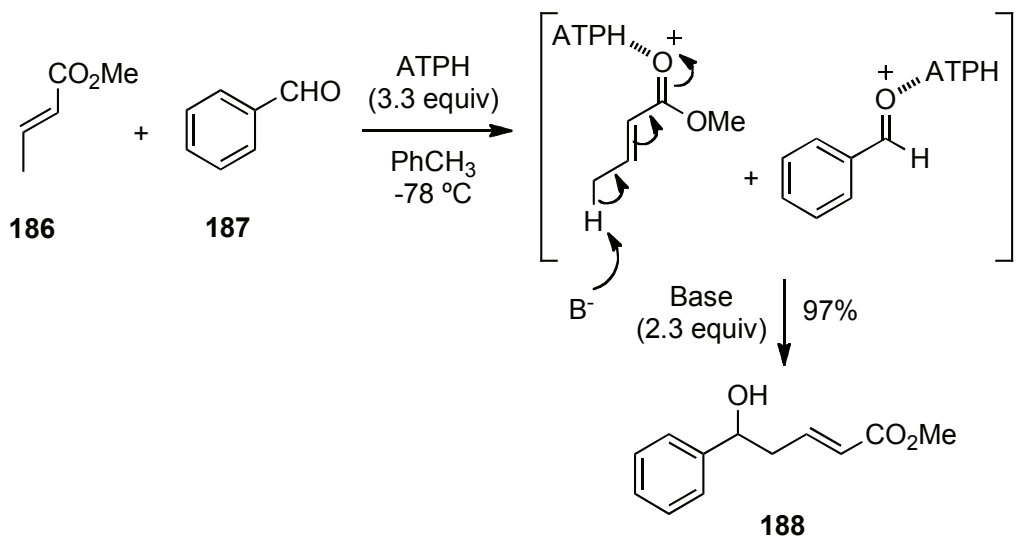

Scheme 4.3.1 Yamamoto vinylogous aldol reaction.

Investigation of the Yamamoto protocol began with oxidation of the alcohol $\mathbf{1 7 8}$ using Dess-Martin reagent ${ }^{10,111}$ to provide aldehyde $\mathbf{1 7 2}$ in $90 \%$ yield. The isolation of the aldehyde $\mathbf{1 7 2}$ proved challenging as it readily decomposed on exposure to light. In order to minimise this exposure, rapid purification of the reaction mixture through a short pad of silica/celite gel, followed by solvent removal and drying under reduced pressure in the dark provided access to $\mathbf{1 7 2}$.

Aldehyde 172 was then added to the freshly prepared mixture of ATPH and methyl 3,3-dimethylacrylate 174 in toluene at $-78{ }^{\circ} \mathrm{C}$. Upon complexation, a freshly prepared solution of LDA was added to the reaction mixture (Scheme 4.3.2). Despite several attempts of the reaction with the highest-purity reagents, no coupled product 189 was observed. Attempts to use aldehyde 190 instead of acrylate 174, also failed to deliver the desired product, only decomposition of the iodo-aldehyde 172 occurred.

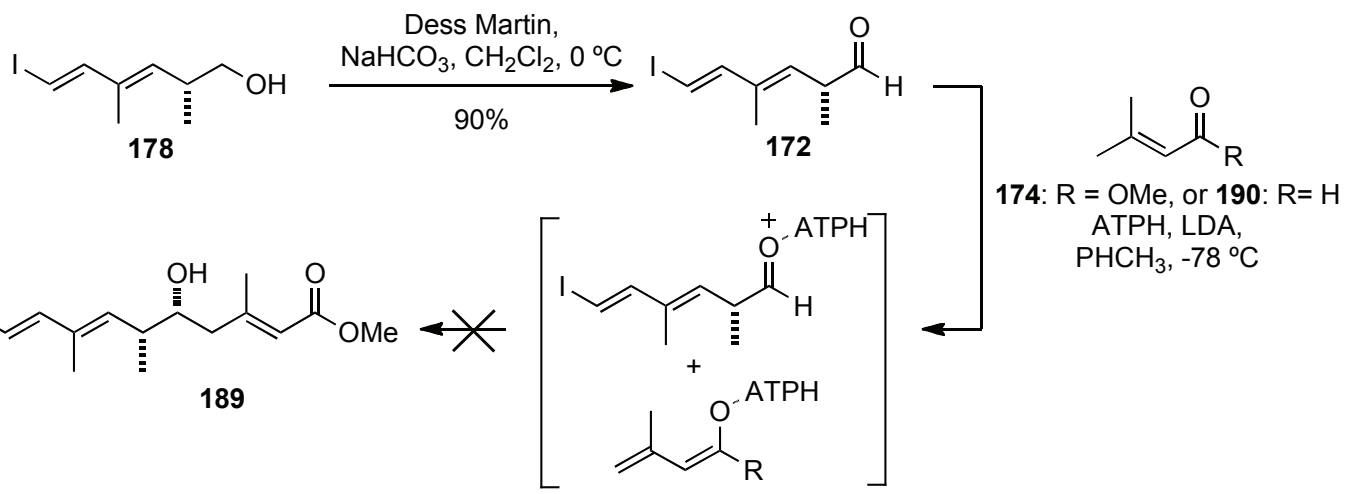

Scheme 4.3.2 Attempted vinylogous Yamamoto aldol reaction to form 189 . 


\subsection{Vinylogous Mukaiyama aldol reaction}

Attention was then focused on the investigation of a vinylogous Mukaiyama aldol reaction (VMAR) between the silyl ketene acetal $\mathbf{1 7 3}$ and aldehyde $\mathbf{1 7 2} .^{112}$ The observed regioselectivity in this reaction can be rationalised upon consideration of the electronic and steric effects of the silyl ketene acetal 173. As described by Denmark, ${ }^{113} \mathbf{1 7 3}$ is a highly electron rich species and its reactions are governed by electrostatic interactions. Relying on the computational calculations for the orbital coefficients (O.C.) and electrophilic susceptibilities (E.S.) of $\mathbf{1 7 3}$ and similar species (silyl enol ether 190, lithium enolate of the methyl crotonate 191), Denmark postulated that $\gamma$-selectivity could be predicted due to the higher electron density observed around C4 rather than the C2 position (Figure 4.4.1). Steric effects contribute to the site selectivity; C2 in $\mathbf{1 7 3}$ is a more sterically hindered site owing to its proximity to the silyl group and the alkyl group of the ester. Therefore, the approach of the electrophile to the less sterically demanding $\mathrm{C} 4$ position is favoured when C4 is not similarly substituted. ${ }^{113}$

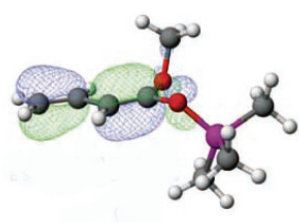

silyl ketene acetal $\mathbf{1 7 3}$

C4 4 C3 C2 21

O.C: $0.302,0.090,0.230,0.219$

E.S: $0.592,0.177,0.451,0.429$

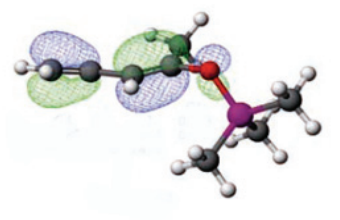

silyl enol ether 190

C4 4 C3 $22 \quad \mathrm{C} 1$

O.C: $0.280,0.083,0.243,0.235$

E.S: $0.550,0.165,0.478,0.461$

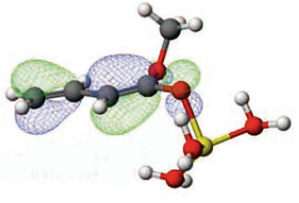

lithium dienolate 191

C4 43 C2 21

O.C: $0.289,0.042,0.311,0.130$

E.S: $0.572,0.084,0.614,0.257$

Figure 4.4.1 Structures of silyl ketene acetal 173, silyl enol ether 190 and lithium dienolate 191, reproduced from ref ${ }^{113}$.

With the aldehyde $\mathbf{1 7 2}$ in hand, the investigation of the VMAR began. A solution of $\mathbf{1 7 2}$ in dichloromethane and silyl ketene acetal 173 in the presence of calcium hydride was cooled down to $-95{ }^{\circ} \mathrm{C}$. Dropwise addition of the $\mathrm{BF}_{3} \cdot \mathrm{Et}_{2} \mathrm{O}$ solution in dichloromethane and controlled elevation of the temperature to allow the reaction to occur, but not above $-80^{\circ} \mathrm{C}$, promoted the formation of the aldol adduct 189 (Scheme 4.4.1). Under the optimised conditions, the $\alpha$-regioisomer was not observed and stereoisomers of the $\gamma$-product were isolated in total $70 \%$ yield with an $E: Z$ ratio of $5: 1$ and syn : anti $6: 1$ for the major $(E)$-diastereoisomer. 


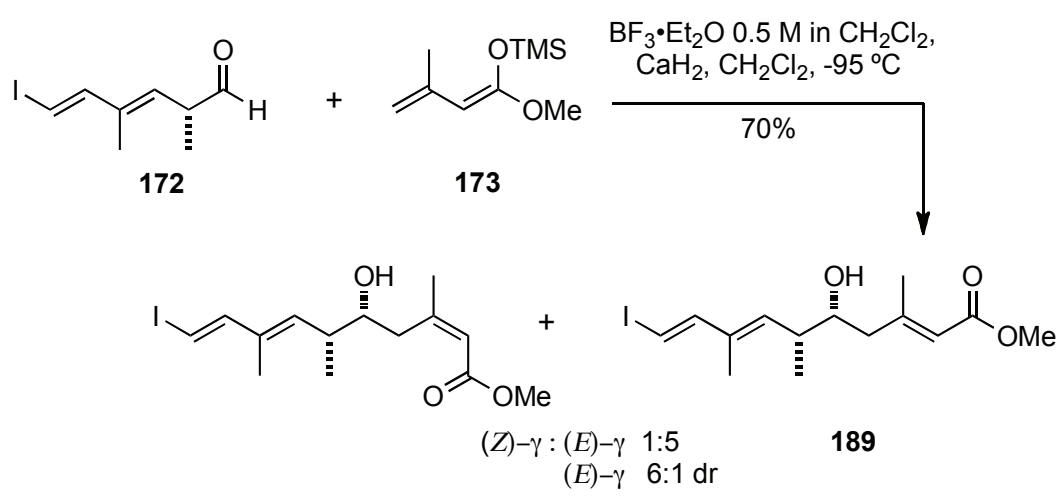

Scheme 4.4.1 Vinylogous Mukaiyama aldol reaction to form 189.

The selectivity of the newly installed C19 stereocentre arose from Felkin-Anh addition to the $\alpha$-chiral aldehyde 172. ${ }^{102,114}$ The transition state TS I presented in Figure 4.4.2 shows the approaching nucleophile with respect to the Bürgi-Dunitz trajectory. The carbonyl compound placed with the largest substituent perpendicular to the $\mathrm{C}=\mathrm{O}$ group and excluding all possible eclipsing interactions.

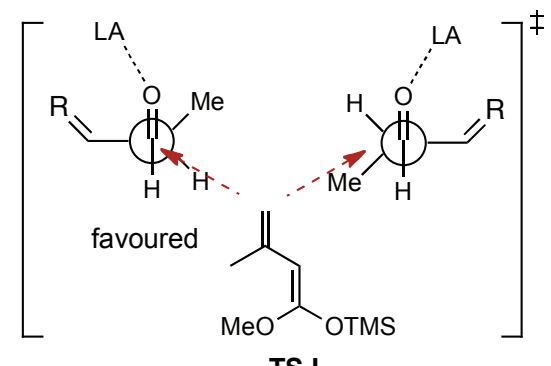

Figure 4.4.2 Felkin-Ahn controlled addition of silyl ketene acetal 173 to the $\alpha$-chiral aldehyde 172 .

Confirmation of the $\mathrm{C} 19$ hydroxyl stereochemistry was provided by Mosher ester analysis (Table 4.4.1). Accordingly shifted protons in (R)- and (S)-MTPA esters 192 and 193 returned positive values for H16 to H18 and negative values for H20 to H24 confirming the expected (R)-configuration of the $\mathrm{C} 19$ stereocentre in $\mathbf{1 8 9}$, consistent with the VMAR proceeding with good levels of Felkin-Anh control. 


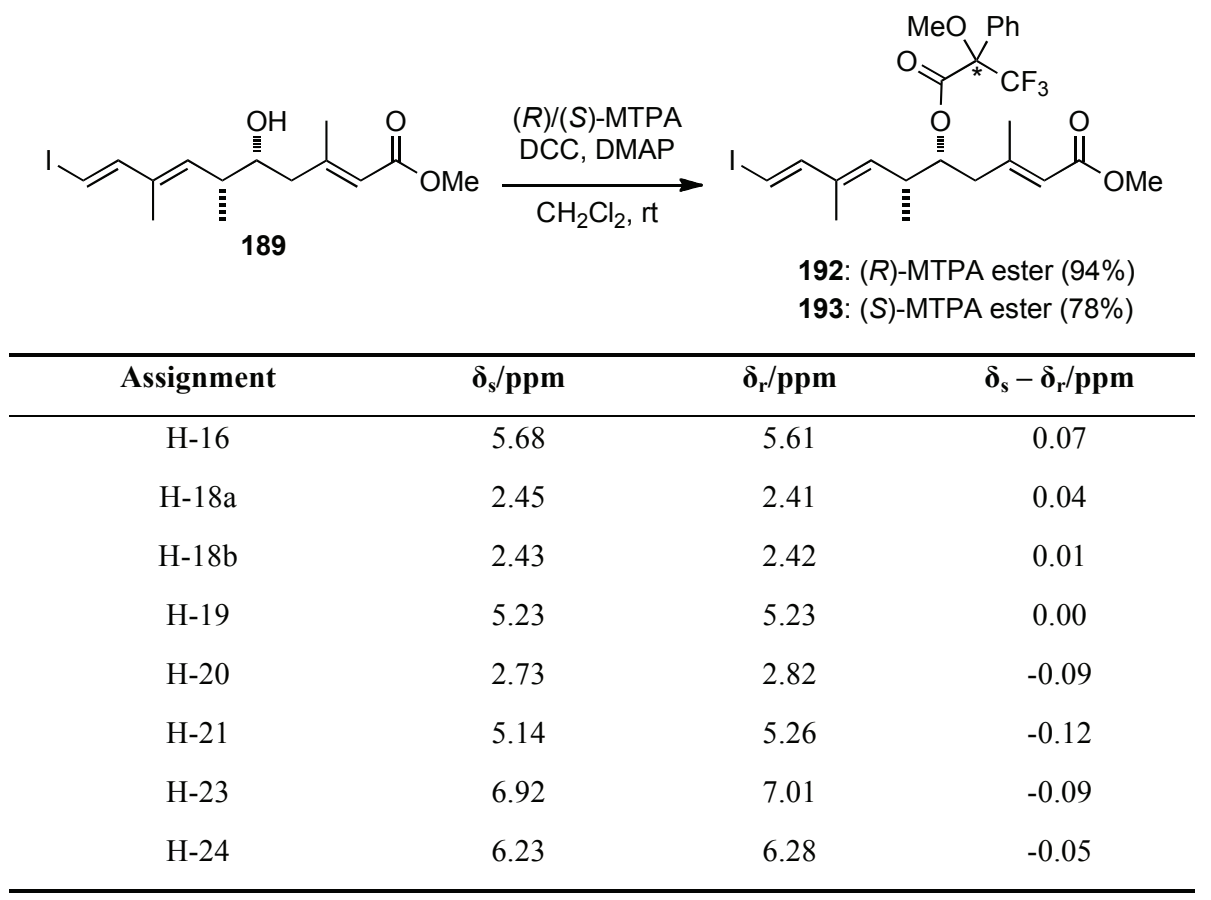

Table 4.4.1 Formation and analysis of the $(R)$ - and $(S)$-Mosher esters 192 and 193.

\subsection{Completion of C15-C24 fragment 171}

Having successfully installed the C18-C19 connection with requisite C19 stereochemistry, all that remained to complete sulfone $\mathbf{1 7 1}$ was C19 protection and manipulation of the $\mathrm{C} 15$ terminus. The C19 hydroxyl of $\mathbf{1 8 9}$ was protected using triethylsilyl triflate and 2,6-lutidine to form enoate 194 in excellent yield (Scheme 4.5.1). Subsequent reduction of the ester moiety with DIBAL-H afforded allylic alcohol 195 in $81 \%$ yield (Scheme 4.5.1).

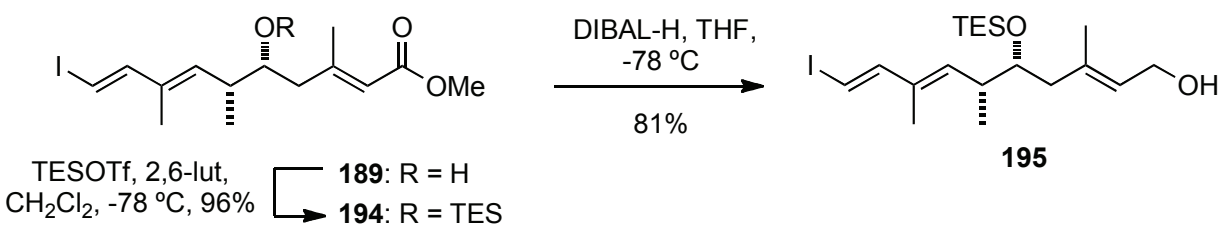

Scheme 4.5.1 Formation of the allylic alcohol 195.

Introduction of the sulfide functionality was accomplished under Mitsunobu conditions with $1 H$-mercaptophenyltetrazole to provide compound 196 in $80 \%$ yield (Scheme 4.5.2). Given the reliable sequence to the advanced synthetic intermediate of the C15-C24 fragment 171, we were now approaching the oxidation of sulfide 196 to complete the Julia-Kocienski coupling partner. 


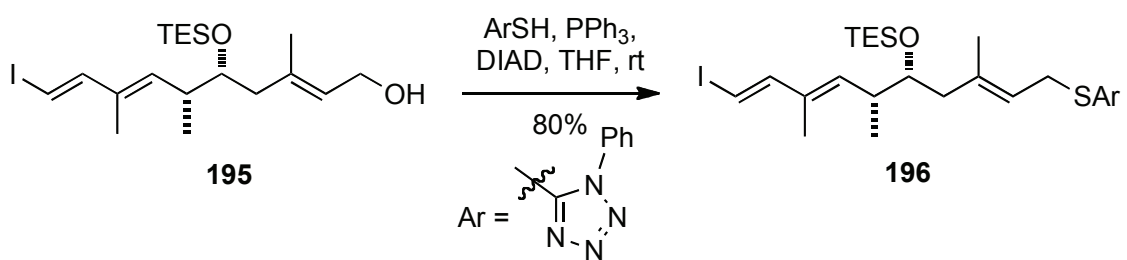

Scheme 4.5.2 Mitsunobu reaction introducing sulfide 196 .

The ammonium molybdate-catalysed oxidation with hydrogen peroxide was investigated first and unfortunately it had a destructive effect on the starting material 196 (Table 4.5.1, entry 1). We then examined different solvents in combination with hydrogen peroxide and molybdenum salt (Table 4.5.1, entries 2-4). In all conditions investigated sulfone 171 was not formed and either sulfide 196 decomposed under the reaction conditions or was partly recovered. Treatment of the substrate 196 with Oxone ${ }^{\circledR}$ in a mixture of water and methanol at $0{ }^{\circ} \mathrm{C}$ did not promote formation of the desired product 171, although starting material was recovered (Table 4.5.1, entry 5). The procedure utilising catalytic scandium triflate in combination with hydrogen peroxide was then attempted and resulted in sulfoxide formation, and sulfone $\mathbf{1 7 1}$ was not detected in the reaction mixture (Table 4.5.1, entry 6).

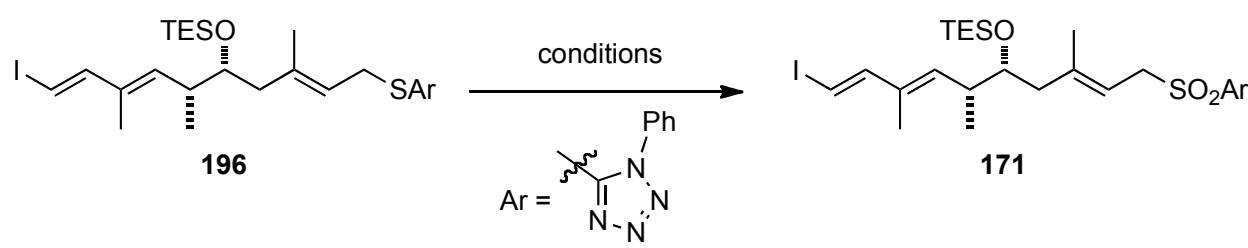

\begin{tabular}{|c|c|c|c|c|}
\hline Entry & Reagent & Solvent & Temp. & Yield (\%) \\
\hline 1 & $20 \mathrm{~mol} \%\left(\mathrm{NH}_{4}\right)_{6} \mathrm{Mo}_{7} \mathrm{O}_{24} \cdot 7 \mathrm{H}_{2} \mathrm{O}, 10$ eq. $\mathrm{H}_{2} \mathrm{O}_{2}$ & EtOH & $0{ }^{\circ} \mathrm{C}-\mathrm{rt}$ & decomp. \\
\hline 2 & $10 \mathrm{~mol} \%\left(\mathrm{NH}_{4}\right)_{6} \mathrm{Mo}_{7} \mathrm{O}_{24} \cdot 7 \mathrm{H}_{2} \mathrm{O}, 5$ eq. $\mathrm{H}_{2} \mathrm{O}_{2}$ & EtOH & $0{ }^{\circ} \mathrm{C}$ & decomp. \\
\hline 3 & $1 \mathrm{~mol} \%\left(\mathrm{NH}_{4}\right)_{6} \mathrm{Mo}_{7} \mathrm{O}_{24} \cdot 7 \mathrm{H}_{2} \mathrm{O}, 10$ eq. $\mathrm{H}_{2} \mathrm{O}_{2}$ & EtOH/THF $1: 1$ & $0{ }^{\circ} \mathrm{C}$ & st. mat. $196(50 \%)$ \\
\hline 4 & $10 \mathrm{~mol} \%\left(\mathrm{NH}_{4}\right)_{6} \mathrm{Mo}_{7} \mathrm{O}_{24} \cdot 7 \mathrm{H}_{2} \mathrm{O}, 10$ eq. $\mathrm{H}_{2} \mathrm{O}_{2}$ & EtOH/DCM 1:1 & $0{ }^{\circ} \mathrm{C}$ & st. mat. $196(50 \%)$ \\
\hline 5 & 3 eq. Oxone & $\mathrm{MeOH} / \mathrm{H}_{2} \mathrm{O} 1: 3$ & $0{ }^{\circ} \mathrm{C}-\mathrm{rt}$ & st. mat. $196(50 \%)$ \\
\hline 6 & $20 \mathrm{~mol} \% \mathrm{La}(\mathrm{OTf})_{3}, 20$ eq. $\mathrm{H}_{2} \mathrm{O}_{2}$ & $\mathrm{CH}_{2} \mathrm{Cl}_{2} / \mathrm{EtOH} 9: 1$ & $0{ }^{\circ} \mathrm{C}$ & sulfoxide \\
\hline
\end{tabular}

Table 4.5.1 Oxidation of sulfide 196. 
Vedejs ${ }^{115}$ studies on stabilisation of the peroxy-molybdenum complexes by the hexamethylphosphoroamide (HMPA) prompted the investigation of the initially studied conditions (Table 4.5.1, entry 2), but in the presence of HMPA. The performed protocol employing $10 \mathrm{~mol} \%$ of ammunium molybdate hydrate with 10 equivalents of the hydrogen peroxide relative to the substrate 196 was premixed with HMPA (v/v). The bright yellow solution was then added dropwise to a $0.1 \mathrm{M}$ solution of sulfide 196 in methanol at $0{ }^{\circ} \mathrm{C}$ (Scheme 4.5.3). The reaction mixture was stirred for 24 hours in an ice-water bath providing the desired sulfone $\mathbf{1 7 1}$ upon aqueous work-up and column chromatography. While this procedure proved to be reliable and convenient on a small scale (up to $0.2 \mathrm{mmol}$ of 196), no full conversion of starting material 196 and partial decomposition was observed when reaction was carried out on bigger quantities $(>0.2 \mathrm{mmol}$ of 196). Nonetheless, performing the reaction in parallel on an adequate scale $(<0.2 \mathrm{mmol}$ of 196) produced a satisfactory amount of sulfone $\mathbf{1 7 1}$.

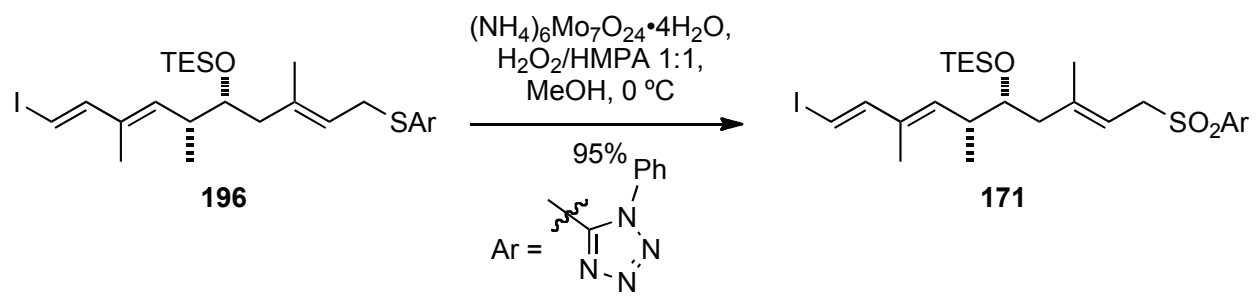

Scheme 4.5.3 Molybdenum catalysed oxidation to sulfone 171.

\subsection{Summary}

The western hemisphere of palmerolide $\mathrm{C}$, the $\mathrm{C} 15-\mathrm{C} 24$ subunit $\mathbf{1 7 1}$ was synthesised in fourteen steps in 16\% overall yield starting from the Weinreb amide derivative of $(S)$-Roche ester 177. The crucial transformation to build the C18-C19 bond relied on Mukaiyama vinylogous aldol reaction, which allowed for controlled introduction of the $\mathrm{C} 19$ stereocentre and was compatible with a sensitive iodo-aldehyde 172. Final oxidation of sulfide 196 was achieved after the addition of HMPA to the ammonium molybdate-catalysed reaction with hydrogen peroxide. 


\section{Chapter 5}

\subsection{C1-C14 and C15-C24 fragment union}

Having completed the synthesis of the C1-C14 and C15-C24 subunits $\mathbf{1 6 9}$ and $\mathbf{1 7 1}$, investigation of their coupling reaction began. As outlined in our retrosynthesis, the JuliaKocienski olefination coupling would be employed as an efficient approach to construct the $\mathrm{C} 14 \mathrm{E}$ alkene of the $\mathrm{C} 14-\mathrm{C} 17$ diene.

\subsubsection{Introduction to the Julia olefination}

In 1973 Julia et al. reported a process to form a $\mathrm{C}-\mathrm{C}$ double bond using a phenyl sulfone anion and carbonyl compounds involved a sequence of several synthetic operations: addition, acylation of the resulting $\beta$-alkoxysulfone and reductive elimination of the $\beta$-acyloxysulfone with $\mathrm{Na}(\mathrm{Hg})$ or $\mathrm{SmI}_{2}$ (Scheme 5.1.1). ${ }^{116}$

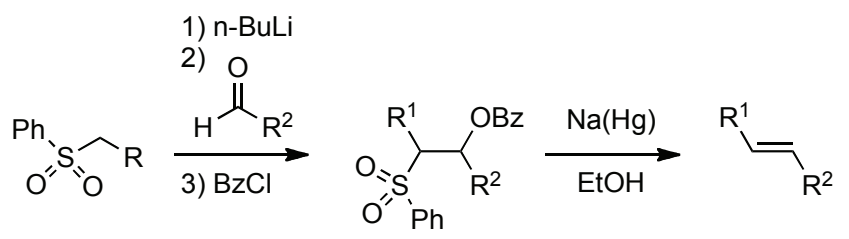

Scheme 5.1.1 Olefination reaction between phenyl sulfone and aldehyde developed by Marc Julia.

The modified Julia reaction developed by Julia, ${ }^{117}$ enabled the preparation of alkenes from benzothiazol-2-yl (BT) sulfones and aldehydes in a single step, but the stereochemical outcome is a direct consequence of the initial carbonyl addition (Scheme 5.1.2). Although this first step is reversible, the formation of the anti or syn intermediate might be influenced by the reaction conditions. A syn intermediate could be formed as a result of chelation control (TS I), which occurs upon addition of the base with small counterions $(\mathrm{Li})$ and in an apolar solvent. The addition of base with a larger counterion $(\mathrm{K})$ 
in polar solvent will promote the formation of the anti intermediate via the open transition state TS II. ${ }^{117}$

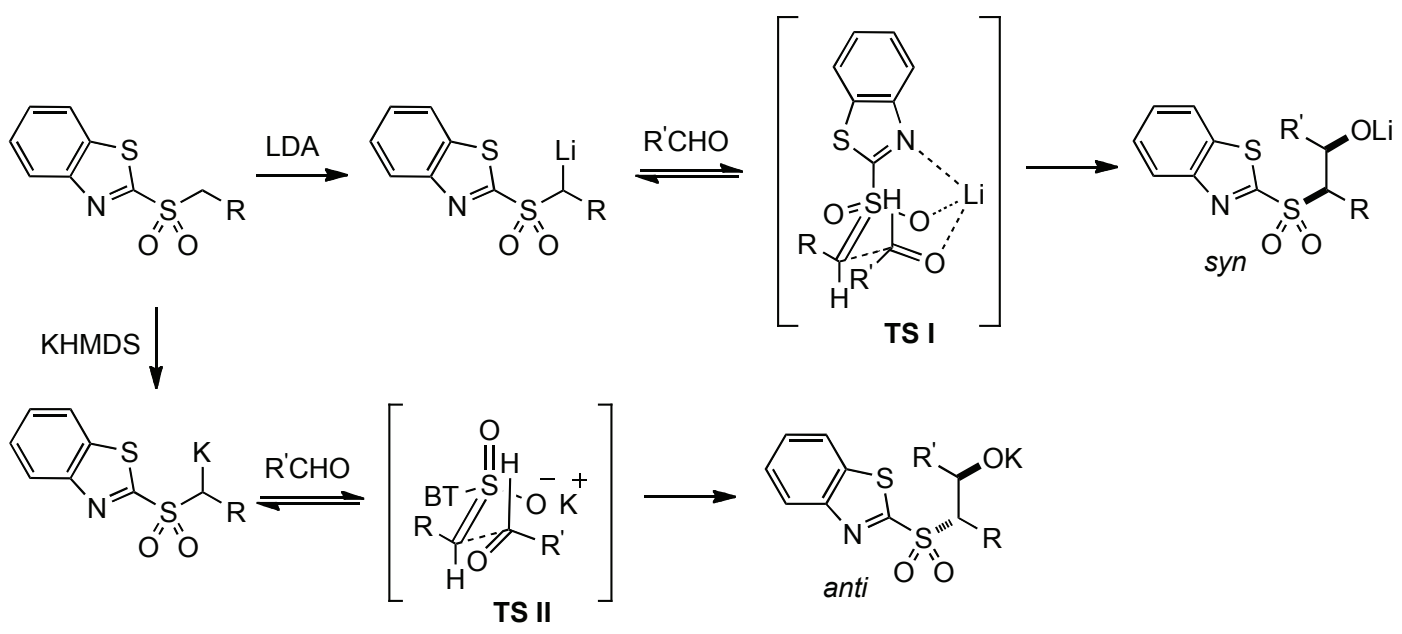

Scheme 5.1.2 Influence of the reaction conditions on modified Julia reaction with BT-sulfone.

In 1993 Julia investigated the base mediated elimination of anti $\beta$-hydroxy-BTsulfones with alkyl substituents, which decompose to the (E)- and (Z)-alkenes, with noticeable propensity to form the $(Z)$-olefins. This can be explained due to the high-energy conformer 197 with the gauche-like arrangement of substituents forming slower than $\mathbf{1 9 8}$ (Scheme 5.1.3). ${ }^{118}$ The initial addition of the metallated sulfone to the carbonyl compound dictates the stereochemical outcome. The observed results reflected the ratio of spirointermediates 197 and 198, which upon elimination of the antiperiplanar lithium benzylthiozolone and sulfur dioxide furnished cis- or trans-olefins. However, when $\beta$ hydroxy-BT-sulfones with R' vinyl/aryl substituents were examined, the tendency towards (E)-olefin formation was observed. If equilibration of the intermediates is precluded, there must be different mechanism effecting predominant formation of the $(E)$-configured olefins. 


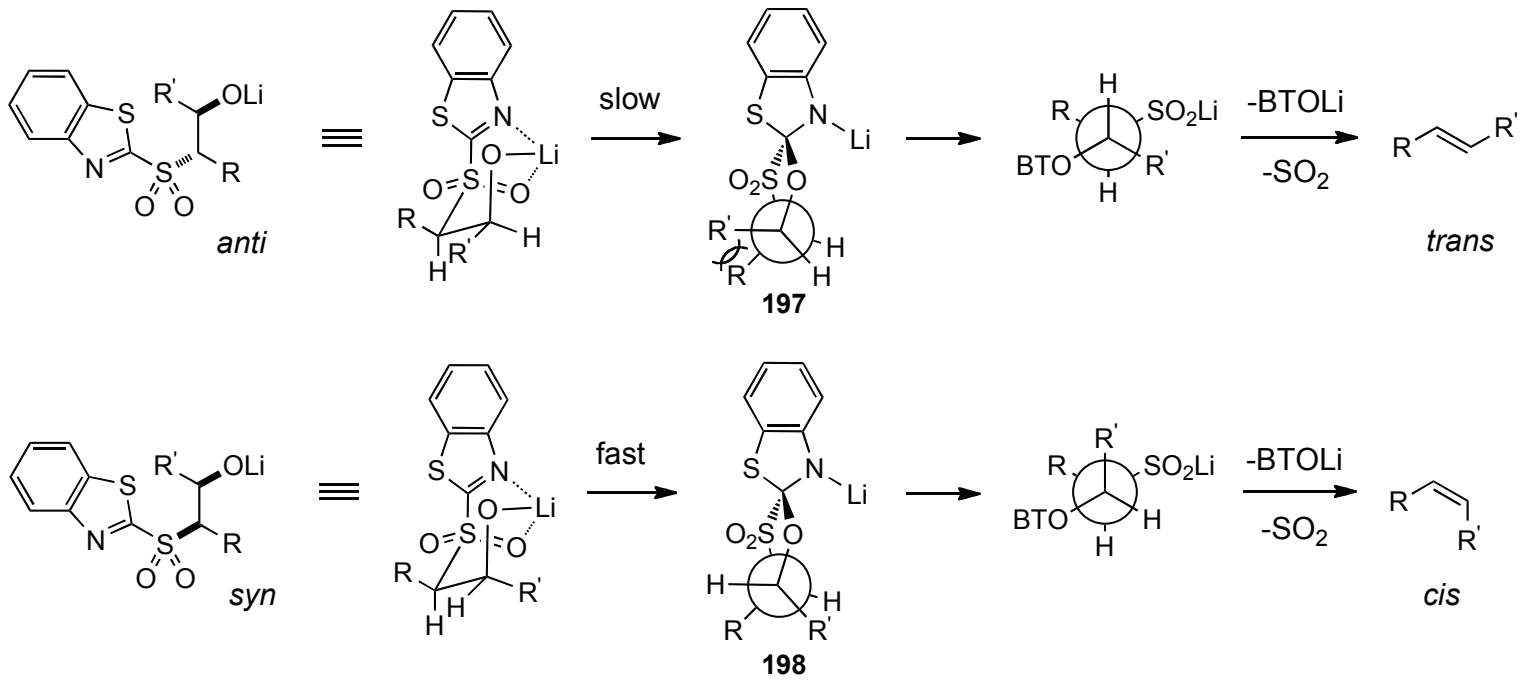

Scheme 5.1.3 Mechanism of modified Julia reaction.

This is explained by the direct loss of the lithium benzylthiozolone from intermediates 197 and 198 (or a similar event immediately following spirocycle opening) yielding zwitterionic conformers 199 and $\mathbf{2 0 0}$ (Scheme 5.1.4). Equilibration of the betaine intermediates towards the more energetically favoured conformer 200, provides an (E)-alkene product upon loss of sulfur dioxide. Unsaturated residues in $\mathrm{R}^{\prime}$ provide stabilisation for the carbenium ion present in $\mathbf{1 9 9 / 2 0 0}$ and therefore promote the suggested pathway. ${ }^{119}$

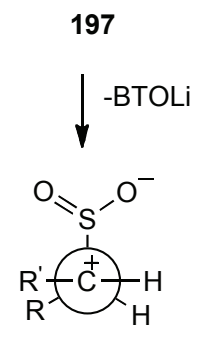

199

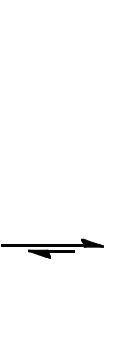

198
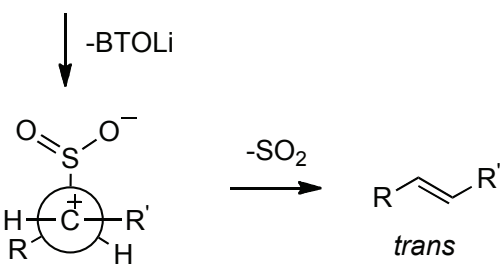

Scheme 5.1.4 Mechanistic rational for observed selectivity in the modified Julia reaction.

The Julia-Kocienski olefination is a later modification of the Julia reaction. Kocienski's studies resulted in the development of an $(E)$-alkene selective system utilising phenyltetrazolyl sulfone (PT) instead of benzothiazolyl (BT) sulfone. Later, Blakemore extended the library of heteroaromatic sulfones compatible with Julia reaction to 1isoquinolinoyl, 1-methyl-2-imidazolyl, 4-methyl-2-imidazolyl and 4-methyl-1,2,4-triazol3-yl sulfones. ${ }^{120}$ 
Kocienski observed that the 1-phenyl-1H-tetrazol-5-yl sulfone anion 201 was not prone to self-condensation, therefore it could first be deprotonated with base (201) and then reacted with an aldehyde (Scheme 5.1.5). This extended the scope of compatible carbonyl substrates, including reactions with base-sensitive aldehydes. The transient $\beta$ alkoxysulfone $\mathbf{2 0 2}$ reacts further to form spiro-intermediate 203, which breaks down via a Smiles rearrangement to deliver the olefinic product with extrusion of sulfur dioxide and lithium phenyltetrazolone. An increase in $(E: Z)$ ratio was generally observed upon changing the counter-ion $(\mathrm{Li}<\mathrm{Na}<\mathrm{K})$ of the base. In addition, an increase in polarity and coordinating ability of the solvent $\left(\mathrm{PhMe}^{2} \mathrm{Et}_{2} \mathrm{O}<\mathrm{THF}<\mathrm{DME}\right)$ favours formation of the (E)-alkene. ${ }^{121}$
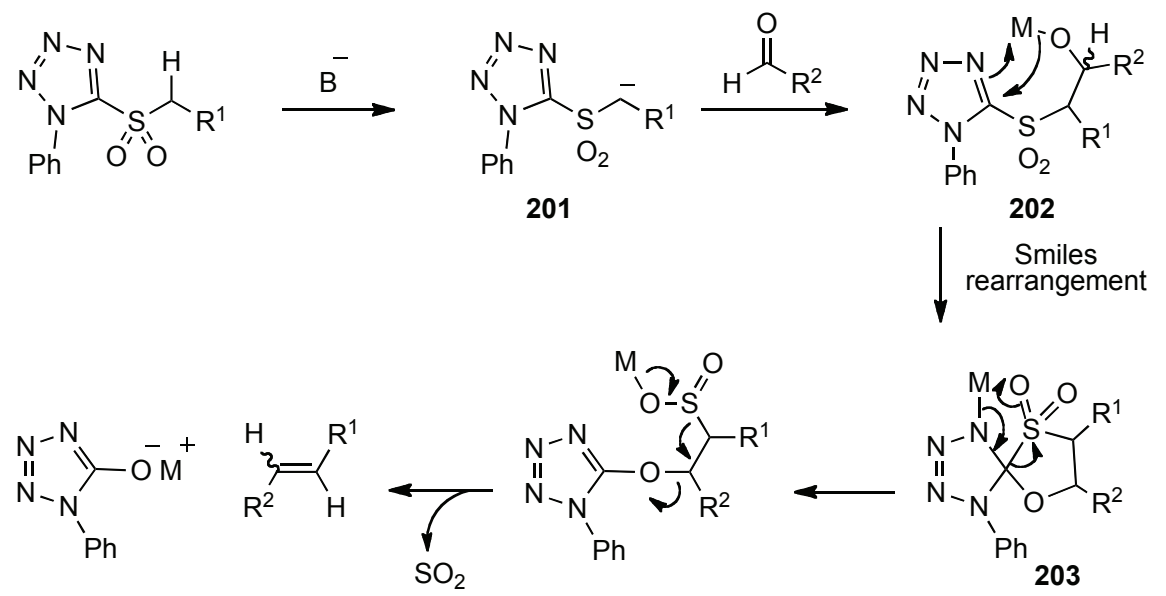

Scheme 5.1.5 Mechanism of Julia-Kocienski reaction.

In 1996, Kocienski reported the synthesis of the C10-C26 fragment 204 of rapamycin, ${ }^{122}$ investigating substrate dependent aspects of the selectivity in Julia olefination. As shown in Scheme 5.1.6, selective formation of the triene portion of 204 was significantly influenced by the choice of starting materials. This study demonstrated that while using BT-sulfone 205, the (Z)-olefin was formed preferentially, which supports the hypothesis that stabilised BT-sulfone anions have a tendency to form (Z)-configured products. When sulfone 206 was used together with conjugated aldehyde 207, formation of the $(E)$-olefin was observed with almost complete selectivity when LiHMDS was employed. 


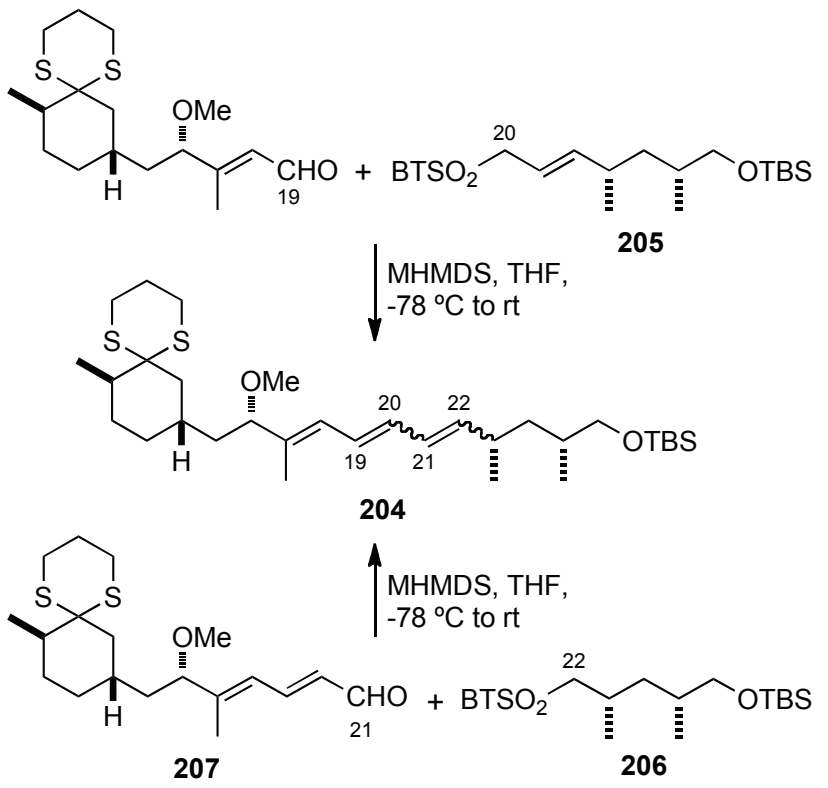

\begin{tabular}{ccc}
\hline $\mathrm{M}$ & Yield (\%) & $E: Z$ \\
\hline \hline $\mathrm{Li}$ & 75 & $29: 71$ \\
$\mathrm{Na}$ & 79 & $43: 57$ \\
$\mathrm{~K}$ & -- & $18: 82$ \\
\hline
\end{tabular}

Scheme 5.1.6 Substrate dependence in modified Julia reaction.

\subsubsection{Model system for Julia-Kocienski coupling}

Given the lengthy synthesis of aldehyde 169 it was decided to pursue model studies to develop the optimal conditions for the olefination reaction. Using sulfone 171 and benzyl-protected aldehyde $\mathbf{2 0 8}$ as a model substrate, a range of reaction conditions were explored as summarised in Table 5.1.1. It was initially thought that by reacting the anion of sulfone 171 with an excess of aldehyde 208 would assure complete formation of the coupled product 209 (Table 5.1.1, entry 1, 2). However, unreacted sulfone 171 was always recovered upon purification, delivering only small amount of the diene 209, regardless of the solvent used. When an excess of sulfone $\mathbf{1 7 1}$ in combination with 1.3 equivalents of base was reacted with a 1.0 equivalent of aldehyde $\mathbf{2 0 8}$, instant formation of the product 209 was detected by TLC (Table 5.1.1, entry 3). Prior to the aqueous work-up the reaction mixture was quenched by the addition of a few drops of methanol, consequently the product 209 was isolated in $80 \%$ yield. Following the assignment by ${ }^{1} \mathrm{H}-{ }^{1} \mathrm{H}$ COSY experiment, ratio of the $(E)$ - and (Z)-isomers was determined by analysis of ${ }^{1} \mathrm{H}$ NMR. Diagnostic C15 resonance $J$ value for $(E, E)$-diene were 15.0 and $10.8 \mathrm{~Hz}$, whereas the (Z)-olefin showed a smaller coupling constant at $6.2 \mathrm{~Hz}$, both values were consistent with the $J$ coupling constants reported for $(E)$ - and (Z)-2-methylhexa-2,4-diene at 14.4 and $5.6 \mathrm{~Hz}$ respectively. ${ }^{123}$ 


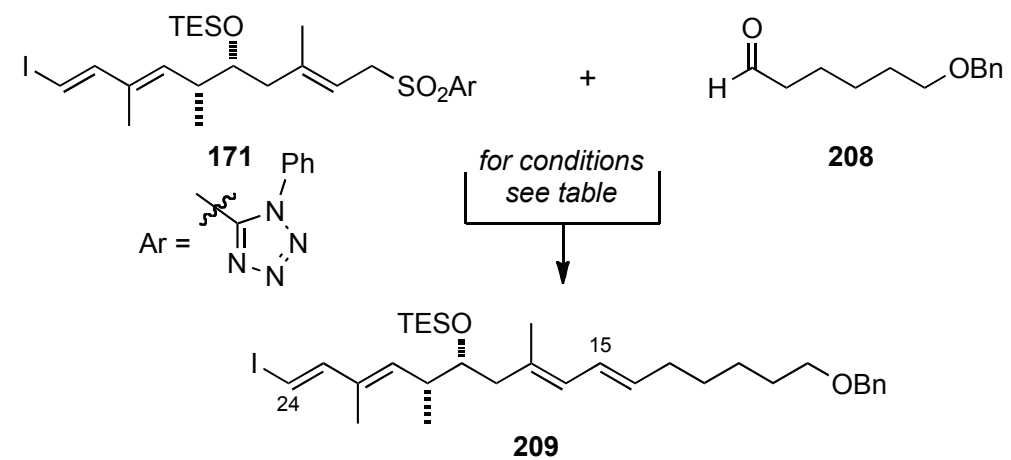

\begin{tabular}{cccccc}
\hline Entry & $\mathbf{1 7 1}: \mathbf{2 0 8}(\mathbf{e q})$ & Base $(\mathbf{e q})$ & Solvent & Temp. $\left({ }^{\mathbf{0}} \mathbf{C}\right)$ & Yield $(\mathbf{\%}, \boldsymbol{E}: \boldsymbol{Z})$ \\
\hline 1 & $1: 1.5$ & NaHMDS $(1.3)$ & THF & -78 to -50 & $10(2: 1)$ \\
2 & $1: 1.5$ & NaHMDS $(1.3)$ & DME & -78 to -50 & $5(2: 1)$ \\
3 & $1.5: 1$ & NaHMDS $(1.3)$ & THF & -78 to -50 & $80(2: 1)$ \\
\hline
\end{tabular}

Table 5.1.1 Julia-Kocienski coupling reaction, model studies.

\subsubsection{C14-C15 bond formation via Julia-Kocienski coupling}

The optimised conditions were now translated to the real system. In the event, deprotonation of the sulfone 171 with NaHMDS in THF at $-78{ }^{\circ} \mathrm{C}$ was followed by addition of aldehyde 169 (Scheme 5.1.7). The reaction was allowed to warm to $-50{ }^{\circ} \mathrm{C}$ within one hour and then quenched with methanol. Aqueous work-up and subsequent column chromatography provided the C1-C24 carbon framework $\mathbf{2 1 0}$ of palmerolide $\mathrm{C}$ in $78 \%$ yield with $7: 1(E: Z)$ selectivity. The improved selectivity compared to the model system might be attributed to the higher complexity and steric demand of the aldehyde component. Confirmation of the olefin geometry, as in the model study, was revealed upon the inspection of the ${ }^{1} \mathrm{H}$ NMR spectra of $\mathbf{2 1 0}$. The $J$ coupling constant values for the $\mathrm{C} 15$ resonance were found to be 15.0 and $10.8 \mathrm{~Hz}$. These values were in good agreement with the values reported for the analogous system in the synthesis of palmerolide A. ${ }^{6}$ 

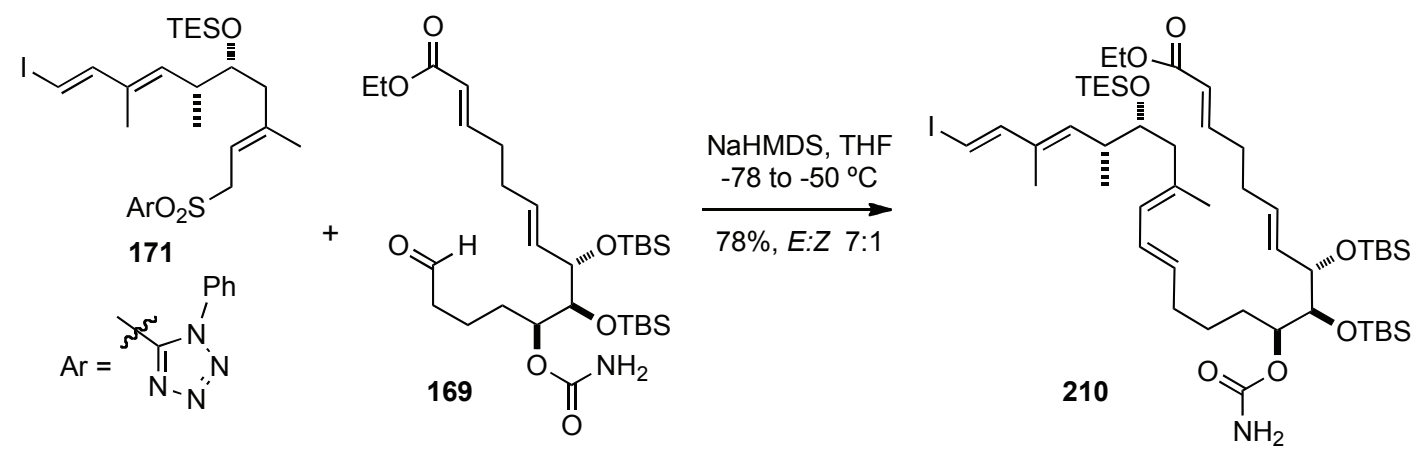

Scheme 5.1.7 Formation of the C1-C24 carbon backbone of palmerolide C via Julia-Kocienski reaction.

\subsection{Advancing the palmerolide $\mathrm{C}$ carbon framework}

With the advanced C1-C24 intermediate $\mathbf{2 1 0}$ in hand, all that remained was to close the macrocyclic ring and deprotect the $\mathrm{C} 8$ and $\mathrm{C} 9$ hydroxyl groups prior to the final installation of the enamide side chain.

\subsubsection{Formation of the macrolactone}

The selective cleavage of the C19 triethylsilyl group in $\mathbf{2 1 0}$ was accomplished using HF•pyridine in pyridine. ${ }^{124}$ A reagent solution was prepared prior to the reaction by mixing $2 \mathrm{~mL}$ of hydrogenfluoride-pyridine complex with $4 \mathrm{~mL}$ of pyridine and $16 \mathrm{~mL}$ of THF. Column chromatography at this point enabled the separation of the C14-C15 (E,Z)diene from the Julia-Kocienski coupling reaction delivering pure alcohol 211 in $88 \%$ yield (Scheme 5.2.1).
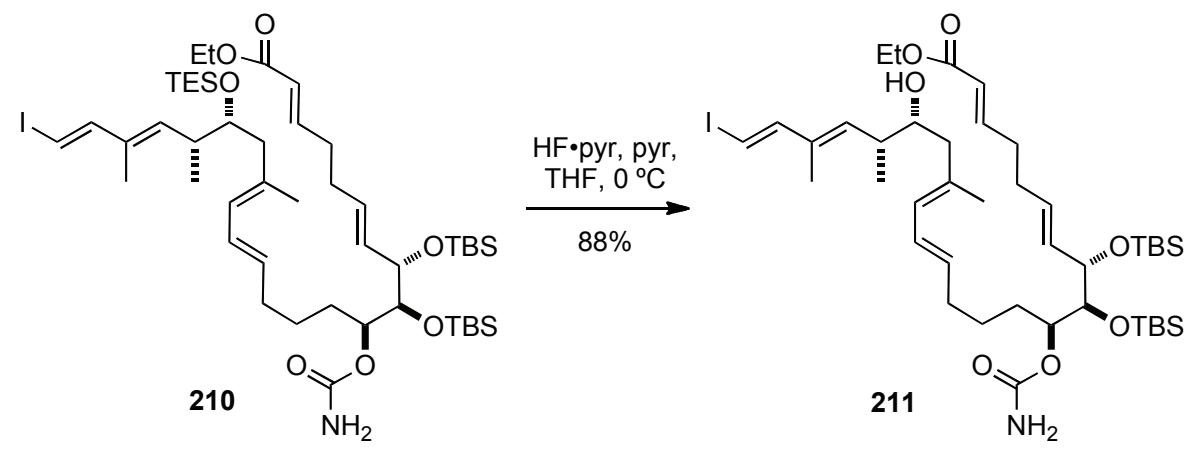

Scheme 5.2.1 Selective deprotection of the triethylsilyl protecting group to form 211 . 
Tentative experimentations with ester hydrolysis using potassium trimethylsilanolate ${ }^{125}$ resulted in a poor yield of seco-acid 212. The reason behind this was related to the potassium salt formed upon the reaction, which required relatively strong acidic conditions to be protonated. However, presence of the acid-sensitive conjugated iodo-diene hindered our chance of succeeding in this approach. The initially investigated aqueous work-up with ammonium chloride did not result in a formation of the expected product. The use of an aqueous citric acid work-up gave the seco-acid 212, although a chromatography column to remove citrate residues was necessary. The best results were obtained upon quenching the reaction mixture with $0.1 \mathrm{M}$ sodium hydrogen sulfate and subsequent addition of solid sodium sulfate. In this way, exposure to acid was minimised and filtration delivered the crude product without requirement for further purification by column chromatography. Under these optimised conditions, a mild saponification protocol employing potassium trimethylsilanolate ${ }^{125}$ was successfully executed and upon formation of the potassium salt of 211, acidic work-up with sodium hydrogen sulfate returned the desired seco-acid 212 (Scheme 5.2.2).

With the seco-acid 212 in hand, macrolactonisation under Yamaguchi conditions ${ }^{31}$ was explored. Preparation of the mixed anhydride commenced with addition of the trichlorobenzoyl chloride to the solution of the seco-acid 212 and triethylamine in toluene. Upon completion, the reaction mixture was diluted and added drop-wise over three hours to a $0.001 \mathrm{M}$ solution of DMAP in toluene. High dilution together with slow addition are important modifications promoting intra- rather then intermolecular esterification of the activated acid. After the addition was completed the reaction mixture was allowed to stir for a further nine hours and subsequent purification delivered macrolactone 213 in $71 \%$ yield over two steps (Scheme 5.2.2).
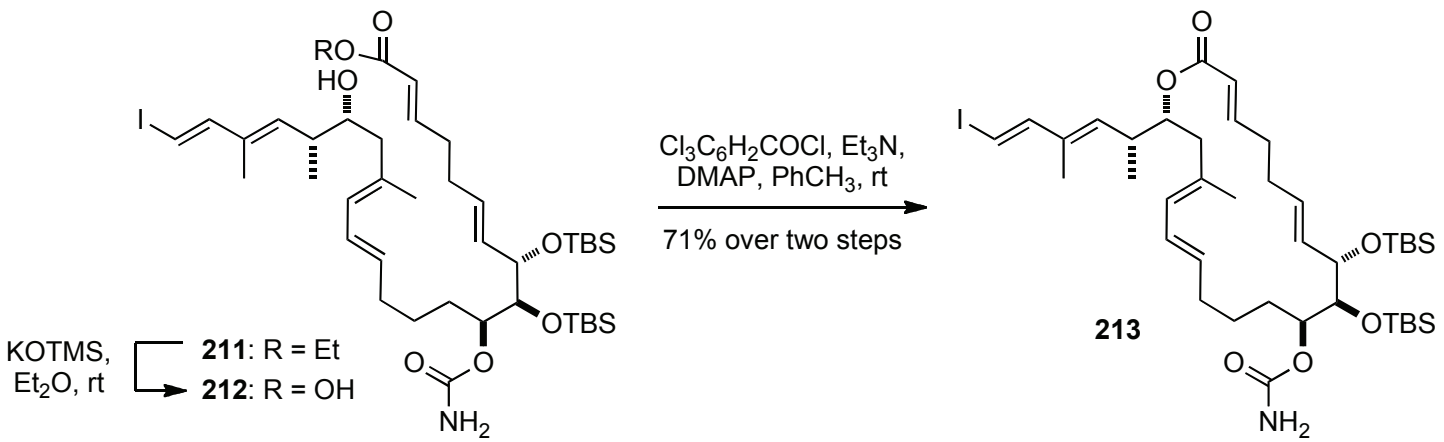

Scheme 5.2.2 Formation of 213 via Yamaguchi macrolactonisation. 


\subsubsection{Final deprotection}

As we were approaching the final stages of the synthesis, deprotection of the remaining silyl groups was required. TBAF was chosen, as it would not affect the iododiene motif and was used in the final phase of palmerolide A synthesis by Hall and de Brabander. Cleavage of the C8 and C9 TBS groups in 213 was accomplished with only five equivalents of TBAF providing the direct precursor 214 to palmerolide $\mathrm{C}$ in $85 \%$ yield (Scheme 5.2.3).
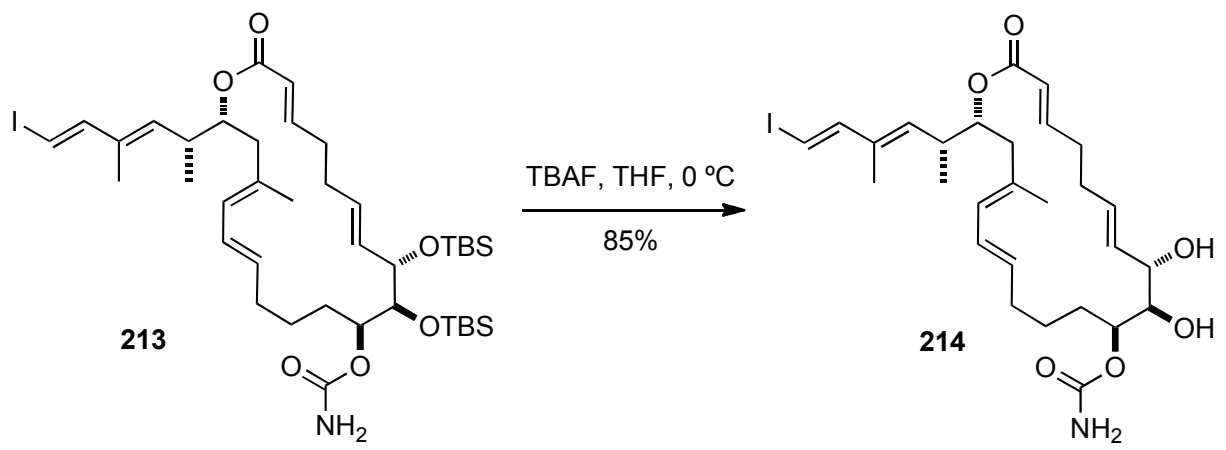

Scheme 5.2.3 Final deprotection to deliver diol 214.

\subsection{Introduction of the enamide side chain to complete palmerolide $\mathrm{C}$}

At this stage, we were facing the final transformation to introduce the enamide side chain. In 2003, Buchwald and Jiang reported the Goldberg-like amidation reaction of vinyl halides using copper iodide as a catalyst and $N, N^{\prime}$-dimethylethylenediamine as a ligand. ${ }^{126}$ They demonstrated the generality of the procedure, where a broad range of substrates with primary and secondary amides were successfully coupled with mono-, di- or tri-substituted vinyl bromides or iodides under optimised catalytic conditions (Scheme 5.3.1).

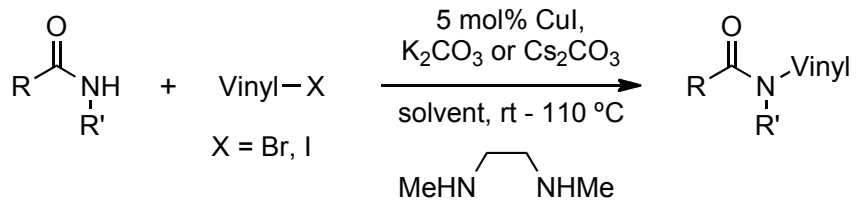

Scheme 5.3.1 Cu(I)-catalysed formation of enamides. 
The mechanism of this transformation is not fully understood, but computational calculations and kinetic experiments performed by Buchwald provide some insight ${ }^{38,127}$ (Scheme 5.3.2). A mechanism based on the oxidative addition/reductive elimination involving $\mathrm{Cu}(\mathrm{I}) / \mathrm{Cu}(\mathrm{III})$ species is proposed. The diamine ligand coordinates the $\mathrm{CuI}(\mathbf{2 1 5})$, therefore, prevents the ligation of the excess amide used and leaves the activation of the halide-substrate as a rate-limiting step. At low concentration of diamine, muliple ligation of the amide on copper occurs (216). To allow formation of the most active diamineligated copper(I) amidate species (217), the dissociation of the amide has to precede coordination to the ligand, which ultimately was identified as another rate-limiting step.

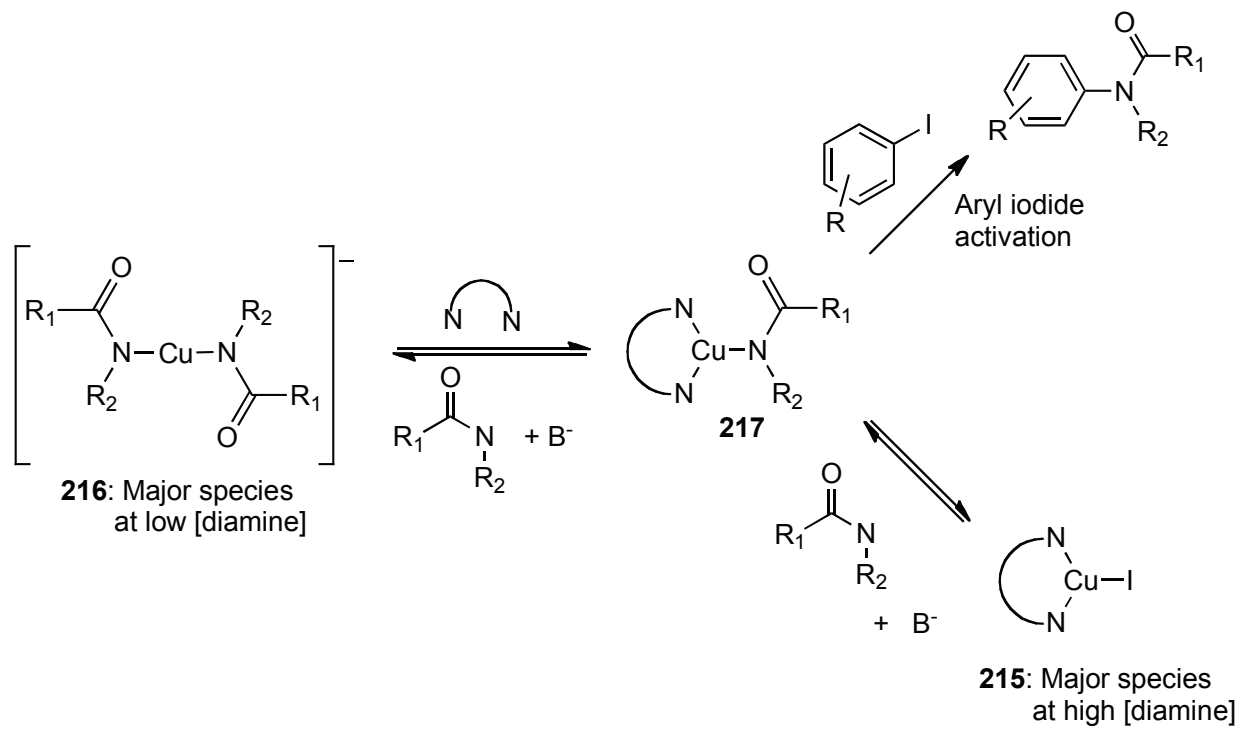

Scheme 5.3.2 Mechanism proposed by Buchwald.

As a prelude to examining this approach, the enamide formation was investigated in a model system. In accord with Buchwald protocol, ${ }^{126}$ catalytic conditions were investigated first using iodo-alcohol $\mathbf{1 7 8}$ as a model substrate. To a mixture of $\mathbf{1 7 8}$, freshly prepared 3,3-dimethylacrylamide 218 was added $5 \mathrm{~mol} \%$ of copper(I) iodide, $10 \mathrm{~mol} \%$ of $N, N^{\prime}$-dimethylethylenediamine and 1.5 equivalents of potassium carbonate in tetrahydrofuran. However, the conditions applied did not promote formation of 219. Following the disappointing results of the catalytic system, a stoichiometric amount of copper(I) iodide and $N, N^{\prime}$-dimethylethylenediamine was employed. Hence, to iodo-alcohol 178 in tetrahydrofuran was added 2 equivalents of amide 218 followed by 1.5 equivalents of copper(I) iodide and an excess of potassium carbonate. The order of addition of the reagents turned out to be important, requiring the diamine ligand as the final addition, 
otherwise the expected product was not formed. Gratifyingly, under stoichiometric conditions the desired enamide 219 was produced in 63\% yield (Table 5.3.1).

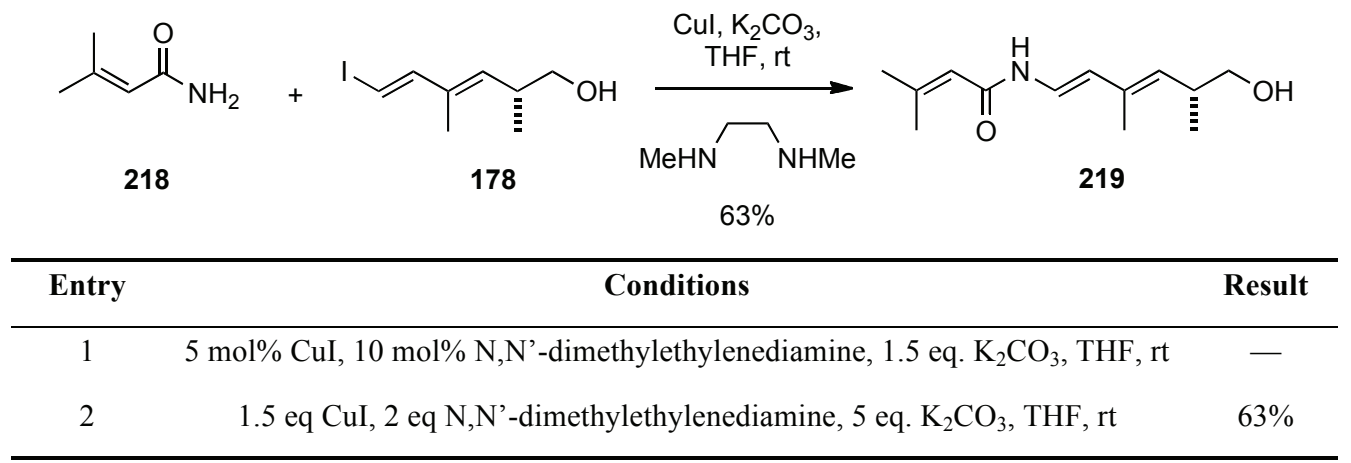

Table 5.3.1 Buchwald enamide formation, model study.

Having established the feasibility of forming the enamide under the Buchwald conditions, efforts turned to the coupling of 214 and 218. Direct translation of the reaction conditions developed during the model study onto substrate $\mathbf{2 1 4}$ promoted formation of the final product $\mathbf{3 b}$. Due to the solubility issues, the reaction was carried out in dimethylformamide. Subsequent purification by column chromatography provided $\mathbf{3 b}$ in 53\% yield and returned unreacted starting material 214 (Scheme 5.3.3).
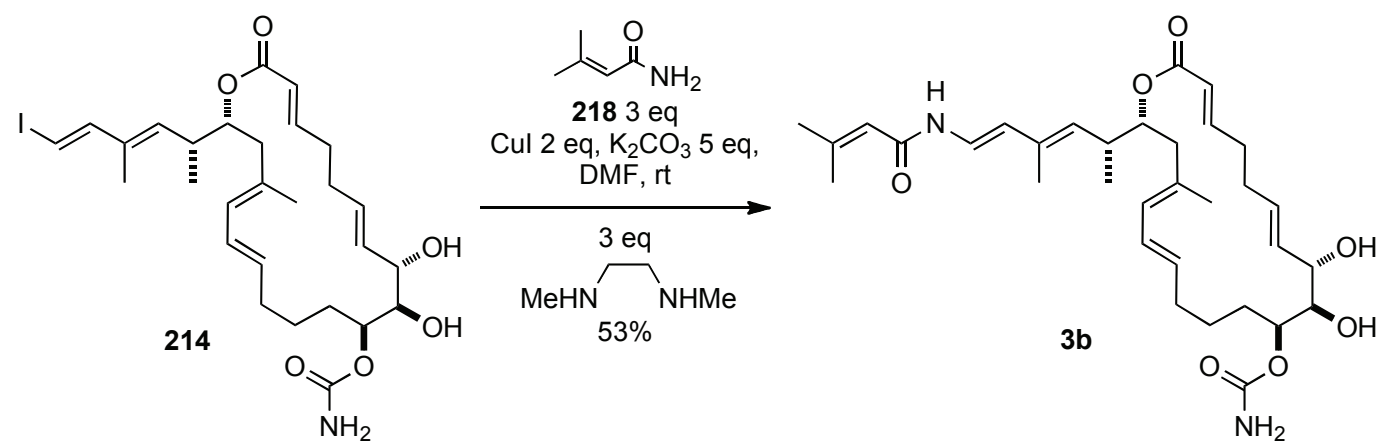

Scheme 5.3.3 Introduction of enamide to form 3 via Buchwald $\mathrm{Cu}(\mathrm{I})$-catalysed reaction.

\subsection{Comparison of synthetic and natural palmerolide $C$}

Having synthesised the target molecule $\mathbf{3 b}$, confirmation of its identity was now the focus. High resolution mass spectrometry revealed the expected ion at $\mathrm{m} / \mathrm{z} 607.3364$, which corresponds to the $[\mathrm{M}+\mathrm{Na}]^{+}$of $\mathrm{C}_{33} \mathrm{H}_{48} \mathrm{~N}_{2} \mathrm{O}_{7} \mathrm{Na}$ and to the reported value of 607.3367. Next, the specific optical rotation was measured at a concentration of $0.2 \mathrm{~g} / \mathrm{mL}$ in methanol to be -19.5 , which correlates with the reported -27.1 value at a concentration 
of $0.1 \mathrm{~g} / \mathrm{mL}$ of methanol. The IR spectrum of the synthetic material was in good agreement with the IR of the natural palmerolide $\mathrm{C}^{21}$

The ${ }^{1} \mathrm{H}$ NMR data provided for the natural sample of palmerolide $\mathrm{C} \mathbf{3}$ was acquired in deuterated dimethylsulfoxide. Unfortunately, due to the small quantity of the synthetic material produced and the difficulties associated with sample recovery from dimethylsulfoxide, we decided to perform our first spectroscopic experiments in a different solvent. The ${ }^{1} \mathrm{H}$ and ${ }^{1} \mathrm{H}-{ }^{1} \mathrm{H}$ COSY NMR were carried out in acetonitrile and allowed for the structural assignment (Table 5.4.1). The initially applied parameters to obtain ${ }^{13} \mathrm{C} \mathrm{NMR}$ were not optimal and further experiments were postponed due to the availability of sufficient time required for the analysis, and thus data was extracted from HSQC and HMBC NMR spectra. 


\begin{tabular}{|c|c|c|}
\hline $\begin{array}{c}\text { Proton } \\
\text { No. }\end{array}$ & $\begin{array}{l}\text { Synthetic palmerolide C } \\
d_{3}-\mathrm{MeCN} 500 \mathrm{MHz}\left(\delta_{\mathrm{H}}\right)\end{array}$ & $\begin{array}{c}\text { Synthetic palmerolide C } \\
d_{3} \text {-MeCN } 800 \mathrm{MHz} \text { HSQC }\left(\delta_{\mathrm{C}}\right)\end{array}$ \\
\hline 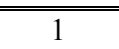 & - & 166.20* \\
\hline 2 & $5.71 \mathrm{~d}(15.7)$ & 123.26 \\
\hline 3 & $6.84-6.78 \mathrm{~m}$ & 142.43 \\
\hline $4 a$ & $2.18-2.12 \mathrm{~m}$ & \multirow{2}{*}{32.43} \\
\hline $4 b$ & $2.45-2.37 \mathrm{~m}$ & \\
\hline $5 a$ & $2.18-2.12 \mathrm{~m}$ & \multirow{2}{*}{30.75} \\
\hline $5 b$ & $2.33-2.24 \mathrm{~m}$ & \\
\hline 6 & $5.49-5.46 \mathrm{~m}$ & 132.6 \\
\hline 7 & $5.36 \mathrm{dd}(15.2,8.0)$ & 130.08 \\
\hline 8 & $3.75-3.70 \mathrm{~m}$ & 74.18 \\
\hline 9 & $3.47-3.43 \mathrm{~m}$ & 75.98 \\
\hline 10 & $4.52 \mathrm{dt}(10.4,3.1)$ & 75.26 \\
\hline $11 \mathrm{a}$ & $1.43-1.36 \mathrm{~m}$ & \multirow{2}{*}{26.09} \\
\hline $11 \mathrm{~b}$ & $1.54-1.45 \mathrm{~m}$ & \\
\hline $12 \mathrm{a}$ & $1.43-1.10 \mathrm{~m}$ & 24.85 \\
\hline 13 & $1.93-1.84 \mathrm{~m}$ & $31.83^{*}$ \\
\hline 14 & $5.46-5.42 \mathrm{~m}$ & 133.24 \\
\hline 15 & $6.20 \mathrm{dd}(14.7,10.9)$ & 127.95 \\
\hline 16 & $5.76 \mathrm{~d}(10.7)$ & 128.6 \\
\hline 17 & - & $132.52 *$ \\
\hline $18 \mathrm{a}$ & $2.20 \mathrm{dd}(14.2,11.1)$ & \multirow{2}{*}{43.15} \\
\hline $18 \mathrm{~b}$ & $2.33-2.24 \mathrm{~m}$ & \\
\hline 19 & 4.94 ddd $(10.5,7.7,2.4)$ & 75.26 \\
\hline 20 & $2.78 \mathrm{dt}(9.85,6.92)$ & 37.81 \\
\hline 21 & $5.20 \mathrm{~d}(9.7)$ & 130.94 \\
\hline 22 & - & $130.26^{*}$ \\
\hline 23 & $5.87 \mathrm{~d}(14.6)$ & 117.18 \\
\hline 24 & $6.93 \mathrm{dd}(14.5,10.5)$ & 122.33 \\
\hline $8-\mathrm{OH}$ & $3.05 \mathrm{~s}$ & - \\
\hline $9-\mathrm{OH}$ & $3.26 \mathrm{~s}$ & - \\
\hline $17-\mathrm{CH}_{3}$ & $1.64 \mathrm{~s}$ & 12.88 \\
\hline $20-\mathrm{CH}_{3}$ & $0.95 \mathrm{~d}(6.7)$ & 17.36 \\
\hline $22-\mathrm{CH}_{3}$ & $1.76 \mathrm{~d}(1.2)$ & 16.16 \\
\hline $\mathrm{CONH}_{2}$ & $5.18-5.09 \mathrm{~m}$ & $164.23 *$ \\
\hline $\mathrm{CONH}$ & $8.16 \mathrm{~d}(10.6)$ & $163.4^{*}$ \\
\hline $2^{\prime}$ & $5.63 \mathrm{~s}$ & 118.13 \\
\hline $3^{\prime}$ & - & $154.09^{*}$ \\
\hline $4^{\prime}$ & $2.16 \mathrm{~d}(1.1)$ & 19.76 \\
\hline 5 & $1.86 \mathrm{~d}(1.1)$ & 27.25 \\
\hline
\end{tabular}

Table 5.4.1 ${ }^{1} \mathrm{H}$ and ${ }^{13} \mathrm{C}$ NMR of synthetic palmerolide C $3 \mathrm{~b}$ 
At this point, with analytical confirmation of the synthesised product in hand we attempted to obtain the NMR spectra in dimethylsulfoxide for a direct comparison with data reported for the natural palmerolide C., ${ }^{4,10}$ The obtained ${ }^{1} \mathrm{H}$ NMR spectrum made the comparison possible however, it showed significant differences in signal shifts (Figure 5.4.1, Table 5.4.2). It appeared difficult to unambiguously justify exact correlation of the H8 and H9 signals as the presence of significant amount of water obscured these resonances in the spectra for the synthetic material, but it was clear that they did not match the corresponding signals in the spectra for the natural sample.

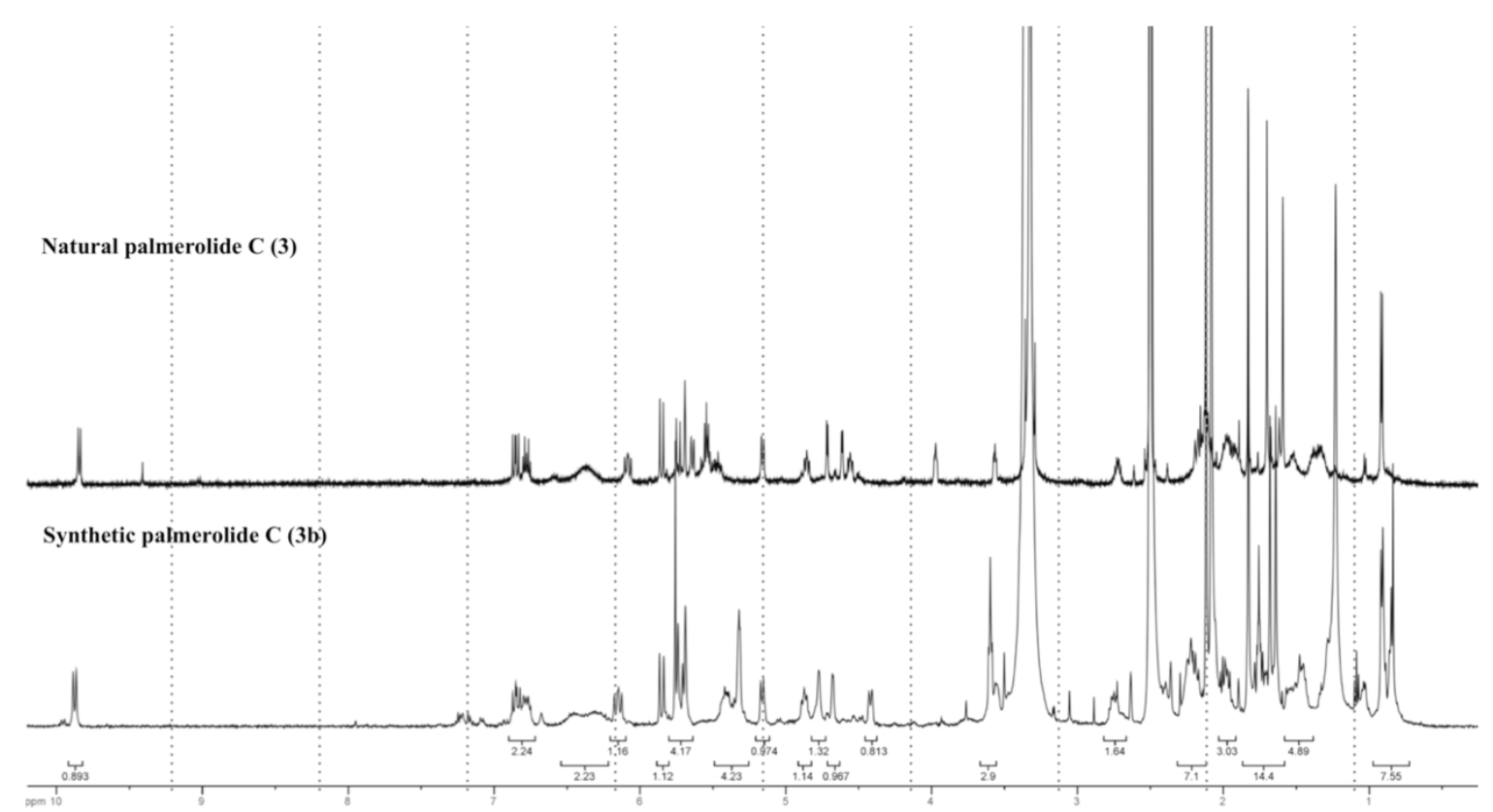

Figure 5.4.1 Comparison of the natural and synthetic palmerolide C ${ }^{1} \mathrm{H}$ NMR in $d_{6}$-DMSO 


\begin{tabular}{|c|c|c|c|}
\hline $\begin{array}{c}\text { Proton } \\
\text { No. }\end{array}$ & $\begin{array}{l}\text { Natural palmerolide C } 3 \\
d_{6} \text {-DMSO } 600 \mathrm{MHz}\left(\delta_{\mathrm{H}}\right)\end{array}$ & $\begin{array}{l}\text { Natural palmerolide C } 3 \\
d_{6} \text {-DMSO } 125 \mathrm{MHz}\left(\delta_{\mathrm{C}}\right)\end{array}$ & $\begin{array}{c}\text { Synthetic palmerolide C 3b } \\
d_{6}-\mathrm{DMSO} 500 \mathrm{MHz}\left(\delta_{\mathrm{H}}\right)\end{array}$ \\
\hline 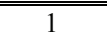 & - & lactone 166.9 & - \\
\hline 2 & $5.73 \mathrm{~d}(15.5)$ & 122.0 & $5.76-569 \mathrm{~m}$ \\
\hline 3 & 6.77 ddd $(15.4,7.5,7.4)$ & 149.8 & $6.78 \mathrm{dt}(15.6,7.4)$ \\
\hline $4 \mathrm{a}$ & $1.30 \mathrm{~m}$ & 31.7 & $200102 \mathrm{~m}$ \\
\hline $4 \mathrm{~b}$ & $2.13 \mathrm{~m}$ & & $2.08-1.93 \mathrm{~m}$ \\
\hline $5 \mathrm{a}$ & $1.89 \mathrm{~m}$ & 32.2 & $2.26-2.22 \mathrm{~m}$ \\
\hline $5 b$ & $1.98 \mathrm{~m}$ & & $2.03-1.97 \mathrm{~m}$ \\
\hline 6 & $5.54 \mathrm{~m}$ & 131.8 & $5.46-5.35 \mathrm{~m}$ \\
\hline 7 & $5.58 \mathrm{~m}$ & 131.1 & $5.35-5.27 \mathrm{~m}$ \\
\hline 8 & $3.96 \mathrm{~m}$ & 72.8 & $3.56 \mathrm{~m}_{\mathrm{obs}}$ \\
\hline 9 & $3.56 \mathrm{~m}$ & 75.6 & $3.46 \mathrm{~m}_{\mathrm{obs}}$ \\
\hline 10 & $4.56 \operatorname{ddd}(10.5,7.5,3.0)$ & 74.2 & $4.42 \mathrm{~d}(10.3)$ \\
\hline $11 \mathrm{a}$ & $1.30 \mathrm{~m}$ & 28.7 & $1.57-1.50 \mathrm{~m}$ \\
\hline $11 \mathrm{~b}$ & $1.49 \mathrm{~m}$ & & $1.49-1.42 \mathrm{~m}$ \\
\hline 12 & $1.95 \mathrm{~m}$ & 30.1 & $2.21 \mathrm{~m}$ \\
\hline 13 & $1.99-1.90 \mathrm{~m}$ & 30.1 & $1.82-1.78 \mathrm{~m}$ \\
\hline 14 & $5.46 \mathrm{ddd}(15.0,10.0,5.0)$ & 132.3 & $5.44-5.40 \mathrm{~m}$ \\
\hline 15 & $6.08 \mathrm{dd}(15.0,12.0)$ & 127.2 & $6.15 \mathrm{dd}(14.1,11.3)$ \\
\hline 16 & $5.63 \mathrm{~d}(11.0)$ & 128.8 & $5.76-5.69$ \\
\hline 17 & - & 132.5 & - \\
\hline $18 \mathrm{a}$ & $2.07 \mathrm{~m}$ & 44.1 & $2.25-2.21 \mathrm{~m}$ \\
\hline $18 \mathrm{~b}$ & $2.18 \mathrm{~m}$ & & $2.19-2.15 \mathrm{~m}$ \\
\hline 19 & $4.85 \operatorname{ddd}(10.5,7.8,2.8)$ & 74.7 & $4.87 \mathrm{t}(8.5)$ \\
\hline 20 & 2.70 qdd $(9.8,6.7,6.7)$ & 37.4 & $2.75 \mathrm{~m}$ \\
\hline 21 & $5.15 \mathrm{~d}(9.5)$ & 130.5 & $5.16 \mathrm{~d}(9.5)$ \\
\hline 22 & - & 133.3 & - \\
\hline 23 & $5.85 \mathrm{~d}(14.5)$ & 117.2 & $5.85 \mathrm{~d}(14.5)$ \\
\hline 24 & $6.85 \mathrm{dd}(14.6,9.0)$ & 122.8 & $6.85 \mathrm{dd}(14.0,10.4)$ \\
\hline 8-OH & $4.62 \mathrm{~d}(5.0)$ & - & $4.68 \mathrm{br} \mathrm{s}$ \\
\hline $9-\mathrm{OH}$ & $4.72 \mathrm{~d}(4.5)$ & - & $4.77 \mathrm{br} \mathrm{s}$ \\
\hline $17-\mathrm{CH}_{3}$ & $1.59 \mathrm{~s}$ & 13.3 & $1.64 \mathrm{~s}$ \\
\hline $20-\mathrm{CH}_{3}$ & $0.90 \mathrm{~d}(7.0)$ & 17.8 & $0.91 \mathrm{~d}(6.5)$ \\
\hline $22-\mathrm{CH}_{3}$ & $1.69 \mathrm{~s}$ & 16.5 & $1.68 \mathrm{~s}$ \\
\hline $\mathrm{NH}_{2}$ & $6.37 \mathrm{br}$ & 157.6 & $6.48-6.28 \mathrm{~m}$ \\
\hline $\mathrm{NH}$ & $9.85 \mathrm{~d}(10.0)$ & 164.0 & $9.88 \mathrm{~d}(10.3)$ \\
\hline 2 ' & $5.68 \mathrm{~s}$ & 118.8 & $5.69-5.76 \mathrm{~m}$ \\
\hline 3 , & - & 152.5 & - \\
\hline 4 ' & $1.82 \mathrm{~s}$ & 27.4 & $1.83 \mathrm{~s}$ \\
\hline 5, & $2.11 \mathrm{~s}$ & 20.3 & $2.12 \mathrm{~s}$ \\
\hline
\end{tabular}

Table 5.4.2 ${ }^{1} \mathrm{H}$ and ${ }^{13} \mathrm{C}$ NMR data shifts for natural and synthetic palmerolide $\mathrm{C}$. 
Our efforts to recover the synthetic sample from dimethylsulfoxide culminated in only small fraction of the initial material being retrieved. However, successful re-isolation of the natural palmerolide $\mathrm{C}$ allowed Baker and co-workers to carry out NMR analysis in acetonitrile and allowed direct comparison of the ${ }^{1} \mathrm{H}$ NMR spectra of the synthetic $\mathbf{3 b}$ and natural sample of palmerolide C 3 (Figure 5.4.2).

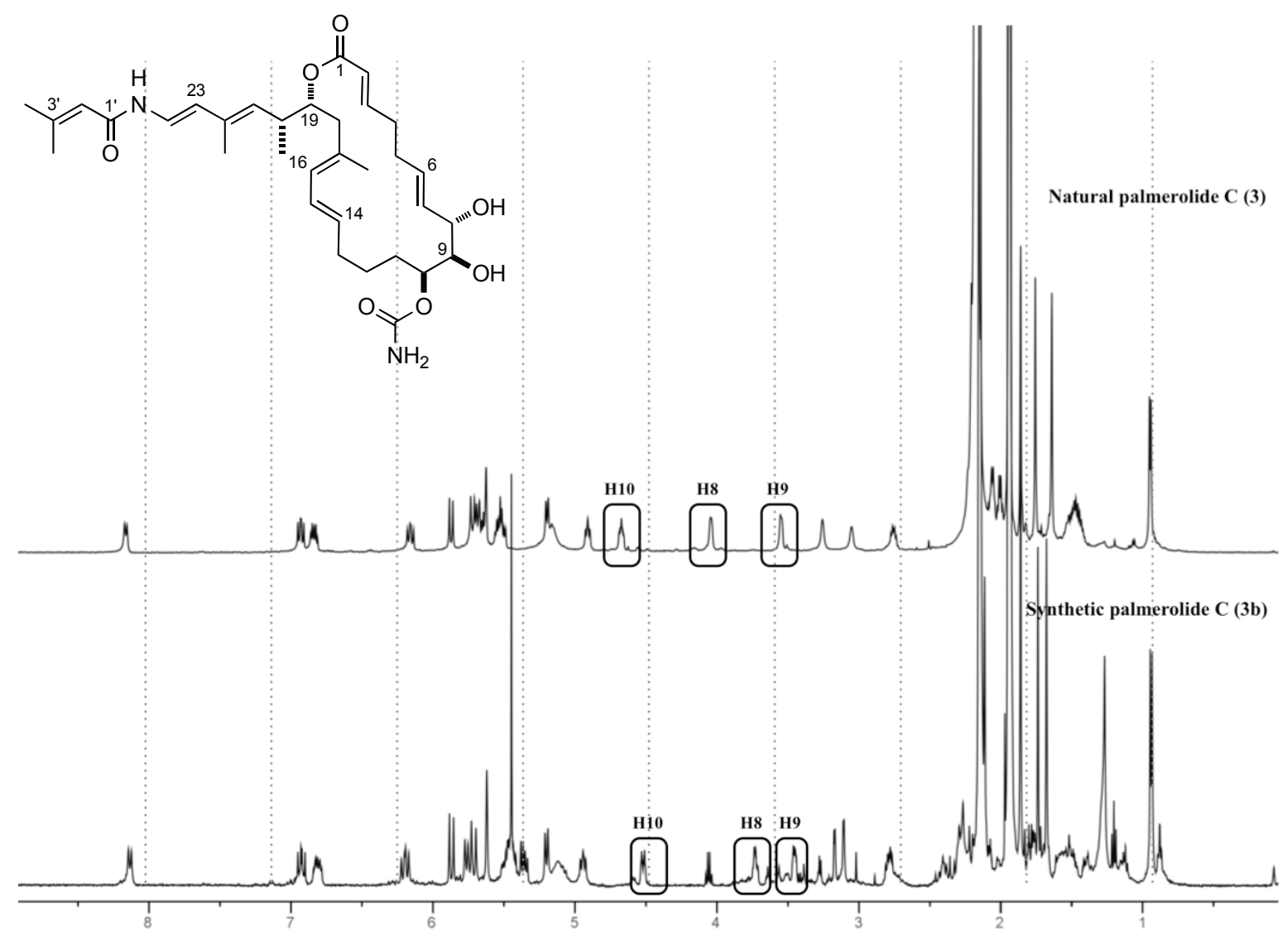

Figure 5.4.2 Comparison of the natural and synthetic palmerolide $\mathrm{C}^{1} \mathrm{H} \mathrm{NMR}$ in $\boldsymbol{d}_{3}-\mathrm{MeCN}$.

The observed differences in chemical shift correspond to the signals from the macrocyclic part of the molecule. The highlighted resonances for the H8-H10 stereocentres, despite following a similar splitting pattern in the natural and synthetic spectra, do not match. On a closer inspection (Figure 5.4.3) the H2-H3 and H6-H7 methine protons appeared to be significantly shifted upfield. Signals corresponding to the H14 and H16 protons were identified at different $\delta_{\mathrm{H}}$ in both natural and synthetic spectra. The discrepancy in chemical shifts of the crucial resonances indicates that the proposed stereochemical assignment of the natural palmerolide $\mathrm{C}$ was incorrect. 


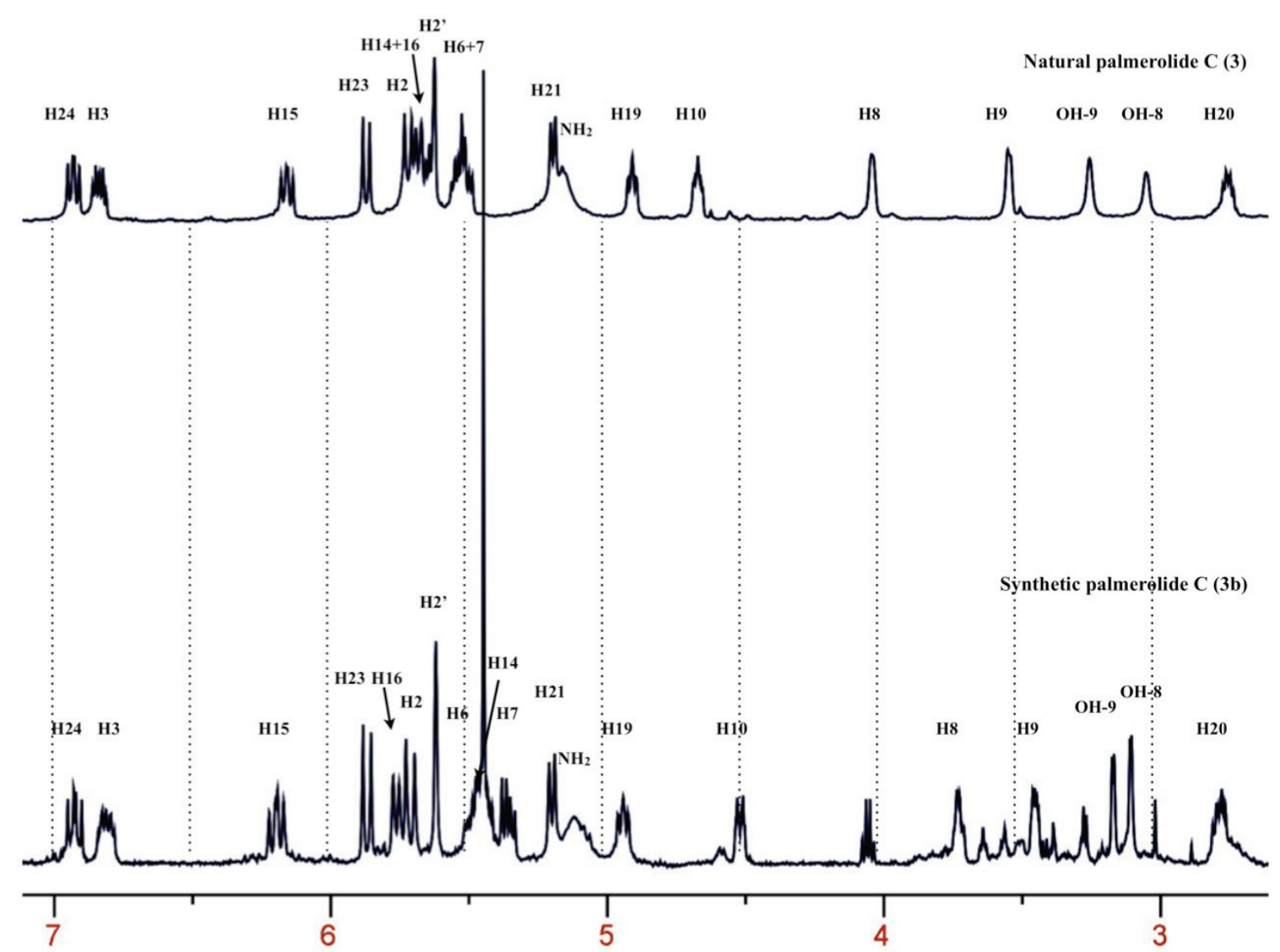

Figure 5.4.3 Comparison of the natural and synthetic palmerolide $\mathrm{C}^{1} \mathrm{H}$ NMR in $\boldsymbol{d}_{3}-\mathrm{MeCN}$.

\subsection{Final stereochemical assignment}

Our re-examination of the experimental data for the Murata $J$-based analysis of the C8-C10 triad of the natural palmerolide C (3) revealed an error (Figure 5.5.1). ${ }^{21}$ The Baker group had misinterpreted the Newman projection of the $J$-coupling based analysis, suggesting the arrangement of the C8-C9 stereocentres to be syn (Figure 5.5.1, A) and the C9-C10 centres anti (Figure 5.5.1, B). Our interpretation indicated that the C8-C9 chiral centres were in fact in an anti relationship, while the arrangement of the C9-C10 stereocentres remained anti as assigned by Baker (Figure 5.5.1). 


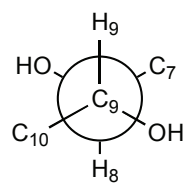

A

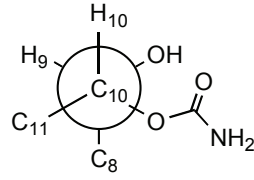

Figure 5.5.1 Newman projections of palmerolide $C$, reported by Baker group. ${ }^{10}$

This misinterpretation of the stereochemical assignment of the C8-C10 centres had a consequence in the synthesis of diastereoisomer $\mathbf{3 b}$ presented in Figure 5.5.2 with the C8-C9 syn and C9-C10 anti configuration. As an outcome of our synthetic investigation and final data evaluation we propose the structure 3 shown in Figure 5.5.2 with revised C8C9 anti and C9-C10 anti stereocentres to be the absolute configuration of the natural palmerolide C.

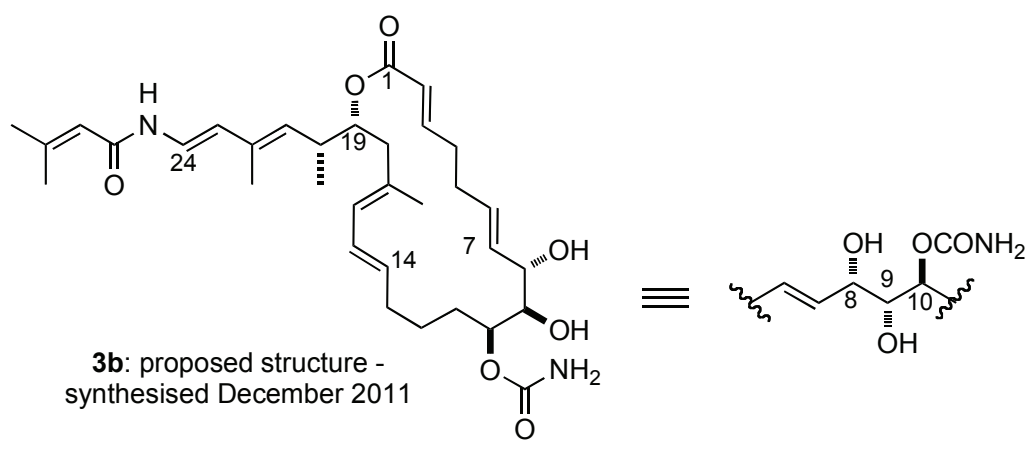

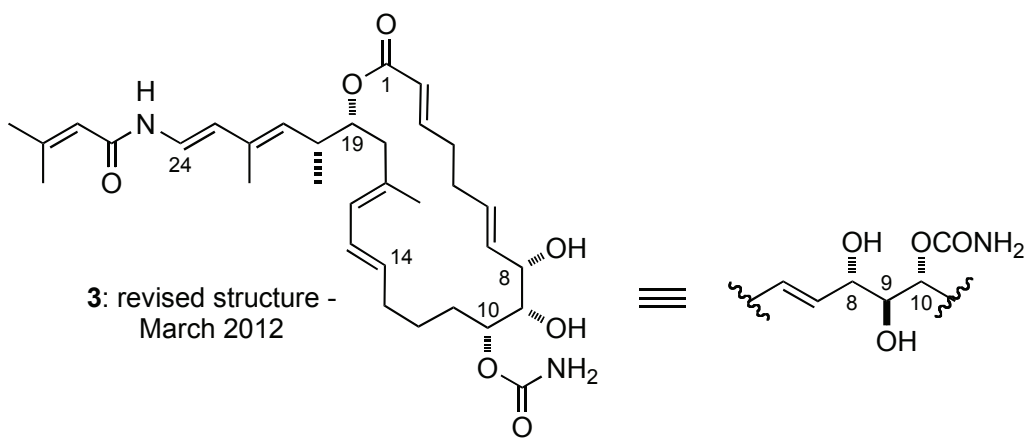

Figure 5.5.2 Proposed and revised structures of palmerolide $C$.

\subsection{Summary}

In summary, the first stereochemical assignment turned out to be incorrect due to the mistaken interpretation of the Mosher esters analysis of the $\mathrm{C} 8$ stereocentre. The error was related to the different priority of the Mosher acid and Mosher acid chloride substituents consequently affecting the entire resolution of the adjacent $\mathrm{C} 8-\mathrm{C} 10$ centres. 
The second assignment was shown to be incorrect due to the misinterpretation of the Newman projection for $J$-based configurational analysis. The first total synthesis of palmerolide $\mathrm{C}$ 3b helped to assign the relative and absolute configuration of this remarkedly interesting natural product (Figure 5.6.1).

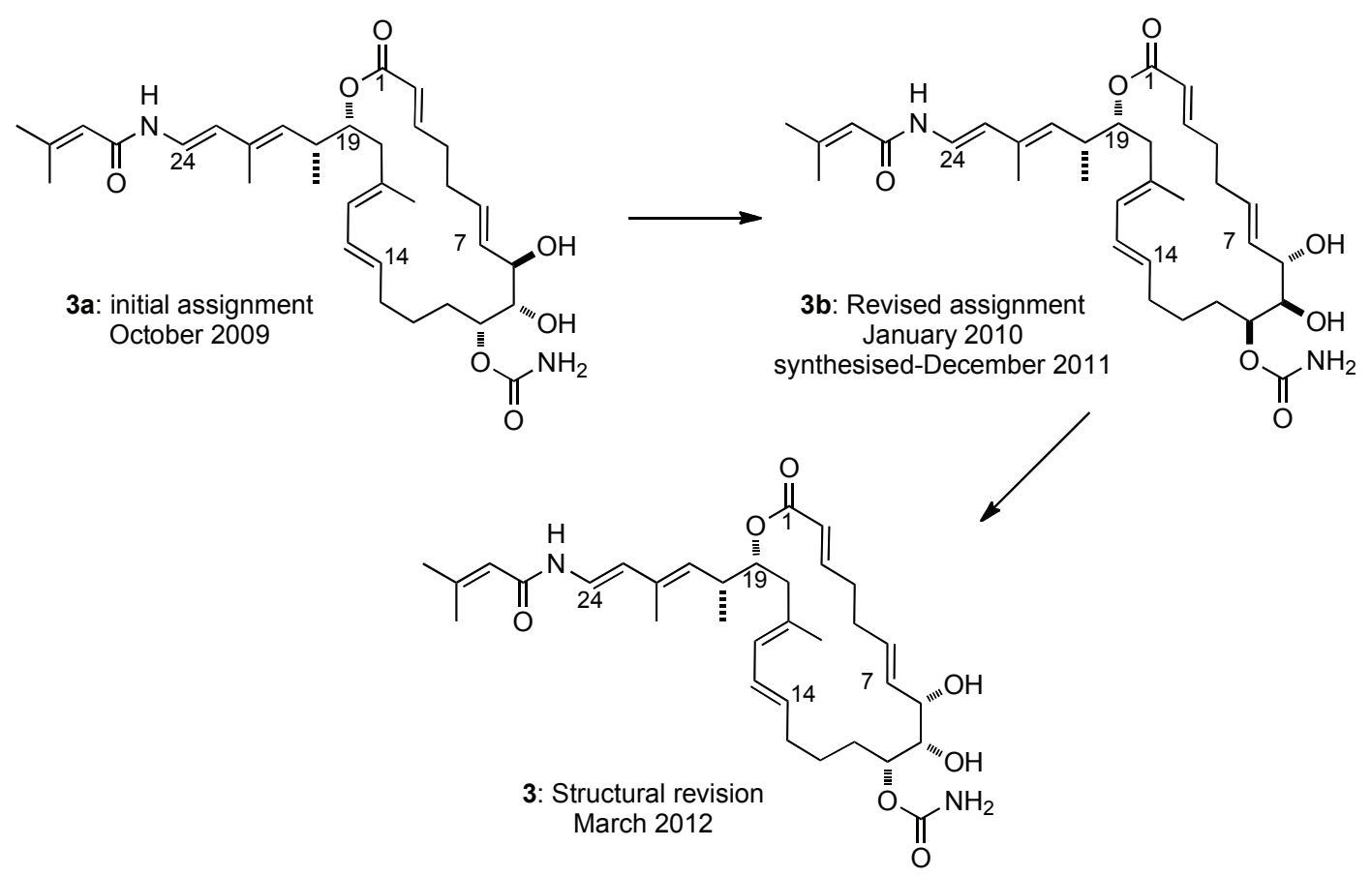

Figure 5.6.1 Summary of the stereochemical outcome. 


\section{Chapter 6}

\subsection{Summary and future work}

The first total synthesis of palmerolide $\mathrm{C} \mathbf{3} \mathbf{b}$ has been accomplished in an efficient and highly convergent manner. Our synthetic strategy evolved significantly during the venture. The first part of the project aimed at the resolution of the putative stereochemistry of the adjacent $\mathrm{C} 8-\mathrm{C} 10$ stereocentres of the natural product $\mathbf{3}$, culminated in the synthesis of the four possible diastereoisomers of $\mathrm{C} 7-\mathrm{C} 14$ degradation fragment. The obtained data set was sent for comparison studies with the natural C7-C14 degradation fragment to the Baker group at the University of South Florida, US. The outcome of their investigation and comparison studies are yet to be concluded.

The original synthetic approach towards the structure with Baker's first stereochemical assignment featured a cross metathesis coupling to assemble the $\mathrm{C} 1-\mathrm{C} 14$ fragment 110 and the Yamamoto vinylogous aldol reaction to advance the construction of the $\mathrm{C} 15-\mathrm{C} 24$ subunit 171. After making significant progress along these routes, lack of success in the attempted cross-metathesis and Yamamoto vinylogous aldol reactions forced the investigation of alternative synthetic plans. A revised synthetic strategy incorporated the secondary stereochemical assignment of the C8-C10 stereotriad, followed by aldol/elimination sequence to construct the $\mathrm{C} 1-\mathrm{C} 14$ fragment 169. At this time the vinylogous Mukaiyama aldol reaction was employed to form the C18-C19 bond.

After the successful elaboration of the key subunits, the critical Julia-Kocienski olefination to assemble the $(E, E)$-diene motif provided direct access to the advanced C1-C24 intermediate 210 comprising the entire carbon backbone of palmerolide $\mathrm{C}$ with all of the stereocentres already incorporated. Selective deprotection, mild saponification and macrolactonisation under Yamaguchi conditions completed the formation of the 20membered macrolactone. Cleavage of the silyl groups set the stage for the Buchwald $\mathrm{Cu}$ (I)-catalysed enamide formation under stoichiometric conditions. In total the synthesis of 
3b contains forty-one steps from commercially available materials with a longest linear sequence of twenty-three steps, starting from the proline-catalysed aldol reaction. The total synthesis of unnatural palmerolide C $3 \mathbf{b}$ was completed with $0.4 \%$ overall yield.

With the stereochemical assignment of the C8-C10 stereotriad and completion of the total synthesis of the diastereoisomer of palmerolide $\mathrm{C} \mathbf{3 b}$, the two major goals of this project have been accomplished. In addition, the convergent synthetic route provided a working platform for the synthesis of the other members of the palmerolide family (Figure 6.1.1).
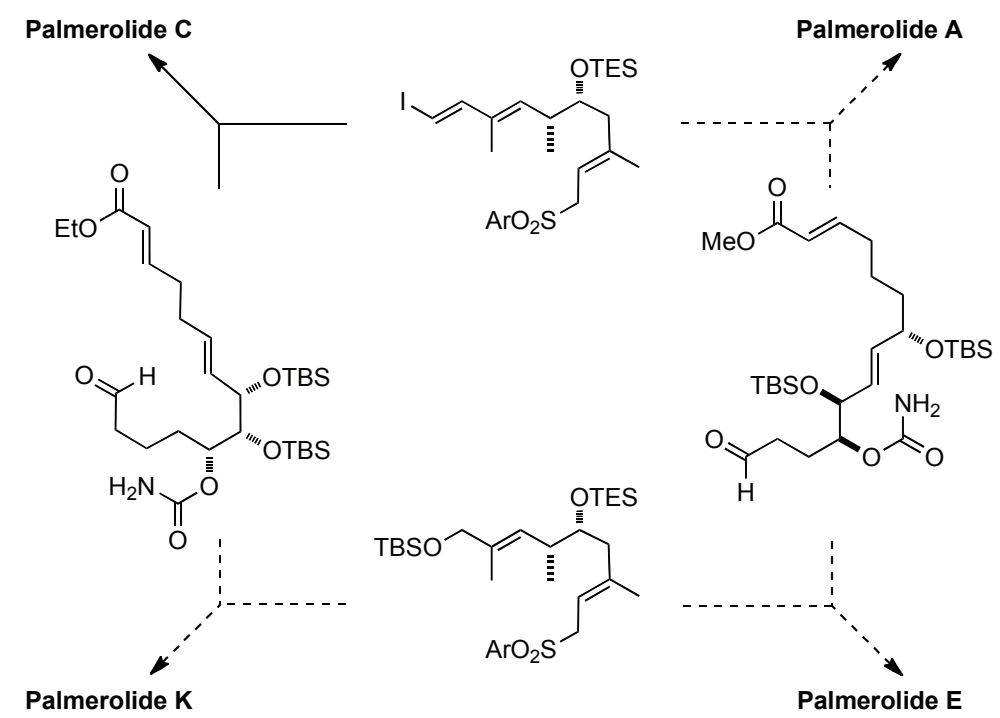

Figure 6.1.1 Synthesis of palmerolides from common intermediates.

However, there are areas of our research on palmerolide $\mathrm{C}$ that could warrant further attention. First, the synthesis of palmerolide $\mathrm{C} \mathbf{3}$ with a revised stereochemistry of the $\mathrm{C} 8$ C10 stereotriad could be readily accomplished. Although, a synthetic route towards the C7-C14 subunit $\mathbf{1 2 8}$ with the natural product configuration has been developed, the coupling of the C3-C6 220 and C7-C14 128 fragments would require attention. The possibility of utilising the cross-metathesis approach could be revisited using C3-C6 subunit 220 followed by incorporation of the $\mathrm{C} 1-\mathrm{C} 3$ enone after the coupling reaction to form $\mathrm{C} 1-\mathrm{C} 14$ subunit. Alternative plan would rely on the Zr-mediated coupling utilising Schwartz reagent and dimethylzinc to introduce C6-C7 alkene. Other possibility to form C6-C7 junction would be the addition of the lithium anion of C1-C7 alkyne 221 to aldehyde 127. Further exploration of the established synthetic route would provide access to the natural palmerolide $\mathrm{C} \mathbf{3}$. 

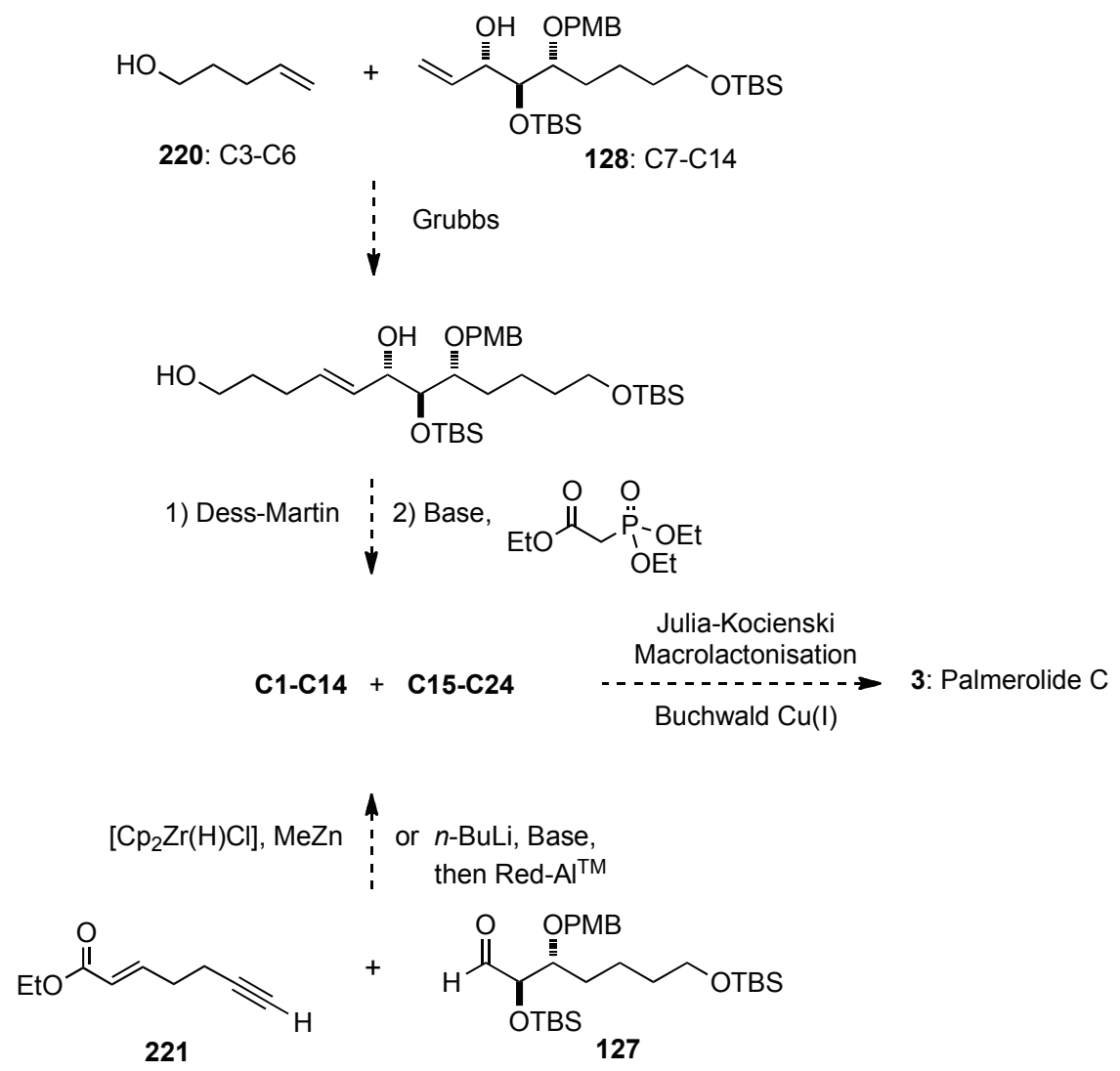

Scheme 6.1.1 Alternative route to C1-C14 fragment.

Secondly, our synthesis of C15-C24 subunit 171 could be modified to provide access to the non-iodinated fragment of the palmerolide E and K. Finally, in a broader investigation, this strategy could be used for the synthesis of the other members of the palmerolide family and their derivatives, leading to a library of potential anticancer agents with unique biological properties and selective efficacy against human melanoma cancers. 


\section{Chapter 7}

\subsection{General comments}

All reactions were performed in flame-dried glassware under positive pressure of Ar with magnetic stirring unless otherwise stated.

${ }^{1}$ H NMR spectra were recorded on a Bruker Avance $300(300 \mathrm{MHz})$ instrument, Bruker Avance II 400 (400 MHz) instrument or Bruker Avance 500 (500 MHz) instrument, using $\mathrm{CDCl}_{3}$ (or other indicated solvent) as reference and internal deuterium lock. The chemical shift data for each signal are given as $\delta$ in units of parts per million (ppm) relative to tetramethylsilane (TMS) where $\delta_{\mathrm{TMS}}=0.00 \mathrm{ppm}$. The multiplicity of each signal is indicated by: s (singlet); br s (broad singlet); d (doublet); t (triplet); q (quartet); qn (quintet); sp (septet) or $\mathrm{m}$ (multiplet). The number of protons (n) for a given resonance is indicated by $\mathrm{nH}$. Coupling constants $(J)$ are quoted in $\mathrm{Hz}$ and are recorded to the nearest $0.1 \mathrm{~Hz}$.

${ }^{13}$ C NMR spectra were recorded on a Bruker Avance $300(75 \mathrm{MHz})$ instrument, Bruker Avance II 400 (100 MHz) instrument or Bruker Avance 500 (125 MHz) instrument using the PENDANT sequence and internal deuterium lock. The chemical shift data for each signal are given as $\delta$ in units of ppm relative to TMS where $\delta_{\mathrm{TMS}}=0.00 \mathrm{ppm}$.

${ }^{19}$ F NMR spectra were recorded on a Bruker Avance $500(500 \mathrm{MHz})$ instrument. Resonances were assigned according to chemical shift, multiplicity, and reference to the literature.

HRMS and LRMS (High and Low resolution mass spectrometry) were recorded using a 
Thermofisher LTQ Orbitrap XL mass spectrometer, Finnigan MAT 900 XLT mass spectrometer, Micromass Quattro II mass spectrometer, Waters ZQ4000 mass spectrometer or a Thermofisher DSQ-II mass spectrometer by EPSRC national mass spectrometry service (Swansea, UK) using Electron Impact (EI), Electrospray Ionisation (ES), Chemical Ionisation (CI), Fast Atom Bombardment (FAB), Atmospheric Pressure Chemical Ionisation (APCI) or Atmospheric solids analysis probe (ASAP) techniques. Other spectra were recorded on a Micromass LCT mass spectrometer by the University of St Andrews mass spectrometry service (School of Chemistry and Biomolecular Sciences). The parent ion $\left(\mathrm{M}^{+},[\mathrm{M}+\mathrm{H}]^{+},[\mathrm{M}-\mathrm{H}]^{+},[\mathrm{M}+\mathrm{Na}]^{+}\right.$or $\left.\left[\mathrm{M}+\mathrm{NH}_{4}\right]^{+}\right)$is quoted.

IR spectra were recorded on a Perkin-Elmer Paragon Series 1000 FTIR spectrometer as thin films between sodium chloride discs or PTFE thin films as indicated. Absorption maxima are reported in wavenumbers $\left(\mathrm{cm}^{-1}\right)$. Intensities of the maxima are quoted as strong (s), medium (m) or weak (w).

Optical rotations were measured using an Perkin-Elmer Model 341 automatic polarimeter, in cells with a path length of $1 \mathrm{dm}$. The concentration (c) is expressed in $\mathrm{g} / 100 \mathrm{~mL}$ (equivalent to $\mathrm{g} / 0.1 \mathrm{dm}^{3}$ ). Specific rotations are denoted $[\alpha]_{\mathrm{D}}^{T}$ and are given in implied units of $10^{-1} \mathrm{deg} \mathrm{cm}^{2} \mathrm{~g}^{-1}$, where $\mathrm{T}$ is temperature in ${ }^{\circ} \mathrm{C}$.

$X$-ray analysis of single crystals was conducted by Prof. Alexandra Slawin at the University of St Andrews on a Rigaku Cu MM007 high brilliance generator with Saturn 92 CCD and XStream LT accessories.

Analytical thin layer chromatography (TLC) was carried out on pre-coated $0.25 \mathrm{~mm}$ Merck Kieselgel $60 \mathrm{~F}_{254}$ plates. Visualisation was by absorption of UV light, or thermal development after dipping in either an aqueous solution of potassium permanganate, potassium carbonate and sodium hydroxide, an ethanolic solution of phosphomolybdic acid (PMA) or a solution of ninhydrin in butan-2-ol.

Flash column chromatography was carried out on silica gel 60 (E. Merck, 40-63 micron) or on activated aluminium oxide (Acros, 50-200 micron, neutral) as indicated, under a positive pressure of compressed air. 
Kugelrohr bulb-to-bulb distillations were carried out using a Büchi Glass Oven B-585 machine. Boiling points are the actual oven temperatures.

Reagents and solvents were purified by standard means. Methanol was distilled from $\mathrm{CaH}_{2}$ in a recycling still under nitrogen. Dichloromethane $\left(\mathrm{CH}_{2} \mathrm{Cl}_{2}\right)$, toluene, tetrahydrofuran (THF) and diethyl ether $\left(\mathrm{Et}_{2} \mathrm{O}\right)$ were dried by passage through two columns of alumina using a MBRAUN SPS-800 solvent purification system under Ar. Anhydrous $N, N$ 'dimethylformamide (DMF) was purchased from Aldrich UK and dried by distillation from $4 \AA$ molecular sieves under $\mathrm{Ar}$ atmosphere. Chloroform was passed through alumina column and dried by distillation from $4 \AA$ molecular sieves under Ar atmosphere. Triethyl amine $\left(\mathrm{Et}_{3} \mathrm{~N}\right), \mathrm{BF}_{3} \cdot \mathrm{Et}_{2} \mathrm{O}$ and 2,6-lutidine were distilled from $\mathrm{CaH}_{2}$ under Ar. An Lerythrulose hydrate solution was desiccated according to the published procedure. Dowex 50 resin was washed with water, $3 \mathrm{M} \mathrm{HCl}$ and methanol prior to use. Chemicals were purchased from Acros UK, Aldrich UK, Avocado UK, Fisher UK or Fluka UK. All reagents and solvents were purified and dried, where necessary, by standard techniques. Where appropriate and if not stated otherwise, all non- aqueous reactions were performed under an inert atmosphere of argon, using a vacuum manifold with the gas passed through $4 \AA \AA$ molecular sieves and self-indicating silica gel. Under reduced pressure refers to the use of a rotary evaporator attached to a diaphragm pump. Hexane refers to a mixture of hexanes and petroleum ether to the fraction boiling between $40-60{ }^{\circ} \mathrm{C}$. Room temperature (rt) refers to the temperature of approximately $25^{\circ} \mathrm{C}$.

Miscellaneous, brine refers to a saturated solution of sodium chloride in deionised water. Reactions at $-78{ }^{\circ} \mathrm{C}$ were readily achieved with isopropanol and dry ice in a Dewar vacuum flask, $-100{ }^{\circ} \mathrm{C}$ was achieved with $\mathrm{CH}_{3} \mathrm{OH}$ and liquid nitrogen. 


\subsection{Preparation of reagents}

\section{Zinc borohydride $\left(\mathbf{Z n}\left(\mathbf{B H}_{4}\right)_{2}\right)^{128,129}$}

To a round bottom flask equipped with magnetic bar at $\mathrm{rt}$ was added anhydrous $\mathrm{ZnCl}_{2}$ $(8.04 \mathrm{~g}, 58.9 \mathrm{mmol}), \mathrm{NaBH}_{4}(5.12 \mathrm{~g}, 135 \mathrm{mmol})$ and THF $(118 \mathrm{~mL})$. The reaction mixture was stirred for $24 \mathrm{~h}$ to afford the clear solution c. $0.5 \mathrm{M}$ which is stable over a period of 6 months when stored under argon at room temperature.

\section{Thexyl borane (thexylBH$)^{66}$}

To a round bottom flask equipped with magnetic bar at $0{ }^{\circ} \mathrm{C}$ was added $\mathrm{BH}_{3} \bullet \mathrm{THF}(10 \mathrm{~mL}$, $1 \mathrm{M}$ soln. in THF) and 2,3-dimethyl-2-butene (10 mL, 1M soln. in THF) was then added dropwise over $30 \mathrm{~min}$. The reaction mixture was stirred for $1 \mathrm{~h}$ to afford the clear solution c. $0.5 \mathrm{M}$ which is stable for a week when stored under argon in the fridge.

\section{Dicyclohexylboron chloride $\left(c-\mathrm{Hex}_{2} \mathrm{BCl}\right)^{130}$}

To a stirred solution of cyclohexene $\left(20.0 \mathrm{~mL}, 197 \mathrm{mmol}\right.$, distilled from $\left.\mathrm{CaH}_{2}\right)$ in $\mathrm{Et}_{2} \mathrm{O}$ $(120 \mathrm{~mL})$ at $-10^{\circ} \mathrm{C}$ was added monochloroborane $\bullet$ dimethylsulfide $(10.3 \mathrm{~mL}, 98.6 \mathrm{mmol})$. The mildly exothermic reaction was controlled by the rate of addition. The resulting solution was stirred at $0{ }^{\circ} \mathrm{C}$ for $1 \mathrm{~h}$. The solvent and volatiles were removed by distillation at atmospheric pressure and the product purified by distillation under reduced pressure to afford the title compound as a colourless oil $(16.0$ g, $76 \%)$; B.pt. $105-110{ }^{\circ} \mathrm{C}$ at $0.5 \mathrm{mmHg}$. Lit. $104-105^{\circ} \mathrm{C} / 0.5 \mathrm{mmHg}$.

\section{Lithium methoxyborohydride $\left(\mathrm{LiBH}_{3}(\mathrm{OMe})\right)^{71-73}$}

To a lithium borohydride solution $(0.5 \mathrm{~mL}, 2 \mathrm{M}$ soln. in THF, $1.0 \mathrm{mmol})$ in THF $(4.5 \mathrm{~mL})$ at $0{ }^{\circ} \mathrm{C}$ was added $\mathrm{MeOH}(40.5 \mu \mathrm{L}, 1.0 \mathrm{mmol})$. Stirring was continued for $1.5 \mathrm{~h}$ at $\mathrm{rt}$ to provide a colourless solution c. $0.2 \mathrm{M}$ which could be stored for $48 \mathrm{~h}$ under argon.

\section{Dicyclohexylboron chloride•Triethyamine complex $\left(c-\mathrm{Hex}_{2} \mathbf{B C l} \cdot \mathrm{NEt}_{3}\right)^{71-73}$}

To a stirred solution of dicyclohexylboron chloride $(120 \mu \mathrm{L}, 0.58 \mathrm{mmol})$ in THF $(3 \mathrm{~mL})$ at $0{ }^{\circ} \mathrm{C}$ was added $\mathrm{Et}_{3} \mathrm{~N}(75 \mu \mathrm{L}, 1.02 \mathrm{mmol})$ to provide a colourless solution $c .0 .19 \mathrm{M}$ which was used immediately in 1,3-syn reduction. 


\section{p-Methoxybenzyl trichloroacetimidate (PMBTCA) ${ }^{86}$}

To a solution of $p$-methoxybenzyl alcohol $(14.0 \mathrm{~mL}, 15.6 \mathrm{~g}, 113 \mathrm{mmol})$ in $\mathrm{CH}_{2} \mathrm{Cl}_{2}(100$ $\mathrm{mL})$ at $-10 \quad{ }^{\circ} \mathrm{C}$ was added aqueous solution of $\mathrm{KOH}(100 \mathrm{~g} / 100 \mathrm{~mL})$ and tetrabutylammonium hydrogen sulfate $(384 \mathrm{mg}, 1.13 \mathrm{mmol})$ followed by dropwise addition of trichloroacetonitrile $(12.5 \mathrm{~mL}, 18.0 \mathrm{~g}, 125 \mathrm{mmol})$. The reaction mixture was then allowed to warmed to $\mathrm{rt}$ over $2 \mathrm{~h}$ and the layers were separated, the aqueous phase was extracted with $\mathrm{CH}_{2} \mathrm{Cl}_{2}(3 \times 100 \mathrm{~mL})$, the combined organic extracts were dried $\left(\mathrm{Na}_{2} \mathrm{SO}_{4}\right)$, filtered and concentrated under reduced pressure. Purification by distillation afforded the title compound $(28.0 \mathrm{~g}, 88 \%)$ as a colourless solution. B.pt. $120-145^{\circ} \mathrm{C}$ at 0.5 mmHg. Lit. $135^{\circ} \mathrm{C}$ at $0.7 \mathrm{mmHg} .{ }^{86}{ }^{1} \mathbf{H}$ NMR $\left(300 \mathrm{MHz}, \mathrm{CDCl}_{3}\right) \delta 7.38(2 \mathrm{H}, \mathrm{d}, J=8.8 \mathrm{~Hz}$, $\operatorname{Ar}-\underline{\mathrm{H}}), 6.91(2 \mathrm{H}, \mathrm{d}, J=8.8 \mathrm{~Hz}, \mathrm{Ar}-\underline{\mathrm{H}}), 5.28\left(2 \mathrm{H}, \mathrm{s}, \underline{\mathrm{CH}}_{2}\right), 3.81\left(3 \mathrm{H}, \mathrm{s}, \mathrm{OC}_{3}\right)$.

\section{1-Hydroxy-1,2-benziodoxol-3(1H)-one 1-Oxide (IBX) ${ }^{110}$}

To a solution of Oxone $(83.0 \mathrm{~g}, 0.135 \mathrm{~mol})$ in deionized water $(225 \mathrm{~mL}, 0.45 \mathrm{M})$ at rt was added 2-iodobenzoic acid $(25.0 \mathrm{~g}, 0.10 \mathrm{~mol})$. The reaction mixture was warmed to $70-73$ ${ }^{\circ} \mathrm{C}$ over $20 \mathrm{~min}$ and mechanically stirred at this temperature for $3 \mathrm{~h}$. The suspension was then cooled to $5{ }^{\circ} \mathrm{C}$ and left at this temperature for $1.5 \mathrm{~h}$ with slow stirring. The mixture was filtered and the solid was repeatedly rinsed with water $(6 \times 50 \mathrm{~mL})$ and acetone $(2 \times$ $50 \mathrm{~mL})$. The white, crystalline solid $(24.0 \mathrm{~g}, 86 \%)$ was left to dry at $\mathrm{rt}$ for $16 \mathrm{~h}$.

\section{1,1,1-Triacetoxy-1,1-dihyd-1,2-benziodoxol-3(1H)-one (Dess-Martin reagent) ${ }^{111}$}

To a solution of acetic anhydride $(82.0 \mathrm{~mL}, 88.7 \mathrm{~g}, 869 \mathrm{mmol})$ and $\mathrm{TsOH} \cdot \mathrm{H}_{2} \mathrm{O}(120 \mathrm{mg}$, $0.631 \mathrm{mmol})$ at $\mathrm{rt}$ was added IBX $(24.0 \mathrm{~g}, 85.7 \mathrm{mmol})$ and the reaction mixture equipped with a drying tube was heated at $80{ }^{\circ} \mathrm{C}$ for $2 \mathrm{~h}$. The mixture was then cooled in ice-water bath and the suspension was filtered and washed with $\mathrm{Et}_{2} \mathrm{O}(5 \times 20 \mathrm{~mL})$. The resulting white, crystalline solid (30.0 g, 83\%) was quickly transferred to an argon flushed amberglass bottle and stored at $-25^{\circ} \mathrm{C}$. 


\section{(1-Methoxy-3-methylbuta-1,3-dienyloxy)-trimethylsilane (173) ${ }^{131}$}

To a stirred solution of diisopropylamine $(8.41 \mathrm{~mL}, 60.0 \mathrm{mmol})$ in THF $(50 \mathrm{~mL})$ at $0{ }^{\circ} \mathrm{C}$ was added n-BuLi $(32.5 \mathrm{~mL}, 1.6 \mathrm{M}$ soln. in hexane, $52.5 \mathrm{mmol})$ and the reaction mixture was stirred at $0{ }^{\circ} \mathrm{C}$ for $15 \mathrm{~min}$ before cooling to $-78^{\circ} \mathrm{C}$. Methyl 3,3-dimethylacrylate (6.54 $\mathrm{mL}, 50.0 \mathrm{mmol})$ was then added. After $1 \mathrm{~h}$ trimethylsilyl chloride $(7.61 \mathrm{~mL}, 60.0 \mathrm{mmol})$ was added as a solution in THF $(5 \mathrm{~mL})$ and the reaction mixture was warmed to $0{ }^{\circ} \mathrm{C}$ and stirring continued for $1 \mathrm{~h}$. The reaction mixture was concentrated under reduced pressure to a volume of $20 \mathrm{~mL}$ and pentane $(100 \mathrm{~mL})$ was added. The suspension was filtered through celite and concentrated under reduced pressure. The residue was distilled under vacuum (bp $48{ }^{\circ} \mathrm{C}$ at $0.75 \mathrm{mmHg}$ ) to give the silyl enol ether 173 as a colourless oil (7.32 g, 79\%). ${ }^{1} \mathbf{H}$ NMR $\left(400 \mathrm{MHz} ; \mathrm{CDCl}_{3}\right) \delta 4.78(1 \mathrm{H}, \mathrm{dt}, J=2.7,0.7 \mathrm{~Hz}, \mathrm{H}-4 \mathrm{a}), 4.54(1 \mathrm{H}, \mathrm{dq}, J=2.7$, $1.4 \mathrm{H}-4 \mathrm{~b}), 4.26$ (1H, s, H-2), 3.57 (3H, s, OC$\left.\underline{H}_{3}\right), 1.93\left(3 \mathrm{H}, \mathrm{dd}, J=1.4,0.7 \mathrm{~Hz}, \mathrm{C}_{3}\right), 0.23$ $\left(9 \mathrm{H}, \mathrm{s}, \mathrm{Si}-\left(\mathrm{CH}_{3}\right)_{3}\right)$.

\section{3-Methyl but-2-enamide (218). ${ }^{132}$}

To a suspension of 3,3-dimethylacrylic acid $(5.0 \mathrm{~g}, 50 \mathrm{mmol})$ in $\mathrm{CH}_{2} \mathrm{Cl}_{2}(100 \mathrm{~mL})$ at $0{ }^{\circ} \mathrm{C}$ was added a catalytic amount of DMF $(60 \mu \mathrm{L})$ and oxalyl chloride $(4.7 \mathrm{~mL}, 55 \mathrm{mmol})$. The resulting reaction mixture was stirred at $0{ }^{\circ} \mathrm{C}$ for $15 \mathrm{~min}$ and then at $\mathrm{rt}$ for a further $90 \mathrm{~min}$. $\mathrm{CH}_{2} \mathrm{Cl}_{2}(100 \mathrm{~mL})$ at $-78^{\circ} \mathrm{C}$, which had been saturated with ammonia gas for $20 \mathrm{~min}$, was then cannulated into the cooled to $-78^{\circ} \mathrm{C}$ reaction mixture. After stirring for an additional $20 \mathrm{~min}$, the reaction mixture was quenched with water $(100 \mathrm{~mL})$ and the aqueous phase was extracted with $\mathrm{CH}_{2} \mathrm{Cl}_{2}(3 \times 100 \mathrm{~mL})$. The combined organic extracts were dried $\left(\mathrm{Na}_{2} \mathrm{SO}_{4}\right)$, filtered and concentrated under reduced pressure to give 3-methyl but-2enamide 218 ( $2.8 \mathrm{~g}, 57 \%)$ as white crystals, which could be used without any further purification.

${ }^{1}$ H NMR (400 MHz, $\left.\mathrm{CDCl}_{3}\right) \delta 5.89\left(1 \mathrm{H}, \mathrm{br} \mathrm{s}, \mathrm{NH}_{2}\right), 5.61(1 \mathrm{H}, \mathrm{dt}, J=2.7,1.3 \mathrm{~Hz}, \mathrm{H}-2)$, $5.51\left(1 \mathrm{H}\right.$, br s, $\left.\underline{\mathrm{H}}_{2}\right), 2.12(3 \mathrm{H}, \mathrm{d}, J=1.3 \mathrm{~Hz}, \mathrm{H}-4), 1.83\left(3 \mathrm{H}, \mathrm{d}, J=1.4 \mathrm{~Hz}, \mathrm{C}_{3}\right)$; ${ }^{13}$ C NMR $\left(101 \mathrm{MHz}, \mathrm{CDCl}_{3}\right) \delta 169.3,152.6,117.7,27.3,19.9$. 


\subsection{Experimental procedures for Chapter 2}

\section{2,2-Dimethyl-5-amino-5-hydroxymethyl-1,3-dioxane $(71)^{51}$}

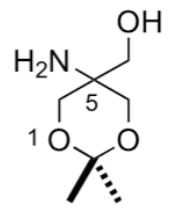

Trizma ${ }^{\circledR}$ base (50 g, $\left.0.30 \mathrm{~mol}\right)$, 2,2-dimethoxypropane (40 g, $\left.0.40 \mathrm{~mol}\right)$, CSA (2.6 g, 11 mmol) in dimethylformamide $(100 \mathrm{~mL})$ were stirred at $\mathrm{rt}$ for $24 \mathrm{~h}$. After addition of $\mathrm{Et}_{3} \mathrm{~N}$ $(2.5 \mathrm{~mL}, 18 \mathrm{mmol})$ the solvent was removed under reduced pressure. The residue was dissolved in EtOAc $(700 \mathrm{~mL})$ followed by addition of $\mathrm{Et}_{3} \mathrm{~N}(40 \mathrm{~mL}, 0.30 \mathrm{~mol})$ and stirring was continued for $15 \mathrm{~min}$. The precipitate was filtered and washed with EtOAc. The filtrate was evaporated to afford 2,2-dimethyl-5-amino-5-hydroxymethyl-1,3-dioxane 71 (37.1 $\mathrm{g}, 90 \%)$ as a colourless solid.

${ }^{1}$ H NMR (300 MHz, CDCl $) \delta 3.82(2 \mathrm{H}, \mathrm{d}, J=11.8 \mathrm{~Hz}, \mathrm{H}-4 \mathrm{a}, \mathrm{H}-6 \mathrm{a}), 3.62(2 \mathrm{H}, \mathrm{d}, J=11.8$

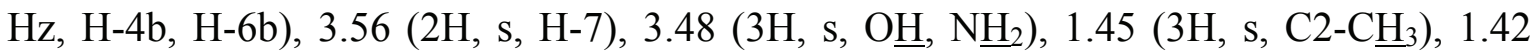
$\left(3 \mathrm{H}, \mathrm{s}, \mathrm{C} 2-\mathrm{C}_{3}\right) ;{ }^{13} \mathbf{C} \mathbf{N M R}\left(75 \mathrm{MHz}, \mathrm{CDCl}_{3}\right) \delta 98.4,66.7,64.2,50.6,25.0,22.1$.

\section{2,2-Dimethyl-1,3-dioxan-5-one (dioxanone) $(63)^{51}$}

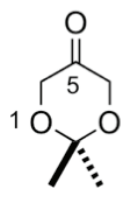

To a solution of $48(20.0 \mathrm{~g}, 12.4 \mathrm{mmol})$ and $\mathrm{KH}_{2} \mathrm{PO}_{4}(16.9 \mathrm{~g}, 12.4 \mathrm{mmol})$ in water $(200$ $\mathrm{mL})$ at $5{ }^{\circ} \mathrm{C}$ was added $\mathrm{NaIO}_{4}\left(370 \mathrm{~mL}, 0.5 \mathrm{M}\right.$ soln. in $\left.\mathrm{H}_{2} \mathrm{O}, 18.5 \mathrm{mmol}\right)$ dropwise over $2 \mathrm{~h}$, while the temperature was maintained at 5-10 ${ }^{\circ} \mathrm{C}$. After $12 \mathrm{~h}$ at rt, $\mathrm{CH}_{2} \mathrm{Cl}_{2}(200 \mathrm{~mL})$ was then added and the layers were separated. The aqueous phase was extracted with $\mathrm{CH}_{2} \mathrm{Cl}_{2}$ $(10 \times 30 \mathrm{~mL})$, the combined extracts were dried $\left(\mathrm{MgSO}_{4}\right)$, filtered and concentrated under reduced pressure. Purification by distillation (bp. $25^{\circ} \mathrm{C} / 1$ torr) afforded 2,2-dimethyl-1,3- 
dioxan-5-one $63(9.68 \mathrm{~g}, 60 \%)$ as a colourless oil.

$\boldsymbol{R}_{f} 0.51$ (10\% EtOAc/Hexanes); ${ }^{1} \mathbf{H}$ NMR (400 MHz, $\left.\mathrm{CDCl}_{3}\right) \delta 4.16(4 \mathrm{H}, \mathrm{s}, \mathrm{H}-4, \mathrm{H}-6)$ and $1.46\left(6 \mathrm{H}, \mathrm{s}, \mathrm{C} 2-\mathrm{CH}_{3}\right) ;{ }^{13} \mathbf{C} \mathbf{N M R}\left(100 \mathrm{MHz}, \mathrm{CDCl}_{3}\right) \delta 208.0,100.1,66.8,23.5$.

\section{5-(tert-Butyldimethylsilyloxy)pentanal (69) $)^{133}$}

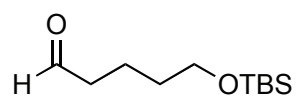

To a solution of 1,4-butanediol $23(6.06 \mathrm{~mL}, 60.5 \mathrm{mmol}), \mathrm{Et}_{3} \mathrm{~N}(3.66 \mathrm{~mL}, 26.3 \mathrm{mmol})$ and DMAP (246 mg, $2.00 \mathrm{mmol}$ ) in $\mathrm{CH}_{2} \mathrm{Cl}_{2}(30.0 \mathrm{~mL})$ at $\mathrm{rt}$ was slowly added a solution of TBSCl $(3.04 \mathrm{~g}, 20.1 \mathrm{mmol})$ in $\mathrm{CH}_{2} \mathrm{Cl}_{2}(10.0 \mathrm{~mL})$. After $16 \mathrm{~h}$, the reaction mixture was poured onto brine $(30 \mathrm{~mL})$ and layers were separated. The aqueous phase was extracted with $\mathrm{CH}_{2} \mathrm{Cl}_{2}(3 \times 30 \mathrm{~mL})$ and the combined organic extracts were washed with water $(50.0$ $\mathrm{mL})$, brine $(50 \mathrm{~mL})$, dried $\left(\mathrm{MgSO}_{4}\right)$, filtered and concentrated under reduced pressure. Purification by flash column chromatography (5\% EtOAc/Hexanes) provided alcohol $\mathbf{7 3}$ $(11.1 \mathrm{~g}, 85 \%)$ as a colourless oil.

$\boldsymbol{R}_{f} 0.48$ (50\% EtOAc/Hexanes); ${ }^{1} \mathbf{H}$ NMR $\left(300 \mathrm{MHz}, \mathrm{CDCl}_{3}\right) \delta$ 3.66-3.60 (4H, m, H-1, 5), 1.65-1.52 (4H, m, H-2, 4), 1.45-1.35 (2H, m, H-3), 0.90 (9H, s, Si-C(C $\left.\left.\underline{\mathrm{H}}_{3}\right)_{3}\right), 0.05$ (6H, m, $\left.\mathrm{Si}-\left(\mathrm{C}_{3}\right)_{2}\right)$.

To a solution of oxalyl chloride $(0.92 \mathrm{~mL}, 11.0 \mathrm{mmol})$ in $\mathrm{CH}_{2} \mathrm{Cl}_{2}(20.0 \mathrm{~mL})$ at $-78{ }^{\circ} \mathrm{C}$ was slowly added DMSO (1.75 mL, $24.1 \mathrm{mmol})$. After $30 \mathrm{~min}$, a solution of alcohol 73 (2.18 g, $10.1 \mathrm{mmol})$ in $\mathrm{CH}_{2} \mathrm{Cl}_{2}(20.0 \mathrm{~mL})$ was added, dropwise. After $1 \mathrm{~h}, \mathrm{Et}_{3} \mathrm{~N}(19.1 \mathrm{~mL}, 137$ mmol) was added and the slurry was allowed to attain rt. After $1 \mathrm{~h}$, saturated $\mathrm{NH}_{4} \mathrm{Cl}(20$ $\mathrm{mL}$ ) solution was added and layers were separated. The aqueous phase was extracted with $\mathrm{CH}_{2} \mathrm{Cl}_{2}(3 \times 40 \mathrm{~mL})$ and the combined organic extracts were washed with $\mathrm{H}_{2} \mathrm{O}(30 \mathrm{~mL})$, brine $(30 \mathrm{~mL})$, dried $\left(\mathrm{MgSO}_{4}\right)$, filtered and concentrated under reduced pressure. The crude aldehyde 69 (1.73 g, 80\%) was used without further purification.

$\boldsymbol{R}_{f} 0.75$ (25\% EtOAc/Hexanes); ${ }^{1} \mathbf{H}$ NMR $\left(400 \mathrm{MHz}, \mathrm{CDCl}_{3}\right) \delta 9.7(1 \mathrm{H}, \mathrm{t}, J=1.7 \mathrm{~Hz}, \mathrm{H}-$ 1), 3.63 (2H, t, $J=6.2 \mathrm{~Hz}, \mathrm{H}-5), 2.46$ (2H, td, $J=7.29,1.76 \mathrm{~Hz}, \mathrm{H}-2), 1.66-1.75$ (2H, m, H-3), 1.50-1.59 (2H, m, H-4), 0.89 (9H, s, Si-C(C $\left.\left.\underline{\mathrm{C}}_{3}\right)_{3}\right), 0.05$ (6H, s, Si-( $\left.\left.\mathrm{C}_{3}\right)_{2}\right)$. 


\section{5-(Benzyloxy)pentan-1-al (76) ${ }^{134}$}<smiles>O=CCCCCCOCc1ccccc1</smiles>

To a solution of pentane-1,5-diol $(6.70 \mathrm{~mL}, 64.0 \mathrm{mmol})$ in THF $(100 \mathrm{~mL})$ at $0{ }^{\circ} \mathrm{C}$ was added $\mathrm{NaH}(1.30 \mathrm{~g}, 54.0 \mathrm{mmol})$. The reaction mixture was stirred at $0{ }^{\circ} \mathrm{C}$ and benzyl bromide (4.60 mL, $34.0 \mathrm{mmol})$ in THF $(20 \mathrm{~mL})$ was added via cannula. After $12 \mathrm{~h}$ at $\mathrm{rt}$, $\mathrm{MeOH}(10 \mathrm{~mL})$ was added and the reaction mixture was concentrated under reduced pressure to the $c a .50 \mathrm{~mL}$ volume. The residue was extracted with $\mathrm{CH}_{2} \mathrm{Cl}_{2}(3 \times 20 \mathrm{~mL})$, washed with brine $(50 \mathrm{~mL})$, dried $\left(\mathrm{Na}_{2} \mathrm{SO}_{4}\right)$, filtered and concentrated under reduced pressure. Purification by flash column chromatography (20-40\% EtOAc/Hexanes) afforded product $77(4.1 \mathrm{~g}, 66 \%)$ as a colourless oil.

$\boldsymbol{R}_{f} 0.31$ (20\% EtOAc/Hexanes); ${ }^{1} \mathbf{H}$ NMR (400 MHz, $\left.\mathrm{CDCl}_{3}\right): \delta$ 7.30-7.19 (5H, m, Ar-H), $4.43\left(2 \mathrm{H}, \mathrm{s}, \mathrm{OCH}_{2} \mathrm{Ph}\right), 3.53(2 \mathrm{H}, \mathrm{t}, J=6.52 \mathrm{~Hz}, \mathrm{H}-5), 3.41$ (2H, t, $\left.J=6.49 \mathrm{~Hz}, \mathrm{H}-1\right), 1.88$ (1H, bs, O프), 1.61-1.54 (2H, m, H-3), 1.53-1.46 (2H, m, H-2), 1.41-1.33 (2H, m, H-4).

To a solution of oxalyl chloride $(1.44 \mathrm{~mL}, 17.0 \mathrm{mmol})$ in $\mathrm{CH}_{2} \mathrm{Cl}_{2}(10.0 \mathrm{~mL})$ at $-78^{\circ} \mathrm{C}$ was added a solution of DMSO $(2.64 \mathrm{~mL}, 34.0 \mathrm{mmol})$ dropwise. The reaction mixture was stirred at $-78{ }^{\circ} \mathrm{C}$ for $15 \mathrm{~min}$ and a solution of alcohol 77 (3.00 g, $\left.15.5 \mathrm{mmol}\right)$ in $\mathrm{CH}_{2} \mathrm{Cl}_{2}$ $(20.0 \mathrm{~mL})$ was added. The reaction mixture was stirred at $-78^{\circ} \mathrm{C}$ for an additional $15 \mathrm{~min}$. and $\mathrm{Et}_{3} \mathrm{~N}(10.8 \mathrm{~mL}, 77.0 \mathrm{mmol})$ was then added, and the mixture was allowed to warm to rt. The reaction was quenched by addition of water $(30 \mathrm{~mL})$ and extracted with $\mathrm{CH}_{2} \mathrm{Cl}_{2}(3$ $\times 50 \mathrm{~mL})$. The combined organic extracts were washed with saturated $\mathrm{NH}_{4} \mathrm{Cl}(50 \mathrm{~mL})$ solution and brine $(50 \mathrm{~mL})$, dried $\left(\mathrm{Na}_{2} \mathrm{SO}_{4}\right)$ and concentrated under reduced pressure. The resulting oil was purified by flash column chromatography (20\% EtOAc/Hexanes) to give $76(1.90 \mathrm{~g}, 65 \%)$ as a colourless oil.

$\boldsymbol{R}_{\boldsymbol{f}} 0.41\left(20 \%\right.$ EtOAc/Hexanes); ${ }^{1} \mathbf{H}$ NMR (400 MHz, $\left.\mathrm{CDCl}_{3}\right): \delta 9.76(1 \mathrm{H}, \mathrm{t}, J=1.7 \mathrm{~Hz}$, H-1), 7.35-7.27 (5H, m, ArH), 4.49 (2H, s, $\left.\mathrm{OC}_{2} \mathrm{Ph}\right), 3.48$ (2H, t, J=6.1 Hz, H-5), 2.45 $(2 \mathrm{H}, \mathrm{td}, J=7.2,1.7 \mathrm{~Hz}, \mathrm{H}-2), 1.78-1.70(2 \mathrm{H}, \mathrm{m}, \mathrm{H}-3)$ and 1.65-1.61 (2H, m, H-4). 


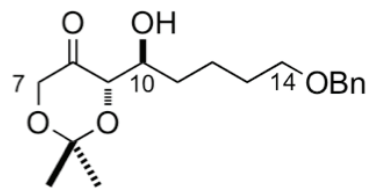

To a solution of dioxanone $63(1.00 \mathrm{~g}, 7.69 \mathrm{mmol})$ in DMF $(5.0 \mathrm{~mL})$ at $\mathrm{rt}$ was added (S)-proline $(221 \mathrm{mg}, 1.92 \mathrm{mmol})$. The resulting mixture was stirred for $15 \mathrm{~min}$ and aldehyde $76(880 \mathrm{mg}, 7.69 \mathrm{mmol})$ was added. After 5 days, saturated $\mathrm{NH}_{4} \mathrm{Cl}(5.0 \mathrm{~mL})$ solution was added and the layers were separated. The aqueous phase was extracted with EtOAc $(3 \times 10 \mathrm{~mL})$ and the combined organic extracts were washed with brine $(20 \mathrm{~mL})$, dried $\left(\mathrm{Na}_{2} \mathrm{SO}_{4}\right)$, filtered and concentrated under reduced pressure. Purification by flash column chromatography (10\% EtOAc/Hexanes) afforded aldol product 78 (237 mg, 23\%) as a colourless oil.

$\boldsymbol{R}_{f} 0.21\left(10 \%\right.$ EtOAc/Hexanes); $[\alpha]_{\mathrm{D}}^{20}-16.0\left(c 0.10, \mathrm{CHCl}_{3}\right)$; IR $\boldsymbol{v}_{\max }\left(\right.$ thin film $\left./ \mathrm{cm}^{-1}\right) 3395$, 2912, 1736, 1652, 1392, 1225, 1197, 1067, 911; ${ }^{1} \mathbf{H}$ NMR (400 MHz, $\left.\mathrm{CDCl}_{3}\right): \delta 7.35-7.31$ $(5 \mathrm{H}, \mathrm{m}, \mathrm{Ar}-\mathrm{H}), 4.50,\left(2 \mathrm{H}, \mathrm{s}, \mathrm{OC}_{2} \mathrm{Ph}\right), 4.25(1 \mathrm{H}, \mathrm{dd}, J=17.3,1.5 \mathrm{~Hz}, \mathrm{H}-7 \mathrm{~A}), 4.07(1 \mathrm{H}$, dd, $J=6.9,1.5 \mathrm{~Hz}, \mathrm{H}-9), 4.01$ (1H, d, $J=17.3 \mathrm{~Hz}, \mathrm{H}-7 \mathrm{~B}), 3.92-3.84$ (1H, m, H-10), 3.53$3.44(2 \mathrm{H}, \mathrm{t}, J=6.4 \mathrm{~Hz}, \mathrm{H}-14), 2.97$ (1H, d, $J=3.9 \mathrm{~Hz}, \mathrm{OH}), 1.70-1.56$ (6H, m, H-11, 12 , 13), $1.46\left(3 \mathrm{H}, \mathrm{s}, \mathrm{C}-\mathrm{C}_{3}\right), 1.42\left(3 \mathrm{H}, \mathrm{s}, \mathrm{C}-\mathrm{CH}_{3}\right) ;{ }^{13} \mathbf{C}$ NMR (100 MHz, $\left.\mathrm{CDCl}_{3}\right): 211.6,139.0$, $128.7,127.9,127.8,101.3,76.2,73.2,70.87,70.69,67.1,32.4,30.0,24.2,23.9,22.1$; HRMS $\left(\mathrm{ES}^{+}\right.$) calcd. for $\mathrm{C}_{18} \mathrm{H}_{27} \mathrm{O}_{5}[\mathrm{M}+\mathrm{H}]^{+}$323.1853, found 323.1857 .

\section{(S)-4-((S)-1-Hydroxypent-4-enyl)-2,2-dimethyl-1,3-dioxan-5-one (83)}

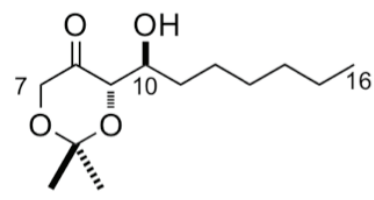

To the stirred dioxanone $\mathbf{6 3}(1.00 \mathrm{~g}, 7.69 \mathrm{mmol})$ in $\mathrm{CHCl}_{3}(5.0 \mathrm{~mL})$ at rt, heptanal $82(0.88$ g, $7.69 \mathrm{mmol})$ was added. After 5 days, saturated $\mathrm{NH}_{4} \mathrm{Cl}(5.0 \mathrm{~mL})$ solution was added and the layers were separated. The aqueous phase was extracted with EtOAc $(3 \times 10 \mathrm{~mL})$ and the combined organic extracts were washed with brine $(20 \mathrm{~mL})$, dried $\left(\mathrm{Na}_{2} \mathrm{SO}_{4}\right)$, filtered 
and concentrated under reduced pressure. Purification by flash column chromatography (10\% EtOAc/Hexanes) afforded aldol product 83 (0.42 g, 45\%).

$\boldsymbol{R}_{f} 0.38\left(10 \%\right.$ EtOAc/Hexanes); $[\alpha]_{\mathrm{D}}^{20}-12.0\left(c 0.10, \mathrm{CHCl}_{3}\right)$; IR $\boldsymbol{v}_{\max }\left(\right.$ thin film $\left./ \mathrm{cm}^{-1}\right) 3514$, 2948, 2921, 2862, 1739, 1373, 1223, 1094, 860; ${ }^{1} \mathbf{H}$ NMR $\left(300 \mathrm{MHz}, \mathrm{CDCl}_{3}\right): \delta 4.25(1 \mathrm{H}$, dd, $J=17.3,1.5 \mathrm{~Hz}, \mathrm{H}-7 \mathrm{~A}), 4.07$ (1H, dd, $J=6.9,1.5 \mathrm{~Hz}, \mathrm{H}-9), 4.01$ (1H, d, $J=17.3 \mathrm{~Hz}$, H-7B), 3.90-3.85 (1H, m, H-10), 2.95 (1H, d, $J=2.6 \mathrm{~Hz}, \mathrm{OH}), 1.55-1.42$ (2H, m, H-11), $1.46\left(3 \mathrm{H}, \mathrm{s}, \mathrm{C}-\mathrm{CH}_{3}\right), 1.43$ (3H, s, C-C $\left.\underline{H}_{3}\right), 1.25-1.19$ (8H, m, H-12, H-13, H-14, H-15), 0.84-0.78 (3H, m, H-16); ${ }^{13} \mathbf{C}$ NMR (75 MHz, $\left.\mathrm{CDCl}_{3}\right)$ : $\delta$ 211.4, 101.1, 76.1, 70.7, 66.9, 32.4, 31.9, 29.4, 25.1, 24.0, 23.6, 22.7, 14.2; HRMS (ES ${ }^{+}$) calcd. for $\mathrm{C}_{13} \mathrm{H}_{28} \mathrm{NO}_{4}[\mathrm{M}+$ $\left.\mathrm{NH}_{4}\right]^{+}$262.2013, found 262.2011.

\section{4-Pentenal (84) ${ }^{135}$}

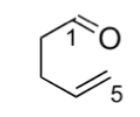

Neat allyl vinyl ether $85(4.0 \mathrm{~g}, 47 \mathrm{mmol})$ sealed in a microwave tube was heated at $160{ }^{\circ} \mathrm{C}$ in a microwave reactor $(200 \mathrm{~W})$ for $2 \mathrm{~h}$ to give aldehyde $84(3.9 \mathrm{~g}, 98 \%)$ as a colourless oil.

${ }^{1} \mathbf{H}$ NMR $\left(400 \mathrm{MHz}, \mathrm{CDCl}_{3}\right) \delta 9.78(1 \mathrm{H}, \mathrm{t}, J=1.6 \mathrm{~Hz}, \mathrm{H}-1), 5.83(1 \mathrm{H}, \mathrm{ddt}, J=17.1,10.3$, 6.6 Hz, H-4), 5.08-4.98 (2H, m, H-5), 2.55-2.50 (2H, m, H-2), 2.41-2.34 (2H, m, H-3).

(S)-4-((S)-1-Hydroxypent-4-enyl)-2,2-dimethyl-1,3-dioxan-5-one (86)

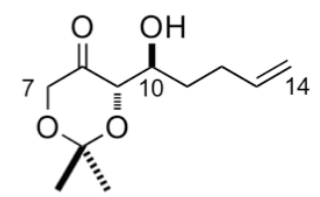

To a solution of dioxanone $63(1.00 \mathrm{~g}, 7.69 \mathrm{mmol})$ in $\mathrm{CHCl}_{3}(5.0 \mathrm{~mL})$ at $0{ }^{\circ} \mathrm{C}$ was added $(S)$-proline $(221 \mathrm{mg}, 1.92 \mathrm{mmol})$. The resulting mixture was stirred for $15 \mathrm{~min}$ and aldehyde 84 (539 mg, $6.41 \mathrm{mmol})$ was then added. After 5 days, saturated $\mathrm{NH}_{4} \mathrm{Cl}(5.0 \mathrm{~mL})$ solution was added and the layers were separated. The aqueous phase was extracted with EtOAc $(3 \times 15 \mathrm{~mL})$ and the combined organic extracts were washed with brine $(20 \mathrm{~mL})$, dried $\left(\mathrm{Na}_{2} \mathrm{SO}_{4}\right)$, filtered and concentrated under reduced pressure. Purification by flash 
column chromatography (10\% EtOAc/Hexanes) afforded aldol product 86 (576 mg, 44\%) as a colourless oil.

$\boldsymbol{R}_{\boldsymbol{f}} 0.26\left(10 \%\right.$ EtOAc/Hexanes); $[\alpha]_{\mathrm{D}}^{20}-4.0\left(c\right.$ 0.25, $\left.\mathrm{CHCl}_{3}\right) ; \mathbf{I R} \boldsymbol{v}_{\max }\left(\right.$ thin film $\left./ \mathrm{cm}^{-1}\right) 3411$, 2922, 1732, 1640, 1375, 1195, 1067, 911; ${ }^{1} \mathbf{H}$ NMR $\left(400 \mathrm{MHz}, \mathrm{CDCl}_{3}\right) \delta 5.82(1 \mathrm{H}, \mathrm{ddt}, J$ $=17.2,10.3,6.8 \mathrm{~Hz}, \mathrm{H}-13), 5.06-4.94(2 \mathrm{H}, \mathrm{m}, \mathrm{H}-14), 4.24(1 \mathrm{H}, \mathrm{dd}, J=17.4,1.5 \mathrm{~Hz}, \mathrm{H}-7 \mathrm{a})$, 4.08 (1H, dd, $J=7.0,1.5 \mathrm{~Hz}, \mathrm{H}-9), 4.01(1 \mathrm{H}, \mathrm{d}, J=17.3 \mathrm{~Hz}, \mathrm{H}-7 \mathrm{~b}), 3.93-3.85$ (1H, ddt, $J$ = 9.1, 6.4, 3.0 Hz, H-10), 2.94-2.89 (1H, d, $J=3.9 \mathrm{~Hz}, \mathrm{OH}), 2.34-2.22(1 \mathrm{H}, \mathrm{m}, \mathrm{H}-12 \mathrm{a})$, 2.20-2.08 (1H, m, H-12b), 1.78-1.68 (1H, m, H-11a), 1.64-1.53 (1H, m, H-11b), 1.46 (3H, s, C-C $\left.\underline{H}_{3}\right)$ and $1.42\left(3 \mathrm{H}, \mathrm{s}, \mathrm{C}-\mathrm{CH}_{3}\right) ;{ }^{13} \mathbf{C} \mathbf{N M R}\left(100 \mathrm{MHz}, \mathrm{CDCl}_{3}\right) \delta 211.3,138.4,115.1$, 101.1, 76.0, 70.1, 66.8, 31.6, 29.4, 23.9, 23.7; HRMS (ES ${ }^{+}$) calcd. for $\mathrm{C}_{11} \mathrm{H}_{18} \mathrm{NaO}_{4}$ $[\mathrm{M}+\mathrm{Na}]^{+}$237.1097, found 237.1101.

\section{(R)-Mosher ester of $86(87)$}

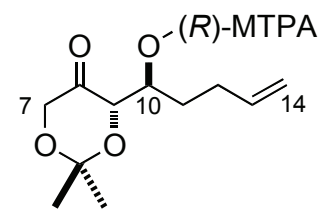

To a stirred mixture of alcohol $86(10.0 \mathrm{mg}, 46.6 \mu \mathrm{mol})$, DCC (47.0 mg, $0.232 \mathrm{mmol})$ and DMAP $(11.0 \mathrm{mg}, 93.3 \mu \mathrm{mol})$ in $\mathrm{CH}_{2} \mathrm{Cl}_{2}(1.0 \mathrm{~mL})$ at $\mathrm{rt}$ was added $(+)-(R)$ - $\alpha$-methoxy- $\alpha$ (trifluoromethyl)-phenylacetic acid $(23.0 \mathrm{mg}, 93.3 \mu \mathrm{mol})$ in one portion. After $16 \mathrm{~h}$, the crude reaction mixture was directly purified by flash column chromatography $(5 \%$ EtOAc/Hexanes) to give $(R)$-MTPA ester 87 (9.0 mg, 45\%).

$\boldsymbol{R}_{\boldsymbol{f}} 0.56$ (10\% EtOAc/Hexanes); ${ }^{1} \mathbf{H}$ NMR (400 MHz, $\left.\mathrm{CDCl}_{3}\right) \delta$ 7.63-7.52 (2H, m, Ar- $\underline{\mathrm{H}}$ ), 7.48-7.36 (3H, m, Ar- $\underline{\mathrm{H}}), 5.81-5.71(1 \mathrm{H}, \mathrm{m}, \mathrm{H}-13), 5.54-5.51$ (1H, m, H-10), 5.04-5.01 (1H, m, H-14a), 5.01-4.98 (1H, m, H-14b), 4.43 (1H, dd, $J=2.8,1.5$ Hz, H-9), $4.13(1 \mathrm{H}$, dd, $J=16.9,1.5 \mathrm{~Hz}, \mathrm{H}-7 \mathrm{a}), 3.93(1 \mathrm{H}, \mathrm{d}, J=16.8 \mathrm{~Hz}, \mathrm{H}-7 \mathrm{~b}), 3.54\left(3 \mathrm{H}, \mathrm{s}, \mathrm{MTPA}-\mathrm{OCH}_{3}\right)$ 2.11-1.95 (2H, m, H-11), 1.791 .63 (2H, m, H-12), 1.37 (3H, s, C-C $\left.\underline{H}_{3}\right), 1.31$ (3H, s, C$\left.\mathrm{CH}_{3}\right) ;{ }^{19} \mathbf{F}$ NMR $\left(376 \mathrm{MHz} ; \mathrm{CDCl}_{3}\right) \delta_{\mathrm{F}}-71.27,-71.36,97 \%$ de. 
(S)-Mosher ester of $86(88)$

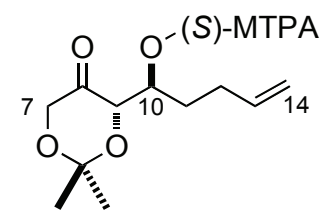

To a stirred mixture of alcohol $86(5.0 \mathrm{mg}, 23 \mu \mathrm{mol})$, DCC $(23.0 \mathrm{mg}, 110 \mu \mathrm{mol})$ and DMAP $(5.0 \mathrm{mg}, 46.0 \mu \mathrm{mol})$ in $\mathrm{CH}_{2} \mathrm{Cl}_{2}(1.0 \mathrm{~mL})$ at $\mathrm{rt}$ was added $(+)-(S)$ - $\alpha$-methoxy- $\alpha-$ (trifluoromethyl)-phenylacetic acid $(12.0 \mathrm{mg}, 46.0 \mu \mathrm{mol})$ in one portion. After $16 \mathrm{~h}$, the crude reaction mixture was purified directly by flash column chromatography $(5 \%$ EtOAc/Hexanes) to give $(S)$-MTPA ester $\mathbf{8 8}(6.0 \mathrm{mg}, 60 \%)$.

$\boldsymbol{R}_{f} 0.56$ (10\% EtOAc/Hexanes); ${ }^{1} \mathbf{H}$ NMR (400 MHz, $\left.\mathrm{CDCl}_{3}\right) \delta$ 7.62-7.48 (2H, m, Ar- $\underline{\mathrm{H}}$ ), 7.46-7.36 (3H, m, Ar- $\underline{\mathrm{H}}), 5.76-5.66(1 \mathrm{H}, \mathrm{m}, \mathrm{H}-13), 5.48(1 \mathrm{H}, \mathrm{td}, J=6.1,2.7 \mathrm{~Hz}, \mathrm{H}-10)$, 4.98 (1H, m, H-14a), 4.94 (1H, m, H-14b), 4.57 (1H, dd, J=3.1, $1.3 \mathrm{~Hz}, \mathrm{H}-9), 4.20$ (1H, dd, $J=17.0,1.3 \mathrm{~Hz}, \mathrm{H}-7 \mathrm{a}), 3.98(1 \mathrm{H}, \mathrm{d}, J=17.0 \mathrm{~Hz}, \mathrm{H}-7 \mathrm{~b}), 3.60$ (3H, s, MTPA-OC$\left.\underline{H}_{3}\right)$, 2.05-1.87 (2H, m, H-11), 1.69-1.57 (2H, m, H-12), 1.45 (3H, s, C-C $\left.\underline{H}_{3}\right), 1.42(3 \mathrm{H}, \mathrm{s}, \mathrm{C}-$ $\left.\mathrm{C}_{3}\right)$.

(4S,5R)-4-((S)-1-Hydroxypent-4-en-1-yl)-2,2-dimethyl-1,3-dioxan-5-ol (92), $(4 S, 5 S)-4-((S)-1-H y d r o x y p e n t-4-e n-1-y l)-2,2-d i m e t h y l-1,3-d i o x a n-5-o l ~(93)$

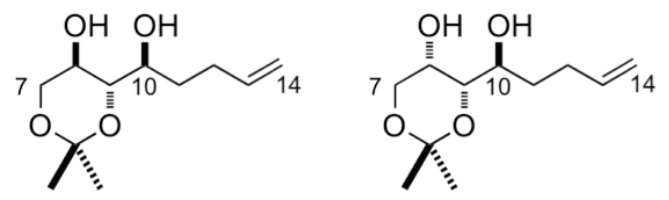

\section{Method A}

To a solution of aldol adduct $86(50.0 \mathrm{mg}, 0.233 \mathrm{mmol})$ in $\mathrm{MeOH}(2.0 \mathrm{~mL})$ at $-78{ }^{\circ} \mathrm{C}$ was added $\mathrm{NaBH}_{4}(10.0 \mathrm{mg}, 0.279 \mathrm{mmol})$. After $1 \mathrm{~h}$ the reaction mixture was quenched with saturated $\mathrm{NH}_{4} \mathrm{Cl}(5.0 \mathrm{~mL})$ solution and the aqueous layer was extracted with $\mathrm{Et}_{2} \mathrm{O}(3 \times 10$ $\mathrm{mL})$. The combined organic extracts were dried $\left(\mathrm{Na}_{2} \mathrm{SO}_{4}\right)$, filtered and concentrated under reduced pressure. The resulting residue was purified by flash column chromatography (10\% EtOAc/Hexanes) to give compounds 92 (20.0 mg, $40 \%)$ and 93 (7.0 $\mathrm{mg}, 14 \%)$ as colourless oils. 


\section{Method B}

To a solution of $\mathrm{Zn}\left(\mathrm{BH}_{4}\right)_{2}\left(5.0 \mathrm{~mL}, 0.5 \mathrm{M}\right.$ in $\left.\mathrm{Et}_{2} \mathrm{O}, 2.5 \mathrm{mmol}\right)$ at $-78{ }^{\circ} \mathrm{C}$ was added aldol adduct 86 (90.0 $\mathrm{mg}, 0.47 \mathrm{mmol})$. After $1 \mathrm{~h}$ the reaction mixture was quenched with saturated $\mathrm{NH}_{4} \mathrm{Cl}(5.0 \mathrm{~mL})$ solution and the aqueous layer was extracted with $\mathrm{Et}_{2} \mathrm{O}(3 \times 20$ $\mathrm{mL})$. The combined organic extracts were dried $\left(\mathrm{Na}_{2} \mathrm{SO}_{4}\right)$, filtered and concentrated under reduced pressure. The residue was purified by flash column chromatography $(10 \%$ EtOAc/Hexanes) to give compounds $92(42.0 \mathrm{mg}, 44 \%)$ and $93(8.0 \mathrm{mg}, 10 \%)$ as colourless oils.

\section{Method C}

To a solution of aldol adduct $86(50.0 \mathrm{mg}, 0.233 \mathrm{mmol})$ in $\mathrm{CH}_{3} \mathrm{CN}(2.0 \mathrm{~mL})$ at $-40{ }^{\circ} \mathrm{C}$ was added $\mathrm{Me}_{4} \mathrm{NBH}(\mathrm{OAc})_{3}(18.0 \mathrm{mg}, 0.689 \mathrm{mmol})$ and glacial acetic acid $(0.2 \mathrm{~mL})$. After $10 \mathrm{~h}$ the reaction mixture was warmed up and quenched with saturated $\mathrm{NaHCO}_{3}(5.0 \mathrm{~mL})$ solution and the aqueous layer was extracted with $\mathrm{CH}_{2} \mathrm{Cl}_{2}(3 \times 10 \mathrm{~mL})$. The combined organic extracts were dried $\left(\mathrm{Na}_{2} \mathrm{SO}_{4}\right)$, filtered and concentrated under reduced pressure. The residue was purified by flash column chromatography (10\% EtOAc/Hexanes) to give compounds 92 (14.0 mg, $28 \%$ ) and 93 (14.0 mg, 28\%) as colourless oils.

92 1,3-syn: $\boldsymbol{R}_{\boldsymbol{f}} 0.16(20 \% \mathrm{EtOAc} / \mathrm{Hexanes}) ;[\alpha]_{\mathrm{D}}^{20}-30.7$ (c 1.8, $\left.\mathrm{CHCl}_{3}\right)$; IR $\boldsymbol{v}_{\max }$ (thin film $\left./ \mathrm{cm}^{-1}\right)$ 3398, 2991, 2923, 1444, 1378, 1207, 1067, 914, 863; ${ }^{1} \mathbf{H}$ NMR (400 MHz, $\left.\mathrm{CDCl}_{3}\right) \delta 5.86(1 \mathrm{H}, \mathrm{ddt}, J=17.1,10.3,6.8 \mathrm{~Hz}, \mathrm{H}-13), 5.11-4.98(2 \mathrm{H}, \mathrm{m}, \mathrm{H}-14), 3.88(1 \mathrm{H}$, dd, $J=11.3,5.4 \mathrm{~Hz}, \mathrm{H}-7 \mathrm{a}), 3.77$ (2H, m, H-8, H-10), 3.61 (1H, dd, $J=11.2,8.5 \mathrm{~Hz}, \mathrm{H}-$ 7b), 3.46 (1H, dd, $J=8.7,7.1 \mathrm{~Hz}, \mathrm{H}-9), 3.32(1 \mathrm{H}$, br s, C8-O프), 2.49 (1H, br s, C10-O프), 2.12-2.29 (2H, m, H-12), 1.88-1.80 (1H, m, H-11a), 1.58-1.49 (1H, m, H-11b), 1.45 (3H, s, C-C $\left.\underline{H}_{3}\right), 1.36\left(3 \mathrm{H}, \mathrm{s}, \mathrm{C}-\mathrm{C}_{3}\right) ;{ }^{13} \mathbf{C}$ NMR $\left(100 \mathrm{MHz}, \mathrm{CDCl}_{3}\right) \delta$ 138.6, 115.3, 98.9, 75.4, 75.0, 67.3, 64.3, 32.6, 29.7, 28.3, 19.8; HRMS $\left(\mathrm{ES}^{+}\right)$calcd. for $\mathrm{C}_{11} \mathrm{H}_{20} \mathrm{NaO}_{4}[\mathrm{M}+\mathrm{Na}]^{+}$ 239.1254, found 239.1254 .

93 1,3-anti: $\boldsymbol{R}_{\boldsymbol{f}} 0.15$ (20\% EtOAc/Hexanes); $[\alpha]_{\mathrm{D}}^{20}+3.7\left(c 1.1, \mathrm{CHCl}_{3}\right)$; IR $\boldsymbol{v}_{\max }$ (thin film $\left./ \mathrm{cm}^{-1}\right)$ 3401, 2920, 2873, 1455, 1380, 1332, 1277, 911, 853; ${ }^{1} \mathbf{H}$ NMR (400 MHz, $\left.\mathrm{CDCl}_{3}\right) \delta 5.85(1 \mathrm{H}, \mathrm{ddt}, J=17.0,10.3,6.7 \mathrm{~Hz}, \mathrm{H}-13), 5.09-4.97(2 \mathrm{H}, \mathrm{m}, \mathrm{H}-14), 4.02(1 \mathrm{H}$, dd, $J=12.4,1.6 \mathrm{~Hz}, \mathrm{H}-7 \mathrm{a}), 3.85(1 \mathrm{H}, \mathrm{dd}, J=12.4,2.1 \mathrm{~Hz}, \mathrm{H}-7 \mathrm{~b}), 3.82-3.76(1 \mathrm{H}, \mathrm{m}, \mathrm{H}-$ 10), $3.72(1 \mathrm{H}, \mathrm{dq}, J=8.7,1.6 \mathrm{~Hz}, \mathrm{H}-8), 3.62$ (1H, dd, $J=6.7,1.5 \mathrm{~Hz}, \mathrm{H}-9), 3.19$ (1H, d, 
$J=8.7 \mathrm{~Hz}, \mathrm{C} 8-\mathrm{OH}), 2.48(1 \mathrm{H}, \mathrm{d}, J=4.5 \mathrm{~Hz}, \mathrm{C} 10-\mathrm{OH}), 2.33-2.26$ (1H, m, H-12a), 2.212.13 (1H, m, H-12b), 1.77-1.68 (1H, m, H-11a), 1.61-1.51 (1H, m, H-11b), 1.44 (3H, s, C$\left.\mathrm{C}_{3} 3\right), 1.43\left(3 \mathrm{H}, \mathrm{s}, \mathrm{C}-\mathrm{C}_{3}\right) ;{ }^{13} \mathbf{C}$ NMR $\left(100 \mathrm{MHz}, \mathrm{CDCl}_{3}\right) \delta 138.5,115.1,99.0,73.9,71.0$, 65.9, 63.6, 32.0, 30.0, 29.6, 18.7; HRMS $\left(\mathrm{ES}^{+}\right)$calcd. for $\mathrm{C}_{11} \mathrm{H}_{24} \mathrm{NO}_{4}\left[\mathrm{M}+\mathrm{NH}_{4}\right]^{+}$234.1700, found 234.1700 .

((4S,5R)-5-Hydroxy-2,2-dimethyl-1,3-dioxan-4-yl)pentane-1,5-diol (94)

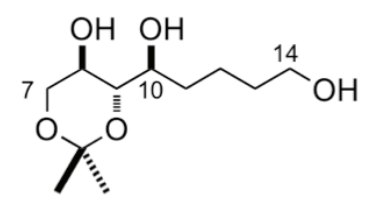

To a solution of thexylBH ${ }_{2}$ in THF $(2.0 \mathrm{~mL}, 0.5 \mathrm{M}, 1.0 \mathrm{mmol})$ at $0{ }^{\circ} \mathrm{C}$ was added olefin 92 $(20.0 \mathrm{mg}, 88.0 \mu \mathrm{mol})$ in THF $(1.0 \mathrm{~mL})$ dropwise. After $1 \mathrm{~h} \mathrm{NaOH}(1.0 \mathrm{~mL}, 10 \% \mathrm{w} / \mathrm{v})$ and $30 \%$ hydrogen peroxide $(1.0 \mathrm{~mL}, 30 \% \mathrm{v} / \mathrm{v})$ were added. The mixture was stirred for 15 min and the aqueous layer was saturated with anhydrous $\mathrm{K}_{2} \mathrm{CO}_{3}$. The organics were extracted with $\mathrm{CH}_{2} \mathrm{Cl}_{2}(3 \times 5 \mathrm{~mL})$, washed with brine $(10.0 \mathrm{~mL})$, dried $\left(\mathrm{Na}_{2} \mathrm{SO}_{4}\right)$, filtered and concentrated under reduced pressure. The resulting residue was purified by flash column chromatography (10\% EtOAc/Hexane) to give compound $94(17.0 \mathrm{mg}, 85 \%)$ as a colourless oil.

$\boldsymbol{R}_{\boldsymbol{f}} 0.11$ (30\% EtOAc/Hexanes); $[\alpha]_{\mathrm{D}}^{20}-32.2\left(c\right.$ 0.5, $\left.\mathrm{CHCl}_{3}\right) ; \mathbf{I R} \boldsymbol{v}_{\max }\left(\right.$ thin film $\left./ \mathrm{cm}^{-1}\right) 3367$, 2931, 2870, 1454, 1376, 1277, 1067, 859; ${ }^{1} \mathbf{H}$ NMR $\left(400 \mathrm{MHz}, \mathrm{CDCl}_{3}\right) \delta 3.88(1 \mathrm{H}, \mathrm{dd}, J=$ 11.2, $5.4 \mathrm{~Hz}, \mathrm{H}-7 \mathrm{a}), 3.79(1 \mathrm{H}, \mathrm{td}, J=8.5,5.4 \mathrm{~Hz}, \mathrm{H}-8), 3.74(1 \mathrm{H}, \mathrm{td}, J=7.6,2.6 \mathrm{~Hz}, \mathrm{H}-$ 10), $3.68(2 \mathrm{H}, \mathrm{t}, J=5.8 \mathrm{~Hz}, \mathrm{H}-14), 3.61(1 \mathrm{H}, \mathrm{dd}, J=11.2,8.4 \mathrm{~Hz}, \mathrm{H}-7 \mathrm{~b}), 3.46(1 \mathrm{H}, \mathrm{dd}, J=$ 8.5, 7.1 Hz, H-9), 1.80-1.71 (1H, m, H-11a), 1.66-1.53 (4H, m, H-12, H-13), 1.52-1.45 (1H, m, H-11b), 1.45 (3H, s, C-C $\left.\underline{H}_{3}\right), 1.36\left(3 \mathrm{H}, \mathrm{s}, \mathrm{C}-\mathrm{CH}_{3}\right) ;{ }^{13} \mathbf{C ~ N M R}\left(100 \mathrm{MHz}, \mathrm{CDCl}_{3}\right) \delta$ 99.0, 75.4, 75.1, 67.2, 64.4, 62.8, 32.8, 32.2, 28.4, 21.4, 19.8; HRMS (ES ${ }^{+}$) calcd. for $\mathrm{C}_{11} \mathrm{H}_{22} \mathrm{NaO}_{5}[\mathrm{M}+\mathrm{Na}]^{+}$257.1359, found 257.1362. 
((4S,5S)-5-Hydroxy-2,2-dimethyl-1,3-dioxan-4-yl)pentane-1,5-diol (95)

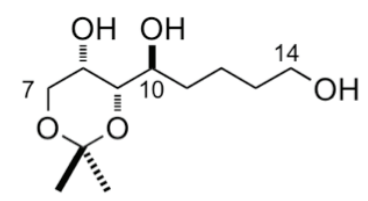

To a solution of thexylBH ${ }_{2}$ in THF $(1.0 \mathrm{~mL}, 0.5 \mathrm{M}, 0.5 \mathrm{mmol})$ at $0{ }^{\circ} \mathrm{C}$ was added olefin 93 $(12.0 \mathrm{mg}, 56 \mu \mathrm{mol})$ in THF $(0.5 \mathrm{~mL})$ dropwise. After $1 \mathrm{~h} \mathrm{NaOH}(0.5 \mathrm{~mL}, 10 \% \mathrm{w} / \mathrm{v})$ and $30 \%$ hydrogen peroxide $(0.5 \mathrm{~mL}, 30 \% \mathrm{v} / \mathrm{v})$ were added. The mixture was stirred for 15 min and the aqueous layer was saturated with anhydrous $\mathrm{K}_{2} \mathrm{CO}_{3}$. The organics were extracted with $\mathrm{CH}_{2} \mathrm{Cl}_{2}(3 \times 5 \mathrm{~mL})$, washed with brine $(10.0 \mathrm{~mL})$, dried $\left(\mathrm{Na}_{2} \mathrm{SO}_{4}\right)$, filtered and concentrated under reduced pressure. The resulting residue was purified by flash column chromatography (10\% EtOAc/Hexane) to give compound $95(10.0 \mathrm{mg}, 77 \%)$ as a colourless oil.

$\boldsymbol{R}_{f} 0.10\left(30 \%\right.$ EtOAc/Hexanes); $[\alpha]_{\mathrm{D}}^{20}-2.4\left(\right.$ c $\left.0.7 \mathrm{CHCl}_{3}\right)$; IR $\boldsymbol{v}_{\max }\left(\right.$ thin film $\left./ \mathrm{cm}^{-1}\right) 3365$, 2925, 2864, 1373, 1279, 1067, 976; ${ }^{1} \mathbf{H}$ NMR (400 MHz, CDCl $) \delta 4.02$ (1H, dd, $J=12.4$, 1.6 Hz, H-7a), 3.85 (1H, dd, $J=12.4,2.0$ Hz, H-7b), 3.79-3.74 (1H, m, H-10), 3.74-3.69 $(1 \mathrm{H}, \mathrm{m}, \mathrm{H}-8), 3.66(2 \mathrm{H}, \mathrm{t}, J=5.7 \mathrm{~Hz}, \mathrm{H}-14), 3.61(1 \mathrm{H}, \mathrm{dd}, J=6.7,1.4 \mathrm{~Hz}, \mathrm{H}-9), 3.32(1 \mathrm{H}$, d, $J=8.6 \mathrm{~Hz}, \mathrm{C} 8-\mathrm{OH}), 2.93$ (1 H, s, C10-Oㅂ) $), 1.69-1.56$ (4H, m, H-12, H-13), 1.52-1.45 (2H, m, H-11), $1.44\left(3 \mathrm{H}, \mathrm{s}, \mathrm{C}-\mathrm{C}_{3}\right), 1.43\left(3 \mathrm{H}, \mathrm{s}, \mathrm{C}-\mathrm{C}_{3}\right) ;{ }^{13} \mathbf{C ~ N M R}\left(100 \mathrm{MHz}, \mathrm{CDCl}_{3}\right) \delta$ 99.0, 74.0, 71.3, 65.9, 63.6, 62.8, 32.61, 32.41, 29.6, 21.9, 19.8; HRMS (ES ${ }^{+}$) calcd. for $\mathrm{C}_{11} \mathrm{H}_{23} \mathrm{O}_{5}[\mathrm{M}+\mathrm{H}]^{+}$235.1540, found 235.1541.

$(2 R, 3 S, 4 S)$-Octane-1,2,3,4,8-pentaol (57)

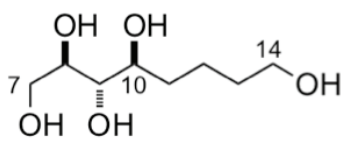

To a solution of triol $94(25.7 \mathrm{mg}, 0.109 \mathrm{mmol})$ in $\mathrm{MeOH}(1.0 \mathrm{~mL})$ and water $(0.1 \mathrm{~mL})$ was added Dowex-50 resin $(40.0 \mathrm{mg})$ and the reaction was refluxed $\left(65-70{ }^{\circ} \mathrm{C}\right)$ for $1 \mathrm{~h}$. The reaction mixture was then cooled to rt, filtered and concentrated under reduced pressure to give 57 (20.8 mg, 98\%) as a colourless viscous oil. 
$\boldsymbol{R}_{\boldsymbol{f}} 0.10\left(10 \% \mathrm{MeOH} / \mathrm{CH}_{2} \mathrm{Cl}_{2}\right) ;[\alpha]_{\mathrm{D}}^{20}-8.6$ (c $\left.0.3, \mathrm{MeOH}\right) ; \mathbf{I R} \boldsymbol{v}_{\max }\left(\right.$ thin film $\left./ \mathrm{cm}^{-1}\right) 3239$, 2860, 1406, 1205, 1149, 799; ${ }^{1}$ H NMR (500 MHz, MeOD) $\delta 3.77$ (1H, dd, $J=11.1,3.2$ Hz, H-7a), 3.67 (2H, m, H-8, H-10), 3.61 (1H, dd, $J=11.1,6.2$ Hz, H-7b), 3.57 (2H, t, $J=$ $6.4 \mathrm{~Hz}, \mathrm{H}-14), 3.47$ (1H, t, $J=6.4 \mathrm{~Hz}, \mathrm{H}-9), 1.72-1.66$ (1H, m, H-11a), 1.65-1.52 (3H, m, H-12a, H-13), 1.51-1.37 (2H, m, H-11b, H-12b); ${ }^{13}$ C NMR (100 MHz, MeOD) $\delta$ C9-76.0, C10-74.4, C8-73.9, C7-64.6, C14-63.0, C11-33.7, C13-33.0, C12-23.0; HRMS (ES $\left.{ }^{+}\right)$ calcd. for $\mathrm{C}_{8} \mathrm{H}_{19} \mathrm{O}_{5}[\mathrm{M}+\mathrm{H}]^{+}$195.1227, found 195.1225.

\section{$(2 S, 3 S, 4 S)$-Octane-1,2,3,4,8-pentaol (58)}

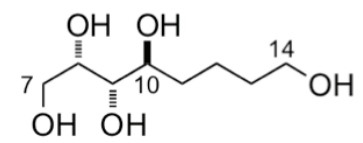

To a solution of triol $95(10.0 \mathrm{mg}, 43 \mu \mathrm{mol})$ in $\mathrm{MeOH}(0.5 \mathrm{~mL})$ and water $(0.05 \mathrm{~mL})$ was added Dowex-50 resin $(30.0 \mathrm{mg})$ and the reaction was refluxed $\left(65-70{ }^{\circ} \mathrm{C}\right)$ for $1 \mathrm{~h}$. The reaction mixture was then cooled to rt, filtered and concentrated under reduced pressure to give 58 (7.5 $\mathrm{mg}, 90 \%)$ as a colourless viscous oil.

$\boldsymbol{R}_{\boldsymbol{f}} 0.10\left(10 \% \mathrm{MeOH} / \mathrm{CH}_{2} \mathrm{Cl}_{2}\right) ;[\alpha]_{\mathrm{D}}^{20}-2.6$ (c $\left.0.8, \mathrm{MeOH}\right) ; \mathbf{I R} \boldsymbol{v}_{\max }\left(\right.$ thin film $\left./ \mathrm{cm}^{-1}\right) 3358$, 2930, 2869, 1454, 1376, 1207, 1157, 1066, 858; ${ }^{1} \mathbf{H}$ NMR (400 MHz, MeOD) $\delta 3.90(1 \mathrm{H}$, td, $J=6.2,2.0 \mathrm{~Hz}, \mathrm{H}-8), 3.62-3.60(3 \mathrm{H}, \mathrm{m}, \mathrm{H}-7, \mathrm{H}-10), 3.57$ (2H, t, $J=6.4 \mathrm{~Hz}, \mathrm{H}-14)$, $3.34(1 \mathrm{H}, \mathrm{dd}, J=6.5,4.5 \mathrm{~Hz}, \mathrm{H}-9), 1.82-1.74$ (1H, m, H-11a), 1.65-1.51 (3H, m, H-12a, H13), 1.47-1.35 (2H, m, H-11b, H-12b); ${ }^{13}$ C NMR (100 MHz, MeOD) $\delta$ C9-74.8, C10-72.6, C8-72.0, C7-65.0, C14-63.0, C11-34.5, C13-33.7, C12-23.0; HRMS (ES ${ }^{+}$calcd. for $\mathrm{C}_{8} \mathrm{H}_{17} \mathrm{O}_{5}[\mathrm{M}-\mathrm{H}]^{-}$193.1081, found 193.1084. 
(S)-4-((S)-1-((tert-Butyldimethylsilyl)oxy)pent-4-en-1-yl)-2,2-dimethyl-1,3-dioxan-5one (96)

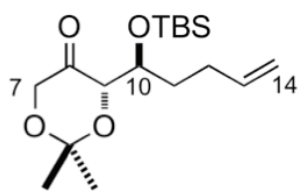

To a solution of alcohol $86(100 \mathrm{mg}, 0.47 \mathrm{mmol})$ in $\mathrm{CH}_{2} \mathrm{Cl}_{2}(5.0 \mathrm{~mL})$ at $-78{ }^{\circ} \mathrm{C}$ was added 2,6-lutidine $(0.11 \mathrm{~mL}, 0.94 \mathrm{mmol})$ and TBSOTf $(0.16 \mathrm{~mL}, 0.71 \mathrm{mmol})$. After $1.5 \mathrm{~h}$, saturated $\mathrm{NH}_{4} \mathrm{Cl}(5.0 \mathrm{~mL})$ solution and $\mathrm{CH}_{2} \mathrm{Cl}_{2}(5.0 \mathrm{~mL})$ were added. The layers were separated and the aqueous phase was extracted with $\mathrm{CH}_{2} \mathrm{Cl}_{2}(3 \times 10 \mathrm{~mL})$. The combined organic extracts were dried $\left(\mathrm{Na}_{2} \mathrm{SO}_{4}\right)$, filtered and concentrated under reduced pressure. Purification by flash column chromatography (5\% EtOAc/Hexane) provided 96 (140 mg, 95\%) as a colourless oil.

$\boldsymbol{R}_{f} 0.43(10 \% \mathrm{EtOAc} /$ Hexanes $) ;[\alpha]_{\mathrm{D}}^{20}-5.7$ ( c 1.0, $\left.\mathrm{CHCl}_{3}\right)$; IR $\boldsymbol{v}_{\max }\left(\right.$ thin film $\left./ \mathrm{cm}^{-1}\right) 2983$, 2934, 1719, 1448, 1370, 1271, 1204, 1041, 981; ${ }^{1} \mathbf{H}$ NMR $\left(400 \mathrm{MHz}, \mathrm{CDCl}_{3}\right) \delta 5.80(1 \mathrm{H}$, ddt, $J=16.9,10.2,6.7 \mathrm{~Hz}, \mathrm{H}-13), 5.02$ (1H, dt, $J=17.1,1.6 \mathrm{~Hz}, \mathrm{H}-14 \mathrm{a}), 4.98-4.94$ (1H, m, H-14b), 4.25 (1H, dd, $J=2.6,1.4$ Hz, H-9), 4.21 (1H, dd, $J=16.5,1.1 \mathrm{~Hz}, \mathrm{H}-7 \mathrm{a}), 4.13-$ 4.09 (1H, m, H-10), 3.93 (1H, d, $J=16.4$ Hz, H-7b), 2.23-2.10 (1H, m, H-12a), 2.09-1.97 (1H, m, H-12b), 1.86-1.76 (1H, m, H-11a), 1.59-1.48 (1H, m, H-11b), 1.46 (3H, s, C-C $\left.\underline{H}_{3}\right)$,

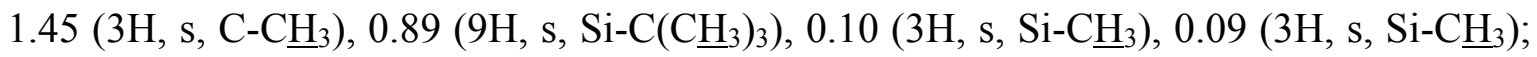
${ }^{13}$ C NMR $\left(100 \mathrm{MHz} \mathrm{CDCl}_{3}\right) \delta$ 208.0, 138.5, 114.8, 100.8, 78.4, 71.5, 67.4, 32.0, 30.2, 26.0, 24.5, 23.6, 18.3, -4.24, -4.43; HRMS $\left(\mathrm{ES}^{+}\right)$calcd. for $\mathrm{C}_{17} \mathrm{H}_{33} \mathrm{O}_{4} \mathrm{Si}[\mathrm{M}+\mathrm{H}]^{+}$329.2143, found 329.2147 . 


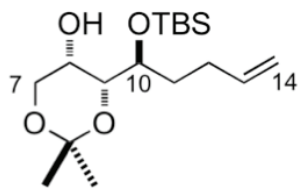

To a solution of ketone $96(84.0 \mathrm{mg}, 0.25 \mathrm{mmol})$ in THF $(2.0 \mathrm{~mL})$ at $-78{ }^{\circ} \mathrm{C}$ was added L-selectride $(0.38 \mathrm{~mL}, 1 \mathrm{M}$ in THF, $0.38 \mathrm{mmol})$. After $1 \mathrm{~h}$ the reaction mixture was quenched with saturated $\mathrm{NH}_{4} \mathrm{Cl}(10.0 \mathrm{~mL})$ solution and the layers were separated. The aqueous layer was extracted with $\mathrm{Et}_{2} \mathrm{O}(3 \times 20 \mathrm{~mL})$ and the combined organic extracts were dried $\left(\mathrm{Na}_{2} \mathrm{SO}_{4}\right)$, filtered and concentrated under reduced pressure. The resulting residue was purified by flash column chromatography (10\% EtOAc/Hexanes) to give compound 97 (78.0 mg, 93\%) as a colourless oil.

$\boldsymbol{R}_{\boldsymbol{f}} 0.42$ (10\% EtOAc/Hexanes); $[\alpha]_{\mathrm{D}}^{20}+2.4$ (c 1.0, $\left.\mathrm{CHCl}_{3}\right)$; IR $\boldsymbol{v}_{\max }\left(\right.$ thin film $\left./ \mathrm{cm}^{-1}\right) 3389$, 2920, 2874, 1456, 1378, 1210, 1152, 850; ${ }^{1} \mathbf{H}$ NMR $\left(400 \mathrm{MHz}, \mathrm{CDCl}_{3}\right) \delta 5.82(1 \mathrm{H}, \mathrm{ddt}, J$ $=17.1,10.4,6.7 \mathrm{~Hz}, \mathrm{H}-13), 5.05-4.94(2 \mathrm{H}, \mathrm{m}, \mathrm{H}-14), 3.99$ (1H, dd, $J=12.4,1.1 \mathrm{~Hz}, \mathrm{H}-$ 7a), 3.96-3.91 (1H, m, H-10), $3.85(1 \mathrm{H}, \mathrm{dd}, J=12.3,1.8 \mathrm{~Hz}, \mathrm{H}-7 \mathrm{~b}), 3.64$ (2H, app d, $J=$ $5.9 \mathrm{~Hz}, \mathrm{H}-8, \mathrm{H}-9), 3.33$ (1H, d, $J=7.4 \mathrm{~Hz}, \mathrm{OH}), 2.18-2.04$ (2H, m, H-12), 1.76-1.67 (1H, m, H-11a), 1.65-1.56 (1H, m, H-11b), 1.44 (3H, s, C-C $\left.\underline{H}_{3}\right), 1.43\left(3 \mathrm{H}, \mathrm{s}, \mathrm{C}-\mathrm{C}_{3}\right), 0.90(9 \mathrm{H}$, s, Si-C(C $\left.\left.\underline{\mathrm{H}}_{3}\right)_{3}\right), 0.14\left(3 \mathrm{H}, \mathrm{s}, \mathrm{Si}-\mathrm{C}_{3}\right), 0.09\left(3 \mathrm{H}, \mathrm{s}, \mathrm{Si}-\mathrm{CH}_{3}\right) ;{ }^{13} \mathbf{C}$ NMR $\left(100 \mathrm{MHz}, \mathrm{CDCl}_{3}\right) \delta$ 138.8, 114.6, 99.0, 72.4, 71.4, 66.3, 63.3, 32.4, 29.7, 28.4, 26.0, 18.6, 18.3, -4.46, -4.51; HRMS $\left(\mathrm{ES}^{+}\right.$) calcd. for $\mathrm{C}_{17} \mathrm{H}_{35} \mathrm{O}_{4} \mathrm{Si}[\mathrm{M}+\mathrm{H}]^{+} 331.2299$, found 331.2302.

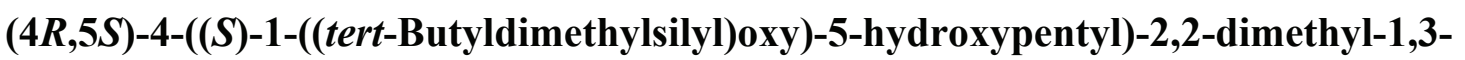
dioxan-5-ol

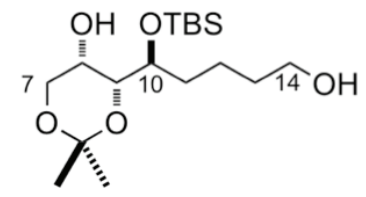

To a solution of thexylBH ${ }_{2}$ in THF $(2.0 \mathrm{~mL}, 0.5 \mathrm{M}, 1.0 \mathrm{mmol})$ at $0{ }^{\circ} \mathrm{C}$ was added olefin 97 $(61.0 \mathrm{mg}, 0.18 \mathrm{mmol})$ in THF $(1.0 \mathrm{~mL})$ dropwise. After $2 \mathrm{~h} \mathrm{NaOH}(1.0 \mathrm{~mL}, 10 \% \mathrm{w} / \mathrm{v})$ and 
$30 \%$ hydrogen peroxide $(1.0 \mathrm{~mL}, 30 \% \mathrm{v} / \mathrm{v})$ were added. The mixture was stirred for 15 min and the aqueous layer was saturated with $\mathrm{NaHCO}_{3}$ and the layers were separated. The aqueous phase was extracted with $\mathrm{CH}_{2} \mathrm{Cl}_{2}(3 \times 5 \mathrm{~mL})$, washed with brine $(10 \mathrm{ml})$, dried $\left(\mathrm{Na}_{2} \mathrm{SO}_{4}\right)$, filtered and concentrated under reduced pressure. The residue was purified by flash column chromatography (20\% EtOAc/Hexanes) to give diol $(56.5 \mathrm{mg}, 88 \%)$ as a colourless oil.

$\boldsymbol{R}_{\boldsymbol{f}} 0.20$ (10\% EtOAc/Hexanes); $[\alpha]_{\mathrm{D}}^{20}+2.2\left(c\right.$ 1.7, $\left.\mathrm{CHCl}_{3}\right) ; \mathbf{I R} \boldsymbol{v}_{\max }\left(\right.$ thin film $\left./ \mathrm{cm}^{-1}\right) 3363$, 2828, 2868, 1455, 1376, 1207, 1157, 1066, 859; ${ }^{1} \mathbf{H}$ NMR $\left(300 \mathrm{MHz}, \mathrm{CDCl}_{3}\right) \delta 3.98(1 \mathrm{H}$, dd, $J=12.3,1.5 \mathrm{~Hz}, \mathrm{H}-7 \mathrm{a}), 3.89-3.94(1 \mathrm{H}, \mathrm{m}, \mathrm{H}-10), 3.84(1 \mathrm{H}, \mathrm{dd}, J=12.3,2.0 \mathrm{~Hz}, \mathrm{H}-$ 7b), 3.60-3.67 (4H, m, H-8, H-9, H-14), 3.42 (1H, d, $J=7.2 \mathrm{~Hz}, \mathrm{C} 8-\mathrm{OH}), 1.52-1.61(6 \mathrm{H}$, m, H-11, H-12, H-13), 1.43 (3H, s, C-C $\left.\underline{H}_{3}\right), 1.42\left(3 \mathrm{H}, \mathrm{s}, \mathrm{C}-\mathrm{CH}_{3}\right), 0.89$ (9H, s, Si-C(C $\left.\left.\underline{\mathrm{C}}_{3}\right)_{3}\right)$, $0.13\left(3 \mathrm{H}, \mathrm{s}, \mathrm{Si}-\mathrm{C}_{3}\right), 0.08\left(3 \mathrm{H}, \mathrm{s}, \mathrm{Si}-\underline{\mathrm{C}}_{3}\right) ;{ }^{13} \mathbf{C} \mathbf{N M R}\left(75 \mathrm{MHz}, \mathrm{CDCl}_{3}\right) \delta 98.8,72.3,71.8$, 66.2, 63.2, 62.8, 33.0, 32.8, 29.6, 25.9 (x3), 20.2, 18.5, 18.1, -4.6 (x2); HRMS (ES ${ }^{+}$) calcd. for $\mathrm{C}_{17} \mathrm{H}_{37} \mathrm{O}_{5} \mathrm{Si}[\mathrm{M}+\mathrm{H}]^{+}$349.2405, found 349.2405.

\section{$(2 S, 3 S, 4 S)$-Octane-1,2,3,4,8-pentaol (58)}

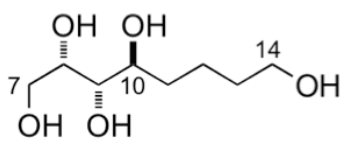

To a solution of diol $(16.0 \mathrm{mg}, 0.07 \mathrm{mmol})$ in $\mathrm{MeOH}(0.5 \mathrm{~mL})$ and water $(0.05 \mathrm{~mL})$ was added Dowex-50 resin $(30.0 \mathrm{mg})$ and the reaction was refluxed $\left(65-70{ }^{\circ} \mathrm{C}\right)$ for $1 \mathrm{~h}$. The reaction mixture was then cooled to rt, filtered and concentrated under reduced pressure to give $(2 R, 3 S, 4 S)$-octane-1,2,3,4,8-pentaol $58(12.0 \mathrm{mg}, 88 \%)$ as a colourless viscous oil. $\boldsymbol{R}_{\boldsymbol{f}} 0.10\left(10 \% \mathrm{MeOH} / \mathrm{CH}_{2} \mathrm{Cl}_{2}\right) ;[\alpha]_{\mathrm{D}}^{20}-2.6$ (c $\left.0.8, \mathrm{MeOH}\right) ; \mathbf{I R} \boldsymbol{v}_{\max }\left(\right.$ thin film $\left./ \mathrm{cm}^{-1}\right) 3358$, 2930, 2869, 1454, 1376, 1207, 1157, 1066; ${ }^{1}$ H NMR (400 MHz, MeOD) $\delta 3.90(1 \mathrm{H}, \mathrm{td}, J$ $=6.2,2.0 \mathrm{~Hz}, \mathrm{H}-8), 3.62-3.60(3 \mathrm{H}, \mathrm{m}, \mathrm{H}-7, \mathrm{H}-10), 3.57$ (2H, t, $J=6.4 \mathrm{~Hz}, \mathrm{H}-14), 3.34$ $(1 \mathrm{H}, \mathrm{dd}, J=6.5,4.5 \mathrm{~Hz}, \mathrm{H}-9), 1.82-1.74$ (1H, m, H-11a), 1.65-1.51 (3H, m, H-12a, H-13), 1.47-1.35 (2H, m, H-11b, H-12b); ${ }^{13}$ C NMR (100 MHz, MeOD) $\delta$ C9-74.8, C10-72.6, C872.0, C7-65.0, C14-63.0, C11-34.5, C13-33.7, C12-23.0; HRMS (ES ${ }^{+}$) calcd. for $\mathrm{C}_{8} \mathrm{H}_{17} \mathrm{O}_{5}$ $[\mathrm{M}-\mathrm{H}]^{+}$193.1081, found 193.1084. 


\section{1,3-Bis[(tert-butyl)dimethylsilyloxy]propan-2-one $(65)^{136}$}

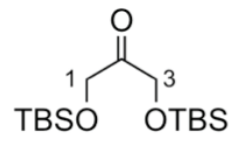

To a solution of dihydroxyacetone dimer 98 (4.00 g, $22.2 \mathrm{mmol})$ in DMF (50 mL) was added TBSCl $(15.9 \mathrm{~g}, 106 \mathrm{mmol})$ and imidazole $(15.0 \mathrm{~g}, 222 \mathrm{mmol})$. After $24 \mathrm{~h}$ the reaction mixture was quenched with cold water and extracted with $\mathrm{Et}_{2} \mathrm{O}(5 \times 50 \mathrm{~mL})$. The combined organic extracts were washed with water $(100 \mathrm{~mL})$, brine $(100 \mathrm{~mL})$, dried $\left(\mathrm{Na}_{2} \mathrm{SO}_{4}\right)$ and concentrated under reduced pressure. The resulting residue was filtered over silica gel (20\% EtOAc/Hexanes) to provide ketone 65 (13.0 g, 95\%) as a colourless oil. $\boldsymbol{R}_{\boldsymbol{f}} 0.80$ (10\% EtOAc/Hexanes); ${ }^{1} \mathbf{H}$ NMR (400 MHz, $\left.\mathrm{CDCl}_{3}\right) \delta 4.42$ (4H, s, H-1, H-3), $0.92\left(18 \mathrm{H}, \mathrm{s}, \mathrm{Si}-\mathrm{C}\left(\mathrm{C}_{3}\right)_{3}\right), 0.09\left(12 \mathrm{H}, \mathrm{s}, \mathrm{Si}-\mathrm{C}_{3}\right)$.

\section{(3R,4S)-1-Hydroxypent-4-en-1-yl)-1,3-bis(tert-butyldimethylsilyloxy)-4-hydroxy-} octane-8-one (99)

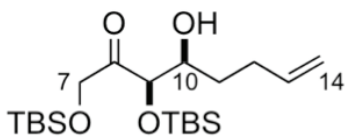

To a solution of ketone $65(1.00 \mathrm{~g}, 3.14 \mathrm{mmol})$ in $\mathrm{CHCl}_{3}(5.0 \mathrm{~mL})$ at $0{ }^{\circ} \mathrm{C}$ was added aldehyde 84 (132 mg, $1.57 \mathrm{mmol}$ ), followed by $\mathrm{H}_{2} \mathrm{O}$ (3 vol\%) and O- $t$ Bu-threonine (55.0 $\mathrm{mg}, 0.31 \mathrm{mmol}$ ) and the reaction mixture was allowed to warm to rt. After 5 days water $(5.0 \mathrm{~mL})$ was added and the layers were separated. The aqueous phase was extracted with EtOAc $(3 \times 10 \mathrm{~mL})$. The combined organic extracts were washed with brine $(20 \mathrm{~mL})$, dried $\left(\mathrm{Na}_{2} \mathrm{SO}_{4}\right)$, filtered and concentrated under reduced pressure. Purification by flash column chromatography (5\% EtOAc/Hexanes) afforded aldol product 99 (180 mg, 28\%) as a colourless oil.

$\boldsymbol{R}_{f} 0.10(10 \%$ EtOAc/Hexanes $) ;[\alpha]_{\mathrm{D}}^{20}+10.0\left(c\right.$ 1.0, $\left.\mathrm{CHCl}_{3}\right) ; \mathbf{I R} \boldsymbol{v}_{\max }\left(\right.$ thin film $\left./ \mathrm{cm}^{-1}\right) 3491$, 3079, 2954, 2931, 2887, 2858, 1735, 1472, 1255, 1100, 838, 779; ${ }^{1} \mathbf{H}$ NMR (400 MHz, $\left.\mathrm{CDCl}_{3}\right) \delta 5.86-5.76(1 \mathrm{H}, \mathrm{m}, \mathrm{H}-13), 5.04(1 \mathrm{H}, \mathrm{dq}, J=17.1,1.7 \mathrm{~Hz}, \mathrm{H}-14 \mathrm{a}), 4.98$ (1H, ddt, $J$ $=10.2,1.9,1.2 \mathrm{~Hz}, \mathrm{H}-14 \mathrm{~b}), 4.48(2 \mathrm{H}, \mathrm{d}, J=3.2 \mathrm{~Hz}, \mathrm{H}-7), 4.30(1 \mathrm{H}, \mathrm{d}, J=2.9 \mathrm{~Hz}, \mathrm{H}-9)$, 3.80 (1H, dddd, $J=10.1,7.1,6.2,2.9 \mathrm{~Hz}, \mathrm{H}-10), 2.32-2.21$ (1H, m, H-12a), 2.19 (1H, d, $J$ 
$=10.0 \mathrm{~Hz}, \mathrm{OH}), 2.17-2.08(1 \mathrm{H}, \mathrm{m}, \mathrm{H}-12 \mathrm{~b}), 1.60-1.55(2 \mathrm{H}, \mathrm{m}, \mathrm{H}-11), 0.94$ (9H, s, Si$\left.\mathrm{C}\left(\mathrm{C}_{3}\right)_{3}\right), 0.92\left(9 \mathrm{H}, \mathrm{s}, \mathrm{Si}-\mathrm{C}\left(\mathrm{C}_{3}\right)_{3}\right), 0.10\left(3 \mathrm{H}, \mathrm{s}, \mathrm{Si}-\mathrm{C}_{3}\right), 0.09$ (3H, s, Si-C $\left.\underline{H}_{3}\right), 0.09$ (3H, s, $\left.\mathrm{Si}-\mathrm{C}_{3}\right), 0.07\left(3 \mathrm{H}, \mathrm{s}, \mathrm{Si}-\mathrm{CH}_{3}\right) ;{ }^{13} \mathbf{C}$ NMR $\left(100 \mathrm{MHz}, \mathrm{CDCl}_{3}\right) \delta 210.3,138.0,115.3,79.3$, 72.5, 68.6, 33.3, 30.1, 26.0, 25.9, -4.7, -4.9, -5.3, -5.4; HRMS $\left(\mathrm{ES}^{+}\right)$calcd. for $\mathrm{C}_{20} \mathrm{H}_{46} \mathrm{NO}_{4} \mathrm{Si}_{2}\left[\mathrm{M}+\mathrm{NH}_{4}\right]^{+} 420.2960$, found 420.2961 .

(R)-Mosher ester of $99(100)$

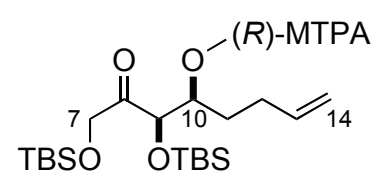

To a stirred mixture of alcohol $99(10.0 \mathrm{mg}, 24.8 \mu \mathrm{mol}), \mathrm{DCC}(20.0 \mathrm{mg}, 99.2 \mu \mathrm{mol})$ and DMAP $(6.1 \mathrm{mg}, 50.0 \mu \mathrm{mol})$ in $\mathrm{CH}_{2} \mathrm{Cl}_{2}(1.0 \mathrm{~mL})$ at $\mathrm{rt}$ was added $(+)-(R)-\alpha$-methoxy- $\alpha$ (trifluoromethyl)-phenylacetic acid $(11.7 \mathrm{mg}, 50.0 \mu \mathrm{mol})$ in one portion. After $16 \mathrm{~h}$, the crude reaction mixture was purified directly by flash column chromatography $(5 \%$ EtOAc/Hexanes) to give (R)-MTPA ester $100(11.0 \mathrm{mg}, 73 \%)$.

$\boldsymbol{R}_{\boldsymbol{f}} 0.26$ (10\% EtOAc/Hexanes); ${ }^{1} \mathbf{H}$ NMR $\left(500 \mathrm{MHz}, \mathrm{CDCl}_{3}\right) \delta$ 7.50-7.54 (2H, m, Ar-H, MTPA), 7.37-7.41 (3H, m, Ar-H, MTPA), 5.77 (1H, ddt, $J=16.8,10.1,6.6$ Hz, H-13), 5.38 (1H, ddd, $J=8.3,5.2,2.2 \mathrm{~Hz}, \mathrm{H}-10), 5.06-5.00(2 \mathrm{H}, \mathrm{m}, \mathrm{H}-14), 4.56(1 \mathrm{H}, \mathrm{d}, J=1.9$ Hz, H-9), 4.35 (1H, d, $J=18.2, \mathrm{H}-7 \mathrm{a}), 4.28(1 \mathrm{H}, \mathrm{d}, J=18.2 \mathrm{~Hz}, \mathrm{H}-7 \mathrm{~b}), 3.47$ (3H, s, MTPA-OCH$\left.{ }_{3}\right), 2.19-2.10$ (1H, m, H-12a), 2.09-2.01 (1H, m, H-12b), 1.88-1.85 (1H, m, H11a), 1.84-1.76 (1 H, m, H-11b), 0.91 (9H, s, Si-C $\left.\left(\underline{\mathrm{C}}_{3}\right)_{3}\right), 0.90\left(9 \mathrm{H}, \mathrm{s}, \mathrm{Si}-\mathrm{C}\left(\mathrm{C}_{3}\right)_{3}\right), 0.08$ $\left(3 \mathrm{H}, \mathrm{s}, \mathrm{Si}-\mathrm{CH}_{3}\right), 0.07\left(3 \mathrm{H}, \mathrm{s}, \mathrm{Si}-\mathrm{C}_{3}\right), 0.04\left(3 \mathrm{H}, \mathrm{s}, \mathrm{Si}-\mathrm{CH}_{3}\right), 0.03\left(3 \mathrm{H}, \mathrm{s}, \mathrm{Si}-\mathrm{CH}_{3}\right) ;{ }^{19} \mathbf{F}$ NMR $\left(470 \mathrm{MHz} ; \mathrm{CDCl}_{3}\right) \delta-72.1,-72.6,91 \%$ de.

(S)-Mosher ester of 99 (101)

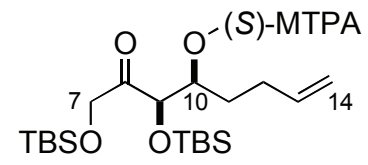

To a stirred mixture of alcohol $99(10.0 \mathrm{mg}, 24.8 \mu \mathrm{mol}), \mathrm{DCC}(20.0 \mathrm{mg}, 99.2 \mu \mathrm{mol})$ and DMAP $(6.1 \mathrm{mg}, 50.0 \mu \mathrm{mol})$ in $\mathrm{CH}_{2} \mathrm{Cl}_{2}(1.0 \mathrm{~mL})$ at $\mathrm{rt}$ was added $(+)-(S)$ - $\alpha$-methoxy- $\alpha$ - 
(trifluoromethyl)-phenylacetic acid $(11.7 \mathrm{mg}, 50.0 \mu \mathrm{mol})$ in one portion. After $16 \mathrm{~h}$, the crude reaction mixture was purified directly by flash column chromatography $(5 \%$ EtOAc/Hexanes) to give (S)-MTPA ester 101 (8.0 mg, 53\%).

$\boldsymbol{R}_{\boldsymbol{f}} 0.26$ (10\% EtOAc/Hexanes); ${ }^{1} \mathbf{H}$ NMR $\left(500 \mathrm{MHz}, \mathrm{CDCl}_{3}\right) \delta$ 7.56-7.58 (2H, m, Ar-H, MTPA), 7.38-7.40 (3H, m, Ar-H, MTPA), 5.69 (1H, ddt, $J=16.8,10.1,6.6$ Hz, H-13), 5.37-5.33 (1H, m, H-10), 4.99-4.89 (2H, m, H-14), 4.67 (1H, d, J=1.6 Hz, H-9), 4.49 (1H, d, $J=18.0 \mathrm{~Hz}, \mathrm{H}-7 \mathrm{a}), 4.28(1 \mathrm{H}, \mathrm{d}, J=18.0 \mathrm{~Hz}, \mathrm{H}-7 \mathrm{~b}), 3.57$ (3H, s, MTPA-OCH$\left.\underline{H}_{3}\right), 1.97-$ 1.86 (3H, m, H-11a, H-12), 1.72-1.64 (1H, m, H-11b), 0.92 (9H, s, Si-C(C $\left.\left.\underline{\mathrm{H}}_{3}\right)_{3}\right), 0.91(9 \mathrm{H}$, s, Si-C( $\left.\left(\underline{\mathrm{C}}_{3}\right)_{3}\right), 0.10\left(3 \mathrm{H}, \mathrm{s}, \mathrm{Si}-\mathrm{C}_{3}\right), 0.08\left(3 \mathrm{H}, \mathrm{s}, \mathrm{Si}-\mathrm{C}_{3}\right), 0.07$ (3H, s, Si-C $\left.\underline{\mathrm{H}}_{3}\right), 0.04(3 \mathrm{H}$, s, $\left.\mathrm{Si}-\underline{\mathrm{C}}_{3}\right)$.

$(2 R, 3 R, 4 S)-1,3-B i s((t e r t-b u t y l d i m e t h y l s i l y l) o x y) o c t-7-e n e-2,4-d i o l ~(102)$

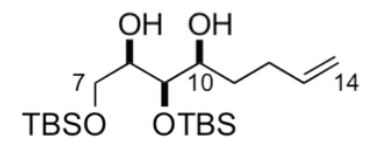

\section{Method A}

To a solution of aldol adduct $99(25.0 \mathrm{mg}, 62 \mu \mathrm{mol})$ in $\mathrm{MeOH}(2.0 \mathrm{~mL})$ at $-78{ }^{\circ} \mathrm{C}$ was added $\mathrm{NaBH}_{4}(2.9 \mathrm{mg}, 75 \mu \mathrm{mol})$. After $1 \mathrm{~h}$, the reaction mixture was quenched with saturated $\mathrm{NH}_{4} \mathrm{Cl}(4.0 \mathrm{~mL})$ solution and the aqueous layer was extracted with $\mathrm{Et}_{2} \mathrm{O}(3 \times 5$ $\mathrm{mL})$. The combined organic extracts were dried $\left(\mathrm{Na}_{2} \mathrm{SO}_{4}\right)$, filtered and concentrated under reduced pressure. The resulting residue was purified by flash column chromatography (5$10 \%$ of EtOAc/Hexanes) to give inseparable mixture of compounds $\mathbf{1 0 2}$ and $\mathbf{1 0 3}$ (20.0 mg, $80 \%)$ as a colourless oil in 10:1 syn:anti ratio.

\section{Method B}

To a solution of aldol adduct $99(50.0 \mathrm{mg}, 0.12 \mathrm{mmol})$ and $\mathrm{CeCl}_{3} \cdot 7 \mathrm{H}_{2} \mathrm{O}(70.0 \mathrm{mg}, 0.18$ mmol) in $\mathrm{MeOH}(2.0 \mathrm{~mL})$ at $-78^{\circ} \mathrm{C}$ was added $\mathrm{NaBH}_{4}(5.6 \mathrm{mg}, 0.15 \mathrm{mmol})$. After $1 \mathrm{~h}$ the reaction mixture was quenched with saturated $\mathrm{NH}_{4} \mathrm{Cl}(4.0 \mathrm{~mL})$ solution and the aqueous layer was extracted with $\mathrm{Et}_{2} \mathrm{O}(3 \times 5 \mathrm{~mL})$. The combined organic extracts were dried $\left(\mathrm{Na}_{2} \mathrm{SO}_{4}\right)$, filtered and concentrated under reduced pressure. The resulting residue was purified by flash column chromatography (5\% of EtOAc/Hexanes) to give inseparable 
mixture of compounds 102 and $103(30.0 \mathrm{mg}, 61 \%)$ as a colourless oil in 10:1 syn:anti ratio.

\section{Method C}

To a solution of aldol adduct $99(26.0 \mathrm{mg}, 65.0 \mu \mathrm{mol})$ in THF $(1.0 \mathrm{~mL})$ at $-78^{\circ} \mathrm{C}$ was added (c-hex $)_{2} \mathrm{BCl} \cdot \mathrm{NEt}_{3}(0.44 \mathrm{~mL}, 0.19 \mathrm{M}$ soln. in THF, $84.0 \mu \mathrm{mol})$ and after stirring for $15 \mathrm{~min}$, $\mathrm{LiBH}_{3} \mathrm{OMe}(0.55 \mathrm{~mL}, 0.2 \mathrm{M}$ soln. in THF, $0.11 \mathrm{mmol})$ was added dropwise. After $3 \mathrm{~h}$ the reaction mixture was quenched by addition of phosphate buffer $\mathrm{pH} 7(1.0 \mathrm{~mL}), \mathrm{MeOH}(1.0$ $\mathrm{mL})$ and $\mathrm{H}_{2} \mathrm{O}_{2}(0.5 \mathrm{~mL}, 30 \%$ aqueous $)$. After 30 min the resulting mixture was partitioned between $\mathrm{H}_{2} \mathrm{O}(5 \mathrm{~mL})$ and $\mathrm{CH}_{2} \mathrm{Cl}_{2}(4 \times 10 \mathrm{~mL})$. The combined organic extracts were dried $\left(\mathrm{Na}_{2} \mathrm{SO}_{4}\right)$, filtered and concentrated under reduced pressure. Purification by flash column chromatography (5\% of EtOAc/Hexanes) provided inseparable mixture of compounds $\mathbf{1 0 2}$ and $103(22.0 \mathrm{mg}, 84 \%)$ as a colourless oil in 10:1 syn:anti ratio.

102 syn: $\boldsymbol{R}_{f} 0.15$ (10\% EtOAc/Hexanes); $[\alpha]_{\mathrm{D}}^{20}-6.0\left(c 0.3, \mathrm{CHCl}_{3}\right)$; IR $\boldsymbol{v}_{\max }$ (thin film/cm ${ }^{-}$ $\left.{ }^{1}\right) 3481,2940,2868,1458,1189,1109,840,766 ;{ }^{1} \mathbf{H}$ NMR $\left(500 \mathrm{MHz}, \mathrm{CDCl}_{3}\right) \delta 5.83(1 \mathrm{H}$, ddt, $J=17.0,10.3,6.6 \mathrm{~Hz}, \mathrm{H}-13)$, 5.07-4.96 (2H, m, H-14), 3.71-3.65 (4H, m, H-7, H-8, H-10), 3.63-3.60 (1H, m, H-9), 2.52 (1H, br s, C8-O프), 2.35 (1H, d, J = 7.0 Hz, C10-O프), 2.31-2.22 (1H, m, H-12a), 2.17-2.08 (1H, m, H-12b), 1.59-1.54 (2H, m, H-11), 0.92 (9H, s, $\left.\mathrm{Si}-\left(\mathrm{C}_{3}\right)_{3}\right), 0.89$ (9H, s, Si- $\left.\left(\mathrm{C}_{3}\right)_{3}\right), 0.14\left(3 \mathrm{H}, \mathrm{s}, \mathrm{Si}-\underline{\mathrm{C}}_{3}\right), 0.12$ (3H, s, Si-C $\left.\underline{\mathrm{H}}_{3}\right), 0.07$ (6H, s, $\left.\mathrm{Si}-\left(\mathrm{C}_{3}\right)_{2}\right) ;{ }^{13} \mathbf{C}$ NMR $\left(125 \mathrm{MHz}, \mathrm{CDCl}_{3}\right) \delta 138.5,115.0,74.7,72.9,70.9,63.7,33.6,30.3$, 26.1, 26.0, 18.4, 18.3, -4.19, -4.21, -5.19, -5.24; HRMS $\left(\mathrm{ES}^{+}\right)$calcd. for $\mathrm{C}_{20} \mathrm{H}_{45} \mathrm{O}_{4} \mathrm{Si}_{2}$ $[\mathrm{M}+\mathrm{H}]^{+}$405.2851, found 405.2853.

\section{$(2 R, 3 R, 4 S)$-Octane-1,2,3,4,8-pentaol (55)}

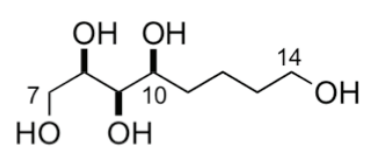

To a solution of thexylBH $\mathrm{H}_{2}$ in $\mathrm{THF}(4.0 \mathrm{~mL}, 0.5 \mathrm{M}, 2.0 \mathrm{mmol})$ at $0{ }^{\circ} \mathrm{C}$ was added olefin $102(145 \mathrm{mg}, 0.36 \mathrm{mmol})$ in THF $(2.0 \mathrm{~mL})$ dropwise. After $1 \mathrm{~h} \mathrm{NaOH}(2.0 \mathrm{~mL}, 10 \% \mathrm{w} / \mathrm{v})$ and hydrogen peroxide $(1 \mathrm{~mL}, 30 \% \mathrm{v} / \mathrm{v})$ were added. The mixture was stirred for $15 \mathrm{~min}$ and the aqueous layer was saturated with anhydrous $\mathrm{K}_{2} \mathrm{CO}_{3}$. The organics were extracted with $\mathrm{CH}_{2} \mathrm{Cl}_{2}(3 \times 10 \mathrm{~mL})$, washed with brine $(20 \mathrm{~mL})$, dried $\left(\mathrm{Na}_{2} \mathrm{SO}_{4}\right)$, filtered and 
concentrated under reduced pressure. The resulting residue was purified by flash column chromatography $(20 \%$ EtOAc/Hexanes) but due to the TBS-migration, the isolated products were directly used in the subsequent step.

To a solution of triol in $\mathrm{MeOH}(1.0 \mathrm{~mL})$ and water $(0.5 \mathrm{~mL})$ was added Dowex-50 resin $(130 \mathrm{mg})$ and the reaction was refluxed $\left(65-70{ }^{\circ} \mathrm{C}\right)$ for $1 \mathrm{~h}$. The reaction mixture was then cooled to rt, filtered and concentrated under reduced pressure to give $\mathbf{5 5}(55.0 \mathrm{mg}, 80 \%)$ as a colourless viscous oil.

$\boldsymbol{R}_{\boldsymbol{f}} 0.10\left(10 \% \mathrm{MeOH} / \mathrm{CH}_{2} \mathrm{Cl}_{2}\right) ;[\alpha]_{\mathrm{D}}^{20}-2.0$ (c 1.2, MeOH); IR $\boldsymbol{v}_{\max }\left(\right.$ thin film $\left./ \mathrm{cm}^{-1}\right) 3259$, 2923, 2899, 1321, 1196, 1127, 1060; ${ }^{1}$ H NMR (400 MHz, MeOD) $\delta$ 3.73-3.66 (2H, m, H8, H-10), 3.63 (2H, dd, $J=12.1,5.6 \mathrm{~Hz}, \mathrm{H}-7), 3.57$ (2H, t, $J=6.5 \mathrm{~Hz}, \mathrm{H}-14), 3.45$ (1H, t, $J$ $=4.0 \mathrm{~Hz}, \mathrm{H}-9), 1.63-1.47$ (6H, m, H-11, H-12, H-13); ${ }^{13} \mathbf{C}$ NMR (100 MHz, MeOD) $\delta$ C9-74.3, C10-74.0, C8-73.4, C7-64.4, C14-62.9, C13-34.2, C11-33.6, C12-23.1; HRMS $\left(\mathrm{ES}^{+}\right)$calcd. for $\mathrm{C}_{8} \mathrm{H}_{18} \mathrm{NaO}_{5}[\mathrm{M}+\mathrm{Na}]^{+} 217.1046$, found 217.1047 .

\section{Dimethyl 2,3-O-Isopropylidene-meso-tartrate ${ }^{74}$}

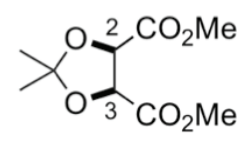

To a solution of dimethyl meso-tartrate $106(1.40 \mathrm{~g}, 7.86 \mathrm{mmol})$ in DMF $(10.0 \mathrm{~mL})$ were added 2,2-dimethoxypropane $(60.0 \mathrm{~mL}), p$-TsOH $(30.0 \mathrm{mg}, 0.16 \mathrm{mmol})$ and the mixture was refluxed for $4 \mathrm{~h}$. The mixture was then cooled to $\mathrm{rt}$ and concentrated to a volume of $\sim 30 \mathrm{~mL}$ and $1 \mathrm{M} \mathrm{NaHCO} 3(50.0 \mathrm{~mL})$ was added and the layers were separated. The aqueous phase was extracted with $\mathrm{CH}_{2} \mathrm{Cl}_{2}(3 \times 50 \mathrm{~mL})$ and the combined organic extracts were washed with water $(50 \mathrm{~mL})$, brine $(50 \mathrm{~mL})$, dried $\left(\mathrm{Na}_{2} \mathrm{SO}_{4}\right)$, filtered and concentrated under reduced pressure. Purification by flash column chromatography $\left(5 \% \mathrm{MeOH} / \mathrm{CH}_{2} \mathrm{Cl}_{2}\right)$ afforded dimethylketal (1.74 g, 98\%).

$\boldsymbol{R}_{\boldsymbol{f}} 0.60\left(10 \% \mathrm{MeOH} / \mathrm{CH}_{2} \mathrm{Cl}_{2}\right) ;{ }^{1} \mathbf{H}$ NMR (300 MHz, MeOD) $\delta 4.84$ (2H, s, H-2, H-3), 3.75 $\left(6 \mathrm{H}, \mathrm{s}, \mathrm{OC}_{3}\right), 1.65\left(3 \mathrm{H}, \mathrm{d}, J=0.6 \mathrm{~Hz}, \mathrm{C}-\mathrm{CH}_{3}\right), 1.42\left(3 \mathrm{H}, \mathrm{d}, J=0.7 \mathrm{~Hz}, \mathrm{C}-\underline{\mathrm{C}}_{3}\right)$. 


\section{2,3-O-Isopropylidenethreitol $(107)^{74}$}

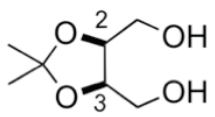

To a solution of dimethylketal $(1.00 \mathrm{~g}, 4.59 \mathrm{mmol})$ in $\mathrm{Et}_{2} \mathrm{O}(50 \mathrm{~mL})$ at $0{ }^{\circ} \mathrm{C}$ was added $\mathrm{LiAlH}_{4}(0.384 \mathrm{~g}, 10.1 \mathrm{mmol})$. The reaction mixture was stirred at $\mathrm{rt}$ for $6 \mathrm{~h}$. Excess of $\mathrm{LiAlH}_{4}$ was destroyed by addition of few drops of water and the mixture was diluted with ether and filtered. The solid residue was washed several times with $\mathrm{MeOH}$ and combined organic filtrates were dried $\left(\mathrm{Na}_{2} \mathrm{SO}_{4}\right)$, filtered and concentrated under reduced pressure. The resulting crude oil was purified by flash column chromatography $\left(5 \% \mathrm{MeOH} / \mathrm{CH}_{2} \mathrm{Cl}_{2}\right)$ to give diol 107 (599 $\mathrm{mg}, 81 \%)$.

$\boldsymbol{R}_{f} 0.30\left(10 \% \mathrm{MeOH} / \mathrm{CH}_{2} \mathrm{Cl}_{2}\right) ;{ }^{1} \mathbf{H}$ NMR $\left(400 \mathrm{MHz}, \mathrm{CDCl}_{3}\right) \delta 4.31-4.26(2 \mathrm{H}, \mathrm{m}, \mathrm{H}-2, \mathrm{H}-$ 3), 3.84-3.72 (4H, m, H-1, H-4), 1.46 (3H, s, C-C $\left.\underline{H}_{3}\right), 1.38\left(3 \mathrm{H}, \mathrm{s}, \mathrm{C}-\mathrm{CH}_{3}\right)$.

\section{4-O-(tert-Butyldimethyl)silyl-2,3-O-isopropylidene-DL-erythritol ${ }^{74}$}

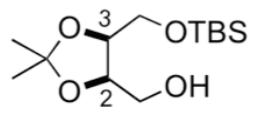

To a solution of diol $107(599 \mathrm{mg}, 3.69 \mathrm{mmol})$ in THF $(10.0 \mathrm{~mL})$ at $0{ }^{\circ} \mathrm{C}$ was added $\mathrm{NaH}$ (212 $\mathrm{mg}, 60 \%$ dispersion in mineral oil, $8.86 \mathrm{mmol}$ ). The mixture was allowed to stir for $15 \mathrm{~min}$ and TBSCl (556 mg, $3.69 \mathrm{mmol})$ was added. After $6 \mathrm{~h}$, water $(10.0 \mathrm{~mL})$ was added and the layers were separated. The aqueous phase was extracted with $\mathrm{CH}_{2} \mathrm{Cl}_{2}(3 \times 20 \mathrm{~mL})$ and the combined organic extracts were washed with brine $(40 \mathrm{~mL})$, dried $\left(\mathrm{Na}_{2} \mathrm{SO}_{4}\right)$, filtered and concentrated under reduced pressure. Purification by flash column chromatography (10\% EtOAc/Hexanes) provided alcohol $(897 \mathrm{mg}, 88 \%)$ as a colourless oil.

$\boldsymbol{R}_{f} 0.25$ (20\% EtOAc/Hexanes); ${ }^{1} \mathbf{H}$ NMR (400 MHz, $\left.\mathrm{CDCl}_{3}\right) \delta 4.35(1 \mathrm{H}, \mathrm{dt}, J=7.3,5.7$ Hz, H-3), 4.23 (1H, ddd, $J=9.1,6.0,4.1 \mathrm{~Hz}, \mathrm{H}-2), 3.84-3.73$ (3H, m, H-1a, H-4), 3.68 $(1 \mathrm{H}, \mathrm{dd}, J=10.5,4.1 \mathrm{~Hz}, \mathrm{H}-1 \mathrm{~b}), 3.01(1 \mathrm{H}, \mathrm{dd}, J=8.4,5.6 \mathrm{~Hz}, \mathrm{O} \underline{\mathrm{H}}), 1.41$ (3H, s, C-C $\left.\underline{H}_{3}\right)$, $1.35\left(3 \mathrm{H}, \mathrm{s}, \mathrm{C}-\mathrm{C}_{3}\right), 0.90\left(9 \mathrm{H}, \mathrm{s}, \mathrm{Si}-\mathrm{C}\left(\mathrm{C}_{3}\right)_{3}\right), 0.11\left(3 \mathrm{H}, \mathrm{s}, \mathrm{Si}-\underline{\mathrm{C}}_{3}\right), 0.10\left(3 \mathrm{H}, \mathrm{s}, \mathrm{Si}-\mathrm{C}_{3}\right)$. 


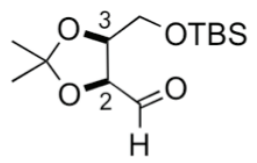

To a solution of oxalyl chloride $(286 \mu \mathrm{L}, 3.38 \mathrm{mmol})$ in $\mathrm{CH}_{2} \mathrm{Cl}_{2}(20.0 \mathrm{~mL})$ at $-78^{\circ} \mathrm{C}$ was added a solution of DMSO $(479 \mu \mathrm{L}, 6.75 \mathrm{mmol})$ in $\mathrm{CH}_{2} \mathrm{Cl}_{2}(5.0 \mathrm{~mL})$ dropwise. The reaction mixture was stirred at $-78{ }^{\circ} \mathrm{C}$ for $15 \mathrm{~min}$ and a solution of alcohol $(850 \mathrm{mg}, 3.07$ mmol) in $\mathrm{CH}_{2} \mathrm{Cl}_{2}(10.0 \mathrm{~mL})$ was added. The reaction mixture was stirred at $-78{ }^{\circ} \mathrm{C}$ for an additional $15 \mathrm{~min}$. and $\mathrm{Et}_{3} \mathrm{~N}(2.14 \mathrm{~mL}, 15.3 \mathrm{mmol})$ was then added, and the mixture was allowed to warm to $\mathrm{rt}$. The reaction was quenched by addition of water $(20.0 \mathrm{~mL})$ and extracted with $\mathrm{CH}_{2} \mathrm{Cl}_{2}(3 \times 30 \mathrm{~mL})$. The combined organic extracts were washed with saturated $\mathrm{NH}_{4} \mathrm{Cl}(50 \mathrm{~mL})$ solution and brine $(50 \mathrm{~mL})$, dried $\left(\mathrm{Na}_{2} \mathrm{SO}_{4}\right)$ and concentrated under reduced pressure. The resulting oil was purified by flash column chromatography (10\% EtOAc/Hexanes) to give $\mathbf{1 0 5}(810 \mathrm{mg}, 96 \%)$ as a colourless oil.

$\boldsymbol{R}_{f} 0.44$ (20\% EtOAc/Hexanes); ${ }^{1} \mathbf{H}$ NMR $\left(400 \mathrm{MHz}, \mathrm{CDCl}_{3}\right) \delta 9.68(1 \mathrm{H}, \mathrm{d}, J=2.0 \mathrm{~Hz}, \mathrm{H}-$ 1), $4.46(1 \mathrm{H}, \mathrm{dd}, J=3.8,2.8 \mathrm{~Hz}, \mathrm{H}-3), 4.42(1 \mathrm{H}, \mathrm{dd}, J=7.9,2.0 \mathrm{~Hz}, \mathrm{H}-2), 3.78(1 \mathrm{H}, \mathrm{dd}, J$ $=11.4,3.8 \mathrm{~Hz}, \mathrm{H}-4 \mathrm{a}), 3.69(1 \mathrm{H}, \mathrm{dd}, J=11.4,2.7 \mathrm{~Hz}, \mathrm{H}-4 \mathrm{~b}), 1.56\left(3 \mathrm{H}, \mathrm{s}, \mathrm{C}-\mathrm{C}_{3}\right), 1.38$

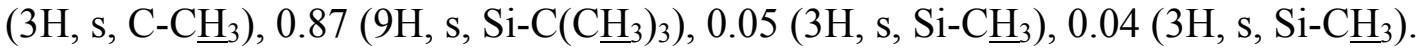

(S)-1-((4R,5S)-5-(((tert-Butyldimethylsilyl)oxy)methyl)-2,2-dimethyl-1,3-dioxolan-4yl)pent-4-en-1-ol (108)

(R)-1-((4R,5S)-5-(((tert-Butyldimethylsilyl)oxy)methyl)-2,2-dimethyl-1,3-dioxolan-4yl)pent-4-en-1-ol (109)

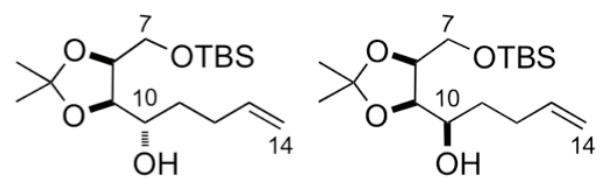

To a flame dried round bottom flask charged with magnesium turnings (1.09 g, $45.2 \mathrm{mmol})$ was added $\mathrm{Et}_{2} \mathrm{O}(5.0 \mathrm{~mL})$ and catalytic amount of iodine. Homoallyl bromide $(5.08 \mathrm{~g}, 37.7$ mmol) dissolved in THF $(12.0 \mathrm{~mL})$ was added dropwise as the temperature was increased to $50{ }^{\circ} \mathrm{C}$. The reaction mixture was stirred for $2 \mathrm{~h}$. 
To a solution of aldehyde $105(0.25 \mathrm{~g}, 0.91 \mathrm{mmol})$ in THF $(5.0 \mathrm{~mL})$ at $-40{ }^{\circ} \mathrm{C}$ was added freshly prepared homoallyl magnesium bromide (1.24 mL, 2M soln. in THF, $2.73 \mathrm{mmol})$ via cannula. The resulting black solution was allowed to warm to $-20^{\circ} \mathrm{C}$ for $1 \mathrm{~h}$, and finally to $0{ }^{\circ} \mathrm{C}$ for $2 \mathrm{~h}$, before being quenched by the addition of saturated $\mathrm{NH}_{4} \mathrm{Cl}(10.0$ $\mathrm{mL})$ solution and diluted with $\mathrm{Et}_{2} \mathrm{O}(20 \mathrm{~mL})$. The organic phase was separated, and the aqueous layer extracted with $\mathrm{Et}_{2} \mathrm{O}(3 \times 20 \mathrm{~mL})$. The combined organic extracts were dried $\left(\mathrm{Na}_{2} \mathrm{SO}_{4}\right)$, filtered and concentrated under reduced pressure. Purification by flash column chromatography (5\% EtOAc/Hexanes) gave hydroxy olefins 108 (230 mg, 70\%) and 109 (80.0 mg, $28 \%$ ) as colourless oils.

108: $\boldsymbol{R}_{\boldsymbol{f}} 0.25$ (20\% EtOAc/Hexanes); IR $\boldsymbol{v}_{\max }$ (thin film $\left./ \mathrm{cm}^{-1}\right)$ 3484, 2953, 2931, 2860, 1472, 1368, 1177, 1082, 837, 779; ${ }^{1} \mathbf{H}$ NMR (400 MHz, $\left.\mathrm{CDCl}_{3}\right) \delta$ 5.81-5.91 (1H, m, H13), 5.05 (1H, dq, $J=17.1,1.8 \mathrm{~Hz}, \mathrm{H}-14 \mathrm{a}), 4.96$ (1H, ddt, $J=10.2,2.2,1.1 \mathrm{~Hz}, \mathrm{H}-14 \mathrm{~b})$, $4.23(1 \mathrm{H}, \mathrm{ddd}, J=10.2,5.4,3.4 \mathrm{~Hz}, \mathrm{H}-8), 4.02(1 \mathrm{H}, \mathrm{dd}, J=9.3,5.4 \mathrm{~Hz}, \mathrm{H}-7 \mathrm{a}), 3.93(1 \mathrm{H}$, dd, $J=3.1,1.6 \mathrm{~Hz}, \mathrm{H}-9), 3.82-3.76$ (2H, m, H-10, O프), 3.57 (1H, dd, $J=10.4,3.1 \mathrm{~Hz}, \mathrm{H}-$ 7b), 2.40-2.30 (1H, m, H-12a), 2.23-2.13 (1H, m, H-12b), 1.89-1.80 (1H, m, H-11a), 1.62$1.56(1 \mathrm{H}, \mathrm{m}, \mathrm{H}-11 \mathrm{~b}), 1.36\left(3 \mathrm{H}, \mathrm{s}, \mathrm{C}-\mathrm{CH}_{3}\right), 1.33\left(3 \mathrm{H}, \mathrm{s}, \mathrm{C}-\mathrm{C}_{3}\right), 0.91\left(9 \mathrm{H}, \mathrm{s}, \mathrm{Si}-\mathrm{C}\left(\mathrm{C}_{3}\right)_{3}\right)$, $0.12\left(3 \mathrm{H}, \mathrm{s}, \mathrm{Si}-\underline{\mathrm{H}}_{3}\right), 0.12\left(3 \mathrm{H}, \mathrm{s}, \mathrm{Si}-\mathrm{C}_{3}\right) ;{ }^{13} \mathbf{C}$ NMR $\left(100 \mathrm{MHz}, \mathrm{CDCl}_{3}\right) \delta 138.9,114.7$, 108.5, 80.9, 68.4, 62.1, 33.3, 29.6, 28.2, 25.9, 25.5, 18.3, -5.45, -5.54; HRMS (ES ${ }^{+}$) calcd. for $\mathrm{C}_{17} \mathrm{H}_{35} \mathrm{O}_{4} \mathrm{Si}[\mathrm{M}+\mathrm{H}]^{+}$331.2299, found 331.2303.

109: $\boldsymbol{R}_{\boldsymbol{f}} 0.24$ (20\% EtOAc/Hexanes); IR $\boldsymbol{v}_{\max }\left(\right.$ thin film $\left./ \mathrm{cm}^{-1}\right)$ 3484, 2953, 2931, 2860, 1472, 1368, 1177, 1082, 837, 779; ${ }^{1} \mathbf{H}$ NMR (400 MHz, $\left.\mathrm{CDCl}_{3}\right) \delta 5.83$ (1H, ddt, $J=17.0$, 10.3, 6.7 Hz, H-13), 5.04 (1H, dq, $J=17.1,1.8 \mathrm{~Hz}, \mathrm{H}-14 \mathrm{a}), 4.96$ (1H, ddt, $J=10.1,2.1$, $1.1 \mathrm{~Hz}, \mathrm{H}-14 \mathrm{~b}), 4.15$ (1H, ddd, $J=7.2,6.4,4.2 \mathrm{~Hz}, \mathrm{H}-8), 4.02$ (1H, dd, $J=6.4,3.6 \mathrm{~Hz}, \mathrm{H}-$ 9), $3.91(1 \mathrm{H}, \mathrm{dd}, J=10.8,7.3 \mathrm{~Hz}, \mathrm{H}-7 \mathrm{a}), 3.81(1 \mathrm{H}, \mathrm{td}, J=8.8,4.2 \mathrm{~Hz}, \mathrm{H}-10), 3.72(1 \mathrm{H}$, dd, $J=10.8,4.2 \mathrm{~Hz}, \mathrm{H}-7 \mathrm{~b}), 2.81$ (1H, d, $J=5.1 \mathrm{~Hz}, \mathrm{OH}), 2.30-2.26$ (1H, m, H-12a), 2.182.14 (1H, m, H-12b), 1.70-1.61 (2H, m, H-11), 1.47 (3H, s, C-C $\left.\underline{H}_{3}\right), 1.36\left(3 \mathrm{H}, \mathrm{s}, \mathrm{C}-\mathrm{C}_{3}\right)$, $0.90\left(9 \mathrm{H}, \mathrm{s}, \mathrm{Si}-\mathrm{C}\left(\mathrm{C}_{3}\right)_{3}\right), 0.09\left(6 \mathrm{H}, \mathrm{s}, \mathrm{Si}-\left(\mathrm{C}_{\mathrm{H}_{3}}\right)_{2}\right) ;{ }^{13} \mathbf{C}$ NMR $\delta\left(100 \mathrm{MHz}, \mathrm{CDCl}_{3}\right) 138.5$, 114.9, 108.2, 79.8, 68.4, 61.9, 33.8, 30.2, 27.5, 26.0, 25.2, 18.4, -5.3; HRMS (ES ${ }^{+}$) calcd. for $\mathrm{C}_{17} \mathrm{H}_{35} \mathrm{O}_{4} \mathrm{Si}[\mathrm{M}+\mathrm{H}]^{+}$331.2299, found 331.2305. 


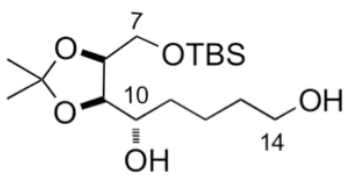

To a solution of thexylBH $\mathrm{H}_{2}$ in $\mathrm{THF}(4.0 \mathrm{~mL}, 0.5 \mathrm{M}, 2.0 \mathrm{mmol})$ at $0{ }^{\circ} \mathrm{C}$ was added olefin $108(110 \mathrm{mg}, 0.33 \mathrm{mmol})$ in THF $(1.0 \mathrm{~mL})$ dropwise. After $1 \mathrm{~h} \mathrm{NaOH}(0.5 \mathrm{~mL}, 10 \% \mathrm{w} / \mathrm{v})$ and hydrogen peroxide $(0.5 \mathrm{~mL}, 30 \% \mathrm{v} / \mathrm{v})$ were added. The mixture was stirred for $15 \mathrm{~min}$ and the aqueous layer was saturated with anhydrous $\mathrm{K}_{2} \mathrm{CO}_{3}$. The organics were extracted with $\mathrm{CH}_{2} \mathrm{Cl}_{2}(3 \times 5 \mathrm{~mL})$, washed with brine $(15.0 \mathrm{~mL})$, dried $\left(\mathrm{Na}_{2} \mathrm{SO}_{4}\right)$, filtered and concentrated under reduced pressure. The residue was purified by flash column chromatography (10\% EtOAc/Hexanes) to give diol (114 mg, 95\%) as a colourless oil.

$\boldsymbol{R}_{\boldsymbol{f}} 0.16$ (20\% EtOAc/Hexanes); IR $\boldsymbol{v}_{\max }$ (thin film/ $\mathrm{cm}^{-1}$ ) 3448, 3409, 2950, 2930, 2858, 1472, 1380, 1275, 1083, 837, 777; ${ }^{1} \mathbf{H}$ NMR (400 MHz, $\left.\mathrm{CDCl}_{3}\right) \delta 4.17-4.11$ (1H, m, H-8), $4.02(1 \mathrm{H}, \mathrm{dd}, J=6.3,3.5 \mathrm{~Hz}, \mathrm{H}-9), 3.91(1 \mathrm{H}, \mathrm{dd}, J=10.8,7.1 \mathrm{~Hz}, \mathrm{H}-7 \mathrm{a}), 3.81-3.77(1 \mathrm{H}$, m, H-10), 3.73 (1H, dd, $J=10.8,4.1 \mathrm{~Hz}, \mathrm{H}-7 \mathrm{~b}), 3.66$ (2H, t, $J=6.3 \mathrm{~Hz}, \mathrm{H}-14), 1.66-1.56$ (6H, m, H-11, H-12, H-13), 1.47 (3H, s, C-C $\left.\underline{H}_{3}\right), 1.36\left(3 \mathrm{H}, \mathrm{s}, \mathrm{C}-\mathrm{C}_{3}\right), 0.90$ (9H, s, Si$\left.\mathrm{C}\left(\mathrm{C}_{3}\right)_{3}\right), 0.09$ (6H, s, Si- $\left.\left(\underline{\mathrm{C}}_{3}\right)_{2}\right) ;{ }^{13} \mathbf{C}$ NMR $\left(100 \mathrm{MHz}, \mathrm{CDCl}_{3}\right) \delta$ 108.2, 79.8, 69.0, 63.0, 61.8, 34.3, 32.8, 31.1, 27.5, 26.0, 25.2, 22.3, 18.4, -5.3; HRMS (ES ${ }^{+}$) calcd. for $\mathrm{C}_{17} \mathrm{H}_{37} \mathrm{O}_{5} \mathrm{Si}[\mathrm{M}+\mathrm{H}]^{+}$349.2405, found 349.2407.

$(2 S, 3 R, 4 S)$-Octane-1,2,3,4,8-pentaol (56)

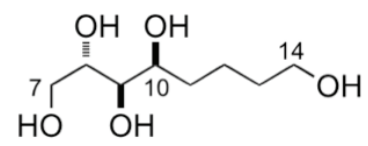

To a solution of diol $(90.0 \mathrm{mg}, 0.26 \mathrm{mmol})$ in $\mathrm{MeOH}(1.0 \mathrm{~mL})$ and water $(0.5 \mathrm{~mL})$ was added Dowex-50 resin $(90.0 \mathrm{mg})$ and the reaction was refluxed $\left(65-70{ }^{\circ} \mathrm{C}\right)$ for $1 \mathrm{~h}$. The reaction mixture was then cooled to rt, filtered and concentrated under reduced pressure to give 56 (49.0 mg, 98\%) as a colourless viscous oil.

$\boldsymbol{R}_{\boldsymbol{f}} 0.10\left(10 \% \mathrm{MeOH} / \mathrm{CH}_{2} \mathrm{Cl}_{2}\right)$; IR $\boldsymbol{v}_{\max }\left(\right.$ thin film $\left./ \mathrm{cm}^{-1}\right)$ 3342, 3244, 2926, 2911, 2860, 1405, 1281, 1087, 1026; ${ }^{1} \mathbf{H}$ NMR (400 MHz, MeOD) $\delta 3.82(1 \mathrm{H}, \mathrm{td}, J=4.3,1.7 \mathrm{~Hz}, \mathrm{H}-$ 
10), 3.79 (1H, dd, $J=11.1,3.5 \mathrm{~Hz}, \mathrm{H}-7 \mathrm{a}), 3.68$ (1H, ddd, $J=8.0,5.9,3.5 \mathrm{~Hz}, \mathrm{H}-8), 3.61$ $(1 \mathrm{H}, \mathrm{dd}, J=11.1,6.0 \mathrm{~Hz}, \mathrm{H}-7 \mathrm{~b}), 3.57$ (2H, t, $J=6.5 \mathrm{~Hz}, \mathrm{H}-14), 3.36$ (1H, dd, $J=10.9,5.9$ Hz, H-9), 1.63-1.49 (5H, m, H-11, 12a, 13), 1.45-1.37 (1H, m, H-12b); ${ }^{13}$ C NMR (100 MHz, MeOD) $\delta$ C9-74.5, C10-73.2, C8-71.4, C7-65.1, C14-62.9, C11-34.5, C13-33.7, C12-23.4; HRMS $\left(\mathrm{ES}^{+}\right)$calcd. for $\mathrm{C}_{8} \mathrm{H}_{19} \mathrm{O}_{5}[\mathrm{M}+\mathrm{H}]^{+}$195.1227, found 195.1227.

\subsection{Experimental procedures for Chapter 3}

(E)-Ethyl-hepta-2,6-dienoate (111) ${ }^{137}$

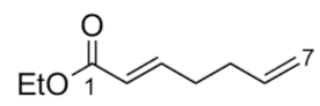

To a solution of triethyl phosphonoacetate $112(3.54 \mathrm{~mL}, 17.8 \mathrm{mmol})$, lithium chloride (2.01 g, $47.6 \mathrm{mmol})$ and Hünig's base $(1.88 \mathrm{~mL}, 10.8 \mathrm{mmol})$ in $\mathrm{MeCN}(40.0 \mathrm{~mL})$ at rt was added a solution of aldehyde $84(1.00 \mathrm{~g}, 11.9 \mathrm{mmol})$ in $\mathrm{MeCN}(5.0 \mathrm{~mL})$. After $18 \mathrm{~h}$, the reaction was quenched by addition of $\mathrm{H}_{2} \mathrm{O}(50.0 \mathrm{~mL})$ and layers were separated. The aqueous phase was extracted with $\mathrm{Et}_{2} \mathrm{O}(3 \times 40 \mathrm{~mL})$ and the combined organic extracts were dried $\left(\mathrm{MgSO}_{4}\right)$, filtered and concentrated under reduced pressure. Purification by flash column chromatography $\left(5 \% \mathrm{Et}_{2} \mathrm{O} /\right.$ Pet. Ether) provided $19(1.51 \mathrm{~g}, 81 \%)$ as a colourless oil.

$\boldsymbol{R}_{\boldsymbol{f}} 0.42\left(20 \% \mathrm{Et}_{2} \mathrm{O} /\right.$ Pet. Ether); ${ }^{1} \mathbf{H}$ NMR (400 MHz, $\left.\mathrm{CDCl}_{3}\right) \delta 6.95(1 \mathrm{H}, \mathrm{dt}, J=15.7,6.7$ Hz, H-3), 5.82 (1H, dt, $J=15.6,1.6 \mathrm{~Hz}, \mathrm{H}-2), 5.83-5.74$ (1H, m, H-6), 5.04 (1H, dq, $J=$ 17.1, $1.7 \mathrm{~Hz}, \mathrm{H}-7 \mathrm{a}), 5.00$ (1H, ddt, $J=10.2,1.8,1.2 \mathrm{~Hz}, \mathrm{H}-7 \mathrm{~b}), 4.17$ (2H, q, $J=7.1 \mathrm{~Hz}$, $\left.\mathrm{OCH}_{2} \mathrm{CH}_{3}\right), 2.33-2.27(2 \mathrm{H}, \mathrm{m}, \mathrm{H}-4), 2.24-2.18(2 \mathrm{H}, \mathrm{m}, \mathrm{H}-5), 1.27(3 \mathrm{H}, \mathrm{t}, J=7.1 \mathrm{~Hz}$, $\left.\mathrm{OCH}_{2} \underline{\mathrm{CH}}_{3}\right)$. 


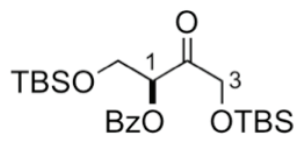

To a solution of pre-dried L-erythrulose $118(6.00 \mathrm{~g}, 50.0 \mathrm{mmol})$ in DMF (60.0 $\mathrm{mL})$ was added TBSCl (16.6 g, $110 \mathrm{mmol})$ and imidazole $(10.2 \mathrm{~g}, 150 \mathrm{mmol})$. The reaction mixture was then stirred at $\mathrm{rt}$ for $2 \mathrm{~h}$ and a saturated $\mathrm{NH}_{4} \mathrm{Cl}(100 \mathrm{~mL})$ solution was added. The layers were separated and the aqueous phase was extracted with $\mathrm{Et}_{2} \mathrm{O}(3 \times 100 \mathrm{~mL})$. The combined organic extracts were washed with $\mathrm{H}_{2} \mathrm{O}(50 \mathrm{~mL})$, brine $(100 \mathrm{~mL})$, dried $\left(\mathrm{Na}_{2} \mathrm{SO}_{4}\right)$, filtered and concentrated under reduced pressure. Purification by flash column chromatography (2-10\% EtOAc/Hexanes) provided bis-TBS-ether $(12.2 \mathrm{~g}, 70 \%)$ as a colourless oil, which was directly used in the next step.

$\boldsymbol{R}_{\boldsymbol{f}} 0.36$ (10\% EtOAc/Hexanes); ${ }^{1} \mathbf{H}$ NMR (400 MHz; $\left.\mathrm{CDCl}_{3}\right) \delta 4.45(2 \mathrm{H}, \mathrm{d}, J=17.9 \mathrm{~Hz}$, H-3a), 4.44 (1H, dt, $J=6.7,3.4$ Hz, H-1), 4.38 (1H, d, $J=17.8$ Hz, H-3b), 4.09 (1H, dd, $J$ $\left.=10.6,3.3 \mathrm{~Hz}, \underline{\mathrm{CH}}_{2}-\mathrm{OTBS}\right), 3.82\left(1 \mathrm{H}, \mathrm{dd}, J=10.6,3.7 \mathrm{~Hz}, \underline{\mathrm{C}}_{2}-\mathrm{OTBS}\right), 3.44(1 \mathrm{H}, \mathrm{d}, J=$ $6.7 \mathrm{~Hz}, \mathrm{O} \underline{\mathrm{H}}), 0.91\left(9 \mathrm{H}, \mathrm{s}, \mathrm{Si}-\mathrm{C}\left(\mathrm{C}_{3}\right)_{3}\right), 0.85\left(9 \mathrm{H}, \mathrm{s}, \mathrm{Si}-\mathrm{C}\left(\mathrm{C}_{3}\right)_{3}\right), 0.09$ (3H, s, Si-C $\left.\underline{\mathrm{H}}_{3}\right), 0.08$ $\left(3 \mathrm{H}, \mathrm{s}, \mathrm{Si}-\mathrm{C}_{3}\right), 0.04\left(6 \mathrm{H}, \mathrm{s}, \mathrm{Si}-\left(\mathrm{C}_{3}\right)_{2}\right)$.

To a solution of bis-TBS-ether $(12.1 \mathrm{~g}, 34.7 \mathrm{mmol})$ in $\mathrm{CH}_{2} \mathrm{Cl}_{2}(200 \mathrm{~mL})$ was added $\mathrm{Et}_{3} \mathrm{~N}$ (6.25 mL, $44.7 \mathrm{mmol})$, DMAP (1.26 g, $10.3 \mathrm{mmol})$ and benzoyl chloride (4.40 mL, 38.0 mmol) dropwise. The reaction mixture was then stirred at $\mathrm{rt}$ for $5 \mathrm{~h}$ and quenched with water $(200 \mathrm{~mL})$ and the layers were separated. The aqueous phase was extracted with $\mathrm{CH}_{2} \mathrm{Cl}_{2}(3 \times 200 \mathrm{~mL})$, and the combined organic extracts were washed with saturated $\mathrm{NH}_{4} \mathrm{Cl}(50 \mathrm{~mL})$ solution, brine $(100 \mathrm{~mL})$, dried $\left(\mathrm{Na}_{2} \mathrm{SO}_{4}\right)$, filtered and concentrated under reduced pressure. Purification by flash column chromatography (2\% EtOAc/Hexanes) provided ketone $119(9.34 \mathrm{~g}, 60 \%)$ as a colourless oil.

$\boldsymbol{R}_{\boldsymbol{f}} 0.68$ (10\% EtOAc/Hexanes); ${ }^{1} \mathbf{H}$ NMR (400 MHz, $\left.\mathrm{CDCl}_{3}\right) \delta 8.08$ (2H, m, Ar-H), 7.58 (1H, m, Ar-H), 7.46 (2H, m, Ar-H), 5.57 (1H, dd, J=4.6, $3.6 \mathrm{~Hz}, \mathrm{H}-1), 4.49$ (2H, s, H-3), 4.20 (1H, dd, $J=11.1,4.6 \mathrm{~Hz}, \mathrm{CH}_{2}$-OTBS), 4.07 (1H, dd, $\left.J=11.1,3.6 \mathrm{~Hz}, \mathrm{CH}_{2}-\mathrm{OTBS}\right)$, $0.93\left(9 \mathrm{H}, \mathrm{s}, \mathrm{Si}-\mathrm{C}\left(\mathrm{CH}_{3}\right)_{3}\right), 0.88\left(9 \mathrm{H}, \mathrm{s}, \mathrm{Si}-\mathrm{C}\left(\mathrm{CH}_{3}\right)_{3}\right), 0.11\left(3 \mathrm{H}, \mathrm{s}, \mathrm{Si}-\mathrm{CH}_{3}\right), 0.11(3 \mathrm{H}, \mathrm{s}, \mathrm{Si}-$ $\left.\mathrm{CH}_{3}\right), 0.08\left(3 \mathrm{H}, \mathrm{s}, \mathrm{Si}-\mathrm{CH}_{3}\right), 0.07\left(3 \mathrm{H}, \mathrm{s}, \mathrm{Si}-\mathrm{CH}_{3}\right)$. 


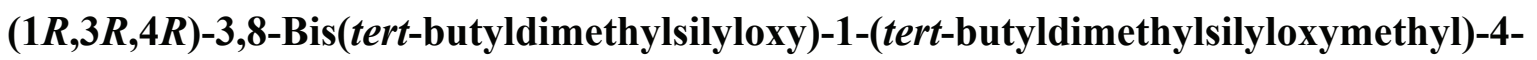
hydroxy-2-oxooctyl benzoate (123)

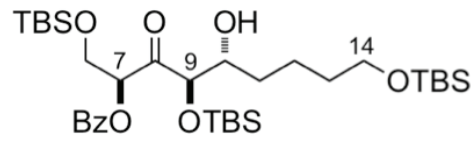

To a stirred solution of ketone $119(1.18 \mathrm{~g}, 2.62 \mathrm{mmol})$ in $\mathrm{Et}_{2} \mathrm{O}(20.0 \mathrm{~mL})$ at $-78{ }^{\circ} \mathrm{C}$ was added dicyclohexylboron chloride $(1.03 \mathrm{~mL}, 4.71 \mathrm{mmol})$ and $\mathrm{Et}_{3} \mathrm{~N}(0.728 \mathrm{~mL}, 5.24 \mathrm{mmol})$ and the reaction mixture was stirred for $0.5 \mathrm{~h}$. Aldehyde $69(0.85 \mathrm{~g}, 3.9 \mathrm{mmol})$ in $\mathrm{Et}_{2} \mathrm{O}$ $(10.0 \mathrm{~mL})$ was then added and stirring was continued for $2 \mathrm{~h}$. The reaction mixture was then diluted with $\mathrm{MeOH}(5.0 \mathrm{~mL})$ and phosphate buffer $\mathrm{pH} 7(5.0 \mathrm{~mL})$ was added followed by $\mathrm{H}_{2} \mathrm{O}_{2}(5.0 \mathrm{~mL}, 30 \% \mathrm{v} / \mathrm{v})$. The biphasic mixture was allowed to warm to $\mathrm{rt}$ and stirred vigorously for $10 \mathrm{~min}$. The layers were separated and the aqueous phase was extracted with $\mathrm{Et}_{2} \mathrm{O}(3 \times 30 \mathrm{~mL})$, washed with brine $(30 \mathrm{~mL})$, dried $\left(\mathrm{Na}_{2} \mathrm{SO}_{4}\right)$, filtered and concentrated under reduced pressure. Flash column chromatography (5\% EtOAc/Hexanes) provided aldol adduct $123(1.40 \mathrm{~g}, 81 \%)$ as a 4:1 inseparable mixture of diastereoisomers. $\boldsymbol{R}_{\boldsymbol{f}} 0.34$ (10\% EtOAc/Hexanes); IR $\boldsymbol{v}_{\max }\left(\right.$ thin film $\left./ \mathrm{cm}^{-1}\right)$ 3491, 2938, 2892, 2861, 1724, 1463, 1267, 1105, 839, 778; ${ }^{1} \mathbf{H}$ NMR (400 MHz, $\left.\mathrm{CDCl}_{3}\right) \delta$ 8.10-8.06 (2H, m, Ar-H), 7.61$7.56(1 \mathrm{H}, \mathrm{m}, \mathrm{Ar}-\mathrm{H}), 7.48-7.43(2 \mathrm{H}, \mathrm{m}, \mathrm{Ar}-\mathrm{H}), 5.72(1 \mathrm{H}, \mathrm{dd}, J=4.9,3.5 \mathrm{~Hz}, \mathrm{H}-7), 4.20$ $(1 \mathrm{H}, \mathrm{d}, J=5.4 \mathrm{~Hz}, \mathrm{H}-9), 4.15$ (2H, dd, $J=4.2,1.9 \mathrm{~Hz}, \mathrm{H}-6), 3.92-3.86(1 \mathrm{H}, \mathrm{m}, \mathrm{H}-10), 3.60$ $(2 \mathrm{H}, \mathrm{t}, J=6.3 \mathrm{~Hz}, \mathrm{H}-14), 3.08$ (1H, d, $J=7.5 \mathrm{~Hz}, \mathrm{OH}), 1.64-1.49$ (5H, m, H-11, H-12a, H13), 1.49-1.37 (1H, m, H-12b), $0.93\left(9 \mathrm{H}, \mathrm{s}, \mathrm{Si}-\mathrm{C}\left(\mathrm{CH}_{3}\right)_{3}\right), 0.88\left(9 \mathrm{H}, \mathrm{s}, \mathrm{Si}-\mathrm{C}\left(\mathrm{CH}_{3}\right)_{3}\right), 0.87$ $\left(9 \mathrm{H}, \mathrm{s}, \mathrm{Si}-\mathrm{C}\left(\mathrm{CH}_{3}\right)_{3}\right), 0.12\left(6 \mathrm{H}, \mathrm{s}, \mathrm{Si}-\left(\mathrm{CH}_{3}\right)_{2}\right), 0.10\left(3 \mathrm{H}, \mathrm{s}, \mathrm{Si}-\mathrm{CH}_{3}\right), 0.09$ (3H, s, Si-CH 3$)$, $0.07\left(3 \mathrm{H}, \mathrm{s}, \mathrm{Si}-\mathrm{CH}_{3}\right), 0.04\left(3 \mathrm{H}, \mathrm{s}, \mathrm{Si}-\mathrm{CH}_{3}\right) ;{ }^{13} \mathbf{C} \mathbf{N M R}\left(75 \mathrm{MHz}, \mathrm{CDCl}_{3}\right) \delta 205.1,166.5$, 133.6, 130.1, 129.4, 128.6, 81.9, 79.1, 73.9, 63.2, 62.8, 32.8, 32.6, 26.1, 26.0, 25.9, 22.3, 18.5, 18.4, 18.3, -4.4, -4.6, -5.1, -5.2; HRMS $\left(\mathrm{ES}^{+}\right)$calcd. for $\mathrm{C}_{34} \mathrm{H}_{65} \mathrm{O}_{7} \mathrm{Si}_{3}[\mathrm{M}+\mathrm{H}]^{+}$ 669.4033, found 669.4020. 
(1R,3R,4R)-3,8-Bis(tert-butyldimethylsilyloxy)-1-(tert-butyldimethylsilyloxymethyl)-4(methoxybenzyl)oxy-2-oxooctyl benzoate (125)

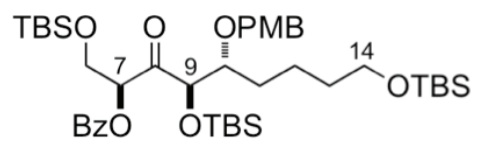

To a solution of alcohol 123 (1.40 g, $2.09 \mathrm{mmol})$ and PMBTCA (886 mg, $3.14 \mathrm{mmol})$ in $\mathrm{PhMe}(20.0 \mathrm{~mL})$ at $0{ }^{\circ} \mathrm{C}$ was added lanthanum (III) triflate $(61.0 \mathrm{mg}, 0.104 \mathrm{mmol})$. After 6 $\mathrm{h}$, cold hexane $(40.0 \mathrm{~mL})$ was added and the precipitate was filtered. The resulting mixture was concentrated under reduced pressure and purified by flash column chromatography (5\% EtOAc/Hexanes) to give PMB ether 125 (1.08 g, 65\%) as a colourless oil.

$\boldsymbol{R}_{\boldsymbol{f}} 0.44\left(10 \%\right.$ EtOAc/Hexanes); $[\alpha]_{\mathrm{D}}^{20}+7.6$ ( 2 2.5, $\left.\mathrm{CHCl}_{3}\right)$; IR $\boldsymbol{v}_{\max }\left(\right.$ thin film/ $\left./ \mathrm{cm}^{-1}\right) 2933$, 2891, 2859, 1725, 1610, 1466, 1255, 1104, 837, 778, 711; ${ }^{1} \mathbf{H}$ NMR (400 MHz, $\left.\mathrm{CDCl}_{3}\right)$ $\delta 8.08(2 \mathrm{H}, \mathrm{dd}, J=8.4,1.3 \mathrm{~Hz}, \operatorname{Ar}-\underline{\mathrm{H}}), 7.56(1 \mathrm{H}, \mathrm{s}, \operatorname{Ar}-\underline{\mathrm{H}}), 7.45(2 \mathrm{H}, \mathrm{t}, J=7.7 \mathrm{~Hz}, \operatorname{Ar}-\underline{\mathrm{H}})$, $7.19(2 \mathrm{H}, \mathrm{d}, J=8.7 \mathrm{~Hz}, \mathrm{PMB}$ Ar- $\underline{\mathrm{H}}), 6.83$ (2H, d, $J=8.7 \mathrm{~Hz}, \mathrm{PMB}$ Ar- $\underline{\mathrm{H}}), 5.87$ (1H, t, $J=$ $4.5 \mathrm{~Hz}, \mathrm{H}-7), 4.63(1 \mathrm{H}, \mathrm{d}, J=3.4 \mathrm{~Hz}, \mathrm{H}-9), 4.50\left(1 \mathrm{H}, \mathrm{d}, J=11.2 \mathrm{~Hz}, \mathrm{CH}_{\mathrm{a}} \mathrm{H}_{\mathrm{b}} \mathrm{Ar}\right), 4.36(1 \mathrm{H}$, d, $\left.J=11.2 \mathrm{~Hz}, \mathrm{CH}_{\mathrm{a}} \underline{\mathrm{H}}_{\mathrm{b}} \mathrm{Ar}\right), 4.08(2 \mathrm{H}, \mathrm{d}, J=4.5 \mathrm{~Hz}, \mathrm{H}-6), 3.79\left(3 \mathrm{H}, \mathrm{s}, \mathrm{Ar}-\mathrm{OCH}_{3}\right), 3.71(1 \mathrm{H}$, dt, $J=8.4,3.3 \mathrm{~Hz}, \mathrm{H}-10), 3.54$ (2H, td, $J=6.3,1.5 \mathrm{~Hz}, \mathrm{H}-14), 1.73-1.63$ (1H, m, H-11a), 1.51-1.38 (4H, m, H-11b, H-12a, H-13), 1.33-1.21 (1H, m, H-12b), 0.94 (9H, s, Si$\left.\mathrm{C}\left(\mathrm{C}_{3}\right)_{3}\right), 0.88\left(9 \mathrm{H}, \mathrm{s}, \mathrm{Si}-\mathrm{C}\left(\mathrm{C}_{3}\right)_{3}\right), 0.84\left(9 \mathrm{H}, \mathrm{s}, \mathrm{Si}-\mathrm{C}\left(\mathrm{C}_{3}\right)_{3}\right), 0.12\left(3 \mathrm{H}, \mathrm{s}, 2 \times \mathrm{Si}-\underline{\mathrm{H}}_{3}\right)$, $0.09\left(3 \mathrm{H}, \mathrm{s}, 2 \times \mathrm{Si}-\underline{\mathrm{C}}_{3}\right), 0.05\left(3 \mathrm{H}, \mathrm{s}, \mathrm{Si}-\underline{\mathrm{C}}_{3}\right), 0.02\left(6 \mathrm{H}, \mathrm{s}, 2 \times \mathrm{Si}^{-} \underline{\mathrm{H}}_{3}\right), 0.02(3 \mathrm{H}, \mathrm{s}, \mathrm{Si}-$ $\left.\mathrm{C}_{3}\right) ;{ }^{13} \mathrm{C}$ NMR $\left(100 \mathrm{MHz}, \mathrm{CDCl}_{3}\right) \delta 205.7,165.6,159.2,133.3,130.4,130.0,129.9$, $129.5,128.5,113.8,81.4,78.3,78.0,71.9,63.3,62.1,55.3,33.0,29.8,26.14,26.12,25.9$, $21.9,18.51,18.48,18.37,-4.0,-4.8,-5.1,-5.3$; HRMS $\left(\mathrm{ES}^{+}\right)$calcd. for $\mathrm{C}_{42} \mathrm{H}_{76} \mathrm{NO}_{8} \mathrm{Si}_{3}$ $\left[\mathrm{M}+\mathrm{NH}_{4}\right]^{+} 806.4873$, found 806.4875 . 
(1R,3S,4R)-3,8-Bis(tert-butyldimethylsilyloxy)-1-(tert-butyldimethylsilyloxymethyl)-4(methoxybenzyl)oxy-octane-1,2-diol (126)

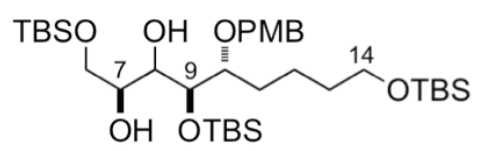

To a solution of keto-ester $125(729 \mathrm{mg}, 0.92 \mathrm{mmol})$ in $\mathrm{Et}_{2} \mathrm{O}(20.0 \mathrm{~mL})$ at $-78{ }^{\circ} \mathrm{C}$ was added $\mathrm{LiBH}_{4}(1.85 \mathrm{~mL}, 2 \mathrm{M}$ soln. in THF, $3.69 \mathrm{mmol})$ dropwise. The reaction mixture was stirred for $2 \mathrm{~h}$ and quenched with saturated $\mathrm{NaHCO}_{3}(50.0 \mathrm{~mL})$ solution. The layers were separated and the aqueous phase was extracted with $\mathrm{Et}_{2} \mathrm{O}(3 \times 50 \mathrm{~mL})$. The combined organic extracts were washed with brine $(50 \mathrm{~mL})$, dried $\left(\mathrm{Na}_{2} \mathrm{SO}_{4}\right)$ and concentrated under reduced pressure. Flash column chromatography (10\% EtOAc/Hexanes) provided diol 126 (415 mg, 66\%) as a colourless oil.

$\boldsymbol{R}_{\boldsymbol{f}} 0.24$ (10\% EtOAc/Hexanes); $[\alpha]_{\mathrm{D}}^{20}+10.1\left(c 0.8 \mathrm{CDCl}_{3}\right) ; \mathbf{I R} \boldsymbol{v}_{\max }$ (thin film $\left./ \mathrm{cm}^{-1}\right) 3316$, 3259, 2923, 2899, 1321, 1196, 1127, 1060; ${ }^{1} \mathbf{H}$ NMR $\left(300 \mathrm{MHz}, \mathrm{CDCl}_{3}\right) \delta 7.26(2 \mathrm{H}, \mathrm{d}, J=$ $8.6 \mathrm{~Hz}, \operatorname{Ar}-\underline{\mathrm{H}}), 6.86(2 \mathrm{H}, \mathrm{d}, J=8.7 \mathrm{~Hz}, \mathrm{Ar}-\underline{\mathrm{H}}), 4.65\left(1 \mathrm{H}, \mathrm{d}, J=10.8 \mathrm{~Hz}, \mathrm{C}_{\mathrm{H}} \mathrm{H}_{\mathrm{b}} \mathrm{Ar}\right), 4.44$ $\left(1 \mathrm{H}, \mathrm{d}, J=10.9 \mathrm{~Hz}, \mathrm{CH}_{\mathrm{a}} \underline{\mathrm{H}}_{\mathrm{b}} \mathrm{Ar}\right), 3.96(1 \mathrm{H}, \mathrm{dd}, J=4.8,3.2, \mathrm{H}-10), 3.80\left(3 \mathrm{H}, \mathrm{s}, \mathrm{Ar}-\mathrm{OC}_{3}\right)$, 3.71-3.52 (7H, m, H-6, H-7, H-8, H-9, H-14), 3.19 (1H, d, J=5.0 Hz, C8-O프), 2.95 (1H, $\mathrm{d}, J=4.5 \mathrm{~Hz}, \mathrm{C} 7-\mathrm{O} \underline{\mathrm{H}}), 1.59-1.47$ (5H, m, H-11, H-12a, H-13), 1.28-1.26 (1H, m, H-12b), $0.92\left(9 \mathrm{H}, \mathrm{s}, \mathrm{Si}-\mathrm{C}\left(\mathrm{CH}_{3}\right)_{3}\right), 0.90\left(18 \mathrm{H}, \mathrm{s}, \mathrm{Si}-\mathrm{C}\left(\mathrm{CH}_{3}\right)_{3}\right), 0.12\left(3 \mathrm{H}, \mathrm{s}, \mathrm{Si}-\mathrm{CH}_{3}\right), 0.11(3 \mathrm{H}, \mathrm{s}, \mathrm{Si}-$ $\left.\mathrm{C}_{3}\right), 0.07\left(6 \mathrm{H}, \mathrm{s}, 2 \times \mathrm{Si}-\underline{\mathrm{H}}_{3}\right), 0.04\left(6 \mathrm{H}, \mathrm{s}, 2 \times \mathrm{Si}-\underline{\mathrm{C}}_{3}\right) ;{ }^{13} \mathbf{C} \mathbf{N M R}\left(75 \mathrm{MHz}, \mathrm{CDCl}_{3}\right) \delta$ $159.3,130.5,129.6,113.9,82.3,73.8,72.7,71.1,70.9,64.8,63.3,55.4,33.1,30.7,26.15$, 26.13, 26.10, 22.5, 18.53, 18.49, 18.33, -3.9, -4.6, -5.10, -5.21; HRMS (ES ${ }^{+}$) calcd. for $\mathrm{C}_{35} \mathrm{H}_{72} \mathrm{O}_{7} \mathrm{Si}_{3}[\mathrm{M}+\mathrm{H}]^{+}$687.4502, found 687.4495. 
(3S,4S,5R)-4,9-Bis((tert-butyldimethylsilyl)oxy)-5-((4-methoxybenzyl)oxy)nonen-3-ol (128)

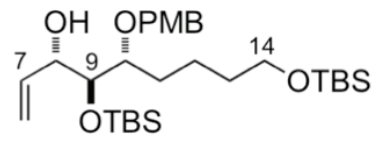

To a solution of diol $126(0.39 \mathrm{~g}, 0.58 \mathrm{mmol})$ in EtOAc $(20.0 \mathrm{~mL})$ was added $\mathrm{Pb}(\mathrm{OAc})_{4}$ $(0.41 \mathrm{mg}, 0.58 \mathrm{mmol})$, resulting in a light yellow suspension. After $10 \mathrm{~min}$ of stirring, the mixture was filtered through a plug of silica gel, eluting with EtOAc to afford aldehyde $127(0.28 \mathrm{~g}, 97 \%)$ as a colourless oil immediately used without further purification.

$\boldsymbol{R}_{f} 0.36\left(10 \%\right.$ EtOAc/Hexanes); ${ }^{1} \mathbf{H}$ NMR $\left(300 \mathrm{MHz}, \mathrm{CDCl}_{3}\right) \delta 9.67(1 \mathrm{H}, \mathrm{d}, J=1.6 \mathrm{~Hz}, \mathrm{H}-$ 8), $7.24(2 \mathrm{H}, \mathrm{d}, J=8.7 \mathrm{~Hz}, \mathrm{Ar}-\underline{\mathrm{H}}), 6.86(2 \mathrm{H}, \mathrm{d}, J=8.7 \mathrm{~Hz}, \mathrm{Ar}-\underline{\mathrm{H}}), 4.60$ (1H, d, $J=11.1$ $\left.\mathrm{Hz}, \underline{\mathrm{C}}_{\mathrm{a}} \mathrm{H}_{\mathrm{b}} \mathrm{Ar}\right), 4.47\left(1 \mathrm{H}, \mathrm{d}, J=11.2 \mathrm{~Hz}, \mathrm{CH}_{\mathrm{a}} \underline{\mathrm{H}}_{\mathrm{b}} \mathrm{Ar}\right), 4.09(1 \mathrm{H}, \mathrm{dd}, J=3.6,1.7 \mathrm{~Hz}, \mathrm{H}-9)$, $3.80\left(3 \mathrm{H}, \mathrm{s}, \mathrm{Ar}-\mathrm{OCH}_{3}\right), 3.62(1 \mathrm{H}, \mathrm{dt}, J=5.7,2.9 \mathrm{~Hz}, \mathrm{H}-10), 3.58(1 \mathrm{H}, \mathrm{t}, J=6.1 \mathrm{~Hz}, \mathrm{H}-14)$, 1.72-1.63 (1H, m, H-11a), 1.56-1.42 (5H, m, H-11b, H-12, H-13), 0.93 (9H, s, Si$\left.\mathrm{C}\left(\mathrm{CH}_{3}\right)_{3}\right), 0.89\left(9 \mathrm{H}, \mathrm{s}, \mathrm{Si}-\mathrm{C}\left(\mathrm{CH}_{3}\right)_{3}\right), 0.08\left(6 \mathrm{H}, \mathrm{s}, 2 \times \mathrm{Si}^{-\mathrm{CH}_{3}}\right), 0.04\left(6 \mathrm{H}, \mathrm{s}, 2 \times \mathrm{Si}-\mathrm{C}_{3}\right)$; ${ }^{13}$ C NMR $\left(75 \mathrm{MHz}, \mathrm{CDCl}_{3}\right) \delta$ 203.8, 159.4, 130.4, 129.6, 113.9, 81.1, 79.6, 72.3, 63.2, $55.4,32.9,30.8,26.1,25.9,22.0,18.52,18.36,-4.58,-4.71,-5.1$.

To a solution of aldehyde $127(370 \mathrm{mg}, 0.72 \mathrm{mmol})$ in THF $(20.0 \mathrm{~mL})$ at $-78{ }^{\circ} \mathrm{C}$ was added vinylmagnesium bromide $(6.2 \mathrm{~mL}, 0.7 \mathrm{M}$ soln. in THF, $4.35 \mathrm{mmol})$. The reaction mixture was allowed to warm up to $0{ }^{\circ} \mathrm{C}$ over $12 \mathrm{~h}$. The reaction was quenched with saturated $\mathrm{NH}_{4} \mathrm{Cl}(20.0 \mathrm{~mL})$ solution and the layers were separated. The aqueous phase was extracted with $\mathrm{Et}_{2} \mathrm{O}(3 \times 50 \mathrm{~mL})$ and the combined organic extracts were washed with brine $(50$ $\mathrm{mL})$, dried $\left(\mathrm{Na}_{2} \mathrm{SO}_{4}\right)$, filtered and concentrated under reduced pressure. Flash column chromatography (5\% EtOAc/Hexanes) provided allylic alcohol 128 (199 $\mathrm{mg}, 52 \%)$ as a colourless oil.

$\boldsymbol{R}_{\boldsymbol{f}} 0.34\left(20 \%\right.$ EtOAc/Hexanes); $[\alpha]_{\mathrm{D}}^{20}+15.3\left(c 1.6, \mathrm{CHCl}_{3}\right)$; IR $\mathbf{v}_{\max }\left(\right.$ thin film/ $\left./ \mathrm{cm}^{-1}\right) 3364$, 2933, 2891, 2860, 1464, 1317, 1099, 837, 774; ${ }^{1}$ H NMR (400 MHz; $\left.\mathrm{CDCl}_{3}\right) \delta 7.25$ (2H, d, $J=8.7 \mathrm{~Hz}, \mathrm{Ar}-\underline{\mathrm{H}}), 6.86(2 \mathrm{H}, \mathrm{d}, J=8.7 \mathrm{~Hz}, \mathrm{Ar}-\underline{\mathrm{H}}), 5.97$ (1H, ddd, $J=17.3,10.5,6.1 \mathrm{~Hz}, \mathrm{H}-$ 2), $5.30(1 \mathrm{H}, \mathrm{dt}, J=17.3,1.6 \mathrm{~Hz}, \mathrm{H}-6 \mathrm{a}), 5.18(1 \mathrm{H}, \mathrm{dt}, J=10.5,1.5 \mathrm{~Hz}, \mathrm{H}-6 \mathrm{~b}), 4.53(1 \mathrm{H}, \mathrm{d}$, $\left.J=11.0 \mathrm{~Hz}, \mathrm{CH}_{\mathrm{a}} \mathrm{H}_{\mathrm{b}} \mathrm{Ar}\right), 4.41\left(1 \mathrm{H}, \mathrm{d}, J=10.9 \mathrm{~Hz}, \mathrm{CH}_{\mathrm{a}} \underline{\mathrm{H}}_{\mathrm{b}} \mathrm{Ar}\right), 4.26-4.22(1 \mathrm{H}, \mathrm{m}, \mathrm{H}-8), 3.80$ $\left(3 \mathrm{H}, \mathrm{s}, \mathrm{Ar}-\mathrm{OC}_{3}\right), 3.75$ (1H, dd, $\left.J=4.9,4.1 \mathrm{~Hz}, \mathrm{H}-9\right), 3.59$ (2H, t, $\left.J=6.3 \mathrm{~Hz}, \mathrm{H}-14\right), 3.50$ $3.46(1 \mathrm{H}, \mathrm{m}, \mathrm{H}-10), 2.26(1 \mathrm{H}, \mathrm{d}, J=4.0 \mathrm{~Hz}, \mathrm{OH}), 1.66-1.57$ (2H, m, H-11), 1.54-1.45 (3H, 
m, H-12a, H-13), 1.40-1.30 (1H, m, H-12b), 0.90 (9H, s, Si-C $\left.\left(\mathrm{CH}_{3}\right)_{3}\right), 0.89$ (9H, s, Si$\left.\mathrm{C}\left(\mathrm{C}_{3}\right)_{3}\right), 0.08\left(6 \mathrm{H}, \mathrm{s}, 2 \times \mathrm{Si}-\underline{\mathrm{C}}_{3}\right), 0.04\left(6 \mathrm{H}, \mathrm{s}, 2 \times \mathrm{Si}-\underline{\mathrm{C}}_{3}\right) ;{ }^{13} \mathbf{C}$ NMR (100 MHz; $\left.\mathrm{CDCl}_{3}\right) \delta 159.3,137.9,130.9,129.5,116.2,113.8,80.9,76.8,75.0,72.1,63.4,55.4,33.2$, $30.8,26.15,26.13,22.1,18.5,18.4,-4.1,-4.2,-5.1$; HRMS $\left(\mathrm{ES}^{+}\right)$calcd. for $\mathrm{C}_{29} \mathrm{H}_{55} \mathrm{O}_{5} \mathrm{Si}_{2}$ $[\mathrm{M}+\mathrm{H}]^{+}$539.3583, found 539.3598.

\section{Diethyl 2,6,10-dodecatrienedioate $(\mathbf{1 3 5})^{138}$}

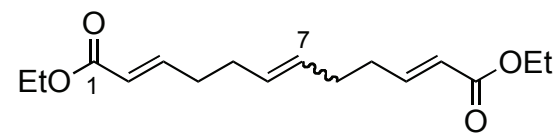

To a stirred solution of Grubbs II cat. (7.60 mg, $9.27 \mu \mathrm{mol})$ in $\mathrm{CH}_{2} \mathrm{Cl}_{2}(5.0 \mathrm{~mL})$ at $\mathrm{rt}$ was added a solution of olefin $111(57.0 \mathrm{mg}, 0.371 \mathrm{mmol})$ and $128(50.0 \mathrm{mg}, 92.8 \mu \mathrm{mol})$ in $\mathrm{CH}_{2} \mathrm{Cl}_{2}(5.0 \mathrm{~mL})$ and the mixture was stirred for $24 \mathrm{~h}$. Following concentration, the crude product was purified by flash column chromatography (10\% EtOAc:Pet.Ether) to provide dimer of the olefin $\mathbf{1 7 6}$ as a colourless oil $(30.0 \mathrm{mg}, 30 \%)$ and returned unreacted olefin $128(20.0 \mathrm{mg})$.

$\boldsymbol{R}_{f} 0.38$ (20\% EtOAc/Hexanes); ${ }^{1} \mathbf{H}$ NMR $\left(400 \mathrm{MHz}, \mathrm{CDCl}_{3}\right) \delta 6.94(2 \mathrm{H}, \mathrm{dt}, J=15.7,6.8$ Hz, H-3, 3'), 5.81 (2H, dt, $\left.J=15.7,1.6 \mathrm{~Hz}, \mathrm{H}-2,2^{\prime}\right), 5.44$ (2H, tt, $J=3.4,1.7 \mathrm{~Hz}, \mathrm{H}-6$, H-7), 4.18 (4H, q, $\left.J=7.1 \mathrm{~Hz}, \mathrm{OCH}_{2} \mathrm{CH}_{3}\right), 2.28-2.23$ (4H, m, H-4, 4'), 2.18-2.14 (4H, m, $\left.\mathrm{H}-5,5^{\prime}\right), 1.28\left(6 \mathrm{H}, \mathrm{t}, J=7.1 \mathrm{~Hz}, \mathrm{OCH}_{2} \mathrm{CH}_{3}\right) ;{ }^{13} \mathbf{C ~ N M R}\left(100 \mathrm{MHz} ; \mathrm{CDCl}_{3}\right) \delta 166.8,148.5$, $129.9,121.8,60.3,32.2,31.0,14.4$.

\section{4-(tert-Butyldimethylsilyl)oxy-butanal (145) $)^{139,140}$}

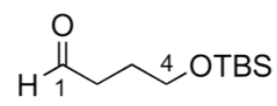

To a solution of 1,4-butanediol $146(9.80 \mathrm{~mL}, 0.11 \mathrm{~mol}), \mathrm{Et}_{3} \mathrm{~N}(6.75 \mathrm{~mL}, 48 \mathrm{mmol})$ and DMAP (450 mg, $3.7 \mathrm{mmol})$ in $\mathrm{CH}_{2} \mathrm{Cl}_{2}(40.0 \mathrm{~mL})$ at $\mathrm{rt}$ was slowly added a solution of TBSCl $(5.60 \mathrm{~g}, 37.1 \mathrm{mmol})$ in $\mathrm{CH}_{2} \mathrm{Cl}_{2}(10.0 \mathrm{~mL})$. After $16 \mathrm{~h}$, the reaction mixture was poured onto brine $(50.0 \mathrm{~mL})$ and layers were separated. The aqueous phase was extracted with $\mathrm{CH}_{2} \mathrm{Cl}_{2}(3 \times 50 \mathrm{~mL})$ and the combined organic extracts were washed with water $(100$ 
$\mathrm{mL})$, brine $(50 \mathrm{~mL})$, dried $\left(\mathrm{MgSO}_{4}\right)$, filtered and concentrated under reduced pressure. Purification by flash column chromatography (5\% EtOAc/Hexanes) provided alcohol (6.42 g, 86\%) as a colourless oil.

$\boldsymbol{R}_{\boldsymbol{f}} 0.43$ (50\% EtOAc/Hexanes); ${ }^{1} \mathbf{H}$ NMR $\left(300 \mathrm{MHz}, \mathrm{CDCl}_{3}\right) \delta 3.67$ (4H, m, H-1, H-4), $2.51(1 \mathrm{H}, \mathrm{t}, J=5.8 \mathrm{~Hz}, \mathrm{O} \underline{\mathrm{H}}), 1.66(4 \mathrm{H}, \mathrm{m}, \mathrm{H}-2, \mathrm{H}-3), 0.91\left(9 \mathrm{H}, \mathrm{s}, \mathrm{Si}-\mathrm{C}\left(\mathrm{C}_{3}\right)_{3}\right), 0.08(6 \mathrm{H}$, m, Si- $\left.\left(\mathrm{CH}_{3}\right)_{2}\right)$.

To a solution of oxalyl chloride $(3.39 \mathrm{~mL}, 39.2 \mathrm{mmol})$ in $\mathrm{CH}_{2} \mathrm{Cl}_{2}(50.0 \mathrm{~mL})$ at $-78{ }^{\circ} \mathrm{C}$ was slowly added DMSO (3.33 mL, $47.1 \mathrm{mmol})$. After $30 \mathrm{~min}$, a solution of alcohol (4.00 g, $19.6 \mathrm{mmol})$ in $\mathrm{CH}_{2} \mathrm{Cl}_{2}(20.0 \mathrm{~mL})$ was added, dropwise. After $1 \mathrm{~h}, \mathrm{Et}_{3} \mathrm{~N}(19.1 \mathrm{~mL}, 137$ mmol) was added and the slurry was allowed to attain rt. After $1 \mathrm{~h}$, saturated $\mathrm{NH}_{4} \mathrm{Cl}(50$ $\mathrm{mL})$ solution was added and layers were separated. The aqueous phase was extracted with $\mathrm{CH}_{2} \mathrm{Cl}_{2}(3 \times 40 \mathrm{~mL})$ and the combined organic extracts were washed with $\mathrm{H}_{2} \mathrm{O}(100 \mathrm{~mL})$, brine $(100 \mathrm{~mL})$, dried $\left(\mathrm{MgSO}_{4}\right)$, filtered and concentrated under reduced pressure. The crude aldehyde 145 (3.17 g, 80\%) was used without further purification.

$\boldsymbol{R}_{\boldsymbol{f}} 0.71$ (25\% EtOAc/Hexanes); ${ }^{1} \mathbf{H}$ NMR $\left(400 \mathrm{MHz}, \mathrm{CDCl}_{3}\right) \delta 9.80(1 \mathrm{H}, \mathrm{t}, J=1.7 \mathrm{~Hz}$, H-1), 3.66 (2H, t, $J=5.9$ Hz, H-4), 2.51 (2H, dt, $J=7.2,1.7$ Hz, H-2), 1.87 (2H, m, H-3), $0.89\left(9 \mathrm{H}, \mathrm{s}, \mathrm{Si}-\mathrm{C}\left(\mathrm{CH}_{3}\right)_{3}\right), 0.05\left(6 \mathrm{H}, \mathrm{m}, 2 \times \mathrm{Si}^{-\mathrm{CH}_{3}}\right)$.

\section{(E)-Methyl-6-(tert-Butyldimethylsilyl)oxy-hex-2-enoate $(147)^{141}$}

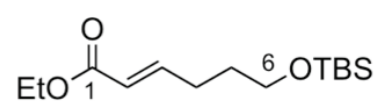

To a solution of triethyl phosphonoacetate 112 (4.04 mL, $20.3 \mathrm{mmol})$, lithium chloride (3.71 g, $87.6 \mathrm{mmol})$ and Hünig's base $(2.28 \mathrm{~mL}, 13.1 \mathrm{mmol})$ in $\mathrm{MeCN}(50.0 \mathrm{~mL})$ at rt was added a solution of aldehyde 145 (3.15 g, $15.6 \mathrm{mmol})$ in $\mathrm{MeCN}(20.0 \mathrm{~mL})$. After $18 \mathrm{~h}$, $\mathrm{H}_{2} \mathrm{O}(50.0 \mathrm{~mL})$ was added and the layers were separated. The aqueous phase was extracted with EtOAc $(3 \times 40 \mathrm{~mL})$ and the combined organic extracts were dried $\left(\mathrm{MgSO}_{4}\right)$, filtered and concentrated under reduced pressure. Purification by flash column chromatography (5\% EtOAc/Hexanes) provided 147 (3.29 g, 77\%) as a colourless oil.

$\boldsymbol{R}_{f} 0.52$ (20\% EtOAc/Hexanes); ${ }^{1} \mathbf{H}$ NMR $\left(400 \mathrm{MHz}, \mathrm{CDCl}_{3}\right) \delta 6.95(1 \mathrm{H}, \mathrm{dt}, J=15.6,6.9$ $\mathrm{Hz}, \mathrm{H}-3), 5.85$ (1H, dt, $J=15.6,1.6 \mathrm{~Hz}, \mathrm{H}-2), 4.15\left(2 \mathrm{H}, \mathrm{q}, J=7.1 \mathrm{~Hz}, \mathrm{OCH}_{2} \mathrm{CH}_{3}\right), 3.60$ $(2 \mathrm{H}, \mathrm{t}, J=7.1 \mathrm{~Hz}, \mathrm{H}-6), 2.3$ (2H, m, H-4), 1.65 (2H, m, H-5), 1.25 (3H, t, $J=7.1 \mathrm{~Hz}$, $\left.\mathrm{OCH}_{2} \underline{\mathrm{C}}_{3}\right), 0.90\left(9 \mathrm{H}, \mathrm{s}, \mathrm{Si}-\mathrm{C}\left(\mathrm{CH}_{3}\right)_{3}\right), 0.05\left(6 \mathrm{H}, \mathrm{s}, \mathrm{Si}-\left(\mathrm{C}_{3}\right)_{2}\right)$. 
(E)-Ethyl 6-hydroxyhex-2-enoate ${ }^{142}$

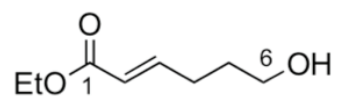

To a solution of TBS ether $147(2.00 \mathrm{~g}, 7.3 \mathrm{mmol})$ in $\mathrm{MeOH} / \mathrm{CH}_{2} \mathrm{Cl}_{2}(1: 4,20.0 \mathrm{~mL})$ at $0{ }^{\circ} \mathrm{C}$ was added $( \pm)$-CSA $(170 \mathrm{mg}, 0.73 \mathrm{mmol})$. After $1.5 \mathrm{~h}$, saturated $\mathrm{NaHCO}_{3}(20.0 \mathrm{~mL})$ solution and $\mathrm{CH}_{2} \mathrm{Cl}_{2}(20.0 \mathrm{~mL})$ were added and the layers were separated. The aqueous phase was extracted with $\mathrm{CH}_{2} \mathrm{Cl}_{2}(3 \times 30 \mathrm{~mL})$ and the combined organic extracts were dried $\left(\mathrm{Na}_{2} \mathrm{SO}_{4}\right)$, filtered and concentrated under reduced pressure. Purification by flash column chromatography (10\% EtOAc/Hexanes) provided alcohol $(1.13 \mathrm{~g}, 98 \%)$ as a colourless oil.

$\boldsymbol{R}_{\boldsymbol{f}} 0.22$ (20\% EtOAc/Hexanes); ${ }^{1} \mathbf{H}$ NMR $\left(300 \mathrm{MHz}, \mathrm{CDCl}_{3}\right) \delta 6.97(1 \mathrm{H}, \mathrm{dt}, J=15.6,7.0$, H-3), $5.84(1 \mathrm{H}, \mathrm{dt}, J=15.6,1.6 \mathrm{~Hz}, \mathrm{H}-2), 4.18\left(2 \mathrm{H}, \mathrm{q}, J=7.1 \mathrm{~Hz}, \mathrm{OCH}_{2} \mathrm{CH}_{3}\right), 3.67$ (2H, t, $J=6.4 \mathrm{~Hz}, \mathrm{H}-6), 2.34-2.27$ (2H, m, H-4), 1.77-1.68 (2H, m, H-5), 1.28 (3H, t, $J=7.1 \mathrm{~Hz}$, $\left.\mathrm{OCH}_{2} \underline{\mathrm{CH}}_{3}\right)$.

(E)-Ethyl 6-oxohex-2-enoate (143) ${ }^{95}$<smiles>CCOC(=O)/C=C/CCC=O</smiles>

To a solution of oxalyl chloride $(820 \mu \mathrm{L}, 9.74 \mathrm{mmol})$ in $\mathrm{CH}_{2} \mathrm{Cl}_{2}(10.0 \mathrm{~mL})$ at $-78^{\circ} \mathrm{C}$ was slowly added DMSO (830 $\mu \mathrm{L}, 11.7 \mathrm{mmol})$. After $30 \mathrm{~min}$, a solution of alcohol (768 mg, $4.87 \mathrm{mmol})$ in $\mathrm{CH}_{2} \mathrm{Cl}_{2}(10.0 \mathrm{~mL})$ was added, dropwise. After $1 \mathrm{~h}, \mathrm{Et}_{3} \mathrm{~N}$ (3.36 mL, 24.1 mmol) was added and the slurry was allowed to attain rt. After $1 \mathrm{~h}$, a saturated $\mathrm{NH}_{4} \mathrm{Cl}$ (20.0 mL) solution was added and the layers were separated. The aqueous phase was extracted with $\mathrm{CH}_{2} \mathrm{Cl}_{2}(3 \times 30 \mathrm{~mL})$ and the combined organic extracts were washed with $\mathrm{H}_{2} \mathrm{O}(50 \mathrm{~mL})$, brine $(50 \mathrm{~mL})$, dried $\left(\mathrm{MgSO}_{4}\right)$, filtered and concentrated under reduced pressure. Purification by flash column chromatography $\left(5 \% \mathrm{Et}_{2} \mathrm{O} / \mathrm{Pet}\right.$. Ether) provided aldehyde 143 (660 mg, 87\%) as a colourless oil.

$\boldsymbol{R}_{\boldsymbol{f}} 0.31$ (15\% EtOAc/Hexanes); ${ }^{1} \mathbf{H}$ NMR $\left(300 \mathrm{MHz}, \mathrm{CDCl}_{3}\right) \delta 9.80(1 \mathrm{H}, \mathrm{d}, J=1.0 \mathrm{~Hz}$, H-6), 6.94 (1H, dt, $J=15.7,6.6 \mathrm{~Hz}, \mathrm{H}-3), 5.85$ (1H, dt, $J=15.7,1.5 \mathrm{~Hz}, \mathrm{H}-2), 4.18$ (2H, q, 
$\left.J=7.1 \mathrm{~Hz}, \mathrm{OCH}_{2} \mathrm{CH}_{3}\right), 2.67-2.62(2 \mathrm{H}, \mathrm{m}, \mathrm{H}-4), 2.57-2.52(2 \mathrm{H}, \mathrm{m}, \mathrm{H}-5), 1.28(3 \mathrm{H}, \mathrm{t}, J=$ $\left.7.1 \mathrm{~Hz}, \mathrm{OCH}_{2} \underline{\mathrm{C}}_{3}\right)$.

$(S)-4-((S)-1-((4-M e t h o x y b e n z y l) o x y) p e n t-4-e n-1-y l)-2,2-d i m e t h y l-1,3-d i o x a n-5-o n e$ (148)

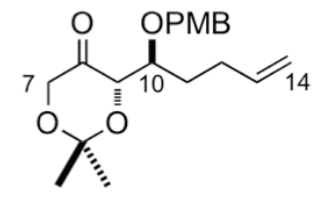

To a solution of alcohol 86 (0.84 g, $3.96 \mathrm{mmol})$ and PMBTCA (1.67 g, $5.94 \mathrm{mmol})$ in toluene $(10.0 \mathrm{~mL})$ at $0{ }^{\circ} \mathrm{C}$ was added lanthanum(III)triflate $(116 \mathrm{mg}, 0.198 \mathrm{mmol})$. After 6 $\mathrm{h}$, cold hexane $(40.0 \mathrm{~mL})$ was added and the precipitate was filtered. The resulting mixture was concentrated under reduced pressure and purified by flash column chromatography (10\% EtOAc/Hexanes) to afford $148(1.02 \mathrm{~g}, 77 \%)$ as a colourless oil.

$\boldsymbol{R}_{f} 0.51\left(20 \%\right.$ EtOAc/Pet. Ether); $[\alpha]_{\mathrm{D}}^{20}-9.3\left(c 0.6, \mathrm{CHCl}_{3}\right)$; IR $\boldsymbol{v}_{\max }\left(\right.$ thin film $\left./ \mathrm{cm}^{-1}\right) 3074$, 3033, 2997, 2935, 2855, 2836, 1723, 1613, 1585, 1526, 1464, 1247, 1035, 821; ${ }^{1}$ H NMR $\left(300 \mathrm{MHz}, \mathrm{CDCl}_{3}\right) \delta 7.27(2 \mathrm{H}, \mathrm{d}, J=9.2 \mathrm{~Hz}, \mathrm{Ar}-\underline{\mathrm{H}}), 6.87(2 \mathrm{H}, \mathrm{d}, J=8.7 \mathrm{~Hz}, \mathrm{Ar}-\underline{\mathrm{H}}), 5.77$ (1H, dddd, $J=17.1,10.1,7.1,6.2 \mathrm{~Hz}, \mathrm{H}-13), 5.03-4.93(2 \mathrm{H}, \mathrm{m}, \mathrm{H}-14), 4.61(1 \mathrm{H}, \mathrm{d}$, $\left.J=11.2 \mathrm{~Hz}, \underline{\mathrm{C}}_{\mathrm{a}} \mathrm{H}_{\mathrm{b}} \mathrm{Ar}\right), 4.50\left(1 \mathrm{H}, \mathrm{d}, J=11.2 \mathrm{~Hz}, \mathrm{CH}_{\mathrm{a}} \underline{\mathrm{H}}_{\mathrm{b}} \mathrm{Ar}\right), 4.42(1 \mathrm{H}, \mathrm{dd}, J=3.1,1.5 \mathrm{~Hz}$, H-9), 4.23 (1H, dd, $J=16.7,1.5$ Hz, H-7a), 3.96 (1H, d, $J=16.7$ Hz, H-7b), 3.88 (1H, dt, $J=9.4,3.2 \mathrm{~Hz}, \mathrm{H}-10), 3.80$ (3H, s, Ar-OCH 3$), 2.29-2.16(1 \mathrm{H}, \mathrm{m}, \mathrm{H}-12 \mathrm{a}), 2.12-1.98(1 \mathrm{H}$, m, H-12b), 1.86 (1H, m, H-11a), $1.46\left(3 \mathrm{H}, \mathrm{s}, \mathrm{C}-\mathrm{CH}_{3}\right), 1.45\left(3 \mathrm{H}, \mathrm{s}, \mathrm{C}-\mathrm{CH}_{3}\right), 1.50-1.39(1 \mathrm{H}$, m, H-11b); ${ }^{13}$ C NMR (75 MHz; $\left.\mathrm{CDCl}_{3}\right) \delta 208.8,159.7,138.7,130.7,130.1,129.8,115.3$, 114.2, 101.2, 76.3, 72.6, 67.5, 55.7, 30.4, 30.0, 24.7, 23.7; HRMS (ES ${ }^{+}$calcd. for $\mathrm{C}_{19} \mathrm{H}_{28} \mathrm{O}_{5}[\mathrm{M}+\mathrm{H}]^{+}$335.1853, found 335.1855. 


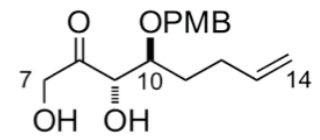

To a solution of acetonide $148(280 \mathrm{mg}, 0.854 \mathrm{mmol})$ in $\mathrm{MeOH}(8.5 \mathrm{~mL})$ at $\mathrm{rt}$ was added p-toluenesulfonic acid monohydrate $(16.0 \mathrm{mg}, 0.085 \mathrm{mmol})$. After $3.5 \mathrm{~h}$ of stirring, $\mathrm{Et}_{3} \mathrm{~N}$ $(0.36 \mathrm{~mL}, 2.56 \mathrm{mmol})$ was added and the mixture was concentrated under reduced pressure. The resulting residue was purified by flash column chromatography $(30 \%$ EtOAc/Hexanes) to give diol $\mathbf{1 5 0}$ (234 $\mathrm{mg}, 92 \%)$ as a colourless oil.

$\boldsymbol{R}_{\boldsymbol{f}} 0.11\left(30 \%\right.$ EtOAc/Hexanes); $[\alpha]_{\mathrm{D}}^{20}+3.7$ (c 3.7, $\left.\mathrm{CHCl}_{3}\right)$; IR v $\max \left(\right.$ thin film $\left./ \mathrm{cm}^{-1}\right) 3446$, 2936, 2839, 1717, 1627, 1586, 1516, 1249, 1029, 823; ${ }^{1} \mathbf{H}$ NMR $\left(300 \mathrm{MHz}, \mathrm{CDCl}_{3}\right) \delta 7.24$ $(2 \mathrm{H}, \mathrm{d}, J=8.8 \mathrm{~Hz}, \mathrm{Ar}-\underline{\mathrm{H}}), 6.90(2 \mathrm{H}, \mathrm{d}, J=8.7 \mathrm{~Hz}, \operatorname{Ar}-\underline{\mathrm{H}}), 5.77$ (1H, dddd, $J=17.14$, 10.16, 7.02, 6.23 Hz, H-13), 5.07-4.95 (2H, m, H-14), 4.54-4.31 (5H, m, $\underline{\mathrm{C}}_{\mathrm{a}} \underline{\mathrm{H}}_{\mathrm{b}} \mathrm{Ar}, \mathrm{H}-7$, $\mathrm{H}-9), 3.81$ (3H, s, Ar-OCH $\left.\underline{H}_{3}\right), 3.67$ (1H, ddd, $\left.J=7.9,4.9,3.9 \mathrm{~Hz}, \mathrm{H}-10\right), 3.04$ (1H, dd, $J=$ $5.8,4.8 \mathrm{~Hz}, \mathrm{C} 7-\mathrm{OH}), 2.83$ (1H, d, $J=4.8 \mathrm{~Hz}, \mathrm{C} 9-\mathrm{O} \underline{\mathrm{H}}), 2.25-2.06$ (2H, m, H-12), 1.88-1.75 (1H, m, H-11a), 1.44-1.54 (1H, m, H-11b); ${ }^{13} \mathbf{C}$ NMR (75 MHz, $\left.\mathrm{CDCl}_{3}\right) \delta 211.8,159.8$, 137.9, 129.8, 129.4, 115.5, 114.2, 79.8, 75.9, 71.9, 67.8, 55.5, 29.2, 29.0; HRMS $\left(\mathrm{ES}^{+}\right)$ calcd. for $\mathrm{C}_{16} \mathrm{H}_{26} \mathrm{NO}_{5}\left[\mathrm{M}+\mathrm{NH}_{4}\right]^{+} 312.1805$, found 312.1809 .

(2S,3S)-Methyl 2-hydroxy-3-((4-methoxybenzyl)oxy)hept-6-enoate (149)<smiles>C=CCCC(O)C(O)C(=O)OC</smiles>

To a solution of diol $150(190 \mathrm{mg}, 0.645 \mathrm{mmol})$ in $\mathrm{THF} / \mathrm{H}_{2} \mathrm{O}(2: 1,20.0 \mathrm{~mL}, 0.033 \mathrm{M})$, at rt was added $\mathrm{H}_{5} \mathrm{IO}_{6}(220 \mathrm{mg}, 0.968 \mathrm{mmol})$. After $24 \mathrm{~h}, \mathrm{Et}_{2} \mathrm{O}(20.0 \mathrm{~mL})$ was added and the layers were separated and the aqueous phase was extracted with $\mathrm{Et}_{2} \mathrm{O}(3 \times 30 \mathrm{~mL})$. The combined organic extracts were washed with water $(20 \mathrm{~mL})$, brine $(20 \mathrm{~mL})$, dried $\left(\mathrm{Na}_{2} \mathrm{SO}_{4}\right)$, filtered and concentrated under reduced pressure.

To a suspension of the resulting residue and $\mathrm{K}_{2} \mathrm{CO}_{3}(110 \mathrm{mg}, 0.774 \mathrm{mmol})$ in DMF (8.0 $\mathrm{mL})$ at $\mathrm{rt}$ was added MeI $(50 \mu \mathrm{L}, 0.774 \mathrm{mmol})$. After $1 \mathrm{~h}$, water $(10.0 \mathrm{~mL})$ was added, and the reaction mixture was extracted with $\mathrm{Et}_{2} \mathrm{O}(3 \times 30 \mathrm{~mL})$. The combined organic layers 
were washed with water $(20 \mathrm{~mL})$, brine $(20 \mathrm{~mL})$, dried $\left(\mathrm{Na}_{2} \mathrm{SO}_{4}\right)$, filtered and concentrated under reduced pressure. The residue was purified by flash column chromatography $(10 \%$ EtOAc/Hexanes) to give methyl ester 149 (120 mg, 64\%).

$\boldsymbol{R}_{\boldsymbol{f}} 0.8\left(30 \%\right.$ EtOAc/Hexanes); $[\alpha]_{\mathrm{D}}^{20}-2.5$ (c $\left.2.5 \mathrm{CHCl}_{3}\right) ;$ IR $\boldsymbol{v}_{\max }\left(\right.$ thin film $\left./ \mathrm{cm}^{-1}\right) 3480$, 2999, 2953, 2838, 1739, 1613, 1520, 1441, 1249, 1174, 1095, 1034, 821; ${ }^{1}$ H NMR (400 $\left.\mathrm{MHz}_{\mathrm{CDCl}}\right) \delta 7.26(2 \mathrm{H}, \mathrm{d}, J=8.8 \mathrm{~Hz}, \mathrm{Ar}-\underline{\mathrm{H}}), 6.88(2 \mathrm{H}, \mathrm{d}, J=8.8 \mathrm{~Hz}, \mathrm{Ar}-\underline{\mathrm{H}}), 5.76(1 \mathrm{H}$, dddd, $J=17.0,10.4,6.8,6.4 \mathrm{~Hz}, \mathrm{H}-13), 5.03-4.94(2 \mathrm{H}, \mathrm{m}, \mathrm{H}-14), 4.61(1 \mathrm{H}, \mathrm{d}, J=11.3$ $\left.\mathrm{Hz}, \underline{\mathrm{CH}}_{\mathrm{a}} \mathrm{H}_{\mathrm{b}} \mathrm{Ar}\right), 4.51\left(1 \mathrm{H}, \mathrm{d}, J=11.3 \mathrm{~Hz}, \mathrm{CH}_{\mathrm{a}} \underline{\mathrm{H}}_{\mathrm{b}} \mathrm{Ar}\right), 4.39(1 \mathrm{H}, \mathrm{dd}, J=5.5,3.0 \mathrm{~Hz}, \mathrm{H}-9)$, $3.81\left(3 \mathrm{H}, \mathrm{s}, \mathrm{Ar}-\mathrm{OCH}_{3}\right), 3.80\left(3 \mathrm{H}, \mathrm{s}, \mathrm{C} 8-\mathrm{OCH}_{3}\right), 3.71(1 \mathrm{H}, \mathrm{ddd}, J=9.0,4.0,3.0 \mathrm{~Hz}, \mathrm{H}-10)$, $2.85(1 \mathrm{H}, \mathrm{d}, J=5.6 \mathrm{~Hz}, \mathrm{C} 9-\mathrm{O} \underline{\mathrm{H}}), 2.27-2.14$ (1H, m, H-12a), 2.13-1.99 (1H, m, H-12b), 1.89-1.74 (1H, m, H-11a), 1.52-1.40 (1H, m, H-11b); ${ }^{13} \mathbf{C}$ NMR (100 MHz, $\left.\mathrm{CDCl}_{3}\right)$ $\delta 173.2,159.4,138.1,130.3,129.7,115.2,114.0,79.9,72.6,72.3,55.4,52.7,29.9,29.6$; HRMS $\left(\mathrm{ES}^{+}\right)$calcd. for $\mathrm{C}_{16} \mathrm{H}_{26} \mathrm{NO}_{5}\left[\mathrm{M}+\mathrm{NH}_{4}\right]^{+}$312.1805, found 312.1808.

(2S,3S)-Methyl-2-((tert-butyldimethylsilyl)oxy)-3-((4-methoxybenzyl)oxy)hept-6enoate (153)

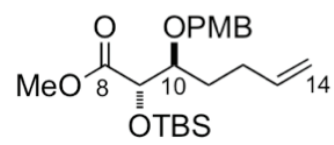

To a solution of alcohol $149(140 \mathrm{mg}, 0.476 \mathrm{mmol})$ in $\mathrm{CH}_{2} \mathrm{Cl}_{2}(5.0 \mathrm{~mL})$ at $-78{ }^{\circ} \mathrm{C}$ was added 2,6-lutidine $(111 \mu \mathrm{L}, 0.952 \mathrm{mmol})$ and TBSOTf $(164 \mu \mathrm{L}, 0.714 \mathrm{mmol})$. After $2 \mathrm{~h}$, saturated $\mathrm{NH}_{4} \mathrm{Cl}(5.0 \mathrm{~mL})$ solution and $\mathrm{CH}_{2} \mathrm{Cl}_{2}(5.0 \mathrm{~mL})$ were added and the layers were separated. The aqueous phase was extracted with $\mathrm{CH}_{2} \mathrm{Cl}_{2}(3 \times 10 \mathrm{~mL})$ and the combined organic extracts were dried $\left(\mathrm{Na}_{2} \mathrm{SO}_{4}\right)$, filtered and concentrated under reduced pressure. Purification by flash column chromatography (5-10\% EtOAc/Hexanes) provided TBS ether 153 (190 mg, 98\%) as a colourless oil.

$\boldsymbol{R}_{f} 0.16\left(20 \%\right.$ EtOAc/Hexanes); $[\alpha]_{\mathrm{D}}^{20}-10.2\left(c\right.$ c $\left.0.5, \mathrm{CHCl}_{3}\right) ; \mathbf{I R} \boldsymbol{v}_{\max }\left(\right.$ thin film $\left./ \mathrm{cm}^{-1}\right) 2954$, 2930, 2858, 1756, 1730, 1514, 1275, 1153, 1122, 837, 779; ${ }^{1} \mathbf{H}$ NMR (400 MHz, $\left.\mathrm{CDCl}_{3}\right) \delta$ $7.24(2 \mathrm{H}, \mathrm{d}, J=8.8 \mathrm{~Hz}, \mathrm{Ar}-\underline{\mathrm{H}}), 6.86(2 \mathrm{H}, \mathrm{d}, J=8.8 \mathrm{~Hz}, \mathrm{Ar}-\underline{\mathrm{H}}), 5.82-5.72$ (1H, m, H-13), 5.01-4.92 (2H, m, H-14), $4.57\left(1 \mathrm{H}, \mathrm{d}, J=11.0 \mathrm{~Hz}, \mathrm{C}_{\mathrm{a}} \mathrm{H}_{\mathrm{b}} \mathrm{Ar}\right), 4.44(1 \mathrm{H}, \mathrm{d}, J=11.0 \mathrm{~Hz}$, $\left.\mathrm{CH}_{\mathrm{a}} \underline{\mathrm{H}}_{\mathrm{b}} \mathrm{Ar}\right), 4.30(1 \mathrm{H}, \mathrm{d}, J=4.6 \mathrm{~Hz}, \mathrm{H}-9), 3.80\left(3 \mathrm{H}, \mathrm{s}, \mathrm{Ar}-\mathrm{OC}_{3}\right), 3.72\left(3 \mathrm{H}, \mathrm{s}, \mathrm{C} 8-\mathrm{OC}_{3}\right)$, $3.68(1 \mathrm{H}, \mathrm{ddd}, J=8.3,4.6,3.7 \mathrm{~Hz}, \mathrm{H}-10), 2.24-2.14(1 \mathrm{H}, \mathrm{m}, \mathrm{H}-12 \mathrm{a}), 2.12-2.02(1 \mathrm{H}, \mathrm{m}$, 
H-12b), 1.80-1.70 (1H, m, H-11a), 1.65-1.56 (1H, m, H-11b), 0.91 (9H, s, Si-C(C $\left.\left.\underline{C H}_{3}\right)_{3}\right)$, $0.07\left(6 \mathrm{H}, \mathrm{s}, \mathrm{Si}-\left(\mathrm{CH}_{3}\right)_{2}\right) ;{ }^{13} \mathrm{C}$ NMR $\left(100 \mathrm{MHz}, \mathrm{CDCl}_{3}\right) \delta 172.9,159.3,138.5,130.6,129.7$, 114.9, 113.8, 80.5, 74.3, 72.2, 55.4, 51.9, 30.0, 29.6, 25.8, 18.4, -4.97, -5.08; HRMS (ES $\left.{ }^{+}\right)$ calcd. for $\mathrm{C}_{22} \mathrm{H}_{40} \mathrm{NO}_{5} \mathrm{Si}\left[\mathrm{M}+\mathrm{NH}_{4}\right]^{+} 426.2670$, found 426.2667.

(2S,3S)-Methyl-2-((tert-butyldimethylsilyl)oxy)-7-hydroxy-3-((4-methoxybenzyl)oxy)heptanoate (154)

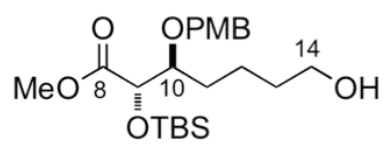

To a solution of thexylBH $\mathrm{BH}_{2}$ in THF $(4.0 \mathrm{~mL}, 0.5 \mathrm{M}, 2.0 \mathrm{mmol})$ at $0{ }^{\circ} \mathrm{C}$ was added olefin $153(200 \mathrm{mg}, 0.489 \mathrm{mmol})$ in THF $(2.0 \mathrm{~mL})$ dropwise. After $1 \mathrm{~h} \mathrm{NaOH}(2.0 \mathrm{~mL}, 10 \% \mathrm{w} / \mathrm{v})$ and $\mathrm{H}_{2} \mathrm{O}_{2}(2 \mathrm{~mL}, 30 \% \mathrm{v} / \mathrm{v})$ were added. The mixture was stirred for $30 \mathrm{~min}$ and the aqueous layer was saturated with anhydrous $\mathrm{K}_{2} \mathrm{CO}_{3}$. The organics were extracted with $\mathrm{CH}_{2} \mathrm{Cl}_{2}(3 \times 10 \mathrm{~mL})$, washed with brine $(30 \mathrm{~mL})$, dried $\left(\mathrm{Na}_{2} \mathrm{SO}_{4}\right)$, filtered and concentrated under reduced pressure. The residue was purified by flash column chromatography $(10 \%$ EtOAc/Hexane) to give alcohol $154(200 \mathrm{mg}, 96 \%)$ as a colourless oil.

$\boldsymbol{R}_{f} 0.38\left(20 \%\right.$ EtOAc/Hexane); $[\alpha]_{\mathrm{D}}^{20}-11.8\left(c \quad 0.8, \mathrm{CHCl}_{3}\right) ; \mathbf{I R} \boldsymbol{v}_{\max }\left(\right.$ thin film $\left./ \mathrm{cm}^{-1}\right) 3446$, 2951, 2930, 2857, 1750, 1514, 1247, 1207, 1155, 1035, 837, 779; ${ }^{1} \mathbf{H}$ NMR (400 MHz, $\left.\mathrm{CDCl}_{3}\right) \delta 7.23(2 \mathrm{H}, \mathrm{d}, J=8.7 \mathrm{~Hz}, \mathrm{Ar}-\underline{\mathrm{H}}), 6.85(2 \mathrm{H}, \mathrm{d}, J=8.7 \mathrm{~Hz}, \mathrm{Ar}-\underline{\mathrm{H}}), 4.57(1 \mathrm{H}, \mathrm{d}, J=$ $\left.11.1 \mathrm{~Hz}, \underline{\mathrm{C}}_{\mathrm{a}} \mathrm{H}_{\mathrm{b}} \mathrm{Ar}\right), 4.43\left(1 \mathrm{H}, \mathrm{d}, J=11.1 \mathrm{~Hz}, \mathrm{CH}_{\mathrm{a}} \underline{\mathrm{H}}_{\mathrm{b}} \mathrm{Ar}\right), 4.30(1 \mathrm{H}, \mathrm{d}, J=4.5 \mathrm{~Hz}, \mathrm{H}-9)$, $3.79\left(3 \mathrm{H}, \mathrm{s}, \mathrm{Ar}-\mathrm{OCH}_{3}\right), 3.71\left(3 \mathrm{H}, \mathrm{s}, \mathrm{C} 8-\mathrm{OC} \underline{H}_{3}\right), 3.67-3.63(1 \mathrm{H}, \mathrm{m}, \mathrm{H}-10), 3.59(2 \mathrm{H}, \mathrm{t}, J=$ 6.3 Hz, H-14), 1.72-1.60 (2H, m, H-11a, H-13a), 1.57-1.46 (2H, m, H-11b, H-13b), 1.391.29 (2H, m, H-12), 0.90 (9H, s, Si-C $\left.\left(\underline{\mathrm{CH}}_{3}\right)_{3}\right), 0.06\left(6 \mathrm{H}, \mathrm{s}, 2 \times \mathrm{Si}_{-} \underline{\mathrm{H}}_{3}\right) ;{ }^{13} \mathbf{C}$ NMR (100 $\left.\mathrm{MHz}, \mathrm{CDCl}_{3}\right) \delta 173.0,159.3,130.5,129.7,113.8,80.9,74.3,72.3,63.0,55.4,52.0,32.8$, $30.4,25.8,21.6,18.4,-4.95,-5.08$; HRMS $\left(\mathrm{ES}^{+}\right)$calcd. for $\mathrm{C}_{22} \mathrm{H}_{42} \mathrm{NO}_{6} \mathrm{Si}\left[\mathrm{M}+\mathrm{NH}_{4}\right]^{+}$ 444.2776, found 444.2779. 
(2S,3S)-Methyl-2,7-bis((tert-butyldimethylsilyl)oxy)-3-((4-methoxybenzyl)oxy)heptanoate (155)

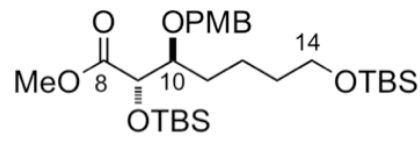

To a solution of TBSCl $(85.0 \mathrm{mg}, 0.564 \mathrm{mmol})$ and imidazole $(40.0 \mathrm{mg}, 0.587 \mathrm{mmol})$ in DMF (1.0 mL) at $0{ }^{\circ} \mathrm{C}$ was added a solution of alcohol $154(200 \mathrm{mg}, 0.468 \mathrm{mmol})$ in DMF $(2.0 \mathrm{~mL})$. After $12 \mathrm{~h}$, a saturated $\mathrm{NH}_{4} \mathrm{Cl}(5.0 \mathrm{~mL})$ solution was added and the layers were separated. The aqueous phase was extracted with $\mathrm{CH}_{2} \mathrm{Cl}_{2}(3 \times 5 \mathrm{~mL})$. The combined organic extracts were washed with $\mathrm{H}_{2} \mathrm{O}(10 \mathrm{~mL})$, brine $(10 \mathrm{~mL})$, dried $\left(\mathrm{Na}_{2} \mathrm{SO}_{4}\right)$, filtered and concentrated under reduced pressure. Purification by flash column chromatography (2\% EtOAc/Hexanes) provided bis-TBS-ether 155 (230 mg, 92\%) as a colourless oil.

$\boldsymbol{R}_{\boldsymbol{f}} 0.72\left(20 \%\right.$ EtOAc/Hexane); $[\alpha]_{\mathrm{D}}^{20}-11.8\left(c 0.8, \mathrm{CHCl}_{3}\right)$; IR $\boldsymbol{v}_{\max }\left(\right.$ thin film $\left./ \mathrm{cm}^{-1}\right) 2951$, 2929, 2896, 2857, 1756, 1514, 1249, 1203, 1150, 1103, 820, 776; ${ }^{1} \mathbf{H}$ NMR (400 MHz, $\left.\mathrm{CDCl}_{3}\right) \delta 7.23(2 \mathrm{H}, \mathrm{d}, J=8.7 \mathrm{~Hz}, \mathrm{Ar}-\underline{\mathrm{H}}), 6.85(2 \mathrm{H}, \mathrm{d}, J=8.7 \mathrm{~Hz}, \mathrm{Ar}-\underline{\mathrm{H}}), 4.55(1 \mathrm{H}, \mathrm{d}, J=$ $\left.11.0 \mathrm{~Hz}, \underline{\mathrm{C}}_{\mathrm{a}} \mathrm{H}_{\mathrm{b}} \mathrm{Ar}\right), 4.44\left(1 \mathrm{H}, \mathrm{d}, J=11.0 \mathrm{~Hz}, \mathrm{CH}_{\mathrm{a}} \underline{\mathrm{H}}_{\mathrm{b}} \mathrm{Ar}\right), 4.28(1 \mathrm{H}, \mathrm{d}, J=4.8 \mathrm{~Hz}, \mathrm{H}-9)$, $3.79\left(3 \mathrm{H}, \mathrm{s}, \mathrm{Ar}-\mathrm{OCH}_{3}\right), 3.71\left(3 \mathrm{H}, \mathrm{s}, \mathrm{C} 8-\mathrm{OCH}_{3}\right), 3.65(1 \mathrm{H}, \mathrm{ddd}, J=8.0,4.6,3.5 \mathrm{~Hz}, \mathrm{H}-10)$, 3.57 (2H, t, $J=6.3 \mathrm{~Hz}, \mathrm{H}-14), 1.68-1.57$ (1H, m, H-11a), 1.52-1.44 (3H, m, H-11b, H-12), 1.37-1.25 (2H, m, H-13), 0.90 (9H, s, Si-C $\left.\left(\mathrm{C}_{3}\right)_{3}\right), 0.89$ (9H, s, Si-C $\left.\left(\mathrm{CH}_{3}\right)_{3}\right), 0.06(6 \mathrm{H}, \mathrm{s}, 2$ $\left.\times \mathrm{Si}-\underline{\mathrm{C}}_{3}\right), 0.04\left(6 \mathrm{H}, \mathrm{s}, 2 \times \mathrm{Si}-\underline{\mathrm{H}}_{3}\right) ;{ }^{13} \mathbf{C} \mathbf{~ N M R}\left(100 \mathrm{MHz}, \mathrm{CDCl}_{3}\right) \delta 173.0,159.3,130.7$, $129.7,113.8,81.2,74.4,72.3,63.2,55.4,51.9,33.1,30.6,26.1,25.8,21.8,18.5,18.4,-4.9$, $-5.08,-5.12$; HRMS $\left(\mathrm{ES}^{+}\right)$calcd. for $\mathrm{C}_{28} \mathrm{H}_{56} \mathrm{NO}_{6} \mathrm{Si}_{2}\left[\mathrm{M}+\mathrm{NH}_{4}\right]^{+}$558.3641, found 558.3635.

(2S,3S)-2,7-Bis((tert-butyldimethylsilyl)oxy)- $N$-methoxy-3-((4-methoxybenzyl)oxy)- $N$ methylheptanamide (156)

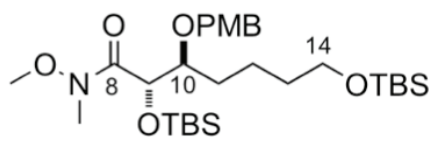

To a solution of methylester $155(0.851 \mathrm{~g}, 1.57 \mathrm{mmol})$ and $\mathrm{N}, \mathrm{O}$-dimethyl hydroxylamine hydrochloride $(0.228 \mathrm{~g}, 2.35 \mathrm{mmol})$ in THF $(50.0 \mathrm{~mL})$ at $-30{ }^{\circ} \mathrm{C}$ was slowly added $i$ - $\mathrm{PrMgCl}(3.54 \mathrm{~mL}, 2 \mathrm{M}$ soln. in THF, $7.08 \mathrm{mmol})$. The reaction mixture was allowed to 
attain $0{ }^{\circ} \mathrm{C}$. After $6 \mathrm{~h}$ a saturated $\mathrm{NH}_{4} \mathrm{Cl}(50.0 \mathrm{~mL})$ solution was added and the layers were separated. The aqueous phase was extracted with $\mathrm{Et}_{2} \mathrm{O}(3 \times 30 \mathrm{~mL})$ and the combined organic extracts were dried $\left(\mathrm{Na}_{2} \mathrm{SO}_{4}\right)$, filtered and concentrated under reduced pressure. Purification by flash column chromatography $(10 \%$ EtOAc/Hexanes) provided Weinreb amide 156 (870 mg, 97\%) as a colourless oil.

$\boldsymbol{R}_{\boldsymbol{f}} 0.57\left(20 \%\right.$ EtOAc/Hexane); $[\alpha]_{\mathrm{D}}^{20}-6.6\left(c \quad 0.6, \mathrm{CHCl}_{3}\right) ; \mathbf{I R} \boldsymbol{v}_{\max }\left(\right.$ thin film $\left./ \mathrm{cm}^{-1}\right) 2950$, 2928, 2856, 1670, 1514, 1250, 1203, 1148, 1103, 823, 775; ${ }^{1} \mathbf{H}$ NMR $\left(300 \mathrm{MHz}, \mathrm{CDCl}_{3}\right) \delta$ $7.19(2 \mathrm{H}, \mathrm{d}, J=8.8 \mathrm{~Hz}, \mathrm{Ar}-\underline{\mathrm{H}}), 6.82(2 \mathrm{H}, \mathrm{d}, J=8.8 \mathrm{~Hz}, \operatorname{Ar}-\underline{\mathrm{H}}), 4.63-4.51$ (1H, m, H-9), $4.43\left(1 \mathrm{H}, \mathrm{d}, J=10.7 \mathrm{~Hz}, \underline{\mathrm{C}}_{\mathrm{a}} \mathrm{H}_{\mathrm{b}} \mathrm{Ar}\right), 4.41\left(1 \mathrm{H}, \mathrm{d}, J=10.7 \mathrm{~Hz}, \mathrm{CH}_{\mathrm{a}} \underline{\mathrm{H}}_{\mathrm{b}} \mathrm{Ar}\right), 3.78(3 \mathrm{H}, \mathrm{s}, \mathrm{Ar}-$ $\left.\mathrm{OCH}_{3}\right), 3.70(1 \mathrm{H}, \mathrm{td}, J=7.4,3.2 \mathrm{~Hz}, \mathrm{H}-10), 3.62-3.59$ (5H, m, N-OCH$\left.\underline{H}_{3}, \mathrm{H}-14\right), 3.21$ (3H, s, N-C $\left.\underline{H}_{3}\right), 1.79-1.65$ (1H, m, H-11a), 1.62-1.45 (4H, m, H-11b, H-12, H-13a), 1.44-1.37 (1H, m, H-13b), 0.89 (9H, s, Si-C $\left.\left(\mathrm{C}_{3}\right)_{3}\right), 0.88\left(9 \mathrm{H}, \mathrm{s}, \mathrm{Si}-\mathrm{C}\left(\mathrm{C}_{3}\right)_{3}\right), 0.06\left(3 \mathrm{H}, \mathrm{s}, \mathrm{Si}-\underline{\mathrm{C}}_{3}\right)$, $0.05\left(3 \mathrm{H}, \mathrm{s}, \mathrm{Si}-\mathrm{CH}_{3}\right), 0.04\left(6 \mathrm{H}, \mathrm{s}, 2 \times \mathrm{Si}_{-} \underline{\mathrm{H}}_{3}\right) ;{ }^{13} \mathbf{C} \mathbf{N M R}\left(75 \mathrm{MHz}, \mathrm{CDCl}_{3}\right) \delta 159.2,130.9$, $129.7,113.7,80.8,73.0,63.4,55.4,33.3,31.1,26.1,25.9,21.4,18.5,18.3,-4.7,-4.97$, -5.11; HRMS $\left(\mathrm{ES}^{+}\right)$calcd. for $\mathrm{C}_{29} \mathrm{H}_{56} \mathrm{O}_{6} \mathrm{NSi}_{2}[\mathrm{M}+\mathrm{H}]^{+}$570.3641, found 570.3638 .

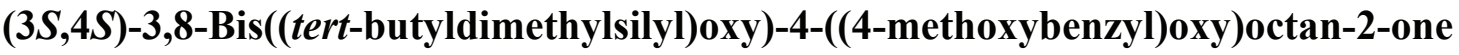
(144)

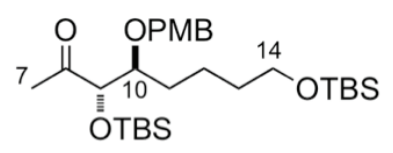

To a solution of Weinreb amide $156(848 \mathrm{mg}, 1.56 \mathrm{mmol})$ in THF $(20.0 \mathrm{~mL})$ at $0{ }^{\circ} \mathrm{C}$ was added $\mathrm{MeMgBr}(2.1 \mathrm{~mL}, 3.0 \mathrm{M}$ soln. in THF, $6.25 \mathrm{mmol})$. The reaction mixture was stirred at $0{ }^{\circ} \mathrm{C}$ for $1 \mathrm{~h}$ and quenched with saturated $\mathrm{NH}_{4} \mathrm{Cl}(20.0 \mathrm{~mL})$ solution. The reaction mixture was allowed to warm to $\mathrm{rt}$ and $\mathrm{H}_{2} \mathrm{O}(5.0 \mathrm{~mL})$ was added to dissolve the precipitate, and the layers were separated. The aqueous phase was extracted with $\mathrm{Et}_{2} \mathrm{O}(3 \times 20 \mathrm{~mL})$ and the combined organic extracts were dried $\left(\mathrm{Na}_{2} \mathrm{SO}_{4}\right)$, filtered and concentrated under reduced pressure. Purification of the residue by flash column chromatography (5\% EtOAc/Hexanes) afforded product $144(785 \mathrm{mg}, 96 \%)$ as a colourless oil.

$\boldsymbol{R}_{\boldsymbol{f}} 0.75\left(20 \%\right.$ EtOAc/Hexanes); $[\alpha]_{\mathrm{D}}^{20}-9.7$ (c $\left.0.8, \mathrm{CHCl}_{3}\right) ; \mathbf{I R} \boldsymbol{v}_{\max }\left(\right.$ thin film $\left./ \mathrm{cm}^{-1}\right) 2954$, 2856, 1714, 1514, 1249, 1209, 1149, 1102, 837, 776; ${ }^{1} \mathbf{H}$ NMR (400 MHz, $\left.\mathrm{CDCl}_{3}\right) \delta 7.25$ $(2 \mathrm{H}, \mathrm{d}, J=8.6 \mathrm{~Hz}, \mathrm{Ar}-\underline{\mathrm{H}}), 6.86(2 \mathrm{H}, \mathrm{d}, J=8.5 \mathrm{~Hz}, \operatorname{Ar}-\underline{\mathrm{H}}), 4.61(1 \mathrm{H}, \mathrm{d}, J=11.2 \mathrm{~Hz}$, 
$\left.\mathrm{C}_{\mathrm{a}} \mathrm{H}_{\mathrm{b}} \mathrm{Ar}\right), 4.45\left(1 \mathrm{H}, \mathrm{d}, J=11.1 \mathrm{~Hz}, \mathrm{CH}_{\mathrm{a}} \underline{\mathrm{H}}_{\mathrm{b}} \mathrm{Ar}\right), 4.10(1 \mathrm{H}, \mathrm{d}, J=3.5 \mathrm{~Hz}, \mathrm{H}-9), 3.80(3 \mathrm{H}, \mathrm{s}$, Ar-OCH$\left.\underline{H}_{3}\right), 3.57$ (3H, dd, $J=7.9,4.5$ Hz, H-10, H-14), 2.19 (3H, s, H-7), 1.64-1.56 (1H, m, H-11a), 1.50-1.42 (3H, m, H-11b, H-13), 1.35-1.22 (2H, m, H-12), 0.93 (9H, s, Si$\left.\mathrm{C}\left(\mathrm{CH}_{3}\right)_{3}\right), 0.88\left(9 \mathrm{H}, \mathrm{s}, \mathrm{Si}-\mathrm{C}\left(\mathrm{CH}_{3}\right)_{3}\right), 0.08\left(3 \mathrm{H}, \mathrm{s}, \mathrm{Si}-\mathrm{C}_{3}\right), 0.05\left(3 \mathrm{H}, \mathrm{s}, \mathrm{Si}-\mathrm{CH}_{3}\right), 0.03(6 \mathrm{H}, \mathrm{s}$, $\left.2 \times \mathrm{Si}-\underline{\mathrm{H}}_{3}\right) .{ }^{13} \mathrm{C} \mathbf{N M R}\left(100 \mathrm{MHz}, \mathrm{CDCl}_{3}\right) \delta 211.8,159.3,130.5,129.6,113.8,81.8,80.7$, 72.4, 63.2, 55.4, 33.0, 30.4, 27.4, 26.1, 25.9, 22.0, 18.5, 18.3, -4.6, -5.0, -5.1; HRMS (ES $\left.{ }^{+}\right)$ calcd. for $\mathrm{C}_{28} \mathrm{H}_{56} \mathrm{NO}_{5} \mathrm{Si}_{2}\left[\mathrm{M}+\mathrm{NH}_{4}\right]^{+}$542.3692, found 542.3687 .

(9S,10S,E)-Ethyl-9,14-bis((tert-butyldimethylsilyl)oxy)-6-hydroxy-10-((4-methoxybenzyl)oxy)-8-oxotetradec-2-enoate (157)

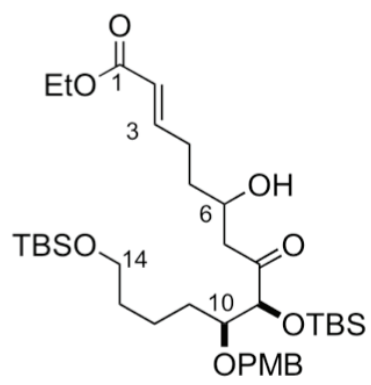

To a stirred solution of ketone $144(320 \mathrm{mg}, 0.610 \mathrm{mmol})$ in $\mathrm{Et}_{2} \mathrm{O}(15.0 \mathrm{~mL})$ at $-78{ }^{\circ} \mathrm{C}$ was added dicyclohexylboron chloride $(240 \mu \mathrm{L}, 1.09 \mathrm{mmol})$ and $\mathrm{Et}_{3} \mathrm{~N}(170 \mu \mathrm{L}, 1.21 \mathrm{mmol})$ and the reaction mixture was stirred for $0.5 \mathrm{~h}$. Aldehyde 143 (143 $\mathrm{mg}, 0.91 \mathrm{mmol})$ in $\mathrm{Et}_{2} \mathrm{O}$ $(5.0 \mathrm{~mL})$ was then added. After $2 \mathrm{~h}$ the reaction mixture was diluted with $\mathrm{MeOH}(3.0 \mathrm{~mL})$, phosphate buffer pH $7(3.0 \mathrm{~mL})$ and $\mathrm{H}_{2} \mathrm{O}_{2}(3 \mathrm{~mL}, 30 \% \mathrm{v} / \mathrm{v})$. The biphasic mixture was allowed to warm to RT and stirred vigorously for $10 \mathrm{~min}$. The layers were separated and the aqueous phase was extracted with $\mathrm{Et}_{2} \mathrm{O}(3 \times 10 \mathrm{~mL})$. The combined organic extracts were washed with brine $(20 \mathrm{~mL})$, dried $\left(\mathrm{NaSO}_{4}\right)$, filtered and concentrated under reduced pressure. Flash column chromatography (10\% EtOAc/Hexanes) gave aldol adduct 157 (373 mg, 90\%) as an inconsequential 1.6:1 mixture of diastereoisomers.

$\boldsymbol{R}_{\boldsymbol{f}} 0.48$ (20\% EtOAc/Hexanes); IR $\boldsymbol{v}_{\max }\left(\right.$ thin film $\left./ \mathrm{cm}^{-1}\right)$ 3513, 2954, 2856, 1720, 1653, 1514, 1250, 1172, 1100, 1040, 837, 778; ${ }^{1} \mathbf{H}$ NMR (400 MHz, $\left.\mathrm{CDCl}_{3}\right) \delta 7.22(2 \mathrm{H}, \mathrm{d}, J=$ $8.7 \mathrm{~Hz}, \mathrm{Ar}-\underline{\mathrm{H}}), 6.98-6.87$ (2H, m, H-3), $6.84(2 \mathrm{H}, \mathrm{d}, J=8.7 \mathrm{~Hz}, \mathrm{Ar}-\underline{\mathrm{H}}), 5.77-5.87$ (2H, m, $\mathrm{H}-2), 4.57\left(1 \mathrm{H}, \mathrm{d}, J=11.1 \mathrm{~Hz}, \mathrm{C}_{\mathrm{a}} \mathrm{H}_{\mathrm{b}} \mathrm{Ar}\right), 4.46\left(1 \mathrm{H}, \mathrm{d}, J=11.1 \mathrm{~Hz}, \mathrm{CH}_{\mathrm{a}} \underline{\mathrm{H}}_{\mathrm{b}} \mathrm{Ar}\right), 4.17(2 \mathrm{H}$, q, $\left.J=7.1 \mathrm{~Hz}, \mathrm{OC}_{2} \mathrm{CH}_{3}\right), 4.08(2 \mathrm{H}, \mathrm{d}, J=3.9 \mathrm{~Hz}, \mathrm{H}-9), 4.02-3.91(1 \mathrm{H}, \mathrm{m}, \mathrm{H}-6), 3.79(3 \mathrm{H}$, s, $\left.\mathrm{Ar}-\mathrm{OC} \underline{H}_{3}\right), 3.59-3.54$ (3H, m, H-10, H-14), 3.06 (1H, d, $\left.J=3.3 \mathrm{~Hz}, \mathrm{C} 6-\mathrm{O} \underline{\mathrm{H}}\right), 2.81-2.67$ 
(2H, m, H-7), 2.64-2.48 (2H, m, H-4), 2.32-2.19 (4H, m, H-5, H-11), 1.61-1.38 (4H, m, H12, H-13), 1.27 (3H, t, $\left.J=7.1 \mathrm{~Hz}, \mathrm{OCH}_{2} \underline{\mathrm{C}}_{3}\right), 0.92\left(9 \mathrm{H}, \mathrm{s}, \mathrm{Si}-\mathrm{C}\left(\mathrm{CH}_{3}\right)_{3}\right), 0.88$ (9H, s, Si$\left.\mathrm{C}\left(\mathrm{C}_{3}\right)_{3}\right), 0.07\left(6 \mathrm{H}, \mathrm{s}, 2 \times \mathrm{Si}-\underline{\mathrm{H}}_{3}\right), 0.02\left(6 \mathrm{H}, \mathrm{s}, 2 \times \mathrm{Si}-\underline{\mathrm{CH}}_{3}\right) ;{ }^{13} \mathbf{C}$ NMR $\left(75 \mathrm{MHz}, \mathrm{CDCl}_{3}\right)$ $\delta 214.3,166.7,159.4,148.7,130.2,129.7,121.8,113.9,81.9,80.5,72.5,66.9,63.1,60.3$, $55.4,45.9,34.9,32.9,30.3,28.3,26.1,25.9,21.9,18.5,18.2,14.4,-4.6,-4.9,-5.1$; HRMS $\left(\mathrm{ES}^{+}\right)$calcd. for $\mathrm{C}_{36} \mathrm{H}_{68} \mathrm{NO}_{8} \mathrm{Si}_{2}\left[\mathrm{M}+\mathrm{NH}_{4}\right]^{+}$698.4478, found 698.4477 .

(2E,6E,9S,10S)-Ethyl-9,14-bis((tert-butyldimethylsilyl)oxy)-10-((4-methoxybenzyl)oxy)-8-oxotetradeca-2,6-dienoate (142)

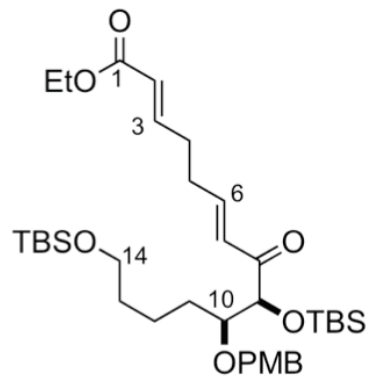

\section{Method A}

To a stirred solution of aldol product $157(350 \mathrm{mg}, 0.514 \mathrm{mmol})$ in $\mathrm{CH}_{2} \mathrm{Cl}_{2}(5.0 \mathrm{~mL})$ at -10 ${ }^{\circ} \mathrm{C}$ was added a solution of Martin's sulfurane $(518 \mathrm{mg}, 0.771 \mathrm{mmol})$ in $\mathrm{CH}_{2} \mathrm{Cl}_{2}(5.0 \mathrm{~mL})$. The resulting pale yellow mixture was stirred for $1 \mathrm{~h}$ and the residual solvent was removed under reduced pressure to yield the crude product as a pale yellow oil. Purification by flash column chromatography (5-10\% EtOAc/Hexanes) furnished the compound $\mathbf{1 4 2}$ as a colourless oil (320 mg, 95\%).

\section{Method B}

To a solution of aldol product $157(80 \mathrm{mg}, 0.117 \mathrm{mmol})$ in $\mathrm{CH}_{2} \mathrm{Cl}_{2}(2.0 \mathrm{~mL})$ at $0{ }^{\circ} \mathrm{C}$ was added $\mathrm{Et}_{3} \mathrm{~N}(40 \mu \mathrm{L}, 0.292 \mathrm{mmol})$ and mesyl chloride $(20 \mu \mathrm{L}, 0.258 \mathrm{mmol})$ and the reaction mixture was allowed to attained rt, DBU $(90 \mu \mathrm{L}, 0.585 \mathrm{mmol})$ was then added and the reaction was stirred for $16 \mathrm{~h}$ before quenching with saturated $\mathrm{NH}_{4} \mathrm{Cl}(5.0 \mathrm{~mL})$ solution. The layers were separated and the aqueous phase was extracted with $\mathrm{CH}_{2} \mathrm{Cl}_{2}(3 \times 10 \mathrm{~mL})$ and the combined organic extracts were washed with brine $(20 \mathrm{~mL})$, dried $\left(\mathrm{Na}_{2} \mathrm{SO}_{4}\right)$, filtered and concentrated under reduced pressure. The resulting residue was purified by 
flash column chromatography (5-10\% EtOAc/Hexanes) to give product $142(46.0 \mathrm{mg}$, $62 \%)$ as a colourless oil.

$\boldsymbol{R}_{\boldsymbol{f}} 0.65\left(20 \%\right.$ EtOAc/Hexane); $[\alpha]_{\mathrm{D}}^{20}-10.0$ (c 3.0, $\left.\mathrm{CHCl}_{3}\right)$; IR $\boldsymbol{v}_{\max }\left(\right.$ thin film $\left./ \mathrm{cm}^{-1}\right) 2954$, 2929, 2856, 1724, 1514, 1250, 1172, 1098, 1040, 837, 775; ${ }^{1} \mathbf{H}$ NMR (400 MHz, $\left.\mathrm{CDCl}_{3}\right) \delta$ $7.23(2 \mathrm{H}, \mathrm{d}, J=8.5 \mathrm{~Hz}, \mathrm{Ar}-\underline{\mathrm{H}}), 6.93(2 \mathrm{H}, \mathrm{dt}, J=15.7,6.4 \mathrm{~Hz}, \mathrm{H}-3, \mathrm{H}-6), 6.85$ (2H, d, $J=$ 8.6 Hz, Ar- $\underline{\mathrm{H}}), 6.66$ (1H, d, $J=15.8 \mathrm{~Hz}, \mathrm{H}-7), 5.84(1 \mathrm{H}, \mathrm{d}, J=15.7 \mathrm{~Hz}, \mathrm{H}-2), 4.57$ (1H, d, $\left.J=11.1 \mathrm{~Hz}, \mathrm{CH}_{\mathrm{a}} \mathrm{H}_{\mathrm{b}} \mathrm{Ar}\right), 4.44\left(1 \mathrm{H}, \mathrm{d}, J=11.2 \mathrm{~Hz}, \mathrm{CH}_{\mathrm{a}} \underline{\mathrm{H}}_{\mathrm{b}} \mathrm{Ar}\right), 4.23(1 \mathrm{H}, \mathrm{d}, J=4.0 \mathrm{~Hz}, \mathrm{H}-9)$, $4.18\left(2 \mathrm{H}, \mathrm{q}, J=7.1 \mathrm{~Hz}, \mathrm{OC}_{2} \mathrm{CH}_{3}\right), 3.80\left(3 \mathrm{H}, \mathrm{s}, \mathrm{Ar}-\mathrm{OC} \underline{H}_{3}\right), 3.56(3 \mathrm{H}, \mathrm{m}, \mathrm{H}-10, \mathrm{H}-14)$, 2.41-2.30 (4H, m, H-4, H-5), 1.65-1.54 (1H, m, H-11a), 1.52-1.41 (4H, m, H-11b, H-12, H-13a), 1.28 (4H, t, $\left.J=7.1 \mathrm{~Hz}, \mathrm{OCH}_{2} \underline{\mathrm{C}}_{3}, \mathrm{H}-13 \mathrm{~b}\right), 0.92$ (9H, s, Si-C(C $\left.\left.\underline{\mathrm{H}}_{3}\right)_{3}\right), 0.89$ (9H, s, $\left.\mathrm{Si}-\mathrm{C}\left(\mathrm{C}_{3}\right)_{3}\right), 0.07\left(3 \mathrm{H}, \mathrm{s}, \mathrm{Si}-\mathrm{C}_{3}\right), 0.03\left(6 \mathrm{H}, \mathrm{s}, 2 \times \mathrm{Si}-\underline{\mathrm{C}}_{3}\right), 0.02$ (3H, s, Si-C $\left.\underline{\mathrm{H}}_{3}\right)$; ${ }^{13}$ C NMR (100 MHz, $\left.\mathrm{CDCl}_{3}\right) \delta 200.7,166.4,159.2,147.1,145.8,130.5,129.7,126.5$, $122.4,113.8,81.6,79.9,72.3,63.2,60.4,55.4,33.0,31.0,30.5,26.1,25.9,22.0,18.5$, 18.3, 14.4, -4.7, -4.9, -5.1; HRMS $\left(\mathrm{ES}^{+}\right)$calcd. for $\mathrm{C}_{36} \mathrm{H}_{66} \mathrm{NO}_{7} \mathrm{Si}_{2}\left[\mathrm{M}+\mathrm{NH}_{4}\right]^{+}$680.4372, found 680.4370 .

(2E,6E,8S,9R,10S)-9,14-bis((tert-butyldimethylsilyl)oxy)-8-hydroxy-10-((4methoxybenzyl)oxy)tetradeca-2,6-dienoate (163)

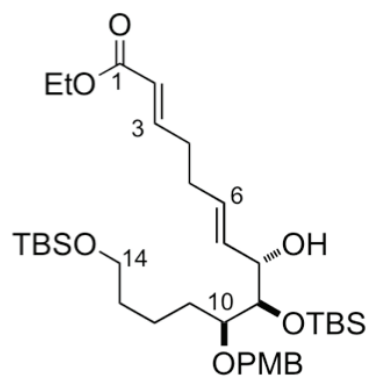

To a solution of enone $142(480 \mathrm{mg}, 0.724 \mathrm{mmol})$ in $\mathrm{MeOH}(20.0 \mathrm{~mL})$ was added $\mathrm{CeCl}_{3} \cdot 7 \mathrm{H}_{2} \mathrm{O}$ (405 mg, $1.08 \mathrm{mmol}$ ) before reaction mixture was cooled to $-78{ }^{\circ} \mathrm{C}$ and $\mathrm{NaBH}_{4}$ (33 mg, $0.868 \mathrm{mmol}$ ) was added. The reaction was stirred for $1 \mathrm{~h}$ before quenching with saturated $\mathrm{NaHCO}_{3}(20.0 \mathrm{~mL})$ solution and the layers were separated. The aqueous phase was extracted with $\mathrm{Et}_{2} \mathrm{O}(3 \times 10 \mathrm{~mL})$ and the combined organic extracts were washed with brine $(20 \mathrm{~mL})$, dried $\left(\mathrm{Na}_{2} \mathrm{SO}_{4}\right)$, filtered and concentrated under reduced pressure. The crude product was purified by flash column chromatography $(10 \%$ EtOAc/Hexanes) to give allylic alcohol 163 (445 mg, 90\%) as a colourless oil. 
$\boldsymbol{R}_{\boldsymbol{f}} 0.56$ (20\% EtOAc/Hexane); $[\alpha]_{\mathrm{D}}^{20}-4.1$ (c 1.1, $\left.\mathrm{CHCl}_{3}\right)$; IR $\boldsymbol{v}_{\max }\left(\right.$ thin film $\left./ \mathrm{cm}^{-1}\right) 3251$, 2926, 2847, 1810, 1719, 1513, 1302, 1250, 1205, 1148, 833, 777; ${ }^{1}$ H NMR (500 MHz, $\left.\mathrm{CDCl}_{3}\right) \delta 7.24(2 \mathrm{H}, \mathrm{d}, J=8.6 \mathrm{~Hz}, \mathrm{Ar}-\underline{\mathrm{H}}), 6.97(1 \mathrm{H}, \mathrm{dt}, J=15.6,6.6 \mathrm{~Hz}, \mathrm{H}-3), 6.86(2 \mathrm{H}, \mathrm{d}$, $J=8.7 \mathrm{~Hz}, \mathrm{Ar}-\underline{\mathrm{H}}), 5.84(1 \mathrm{H}, \mathrm{dt}, J=15.6,1.4 \mathrm{~Hz}, \mathrm{H}-2), 5.68-5.74(1 \mathrm{H}, \mathrm{m}, \mathrm{H}-6), 5.54(1 \mathrm{H}$, dd, $J=15.5,5.7 \mathrm{~Hz}, \mathrm{H}-7), 4.49\left(1 \mathrm{H}, \mathrm{d}, J=10.7 \mathrm{~Hz}, \mathrm{CH}_{\mathrm{a}} \mathrm{H}_{\mathrm{b}} \mathrm{Ar}\right), 4.44(1 \mathrm{H}, \mathrm{d}, J=10.8 \mathrm{~Hz}$, $\left.\mathrm{CH}_{\mathrm{a}} \underline{\mathrm{H}}_{\mathrm{b}} \mathrm{Ar}\right), 4.17\left(2 \mathrm{H}, \mathrm{q}, J=7.1 \mathrm{~Hz}, \mathrm{OC}_{2} \mathrm{CH}_{3}\right), 4.10-4.14(1 \mathrm{H}, \mathrm{m}, \mathrm{H}-8), 3.79$ (3H, s, Ar$\left.\mathrm{OCH}_{3}\right), 3.66(1 \mathrm{H}, \mathrm{dd}, J=4.9,4.2 \mathrm{~Hz}, \mathrm{H}-9), 3.60(2 \mathrm{H}, \mathrm{t}, J=6.2 \mathrm{~Hz}, \mathrm{H}-14), 3.48(1 \mathrm{H}, \mathrm{m}$, H-10), 3.25 (1H, d, $J=6.2 \mathrm{~Hz}, \mathrm{C} 8-\mathrm{OH}), 2.31-2.27$ (2H, m, H-4), 2.24-2.21 (2H, m, H-5), 1.64-1.54 (2H, m, H-11), 1.54-1.45 (3H, m, H-13, H-12a), 1.41-1.32 (1H, m, H-12b), 1.28 $\left(3 \mathrm{H}, \mathrm{t}, J=7.1 \mathrm{~Hz}, \mathrm{OCH}_{2} \underline{\mathrm{C}}_{3}\right), 0.90\left(9 \mathrm{H}, \mathrm{s}, \mathrm{Si}-\mathrm{C}\left(\mathrm{C}_{3}\right)_{3}\right), 0.89\left(9 \mathrm{H}, \mathrm{s}, \mathrm{Si}-\mathrm{C}\left(\mathrm{C}_{3}\right)_{3}\right), 0.09$ $\left(3 \mathrm{H}, \mathrm{s}, \mathrm{Si}-\mathrm{CH}_{3}\right), 0.07\left(3 \mathrm{H}, \mathrm{s}, \mathrm{Si}-\mathrm{CH}_{3}\right), 0.05\left(6 \mathrm{H}, \mathrm{s}, 2 \times \mathrm{Si}_{-} \underline{\mathrm{H}}_{3}\right) ;{ }^{13} \mathbf{C}$ NMR (125 MHz, $\left.\mathrm{CDCl}_{3}\right) \delta 166.7,159.4,148.4,131.1,130.8,130.3,129.7,121.9,113.9,81.1,75.4,73.3$, $72.2,63.3,60.3,55.4,33.2,31.9,31.0,30.2,26.13,26.1,21.6,18.5,18.3,14.4,-4.0,-4.3$, -5.1; HRMS $\left(\mathrm{ES}^{+}\right)$calcd. for $\mathrm{C}_{36} \mathrm{H}_{66} \mathrm{NO}_{7} \mathrm{Si}_{2}\left[\mathrm{M}+\mathrm{NH}_{4}\right]^{+}$680.4372, found 680.4370 .

\section{(R)-Mosher ester of 163 (164)}

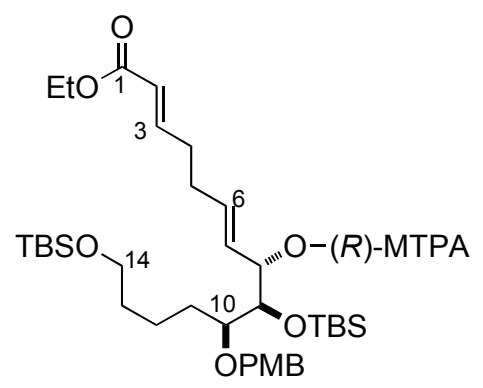

To a stirred mixture of alcohol $163(10.0 \mathrm{mg}, 15 \mu \mathrm{mol})$, DCC (13.0 mg, $61 \mu \mathrm{mol})$ and DMAP $(3.7 \mathrm{mg}, 31 \mu \mathrm{mol})$ in $\mathrm{CH}_{2} \mathrm{Cl}_{2}(1.0 \mathrm{~mL})$ at $\mathrm{rt}$ was added $(+)-(R)$ - $\alpha$-methoxy- $\alpha$ (trifluoromethyl)-phenylacetic acid $(7.0 \mathrm{mg}, 30 \mu \mathrm{mol})$ in one portion. After $16 \mathrm{~h}$, the crude reaction mixture was purified directly by flash column chromatography $(5 \%$ EtOAc/Hexanes) to give ( $R$ )-MTPA ester $164(12.0 \mathrm{mg}, 92 \%)$ as a colourless oil.

$\boldsymbol{R}_{\boldsymbol{f}} 0.66$ (20\% EtOAc/Hexanes); ${ }^{1} \mathbf{H}$ NMR (400 MHz, $\left.\mathrm{CDCl}_{3}\right) \delta$ 7.56-7.54 (2H, m, Ph- $\left.\underline{\mathrm{H}}\right)$, $7.36(3 \mathrm{H}, \mathrm{m}, \mathrm{Ph}-\underline{\mathrm{H}}), 7.21(2 \mathrm{H}, \mathrm{d}, J=8.6 \mathrm{~Hz}, \mathrm{Ar}-\underline{\mathrm{H}}), 6.92(1 \mathrm{H}, \mathrm{dt}, J=15.7,6.3 \mathrm{~Hz}, \mathrm{H}-3)$, $6.85(2 \mathrm{H}, \mathrm{d}, J=8.6 \mathrm{~Hz}, \mathrm{Ar}-\underline{\mathrm{H}}), 5.81(1 \mathrm{H}, \mathrm{dd}, J=15.8,1.3 \mathrm{~Hz}, \mathrm{H}-2), 5.82-5.74(1 \mathrm{H}, \mathrm{m}, \mathrm{H}-$ 6), $5.55(1 \mathrm{H}, \mathrm{d}, J=1.5 \mathrm{~Hz}, \mathrm{H}-8), 5.58-5.50(1 \mathrm{H}, \mathrm{m}, \mathrm{H}-7), 4.27(1 \mathrm{H}, \mathrm{d}, J=10.7 \mathrm{~Hz}$, $\left.\mathrm{C}_{\mathrm{a}} \mathrm{H}_{\mathrm{b}} \mathrm{Ar}\right), 4.22\left(1 \mathrm{H}, \mathrm{d}, J=10.7 \mathrm{~Hz}, \mathrm{CH}_{\mathrm{a}} \underline{\mathrm{H}}_{\mathrm{b}} \mathrm{Ar}\right), 4.17\left(2 \mathrm{H}, \mathrm{q}, J=7.1 \mathrm{~Hz}, \mathrm{OCH}_{2} \mathrm{CH}_{3}\right), 3.80$ 
$\left(3 \mathrm{H}, \mathrm{s}, \mathrm{Ar}-\mathrm{OCH}_{3}\right), 3.76(1 \mathrm{H}, \mathrm{td}, J=3.3,1.7 \mathrm{~Hz}, \mathrm{H}-9), 3.57-3.54$ (2H, m, H-14), 3.54 (3H, $\left.\mathrm{s}, \mathrm{OC}_{3}\right), 3.25(1 \mathrm{H}, \mathrm{q}, J=5.3 \mathrm{~Hz}, \mathrm{H}-10), 2.26-2.18(4 \mathrm{H}, \mathrm{m}, \mathrm{H}-4, \mathrm{H}-5), 1.52-1.45$ (2 H, m, H-11), 1.46-1.39 (2H, m, H-13), 1.27 (3H, t, $\left.J=7.1 \mathrm{~Hz}, \mathrm{OCH}_{2} \underline{\mathrm{H}}_{3}\right), 1.20-1.26$ (2H, m, H12), 0.89 (9H, s, Si-C( $\left.\left.\mathrm{CH}_{3}\right)_{3}\right), 0.85\left(9 \mathrm{H}, \mathrm{s}, \mathrm{Si}-\mathrm{C}\left(\mathrm{CH}_{3}\right)_{3}\right), 0.04$ (3H, s, Si-C $\left.\underline{H}_{3}\right), 0.04(6 \mathrm{H}, \mathrm{s}$, $\left.2 \times \mathrm{Si}_{-} \underline{\mathrm{H}}_{3}\right), 0.00\left(3 \mathrm{H}, \mathrm{s}, \mathrm{Si}-\mathrm{C}_{3}\right)$.

\section{(S)-Mosher ester of 163 (165)}

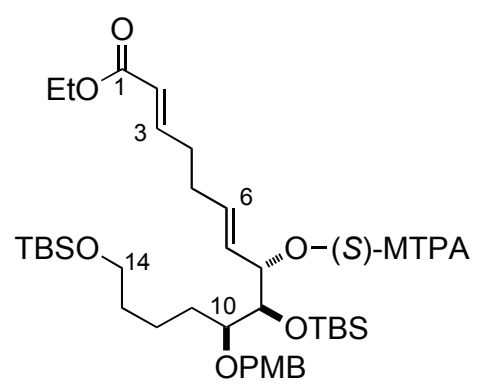

To a stirred mixture of alcohol $163(5.0 \mathrm{mg}, 7.7 \mu \mathrm{mol})$, DCC $(6.3 \mathrm{mg}, 31 \mu \mathrm{mol})$ and DMAP $(1.8 \mathrm{mg}, 15 \mu \mathrm{mol})$ in $\mathrm{CH}_{2} \mathrm{Cl}_{2}(1.0 \mathrm{~mL})$ at $\mathrm{rt}$ was added $(+)-(S)$ - $\alpha$-methoxy- $\alpha-$ (trifluoromethyl)-phenylacetic acid $(3.5 \mathrm{mg}, 15 \mu \mathrm{mol})$ in one portion. After $16 \mathrm{~h}$, the crude reaction mixture was purified directly by flash column chromatography $(5 \%$ EtOAc/Hexanes) to give (S)-MTPA ester 165 (5.0 mg, 75\%).

$\boldsymbol{R}_{f} 0.66$ (20\% EtOAc/Hexanes); ${ }^{1} \mathbf{H}$ NMR $\left(500 \mathrm{MHz}, \mathrm{CDCl}_{3}\right) \delta 7.54(2 \mathrm{H}, \mathrm{d}, J=8.3 \mathrm{~Hz}, \mathrm{Ph}-$ $\underline{\mathrm{H}}), 7.40-7.34(3 \mathrm{H}, \mathrm{m}, \mathrm{Ph}-\underline{\mathrm{H}}), 7.22(2 \mathrm{H}, \mathrm{d}, J=8.7 \mathrm{~Hz}, \mathrm{Ar}-\underline{\mathrm{H}}), 6.91(1 \mathrm{H}, \mathrm{dt}, J=15.7,6.5$ Hz, H-3), 6.84 (2H, d, $J=8.7 \mathrm{~Hz}, \mathrm{Ar}-\underline{\mathrm{H}}), 5.81(1 \mathrm{H}, \mathrm{d}, J=15.7 \mathrm{~Hz}, \mathrm{H}-2), 5.67$ (1H, dt, $J=$ 15.4, 6.3 Hz, H-6), 5.60 (1H, dd, $J=7.5,2.9 \mathrm{~Hz}, \mathrm{H}-8), 5.44$ (1H, dd, $J=16.0,7.4 \mathrm{~Hz}, \mathrm{H}-$ 7), $4.31\left(1 \mathrm{H}, \mathrm{d}, J=10.7 \mathrm{~Hz}, \mathrm{CH}_{\mathrm{a}} \mathrm{H}_{\mathrm{b}} \mathrm{Ar}\right), 4.29\left(1 \mathrm{H}, \mathrm{d}, J=10.7 \mathrm{~Hz}, \mathrm{CH}_{\mathrm{a}} \underline{\mathrm{H}}_{\mathrm{b}} \mathrm{Ar}\right), 4.17$ (2H, q, $\left.J=7.1 \mathrm{~Hz}, \mathrm{OCH}_{2} \mathrm{CH}_{3}\right), 3.79\left(3 \mathrm{H}, \mathrm{s}, \mathrm{Ar}-\mathrm{OCH}_{3}\right), 3.79-3.76(1 \mathrm{H}, \mathrm{m}, \mathrm{H}-9), 3.57(3 \mathrm{H}, \mathrm{t}, J=$ $6.2 \mathrm{~Hz}, \mathrm{H}-14), 3.50\left(3 \mathrm{H}, \mathrm{s}, \mathrm{OCH}_{3}\right), 3.31(1 \mathrm{H}, \mathrm{td}, J=5.9,3.5 \mathrm{~Hz}, \mathrm{H}-10), 2.24-2.19(2 \mathrm{H}, \mathrm{m}$, $\mathrm{H}-4), 2.19-2.13(2 \mathrm{H}, \mathrm{m}, \mathrm{H}-5), 1.51-1.42(2 \mathrm{H}, \mathrm{m}, \mathrm{H}-11), 1.27(3 \mathrm{H}, \mathrm{t}, J=7.2 \mathrm{~Hz}$, $\left.\mathrm{OCH}_{2} \mathrm{CH}_{3}\right), 1.21-1.27(4 \mathrm{H}, \mathrm{m}, \mathrm{H}-12, \mathrm{H}-13), 0.89\left(9 \mathrm{H}, \mathrm{s}, \mathrm{Si}-\mathrm{C}\left(\mathrm{CH}_{3}\right)_{3}\right), 0.85$ (9H, s, Si$\left.\mathrm{C}\left(\mathrm{CH}_{3}\right)_{3}\right), 0.05\left(3 \mathrm{H}, \mathrm{s}, \mathrm{Si}-\left(\mathrm{CH}_{3}\right)_{2}\right), 0.04\left(6 \mathrm{H}, \mathrm{s}, 2 \times \mathrm{Si}_{-} \mathrm{CH}_{3}\right), 0.02\left(3 \mathrm{H}, \mathrm{s}, \mathrm{Si}-\left(\mathrm{CH}_{3}\right)_{2}\right)$. 


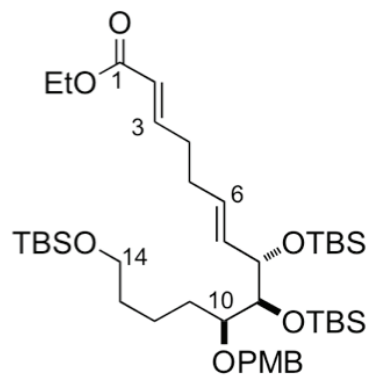

To a solution of alcohol $163(450 \mathrm{mg}, 0.677 \mathrm{mmol})$ in $\mathrm{CH}_{2} \mathrm{Cl}_{2}(10.0 \mathrm{~mL})$ at $-78{ }^{\circ} \mathrm{C}$ was added 2,6-lutidine $(160 \mu \mathrm{L}, 1.35 \mathrm{mmol})$ and TBSOTf $(233 \mu \mathrm{L}, 1.01 \mathrm{mmol})$. After $4 \mathrm{~h}$, saturated $\mathrm{NH}_{4} \mathrm{Cl}(20.0 \mathrm{~mL})$ solution and $\mathrm{CH}_{2} \mathrm{Cl}_{2}(20.0 \mathrm{~mL})$ were added. The aqueous layer was extracted with $\mathrm{CH}_{2} \mathrm{Cl}_{2}(3 \times 20 \mathrm{~mL})$ and the combined organic extracts were dried $\left(\mathrm{Na}_{2} \mathrm{SO}_{4}\right)$, filtered and concentrated under reduced pressure. Purification by flash column chromatography (5\% EtOAc/Hexanes) provided TBS ether 166 (460 g, 87\%) as a colourless oil.

$\boldsymbol{R}_{\boldsymbol{f}} 0.9\left(20 \%\right.$ EtOAc/Hexanes); $[\alpha]_{\mathrm{D}}^{20}-16.3$ (c 1.4, $\left.\mathrm{CHCl}_{3}\right)$; IR $\boldsymbol{v}_{\max }\left(\right.$ thin film/ $\left./ \mathrm{cm}^{-1}\right)$ 2952, 2933, 2893, 2858, 1723, 1512, 1466, 1252, 1101, 1043, 836, 777; ${ }^{1} \mathbf{H}$ NMR (300 MHz, $\left.\mathrm{CDCl}_{3}\right) \delta 7.25(2 \mathrm{H}, \mathrm{d}, J=8.7 \mathrm{~Hz}, \mathrm{Ar}-\underline{\mathrm{H}}), 6.95(1 \mathrm{H}, \mathrm{dt}, J=15.7,6.5 \mathrm{~Hz}, \mathrm{H}-3), 6.85(2 \mathrm{H}, \mathrm{d}$, $J=8.8 \mathrm{~Hz}, \mathrm{Ar}-\underline{\mathrm{H}}), 5.82$ (1H, dt, $J=15.7,1.4 \mathrm{~Hz}, \mathrm{H}-2), 5.58-5.56$ (2H, m, H-6, H-7), 4.52 $\left(1 \mathrm{H}, \mathrm{d}, J=11.1 \mathrm{~Hz}, \underline{\mathrm{CH}}_{\mathrm{a}} \mathrm{H}_{\mathrm{b}} \mathrm{Ar}\right), 4.25\left(1 \mathrm{H}, \mathrm{d}, J=11.1 \mathrm{~Hz}, \mathrm{CH}_{\mathrm{a}} \underline{\mathrm{H}}_{\mathrm{b}} \mathrm{Ar}\right), 4.18(2 \mathrm{H}, \mathrm{q}, J=7.1$ $\left.\mathrm{Hz}, \mathrm{OC}_{2} \mathrm{CH}_{3}\right), 4.11-4.08(1 \mathrm{H}, \mathrm{m}, \mathrm{H}-8), 3.87(1 \mathrm{H}, \mathrm{dd}, J=4.9,1.5 \mathrm{~Hz}, \mathrm{H}-9), 3.80(3 \mathrm{H}, \mathrm{s}$, Ar-OC$\left.\underline{H}_{3}\right), 3.55$ (2H, t, $J=6.5$ Hz, H-14), 3.45-3.42 (1H, m, H-10), 2.28-2.19 (4H, m, H-4, H-5), 1.47-1.42 (4H, m, H-11, H-13), 1.28 (3H, t, $\left.J=7.1 \mathrm{~Hz}, \mathrm{OCH}_{2} \mathrm{CH}_{3}\right), 1.26-1.16(2 \mathrm{H}$, m, H-12), 0.91 (9H, s, Si-C(C $\left.\left.\underline{\mathrm{H}}_{3}\right)_{3}\right), 0.90\left(9 \mathrm{H}, \mathrm{s}, \mathrm{Si}-\mathrm{C}\left(\mathrm{C}_{3}\right)_{3}\right), 0.88\left(9 \mathrm{H}, \mathrm{s}, \mathrm{Si}-\mathrm{C}\left(\mathrm{C}_{3}\right)_{3}\right)$, $0.07\left(3 \mathrm{H}, \mathrm{s}, \mathrm{Si}-\mathrm{CH}_{3}\right), 0.07$ (3H, s, Si-C $\left.\underline{H}_{3}\right), 0.06\left(3 \mathrm{H}, \mathrm{s}, \mathrm{Si}-\mathrm{CH}_{3}\right), 0.04\left(3 \mathrm{H}, \mathrm{s}, \mathrm{Si}-\mathrm{CH}_{3}\right), 0.03$ $\left(6 \mathrm{H}, \mathrm{s}, 2 \times \mathrm{Si}-\underline{\mathrm{H}}_{3}\right) ;{ }^{13} \mathrm{C}$ NMR $\left(75 \mathrm{MHz} ; \mathrm{CDCl}_{3}\right) \delta 166.7,159.0,148.4,131.4,130.9$, $129.4,128.9,121.9,113.7,79.7,74.7,71.3,63.6,60.3,55.4,33.3,32.1,31.0,30.9,26.14$, 26.11, 26.06, 22.7, 18.5, 18.4, 18.3, 14.4, -4.2, -4.3, -4.5, -4.6, -5.09, -5.11; HRMS $\left(\mathrm{ES}^{+}\right)$ calcd. for $\mathrm{C}_{42} \mathrm{H}_{82} \mathrm{NO}_{7} \mathrm{Si}_{3}\left[\mathrm{M}+\mathrm{NH}_{4}\right]^{+}$796.5394, found 796.5394 . 
(2E,6E,8S,9S,10S)-Ethyl 8,9,14-tris((tert-butyldimethylsilyl)oxy)-10-

hydroxytetradeca-2,6-dienoate (167)

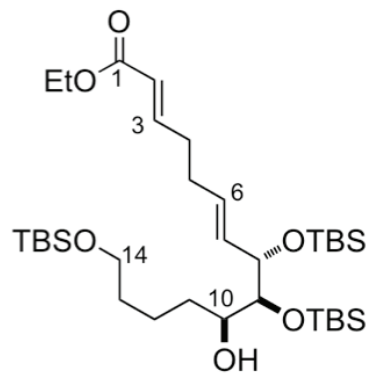

To a solution of PMB ether $166(150 \mathrm{mg}, 0.192 \mathrm{mmol})$ in $\mathrm{CH}_{2} \mathrm{Cl}_{2}(2.0 \mathrm{~mL})$ and phosphate buffer $\mathrm{pH} 7(0.1 \mathrm{~mL})$ at $0{ }^{\circ} \mathrm{C}$ was added DDQ $(65.0 \mathrm{mg}, 0.288 \mathrm{mmol})$. The reaction mixture was stirred for $1 \mathrm{~h}$ before quenching with saturated $\mathrm{NaHCO}_{3}(5.0 \mathrm{~mL})$ solution and the layers were separated. The aqueous phase was extracted with $\mathrm{CH}_{2} \mathrm{Cl}_{2}(3 \times 10 \mathrm{~mL})$, washed with brine $(20 \mathrm{~mL})$, dried $\left(\mathrm{Na}_{2} \mathrm{SO}_{4}\right)$, filtered and concentrated under reduced pressure. Purification by flash column chromatography (5\% EtOAc/Hexanes) provided alcohol 167 (110 mg, $88 \%$ yield) as a colourless oil.

$\boldsymbol{R}_{f} 0.76\left(15 \%\right.$ EtOAc/Hexanes); $[\alpha]_{\mathrm{D}}^{20}-24.4$ (c 1.0, $\left.\mathrm{CHCl}_{3}\right) ; \mathbf{I R} \boldsymbol{v}_{\max }\left(\right.$ thin film $\left./ \mathrm{cm}^{-1}\right) 3418$, 2921, 2847, 1719, 1277, 1126, 835, 766; ${ }^{1} \mathbf{H}$ NMR $\left(300 \mathrm{MHz}, \mathrm{CDCl}_{3}\right) \delta$ 7.00-6.90 (1H, m, H-3), 5.86-5.80 (1H, m, H-2), 5.69-5.67 (2H, m, H-6, H-7), 4.31-4.27 (1H, m, H-8), 4.17 $\left(2 \mathrm{H}, \mathrm{q}, J=7.1 \mathrm{~Hz}, \mathrm{OC}_{2} \mathrm{CH}_{3}\right), 3.91(1 \mathrm{H}, \mathrm{s}, \mathrm{C} 10-\mathrm{O} \underline{\mathrm{H}}), 3.63-3.57$ (3H, m, H-10, H-14), 3.40 $(1 \mathrm{H}, \mathrm{dd}, J=8.5,4.2 \mathrm{~Hz}, \mathrm{H}-9), 2.31-2.26$ (4H, m, H-4, H-5), 1.57-1.51 (4H, m, H-12, H13), 1.35-1.27 (2H, m, H-11), $1.27\left(3 \mathrm{H}, \mathrm{t}, J=7.1 \mathrm{~Hz}, \mathrm{OCH}_{2} \mathrm{CH}_{3}\right), 0.90$ (9H, s, Si$\left.\mathrm{C}\left(\mathrm{CH}_{3}\right)_{3}\right), 0.88\left(9 \mathrm{H}, \mathrm{s}, \mathrm{Si}-\mathrm{C}\left(\mathrm{CH}_{3}\right)_{3}\right), 0.87\left(9 \mathrm{H}, \mathrm{s}, \mathrm{Si}-\mathrm{C}\left(\mathrm{CH}_{3}\right)_{3}\right), 0.09\left(3 \mathrm{H}, \mathrm{s}, \mathrm{Si}-\mathrm{CH}_{3}\right), 0.08$ $\left(6 \mathrm{H}, \mathrm{s}, 2 \times \mathrm{Si}_{-} \underline{\mathrm{H}}_{3}\right), 0.05\left(3 \mathrm{H}, \mathrm{s}, \mathrm{Si}-\mathrm{CH}_{3}\right), 0.03\left(6 \mathrm{H}, \mathrm{s}, 2 \times \mathrm{Si}_{-} \underline{\mathrm{H}}_{3}\right) ;{ }^{13} \mathrm{C}$ NMR $(75 \mathrm{MHz}$, $\left.\mathrm{CDCl}_{3}\right) \delta 166.7,148.3,130.8,128.6,122.0,76.7,75.5,72.6,63.6,60.3,33.8,33.3,32.1$, $30.9,26.2,25.95,25.91,21.5,18.6,18.2,18.1,14.4,-4.0,-4.5,-4.6,-4.9,-5.1$; HRMS $\left(\mathrm{ES}^{+}\right)$calcd. for $\mathrm{C}_{34} \mathrm{H}_{74} \mathrm{O}_{6} \mathrm{NSi}_{3}\left[\mathrm{M}+\mathrm{NH}_{4}\right]^{+}$676.4818, found 676.4802 . 


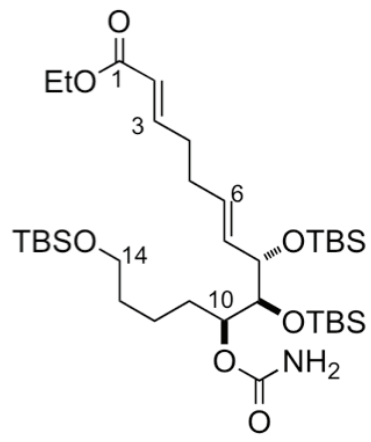

To a stirred solution of alcohol $167(45.0 \mathrm{mg}, 0.683 \mu \mathrm{mol})$ in $\mathrm{CH}_{2} \mathrm{Cl}_{2}(4.0 \mathrm{~mL})$ was added trichloroacetyl isocyanate $(16.3 \mu \mathrm{L}, 0.136 \mathrm{mmol})$ at $0{ }^{\circ} \mathrm{C}$ and the reaction was stirred at $\mathrm{rt}$ for $1 \mathrm{~h} . \mathrm{CH}_{2} \mathrm{Cl}_{2}(2.0 \mathrm{~mL})$ and an excess amount of $\mathrm{Al}_{2} \mathrm{O}_{3}$ was added and the resulting suspension was stirred overnight. The suspension was filtered, concentrated under reduced pressure and purified by flash column chromatography (10-20\% EtOAc/Hexanes) to give carbamate 168 (34.0 $\mathrm{mg}, 72 \%)$ as a colourless oil.

$\boldsymbol{R}_{\boldsymbol{f}} 0.29$ (10\% EtOAc/Hexanes); $[\alpha]_{\mathrm{D}}^{20}-18.5\left(c \quad 0.8, \mathrm{CHCl}_{3}\right) ; \mathbf{I R} \mathbf{v}_{\max }\left(\right.$ thin film $\left./ \mathrm{cm}^{-1}\right) 3276$, 3138, 2926, 2852, 1723, 1283, 1150, 977, 835, 775; ${ }^{1} \mathbf{H}$ NMR $\left(400 \mathrm{MHz}, \mathrm{CDCl}_{3}\right) \delta 6.95$ (1H, dt, $J=15.6,6.5 \mathrm{~Hz}, \mathrm{H}-3), 5.83$ (1H, dt, $J=15.6,1.5 \mathrm{~Hz}, \mathrm{H}-2), 5.61-5.59$ (2H, m, H-6, H-7), 4.92-4.88 (1H, m, H-10), $4.46\left(2 \mathrm{H}\right.$, br s, $\left.\mathrm{NH}_{2}\right), 4.18\left(2 \mathrm{H}, \mathrm{q}, J=7.1 \mathrm{~Hz}, \mathrm{OC}_{2} \mathrm{CH}_{3}\right)$, 4.11-4.08 (1H, m, H-8), 3.77 (1H, dd, $J=5.1,2.4 \mathrm{~Hz}, \mathrm{H}-9), 3.57$ (2H, td, $J=6.6,1.4 \mathrm{~Hz}$, H-14), 2.31-2.25 (2H, m, H-4), 2.23-2.19 (2H, m, H-5), 1.60-1.55 (2H, m, H-11), 1.51$1.44(2 \mathrm{H}, \mathrm{m}, \mathrm{H}-13), 1.28\left(3 \mathrm{H}, \mathrm{t}, J=7.1 \mathrm{~Hz}, \mathrm{OCH}_{2} \underline{\mathrm{C}}_{3}\right), 1.28-1.22(2 \mathrm{H}, \mathrm{m}, \mathrm{H}-12), 0.91$ $\left(9 \mathrm{H}, \mathrm{s}, \mathrm{Si}-\mathrm{C}\left(\mathrm{C}_{3}\right)_{3}\right), 0.90\left(9 \mathrm{H}, \mathrm{s}, \mathrm{Si}-\mathrm{C}\left(\mathrm{C}_{3}\right)_{3}\right), 0.88\left(9 \mathrm{H}, \mathrm{s}, \mathrm{Si}-\mathrm{C}\left(\mathrm{C}_{3}\right)_{3}\right), 0.07$ (3H, s, Si$\left.\mathrm{CH}_{3}\right), 0.05\left(3 \mathrm{H}, \mathrm{s}, \mathrm{Si}-\mathrm{C}_{3}\right), 0.05\left(3 \mathrm{H}, \mathrm{s}, \mathrm{Si}-\mathrm{C}_{3}\right), 0.03\left(6 \mathrm{H}, \mathrm{s}, \mathrm{Si}-\left(\mathrm{CH}_{3}\right)_{2}\right), 0.01(3 \mathrm{H}, \mathrm{s}, \mathrm{Si}-$ $\left.\mathrm{C}_{3}\right) ;{ }^{13} \mathbf{C}$ NMR $\left(100 \mathrm{MHz}, \mathrm{CDCl}_{3}\right) \delta$ 166.7, 156.5, 148.4, 131.0, 129.4, 121.9, 77.4, 76.1, $74.9,63.3,60.3,33.1,32.0,30.9,29.5,26.13,26.06,26.04,22.1,18.36,18.34,14.4,-4.3$, 4.35, -4.4, -4.5, -5.1; HRMS $\left(\mathrm{ES}^{+}\right)$calcd. for $\mathrm{C}_{35} \mathrm{H}_{72} \mathrm{NO}_{7} \mathrm{Si}_{3}[\mathrm{M}+\mathrm{H}]^{+}$702.4611, found 702.4614 


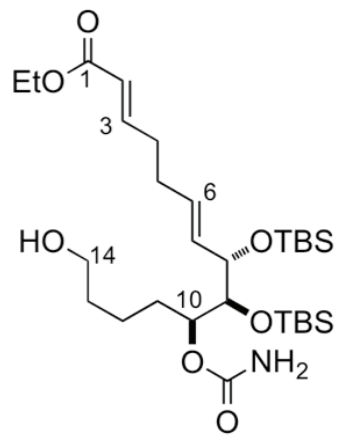

To a solution of tris-tert-butyldimethylsilyl ether $168(130 \mathrm{mg}, 0.185 \mathrm{mmol})$ in $\mathrm{MeOH} / \mathrm{CH}_{2} \mathrm{Cl}_{2}(1: 6,5.0 \mathrm{~mL})$ at $0{ }^{\circ} \mathrm{C}$ was added ( $( \pm)$-CSA $(4.3 \mathrm{mg}, 18.5 \mu \mathrm{mol})$. After $1.5 \mathrm{~h}$, saturated $\mathrm{NaHCO}_{3}(5.0 \mathrm{~mL})$ solution and $\mathrm{CH}_{2} \mathrm{Cl}_{2}(5.0 \mathrm{~mL})$ were added and the layers were separated. The aqueous phase was extracted with $\mathrm{CH}_{2} \mathrm{Cl}_{2}(3 \times 5 \mathrm{~mL})$ and the combined organic extracts were dried $\left(\mathrm{Na}_{2} \mathrm{SO}_{4}\right)$, filtered and concentrated under reduced pressure. Purification by flash column chromatography (10-20\% EtOAC/Hexanes) provided alcohol $170(100 \mathrm{mg}, 93 \%)$ as a colourless oil.

$\boldsymbol{R}_{\boldsymbol{f}} 0.26\left(30 \%\right.$ EtOAc/Hexanes); $[\alpha]_{\mathrm{D}}^{20}-15.0$ ( $\left.c 0.2, \mathrm{CHCl}_{3}\right)$; IR $\boldsymbol{v}_{\max }\left(\right.$ thin film/ $\left.\mathrm{cm}^{-1}\right) 3466$, 3364, 2979, 2929, 2854, 1714, 1366, 1312, 1278, 1188, 1128, 835, 775; ${ }^{1} \mathbf{H}$ NMR (400 $\left.\mathrm{MHz}_{\mathrm{CDCl}}\right) \delta 6.95(1 \mathrm{H}, \mathrm{dt}, J=15.7,6.6 \mathrm{~Hz}, \mathrm{H}-3), 5.83(1 \mathrm{H}, \mathrm{dt}, J=15.7,1.4 \mathrm{~Hz}, \mathrm{H}-2)$, $5.60(2 \mathrm{H}, \mathrm{m}, J=2.2 \mathrm{~Hz}, \mathrm{H}-6,7), 4.92-4.88$ (1H, m, H-10), 4.53-4.42 (2H, br s, $\left.\mathrm{NH}_{2}\right), 4.18$ $\left(2 \mathrm{H}, \mathrm{q}, J=7.1 \mathrm{~Hz}, \mathrm{OCH}_{2} \mathrm{CH}_{3}\right), 4.11-4.08(1 \mathrm{H}, \mathrm{m}, \mathrm{H}-8), 3.78(1 \mathrm{H}, \mathrm{dd}, J=5.2,2.4 \mathrm{~Hz}, \mathrm{H}-$ 9), 3.61 (2H, t, $J=6.6 \mathrm{~Hz}, \mathrm{H}-14), 2.31-2.27$ (2H, m, H-4), 2.24-2.21 (2H, m, H-5), 1.611.52 (4H, m, H-11, H-13), 1.45-1.37 (2H, m, H-12), 1.28 (3H, t, $\left.J=7.1 \mathrm{~Hz}, \mathrm{OCH}_{2} \mathrm{CH}_{3}\right)$, $0.91\left(9 \mathrm{H}, \mathrm{s}, \mathrm{Si}-\mathrm{C}\left(\mathrm{C}_{3}\right)_{3}\right), 0.90\left(9 \mathrm{H}, \mathrm{s}, \mathrm{Si}-\mathrm{C}\left(\mathrm{C}_{3}\right)_{3}\right), 0.07\left(3 \mathrm{H}, \mathrm{s}, \mathrm{Si}-\mathrm{C}_{3}\right), 0.05(6 \mathrm{H}, \mathrm{s}, 2 \times$ $\left.\mathrm{Si}-\mathrm{C}_{3}\right), 0.01$ (3H, s, Si-C $\left.\underline{H}_{3}\right) ;{ }^{13} \mathbf{C}$ NMR $\left(100 \mathrm{MHz}, \mathrm{CDCl}_{3}\right) \delta 166.8,156.5,148.5,130.9$, 129.4, 121.9, 77.4, 75.8, 74.8, 63.0, 60.3, 32.8, 32.0, 30.8, 29.5, 26.05, 26.03, 21.9, 18.36, $18.33,14.4,-4.33,-4.35,-4.4,-4.5$; HRMS $\left(\mathrm{ES}^{+}\right)$calcd. for $\mathrm{C}_{29} \mathrm{H}_{58} \mathrm{NO}_{7} \mathrm{Si}_{2}[\mathrm{M}+\mathrm{H}]^{+}$ 588.3746 , found 588.3743 . 


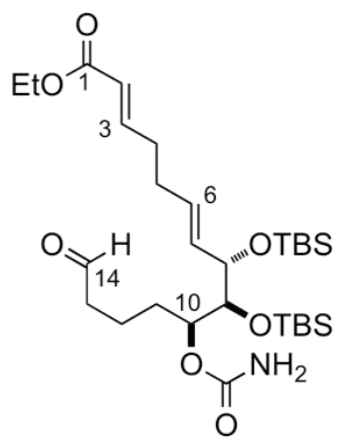

To a solution of alcohol $170(40.0 \mathrm{mg}, 68 \mu \mathrm{mol})$ in $\mathrm{CH}_{2} \mathrm{Cl}_{2}(5.0 \mathrm{~mL})$ at $0{ }^{\circ} \mathrm{C}$ was added $\mathrm{NaHCO}_{3}(28.0 \mathrm{mg}, 0.34 \mathrm{mmol})$ followed by Dess-Martin periodinane $(86.0 \mathrm{mg}, 0.20$ mmol). The reaction mixture was stirred at $0{ }^{\circ} \mathrm{C}$ for $1.5 \mathrm{~h}$, and quenched by addition of cold hexane $(5.0 \mathrm{~mL})$ and cold toluene $(5.0 \mathrm{~mL}) . \mathrm{CH}_{2} \mathrm{Cl}_{2}$ was then removed under reduced pressure and the resultant white suspension was filtered through Celite and washed with excess hexanes. The filtrate was concentrated under reduced pressure and purification by flash column chromatography (10-20\% EtOAc/Hexanes) provided aldehyde 169 (31.0 mg, $80 \%)$ as a colourless oil.

$\boldsymbol{R}_{\boldsymbol{f}} 0.38$ (30\% EtOAc/Hexanes); $[\alpha]_{\mathrm{D}}^{20}-22.2$ (c 3.0, $\left.\mathrm{CHCl}_{3}\right) ; \mathbf{I R} \boldsymbol{v}_{\max }\left(\right.$ thin film $\left./ \mathrm{cm}^{-1}\right) 3361$, 2954, 2929, 2896, 2857, 1722, 1655, 1253, 1124, 836, 776; ${ }^{1} \mathbf{H}$ NMR (400 MHz, $\left.\mathrm{C}_{6} \mathrm{D}_{6}\right) \delta$ $9.32(1 \mathrm{H}, \mathrm{t}, J=1.5 \mathrm{~Hz}, \mathrm{H}-14), 7.01(1 \mathrm{H}, \mathrm{dt}, J=15.6,6.5 \mathrm{~Hz}, \mathrm{H}-3), 5.90-5.86(1 \mathrm{H}, \mathrm{m}, \mathrm{H}-$ 2), $5.71(1 \mathrm{H}, \mathrm{dd}, J=15.5,5.5 \mathrm{~Hz}, \mathrm{H}-7), 5.64-5.59(1 \mathrm{H}, \mathrm{m}, \mathrm{H}-6), 5.17$ (1H, dt, $J=9.5,2.5$ $\mathrm{Hz}, \mathrm{H}-10), 4.25(1 \mathrm{H}, \mathrm{td}, J=5.4,0.9 \mathrm{~Hz}, \mathrm{H}-8), 4.05\left(2 \mathrm{H}, \mathrm{q}, J=7.1 \mathrm{~Hz}, \mathrm{OCH}_{2} \mathrm{CH}_{3}\right), 4.01$ $(1 \mathrm{H}, \mathrm{dd}, J=5.3,2.2 \mathrm{~Hz}, \mathrm{H}-9), 2.02-1.96$ (6H, m, H-4, H-5, H-13), 1.73-1.67 (3H, m, H-11, $\mathrm{H}-12 \mathrm{a}), 1.56-1.47$ (1H, m, H-12b), 1.05 (9H, s, Si-C $\left.\left(\mathrm{CH}_{3}\right)_{3}\right), 1.03\left(9 \mathrm{H}, \mathrm{s}, \mathrm{Si}-\mathrm{C}\left(\mathrm{CH}_{3}\right)_{3}\right)$, 1.03-1.00 (3H, t, $\left.J=7.1 \mathrm{~Hz}, \mathrm{OCH}_{2} \underline{\mathrm{C}}_{3}\right), 0.19\left(3 \mathrm{H}, \mathrm{s}, \mathrm{Si}-\underline{\mathrm{C}}_{3}\right), 0.16\left(3 \mathrm{H}, \mathrm{s}, \mathrm{Si}-\mathrm{C}_{3}\right), 0.13$ $\left(3 \mathrm{H}, \mathrm{s}, \mathrm{Si}-\mathrm{C}_{3}\right), 0.12\left(3 \mathrm{H}, \mathrm{s}, \mathrm{Si}-\mathrm{C}_{3}\right) ;{ }^{13} \mathrm{C}$ NMR $\left(100 \mathrm{MHz}, \mathrm{C}_{6} \mathrm{D}_{6}\right) \delta 200.7,166.1,156.7$, 148.1, 131.0, 130.2, 122.5, 78.1, 75.4, 75.1, 60.1, 43.5, 31.9, 30.9, 29.2, 26.3, 26.2, 18.65, $18.60,18.52,14.4,-4.18,-4.25,-4.28,-4.31$; HRMS $\left(\mathrm{ES}^{+}\right)$calcd. for $\mathrm{C}_{29} \mathrm{H}_{56} \mathrm{NO}_{7} \mathrm{Si}_{2}$ $[\mathrm{M}+\mathrm{H}]^{+}$586.3590, found 586.3589. 


\subsection{Experimental procedures for Chapter 4}

(S)-Methyl-3-((tert-butyldimethylsilyl)oxy)-2-methylpropanoate ${ }^{73}$<smiles>COC(=O)C(C)C(C[SeH2])C(C)=O</smiles>

To a stirred solution of methyl $(S)$-3-hydroxy-2-methylpropionate 177 (17.1 g, $145 \mathrm{mmol})$ and imidazole $(14.8 \mathrm{~g}, 217 \mathrm{mmol})$ in $\mathrm{CH}_{2} \mathrm{Cl}_{2}(300 \mathrm{~mL})$ at $0{ }^{\circ} \mathrm{C}$ was added $\mathrm{TBSCl}(26.2 \mathrm{~g}$, $174 \mathrm{mmol}$ ) in portions. The reaction was stirred at $\mathrm{rt}$ for $12 \mathrm{~h}$ and quenched by addition of saturated $\mathrm{NaHCO}_{3}(300 \mathrm{~mL})$ solution. The aqueous phase was extracted with $\mathrm{CH}_{2} \mathrm{Cl}_{2}(4 \times$ $200 \mathrm{~mL})$ and the combined organic extracts were washed with brine $(300 \mathrm{~mL})$, dried $\left(\mathrm{MgSO}_{4}\right)$, filtered and concentrated under reduced pressure. Purification by flash column chromatography (10\% EtOAc/Hexanes) provided compound (32.0 g, 95\%) as a colourless oil.

$\boldsymbol{R}_{f} 0.52$ (20\% EtOAc/Hexanes); ${ }^{1} \mathbf{H}$ NMR (400 MHz, $\left.\mathrm{CDCl}_{3}\right) \delta 3.77(1 \mathrm{H}, \mathrm{dd}, J=9.7,6.9$ $\mathrm{Hz}, \mathrm{H}-3 \mathrm{a}), 3.68$ (3H, s, $\left.\mathrm{OCH}_{3}\right), 3.65(1 \mathrm{H}, \mathrm{dd}, J=10.0,6.0 \mathrm{~Hz}, \mathrm{H}-3 \mathrm{~b}), 2.73-2.53$ (1H, m, H2), $1.13\left(3 \mathrm{H}, \mathrm{d}, J=7.0 \mathrm{~Hz}, \mathrm{C}_{3}\right), 0.87\left(9 \mathrm{H}, \mathrm{s}, \mathrm{Si}-\mathrm{C}\left(\mathrm{C}_{\mathrm{H}_{3}}\right)_{3}\right), 0.02\left(6 \mathrm{H}, \mathrm{s}, 2 \times \mathrm{Si}-\mathrm{C}_{3}\right)$.

(S)-3-((tert-Butyldimethylsilyl)oxy)- $N$-methoxy- $N$-2-dimethylpropanamide (179) ${ }^{73}$

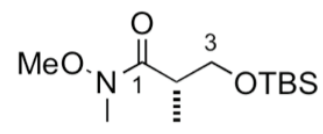

To a stirred slurry of ester $(32.0 \mathrm{~g}, 137 \mathrm{mmol})$ and Weinreb salt $(20.0 \mathrm{~g}, 205 \mathrm{mmol})$ in THF (300 mL) was added $i$-PrMgCl (205 mL, $2 \mathrm{M}$ soln. in THF, $415 \mathrm{mmol})$ dropwise, such that the internal temperature did not exceed $-15^{\circ} \mathrm{C}$. After $1 \mathrm{~h}$ at $-10^{\circ} \mathrm{C}$, saturated $\mathrm{NH}_{4} \mathrm{Cl}(400 \mathrm{~mL})$ solution was added and layers were separated. The aqueous phase was extracted with $\mathrm{Et}_{2} \mathrm{O}(3 \times 300 \mathrm{~mL})$ and the combined organic extracts were dried $\left(\mathrm{MgSO}_{4}\right)$, filtered and concentrated under reduced pressure. Purification by flash column chromatography (5-20\% $\mathrm{Et}_{2} \mathrm{O} /$ Hexanes) afforded the Weinreb amide 179 (30.0 g, 84\%) as a colourless oil. 
$\boldsymbol{R}_{\boldsymbol{f}} 0.52$ (20\% EtOAc/Hexanes); ${ }^{1} \mathbf{H}$ NMR (400 MHz, $\left.\mathrm{CDCl}_{3}\right) \delta 3.83(1 \mathrm{H}, \mathrm{dd}, J=9.5,8.2$ $\mathrm{Hz}, \mathrm{H}-3 \mathrm{a}), 3.70\left(3 \mathrm{H}, \mathrm{s}, \mathrm{N}-\mathrm{OCH}_{3}\right), 3.52(1 \mathrm{H}, \mathrm{dd}, J=9.5,6.1 \mathrm{~Hz}, \mathrm{H}-3 \mathrm{~b}), 3.19$ (3H, s, N$\left.\mathrm{C}_{3}\right), 3.19-3.09$ (1H, m, H-2), $1.06\left(3 \mathrm{H}, \mathrm{d}, J=6.9 \mathrm{~Hz}, \mathrm{C}_{3}\right), 0.86\left(9 \mathrm{H}, \mathrm{s}, \mathrm{Si}-\mathrm{C}\left(\mathrm{CH}_{3}\right)_{3}\right)$, $0.04\left(3 \mathrm{H}, \mathrm{s}, \mathrm{Si}-\mathrm{CH}_{3}\right), 0.03\left(3 \mathrm{H}, \mathrm{s}, \mathrm{Si}-\mathrm{CH}_{3}\right)$.

\section{(S)-3-((tert-Butyldimethylsilyl)oxy)-2-methylpropanal (176) ${ }^{73}$}<smiles>CC(C=O)C(C)C[SeH2]</smiles>

To a stirred solution of Weinreb amide $179(5.00 \mathrm{~g}, 19.1 \mathrm{mmol})$ in THF $(50.0 \mathrm{~mL})$ at -78 ${ }^{\circ} \mathrm{C}$ was added DIBAL-H $(38.3 \mathrm{~mL}, 1 \mathrm{M}$ soln. in Hexane, $38.3 \mathrm{mmol})$. After $1 \mathrm{~h}$ at this temerature the mixture was cannulated into stirred solution of potassium sodium tartrate $(250 \mathrm{~mL})$ salt, further stirred vigorously for $1 \mathrm{~h}$ and extracted with $\mathrm{Et}_{2} \mathrm{O}(3 \times 150 \mathrm{~mL})$. The combined organic extracts were dried $\left(\mathrm{MgSO}_{4}\right)$, filtered and concentrated under reduced pressure. The resultant pale yellow oil was used without purification in the next step. $\boldsymbol{R}_{f} 0.62$ (20\% EtOAc/Hexanes); ${ }^{1} \mathbf{H}$ NMR $\left(300 \mathrm{MHz}, \mathrm{CDCl}_{3}\right) \delta 9.74(1 \mathrm{H}, \mathrm{d}, J=1.6 \mathrm{~Hz}$, H-1), 3.86 (1H, dd, $J=10.2,5.2 \mathrm{~Hz}, \mathrm{H}-3 \mathrm{a}), 3.80$ (1H, dd, $J=10.2,6.3 \mathrm{~Hz}, \mathrm{H}-3 \mathrm{~b}), 2.60-$ $2.47(1 \mathrm{H}, \mathrm{m}, \mathrm{H}-2), 1.09\left(3 \mathrm{H}, \mathrm{d}, J=7.0 \mathrm{~Hz}, \mathrm{CH}_{3}\right), 0.87\left(9 \mathrm{H}, \mathrm{s}, \mathrm{Si}-\mathrm{C}\left(\mathrm{C}_{3}\right)_{3}\right), 0.05(6 \mathrm{H}, \mathrm{s}, 2 \times$ $\left.\mathrm{Si}-\mathrm{C}_{3}\right)$.

$(2 E, 4 R)$-Ethyl 5-((tert-butyldimethylsilyl)oxy)-2,4-dimethylpent-2-enoate $(181)^{143}$<smiles>CCOC(=O)/C(C)=C/[C@H](C)C[Hg+]SC</smiles>

To a slurry of anyhydrous $\mathrm{Ba}(\mathrm{OH})_{2}(4.76 \mathrm{~g}, 27.8 \mathrm{mmol})$ in $\mathrm{THF}(50.0 \mathrm{~mL})$ was added triethylphosphonate $(5.50 \mathrm{~g}, 23.1 \mathrm{mmol}$ ) and the resulting mixture stirred at $\mathrm{rt}$ for $45 \mathrm{~min}$. A solution of aldehyde $176(3.13 \mathrm{~g}, 15.4 \mathrm{mmol})$ in THF $(50.0 \mathrm{~mL})$ and $\mathrm{H}_{2} \mathrm{O}(1.25 \mathrm{~mL})$ was then added via cannula and the reaction mixture was stirred for a further $16 \mathrm{~h}$. The reaction was then quenched with saturated $\mathrm{NH}_{4} \mathrm{Cl}(100 \mathrm{~mL})$ solution and layers were separated. The aqueous phase was extracted with $\mathrm{Et}_{2} \mathrm{O}(3 \times 100 \mathrm{~mL})$. The combined organic extracts were dried $\left(\mathrm{MgSO}_{4}\right)$, filtered and concentrated under reduced pressure. Flash column 
chromatography (10\% EtOAc/Hexanes) afforded enoate 181 (3.27 g, 76\%) as a colourless oil in $7: 1 E: Z$ ratio.

$\boldsymbol{R}_{f} 0.48$ (10\% EtOAc/Hexanes); ${ }^{1} \mathbf{H}$ NMR $\left(300 \mathrm{MHz}, \mathrm{CDCl}_{3}\right) \delta 6.55(1 \mathrm{H}, \mathrm{dq}, J=9.9,1.4$ $\mathrm{Hz}, \mathrm{H}-3), 4.22-4.14\left(2 \mathrm{H}, \mathrm{q}, J=7.1 \mathrm{~Hz}, \mathrm{OCH}_{2} \mathrm{CH}_{3}\right), 3.51-3.43(2 \mathrm{H}, \mathrm{m}, \mathrm{H}-5), 2.68$ (1H, dq, $J=9.9,6.6 \mathrm{~Hz}, \mathrm{H}-4), 1.85\left(3 \mathrm{H}, \mathrm{d}, J=1.4 \mathrm{~Hz}, \mathrm{C} 2-\underline{\mathrm{C}}_{3}\right), 1.28(3 \mathrm{H}, \mathrm{t}, J=7.1 \mathrm{~Hz}$, $\left.\mathrm{OCH}_{2} \underline{\mathrm{C}}_{3}\right), 1.00\left(3 \mathrm{H}, \mathrm{d}, J=6.7 \mathrm{~Hz}, \mathrm{C} 4-\underline{\mathrm{C}}_{3}\right), 0.87\left(9 \mathrm{H}, \mathrm{s}, \mathrm{Si}-\mathrm{C}\left(\mathrm{C}_{3}\right)_{3}\right), 0.03(3 \mathrm{H}, \mathrm{s}, \mathrm{Si}-$ $\left.\mathrm{C}_{3}\right), 0.02\left(3 \mathrm{H}, \mathrm{s}, \mathrm{Si}-\underline{\mathrm{C}}_{3}\right)$.

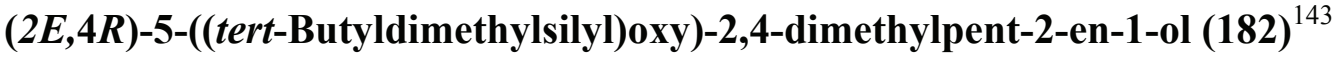

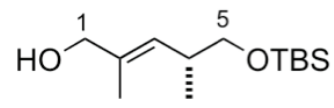

To a stirred solution of enoate $181(2.80 \mathrm{~g}, 9.77 \mathrm{mmol})$ in THF $(40.0 \mathrm{~mL})$ at $-78{ }^{\circ} \mathrm{C}$ was added DIBAL-H (19.5 mL, 1M soln. in hexane, $19.5 \mathrm{mmol})$. After $1 \mathrm{~h}$ at this temperature the mixture was cannulated into stirred solution of potassium sodium tartrate $(150 \mathrm{~mL})$ salt, further stirred vigorously for $1 \mathrm{~h}$ and extracted with $\mathrm{Et}_{2} \mathrm{O}(3 \times 100 \mathrm{~mL})$. The combined organic extracts were dried $\left(\mathrm{MgSO}_{4}\right)$, filtered and concentrated under reduced pressure. Flash column chromatography (10\% EtOAc/Hexanes) afforded E-alcohol 182 (1.93 g, $81 \%)$ as a colourless oil.

$\boldsymbol{R}_{f} 0.28$ (20\% EtOAc/Hexanes); ${ }^{1} \mathbf{H}$ NMR $\left(300 \mathrm{MHz}, \mathrm{CDCl}_{3}\right) \delta 5.18(1 \mathrm{H}, \mathrm{dq}, J=9.4,1.3$, H-3), 3.99 (2H, dd, $J=6.0,0.9$ Hz, H-1), 3.45 (1H, dd, $J=9.8,6.1, \mathrm{H}-5 \mathrm{a}), 3.36$ (1H, dd, $J$ =9.8, 7.2 Hz, H-5b), $2.58(1 \mathrm{H}, \mathrm{dq}, J=9.3,6.7 \mathrm{~Hz}, \mathrm{H}-4), 1.69\left(3 \mathrm{H}, \mathrm{d}, J=1.4 \mathrm{~Hz}, \mathrm{C} 2-\underline{\mathrm{C}}_{3}\right)$, $0.95\left(3 \mathrm{H}, \mathrm{d}, J=6.7 \mathrm{~Hz}, \mathrm{C} 4-\mathrm{C}_{3}\right), 0.88\left(9 \mathrm{H}, \mathrm{s}, \mathrm{Si}-\mathrm{C}\left(\mathrm{C}_{\mathrm{H}_{3}}\right)_{3}\right), 0.03\left(6 \mathrm{H}, \mathrm{s}, 2 \times \mathrm{Si}-\mathrm{C}_{3}\right)$.

(2E,4R)-5-((tert-Butyldimethylsilyl)oxy)-2,4-dimethylpent-2-enal (175)

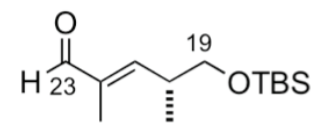

To a solution of alcohol $182(500 \mathrm{mg}, 2.04 \mathrm{mmol})$ in $\mathrm{CH}_{2} \mathrm{Cl}_{2}(20.0 \mathrm{~mL})$ at $0{ }^{\circ} \mathrm{C}$ was added $\mathrm{NaHCO}_{3}(859 \mathrm{mg}, 10.2 \mathrm{mmol})$ followed by Dess-Martin periodinane (2.60 g, $\left.6.13 \mathrm{mmol}\right)$. The reaction mixture was stirred at $0{ }^{\circ} \mathrm{C}$ for $1.5 \mathrm{~h}$, and quenched by addition of cold hexane $(40.0 \mathrm{~mL})$ and cold toluene $(10.0 \mathrm{~mL})$ and $\mathrm{CH}_{2} \mathrm{Cl}_{2}$ was then removed under reduced 
pressure. The resultant white suspension was filtered through short pad of silica gel and Celite, eluting with $20 \%$ EtOAc/Hexanes. The filtrate was concentrated under reduced pressure to provide aldehyde 175 (440 $\mathrm{mg}, 90 \%$ ) as a colourless oil which was immediately used without further purification.

$\boldsymbol{R}_{\boldsymbol{f}} 0.42$ (10\% EtOAc/Hexane); ${ }^{1} \mathbf{H}$ NMR $\left(300 \mathrm{MHz}, \mathrm{CDCl}_{3}\right) \delta 9.40(1 \mathrm{H}, \mathrm{s}, \mathrm{H}-23), 6.32$ $(1 \mathrm{H}, \mathrm{dq}, J=9.7,1.3 \mathrm{~Hz}, \mathrm{H}-21), 3.59(1 \mathrm{H}, \mathrm{dd}, J=9.9,6.0 \mathrm{~Hz}, \mathrm{H}-19 \mathrm{a}), 3.54(1 \mathrm{H}, \mathrm{dd}, J=$ 9.9, $6.5 \mathrm{~Hz}, \mathrm{H}-19 \mathrm{~b}), 2.89$ (1H, ddd, $J=12.6,9.7,6.6 \mathrm{~Hz}, \mathrm{H}-20), 1.77$ (3H, d, $J=1.4 \mathrm{~Hz}$, $\left.\mathrm{C} 22-\mathrm{C}_{3}\right), 1.06\left(3 \mathrm{H}, \mathrm{d}, J=6.8 \mathrm{~Hz}, \mathrm{C} 20-\underline{\mathrm{C}}_{3}\right), 0.87\left(9 \mathrm{H}, \mathrm{s}, \mathrm{Si}-\mathrm{C}\left(\mathrm{CH}_{3}\right)_{3}\right), 0.04$ (3H, s, Si$\left.\mathrm{CH}_{3}\right), 0.03\left(3 \mathrm{H}, \mathrm{s}, \mathrm{Si}-\mathrm{CH}_{3}\right)$.

(2R,3E,5E)-6-Iodo-2,4-dimethylhexa-3,5-dien-1-ol (178)

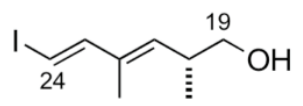

To a solution of flame-dried $\mathrm{CrCl}_{2}(1.12 \mathrm{~g}, 9.07 \mathrm{mmol})$ in THF $(11.0 \mathrm{~mL})$ at $\mathrm{rt}$ was added a solution of aldehyde $175(220 \mathrm{mg}, 0.907 \mathrm{mmol})$ and $\mathrm{CHI}_{3}(1.07 \mathrm{~g}, 2.72 \mathrm{mmol})$ in 1,4dioxane $(65.0 \mathrm{~mL})$. The reaction mixture was stirred for $2 \mathrm{~h}$ before it was quenched with saturated $\mathrm{NaHCO}_{3}(50.0 \mathrm{~mL})$ solution. The resulting mixture was diluted with EtOAc (200 $\mathrm{mL})$, filtered through a short pad of Celite and washed with EtOAc $(250 \mathrm{~mL})$. The layers were separated and the aqueous phase was extracted with EtOAc $(3 \times 50 \mathrm{~mL})$. The combine organic extracts were dried $\left(\mathrm{Na}_{2} \mathrm{SO}_{4}\right)$, filtered and concentrated under reduced pressure. The crude material was directly subjected to TBAF deprotection.

$\boldsymbol{R}_{f} 0.8$ (10\% EtOAc/Hexanes); ${ }^{1} \mathbf{H}$ NMR $\left(400 \mathrm{MHz}, \mathrm{CDCl}_{3}\right) \delta 7.03(1 \mathrm{H}, \mathrm{dd}, J=14.6,0.8$ Hz, H-23), 6.15 (1H, dd, $J=14.6,0.6$ Hz, H-24), 5.27 (1H, d, $J=9.5$ Hz, H-21), 3.43 (2H, qd, $J=10.6,6.5 \mathrm{~Hz}, \mathrm{H}-19), 2.64(1 \mathrm{H}, \mathrm{m}, \mathrm{H}-20), 1.74\left(3 \mathrm{H}, \mathrm{d}, J=1.3 \mathrm{~Hz}, \mathrm{C} 22-\mathrm{CH}_{3}\right), 0.97$ $\left(3 \mathrm{H}, \mathrm{d}, J=6.7 \mathrm{~Hz}, \mathrm{C} 20-\underline{\mathrm{H}}_{3}\right), 0.88\left(9 \mathrm{H}, \mathrm{s}, \mathrm{Si}-\mathrm{C}\left(\mathrm{C}_{3}\right)_{3}\right), 0.03\left(3 \mathrm{H}, \mathrm{s}, \mathrm{Si}-\mathrm{C}_{3}\right), 0.02(3 \mathrm{H}, \mathrm{s}$, $\left.\mathrm{Si}-\underline{\mathrm{C}}_{3}\right)$.

To a solution of crude $185(332 \mathrm{mg}, 0.907 \mathrm{mmol})$ in $\mathrm{THF}(10.0 \mathrm{~mL})$ at $0{ }^{\circ} \mathrm{C}$ was added TBAF $(9.1 \mathrm{~mL}, 1 \mathrm{M}$ in THF, $0.907 \mathrm{mmol})$. The reaction mixture was allowed to warm to $\mathrm{rt}$ and stirred for $12 \mathrm{~h}$. The reaction mixture was diluted with $\mathrm{Et}_{2} \mathrm{O}(10.0 \mathrm{~mL})$, poured onto water $(20.0 \mathrm{~mL})$ and the layers were separated. The aqueous phase was extracted with $\mathrm{Et}_{2} \mathrm{O}(3 \times 20 \mathrm{~mL})$ and the combined organic extracts were washed with brine $(20 \mathrm{~mL})$, dried $\left(\mathrm{Na}_{2} \mathrm{SO}_{4}\right)$, filtered and concentrated under reduced pressure. Purification by flash 
column chromatography (10\% EtOAc/Hexanes) provided iodo-alcohol 178 (200 mg, 90\%) as a pale yellow oil.

$\boldsymbol{R}_{\boldsymbol{f}} 0.32(10 \% \mathrm{EtOAc/Hexane}) ;[\alpha]_{\mathrm{D}}^{20}+40.8\left(c 0.7, \mathrm{CHCl}_{3}\right)$; IR $\boldsymbol{v}_{\max }\left(\right.$ thin film $\left./ \mathrm{cm}^{-1}\right) 3374$, 2959, 2926, 2870, 1726, 1707, 1451, 1178, 1035, 1018, 960, 752; ${ }^{1} \mathbf{H}$ NMR (400 MHz, $\left.\mathrm{CDCl}_{3}\right) \delta 7.06(2 \mathrm{H}, \mathrm{dd}, J=14.7,0.8 \mathrm{~Hz}, \mathrm{H}-23), 6.22(1 \mathrm{H}, \mathrm{dd}, J=14.7,0.6 \mathrm{~Hz}, \mathrm{H}-24), 5.27$ (1H, dt, $J=9.6,0.6$ Hz, H-21), 3.55-3.49 (1H, m, H-19a), 3.44-3.39 (1H, m, H-19b), 2.78$2.67(1 \mathrm{H}, \mathrm{m}, \mathrm{H}-20), 1.78\left(3 \mathrm{H}, \mathrm{d}, J=1.3 \mathrm{~Hz}, \mathrm{C} 22-\mathrm{CH}_{3}\right), 0.98(3 \mathrm{H}, \mathrm{d}, J=6.7 \mathrm{~Hz}, \mathrm{C} 20-$ $\left.\mathrm{CH}_{3}\right) ;{ }^{13} \mathrm{C}$ NMR $\left(100 \mathrm{MHz}, \mathrm{C}_{6} \mathrm{D}_{6}\right) \delta 149.6,136.5,136.0,74.5,67.6,35.7,16.8,12.5$; HRMS $\left(\mathrm{ES}^{+}\right.$) calcd. for $\mathrm{C}_{8} \mathrm{H}_{14} \mathrm{I}_{1} \mathrm{O}[\mathrm{M}+\mathrm{H}]^{+}$253.0084, found 253.0079.

$(2 R, 3 E, 5 E)-6-I o d o-2,4-d i m e t h y l h e x a-3,5-d i e n a l ~(172)$

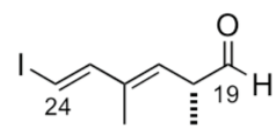

To a solution of alcohol $178(300 \mathrm{mg}, 1.19 \mathrm{mmol})$ in $\mathrm{CH}_{2} \mathrm{Cl}_{2}(40.0 \mathrm{~mL})$ at $0{ }^{\circ} \mathrm{C}$ was added $\mathrm{NaHCO}_{3}$ (499 mg, $\left.5.95 \mathrm{mmol}\right)$ followed by Dess-Martin periodinane $(1.51 \mathrm{~g}, 3.57 \mathrm{mmol})$. The reaction mixture was stirred at $0{ }^{\circ} \mathrm{C}$ for $1.5 \mathrm{~h}$ and quenched by addition of cold hexane $(50.0 \mathrm{~mL})$ and cold toluene $(10.0 \mathrm{~mL}) . \mathrm{CH}_{2} \mathrm{Cl}_{2}$ was then removed under reduced pressure and the resulting white suspension was filtered through Celite and short pad of silica gel (20\% EtOAc/Hexanes) and concentrated under reduced pressure to provide aldehyde 172 (267 mg, 90\%) as pale yellow oil. The resultant compound was immediately used without further purification.

$\boldsymbol{R}_{f} 0.46$ (10\% EtOAc/Hexanes); ${ }^{1} \mathbf{H}$ NMR $\left(400 \mathrm{MHz}, \mathrm{C}_{6} \mathrm{D}_{6}\right) \delta 9.05(1 \mathrm{H}, \mathrm{d}, J=1.6, \mathrm{H}-19)$, $6.81(1 \mathrm{H}, \mathrm{dd}, J=14.7,0.8 \mathrm{~Hz}, \mathrm{H}-23), 5.91(1 \mathrm{H}, \mathrm{dd}, J=14.7,0.6 \mathrm{~Hz}, \mathrm{H}-24), 4.73$ (1H, ddt, $J=9.2,1.3,0.7 \mathrm{~Hz}, \mathrm{H}-21), 2.69$ (1H, ddd, $J=9.0,7.1,1.8 \mathrm{~Hz}, \mathrm{H}-20), 1.19$ (3H, d, $J=1.3$ $\left.\mathrm{Hz}, \mathrm{C} 22-\underline{\mathrm{C}}_{3}\right), 0.79\left(3 \mathrm{H}, \mathrm{d}, J=6.9 \mathrm{~Hz}, \mathrm{C} 20-\underline{\mathrm{C}}_{3}\right)$. 


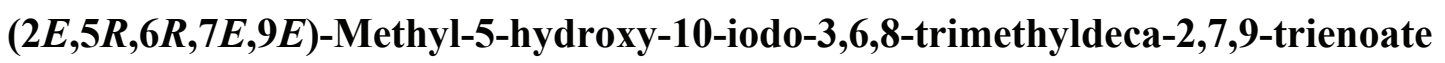
(189)

$(2 E, 5 S, 6 R, 7 E, 9 E)$-Methyl-5-hydroxy-10-iodo-3,6,8-trimethyldeca-2,7,9-trienoate
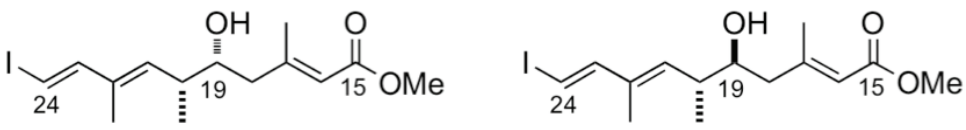

To a stirred solution of aldehyde $172(200 \mathrm{mg}, 0.799 \mathrm{mmol})$ and silyl ketene acetal 173 (253 mg, $1.36 \mathrm{mmol})$ containing $\mathrm{CaH}_{2}(513 \mathrm{mg}, 12.2 \mathrm{mmol})$ in $\mathrm{CH}_{2} \mathrm{Cl}_{2}(40.0 \mathrm{~mL})$ at $-95^{\circ} \mathrm{C}$ was added $\mathrm{BF}_{3} \cdot \mathrm{OEt}_{2}\left(5.0 \mathrm{~mL}, 0.5 \mathrm{M}\right.$ soln. in $\left.\mathrm{CH}_{2} \mathrm{Cl}_{2}, 2.39 \mathrm{mmol}\right)$ dropwise. After $20 \mathrm{~min}$, the reaction mixture was allowed to warm to $-80^{\circ} \mathrm{C}$ and a further aliquot of $\mathrm{BF}_{3} \cdot \mathrm{OEt}_{2}(5.0$ $\mathrm{mL}, 0.5 \mathrm{M}$ soln. in $\mathrm{CH}_{2} \mathrm{Cl}_{2}, 2.39 \mathrm{mmol}$ ) was added. After 20 min the reaction mixture was quenched by the slow addition of saturated $\mathrm{NaHCO}_{3}(50.0 \mathrm{~mL})$ solution and allowed to warm to rt. The layers were separated and the aqueous phase was extracted with $\mathrm{CH}_{2} \mathrm{Cl}_{2}(3$ $\times 50 \mathrm{~mL})$. The combined organic extracts were dried $\left(\mathrm{Na}_{2} \mathrm{SO}_{4}\right)$, filtered and concentrated under reduced pressure. Purification by flash column chromatography (1-10\% EtOAc/Hexanes) provided a mixture of aldol products $(210 \mathrm{mg}, 70 \%)$ as colourless oils in 6:1 syn:anti ratio for $E$ isomer and 3:1 syn:anti ratio for $Z$ isomer.

189 E-syn: $\boldsymbol{R}_{\boldsymbol{f}} 0.26\left(10 \%\right.$ EtOAc/Hexanes); $[\alpha]_{\mathrm{D}}^{20}+17.7$ (c 1.0, $\left.\mathrm{CHCl}_{3}\right)$; IR $\boldsymbol{v}_{\max }$ (thin film/ $\left./ \mathrm{cm}^{-1}\right)$ 3475, 2948, 1715, 1644, 1435, 1223, 1151, 1059, 950; ${ }^{1} \mathbf{H}$ NMR (400 MHz, $\left.\mathrm{C}_{6} \mathrm{D}_{6}\right) \delta 6.92(1 \mathrm{H}, \mathrm{dd}, J=14.7,0.8 \mathrm{~Hz}, \mathrm{H}-23), 5.91(1 \mathrm{H}, \mathrm{dd}, J=14.7,0.5 \mathrm{~Hz}, \mathrm{H}-24), 5.80$ $(1 \mathrm{H}, \mathrm{dd}, J=2.1,1.2 \mathrm{~Hz}, \mathrm{H}-16), 4.89(1 \mathrm{H}, \mathrm{d}, J=10.0 \mathrm{~Hz}, \mathrm{H}-21), 3.44\left(3 \mathrm{H}, \mathrm{s}, \mathrm{OC}_{3}\right), 3.16-$ 3.23 (1H, m, H-19), 2.21-2.11 (1H, m, H-20), 2.18 (H, d, J=1.2 Hz, C17-C $\left.\underline{H}_{3}\right), 1.95(1 \mathrm{H}$, dd, $J=13.4,2.3 \mathrm{~Hz}, \mathrm{H}-18 \mathrm{a}), 1.75$ (1H, dd, $J=13.7,9.8 \mathrm{~Hz}, \mathrm{H}-18 \mathrm{~b}), 1.31$ (3H, d, $J=1.2$ $\left.\mathrm{Hz}, \mathrm{C} 22-\mathrm{C}_{3}\right), 0.87\left(3 \mathrm{H}, \mathrm{d}, J=6.7 \mathrm{~Hz}, \mathrm{C} 20-\mathrm{C}_{3}\right) ;{ }^{13} \mathbf{C} \mathbf{N M R}\left(100 \mathrm{MHz}, \mathrm{C}_{6} \mathrm{D}_{6}\right) \delta 166.6$, $157.2,149.8,136.5,134.8,118.1,74.7,73.1,50.6,46.7,39.4,19.0,16.2,12.1$; HRMS $\left(\mathrm{ES}^{+}\right)$calcd. for $\mathrm{C}_{14} \mathrm{H}_{25} \mathrm{I}_{1} \mathrm{NO}_{3}\left[\mathrm{M}+\mathrm{NH}_{4}\right]^{+}$382.0874, found 382.0869 .

E-anti: $\boldsymbol{R}_{\boldsymbol{f}} 0.27\left(10 \%\right.$ EtOAc/Hexanes); $[\alpha]_{\mathrm{D}}^{20}+5.5\left(c 0.2, \mathrm{CHCl}_{3}\right) ; \mathbf{I R} \boldsymbol{v}_{\max }\left(\right.$ thin film $\left./ \mathrm{cm}^{-1}\right)$ 3496, 2948, 2926, 1711, 1646, 1436, 1227, 1152, 949, 839; ${ }^{1}$ H NMR (400 MHz, $\left.\mathrm{C}_{6} \mathrm{D}_{6}\right) \delta$ $6.95(1 \mathrm{H}, \mathrm{dd}, J=14.7,0.8 \mathrm{~Hz}, \mathrm{H}-23), 5.92(1 \mathrm{H}, \mathrm{dd}, J=14.7,0.6 \mathrm{~Hz}, \mathrm{H}-24), 5.80(1 \mathrm{H}, \mathrm{q}, J$ $=1.2 \mathrm{~Hz}, \mathrm{H}-16), 5.12(1 \mathrm{H}, \mathrm{d}, J=10.0 \mathrm{~Hz}, \mathrm{H}-21), 3.43\left(3 \mathrm{H}, \mathrm{s}, \mathrm{OC}_{3}\right), 3.24(1 \mathrm{H}, \mathrm{dq}, J=$ 8.2, 4.2 Hz, H-19), 2.20 (3H, d, $\left.J=1.3 \mathrm{~Hz}, \mathrm{C} 17-\underline{\mathrm{C}}_{3}\right), 2.17-2.14$ (1H, m, H-20), 1.85 (1H, 
dd, $J=4.1,0.9 \mathrm{~Hz}, \mathrm{H}-18 \mathrm{a}), 1.85-1.84$ (1H, m, H-18b), 1.32 (3H, d, $\left.J=1.3 \mathrm{~Hz}, \mathrm{C} 22-\mathrm{C}_{3}\right)$, $0.77\left(3 \mathrm{H}, \mathrm{d}, J=6.7 \mathrm{~Hz}, \mathrm{C} 20-\mathrm{C}_{3}\right) ;{ }^{13} \mathbf{C}$ NMR $\left(100 \mathrm{MHz}, \mathrm{C}_{6} \mathrm{D}_{6}\right) \delta 166.7,157.0,149.8$, 135.6, 135.2, 118.1, 74.7, 72.8, 50.6, 46.4, 38.7, 19.1, 17.1, 12.1; HRMS (ES ${ }^{+}$) calcd. for $\mathrm{C}_{14} \mathrm{H}_{25} \mathrm{I}_{1} \mathrm{NO}_{3}\left[\mathrm{M}+\mathrm{NH}_{4}\right]^{+}$382.0874, found 382.0871.

(2Z,5R,6R,7E,9E)-Methyl 5-hydroxy-10-iodo-3,6,8-trimethyldeca-2,7,9-trienoate

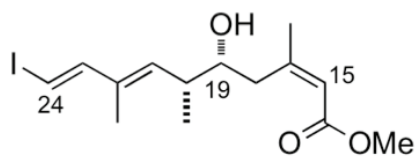

(Z)-syn: $\mathbf{R}_{\mathbf{f}} 0.29$ (10\% EtOAc/Hexanes); $[\alpha]_{\mathrm{D}}^{20}+20.7$ (c 1.7, $\left.\mathrm{CHCl}_{3}\right)$; IR $\boldsymbol{v}_{\max }$ (thin film/cm ${ }^{-}$ $\left.{ }^{1}\right) 3450,3442,2970,2926,1714,1651,1447,1280,1180,1015,949,867 ;{ }^{1}$ H NMR (500 MHz, $\left.\mathrm{C}_{6} \mathrm{D}_{6}\right) \delta 6.98(1 \mathrm{H}, \mathrm{d}, J=14.7 \mathrm{~Hz}, \mathrm{H}-23), 5.90(1 \mathrm{H}, \mathrm{d}, J=14.6 \mathrm{~Hz}, \mathrm{H}-24), 5.77(1 \mathrm{H}$, $\mathrm{d}, J=1.2 \mathrm{~Hz}, \mathrm{H}-16), 5.14(1 \mathrm{H}, \mathrm{d}, J=10.0 \mathrm{~Hz}, \mathrm{H}-21), 3.47(1 \mathrm{H}, \mathrm{dtd}, J=10.4,7.1,2.9 \mathrm{~Hz}$, H-19), $3.28\left(3 \mathrm{H}, \mathrm{s}, \mathrm{OC}_{3}\right), 3.19(1 \mathrm{H}, \mathrm{d}, J=7.4 \mathrm{~Hz}, \mathrm{OH}), 2.98(1 \mathrm{H}, \mathrm{dd}, J=12.6,10.6 \mathrm{~Hz}$, H-18a), 2.44 (1H, dt, $J=10.0,6.7$ Hz, H-20), 2.15 (1H, dd, $J=12.6,2.5$ Hz, H-18b), 1.54 $\left(3 \mathrm{H}, \mathrm{d}, J=1.3 \mathrm{~Hz}, \mathrm{C} 17-\underline{\mathrm{H}}_{3}\right), 1.39\left(3 \mathrm{H}, \mathrm{d}, J=1.1 \mathrm{~Hz}, \mathrm{C} 22-\mathrm{CH}_{3}\right), 1.09(3 \mathrm{H}, \mathrm{d}, J=6.7 \mathrm{~Hz}$, $\left.\mathrm{C} 20-\underline{\mathrm{H}}_{3}\right) ;{ }^{13} \mathrm{C}$ NMR $\left(125 \mathrm{MHz}, \mathrm{C}_{6} \mathrm{D}_{6}\right) \delta$ 168.5, 158.7, 150.0, 137.3, 134.7, 118.1, 74.8, 74.3, 51.0, 41.1, 39.7, 25.5, 16.6, 12.2; HRMS $\left(\mathrm{ES}^{+}\right)$calcd. for $\mathrm{C}_{14} \mathrm{H}_{22} \mathrm{I}_{1} \mathrm{O}_{3}[\mathrm{M}+\mathrm{H}]^{+}$ 365.0608, found 365.0612 .

(R)-Mosher ester of 189 (192)

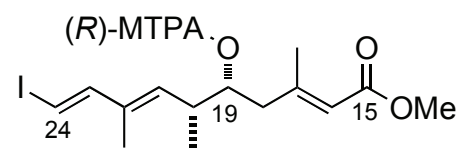

To a stirred mixture of alcohol $189(20.0 \mathrm{mg}, 54.9 \mu \mathrm{mol}), \mathrm{DCC}(45.0 \mathrm{mg}, 219 \mu \mathrm{mol})$ and DMAP $(13.0 \mathrm{mg}, 109 \mu \mathrm{mol})$ in $\mathrm{CH}_{2} \mathrm{Cl}_{2}(2.0 \mathrm{~mL})$ at $\mathrm{rt}$ was added $(+)-(R)-\alpha$-methoxy- $\alpha-$ (trifluoromethyl)-phenylacetic acid $(26.0 \mathrm{mg}, 109 \mu \mathrm{mol})$ in one portion. After $12 \mathrm{~h}$, the crude reaction mixture was directly purified by flash column chromatography $(5 \%$ EtOAc/Hexanes) to give (R)-MTPA ester 192 (30.0 mg, 94\%).

$\boldsymbol{R}_{\boldsymbol{f}} 0.46\left(10 \%\right.$ EtOAc/Hexanes); ${ }^{1} \mathbf{H}$ NMR $\left(400 \mathrm{MHz} ; \mathrm{CDCl}_{3}\right) \delta 7.48(2 \mathrm{H}, \mathrm{d}, J=8.3 \mathrm{~Hz}$, Ph- $\underline{\mathrm{H}}), 7.43-7.37$ (3H, m, Ph- $\underline{\mathrm{H}}), 7.01(1 \mathrm{H}, \mathrm{dd}, J=14.7,0.8 \mathrm{~Hz}, \mathrm{H}-23), 6.28(1 \mathrm{H}, \mathrm{dd}, J=$ 
14.7, $0.5 \mathrm{~Hz}, \mathrm{H}-24), 5.61(1 \mathrm{H}, \mathrm{d}, J=1.2 \mathrm{~Hz}, \mathrm{H}-16), 5.26$ (1H, dd, $J=9.9,0.6 \mathrm{~Hz}, \mathrm{H}-21)$, $5.23(1 \mathrm{H}, \mathrm{dt}, J=7.7,5.8 \mathrm{~Hz}, \mathrm{H}-19), 3.66\left(3 \mathrm{H}, \mathrm{s}, \mathrm{OCH}_{3}\right), 3.46(3 \mathrm{H}, \mathrm{d}, J=1.0 \mathrm{~Hz}, \mathrm{MTPA}-$ $\left.\mathrm{OC}_{3}\right), 2.82(1 \mathrm{H}, \mathrm{dq}, J=9.8,6.6 \mathrm{~Hz}, \mathrm{H}-20), 2.42(1 \mathrm{H}, \mathrm{s}, \mathrm{H}-18 \mathrm{a}), 2.41(1 \mathrm{H}, \mathrm{dd}, J=2.5,0.8$ $\mathrm{Hz}, \mathrm{H}-18 \mathrm{~b}), 2.17$ (3H, d, $\left.J=1.3 \mathrm{~Hz}, \mathrm{C} 17-\mathrm{C}_{3}\right), 1.74\left(3 \mathrm{H}, \mathrm{d}, J=1.3 \mathrm{~Hz}, \mathrm{C} 22-\mathrm{C}_{3}\right), 1.03$ $\left(3 \mathrm{H}, \mathrm{d}, J=6.8 \mathrm{~Hz}, \mathrm{C} 20-\underline{\mathrm{C}}_{3}\right)$.

\section{(S)-Mosher ester of 189 (193)}

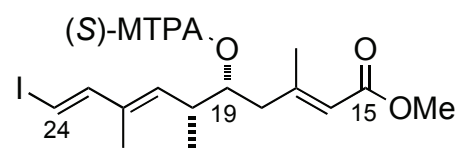

To a stirred mixture of alcohol $189(20.0 \mathrm{mg}, 54.9 \mu \mathrm{mol}), \mathrm{DCC}(45.0 \mathrm{mg}, 219 \mu \mathrm{mol})$ and DMAP $(13.0 \mathrm{mg}, 109 \mu \mathrm{mol})$ in $\mathrm{CH}_{2} \mathrm{Cl}_{2}(2.0 \mathrm{~mL})$ at $\mathrm{rt}$ was added $(+)-(S)$ - $\alpha$-methoxy- $\alpha-$ (trifluoromethyl)-phenylacetic acid $(26.0 \mathrm{mg}, 109 \mu \mathrm{mol})$ in one portion. After $12 \mathrm{~h}$, the crude reaction mixture was purified directly by flash column chromatography (5\% EtOAc/Hexanes) to give (S)-MTPA ester 193 (25.0 mg, 78\%).

$\boldsymbol{R}_{\boldsymbol{f}} 0.46$ (10\% EtOAc/Hexanes); ${ }^{1} \mathbf{H}$ NMR (400 MHz, $\left.\mathrm{CDCl}_{3}\right) \delta$ 7.49-7.47 (2H, m, Ph- $\underline{\mathrm{H}}$ ), 7.37-7.41 (3H, m, Ph- $\underline{\mathrm{H}}), 6.92$ (1H, dd, $J=14.7,0.7 \mathrm{~Hz}, \mathrm{H}-23), 6.23$ (1H, dd, $J=14.7,0.5$ Hz, H-24), 5.68 (1H, q, $J=1.1 \mathrm{~Hz}, \mathrm{H}-16), 5.23$ (1H, dt, $J=7.2,6.1 \mathrm{~Hz}, \mathrm{H}-19), 5.14$ (1H, d, $J=10.4 \mathrm{~Hz}, \mathrm{H}-21), 3.68\left(3 \mathrm{H}, \mathrm{s}, \mathrm{OCH}_{3}\right), 3.51\left(3 \mathrm{H}, \mathrm{d}, J=1.2 \mathrm{~Hz}, \mathrm{MTPA}-\mathrm{OC}_{3}\right), 2.73$ (1H, dq, $J=9.9,6.6 \mathrm{~Hz}, \mathrm{H}-20), 2.45$ (1H, d, $J=0.8 \mathrm{~Hz}, \mathrm{H}-18 \mathrm{a}), 2.43$ (1H, m, H-18b), 2.19 $\left(3 \mathrm{H}, \mathrm{d}, J=1.3 \mathrm{~Hz}, \mathrm{C} 17-\mathrm{CH}_{3}\right), 1.67\left(3 \mathrm{H}, \mathrm{d}, J=1.3 \mathrm{~Hz}, \mathrm{C} 22-\mathrm{CH}_{3}\right), 0.94(3 \mathrm{H}, \mathrm{d}, J=6.8 \mathrm{~Hz}$, $\left.\mathrm{C} 20-\mathrm{C}_{3}\right)$.

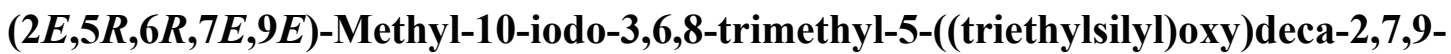
trienoate (194)

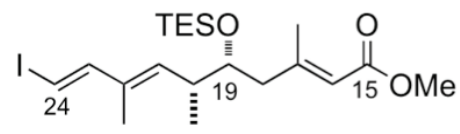

To a solution of alcohol $189(250 \mathrm{mg}, 0.686 \mathrm{mmol})$ in $\mathrm{CH}_{2} \mathrm{Cl}_{2}(7.0 \mathrm{~mL})$ at $-78{ }^{\circ} \mathrm{C}$ was added 2,6-lutidine $(240 \mu \mathrm{L}, 2.06 \mathrm{mmol})$ and TESOTf $(250 \mu \mathrm{L}, 1.10 \mathrm{mmol})$. After $4 \mathrm{~h}$, saturated $\mathrm{NH}_{4} \mathrm{Cl}(15.0 \mathrm{~mL})$ solution and $\mathrm{CH}_{2} \mathrm{Cl}_{2}(15.0 \mathrm{~mL})$ were added and the layers were 
separated. The aqueous layer was extracted with $\mathrm{CH}_{2} \mathrm{Cl}_{2}(3 \times 20 \mathrm{~mL})$ and the combined organic extracts were dried $\left(\mathrm{Na}_{2} \mathrm{SO}_{4}\right)$, filtered and concentrated under reduced pressure. Purification by flash column chromatography (5\% EtOAc/Hexanes) provided TES ether $194(230 \mathrm{mg}, 96 \%)$ as a colourless oil.

$\boldsymbol{R}_{\boldsymbol{f}} 0.73\left(10 \%\right.$ EtOAc/Hexanes); $[\alpha]_{\mathrm{D}}^{20}+33.6\left(c 0.5, \mathrm{CHCl}_{3}\right) ; \mathbf{I R} \boldsymbol{v}_{\max }\left(\right.$ thin film $\left./ \mathrm{cm}^{-1}\right) 2953$, 2911, 2876, 1720, 1647, 1435, 1223, 1150, 1087, 1008, 739, 725; ${ }^{1} \mathbf{H}$ NMR (300 MHz, $\left.\mathrm{C}_{6} \mathrm{D}_{6}\right) \delta 7.02(1 \mathrm{H}, \mathrm{dd}, J=14.6,0.7 \mathrm{~Hz}, \mathrm{H}-23), 5.93(1 \mathrm{H}, \mathrm{d}, J=14.6 \mathrm{~Hz}, \mathrm{H}-24), 5.87(1 \mathrm{H}$, q, $J=1.1 \mathrm{~Hz}, \mathrm{H}-16), 5.12(1 \mathrm{H}, \mathrm{dd}, J=9.7,0.4 \mathrm{~Hz}, \mathrm{H}-21), 3.64(1 \mathrm{H}, \mathrm{dt}, J=6.6,5.4 \mathrm{~Hz}, \mathrm{H}-$ 19), $3.44\left(3 \mathrm{H}, \mathrm{s}, \mathrm{OC}_{3}\right), 2.46-2.39(1 \mathrm{H}, \mathrm{m}, \mathrm{H}-20), 2.25\left(3 \mathrm{H}, \mathrm{d}, J=1.3 \mathrm{~Hz}, \mathrm{C} 17-\underline{\mathrm{C}}_{3}\right)$, 2.08-2.10 (1H, td, $J=6.10,0.95 \mathrm{~Hz}, \mathrm{H}-18 \mathrm{a}), 2.07$ (1 H, d, $J=0.8 \mathrm{~Hz}, \mathrm{H}-18 \mathrm{~b}), 1.36$ (3H, d, $\left.J=1.2 \mathrm{~Hz}, \mathrm{C} 22-\mathrm{C}_{3}\right), 0.96\left(6 \mathrm{H}, \mathrm{m}, 3 \times \mathrm{Si}-\mathrm{C}_{2} \mathrm{CH}_{3}\right), 0.89\left(3 \mathrm{H}, \mathrm{d}, J=6.8 \mathrm{~Hz}, \mathrm{C} 20-\underline{\mathrm{H}}_{3}\right)$, $0.56\left(9 \mathrm{H}, \mathrm{m}, 3 \times \mathrm{Si}_{-}-\mathrm{CH}_{2} \mathrm{CH}_{3}\right) ;{ }^{13} \mathbf{C}$ NMR $\left(75 \mathrm{MHz}, \mathrm{C}_{6} \mathrm{D}_{6}\right) \delta 166.6,156.5,149.9,137.2$, 134.4, 118.7, 74.7, 74.5, 50.6, 46.9, 38.5, 19.5, 15.6, 12.1, 7.2, 5.6; HRMS (ES ${ }^{+}$) calcd. for $\mathrm{C}_{20} \mathrm{H}_{40} \mathrm{INO}_{3} \mathrm{Si}\left[\mathrm{M}+\mathrm{NH}_{4}\right]^{+}$496.1738, found 496.1734.

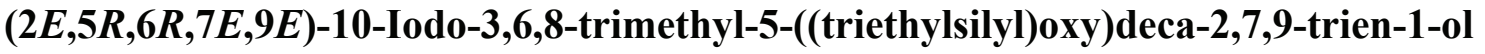
(195)

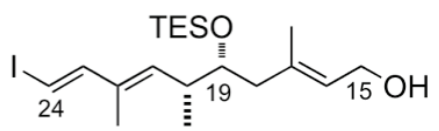

To a stirred solution of enoate $194(330 \mathrm{mg}, 0.689 \mathrm{mmol})$ in THF $(40.0 \mathrm{~mL})$ at $-78{ }^{\circ} \mathrm{C}$ was added DIBAL-H (1.4 mL, 1M soln. in hexane, $1.38 \mathrm{mmol})$. After $1 \mathrm{~h}$ at this temerature the mixture was added via cannula into a stirred solution of potassium sodium tartrate $(50.0$ $\mathrm{mL})$ salt, further stirred vigorously for $1 \mathrm{~h}$ and extracted with $\mathrm{Et}_{2} \mathrm{O}(3 \times 100 \mathrm{~mL})$. The combined organic extracts were dried $\left(\mathrm{MgSO}_{4}\right)$, filtered and concentrated under reduced pressure. Flash column chromatography (10\% EtOAc/Hexanes) afforded alcohol 195 (250 $\mathrm{mg}, 81 \%)$ as a colourless oil.

$\boldsymbol{R}_{f} 0.29$ (20\% EtOAc/Hexanes); $[\alpha]_{\mathrm{D}}^{20}+18.5$ (c $\left.0.7, \mathrm{CHCl}_{3}\right) ; \mathbf{I R} \boldsymbol{v}_{\max }\left(\right.$ thin film $\left./ \mathrm{cm}^{-1}\right) 3342$, 2955, 2911, 1457, 1003, 948, 744, 725; ${ }^{1} \mathbf{H}$ NMR (400 MHz, $\left.\mathrm{C}_{6} \mathrm{D}_{6}\right) \delta 7.07(1 \mathrm{H}, \mathrm{dd}, J=$ 14.6, $0.8 \mathrm{~Hz}, \mathrm{H}-23), 5.93$ (1H, dd, $J=14.6,0.6 \mathrm{~Hz}, \mathrm{H}-24), 5.38$ (1H, ddd, $J=7.8,5.4,1.2$ Hz, H-16), 5.27 (1H, d, $J=9.8$ Hz, H-21), $3.94(2 \mathrm{H}, \mathrm{d}, J=6.8 \mathrm{~Hz}, \mathrm{H}-15), 3.67$ (1H, td, $J=$ 6.2, 4.7 Hz, H-19), 2.50 (1H, ddd, $J=9.8,6.8,4.7, \mathrm{H}-20), 2.14$ (2H, qd, $J=13.6,6.2 \mathrm{~Hz}$, 
$\mathrm{H}-18), 1.51\left(3 \mathrm{H}, \mathrm{t}, J=0.6 \mathrm{~Hz}, \mathrm{C} 17-\mathrm{CH}_{3}\right), 1.41\left(3 \mathrm{H}, \mathrm{d}, J=1.3 \mathrm{~Hz}, \mathrm{C} 22-\mathrm{CH}_{3}\right), 0.97-1.01$ $\left.\left(6 \mathrm{H}, \mathrm{m}, 3 \times \mathrm{Si}-\mathrm{C}_{2} \mathrm{CH}_{3}\right)_{3}, \mathrm{C} 20-\mathrm{CH}_{3}\right), 0.58\left(9 \mathrm{H}, \mathrm{m}, 3 \times \mathrm{Si}_{-} \mathrm{CH}_{2} \mathrm{CH}_{3}\right) ;{ }^{13} \mathbf{C}$ NMR $(100 \mathrm{MHz}$, $\left.\mathrm{C}_{6} \mathrm{D}_{6}\right) \delta 150.0,138.3,135.0,133.9,129.0,74.7,74.2,59.3,45.9,37.8,16.9,15.1,12.1$, 7.3, 5.6; HRMS $\left(\mathrm{ES}^{+}\right)$calcd. for $\mathrm{C}_{19} \mathrm{H}_{40} \mathrm{INO}_{2} \mathrm{Si}\left[\mathrm{M}+\mathrm{NH}_{4}\right]^{+} 468.1789$, found 468.1787

\section{5-(((2E,5R,6R,7E,9E)-10-Iodo-3,6,8-trimethyl-5-((triethylsilyl)oxy)deca-2,7,9-trien-1- yl)thio)-1-phenyl-1H-tetrazole (196)}

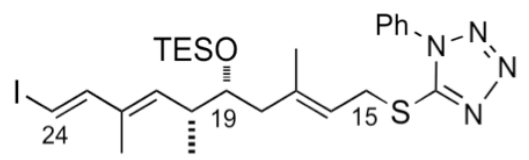

To a stirred solution of alcohol $195(130 \mathrm{mg}, 0.288 \mathrm{mmol})$, in THF $(30.0 \mathrm{~mL})$ at $0{ }^{\circ} \mathrm{C}$ was added $1 H$-mercaptophenyltetrazole $(66.0 \mathrm{mg}, 0.378 \mathrm{mmol}), \mathrm{PPh}_{3}(99.0 \mathrm{mg}, 0.378 \mathrm{mmol})$ followed by DIAD $(74.0 \mu \mathrm{L}, 0.378 \mathrm{mmol})$ dropwise. The reaction mixture was allowed to warm to rt for $1.5 \mathrm{~h}$, before a saturated $\mathrm{NH}_{4} \mathrm{Cl}(20.0 \mathrm{~mL})$ solution and $\mathrm{Et}_{2} \mathrm{O}(30.0 \mathrm{~mL})$ were added. The layers were separated and the aqueous phase was extracted with $\mathrm{Et}_{2} \mathrm{O}(3 \times 50$ $\mathrm{mL})$. The combined organic extracts were dried $\left(\mathrm{Na}_{2} \mathrm{SO}_{4}\right)$, filtered and concentrated under reduced pressure. Purification by flash column chromatography (10\% EtOAc/Hexanes) gave sulfide $196(140 \mathrm{mg}, 80 \%)$ as a colourless oil.

$\boldsymbol{R}_{f} 0.62\left(10 \%\right.$ EtOAc/Hexanes) $[\alpha]_{\mathrm{D}}^{20}+58.5\left(c 1.0, \mathrm{CHCl}_{3}\right)$; IR $\boldsymbol{v}_{\max }\left(\right.$ thin film $\left./ \mathrm{cm}^{-1}\right) 3068$, 2955, 2911, 2875, 1597, 1500, 1384, 1234, 1092, 1015, 950, 759, 742, 725; ${ }^{1}$ H NMR (400 MHz, $\left.\mathrm{C}_{6} \mathrm{D}_{6}\right) \delta$ 7.17-7.19 (2H, m, Ar- $\left.\underline{\mathrm{H}}\right), 7.05(1 \mathrm{H}, \mathrm{dd}, J=14.6,0.7 \mathrm{~Hz}, \mathrm{H}-23), 6.87-6.93$ $(3 \mathrm{H}, \mathrm{m}, \mathrm{Ar}-\underline{\mathrm{H}}), 5.95$ (1H, dd, $J=14.6,0.5 \mathrm{~Hz}, \mathrm{H}-24), 5.37$ (1H, td, $J=7.9,1.1 \mathrm{~Hz}, \mathrm{H}-16)$, $5.22(1 \mathrm{H}, \mathrm{dt}, J=9.8,0.5 \mathrm{~Hz}, \mathrm{H}-21), 3.90(2 \mathrm{H}, \mathrm{qd}, J=14.6,7.9 \mathrm{~Hz}, \mathrm{H}-15), 3.62(1 \mathrm{H}, \mathrm{td}, J$ = 6.2, $4.7 \mathrm{~Hz}, \mathrm{H}-19), 2.42$ (1H, ddd, $J=9.7,6.8,4.6 \mathrm{~Hz}, \mathrm{H}-20), 2.07$ (2H, ddt, $J=20.2$, $13.9,6.6 \mathrm{~Hz}, \mathrm{H}-18), 1.58\left(3 \mathrm{H}, \mathrm{d}, J=1.3 \mathrm{~Hz}, \mathrm{C} 17-\underline{\mathrm{C}}_{3}\right), 1.40(3 \mathrm{H}, \mathrm{d}, J=1.2 \mathrm{~Hz}, \mathrm{C} 22-$ $\left.\mathrm{C}_{3}\right), 0.96\left(6 \mathrm{H}, \mathrm{s}, 3 \times \mathrm{Si}-\mathrm{C}_{2} \mathrm{CH}_{3}\right), 0.92\left(3 \mathrm{H}, \mathrm{d}, J=6.8 \mathrm{~Hz}, \mathrm{C} 20-\underline{\mathrm{H}}_{3}\right), 0.53-0.58(9 \mathrm{H}, \mathrm{m}$, $\left.3 \times \mathrm{Si}-\mathrm{CH}_{2} \mathrm{CH}_{3}\right) ;{ }^{13} \mathbf{C}$ NMR $\left(100 \mathrm{MHz}, \mathrm{C}_{6} \mathrm{D}_{6}\right) \delta 154.1,150.0,139.8,138.0,134.3,134.0$, 129.64, 129.61, 123.9, 120.8, 74.5, 74.3, 45.8, 37.7, 31.6, 16.9, 15.0, 12.1, 7.3, 5.6; HRMS (ASAP) calcd. for $\mathrm{C}_{26} \mathrm{H}_{40} \mathrm{IN}_{4} \mathrm{OSSi}[\mathrm{M}+\mathrm{H}]^{+}$611.1731, found 611.1729 . 
5-(((2E,5R,6R,7E,9E)-10-Iodo-3,6,8-trimethyl-5-((triethylsilyl)oxy)deca-2,7,9-trien-1yl)sulfonyl)-1-phenyl-1H-tetrazole (171)

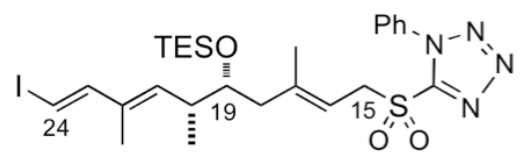

A mixture of ammonium molybdate $(1.6 \mathrm{mg}, 1.31 \mu \mathrm{mol})$, hydrogen peroxide $(0.15 \mathrm{~mL}$, $1.31 \mathrm{mmol}, 30 \% \mathrm{v} / \mathrm{v})$ and HMPA $(0.15 \mathrm{~mL})$ was stirred at $0{ }^{\circ} \mathrm{C}$ for $15 \mathrm{~min}$. The resulting bright yellow solution was added dropwise to a solution of sulfide 196 (80.0 mg, 0.131 mmol $)$ in $\mathrm{MeOH}(2.0 \mathrm{~mL})$ at $0{ }^{\circ} \mathrm{C}$. After $21 \mathrm{~h}$, the reaction mixture was diluted with $\mathrm{MeOH}$ (2.0 mL), and $\mathrm{H}_{2} \mathrm{O}(10.0 \mathrm{~mL})$ was added and the layers were separated. The aqueous phase was extracted with $\mathrm{CH}_{2} \mathrm{Cl}_{2}(3 \times 10 \mathrm{~mL})$ and the combined organic extracts were dried $\left(\mathrm{Na}_{2} \mathrm{SO}_{4}\right)$, filtered and concentrated under reduced pressure. Purification by flash column chromatography (5\% Acetone/Hexanes) provided sulfone $171(79.0 \mathrm{mg}, 95 \%)$ as a colourless oil.

$\boldsymbol{R}_{\boldsymbol{f}} 0.43\left(20 \%\right.$ Acetone/Hexanes); $[\alpha]_{D}^{20}+33.6\left(c \quad 0.5, \mathrm{CHCl}_{3}\right) ; \mathbf{I R} \boldsymbol{v}_{\max }$ (thin film $/ \mathrm{cm}^{-1}$ ) 3069, 2955, 2925, 2875, 1498, 1461, 1343, 1156, 1107, 1015, 950, 761, 739; ${ }^{1} \mathbf{H}$ NMR (400 MHz, $\left.\mathrm{C}_{6} \mathrm{D}_{6}\right) \delta$ 7.26-7.33 (2H, m, Ar- $\left.\underline{\mathrm{H}}\right), 7.05$ (1H, dd, $\left.J=14.6,0.8 \mathrm{~Hz}, \mathrm{H}-23\right), 6.85-$ $6.86(3 \mathrm{H}, \mathrm{m}, \mathrm{Ar}-\underline{\mathrm{H}}), 5.94$ (2H, dd, $J=14.6,0.5 \mathrm{~Hz}, \mathrm{H}-24), 5.29$ (1H, td, $J=7.9,0.9 \mathrm{~Hz}, \mathrm{H}-$ 16), $5.22(1 \mathrm{H}, \mathrm{d}, J=9.3 \mathrm{~Hz}, \mathrm{H}-21), 4.08(2 \mathrm{H}, \mathrm{dd}, J=7.9,3.0 \mathrm{~Hz}, \mathrm{H}-15), 3.62(1 \mathrm{H}, \mathrm{td}, J=$ 6.5, 4.1 Hz, H-19), 2.38-2.35 (1H, m, H-20), 2.10 (1H, dd, $J=13.6,6.9$ Hz, H-18a), 2.01 $(1 \mathrm{H}, \mathrm{dd}, J=13.6,6.1 \mathrm{~Hz}, \mathrm{H}-18 \mathrm{~b}), 1.50\left(3 \mathrm{H}, \mathrm{d}, J=1.2 \mathrm{~Hz}, \mathrm{C} 17-\mathrm{C}_{3}\right), 1.39$ (3H, d, $J=1.2$ $\left.\mathrm{Hz}, \mathrm{C} 22-\underline{\mathrm{C}}_{3}\right), 0.88-1.01\left(6 \mathrm{H}, \mathrm{m}, 3 \times \mathrm{Si}-\mathrm{C}_{2} \mathrm{CH}_{3}\right), 0.86\left(3 \mathrm{H}, \mathrm{d}, J=6.7 \mathrm{~Hz}, \mathrm{C} 20-\underline{\mathrm{H}}_{3}\right)$, 0.46-0.60 (9H, m, $3 \times$ Si-C $\left.\underline{H}_{2} \mathrm{CH}_{3}\right) ;{ }^{13} \mathbf{C}$ NMR $\left(100 \mathrm{MHz}, \mathrm{C}_{6} \mathrm{D}_{6}\right) \delta 154.0,149.8,146.8$, 136.6, 134.8, 133.5, 131.0, 129.4, 125.4, 111.0, 74.6, 72.6, 56.2, 45.9, 39.2, 17.0, 16.3, 12.1, 7.2, 6.9; HRMS $\left(\mathrm{ES}^{+}\right)$calcd. for $\mathrm{C}_{26} \mathrm{H}_{40} \mathrm{IN}_{5} \mathrm{O}_{3} \mathrm{SSi}\left[\mathrm{M}+\mathrm{NH}_{4}\right]^{+} 660.1895$, found 660.1893 . 


\subsection{Experimental procedures for Chapter 5}

(((1E,3E,5R,6R,8E,10E)-16-(Benzyloxy)-1-iodo-3,5,8-trimethylhexadeca-1,3,8,10tetraen-6-yl)oxy)triethylsilane (209)

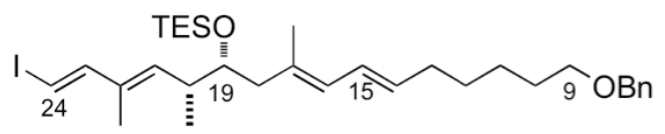

To a solution of sulfone $171(10.0 \mathrm{mg}, 15.7 \mu \mathrm{mol})$ in THF $(1.0 \mathrm{~mL})$ at $-78{ }^{\circ} \mathrm{C}$ was added NaHMDS (12 $\mu \mathrm{L}, 2 \mathrm{M}$ soln. in THF, $12.5 \mu \mathrm{mol})$. After $5 \mathrm{~min}$, a solution of aldehyde 208 (3.0 mg, $10.5 \mu \mathrm{mol})$ in THF $(0.5 \mathrm{~mL})$ was added dropwise and the reaction mixture was allowed to warm to $-60{ }^{\circ} \mathrm{C}$. After $1 \mathrm{~h}, \mathrm{MeOH}(0.5 \mathrm{~mL})$ was added followed by saturated $\mathrm{NH}_{4} \mathrm{Cl}(5.0 \mathrm{~mL})$ solution and the reaction mixture was warmed to $\mathrm{rt}$. The layers were separated and the aqueous phase was extracted with $\mathrm{Et}_{2} \mathrm{O}(3 \times 5 \mathrm{~mL})$. The combined organic extracts were washed with brine $(10 \mathrm{~mL})$, dried $\left(\mathrm{Na}_{2} \mathrm{SO}_{4}\right)$, filtered and concentrated under reduced pressure. Purification by flash column chromatography $(5 \%$ EtOAc/Hexanes) provided alkene $\mathbf{2 0 9}(5.0 \mathrm{mg}, 80 \%)$ as a colourless oil, in 2:1 E:Z ratio. $\boldsymbol{R}_{\boldsymbol{f}} 0.65$ (10\% EtOAc/Hexanes); IR $\boldsymbol{v}_{\max }$ (thin film/ $\mathrm{cm}^{-1}$ ) 3367, 3184, 2919, 1675, 1277, 1181, 1106, 676, 611; ${ }^{1} \mathbf{H}$ NMR (400 MHz, $\left.\mathrm{C}_{6} \mathrm{D}_{6}\right) \delta 7.32(2 \mathrm{H}, \mathrm{d}, J=6.9 \mathrm{~Hz}, \mathrm{Ar}-\underline{\mathrm{H}}), 7.20$ (3H, m, Ar- $\underline{\mathrm{H}}), 7.08$ (1H, dd, $J=14.6,0.8 \mathrm{~Hz}, \mathrm{H}-23), 6.35$ (1H, ddt, $J=14.9,10.7,1.4 \mathrm{~Hz}$, H-15), 5.97 (1H, d, $J=11.0 \mathrm{~Hz}, \mathrm{H}-16), 5.93$ (1H, dd, $J=14.6,0.6 \mathrm{~Hz}, \mathrm{H}-24), 5.61$ (1H, dt, $J=14.8,7.2 \mathrm{~Hz}, \mathrm{H}-14), 5.29(1 \mathrm{H}, \mathrm{d}, J=10.4 \mathrm{~Hz}, \mathrm{H}-21), 4.35\left(2 \mathrm{H}, \mathrm{s}, \mathrm{C}_{\mathrm{a}} \underline{\mathrm{H}}_{\mathrm{b}} \mathrm{Ar}\right), 3.73(1 \mathrm{H}$, td, $J=6.2,4.8$ Hz, H-19), 3.34-3.28 (2H, m, H-9), 2.57-2.50 (1H, m, H-20), 2.29-2.17 (2H, m, H-18), 2.09-2.04 (2H, m, H-13), 1.72 (3H, d, $J=0.9$ Hz, C17-C $\left.\underline{H}_{3}\right), 1.61-1.55$ (2H, m, $\mathrm{H}-10), 1.42$ (3H, d, $\left.J=1.3 \mathrm{~Hz}, \mathrm{C} 22-\mathrm{CH}_{3}\right), 1.33-1.40$ (4H, m, H-11, H-12), 0.97-1.05 (9H, m, $\left.\left.3 \times \mathrm{Si}-\underline{\mathrm{C}}_{2} \mathrm{CH}_{3}\right)_{3}, \mathrm{C} 20-\underline{\mathrm{H}}_{3}\right), 0.57-0.65\left(9 \mathrm{H}, \mathrm{m}, 3 \times \mathrm{Si}_{-} \mathrm{CH}_{2} \mathrm{CH}_{3}\right) ;{ }^{13} \mathbf{C}$ NMR $(100 \mathrm{MHz}$, $\left.\mathrm{C}_{6} \mathrm{D}_{6}\right) \delta 150.1,138.4,133.9,133.4,132.4,128.7,128.6,127.7,127.6,127.1,125.0,75.0$, 74.0, 73.0, 70.5, 46.4, 38.0, 33.4, 30.2, 29.9, 26.3, 17.3, 15.3, 12.1, 7.4, 5.6; HRMS (ES $\left.{ }^{+}\right)$ calcd. for $\mathrm{C}_{32} \mathrm{H}_{55} \mathrm{INO}_{2} \mathrm{Si}\left[\mathrm{M}+\mathrm{NH}_{4}\right]^{+}$640.3041, found 640.3038 . 


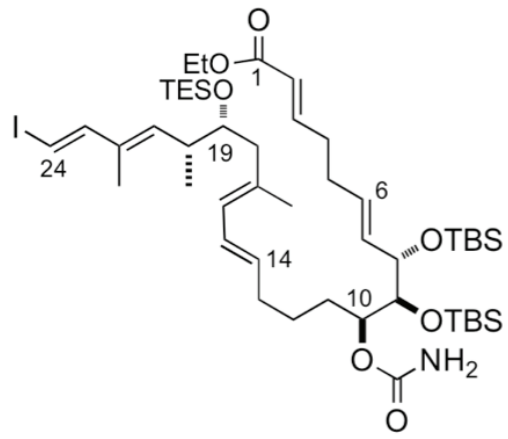

To a solution of sulfone $171(57.0 \mathrm{mg}, 89 \mu \mathrm{mol})$ in THF $(3.0 \mathrm{~mL})$ at $-78{ }^{\circ} \mathrm{C}$ was added NaHMDS $(36.0 \mu \mathrm{L}, 2 \mathrm{M}$ in THF, $71.7 \mu \mathrm{mol})$. After $5 \mathrm{~min}$, a solution of aldehyde $\mathbf{1 6 9}$ $(35.0 \mathrm{mg}, 59.7 \mu \mathrm{mol})$ in THF $(2.0 \mathrm{~mL})$ was added dropwise and the reaction mixture was allowed to warm to $-50{ }^{\circ} \mathrm{C}$. After $1 \mathrm{~h}, \mathrm{MeOH}(1.0 \mathrm{~mL})$ was added followed by saturated $\mathrm{NH}_{4} \mathrm{Cl}(30.0 \mathrm{~mL})$ solution and the reaction mixture was warmed to $\mathrm{rt}$. The layers were separated and the aqueous phase was extracted with $\mathrm{Et}_{2} \mathrm{O}(3 \times 20 \mathrm{~mL})$. The combined organic extracts were washed with brine $(30 \mathrm{~mL})$, dried $\left(\mathrm{Na}_{2} \mathrm{SO}_{4}\right)$, filtered and concentrated under reduced pressure. Purification by flash column chromatography (5\% EtOAc/Hexanes) provided alkene $210(46.0 \mathrm{mg}, \mathbf{7 8 \%})$ as a colourless oil in 7:1 E:Z ratio. $\boldsymbol{R}_{\boldsymbol{f}} 0.65\left(10 \%\right.$ EtOAc/Hexanes); $[\alpha]_{\mathrm{D}}^{20}+35.0\left(c\right.$ 3.5, $\left.\mathrm{CHCl}_{3}\right)$; IR $\boldsymbol{v}_{\max }\left(\right.$ thin film $\left./ \mathrm{cm}^{-1}\right) 2953$, 2911, 2876, 1720, 1647, 1435, 1223, 1150, 1087, 1008, 739, 725; ${ }^{1} \mathbf{H}$ NMR (400 MHz, $\left.\mathrm{C}_{6} \mathrm{D}_{6}\right) \delta 7.08(1 \mathrm{H}, \mathrm{dd}, J=14.6,0.7 \mathrm{~Hz}, \mathrm{H}-23), 7.00(1 \mathrm{H}, \mathrm{dt}, J=15.6,6.5 \mathrm{~Hz}, \mathrm{H}-3), 6.37$ (1H, dd, $J=15.0,10.8 \mathrm{~Hz}, \mathrm{H}-15), 5.99-5.95$ (1H, m, H-14), 5.95 (1H, dd, $J=14.6,0.5 \mathrm{~Hz}$, H-24), 5.89-5.84 (1H, m, H-2), 5.75 (1H, dd, $J=15.6,5.8$ Hz, H-7), 5.67-5.57 (2H, m, H6, H-16), 5.32 (1H, d, $J=9.7 \mathrm{~Hz}, \mathrm{H}-21), 5.27-5.23(1 \mathrm{H}, \mathrm{m}, \mathrm{H}-10), 4.28$ (1H, t, $J=5.3 \mathrm{~Hz}$, $\mathrm{H}-8), 4.07$ (2H, q, $\left.J=7.1 \mathrm{~Hz}, \mathrm{OC}_{2} \mathrm{CH}_{3}\right), 4.03(1 \mathrm{H}, \mathrm{dd}, J=5.0,2.1 \mathrm{~Hz}, \mathrm{H}-9), 3.74(1 \mathrm{H}, \mathrm{td}$, $J=6.2,4.6$ Hz, H-19), 2.55 (1H, m, H-20), 2.22 (2H, m, H-18), 2.00-1.94 (4H, m, H-4, H5), 1.91-1.80 (2H, m, H-11), $1.72\left(3 \mathrm{H}, \mathrm{d}, J=0.8 \mathrm{~Hz}, \mathrm{C} 17-\mathrm{C}_{3}\right), 1.76-1.65$ (2H, m, H-13), 1.60-1.51 (2H, m, H-12), $1.44\left(3 \mathrm{H}, \mathrm{d}, J=1.2 \mathrm{~Hz}, \mathrm{C} 22-\mathrm{C}_{3}\right), 1.06\left(9 \mathrm{H}, \mathrm{s}, \mathrm{Si}-\mathrm{C}\left(\mathrm{C}_{3}\right)_{3}\right)$, 1.05 (9H, s, Si-C(C $\left.\left.\underline{\mathrm{C}}_{3}\right)_{3}\right), 1.06-0.98\left(12 \mathrm{H}, \mathrm{m}, \mathrm{C} 20-\mathrm{CH}_{3}, \mathrm{OCH}_{2} \mathrm{CH}_{3}, \underline{\mathrm{Si}}-\left(\mathrm{CH}_{2} \mathrm{CH}_{3}\right)_{3}\right), 0.64-$ $0.57\left(9 \mathrm{H}, \mathrm{m}, \mathrm{Si}-\left(\mathrm{CH}_{2} \mathrm{C}_{3}\right)_{3}\right), 0.20\left(3 \mathrm{H}, \mathrm{s}, \mathrm{Si}-\mathrm{C}_{3}\right), 0.19\left(3 \mathrm{H}, \mathrm{s}, \mathrm{Si}-\underline{\mathrm{H}}_{3}\right), 0.14$ (3H, s, Si$\left.\mathrm{C}_{3}\right), 0.13\left(3 \mathrm{H}, \mathrm{s}, \mathrm{Si}-\underline{\mathrm{C}}_{3}\right) ;{ }^{13} \mathrm{C}$ NMR $\left(100 \mathrm{MHz}, \mathrm{C}_{6} \mathrm{D}_{6}\right) \delta 166.0,156.6,150.1,148.0$, 
$138.5,133.8,133.2,132.5,131.3,130.0,128.7,127.3,122.4,78.2,75.58,75.56,74.9$, 74.0, 60.0, 46.4, 37.8, 33.4, 32.0, 31.0, 29.8, 26.4, 26.32, 26.26, 18.62, 18.56, 17.3, 15.1, 14.4, 12.1, 7.4, 5.6, -4.1, -4.18, -4.22, -4.3; HRMS $\left(\mathrm{ES}^{+}\right)$calcd. for $\mathrm{C}_{48} \mathrm{H}_{92} \mathrm{IN}_{2} \mathrm{O}_{7} \mathrm{Si}_{3}$ $\left[\mathrm{M}+\mathrm{NH}_{4}\right]^{+} 1019.5252$, found 1019.5258 .

(2E,6E,8S,9S,10S,14E,16E,19R,20R,21E,23E)-Ethyl 8,9-bis((tert-butyldimethylsilyl)oxy)-10-(carbamoyloxy)-19-hydroxy-24-iodo-17,20,22-trimethyltetracosa-

2,6,14,16,21,23-hexaenoate (211)

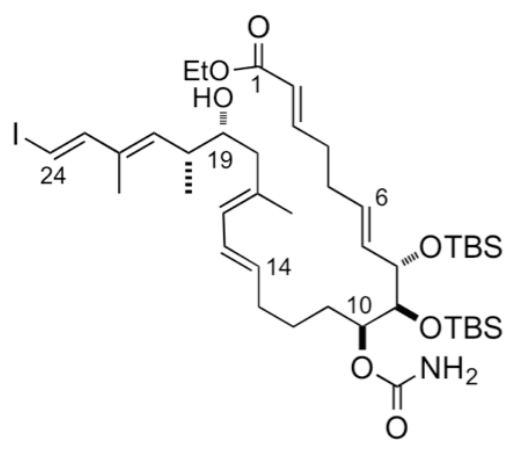

To a solution of $210(5.0 \mathrm{mg}, 0.499 \mu \mathrm{mol})$ in THF $(2.0 \mathrm{~mL})$ at $0{ }^{\circ} \mathrm{C}$ was added a buffered solution of pyridine hydrofluoride $(0.2 \mathrm{~mL}$, stock solution prepared from $0.5 \mathrm{~mL}$ of pyridine hydrofluoride, $1.0 \mathrm{~mL}$ of pyridine, and $4.0 \mathrm{~mL}$ of THF) dropwise. After stirring for $1 \mathrm{~h}$, the reaction was quenched by the addition of saturated $\mathrm{NaHCO}_{3}(5.0 \mathrm{~mL})$ solution, diluted with $\mathrm{Et}_{2} \mathrm{O}(15.0 \mathrm{~mL})$ and brine $(10.0 \mathrm{~mL})$, and finally extracted with $\mathrm{Et}_{2} \mathrm{O}(3 \times 10$ $\mathrm{mL})$. The combined organic extracts were dried $\left(\mathrm{Na}_{2} \mathrm{SO}_{4}\right)$, filtered and concentrated under reduced pressure. Purification by flash column chromatography (10\% EtOAc/Hexanes) gave alcohol 211 (3.9 $\mathrm{mg}, 88 \%)$ as a colourless oil.

$\boldsymbol{R}_{\boldsymbol{f}} 0.30\left(20 \%\right.$ EtOAc/Hexane); $[\alpha]_{\mathrm{D}}^{20}+30.6\left(c\right.$ 1.0, $\left.\mathrm{CHCl}_{3}\right)$; IR $\boldsymbol{v}_{\max }\left(\right.$ thin film $\left./ \mathrm{cm}^{-1}\right) 3509$, $3370,2955,2975,2857,1723,1655,1472,1463,1255,1126,837,776 ;{ }^{1} \mathbf{H}$ NMR (400 $\left.\mathrm{MHz}, \mathrm{C}_{6} \mathrm{D}_{6}\right) \delta 7.04(1 \mathrm{H}, \mathrm{dt}, J=15.7,6.4 \mathrm{~Hz}, \mathrm{H}-3), 6.97(1 \mathrm{H}, \mathrm{dd}, J=14.6,0.7 \mathrm{~Hz}, \mathrm{H}-23)$, $6.34(1 \mathrm{H}, \mathrm{dd}, J=15.0,10.8 \mathrm{~Hz}, \mathrm{H}-15), 5.95-5.86$ (2H, m, H-2, H-16) 5.91 (1H, dd, $J=$ 14.6, 0.5 Hz, H-24), 5.76 (1H, dd, $J=15.5,5.6 \mathrm{~Hz}, \mathrm{H}-7), 5.66-5.59$ (2H, m, H-6, H-14), $5.28(1 \mathrm{H}, \mathrm{ddd}, J=9.3,3.2,2.3 \mathrm{~Hz}, \mathrm{H}-10), 5.10(1 \mathrm{H}, \mathrm{d}, J=9.9 \mathrm{~Hz}, \mathrm{H}-21), 4.29$ (1H, t, $J=$ $4.9 \mathrm{~Hz}, \mathrm{H}-8), 4.09-4.02(1 \mathrm{H}, \mathrm{m}, \mathrm{H}-9) 4.06\left(2 \mathrm{H}, J=7.1 \mathrm{~Hz}, \mathrm{OCH}_{2} \mathrm{CH}_{3}\right), 3.69(2 \mathrm{H}$, br s, $\left.\mathrm{N}_{2}\right), 3.38$ (1H, ddd, $\left.J=9.7,7.0,2.7 \mathrm{~Hz}, \mathrm{H}-19\right), 2.41(1 \mathrm{H}, \mathrm{dt}, J=10.0,6.7 \mathrm{~Hz}, \mathrm{H}-20)$, 2.22-2.17 (2H, m, H-13), 2.14 (1H, dd, $J=13.8,2.4$ Hz, H-18a), 2.04-1.94 (4H, m, H-4, 
H-5), 1.91 (1H, dd, $J=13.9,10.0$ Hz, H-18b), 1.89-1.82 (2H, m, H-11), 1.70-1.57 (5 H, m, $\mathrm{H}-12), 1.62\left(3 \mathrm{H}, \mathrm{s}, \mathrm{C} 17-\underline{\mathrm{H}}_{3}\right), 1.40\left(3 \mathrm{H}, \mathrm{d}, J=1.2 \mathrm{~Hz}, \mathrm{C} 22-\underline{\mathrm{C}}_{3}\right), 1.08-1.05\left(3 \mathrm{H}, \mathrm{m}_{\mathrm{obs}}\right.$, C20- $\left.\underline{\mathrm{H}}_{3}\right), 1.07\left(9 \mathrm{H}, \mathrm{s}, \mathrm{Si}-\mathrm{C}\left(\mathrm{CH}_{3}\right)_{3}\right), 1.06\left(9 \mathrm{H}, \mathrm{s}, \mathrm{Si}-\mathrm{C}\left(\mathrm{C}_{3}\right)_{3}\right), 1.03(3 \mathrm{H}, \mathrm{t}, J=7.1 \mathrm{~Hz}$,

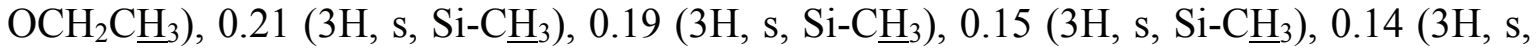
$\left.\mathrm{Si}-\mathrm{CH}_{3}\right) ;{ }^{13} \mathbf{C}$ NMR $\left(100 \mathrm{MHz}, \mathrm{C}_{6} \mathrm{D}_{6}\right) \delta 166.1,156.3,150.1,148.2,137.4,134.5,133.6$, $132.9,131.3,130.0,128.7,127.2,122.4,78.2$, 75.5, 75.4, 74.2, 72.8, 60.1, 46.3, 39.3, 33.3, $32.0,31.0,29.6,26.3,26.2,26.13,18.62,18.55,16.7,16.51,14.4,12.1,-4.15,-4.19,-4.23$, -4.26; HRMS $\left(\mathrm{ES}^{+}\right)$calcd. for $\mathrm{C}_{42} \mathrm{H}_{78} \mathrm{IN}_{2} \mathrm{O}_{7} \mathrm{Si}_{2}\left[\mathrm{M}+\mathrm{NH}_{4}\right]^{+}$905.4387, found 905.4394.

(2E,6E,8S,9S,10S,14E,16E,19R,20R,21E,23E)-8,9-Bis((tert-butyldimethylsilyl)oxy)-10(carbamoyloxy)-19-hydroxy-24-iodo-17,20,22-trimethyltetracosa-2,6,14,16,21,23hexaenoic acid (212)

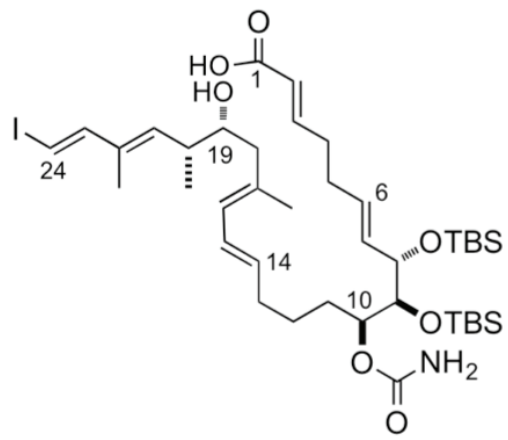

To a solution of ethyl ester $211(14.0 \mathrm{mg}, 15.7 \mu \mathrm{mol})$ in $\mathrm{Et}_{2} \mathrm{O}(1.0 \mathrm{ml})$ was added potassium trimethylsilanolate $(6.1 \mathrm{mg}, 47.3 \mu \mathrm{mol})$ in one portion. The slightly yellowish solution was stirred at $\mathrm{rt}$ for $24 \mathrm{~h}$ and then quenched by addition of $\mathrm{NaHSO}_{4}(100 \mu \mathrm{L}, 0.1$ M), buffered with $\mathrm{Na}_{2} \mathrm{SO}_{4}$, filtered and concentrated under reduced pressure. The resulting crude residue was dried under high vacuum and immediately used in the next step.

$\boldsymbol{R}_{\boldsymbol{f}} 0.23$ (30\% EtOAc/Hexane and drop of $\left.\mathrm{AcOH}\right) ;{ }^{1} \mathbf{H}$ NMR $\left(400 \mathrm{MHz}, \mathrm{C}_{6} \mathrm{D}_{6}\right) \delta 7.14-7.08$ (1H, m, H-3), 7.00 (1 H, dd, $J=14.6,0.8 \mathrm{~Hz}, \mathrm{H}-23), 6.42-6.32$ (1H, m, H-15), 6.15 (1H, d, $J=15.5 \mathrm{~Hz}, \mathrm{H}-2), 6.00$ (1H, d, $J=10.8 \mathrm{~Hz}, \mathrm{H}-16), 5.92$ (1H, d, $J=14.6 \mathrm{~Hz}, \mathrm{H}-24), 5.81-$ $5.74(1 \mathrm{H}, \mathrm{m}, \mathrm{H}-7), 5.71-5.67(1 \mathrm{H}, \mathrm{m}, \mathrm{H}-6), 5.61(1 \mathrm{H}, \mathrm{dd}, J=14.7,7.4 \mathrm{~Hz}, \mathrm{H}-14), 5.30-$ 5.27 (1H, m, H-10), 5.20 (1H, d, J=10.6 Hz, H-21), 4.31-4.26 (1H, m, H-8), 4.06 (1H, dd, $J=5.3,2.2 \mathrm{~Hz}, \mathrm{H}-9), 3.53-3.46$ (1H, m, H-19), 2.52-2.43 (1H, m, H-20), 2.24-2.14 (3H, m, H-13, H-18a), 2.15-2.08 (2H, m, H-4), 2.08-2.00 (3H, m, H-5, H-18b), 1.91-1.82 (2H, m, $\mathrm{H}-11), 1.69$ (3H, s, C17-C $\left.\underline{H}_{3}\right), 1.68-1.66(2 \mathrm{H}, \mathrm{m}, \mathrm{H}-12), 1.43(3 \mathrm{H}, \mathrm{d}, J=1.2 \mathrm{~Hz}, \mathrm{C} 22-$ 
$\left.\mathrm{CH}_{3}\right), 1.12\left(3 \mathrm{H}, \mathrm{d}, J=6.7 \mathrm{~Hz}, \mathrm{C} 20-\mathrm{CH}_{3}\right), 1.08\left(9 \mathrm{H}, \mathrm{s}, \mathrm{Si}-\mathrm{C}\left(\mathrm{CH}_{3}\right)_{3}\right), 1.07$ (9H, s, Si$\left.\mathrm{C}\left(\mathrm{C}_{3}\right)_{3}\right), 0.22\left(3 \mathrm{H}, \mathrm{s}, \mathrm{Si}-\underline{\mathrm{C}}_{3}\right), 0.20\left(3 \mathrm{H}, \mathrm{s}, \mathrm{Si}-\underline{\mathrm{C}}_{3}\right), 0.16\left(3 \mathrm{H}, \mathrm{s}, \mathrm{Si}-\mathrm{C}_{3}\right), 0.15(3 \mathrm{H}, \mathrm{s}, \mathrm{Si}-$ $\left.\mathrm{C}_{3}\right)$.

$(2 R, 4 E, 6 E, 11 S, 12 S, 13 S, 14 E, 18 E)-12,13-B i s((t e r t-b u t y l d i m e t h y l s i l y l) o x y)-2-((R, 3 E, 5 E)-$ 6-iodo-4-methylhexa-3,5-dien-2-yl)-4-methyl-20-oxooxacycloicosa-4,6,14,18-tetraen11-yl carbamate (213)

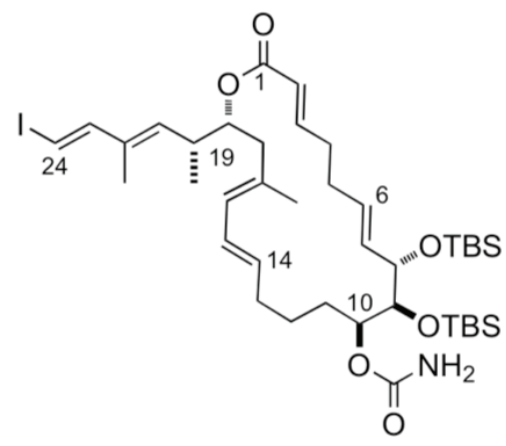

To a stirred solution of acid $212(13.0 \mathrm{mg}, 15.1 \mu \mathrm{mol})$ in toluene $(1.0 \mathrm{~mL})$ at $\mathrm{rt}$ was added $\mathrm{Et}_{3} \mathrm{~N}(42.0 \mu \mathrm{l}, 0.302 \mathrm{mmol})$ and 2,4,6-trichlorobenzoyl chloride $(35.0 \mu \mathrm{L}, 0.226 \mathrm{mmol})$. After $1 \mathrm{~h}$, the reaction mixture was diluted with toluene $(4.0 \mathrm{~mL})$ and added over $3 \mathrm{~h}$ to a stirred solution of DMAP $(74.0 \mathrm{mg}, 0.604 \mathrm{mmol})$ in toluene $(10.0 \mathrm{~mL})$. The reaction mixture was stirred for $12 \mathrm{~h}$ and then quenched with $\mathrm{NaHCO}_{3}(15.0 \mathrm{~mL})$ solution. The layers were separated and the aqueous layer extracted with $\mathrm{CH}_{2} \mathrm{Cl}_{2}(3 \times 15 \mathrm{~mL})$. The combined organic extracts were dried $\left(\mathrm{Na}_{2} \mathrm{SO}_{4}\right)$, filtered and concentrated under reduced pressure. Purification by flash column chromatography (10\% EtOAc/Hexanes) gave macrolactone $213(9.0 \mathrm{mg}, 71 \%)$ as a colourless oil.

$\boldsymbol{R}_{\boldsymbol{f}} 0.44\left(20 \%\right.$ EtOAc/Hexane); $[\alpha]_{\mathrm{D}}^{20}+1.2\left(c \quad 0.9, \mathrm{CHCl}_{3}\right) ; \mathbf{I R} \boldsymbol{v}_{\max }\left(\right.$ thin film $\left./ \mathrm{cm}^{-1}\right) 3510$, $3368,3017,2930,2858,1724,1652,1596,1465,1385,1256,1123,1034,973,837,776$; ${ }^{1}$ H NMR (400 MHz, $\left.\mathrm{C}_{6} \mathrm{D}_{6}\right) \delta$ 6.94-6.87 (1H, mobs, H-3), $6.93(1 \mathrm{H}, \mathrm{dd}, J=14.7,0.7 \mathrm{~Hz}, \mathrm{H}-$ 23), 6.27 (1H, dd, $J=15.5,11.1 \mathrm{~Hz}, \mathrm{H}-15), 5.96-5.83$ (2H, mobs, H-2, H-16), 5.91 (1H, dd, $J=14.6,0.5 \mathrm{~Hz}, \mathrm{H}-24), 5.68-5.63$ (2H, m, H-6, H-7), 5.53 (1H, ddd, $J=14.6,9.3,4.8 \mathrm{~Hz}$, H-14), 5.19 (1H, dt, $J=10.3,2.1 \mathrm{~Hz}, \mathrm{H}-10), 5.12$ (1H, ddd, $J=10.4,7.6,3.1 \mathrm{~Hz}, \mathrm{H}-19)$, $4.97(1 \mathrm{H}, \mathrm{d}, J=10.0 \mathrm{~Hz}, \mathrm{H}-21), 4.21-4.16(1 \mathrm{H}, \mathrm{m}, \mathrm{H}-8), 4.11(1 \mathrm{H}, \mathrm{dd}, J=6.9,1.8 \mathrm{~Hz}, \mathrm{H}-$ 9), 3.64 (2H, br s, $\left.\mathrm{NH}_{2}\right), 2.67-2.77$ (1H, m, H-20), 2.37-2.27 (1H, m, H-13a), 2.18-2.09 (2H, m, H-18), 2.00-1.89 (4H, m, H-4, H-5), 1.89-1.86 (1H, m, H-13b), 1.86-1.81 (2H, m, 
$\mathrm{H}-11), 1.80-1.66$ (2H, m, H-12), 1.65 (3H, s, C17-C $\left.\underline{H}_{3}\right), 1.36(3 \mathrm{H}, \mathrm{d}, J=1.2 \mathrm{~Hz}, \mathrm{C} 22-$ $\left.\mathrm{C}_{3}\right), 1.10\left(9 \mathrm{H}, \mathrm{s}, \mathrm{Si}-\mathrm{C}\left(\mathrm{CH}_{3}\right)_{3}\right), 1.04\left(9 \mathrm{H}, \mathrm{s}, \mathrm{Si}-\mathrm{C}\left(\mathrm{C}_{3}\right)_{3}\right), 0.95(3 \mathrm{H}, \mathrm{d}, J=6.7 \mathrm{~Hz}, \mathrm{C} 20-$ $\left.\mathrm{C}_{3}\right), 0.21\left(3 \mathrm{H}, \mathrm{s}, \mathrm{Si}-\underline{\mathrm{C}}_{3}\right), 0.21\left(3 \mathrm{H}, \mathrm{s}, \mathrm{Si}-\underline{\mathrm{C}}_{3}\right), 0.14\left(3 \mathrm{H}, \mathrm{s}, \mathrm{Si}-\mathrm{C}_{3}\right), 0.10$ (3H, s, Si$\left.\mathrm{CH}_{3}\right) .{ }^{13} \mathrm{C}$ NMR $\left(125 \mathrm{MHz}, \mathrm{C}_{6} \mathrm{D}_{6}\right) \delta 165.4,156.1,149.7,147.4,135.4,133.0,131.58$, 131.39, 130.3, 129.2, 128.1 (obs), 127.5, 123.3, 78.3, 76.6, 75.5, 74.9, 74.3, 43.4, 37.5, $32.16,31.98,31.1,30.2,26.5,26.4,25.2,18.71,18.66,17.2,16.6,12.1,-3.6,-3.7,-3.8,-$ 4.2; HRMS $\left(\mathrm{ES}^{+}\right)$calcd. for $\mathrm{C}_{40} \mathrm{H}_{68} \mathrm{INO}_{6} \mathrm{Si}_{2} \mathrm{Na}[\mathrm{M}+\mathrm{Na}]^{+}$864.3522, found 864.3528.

\section{$N$-((1E,3E,5R)-6-Hydroxy-3,5-dimethylhexa-1,3-dien-1-yl)-3-methylbut-2-enamide} (219)

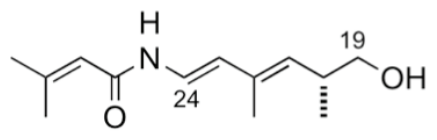

To a mixture of vinyl iodide 178 (100 mg, $0.396 \mathrm{mmol})$, amide 218 (78.6 mg, 0.793 mmol), flame-dried $\mathrm{K}_{2} \mathrm{CO}_{3}(328 \mathrm{mg}, 2.37 \mathrm{mmol})$ and $\mathrm{CuI}(113 \mathrm{mg}, 0.594 \mathrm{mmol})$ in $\mathrm{THF}$ $(20.0 \mathrm{~mL})$ at $\mathrm{rt}$ was added $N, N^{\prime}$-dimethylethylenediamine $(128 \mu \mathrm{L}, 1.18 \mathrm{mmol})$. The reaction mixture was stirred for $1 \mathrm{~h}$ before it was concentrated under reduced pressure. The resulting residue was diluted with EtOAc $(10.0 \mathrm{~mL})$, filtered through a pad of Celite ${ }^{\circledR}$, washed with EtOAc $(10.0 \mathrm{~mL})$ and concentrated under reduced pressure. Purification by flash column chromatography afforded enamide $219(50.0 \mathrm{mg}, 63 \%)$ as a colourless oil, in 5:1 E:Z ratio.

$\boldsymbol{R}_{\boldsymbol{f}} 0.56$ (10\% EtOAc/Hexane); IR $\boldsymbol{v}_{\max }\left(\right.$ thin film/ $\left.\mathrm{cm}^{-1}\right)$ 3425, 2982, 2935, 1718, 1655, $1315,1272,1157,1041,981 ;{ }^{1} \mathbf{H}$ NMR $\left(400 \mathrm{MHz}, \mathrm{CDCl}_{3}\right) \delta 7.78(1 \mathrm{H}, \mathrm{d}, J=10.4, \mathrm{~N}-\underline{\mathrm{H}})$, $6.99(1 \mathrm{H}, \mathrm{dd}, J=14.4,10.5 \mathrm{~Hz}, \mathrm{H}-23), 5.81(1 \mathrm{H}, \mathrm{dd}, J=14.5,0.6 \mathrm{~Hz}, \mathrm{H}-24), 5.60$ (1H, dt, $J=2.6,1.3 \mathrm{~Hz}, \mathrm{H}-27), 5.06$ (1H, d, $J=9.5 \mathrm{~Hz}, \mathrm{H}-21), 3.48-3.42$ (1H, m, H-19a), 3.38-3.32 (1H, m, H-19b), 2.74-2.66 (1H, m, H-20), 2.17 (3H, d, $J=1.2 \mathrm{~Hz}, \mathrm{H}-29), 1.84$ (3H, d, $J=$ $\left.1.2 \mathrm{~Hz},-\mathrm{CH}_{3}\right), 1.77\left(3 \mathrm{H}, \mathrm{d}, J=1.2, \mathrm{C} 22-\mathrm{CH}_{3}\right), 0.93\left(3 \mathrm{H}, \mathrm{d}, J=6.7 \mathrm{~Hz}, \mathrm{C} 20-\mathrm{CH}_{3}\right)$; ${ }^{13} \mathbf{C}$ NMR $\left(100 \mathrm{MHz}, \mathrm{CDCl}_{3}\right) \delta 164.0,153.9,133.9,131.9,121.5,117.8,117.5,67.8,35.7$, 27.5, 20.1, 17.2, 13.0; HRMS $\left(\mathrm{ES}^{+}\right.$) calcd. for $\mathrm{C}_{13} \mathrm{H}_{22} \mathrm{NO}_{2}[\mathrm{M}+\mathrm{H}]^{+}$224.1645, found 224.1646 . 
$(2 R, 4 E, 6 E, 11 S, 12 R, 13 S, 14 E, 18 E)-12,13-D i h y d r o x y-2-((R, 3 E, 5 E)-6-i o d o-4-$

methylhexa-3,5-dien-2-yl)-4-methyl-20-oxooxacycloicosa-4,6,14,18-tetraen-11-yl

carbamate (214)

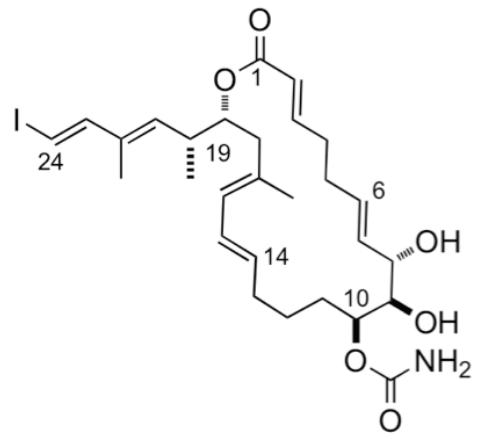

To a solution of bis-TBS-ether $213(9.00 \mathrm{mg}, 10.7 \mu \mathrm{mol})$ in THF $(1.0 \mathrm{~mL})$ at $0{ }^{\circ} \mathrm{C}$ was added TBAF $(54.0 \mu \mathrm{L}, 1 \mathrm{M}$ soln. in THF, $54.0 \mu \mathrm{mol})$. The reaction was stirred for $2 \mathrm{~h}$, quenched with cold water $(1.0 \mathrm{~mL})$ and the layers were separated. The aqueous phase was extracted with EtOAc $(3 \times 5 \mathrm{~mL})$ and the combined organic extracts were dried $\left(\mathrm{Na}_{2} \mathrm{SO}_{4}\right)$, filtered and concentrated under reduced pressure. Purification by flash column chromatography (50-100\% EtOAc/Pet.Ether) provided diol 214 (5.5 mg, 85\%) as a white flakes.

$\boldsymbol{R}_{\boldsymbol{f}} 0.39\left(5 \% \mathrm{MeOH} / \mathrm{CH}_{2} \mathrm{Cl}_{2}\right) ;[\alpha]_{\mathrm{D}}^{20}+9.6\left(c \quad 0.5, \mathrm{CHCl}_{3}\right)$; IR $\boldsymbol{v}_{\max }\left(\right.$ thin film $\left./ \mathrm{cm}^{-1}\right) 3432$, 2923, 2851, 1713, 1651, 1461, 1391, 1276, 1060, 1020, 972; ${ }^{1} \mathbf{H} \mathbf{N M R}\left(500 \mathrm{MHz}, \mathrm{C}_{6} \mathrm{D}_{6}\right) \delta$ $6.93(1 \mathrm{H}, \mathrm{d}, J=14.5 \mathrm{~Hz}, \mathrm{H}-23), 6.86(1 \mathrm{H}, \mathrm{dt}, J=15.6,5.9 \mathrm{~Hz}, \mathrm{H}-3), 6.33(1 \mathrm{H}, \mathrm{dd}, J=$ 14.9, 11.2 Hz, H-15), 5.96-5.92 (1H, m, H-16), 5.92 (1H, d, J=14.7 Hz, H-24), 5.83 (1H, d, $J=15.8 \mathrm{~Hz}, \mathrm{H}-2), 5.57$ (1H, dt, $J=14.6,5.4 \mathrm{~Hz}, \mathrm{H}-6), 5.47-5.39$ (2H, m, H-7, H-14), 5.19-5.13 (1H, m, H-19), 5.00-4.93 (2H, m, H-10, H-21), 4.17-3.98 (2H, br s, $\left.\underline{\mathrm{H}}_{2}\right), 3.91$ $(1 \mathrm{H}, \mathrm{t}, J=7.0 \mathrm{~Hz}, \mathrm{H}-8), 3.68$ (1H, br s, H-9), 2.69 (1H, dt, $J=10.1,7.0 \mathrm{~Hz}, \mathrm{H}-20), 2.30-$ 2.22 (1H, m, H-13a), 2.12-2.18 (2H, m, H-18), 1.91-1.97 (1H, m, H-13b), 1.89-1.77 (4H, m, H-4, H-5), 1.77-1.71 (1H, m, H-11a), 1.73 (3H, s, C17-C $\left.\underline{H}_{3}\right), 1.71-1.68$ (1H, m, H-11b), 1.67-1.62 (1H, m, H-12a), 1.35 (3H, d, $J=0.9$ Hz, C22-C $\left.\underline{H}_{3}\right), 1.34-1.28$ (1H, m, H-12b), $0.92\left(3 \mathrm{H}, \mathrm{d}, J=6.8 \mathrm{~Hz}, \mathrm{C} 20-\mathrm{CH}_{3}\right) ;{ }^{13} \mathbf{C}$ NMR $\left(125 \mathrm{MHz}, \mathrm{C}_{6} \mathrm{D}_{6}\right) \delta 165.6,157.0,149.5$, 147.9 , 135.3, 135.1, 132.3, 131.93, 131.90, 130.2, 128.4, 127.3, 122.9, 75.9, 75.4, 74.82, 74.72, 72.8, 42.8, 37.5, 31.8, 31.6, 30.3, 27.1, 24.7, 16.9, 16.6, 12.0; HRMS (ES ${ }^{+}$) calcd. for $\mathrm{C}_{28} \mathrm{H}_{40} \mathrm{INO}_{6} \mathrm{Na}[\mathrm{M}+\mathrm{Na}]^{+}$636.1785, found 636.1787. 


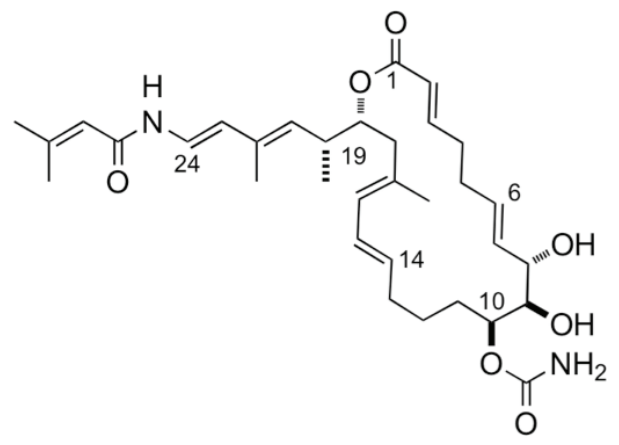

To a mixture of vinyl iodide 214 (4.0 mg, $6.52 \mu \mathrm{mol})$, amide 218 (1.9 mg, $19.5 \mu \mathrm{mol})$, $\mathrm{K}_{2} \mathrm{CO}_{3}(4.5 \mathrm{mg}, 32.6 \mu \mathrm{mol}$, flame-dried) and $\mathrm{CuI}(2.5 \mathrm{mg}, 13.0 \mu \mathrm{mol})$ in degassed DMF $(0.6 \mathrm{~mL})$ was added $N, N$ '-dimethylethylenediamine $(19.5 \mu \mathrm{L}, 0.01 \mathrm{M}$ soln. in DMF, 19.5 $\mu \mathrm{mol})$ at $\mathrm{rt}$. The reaction mixture was stirred for $1 \mathrm{~h}$ before it was diluted with EtOAc (5.0 $\mathrm{mL})$, filtered through a pad of Celite ${ }^{\circledR}$, washed with EtOAc $(5.0 \mathrm{~mL})$ and concentrated under reduced pressure. Purification by flash column chromatography $\left(2 \% \mathrm{MeOH} / \mathrm{CH}_{2} \mathrm{Cl}_{2}\right)$ afforded enamide 3 (2.0 mg, 53\%) as a white solid and recovered starting material (1.5 mg, $39 \%)$.

$\boldsymbol{R}_{\boldsymbol{f}} 0.31\left(5 \% \mathrm{MeOH} / \mathrm{CH}_{2} \mathrm{Cl}_{2}\right) ;[\alpha]_{\mathrm{D}}^{20}-19.5(c \quad 0.2, \mathrm{MeOH}) ; \mathbf{I R} \boldsymbol{v}_{\max }\left(\right.$ thin film $\left./ \mathrm{cm}^{-1}\right) 3319$, $2943,2831,1730,1717,1609,1514,1449,1358,1165,1099,1022,783$;

${ }^{1} \mathbf{H}$ NMR $\left(500 \mathrm{MHz} ; \mathrm{CD}_{3} \mathrm{CN}\right) \delta 8.14(1 \mathrm{H}, \mathrm{d}, J=10.5 \mathrm{~Hz}, \mathrm{NH}), 6.93(1 \mathrm{H}, \mathrm{dd}, J=14.5$, $10.6 \mathrm{~Hz}, \mathrm{H}-24), 6.84-6.78$ (1H, m, H-3), 6.20 (1H, dd, $J=14.7,10.9$ Hz, H-15), 5.87 (1H, d, $J=14.6 \mathrm{~Hz}, \mathrm{H}-23), 5.76(1 \mathrm{H}, \mathrm{d}, J=10.7 \mathrm{~Hz}, \mathrm{H}-16), 5.71(1 \mathrm{H}, \mathrm{d}, J=15.7 \mathrm{~Hz}, \mathrm{H}-2), 5.62$ (1H, s, H-2'), 5.49-5.46 (1H, m, H-6), 5.46-5.42 (1H, m, H-14), 5.36 (1H, dd, $J=15.2,8.0$ $\mathrm{Hz}, \mathrm{H}-7), 5.20$ (1H, d, $J=9.7 \mathrm{~Hz}, \mathrm{H}-21), 5.05-5.16\left(2 \mathrm{H}, \mathrm{m}, \mathrm{N}_{2}\right), 4.94$ (1H, ddd, $J=10.5$, 7.7, 2.4 Hz, H-19), 4.52 (1H, dt, $J=10.4,3.1 \mathrm{~Hz}, \mathrm{H}-10), 3.75-3.70$ (1H, m, H-8), 3.47-3.43 (1H, m, H-9), $3.17(1 \mathrm{H}, \mathrm{d}, J=4.0 \mathrm{~Hz}, \mathrm{C} 9-\mathrm{O} \underline{\mathrm{H}}), 3.11(1 \mathrm{H}, \mathrm{d}, J=3.3 \mathrm{~Hz}, \mathrm{C} 8-\mathrm{O} \underline{\mathrm{H}}), 2.78(1 \mathrm{H}$, dt, $J=9.85,6.92 \mathrm{~Hz}, \mathrm{H}-20), 2.45-2.37$ (1H, m, H-4a), 2.33-2.24 (2H, m, H-18a, H-5a), 2.20 (1H, dd, $J=14.2,11.1 \mathrm{~Hz}, \mathrm{H}-18 \mathrm{~b}), 2.16$ (3H, s, H-4'), 2.18-2.12 (2H, m, H-4b, H5b), 1.93-1.84 (2H, m, H-13), 1.86 (3H, s, H-5'), 1.74 (3H, s, C22- $\left.\underline{H}_{3}\right), 1.68$ (3H, s, C17$\mathrm{C}_{3}$ ), 1.54-1.45 (1H, m, H-11a), 1.43-1.36 (2H, m, H-11b, H-12a), 1.17-1.10 (1H, m, H12b), $0.94\left(3 \mathrm{H}, \mathrm{d}, J=6.7 \mathrm{~Hz}, \mathrm{C} 20-\mathrm{CH}_{3}\right)$.

${ }^{1}$ H NMR (500 MHz; DMSO) $\delta 9.88(1 \mathrm{H}, \mathrm{d}, J=10.3 \mathrm{~Hz}, \mathrm{~N} \underline{\mathrm{H}}), 6.85(1 \mathrm{H}, \mathrm{dd}, J=14.0,10.4$ $\mathrm{Hz}, \mathrm{H}-24), 6.78$ (1H, dt, $J=15.6,7.4 \mathrm{~Hz}, \mathrm{H}-3), 6.48-6.28\left(2 \mathrm{H}, \mathrm{m}, \mathrm{NH}_{2}\right), 6.15(1 \mathrm{H}, \mathrm{dd}, J=$ 
14.1, 11.3 Hz, H-15), 5.85 (1H, d, $J=14.5$ Hz, H-23), 5.69-5.76 (3H, m, H-2, H-16, H-2'), 5.46-5.35 (2H, m, H-6, H-14), 5.35-5.27 (1H, m, H-7), 5.16 (1H, d, $J=9.5$ Hz, H-21), 4.87 $(1 \mathrm{H}, \mathrm{t}, J=8.5 \mathrm{~Hz}, \mathrm{H}-19), 4.77(1 \mathrm{H}, \mathrm{s}, \mathrm{C} 9-\mathrm{O} \underline{\mathrm{H}}), 4.68(1 \mathrm{H}, \mathrm{s}, \mathrm{C} 8-\mathrm{O} \underline{\mathrm{H}}), 4.42(1 \mathrm{H}, \mathrm{d}, J=10.3$ Hz, H-10), 3.56 (1H, obs, H-9), 3.46 (1H, obs, H-8), 2.75 (1H, m, H-20), 2.14-2.25 (2H, m, H-18), 2.12 (3H, s, H-5'), 2.26-2.22 (1H, m, H-5a), 2.21 (2H, m, H-12), 2.08-1.93 (3H, m, H-4, H-5b), 1.83 (3H, s, H-4'), 1.82-1.78 (2H, m, H-13), 1.68 (3H, s, C22- $\left.\underline{\mathrm{H}}_{3}\right), 1.64$ $\left(3 \mathrm{H}, \mathrm{s}, \mathrm{C} 17-\mathrm{C}_{3}\right), 1.57-1.49$ (1H, m, H-11a), 1.49-1.42 (1H, m, H-11b), 0.91 (3H, d, $J=$ $\left.6.5 \mathrm{~Hz}, \mathrm{C} 20-\mathrm{CH}_{3}\right)$.

${ }^{13}$ C NMR (HSQC, HMBC, $\mathrm{CD}_{3} \mathrm{CN}$ ) $\delta 166.2,164.2,164.4,154.1,142.4,133.2,132.6$, $132.5,130.9,130.3,130.1,128.6,127.9,123.3,122.3,118.1,117.2,76.0,75.3,75.26$, 74.2, 43.1, 37.8, 32.4, 31.8, 30.7, 27.25, 26.1, 24.8, 19.8, 17.4, 16.2, 12.9; HRMS $\left(\mathrm{ES}^{+}\right)$ calcd. for $\mathrm{C}_{33} \mathrm{H}_{48} \mathrm{~N}_{2} \mathrm{NaO}_{7}[\mathrm{M}+\mathrm{Na}]^{+}$607.3359, found 607.3364 . 
Appendix: Selected ${ }^{1} \mathrm{H}$ and ${ }^{13} \mathrm{C}$ NMR spectra 


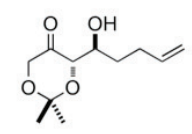

${ }^{1} \mathrm{H}$ NMR $\left(400 \mathrm{MHz}, \mathrm{CDCl}_{3}\right)$
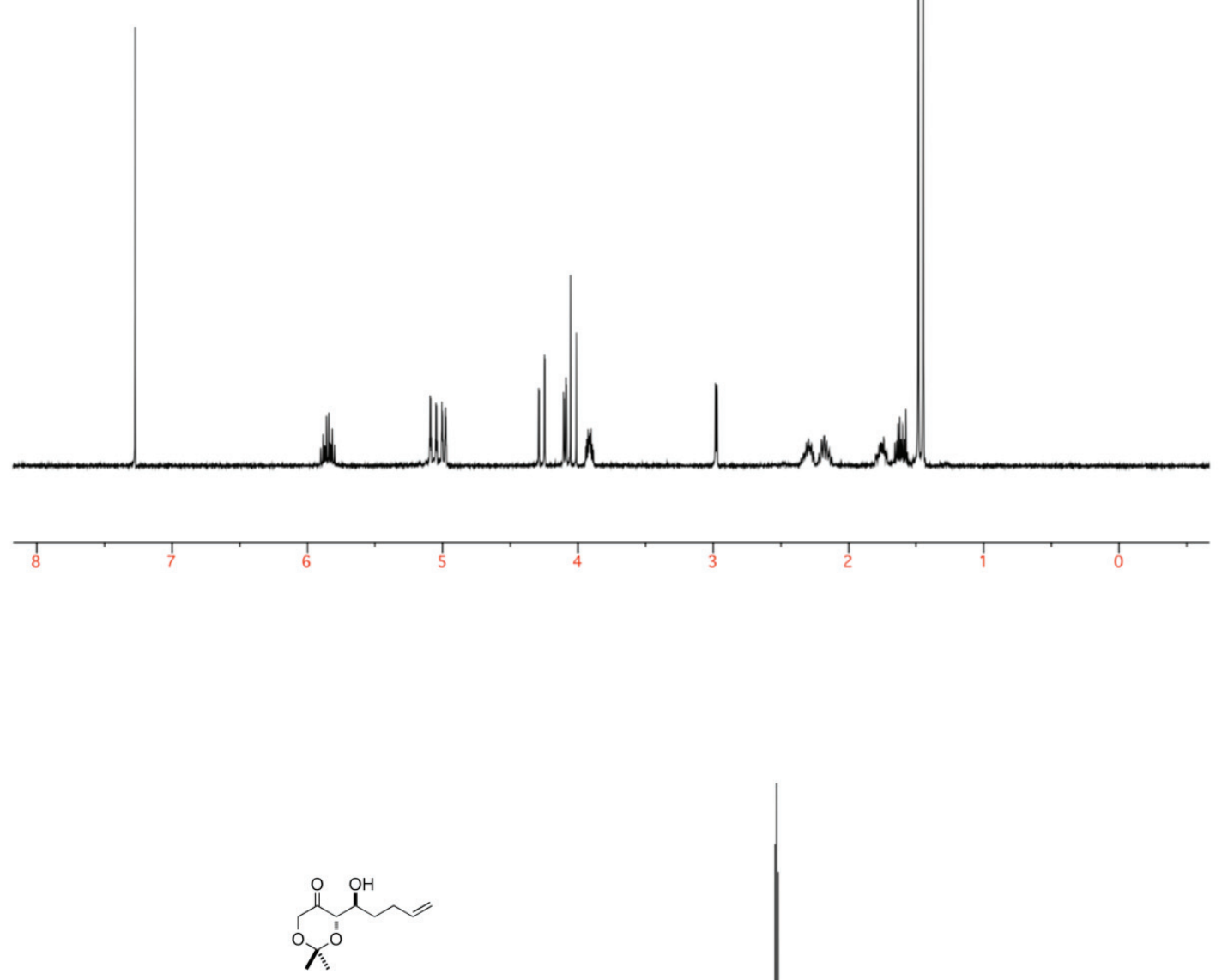

${ }^{13} \mathrm{C}$ NMR $\left(100 \mathrm{MHz}, \mathrm{CDCl}_{3}\right)$

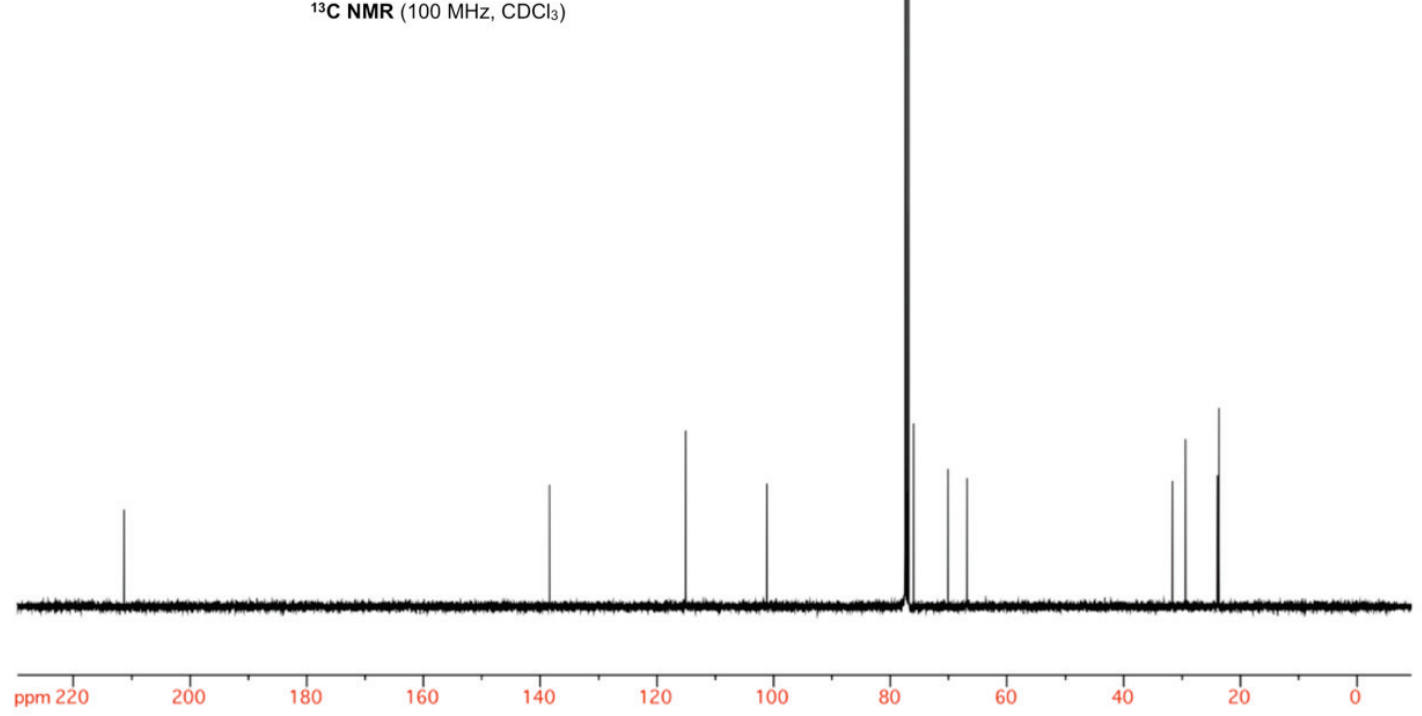


Appendix
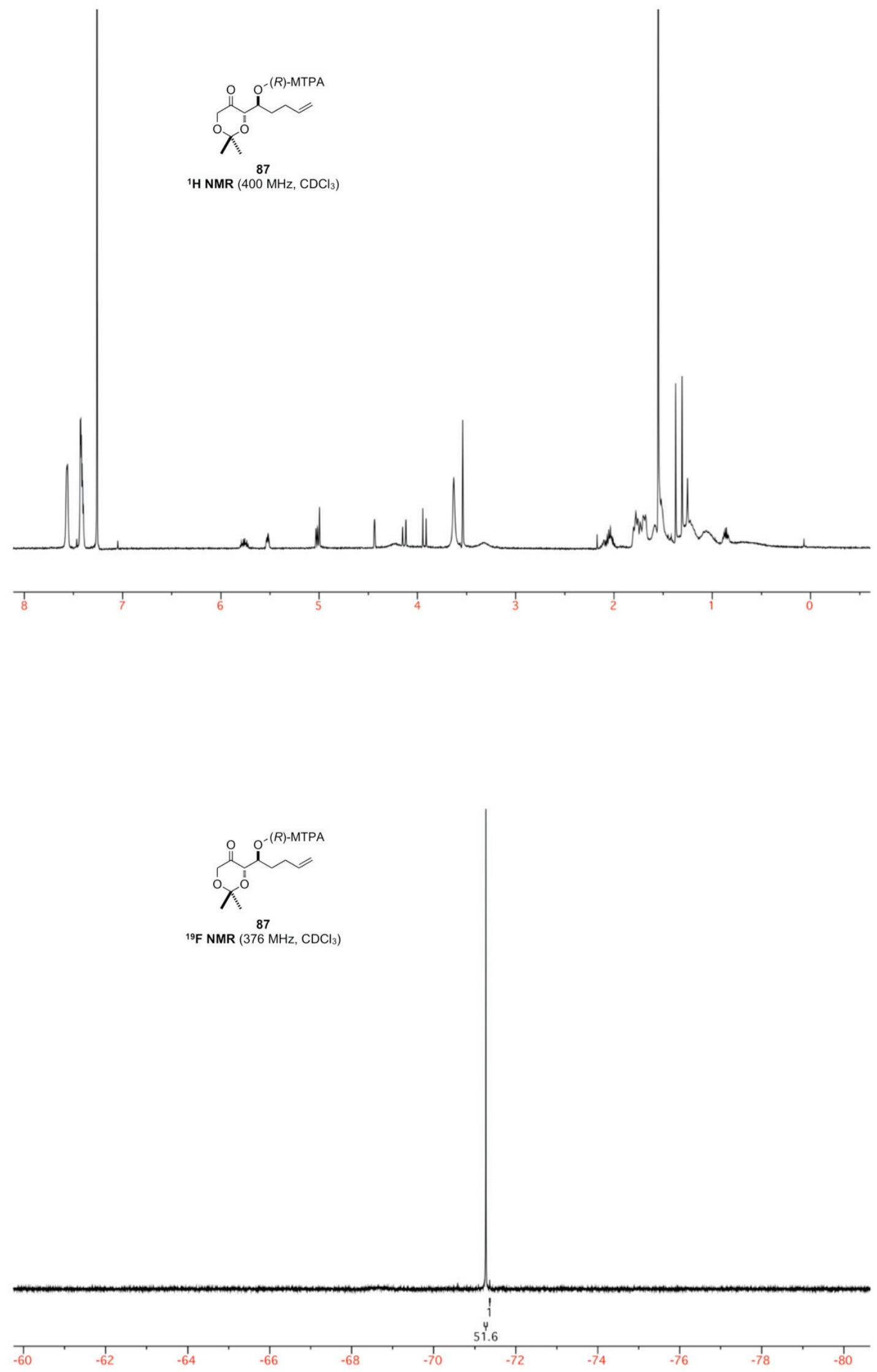

174 

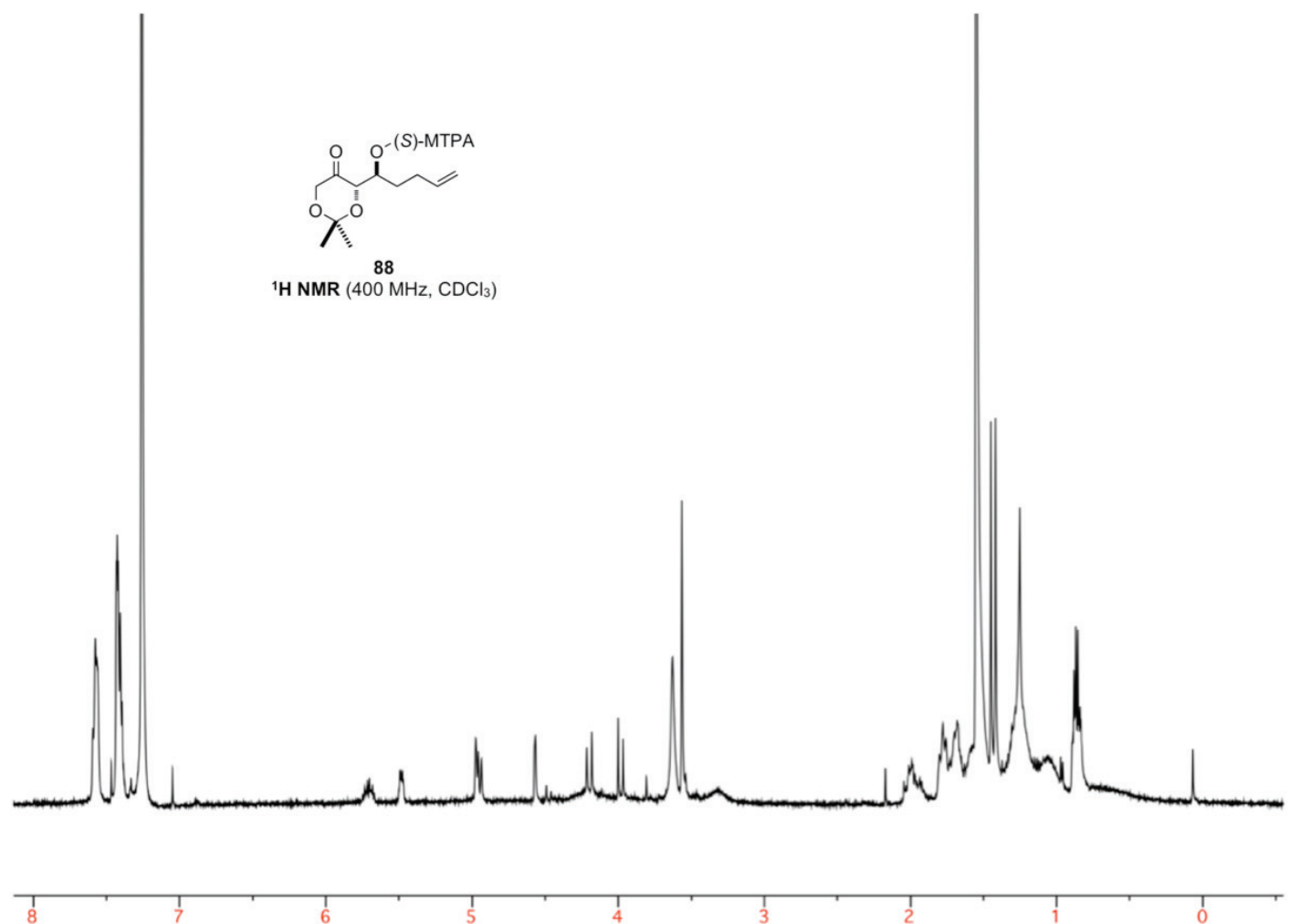

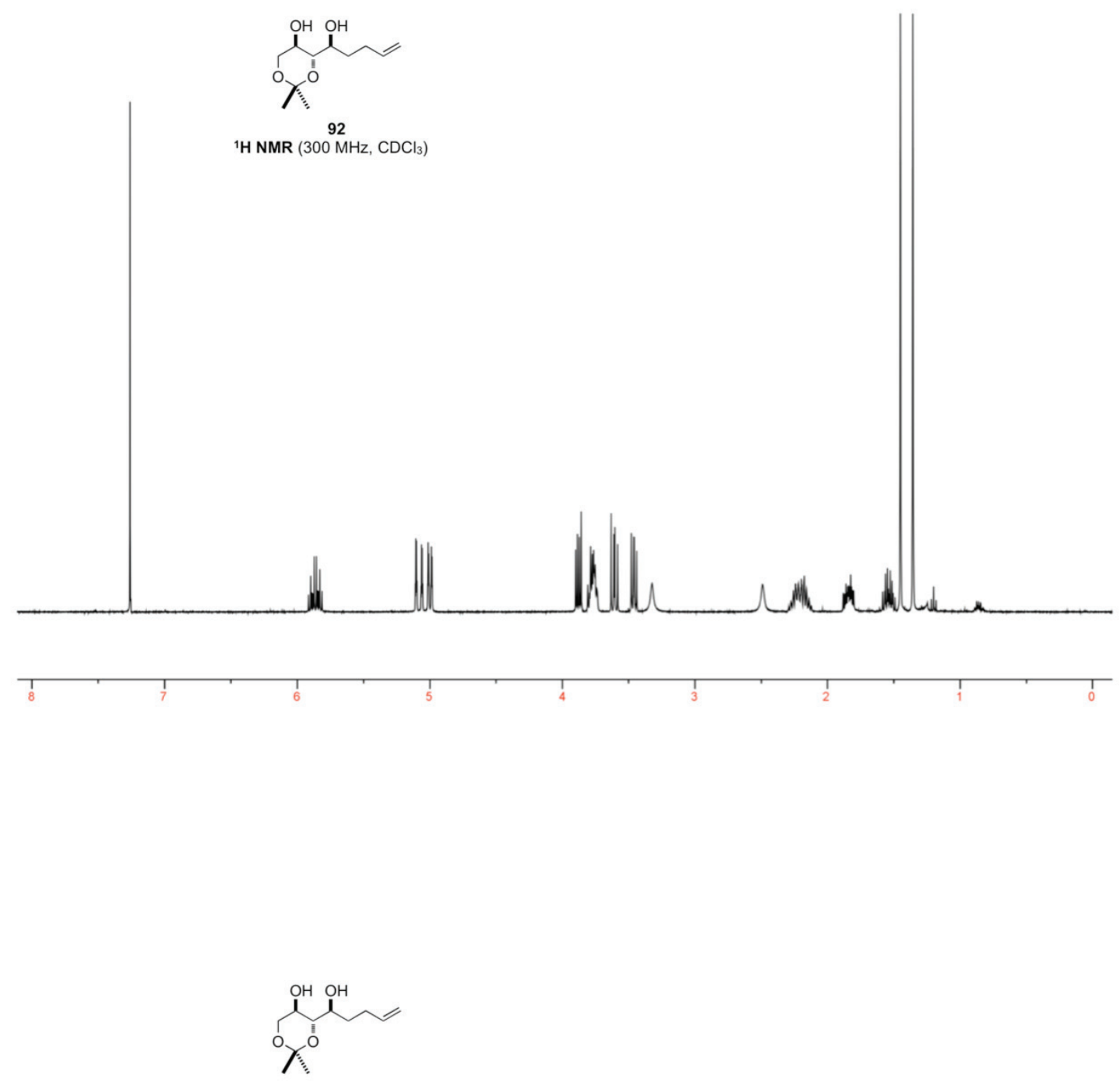

92

${ }^{13} \mathrm{C}$ NMR $\left(100 \mathrm{MHz}, \mathrm{CDCl}_{3}\right)$

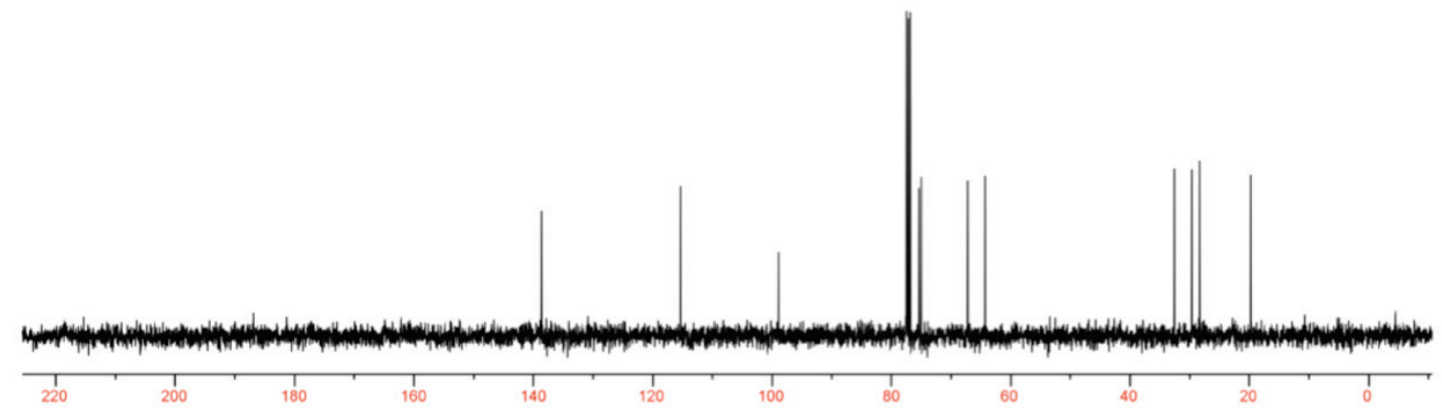




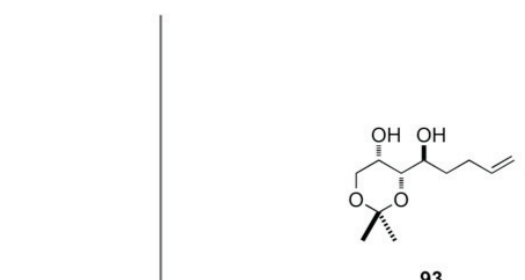

${ }^{1} \mathrm{H}$ NMR $\left(400 \mathrm{MHz}, \mathrm{CDCl}_{3}\right)$
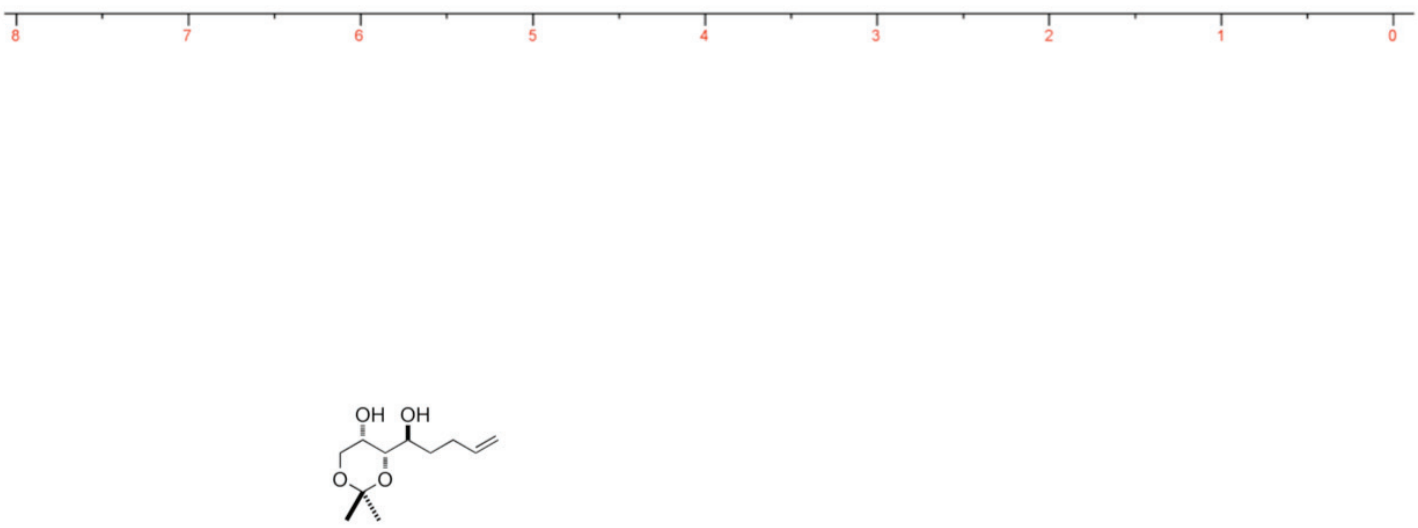

93

${ }^{13} \mathrm{C} \mathrm{NMR}\left(100 \mathrm{MHz}, \mathrm{CDCl}_{3}\right)$
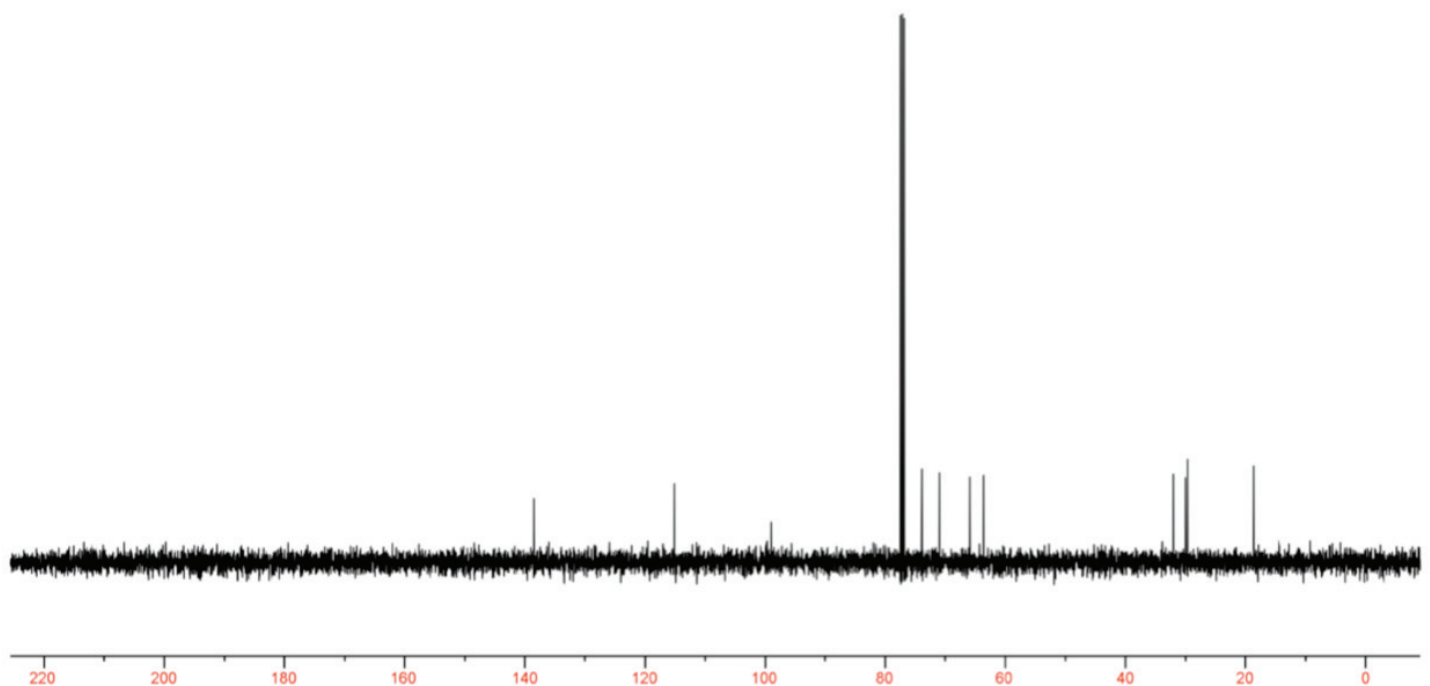

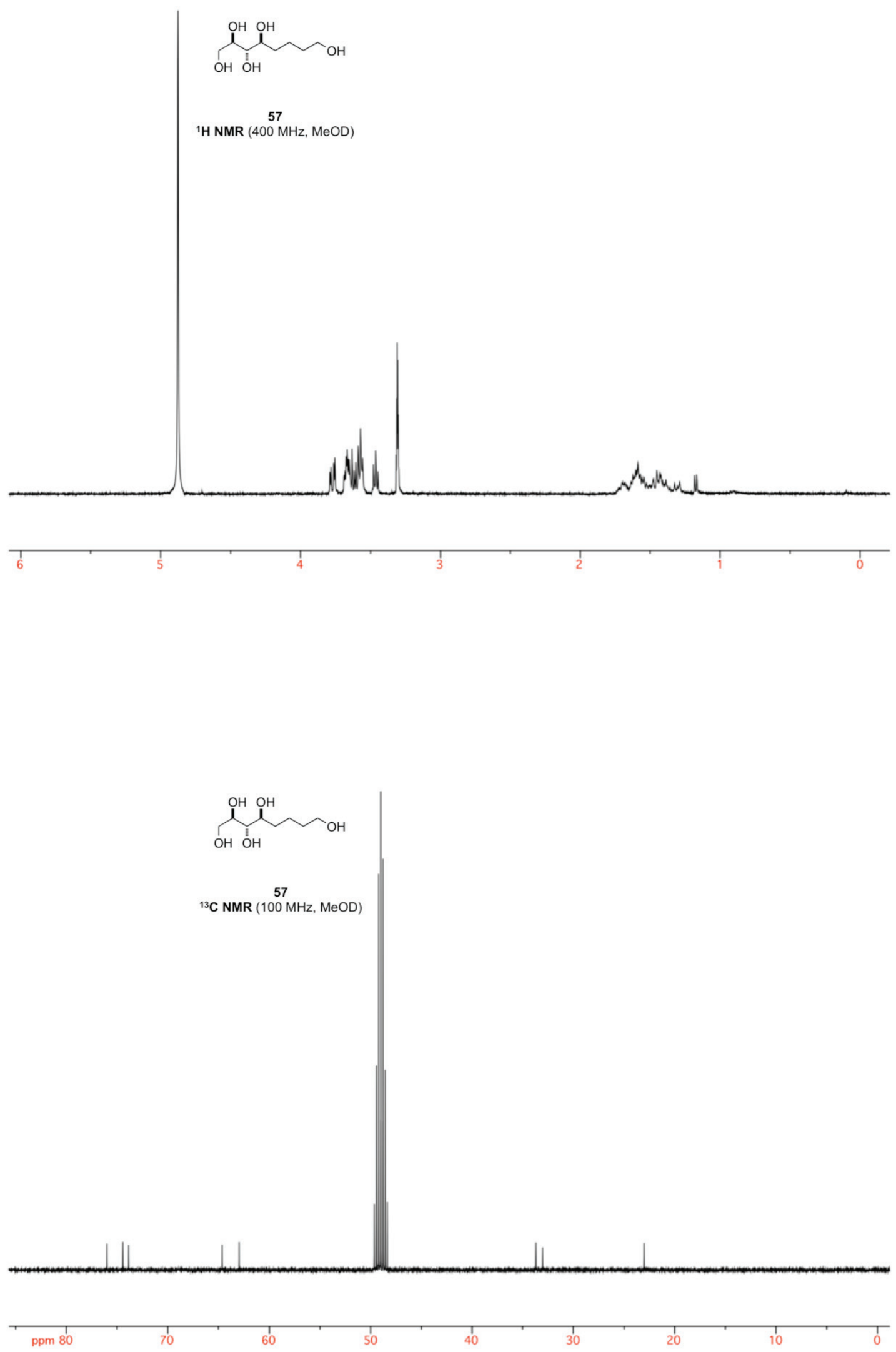


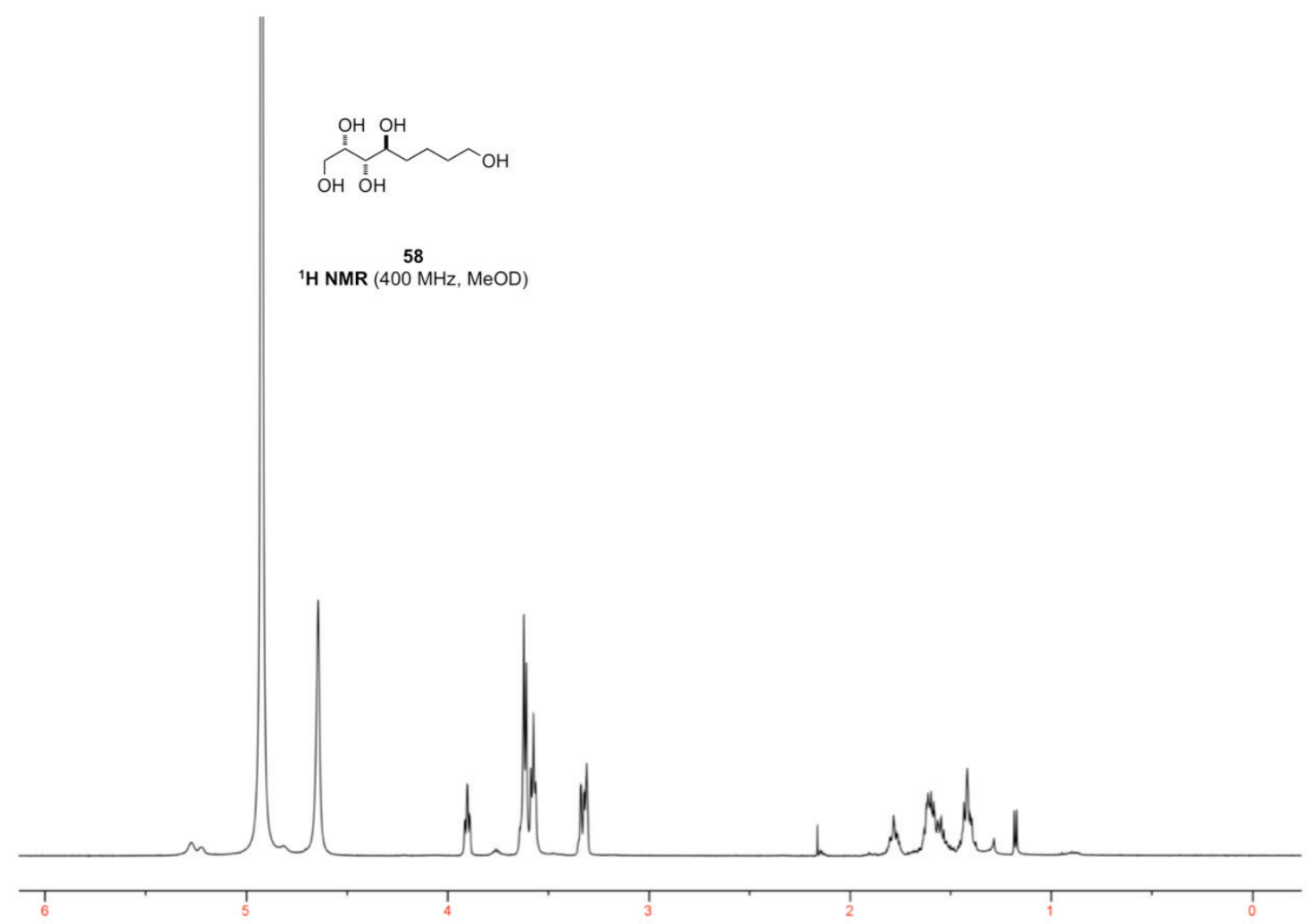

$\overbrace{\mathrm{OH}}^{\mathrm{OH}}$

58

${ }^{13} \mathrm{C}$ NMR (100 MHz, MeOD)

ppm 80

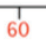

50

40

30

20

10 

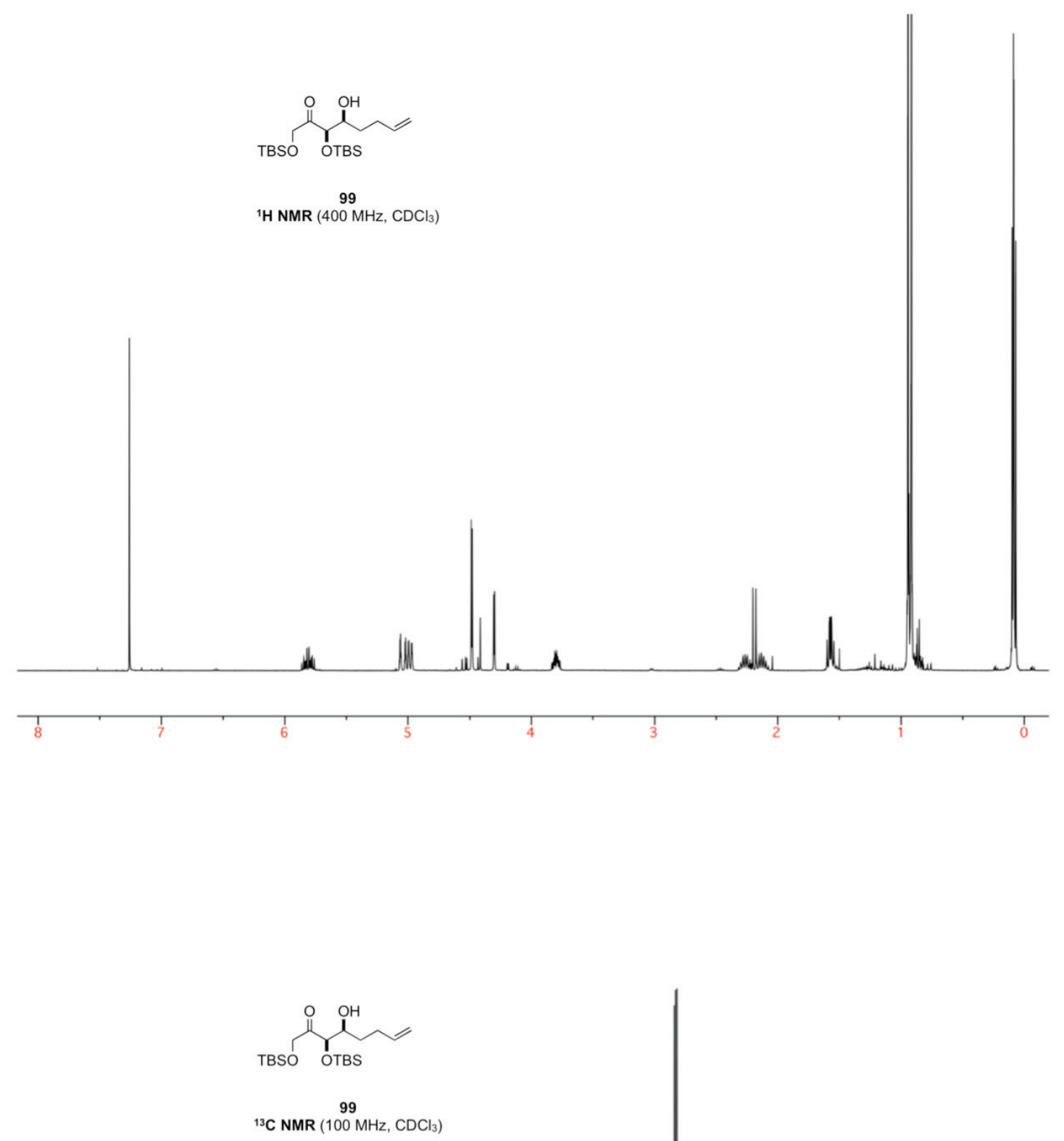

ppm 220 $200+180$ 160 140 120 100 80 60 40 

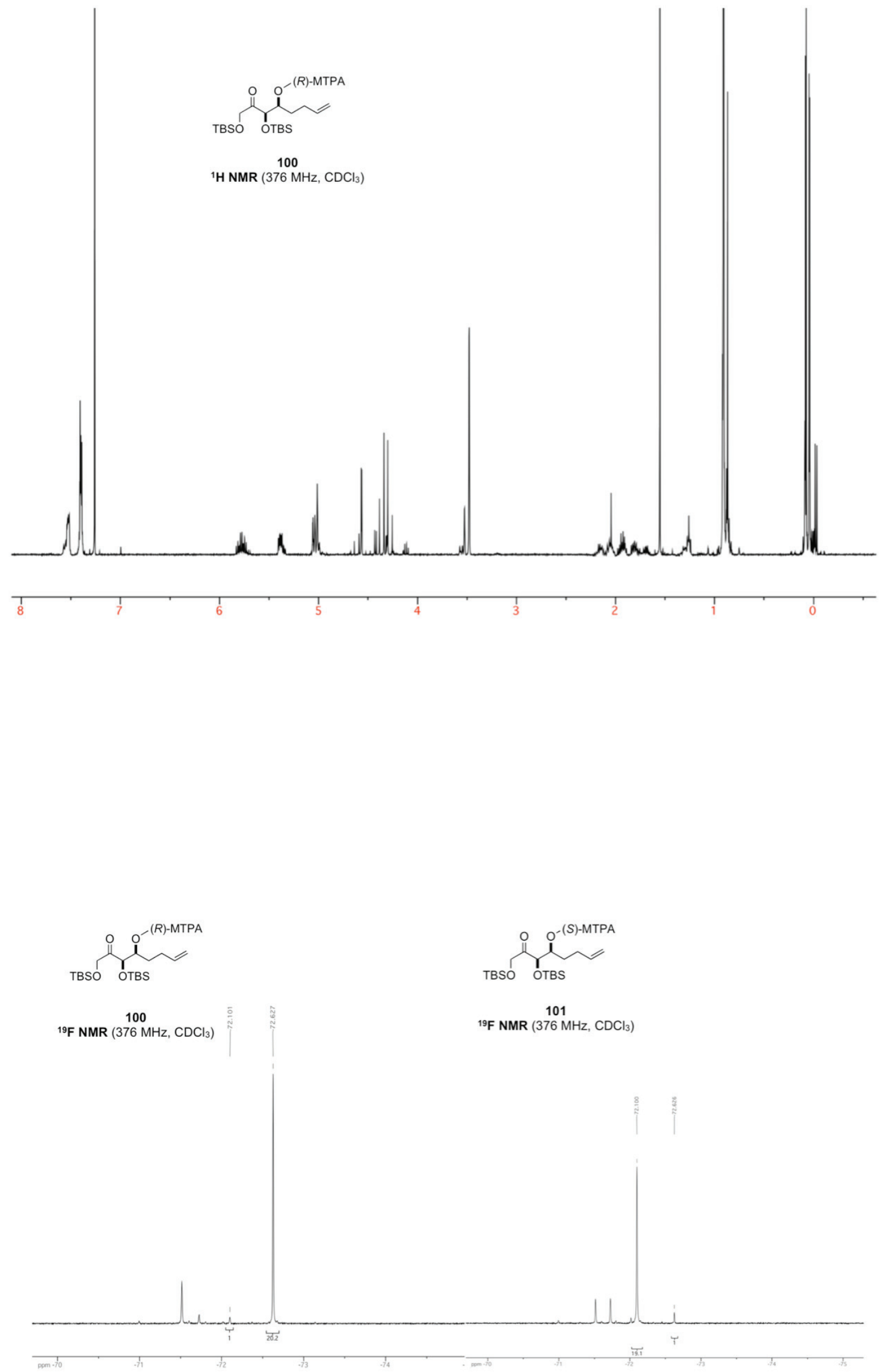


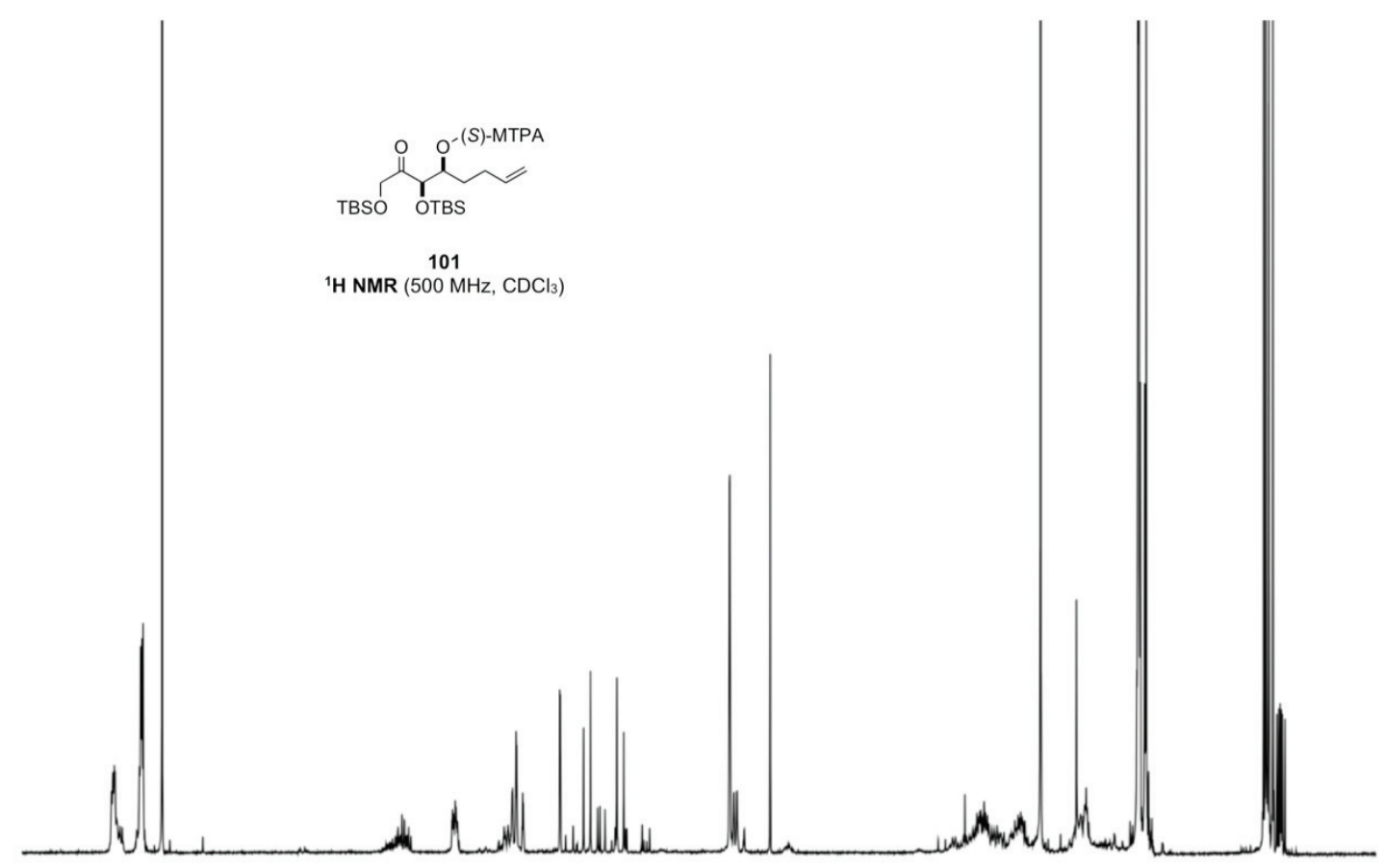

8 

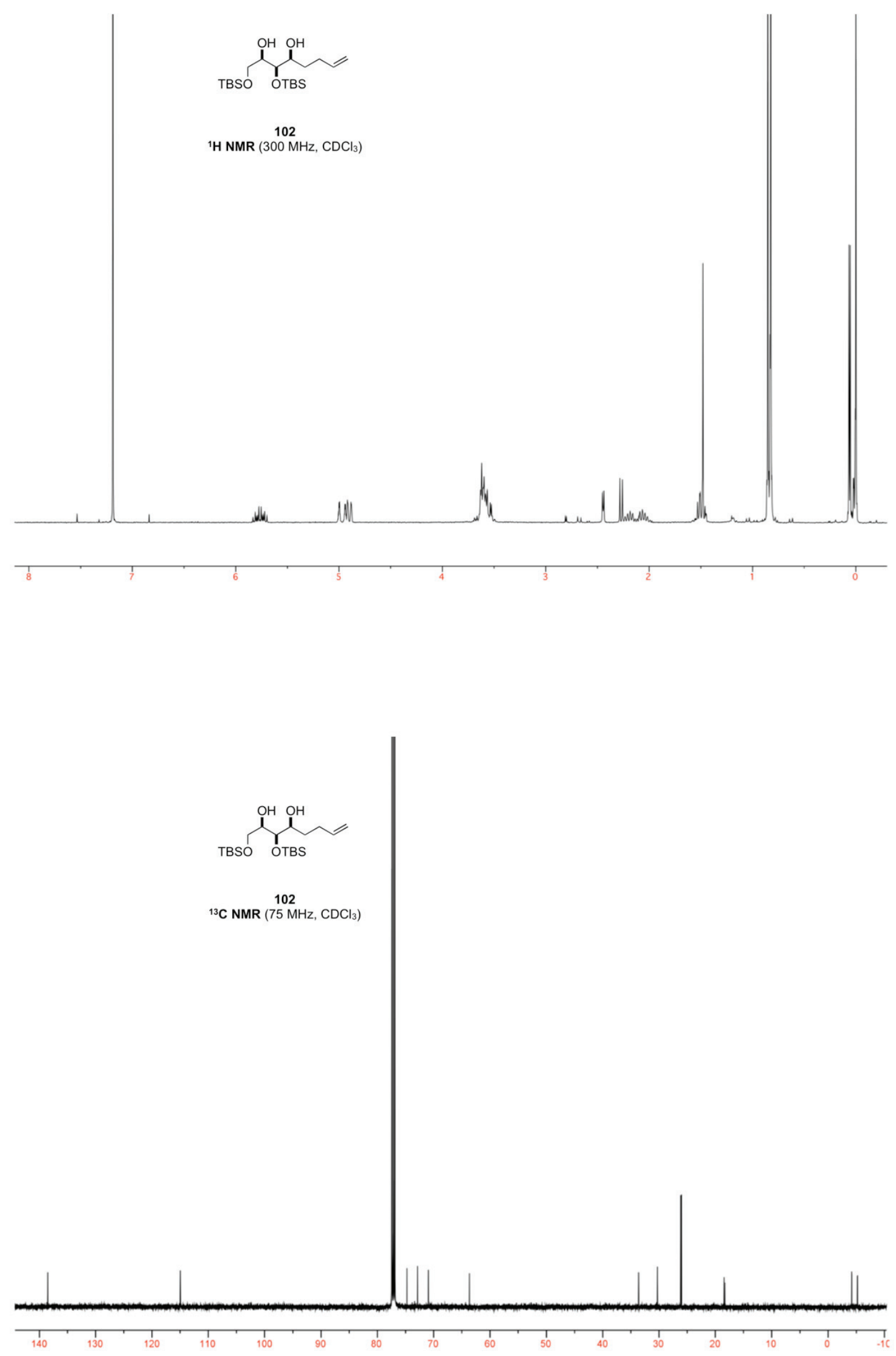


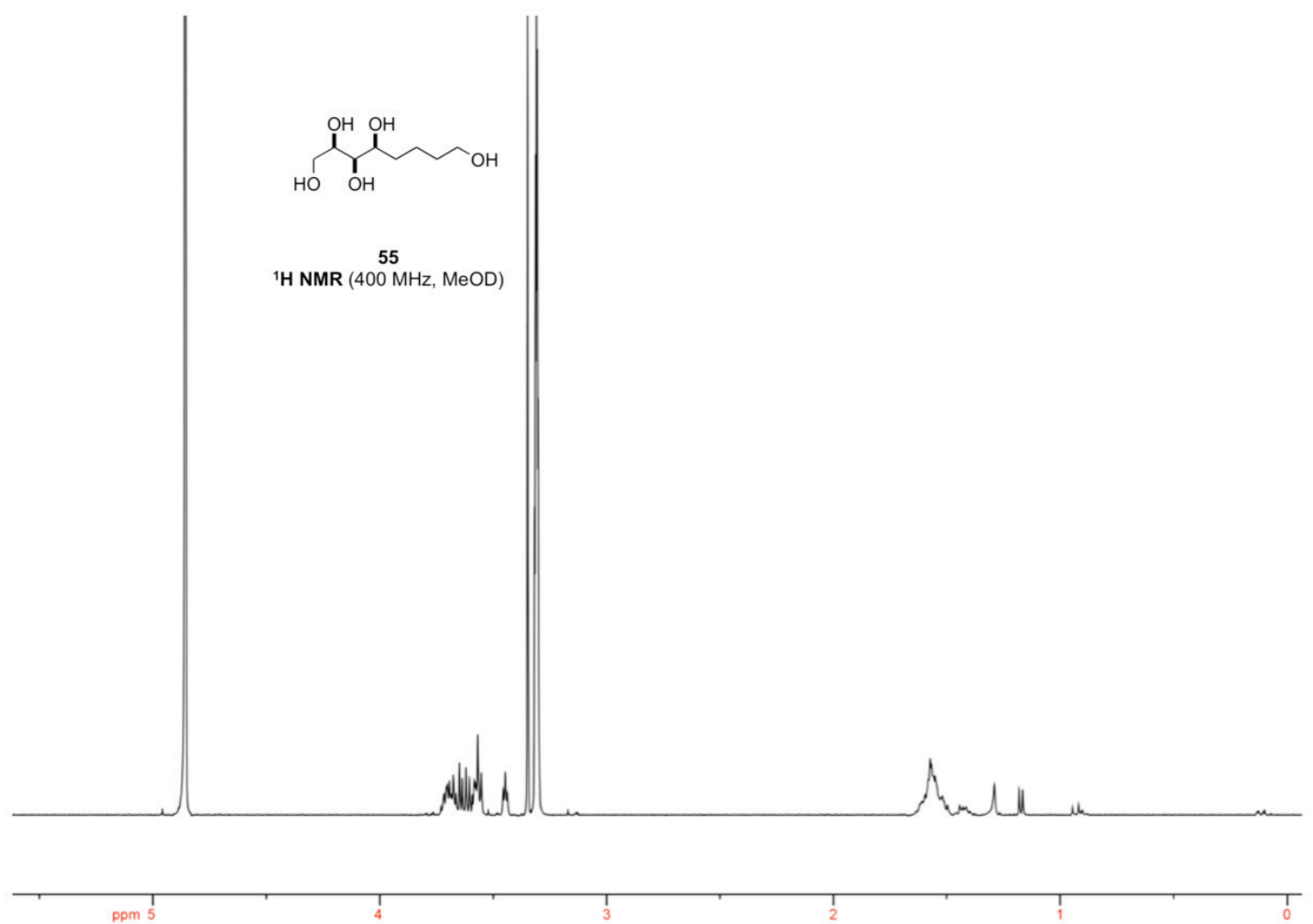

$\overbrace{\mathrm{OH}}^{\mathrm{OH}}$

${ }^{13} \mathrm{C}$ NMR $(100 \mathrm{MHz}, \mathrm{MeOD})$

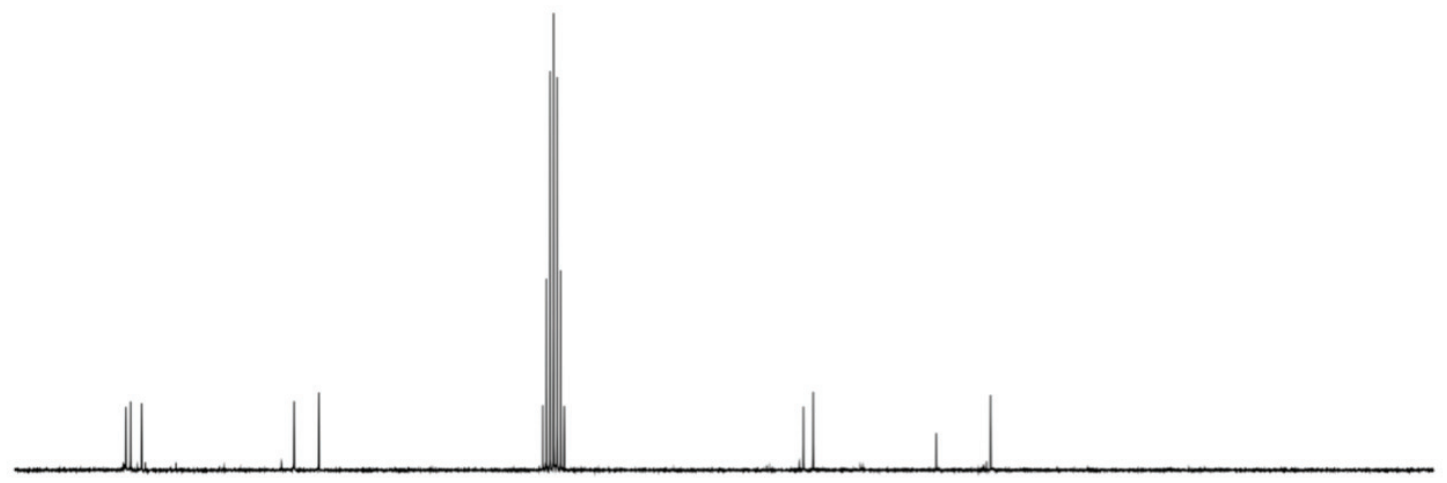

\begin{tabular}{l|l|l|l|l|l|l|l}
\hline & 1 \\
80 & 1 & 1 & 1 & 1 & 1 & 1 \\
\hline
\end{tabular} 


$$
\overbrace{\mathrm{OH}}^{\mathrm{OH}}
$$

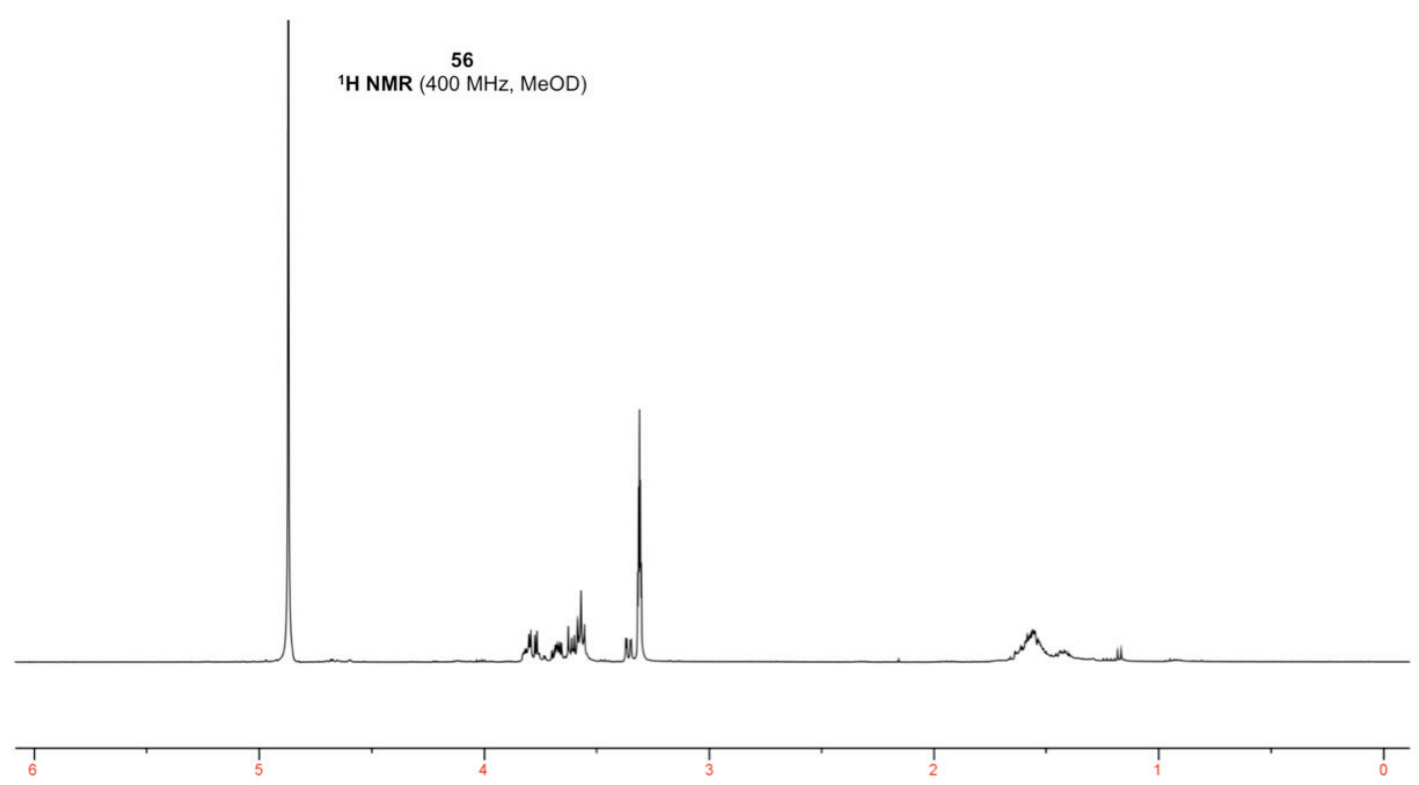

$$
\underbrace{\mathrm{OH}}_{\mathrm{OH}}
$$

${ }^{13} \mathrm{C}$ NMR (100 MHz, MeOD)

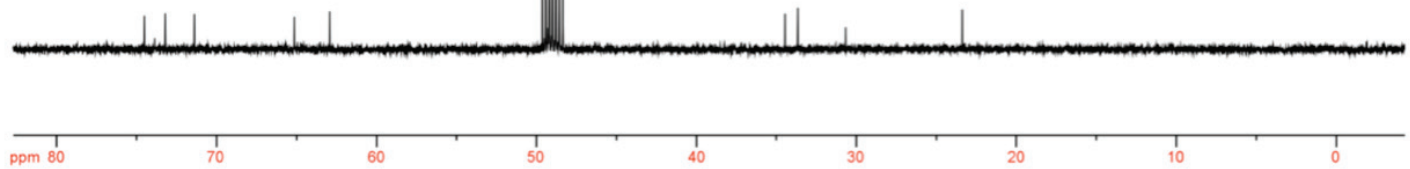



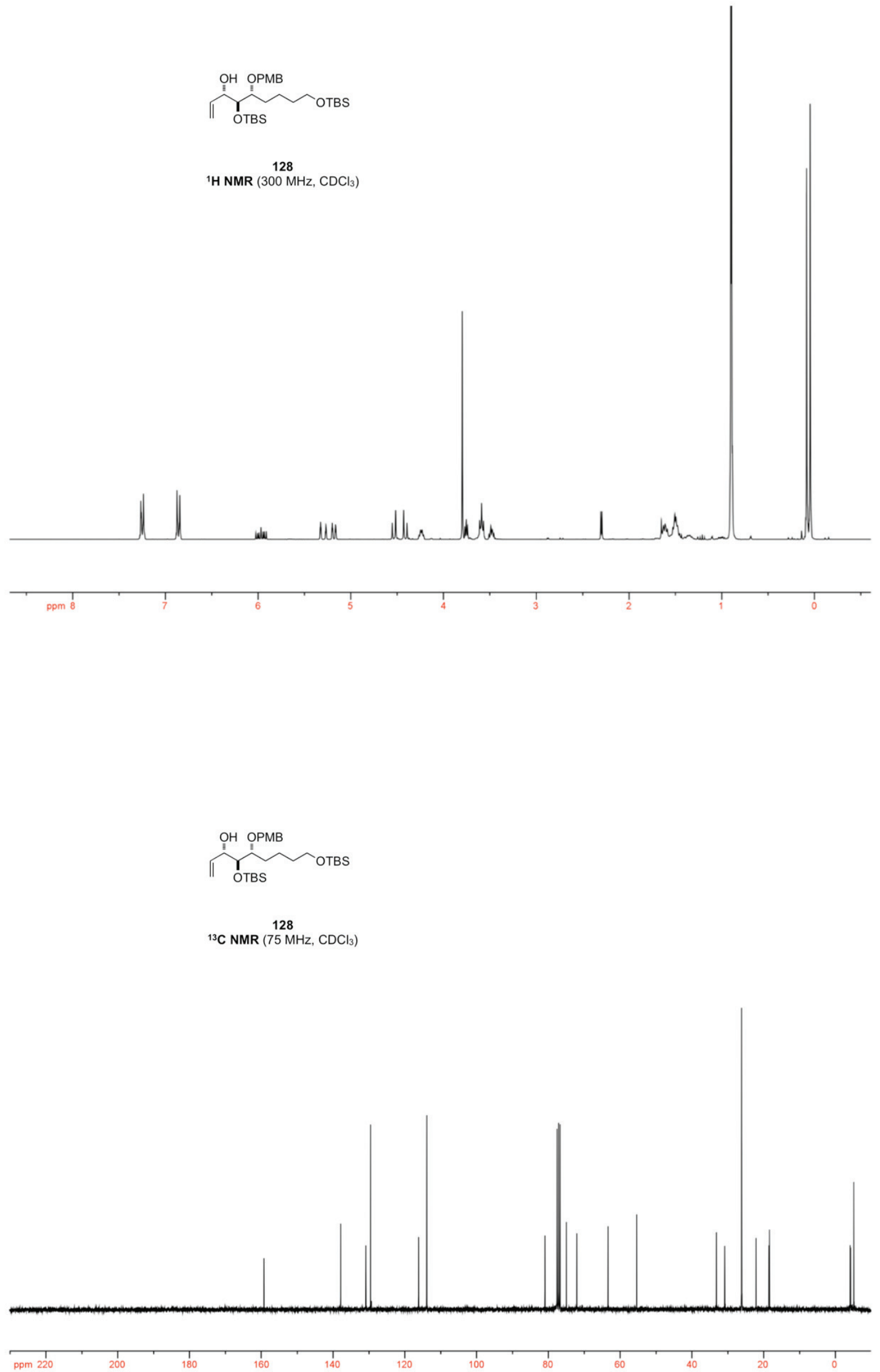

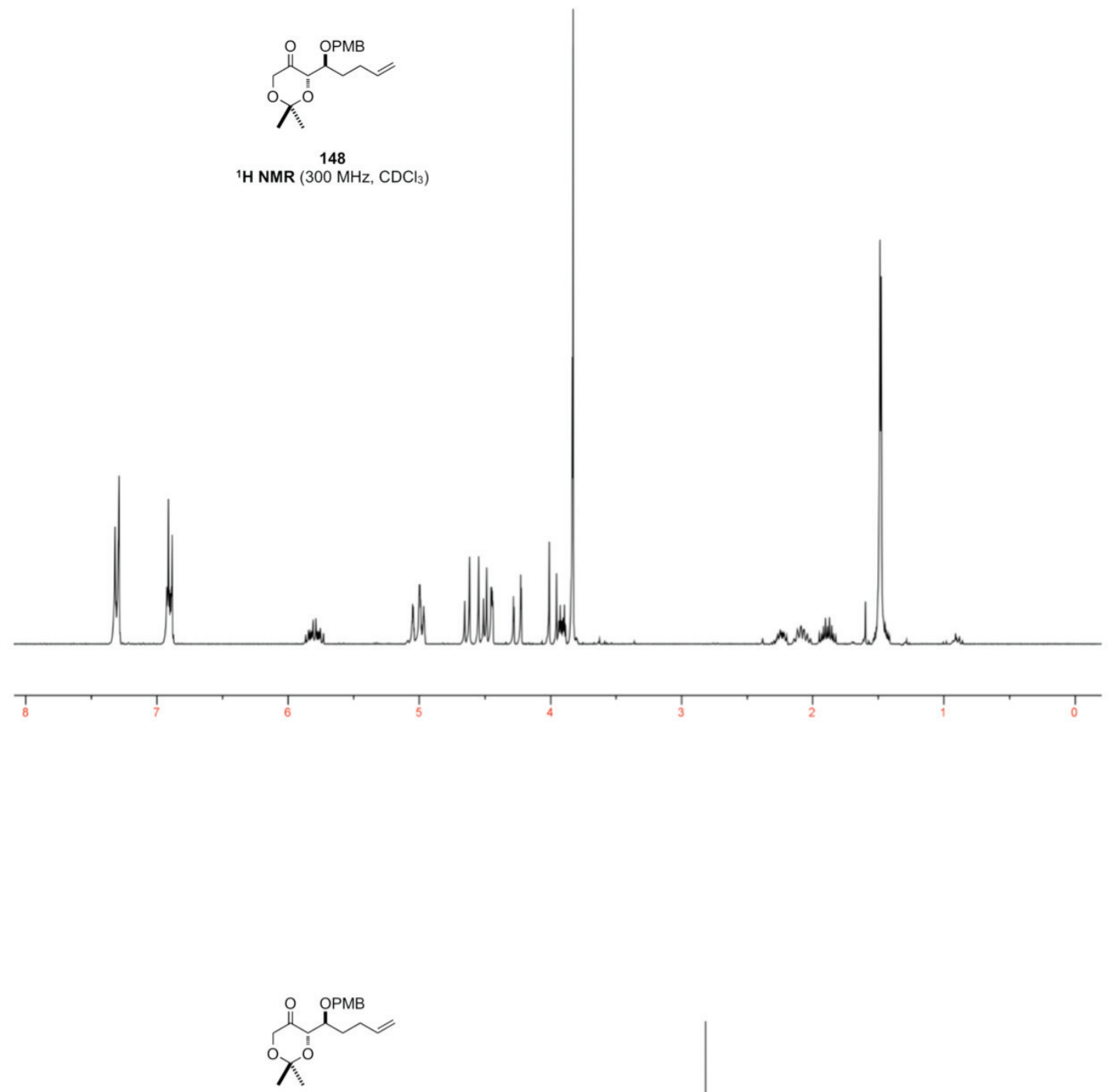

148
${ }^{13} \mathrm{C}$ NMR $\left(75 \mathrm{MHz}, \mathrm{CDCl}_{3}\right)$

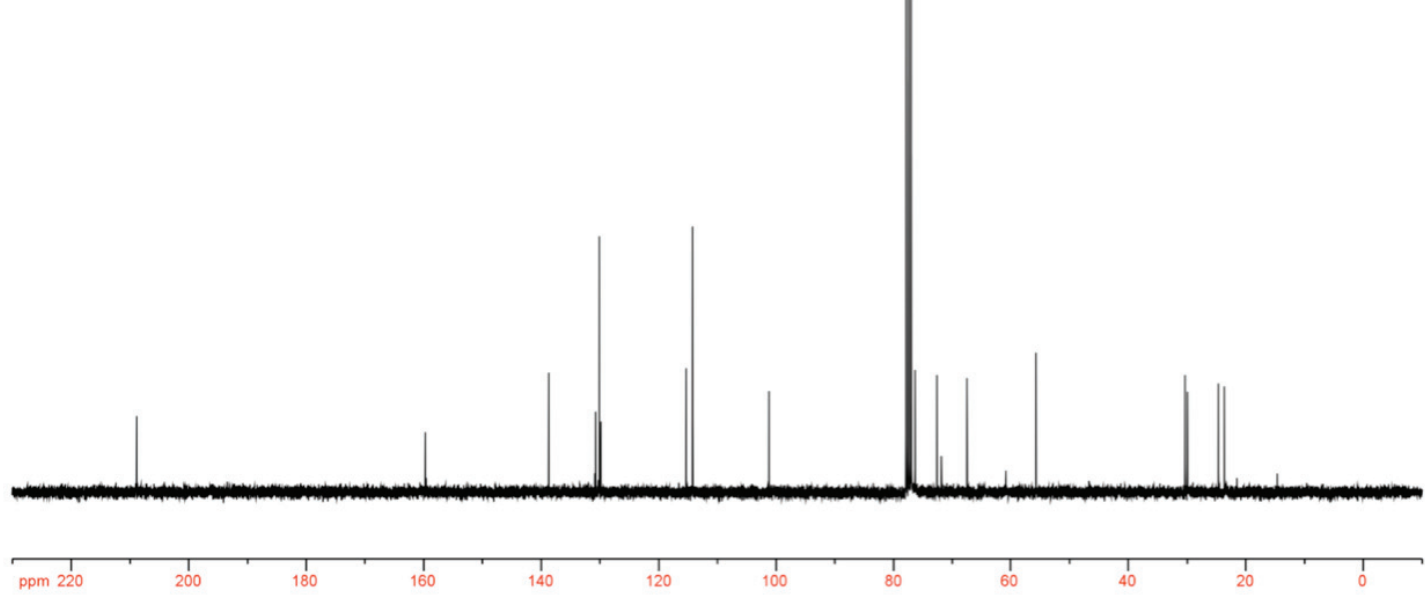



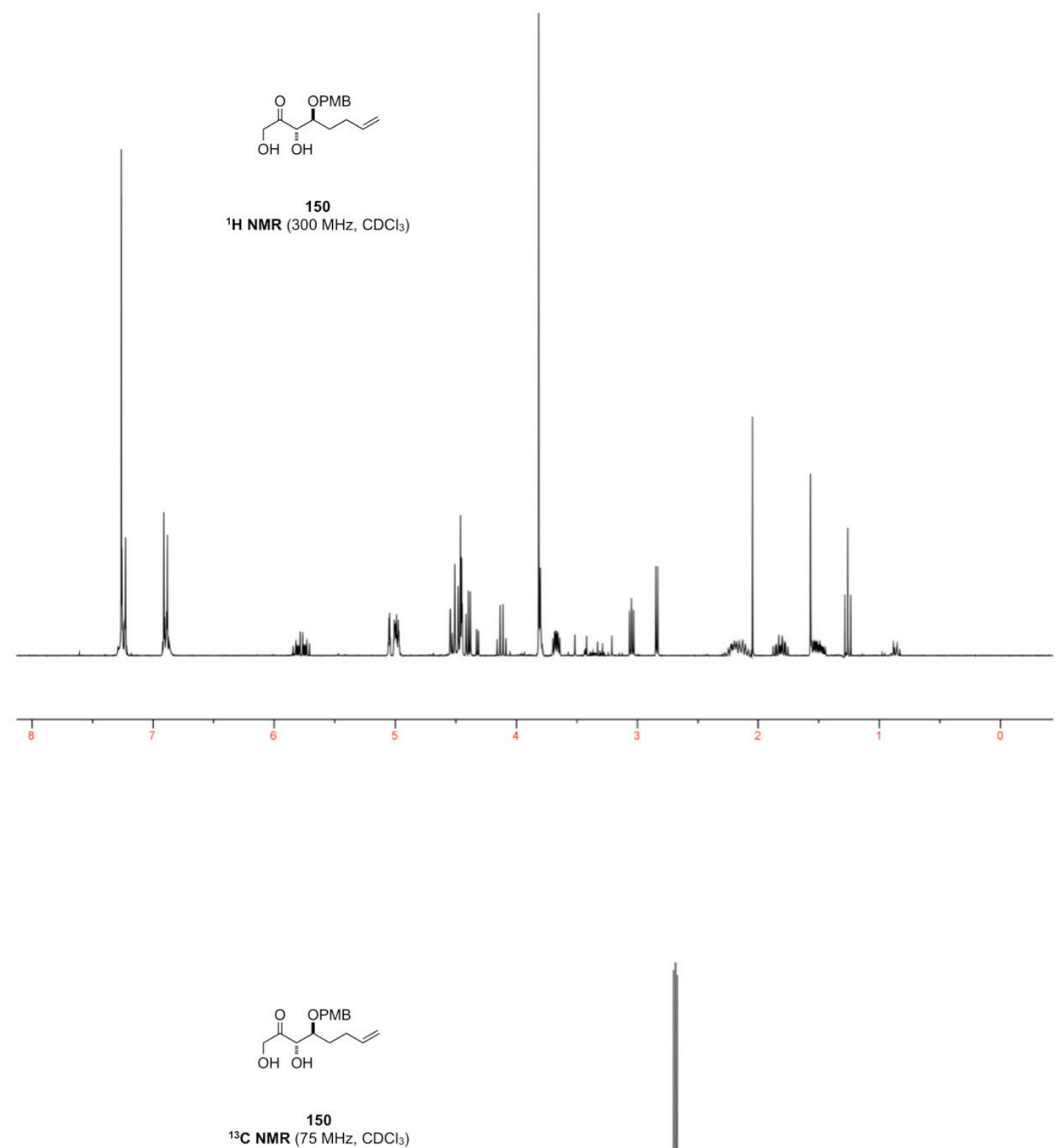

${ }^{13} \mathrm{C}$ NMR $\left(75 \mathrm{MHz}, \mathrm{CDCl}_{3}\right)$

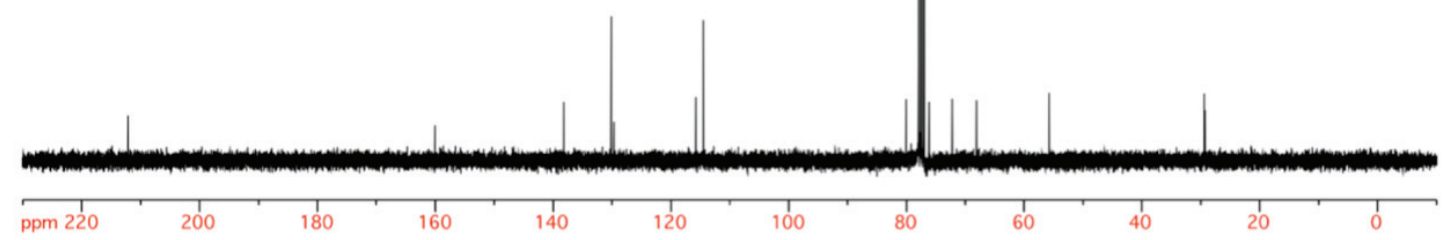




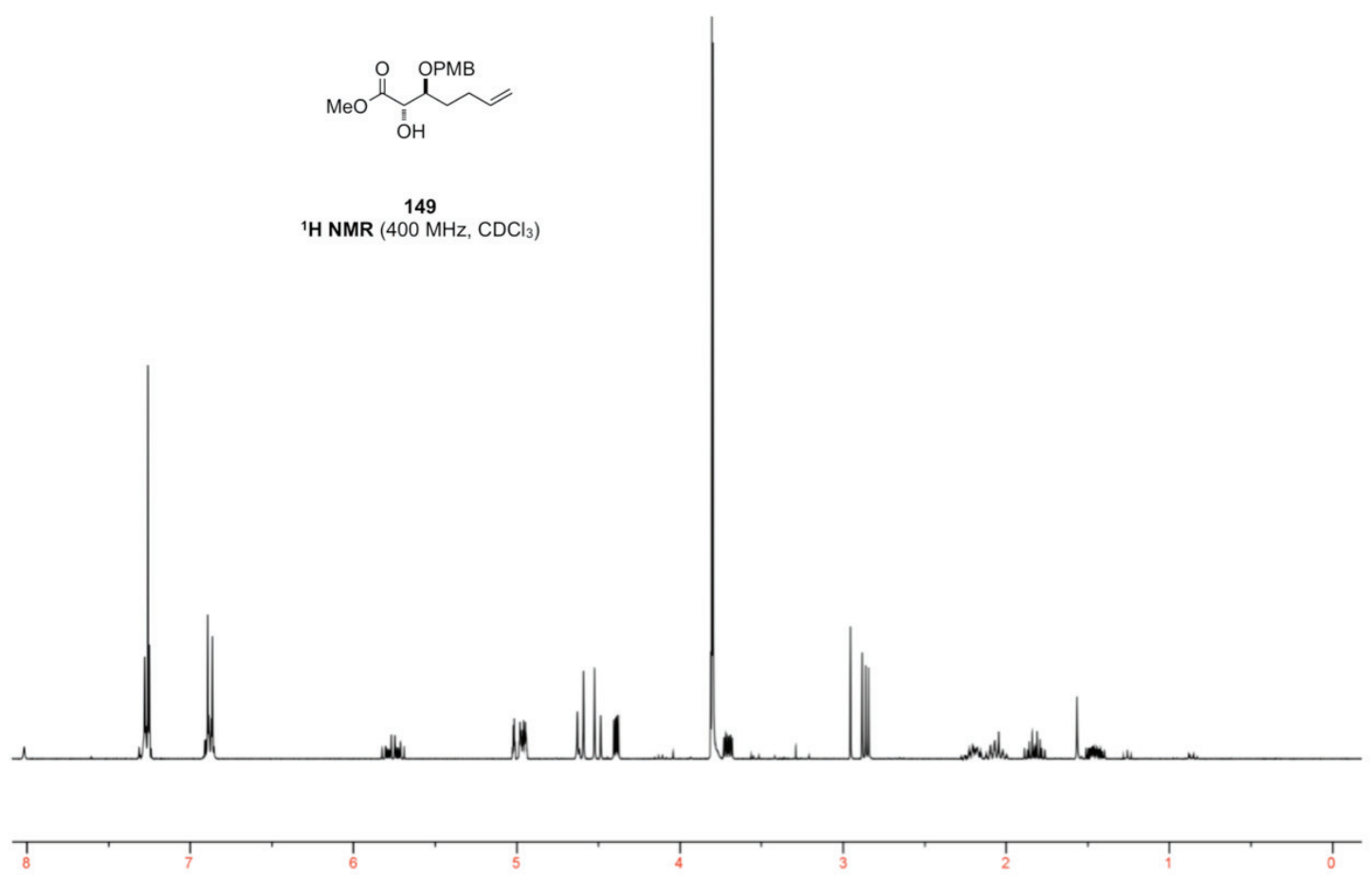

$$
{ }_{\mathrm{MeO}}^{\stackrel{\mathcal{H}_{\mathrm{OH}}}{\mathrm{OPMB}}}
$$

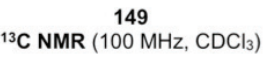

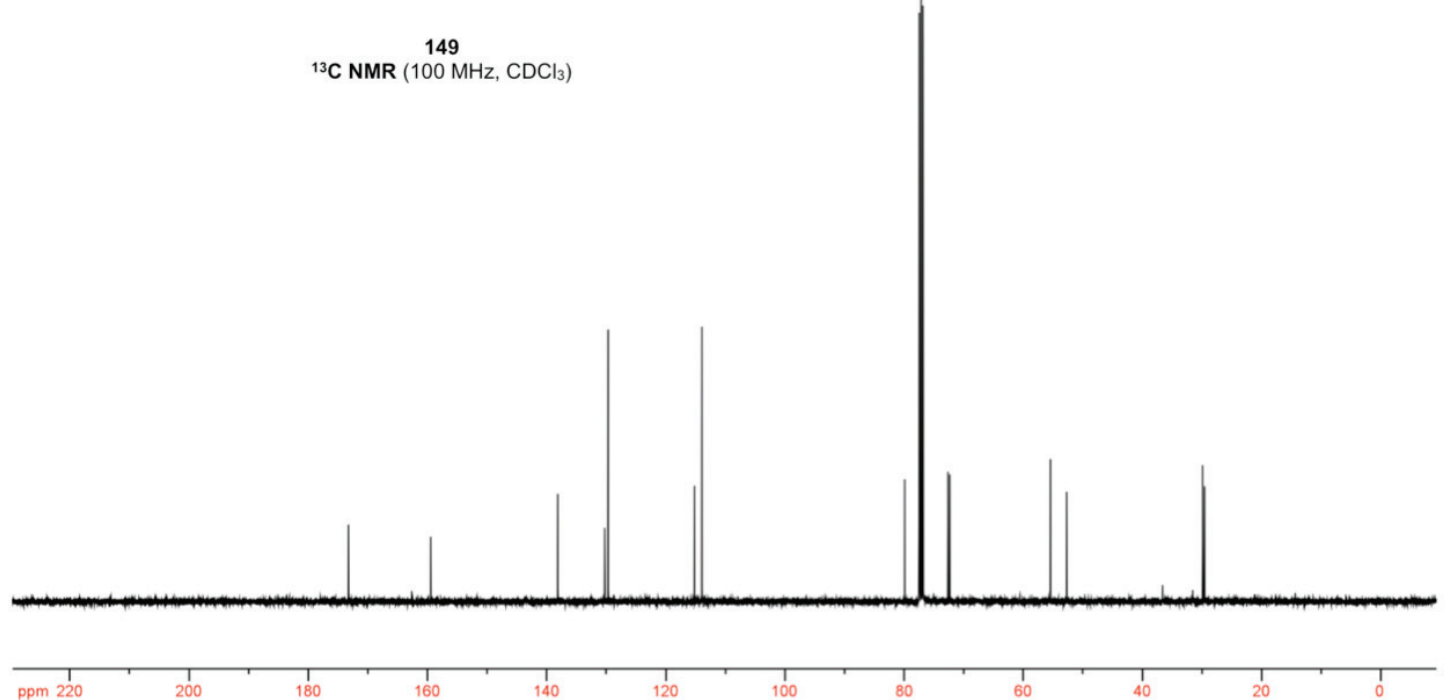




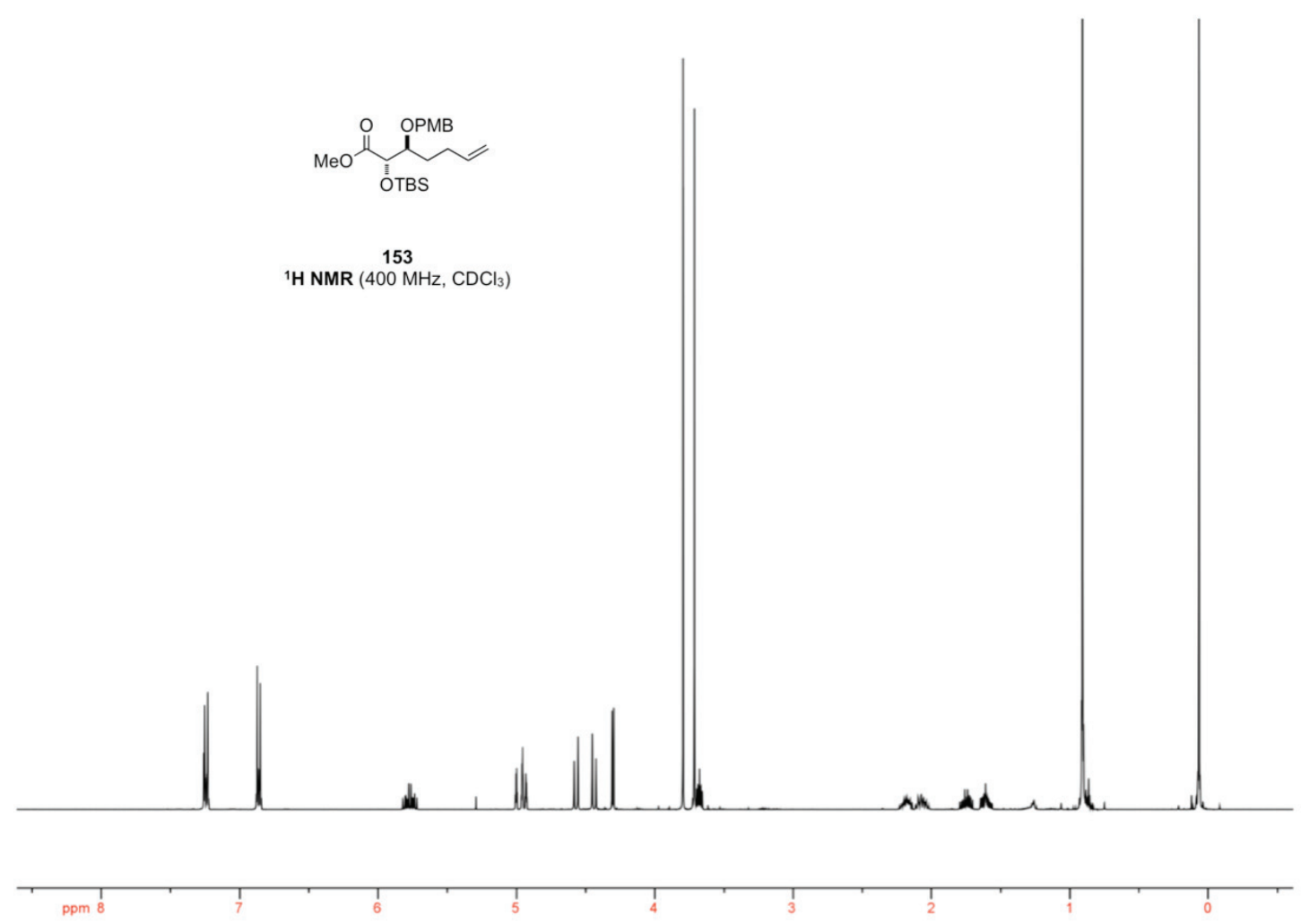

OTBS

${ }^{13} \mathrm{C}$ NMR $\left(100 \mathrm{MHz}, \mathrm{CDCl}_{3}\right)$

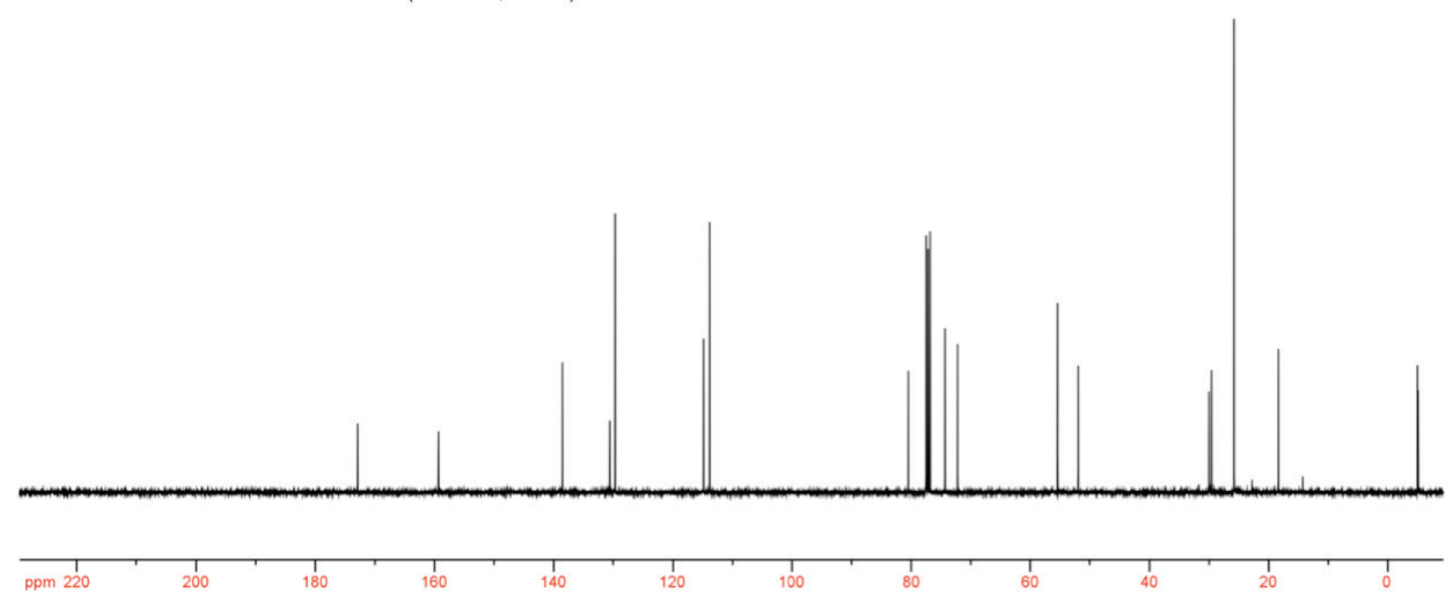



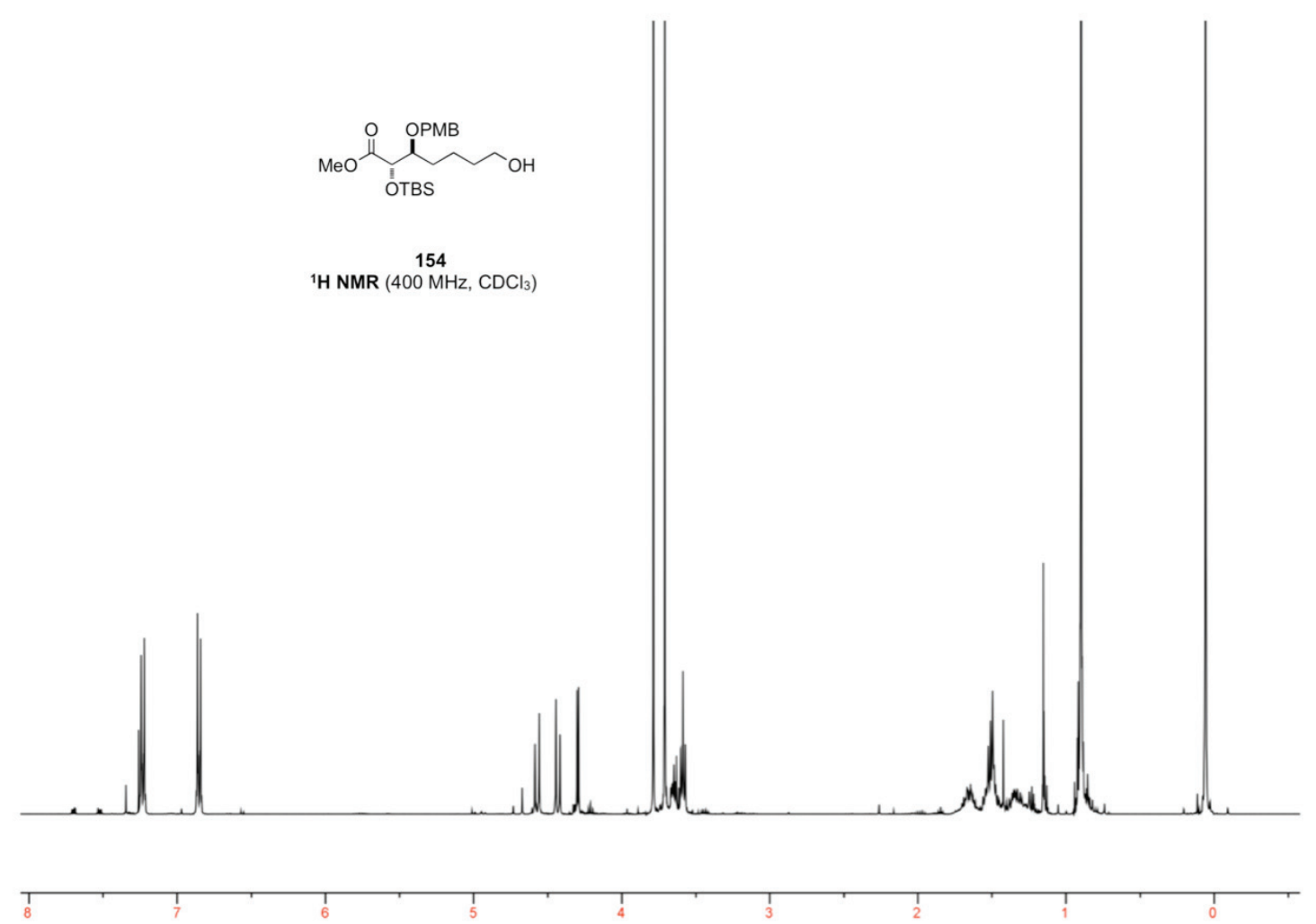

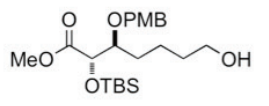

${ }^{13} \mathrm{C}$ NMR $\left(75 \mathrm{MHz}, \mathrm{CDCl}_{3}\right)$ 

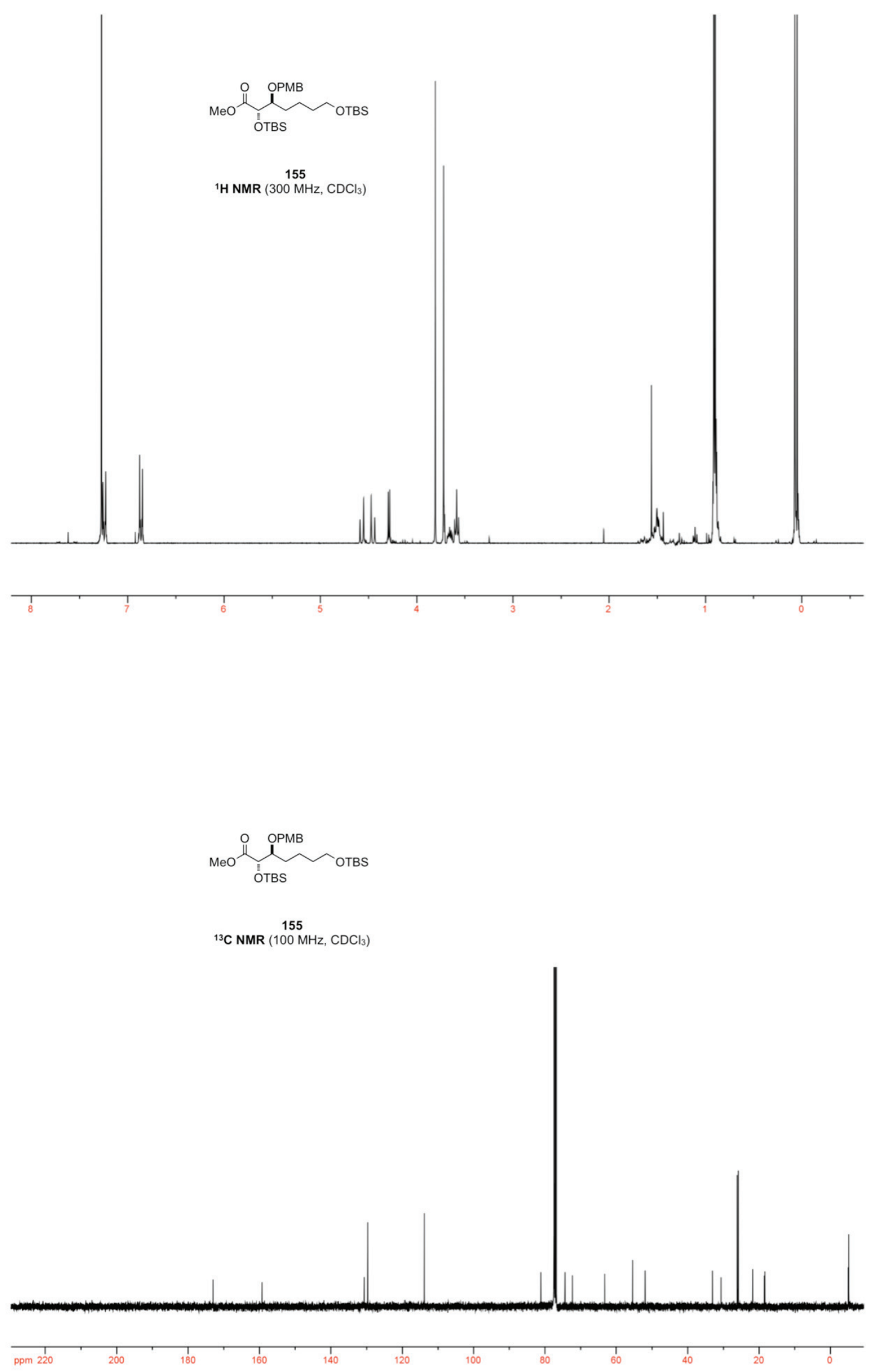

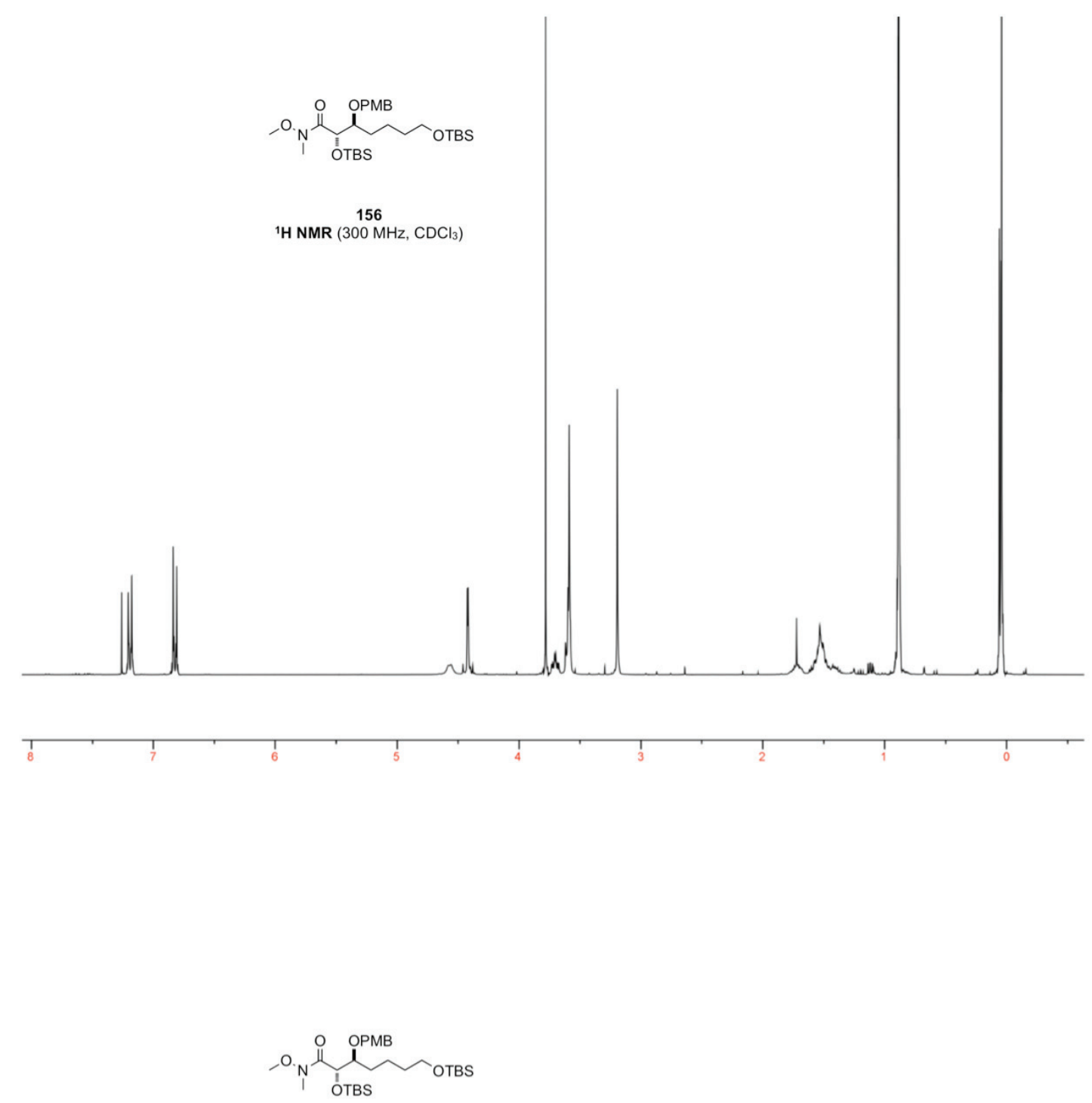

${ }^{13} \mathrm{C}$ NMR $\left(75 \mathrm{MHz}, \mathrm{CDCl}_{3}\right)$

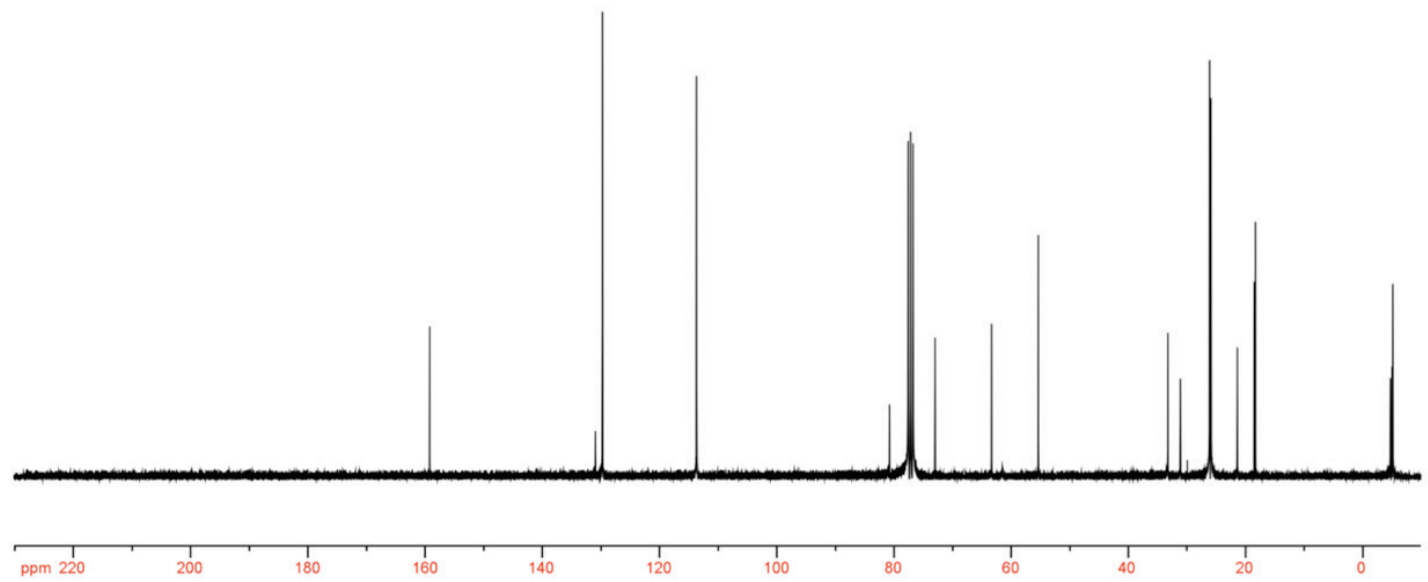



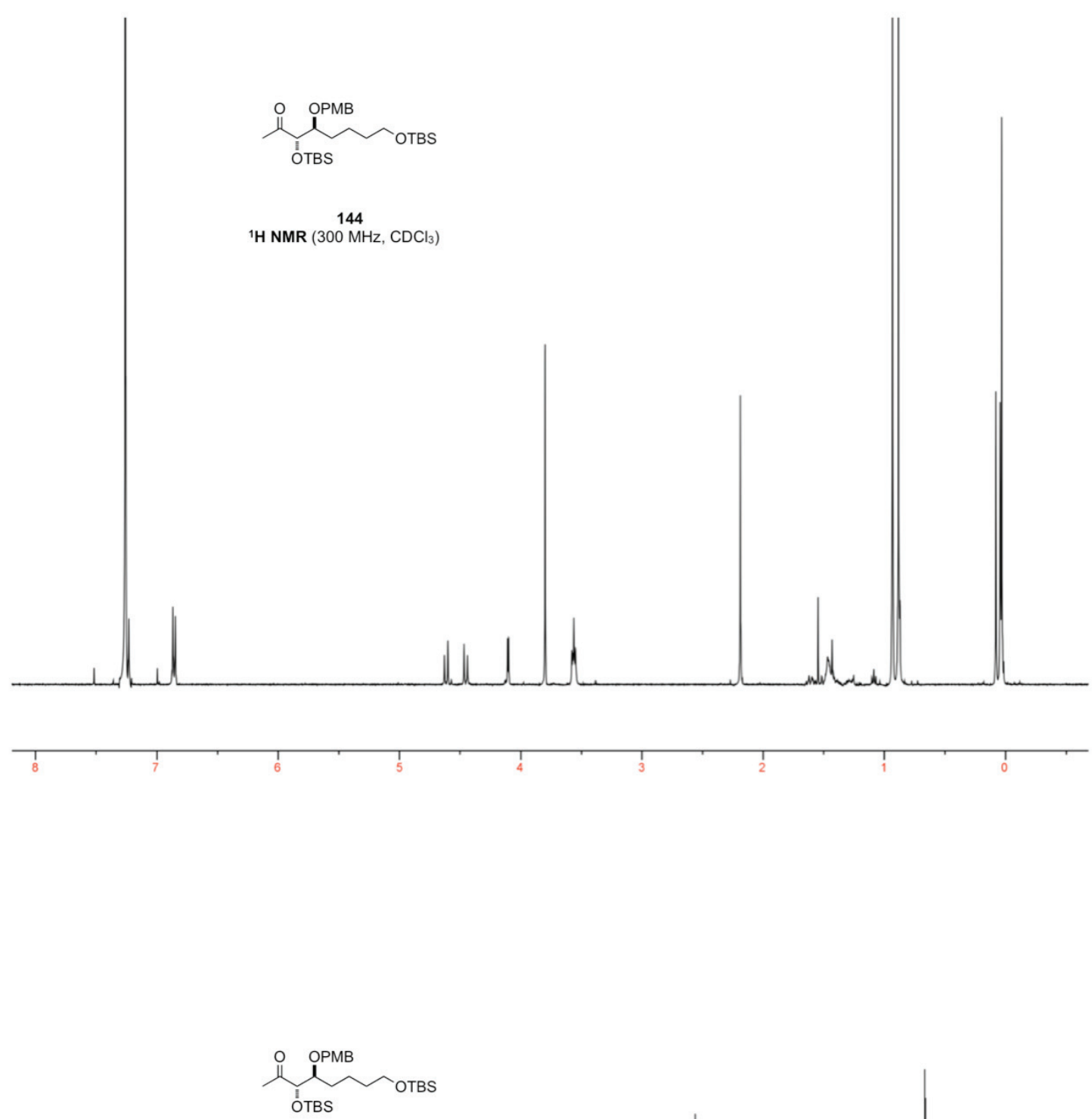

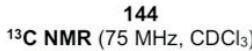

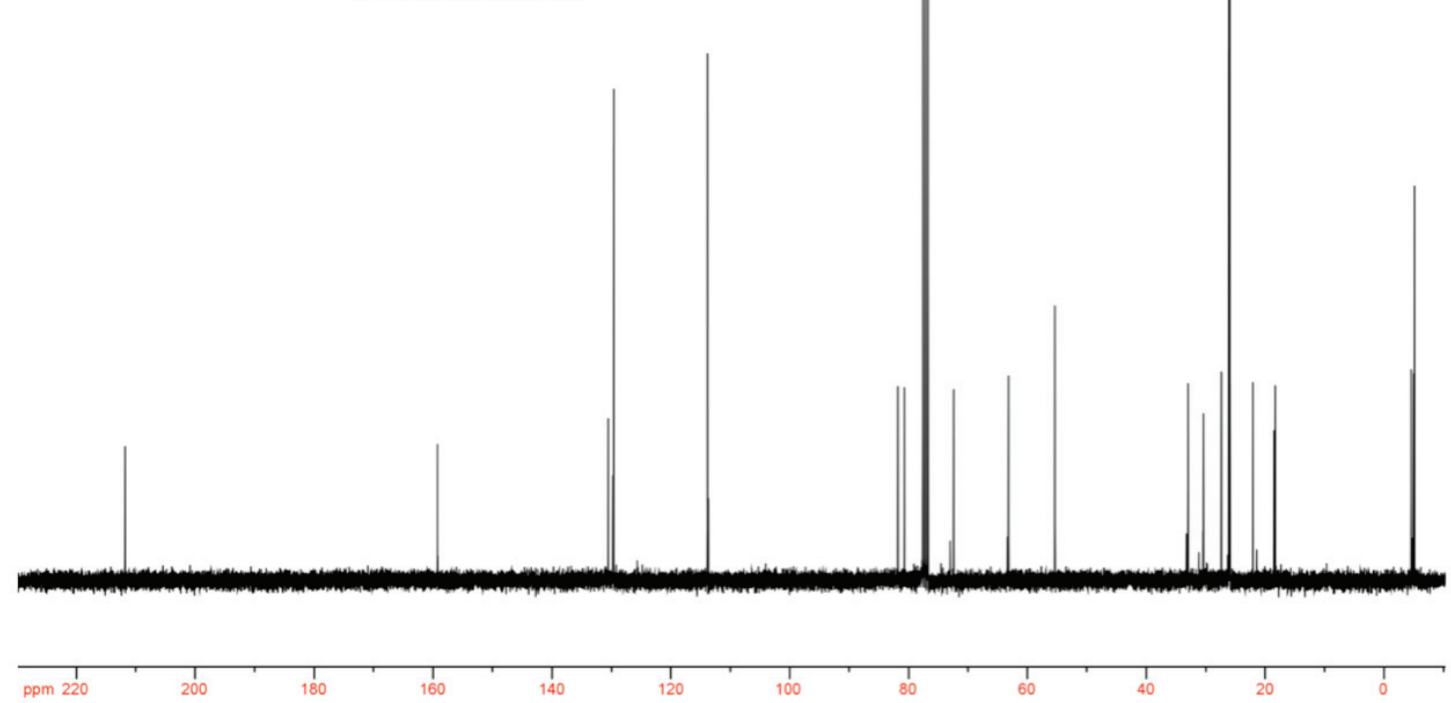



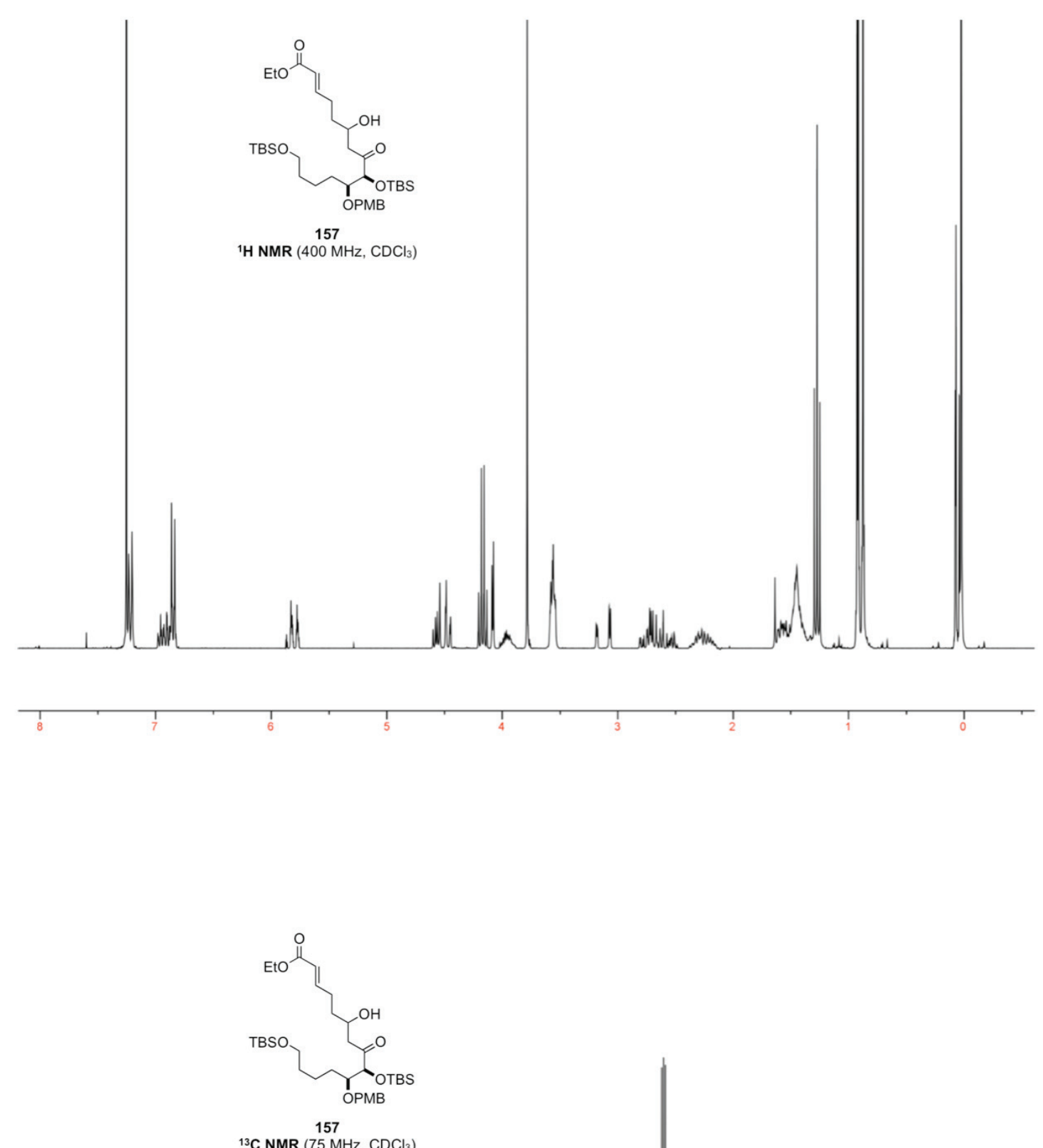

${ }^{13} \mathrm{C}$ NMR $\left(75 \mathrm{MHz}, \mathrm{CDCl}_{3}\right)$
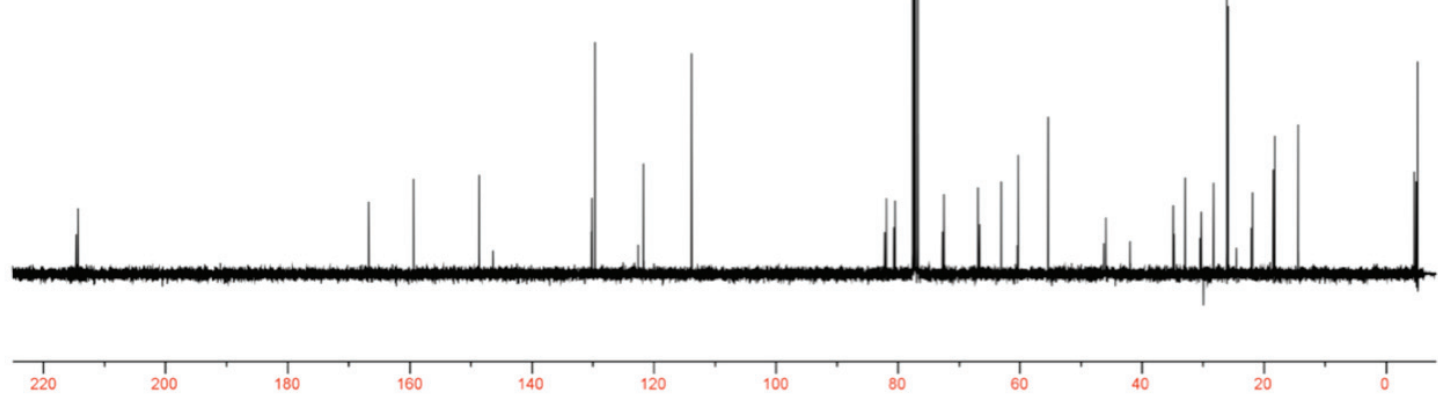

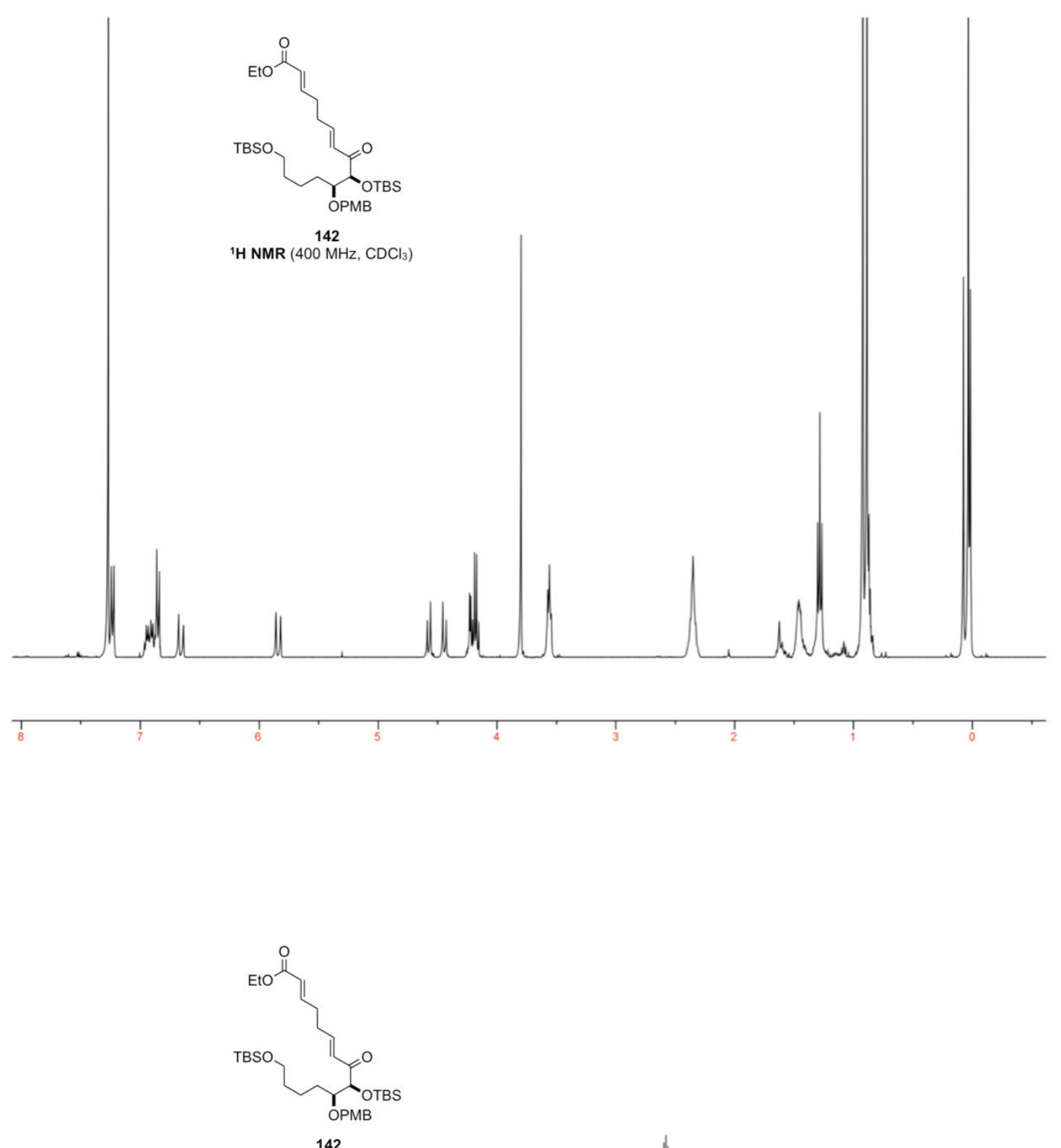

${ }^{13} \mathrm{C} \mathrm{NMR}\left(100 \mathrm{MHz}, \mathrm{CDCl}_{3}\right)$

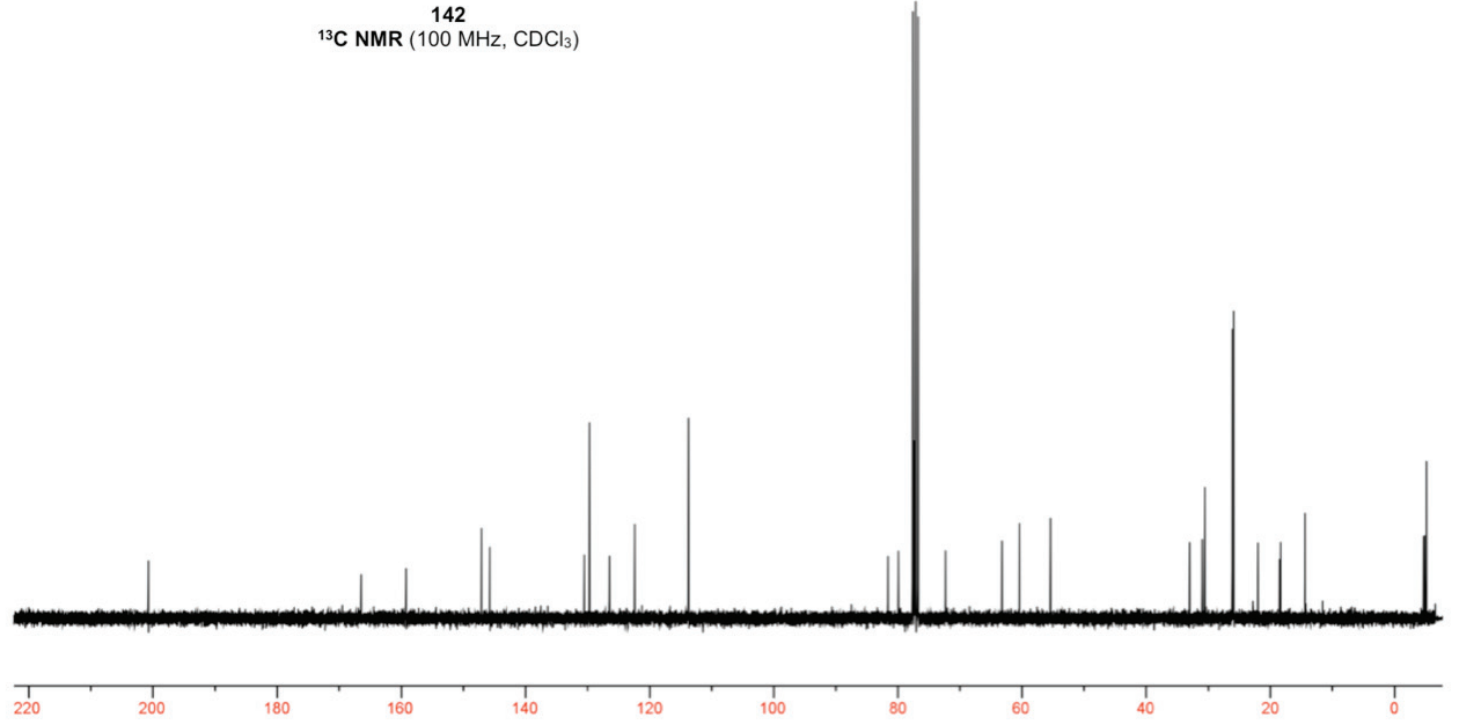




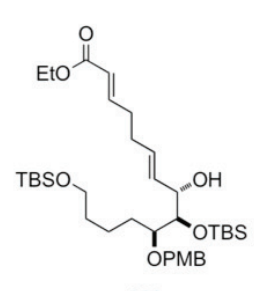

1 $\mathrm{H}$ NMR $\left(500 \mathrm{MHz}, \mathrm{CDCl}_{3}\right)$

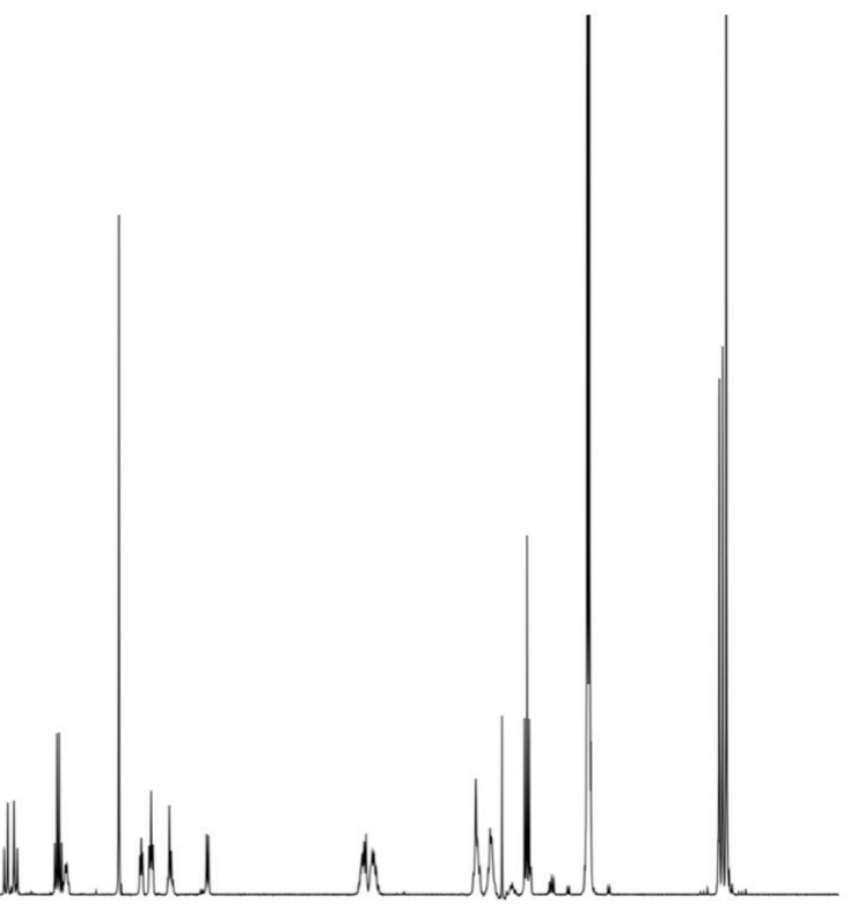

$T_{8}^{1}$

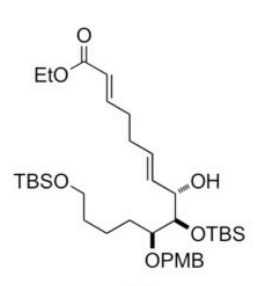

${ }^{13} \mathrm{C}$ NMR $\left(125 \mathrm{MHz}, \mathrm{CDCl}_{3}\right)$
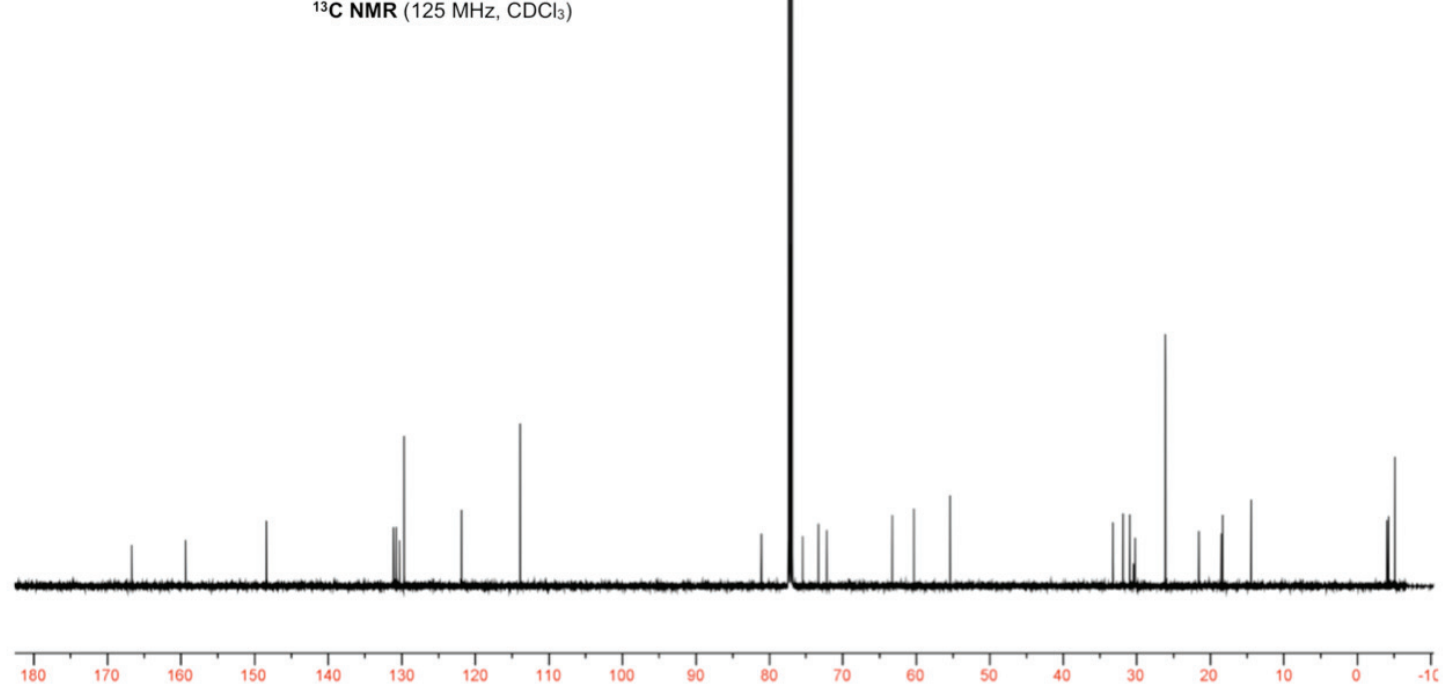

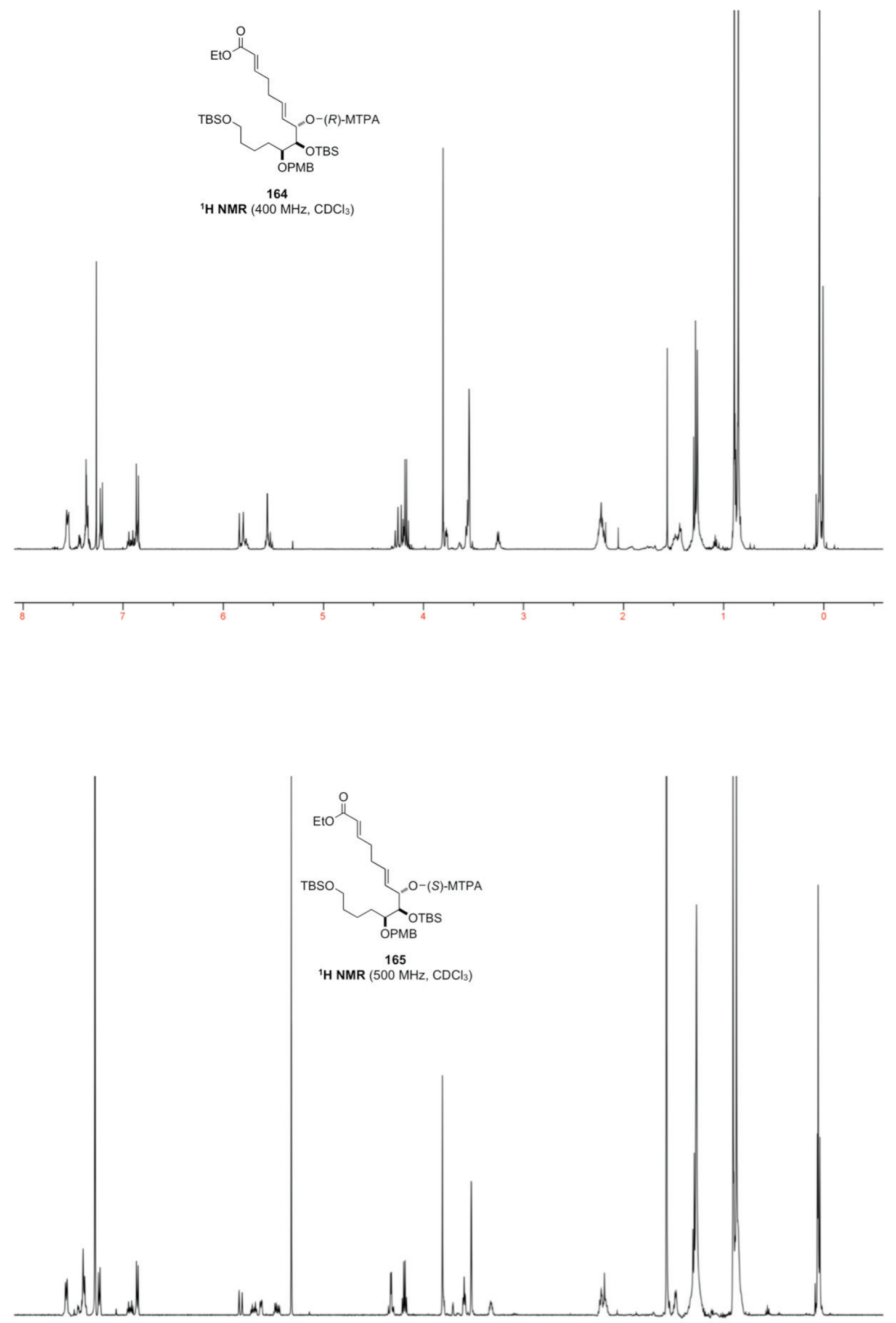

$\underset{8}{1}$

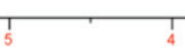



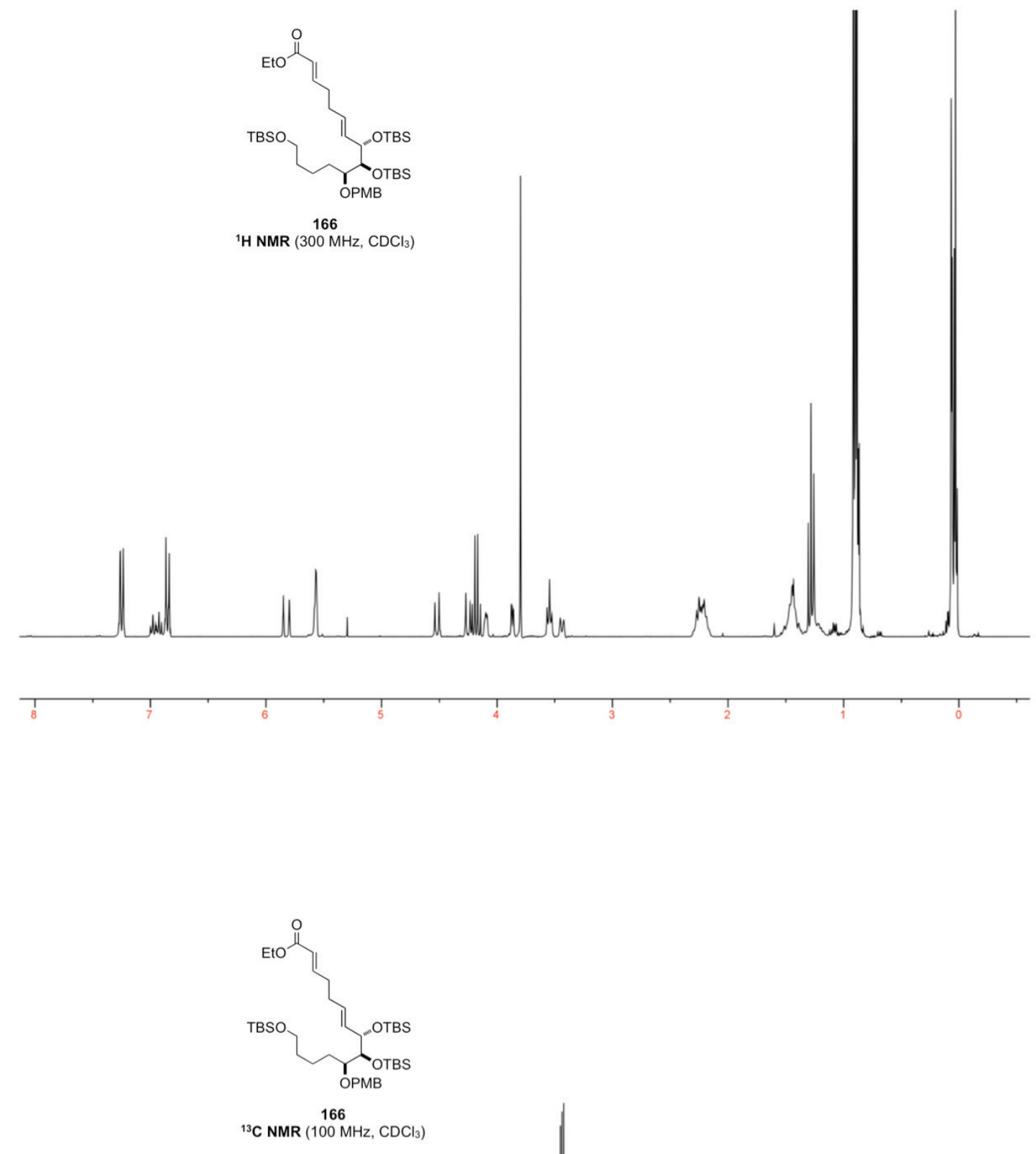

${ }^{13} \mathrm{C}$ NMR $\left(100 \mathrm{MHz}, \mathrm{CDCl}_{3}\right)$
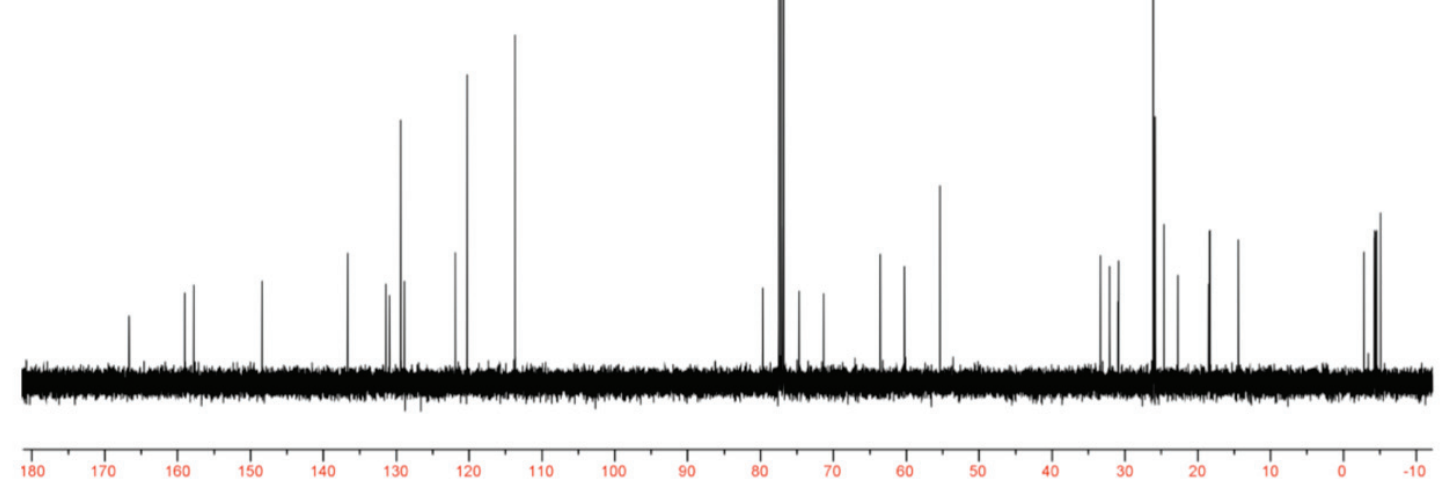


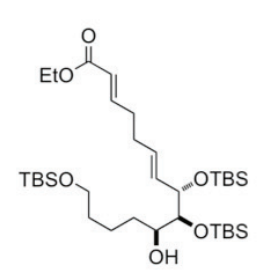

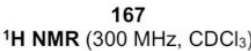
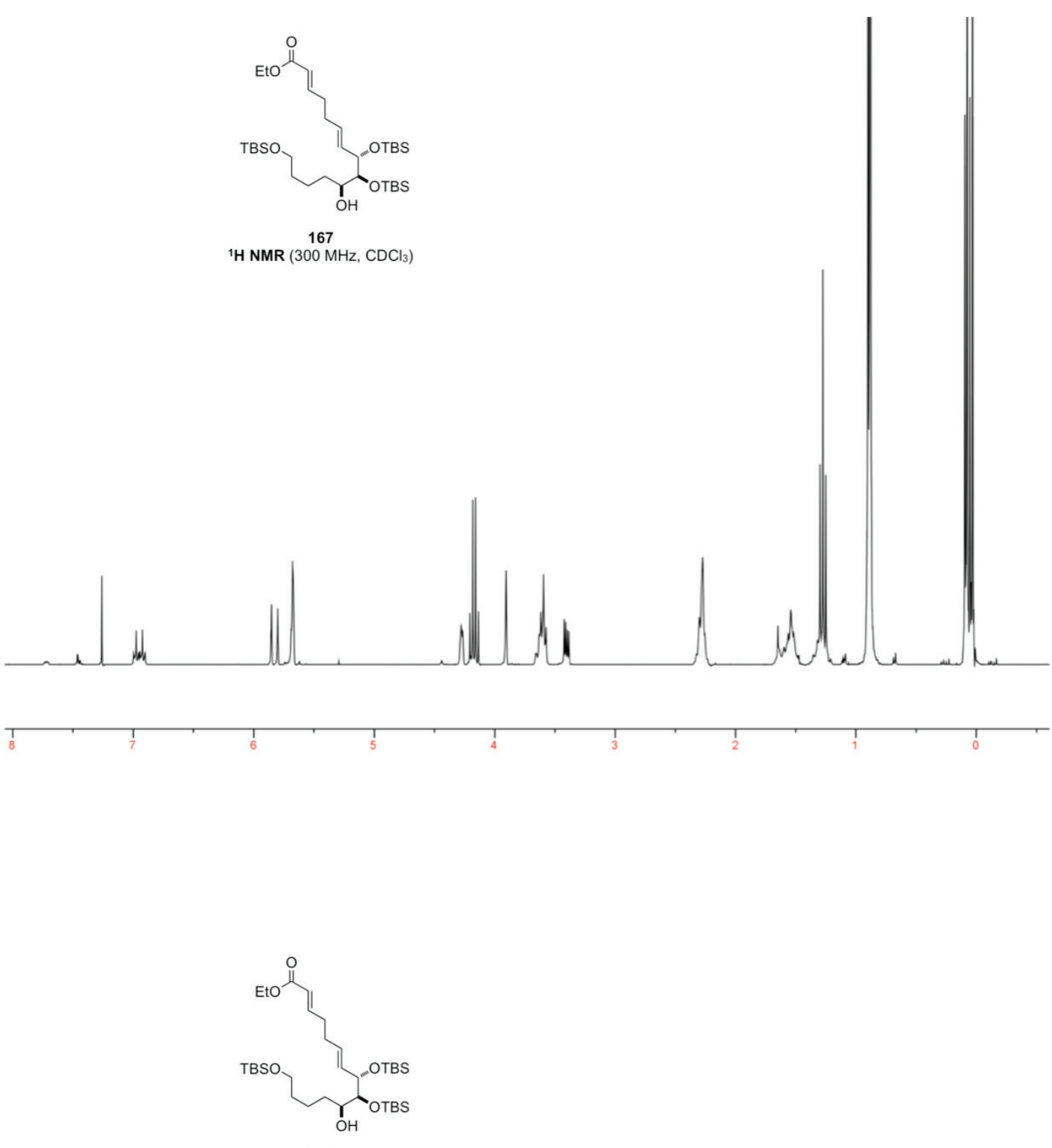

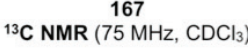

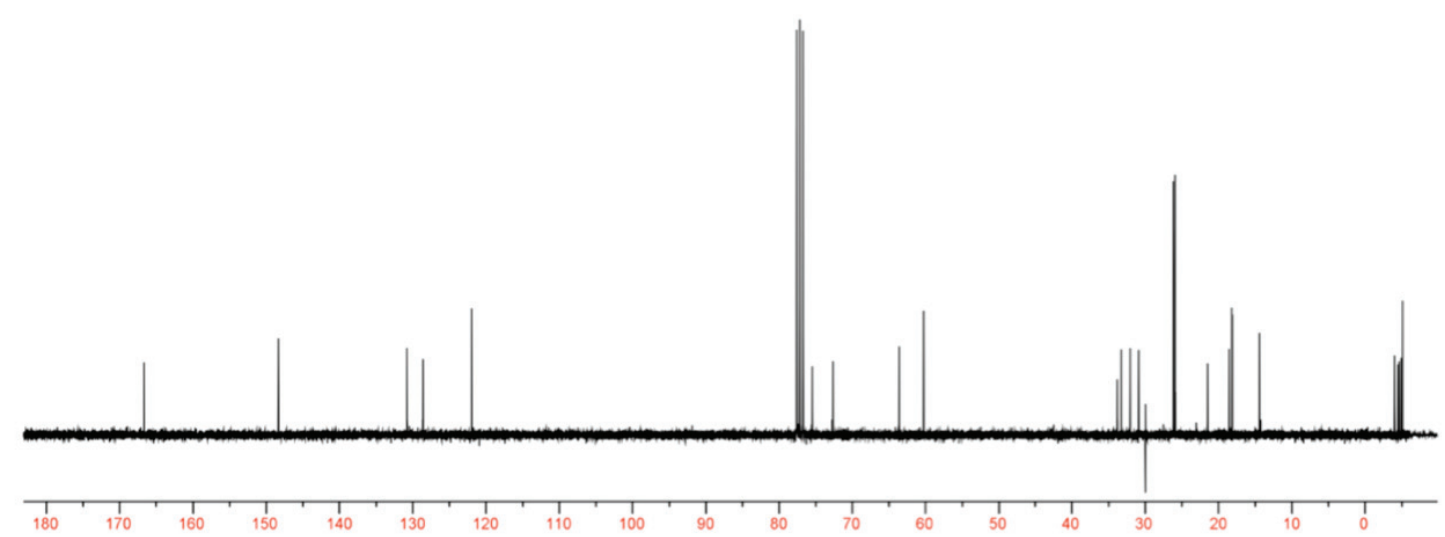




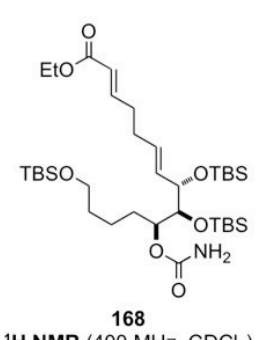

${ }^{1} \mathrm{H}$ NMR (400 MHz, $\left.\mathrm{CDCl}_{3}\right)$
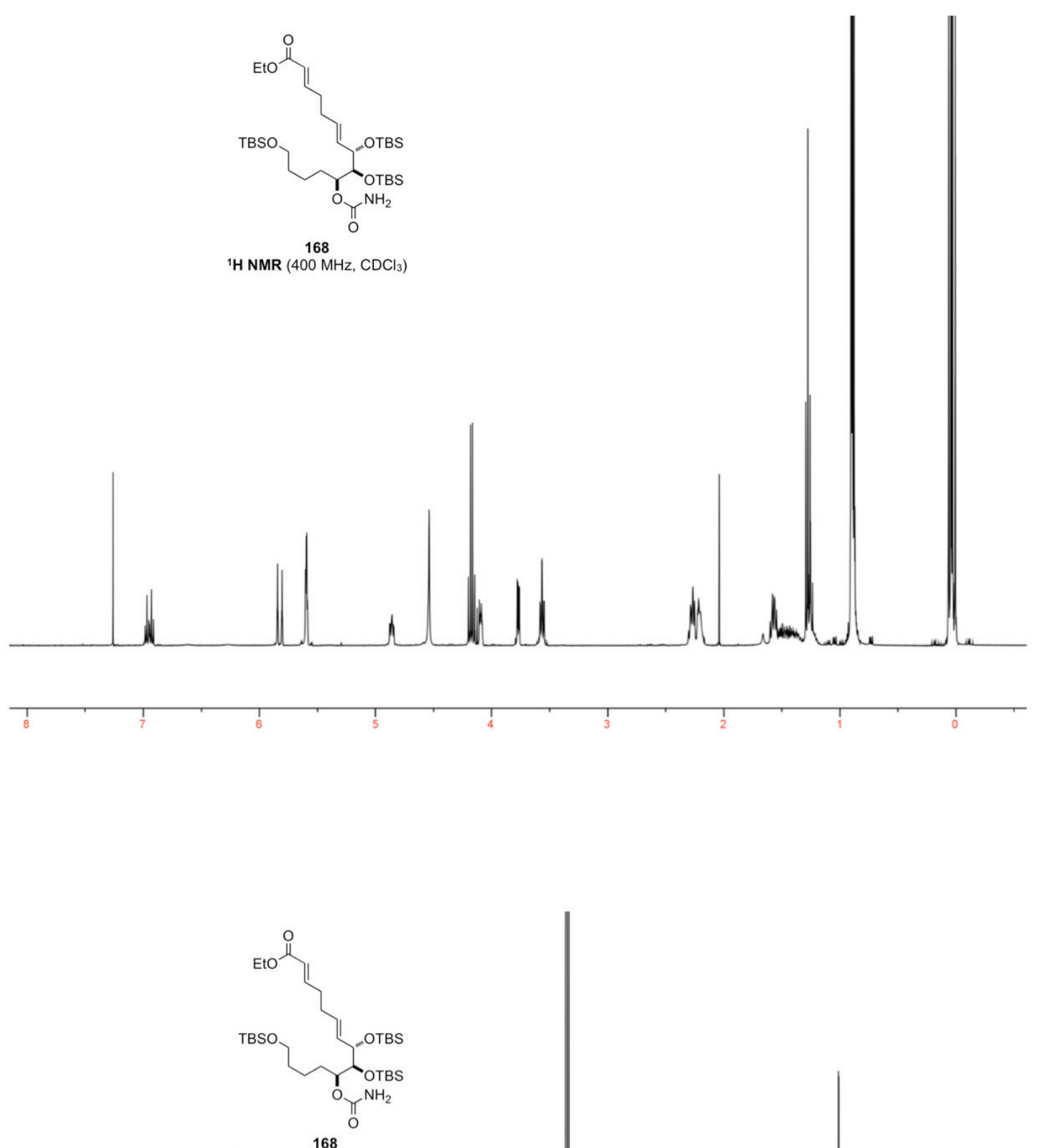

${ }^{13} \mathrm{C}$ NMR $\left(100 \mathrm{MHz}, \mathrm{CDCl}_{3}\right)$

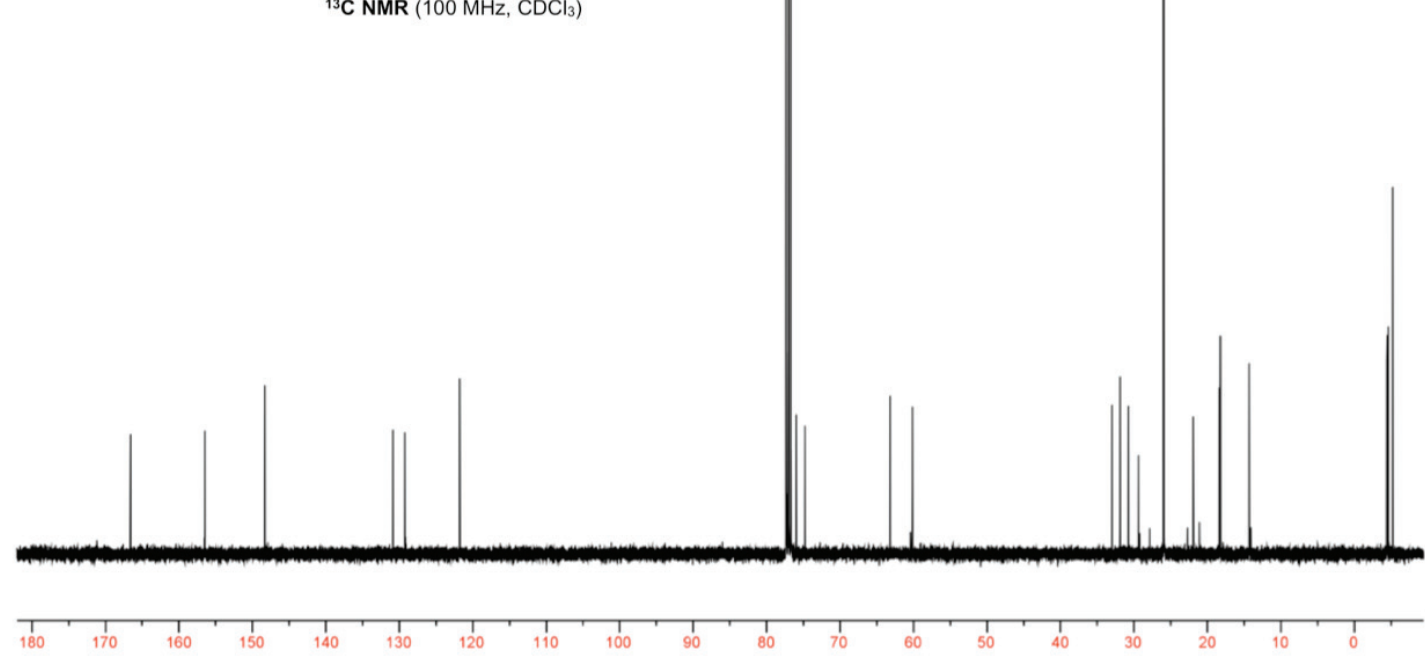



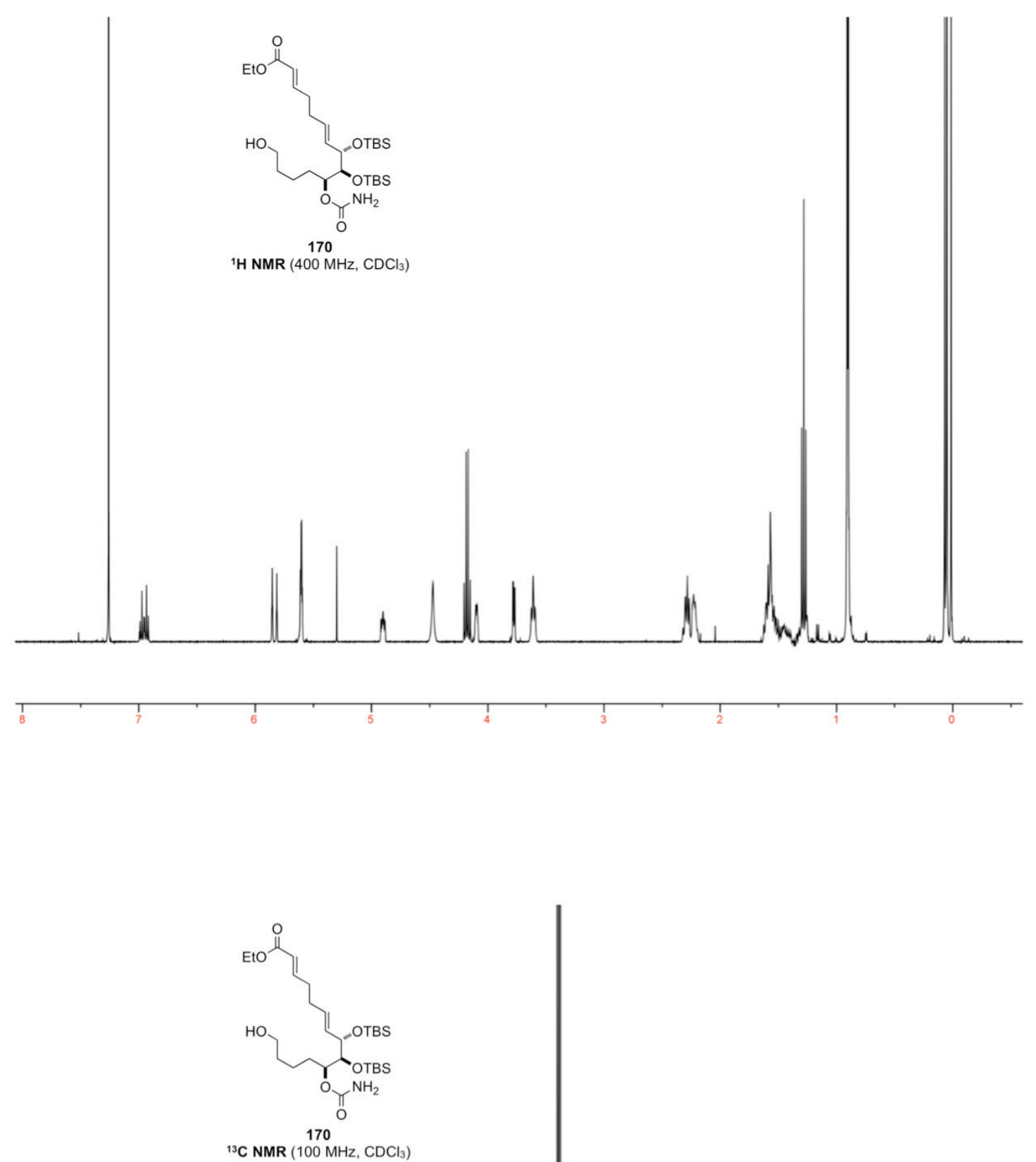

${ }^{13} \mathrm{C}$ NMR $\left(100 \mathrm{MHz}, \mathrm{CDCl}_{3}\right)$
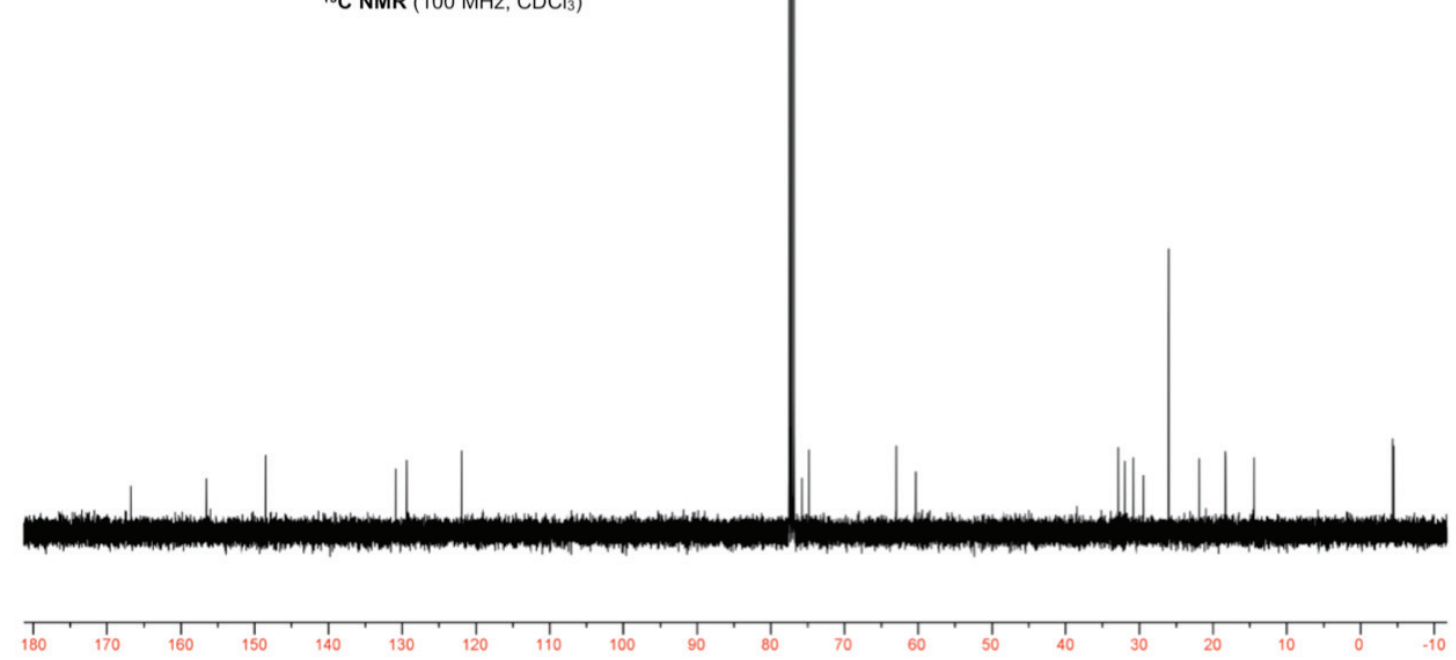

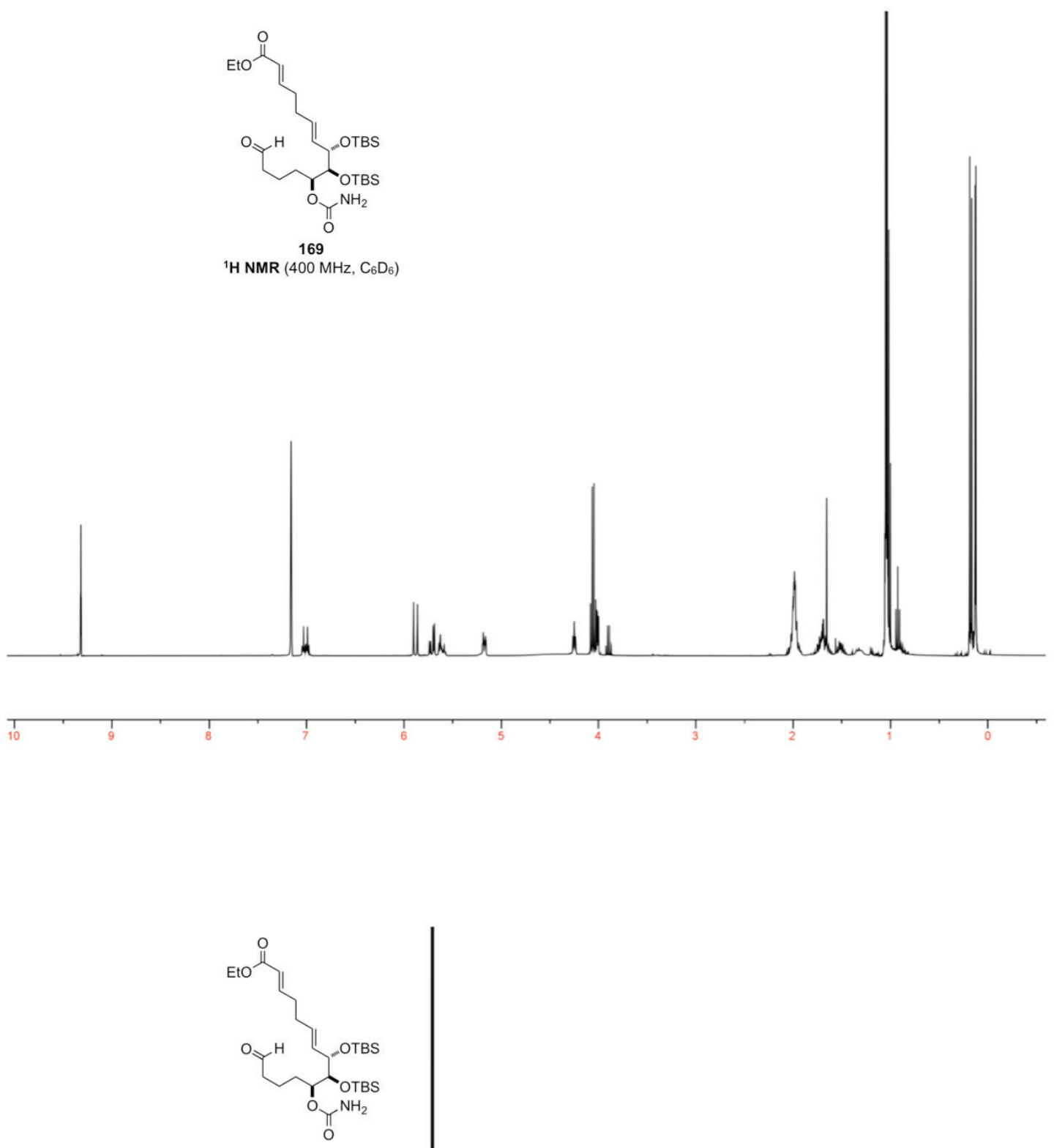

${ }^{13} \mathrm{C}$ NMR $\left(100 \mathrm{MHz}, \mathrm{C}_{6} \mathrm{D}_{6}\right)$

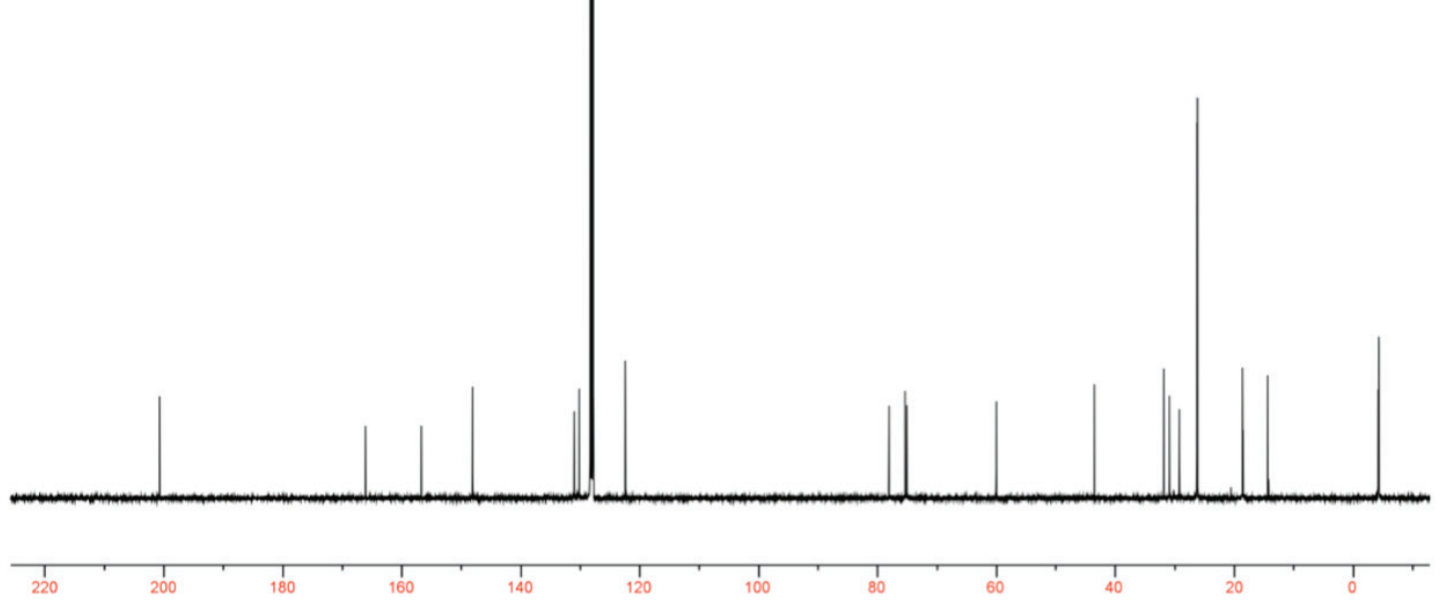


Appendix
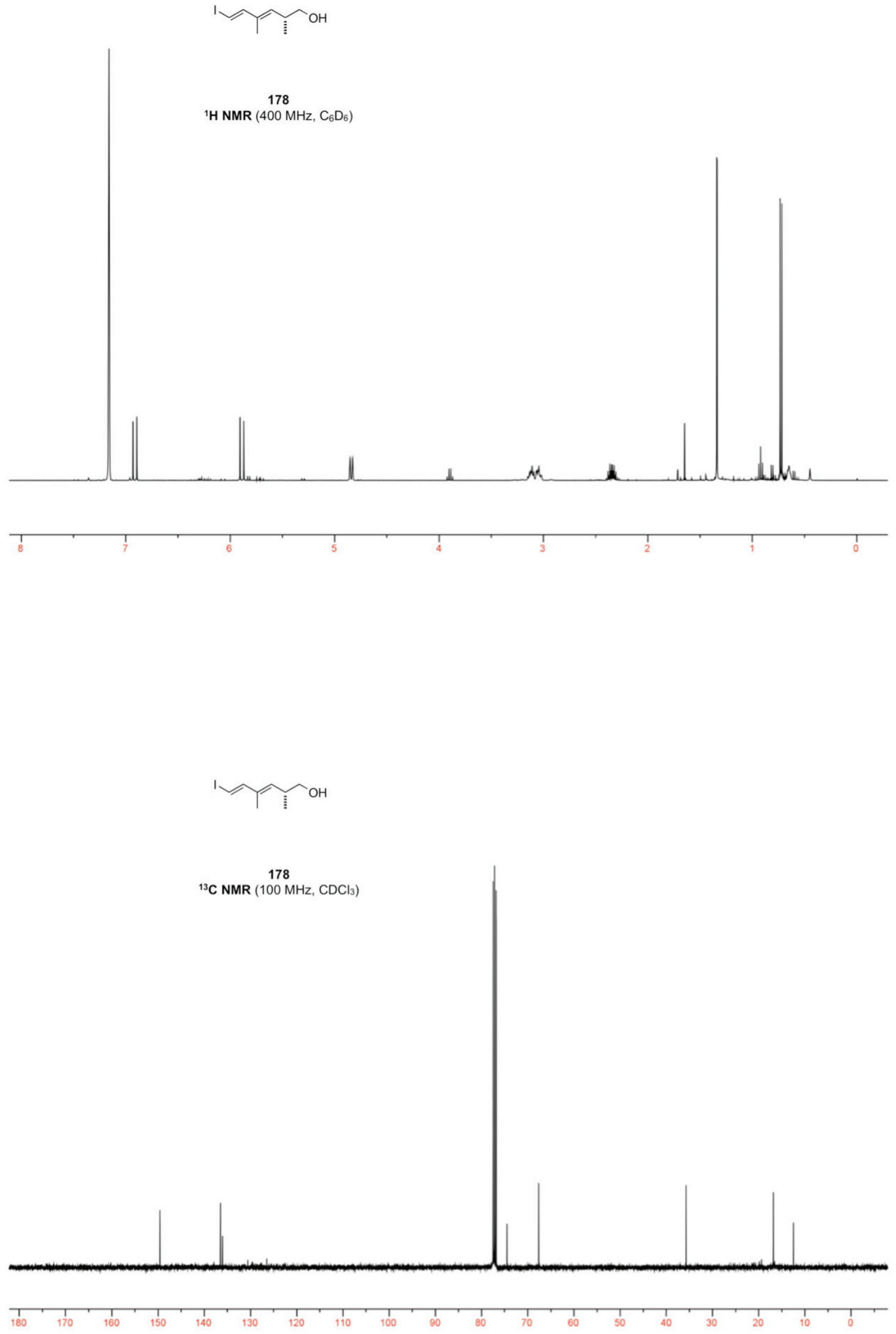

204 
Appendix
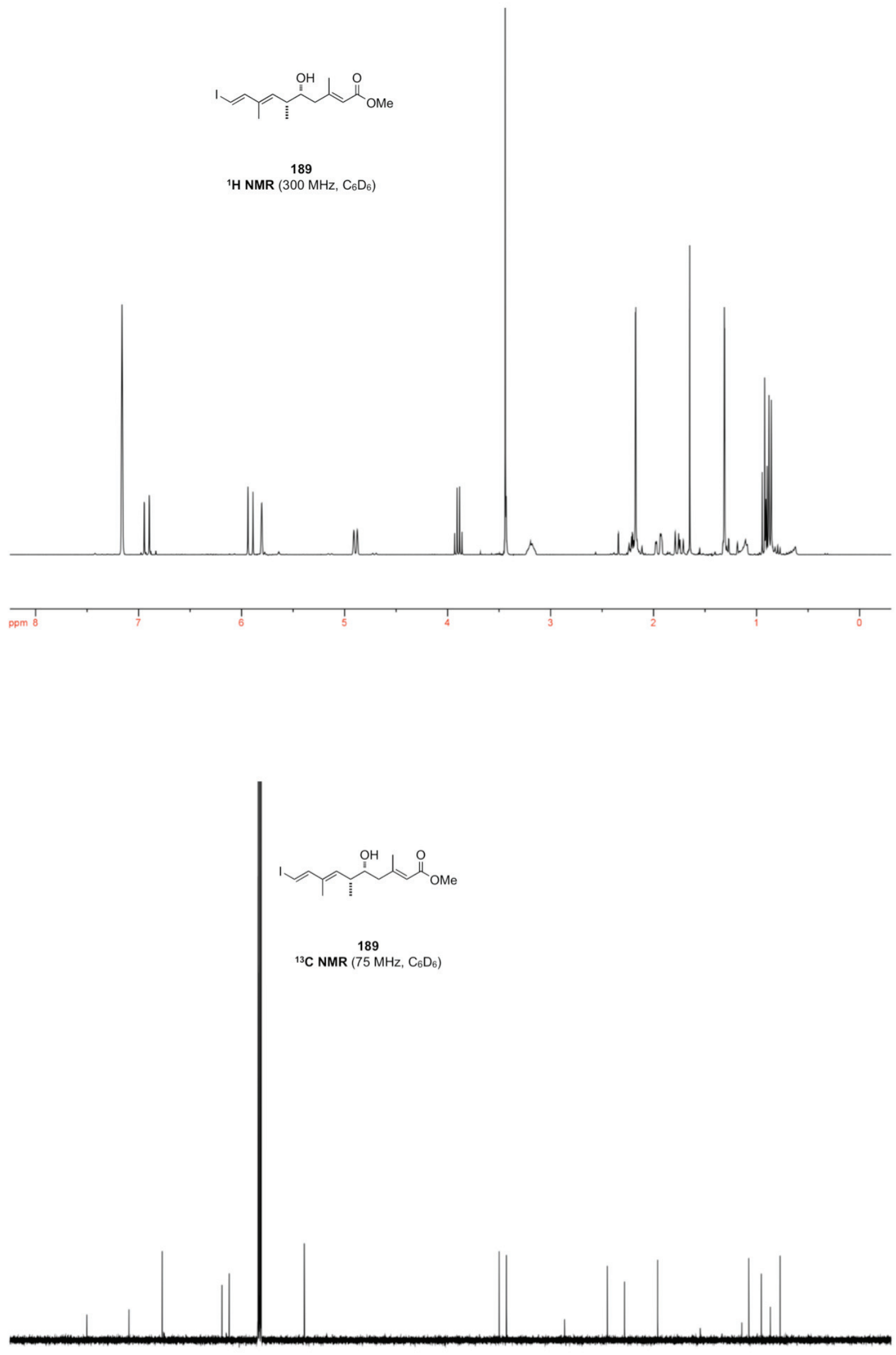

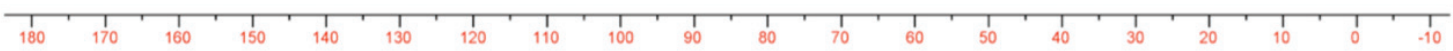

205 

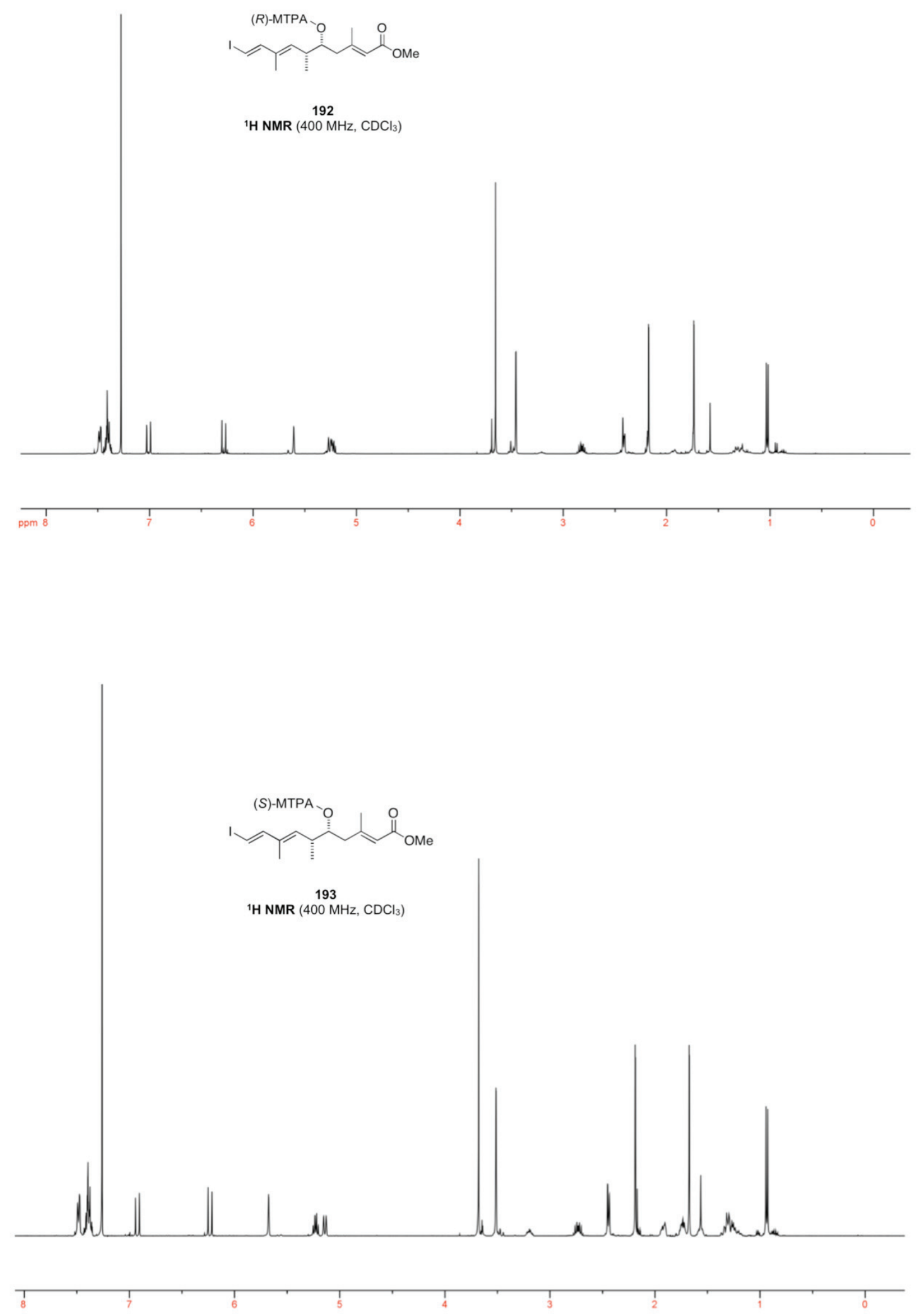

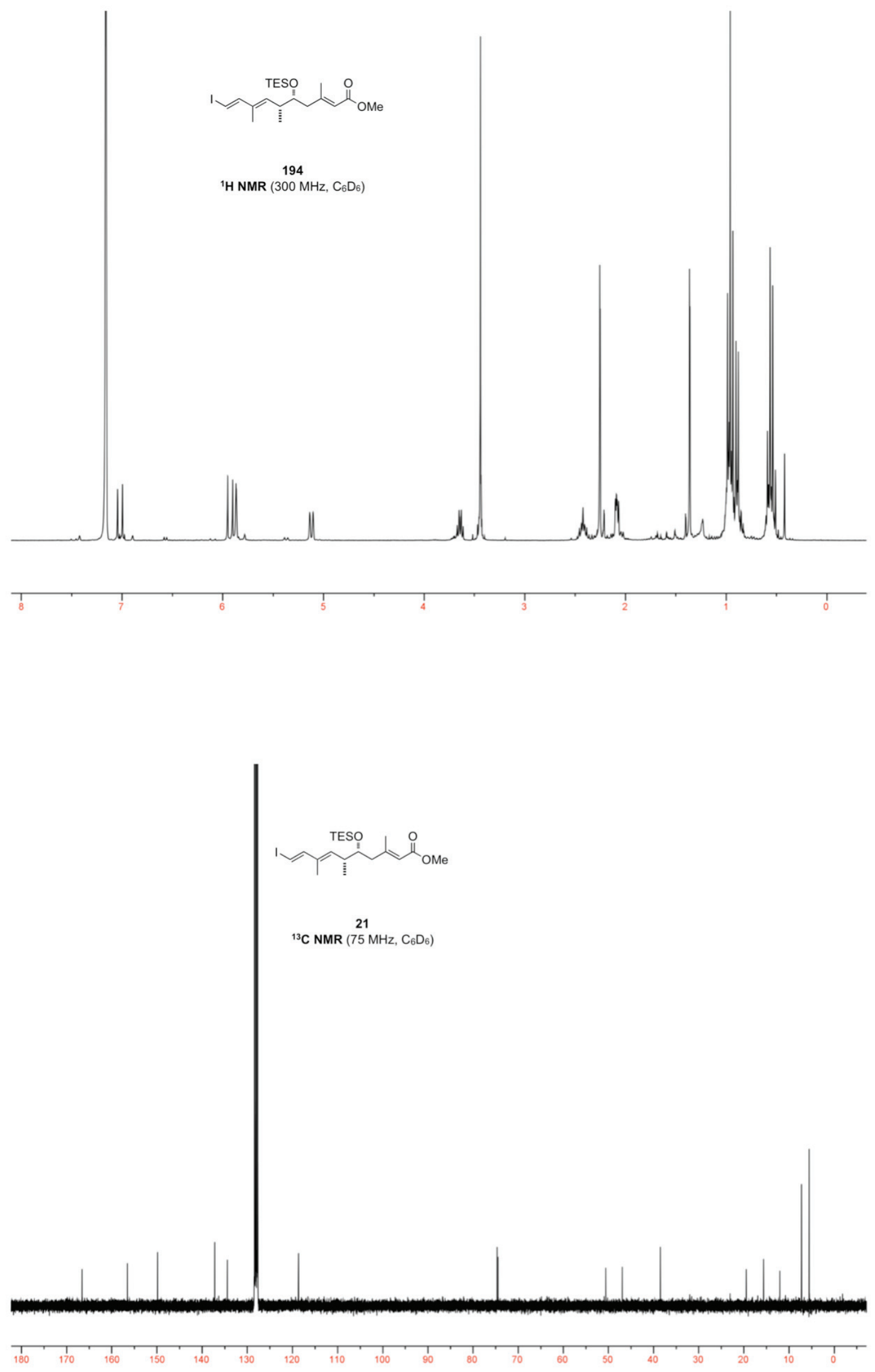

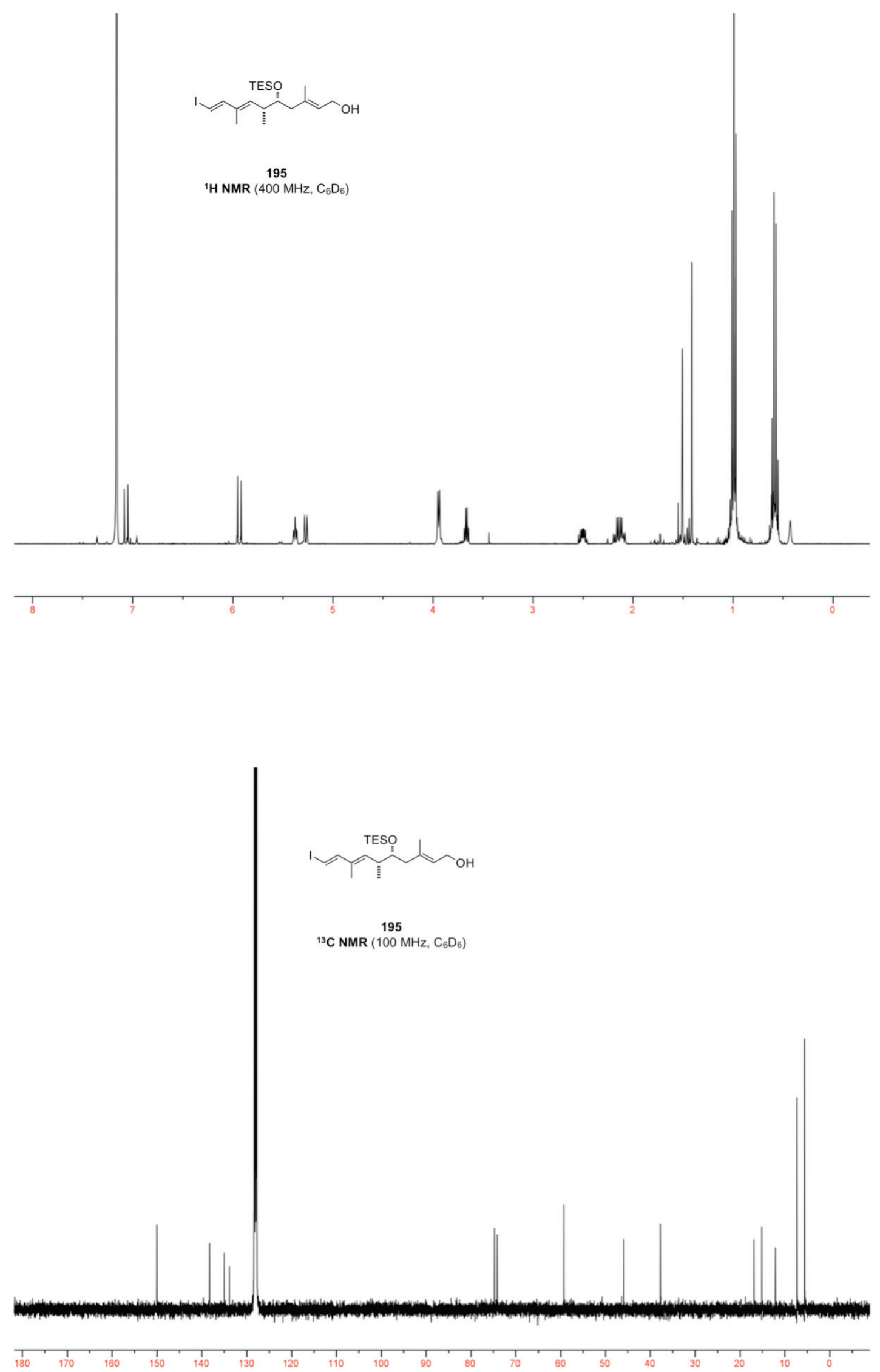

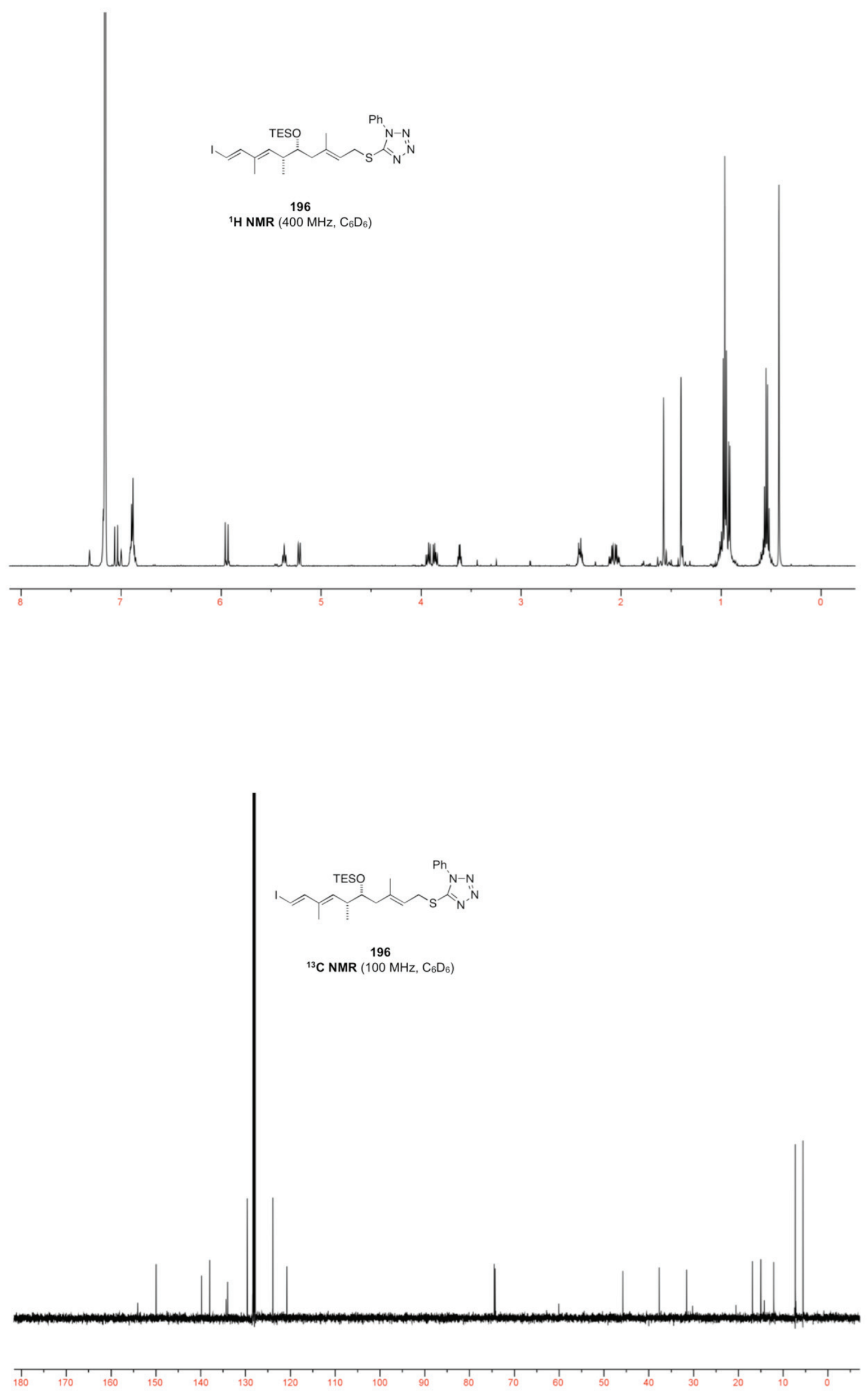

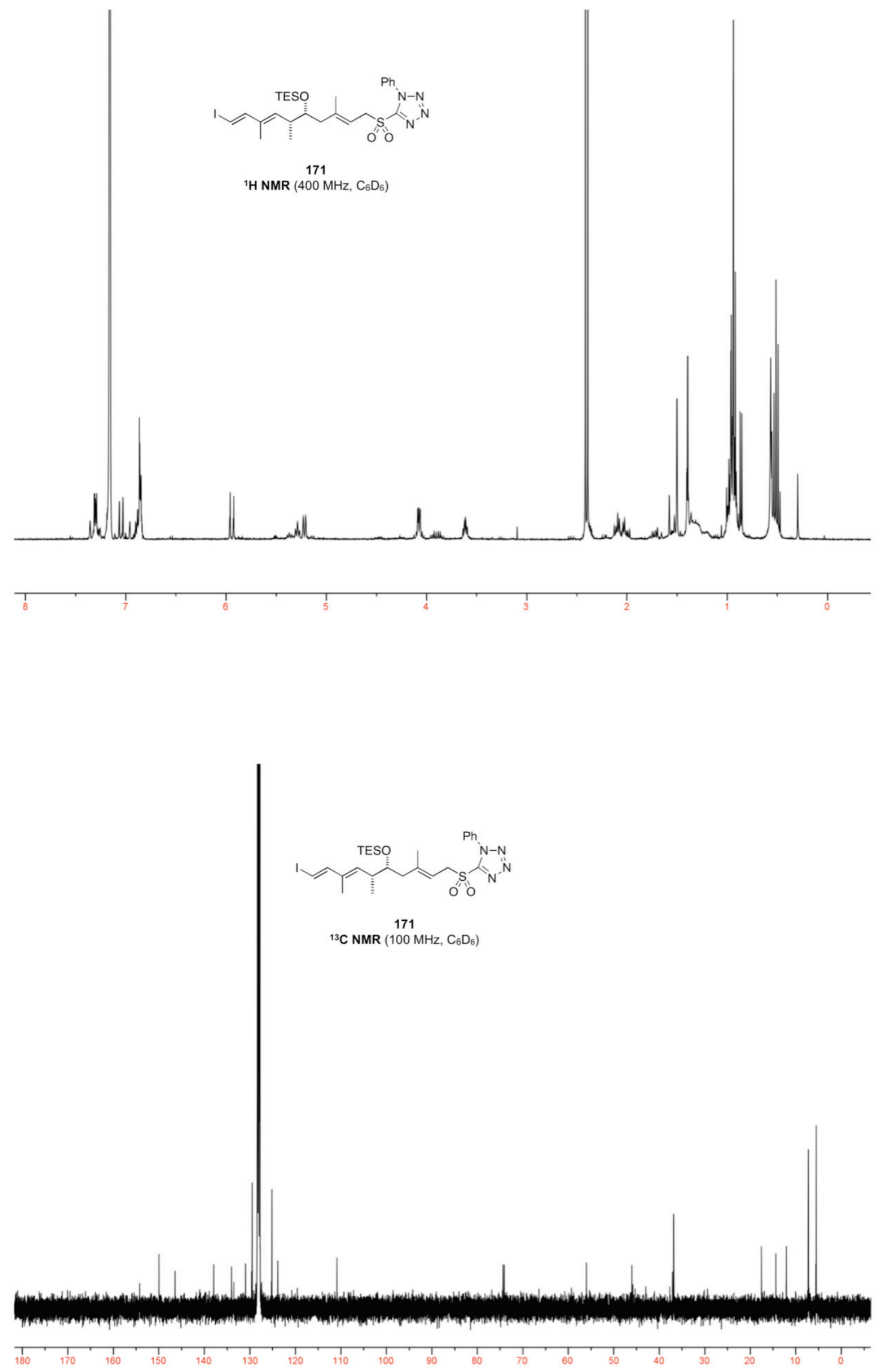

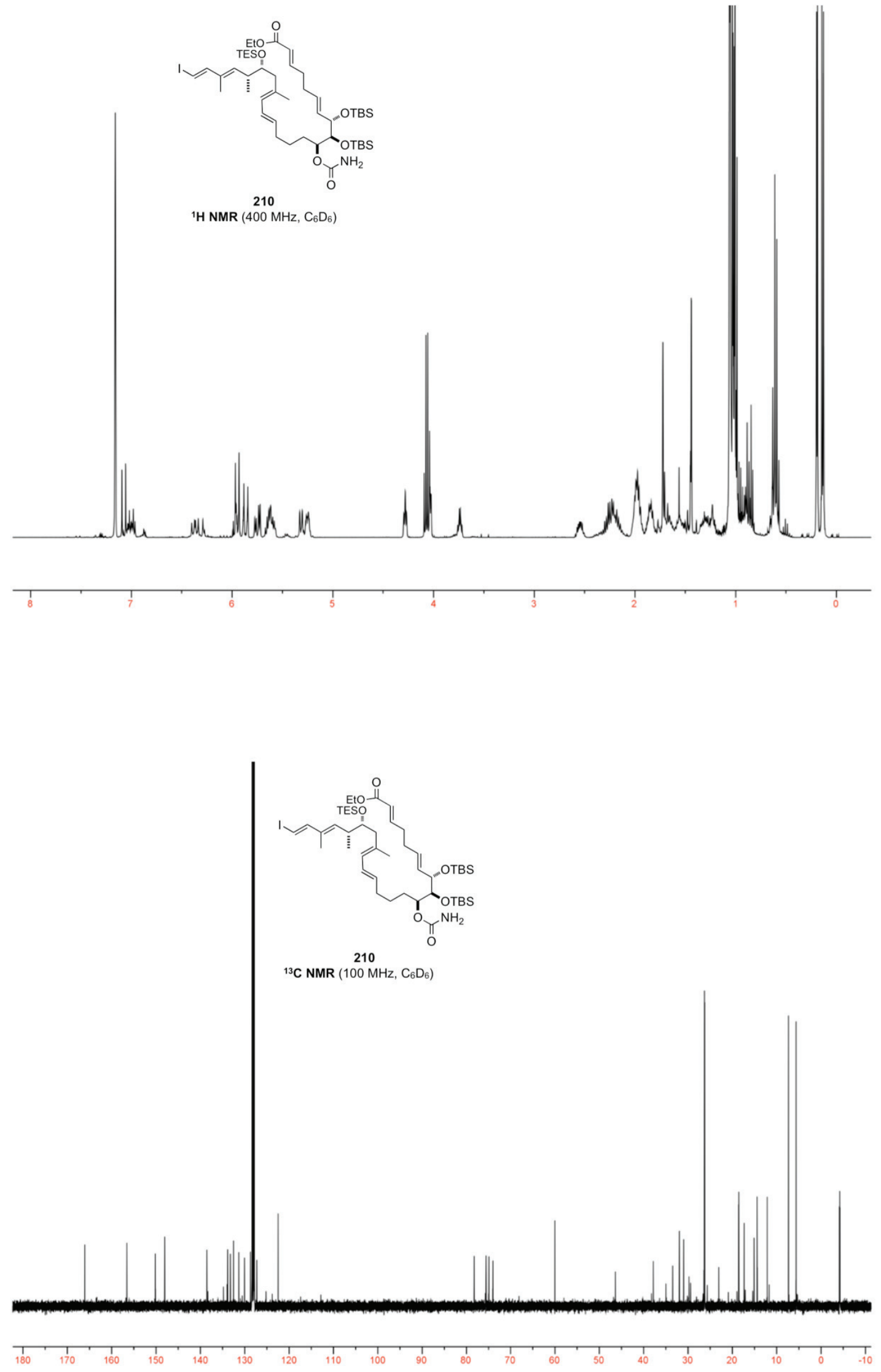

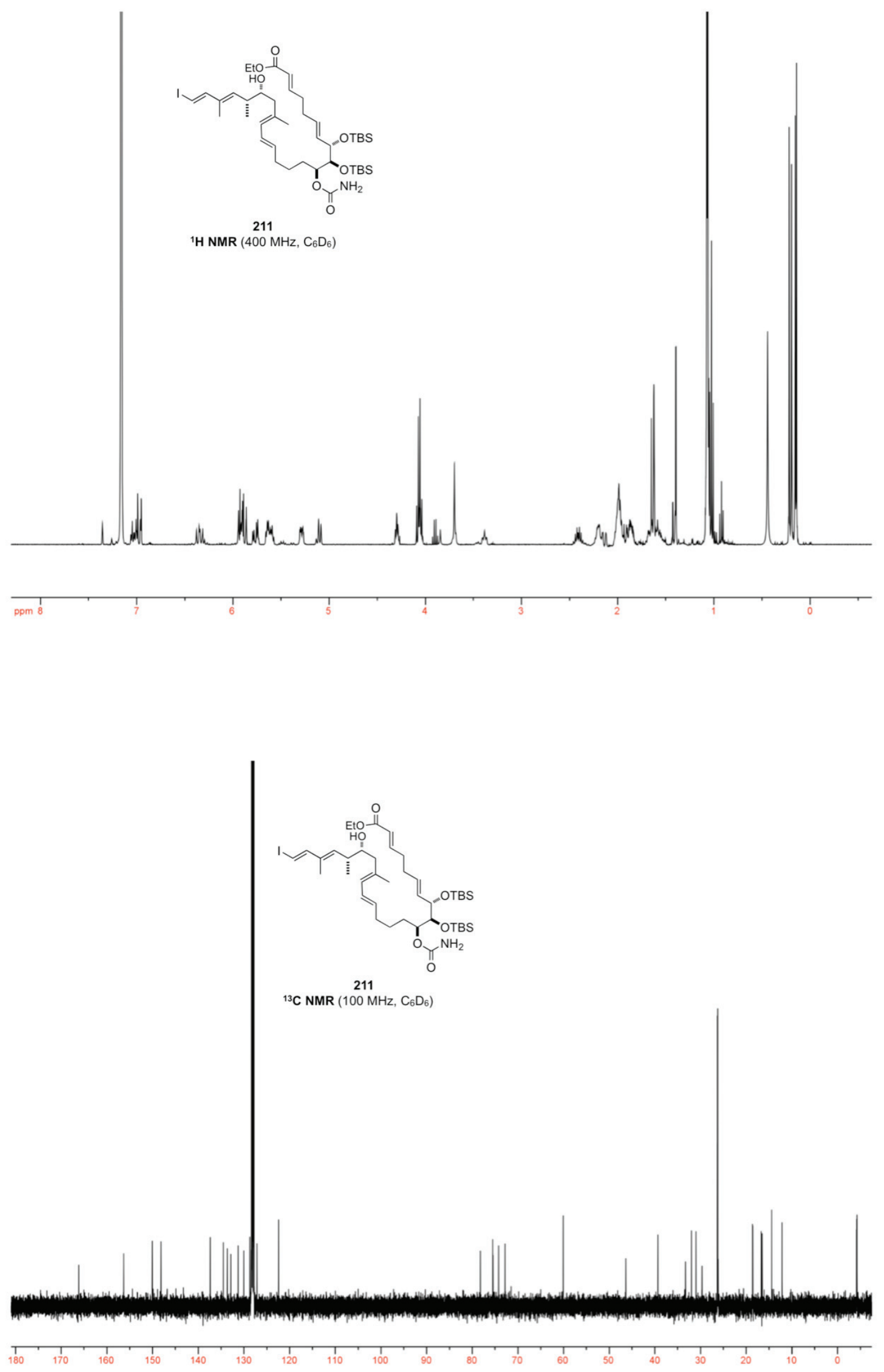

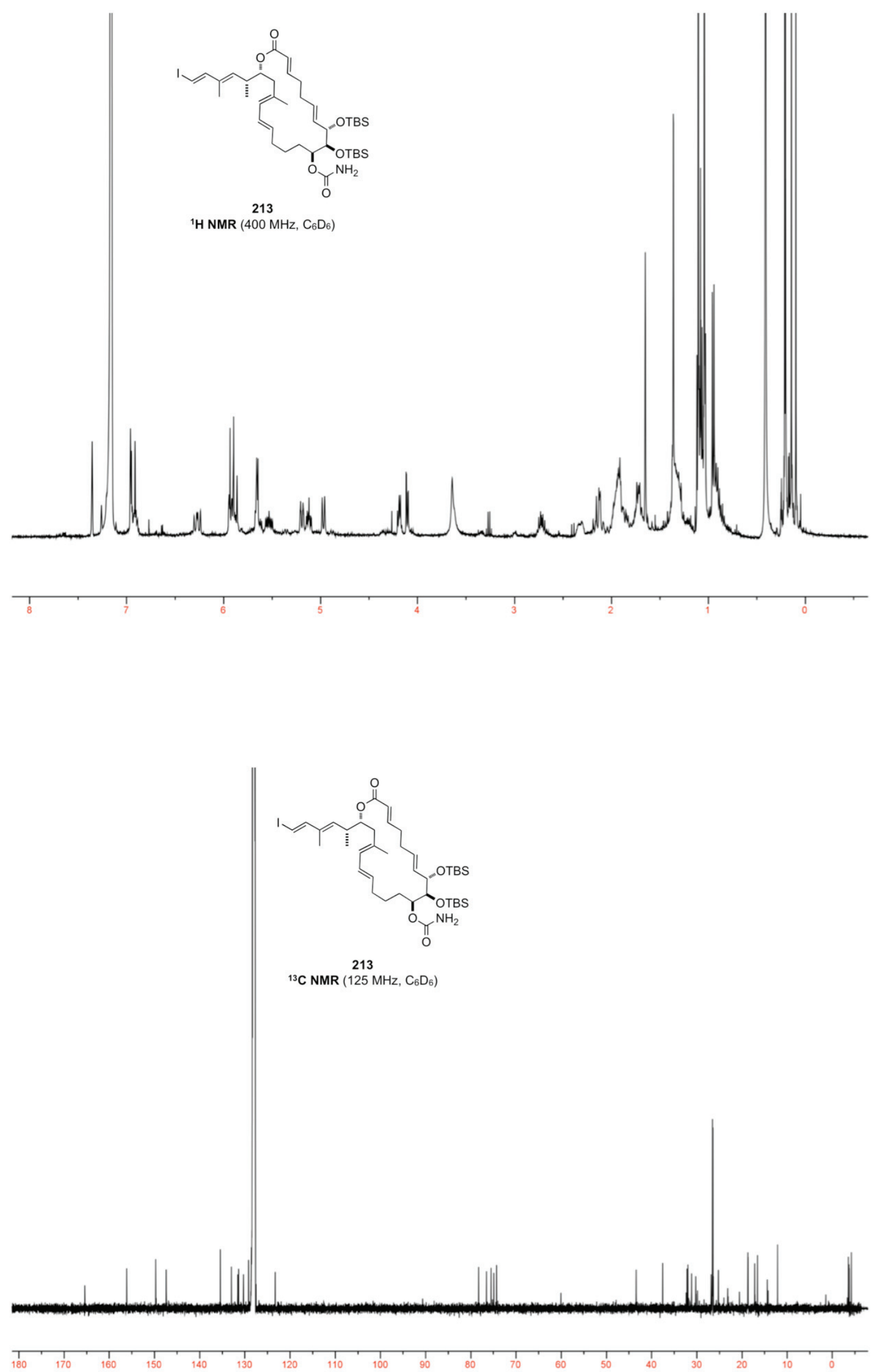

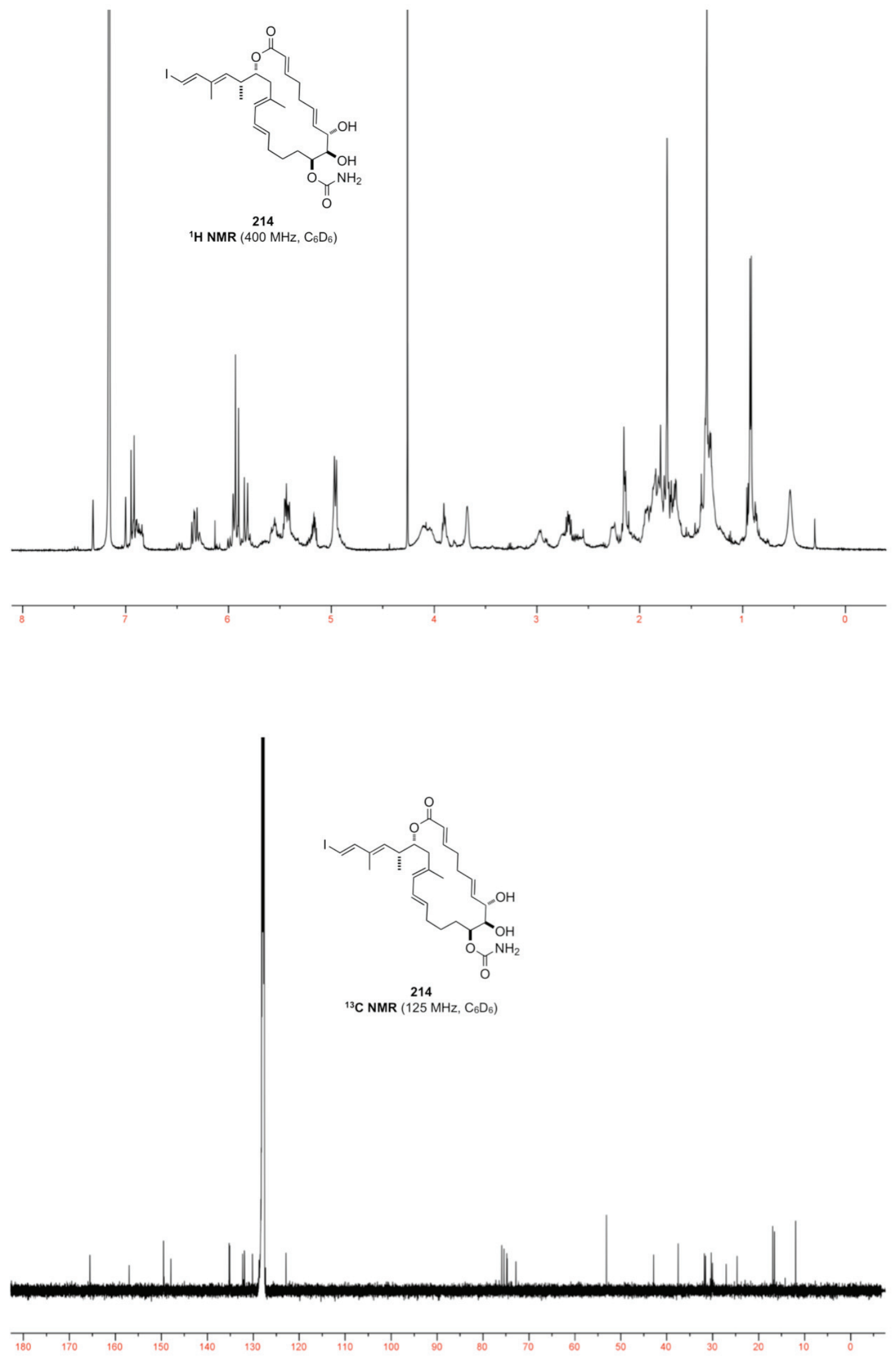


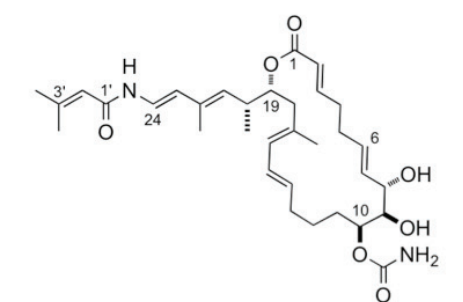

3b: proposed structure $=9,10$-bis-epi-palmerolide $\mathrm{C}$

${ }^{1} \mathrm{H}$ NMR $\left(500 \mathrm{MHz}, d_{6}\right.$-DMSO)
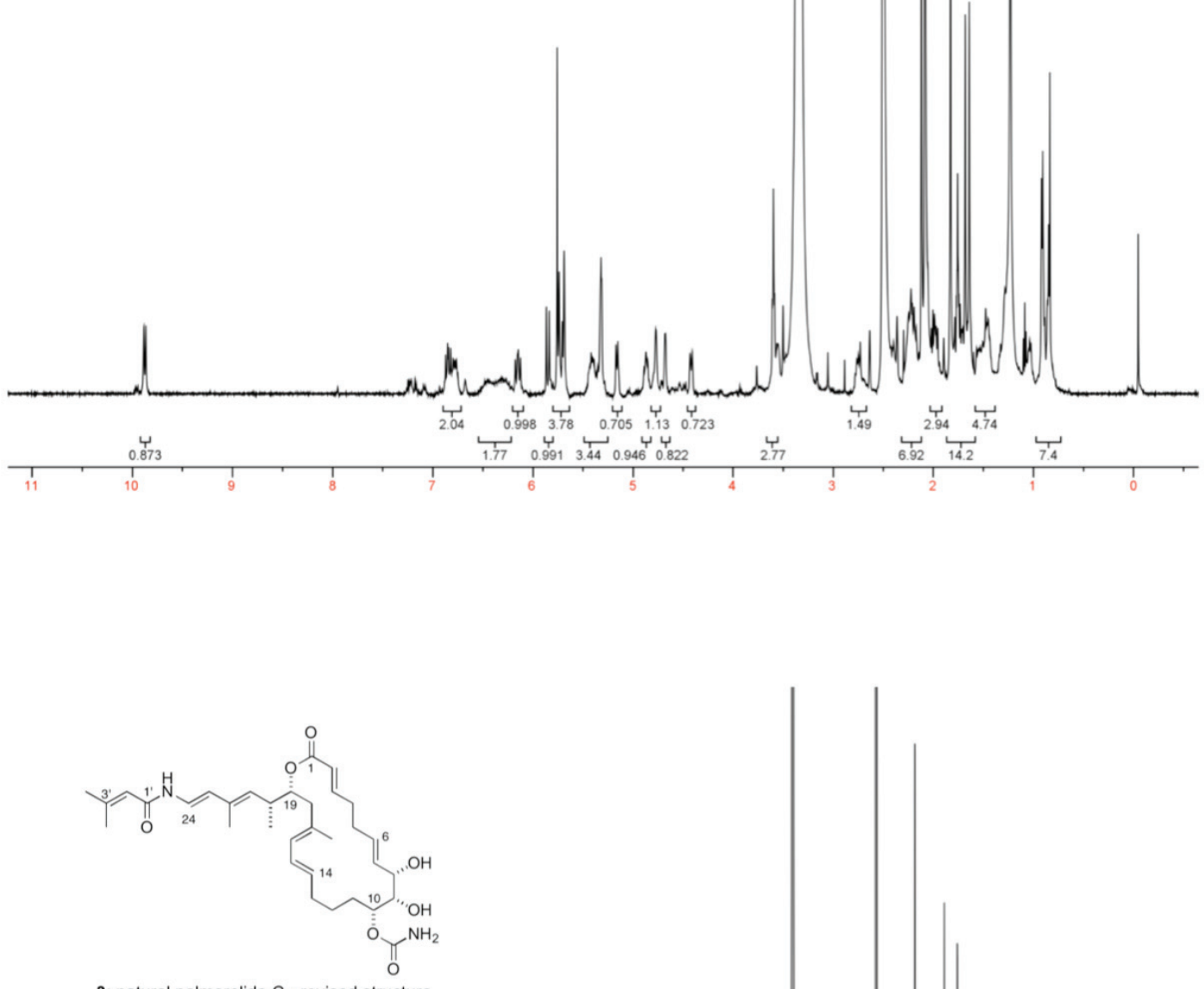

3: natural palmerolide $\mathrm{C}$ - revised structure ${ }^{1} \mathrm{H}$ NMR (500 MHz, d $\left.d_{\text {-DMSO}}\right)$

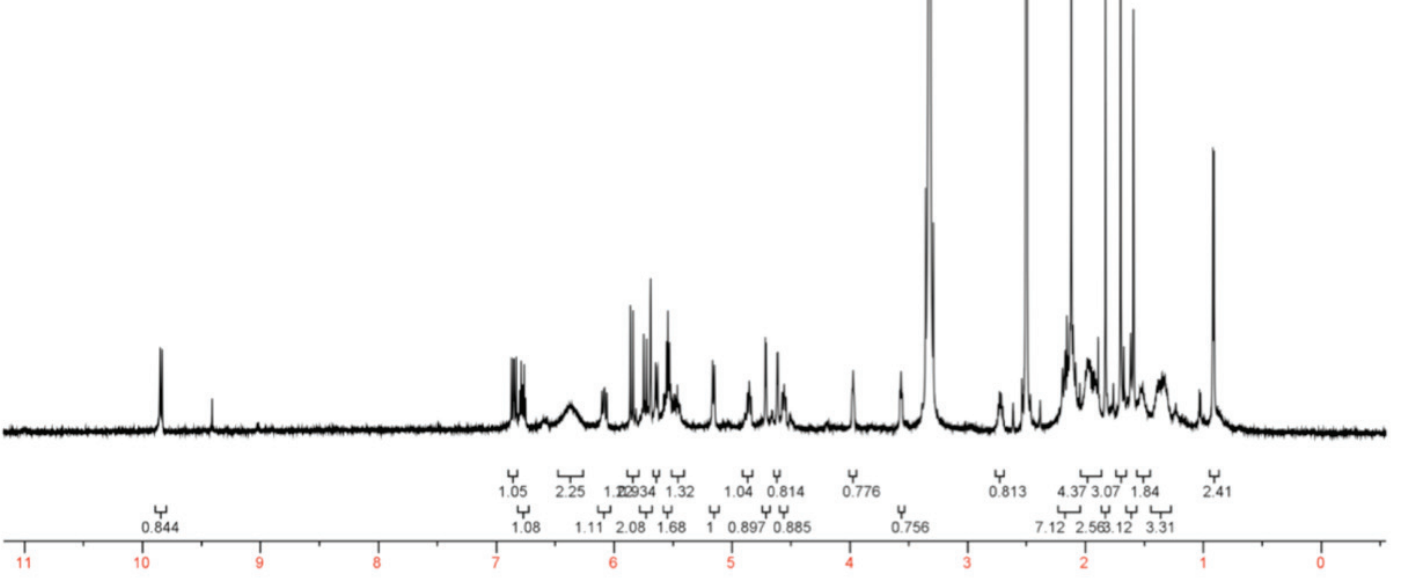



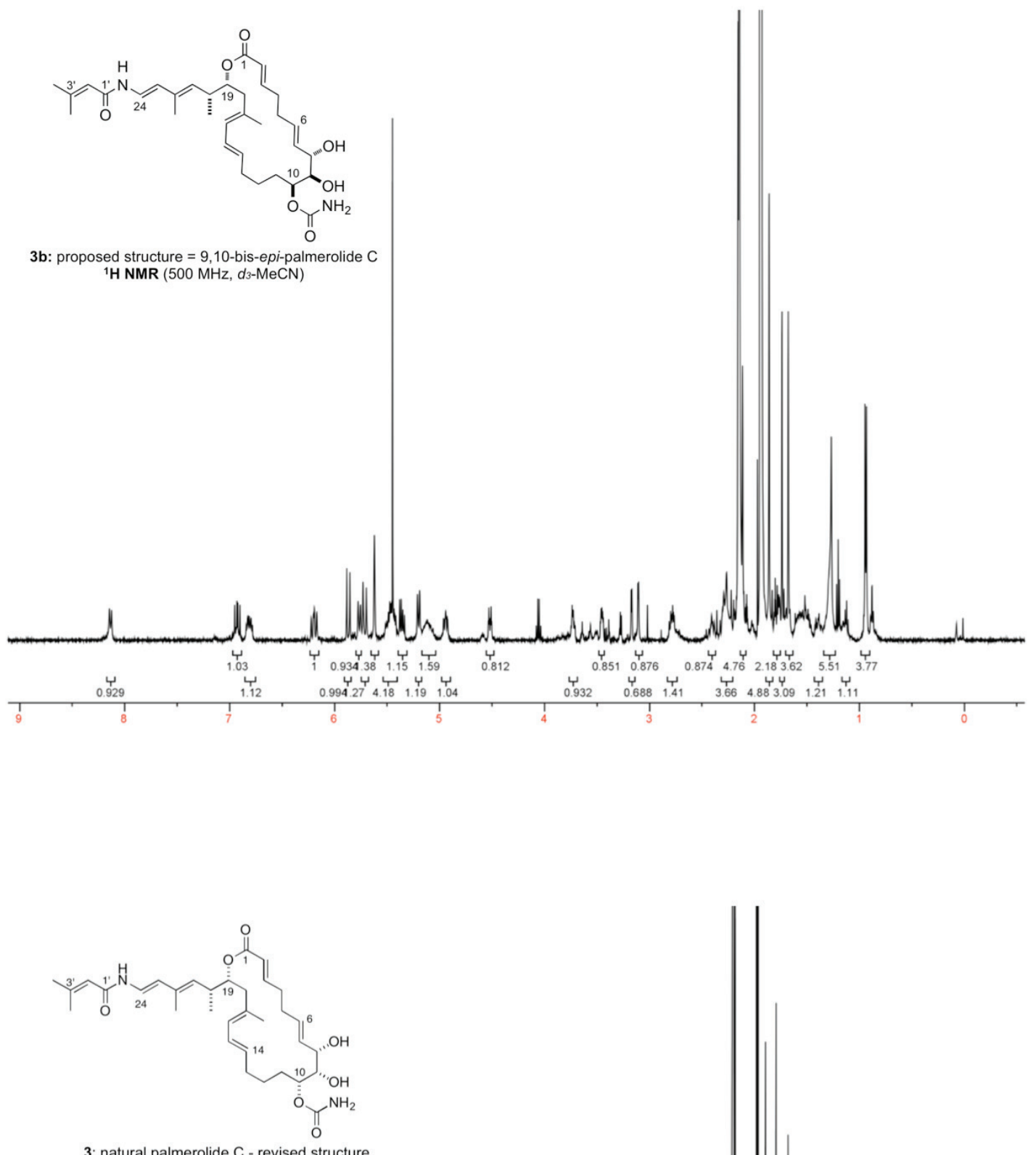

3: natural palmerolide $\mathrm{C}$ - revised structure ${ }^{1} \mathrm{H}$ NMR $\left(600 \mathrm{MHz}, d_{3}-\mathrm{MeCN}\right)$

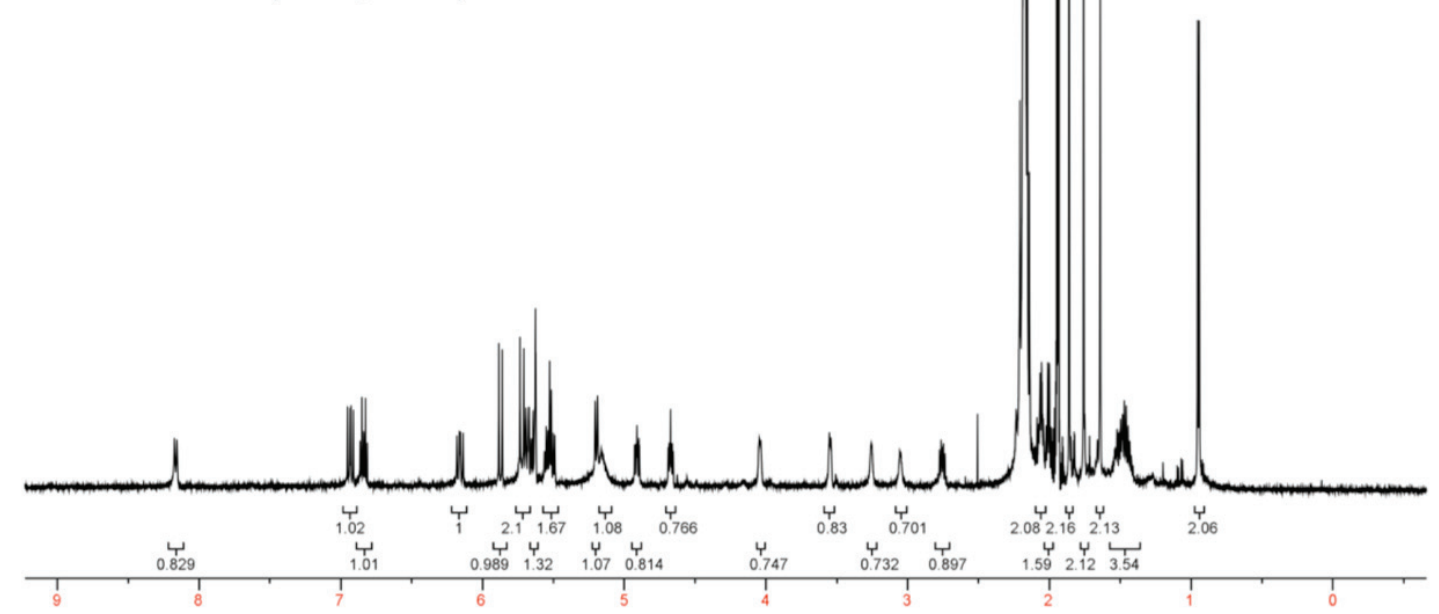



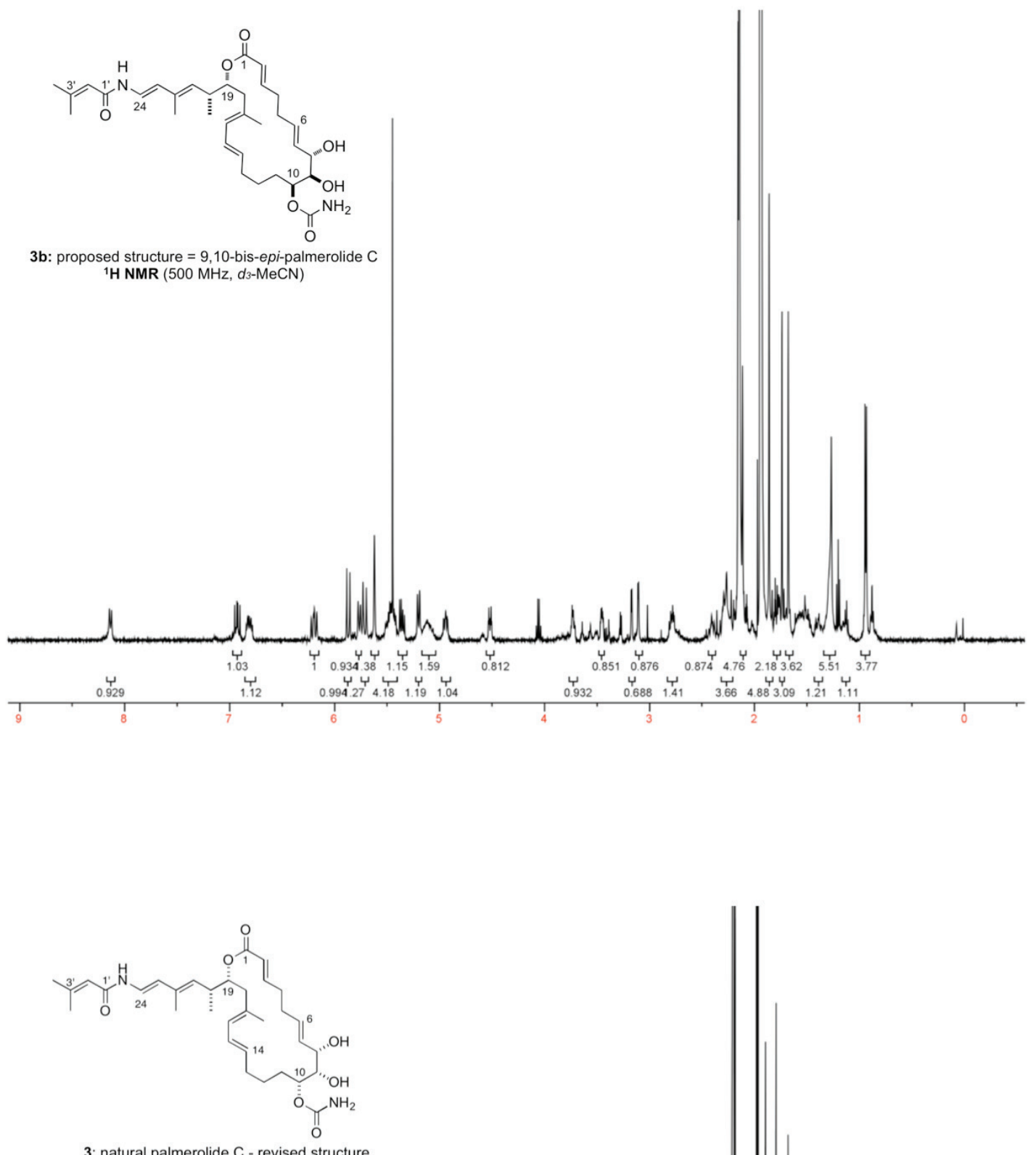

3: natural palmerolide $\mathrm{C}$ - revised structure ${ }^{1} \mathrm{H}$ NMR $\left(600 \mathrm{MHz}, d_{3}-\mathrm{MeCN}\right)$

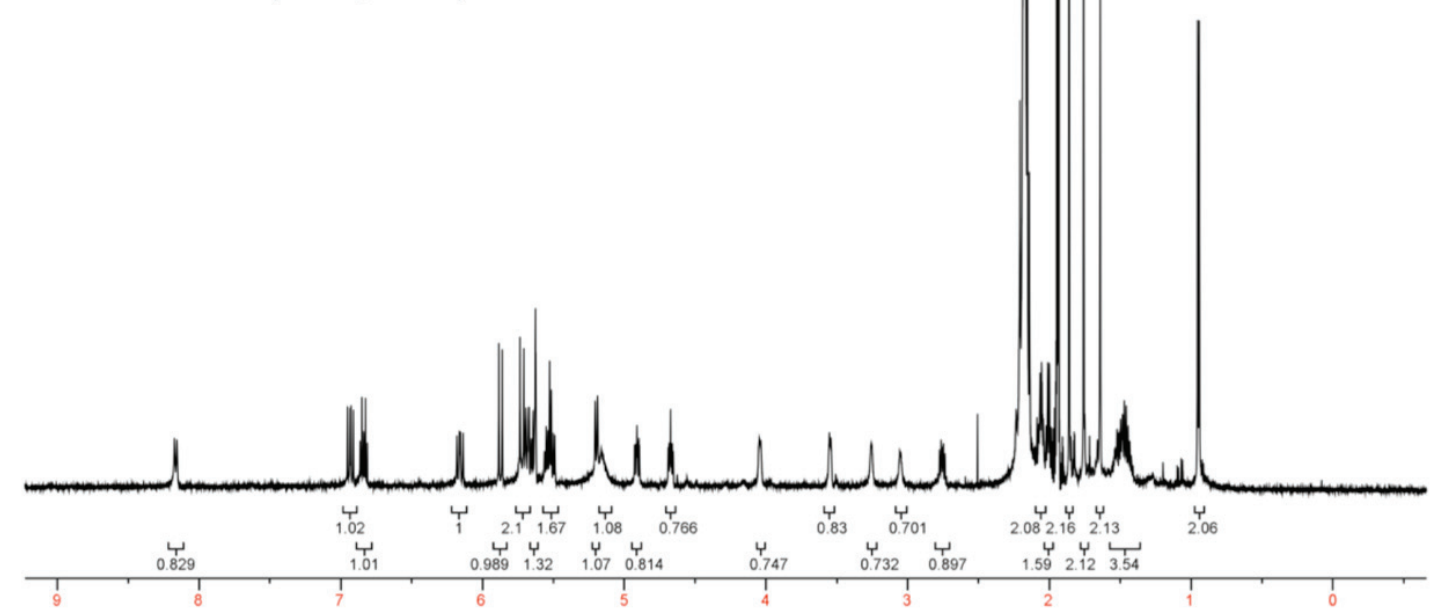



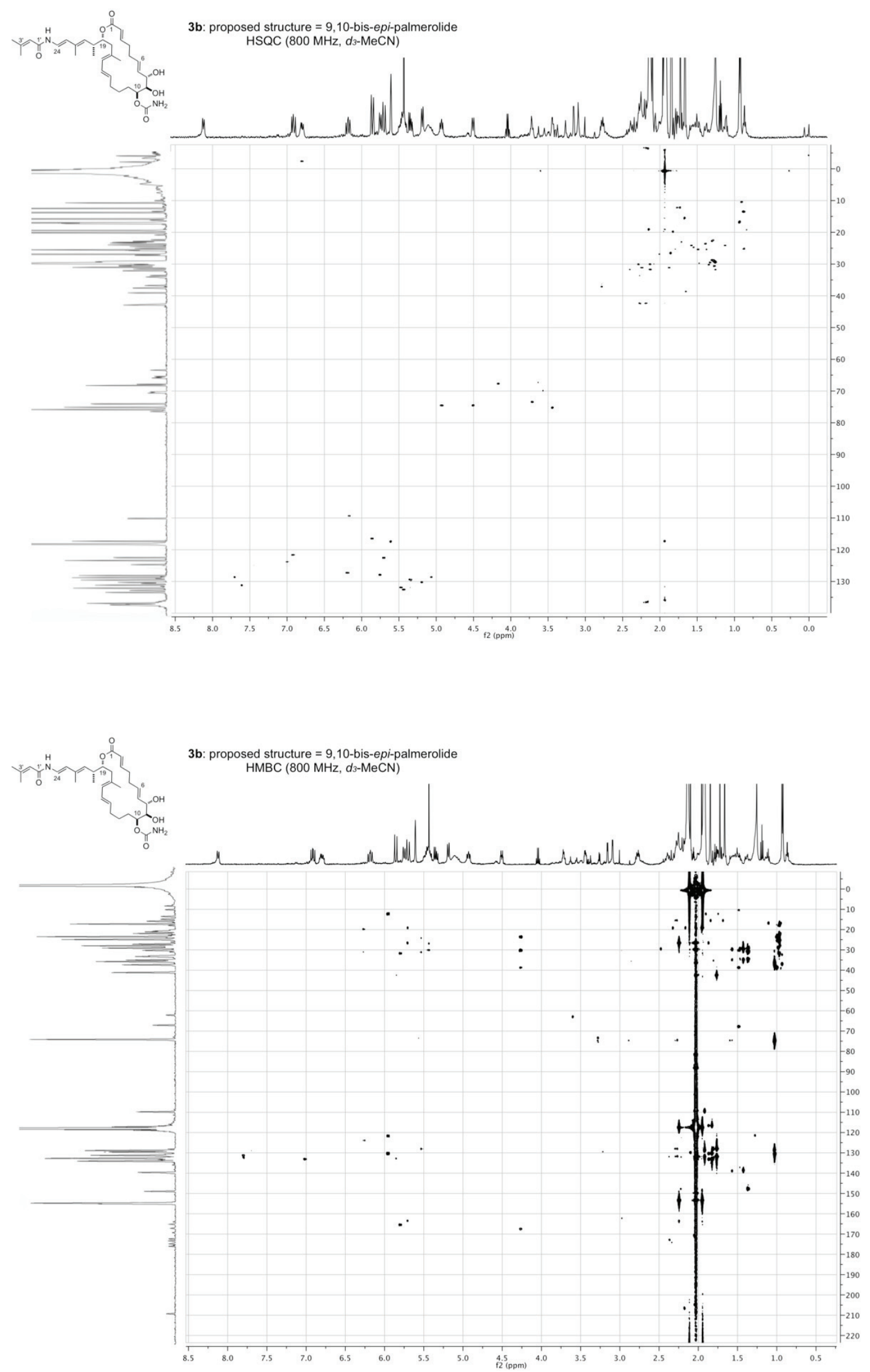


\section{References}

(1) Avila, C.; Taboada, S.; Nunez-Pons, L. Mar. Ecol.-Evol. Persp. 2008, 29, 1.

(2) Gutt, J. Polar Biol. 2001, 24, 553.

(3) Diyabalanage, T.; Amsler, C. D.; McClintock, J. B.; Baker, B. J. J. Am. Chem. Soc. 2006, 128,5630 .

(4) Baker, B. J.; Diyabalanage, T.; McClintock, J. B.; Amsler, C. D.; University of South Florida; The UAB Research Foundation: 2009.

(5) Jiang, X.; Liu, B.; Lebreton, S.; De Brabander, J. K. J. Am. Chem. Soc. 2007, 129, 6386.

(6) Nicolaou, K. C.; Guduru, R.; Sun, Y.-P.; Banerji, B.; Chen, D. Y. K. Angew. Chem. Int. Ed. 2007, 46, 5896.

(7) Nicolaou, K. C.; Sun, Y.-P.; Guduru, R.; Banerji, B.; Chen, D. Y. K. J. Am. Chem. Soc. 2008, 130, 3633.

(8) Penner, M.; Rauniyar, V.; Kaspar, L. T.; Hall, D. G. J. Am. Chem. Soc. 2009, 131, 14216.

(9) Matsumori, N.; Nonomura, T.; Sasaki, M.; Murata, M.; Tachibana, K.; Satake, M.; Yasumoto, T. Tetrahedron Lett. 1996, 37, 1269.

(10) Noguez, J. H.; Diyabalanage, T. K. K.; Miyata, Y.; Xie, X.-S.; Valeriote, F. A.; Amsler, C. D.; McClintock, J. B.; Baker, B. J. Biorg. Med. Chem. 2011, 19, 6608.

(11) Diyabalanage, T. K. K.; Florida, U. o. S. Chemical Investigation of Two Antarctic Invertebrates, Synoicum Adareanum (Chordata: Ascidiaceae: Enterogona: Polyclinidae) and Austrodoris Kergulenensis (Mollusca: Gastropoda: Nudibranchia: Dorididae); University of South Florida, 2006.

(12) Riesenfeld, C. S.; Murray, A. E.; Baker, B. J. J. Nat. Prod. 2008, 71, 1812.

(13) Tang, L.; Shah, S.; Chung, L.; Carney, J.; Katz, L.; Khosla, C.; Julien, B. Science 2000, 287, 640 .

(14) Carmeli, S.; Moore, R. E.; Patterson, G. M. L.; Yoshida, W. Y. Tetrahedron Lett. 1993, 34, 5571.

(15) Butcher, R. A.; Schroeder, F. C.; Fischbach, M. A.; Straight, P. D.; Kolter, R.; Walsh, C. T.; Clardy, J. Proceedings of the National Academy of Sciences 2007, 104, 1506. 
(16) Edwards, D. J.; Marquez, B. L.; Nogle, L. M.; McPhail, K.; Goeger, D. E.; Roberts, M. A.; Gerwick, W. H. Chem. Bio. 2004, 11, 817.

(17) Simunovic, V.; Müller, R. ChemBioChem 2007, 8, 497.

(18) Boyd, M. R.; Farina, C.; Belfiore, P.; Gagliardi, S.; Kim, J. W.; Hayakawa, Y.; Beutler, J. A.; McKee, T. C.; Bowman, B. J.; Bowman, E. J. J. Pharmacol. Exp. Ther. 2001, 297, 114.

(19) Xie, X.-S.; Padron, D.; Liao, X.; Wang, J.; Roth, M. G.; De Brabander, J. K. J. Biol. Chem. 2004, 279, 19755.

(20) Bowman, E. J.; Siebers, A.; Altendorf, K. PNAS 1988, 85, 7972.

(21) Noguez-Heimbegner, J., University of South Florida, 2010.

(22) Kaliappan, K. P.; Gowrisankar, P. Synlett 2007, 2007, 1537.

(23) Chandrasekhar, S.; Vijeender, K.; Chandrashekar, G.; Raji Reddy, C. Tetrahedron: Asymmetry 2007, 18, 2473.

(24) Jagel, J.; Schmauder, A.; Binanzer, M.; Maier, M. E. Tetrahedron 2007, 63, 13006.

(25) Jagel, J.; Maier, M. E. Synthesis 2009, 2009, 2881.

(26) Jones, D. M.; Dudley, G. B. Synlett 2010, 2010, 223.

(27) Gowrisankar, P.; Pujari, S. A.; Kaliappan, K. P. Chem. Eur. J. 2010, 16, 5858.

(28) Prasad, K. R.; Pawar, A. B. Synlett 2010, 2010, 1093.

(29) Prasad, K. R.; Pawar, A. B. Org. Lett. 2011, 13, 4252.

(30) Evans, D. A.; Starr, J. T. J. Am. Chem. Soc. 2003, 125, 13531.

(31) Inanaga, J.; Hirata, K.; Saeki, H.; Katsuki, T.; Yamaguchi, M. Bull. Chem. Soc. Jpn. 1979, 52, 1989.

(32) De Mico, A.; Margarita, R.; Parlanti, L.; Vescovi, A.; Piancatelli, G. J. Org. Chem. 1997, 62, 6974 .

(33) Stork, G.; Nakamura, E. J. Org. Chem. 1979, 44, 4010.

(34) Corey, E. J.; Helal, C. J. Angew. Chem. Int. Ed. 1998, 37, 1986.

(35) Mata, E. G.; Mascaretti, O. A. Tetrahedron Lett. 1988, 29, 6893.

(36) Yin; Liebscher, J. r. Chem. Rev. 2006, 107, 133.

(37) Takai, K.; Nitta, K.; Utimoto, K. J. Am. Chem. Soc. 1986, 108, 7408.

(38) Klapars, A.; Huang, X.; Buchwald, S. L. J. Am. Chem. Soc. 2002, 124, 7421.

(39) Ravu, V. R.; Leung, G. Y. C.; Lim, C. S.; Ng, S. Y.; Sum, R. J.; Chen, D. Y. K. Eur. J. Org. Chem. 2011, 2011, 463.

(40) Nicolaou, K. C.; Leung, G. Y. C.; Dethe, D. H.; Guduru, R.; Sun, Y.-P.; Lim, C. S.; Chen, D. Y. K. J. Am. Chem. Soc. 2008, 130, 10019. 
(41) Gao, X.; Hall, D. G. J. Am. Chem. Soc. 2003, 125, 9308.

(42) Burke, S. D.; Fobare, W. F.; Pacofsky, G. J. J. Org. Chem. 1983, 48, 5221.

(43) Rauniyar, V.; Hall, D. G. J. Org. Chem. 2009, 74, 4236.

(44) Lebar, M. D.; Baker, B. J. Tetrahedron Lett. 2007, 48, 8009.

(45) Regeling, H.; F. Chittenden, G. J. Carbohydr. Res. 1992, 216, 79.

(46) Rychnovsky, S. D.; Griesgraber, G.; Schlegel, R. J. Am. Chem. Soc. 1995, 117, 197.

(47) Rychnovsky, S. D.; Skalitzky, D. J. Tetrahedron Lett. 1990, 31, 945.

(48) Rychnovsky, S. D.; Rogers, B.; Yang, G. J. Org. Chem. 1993, 58, 3511.

(49) Murata, M.; Matsuoka, S.; Matsumori, N.; Paul, G. K.; Tachibana, K. J. Am. Chem. Soc. 1999, 121, 870.

(50) Eberstadt, M.; Gemmecker, G.; Mierke, D. F.; Kessler, H. Angew. Chem. Int. Ed. 1995, 34, 1671.

(51) Enders, D.; Grondal, C. Angew. Chem. Int. Ed. 2005, 44, 1210.

(52) Northrup, A. B.; MacMillan, D. W. C. Science 2004, 305, 1752.

(53) Northrup, A. B.; Mangion, I. K.; Hettche, F.; MacMillan, D. W. C. Angew. Chem. Int. Ed. 2004, 43, 2152.

(54) Enders, D.; Palecek, J.; Grondal, C. Chem. Commun. 2006, 655.

(55) Edlin, C. D.; Faulkner, J.; Quayle, P. Tetrahedron Lett. 2006, 47, 1145.

(56) Dale, J. A.; Mosher, H. S. J. Am. Chem. Soc. 1973, 95, 512.

(57) Seco, J. M.; Quina, E.; Riguera, R. Chem. Rev. 2004, 104, 17.

(58) Bahmanyar, S.; Houk, K. N. J. Am. Chem. Soc. 2001, 123, 12911.

(59) Bahmanyar, S.; Houk, K. N.; Martin, H. J.; List, B. J. Am. Chem. Soc. 2003, 125, 2475.

(60) Benjamin, L. Tetrahedron 2002, 58, 5573.

(61) Heine, A.; DeSantis, G.; Luz, J. G.; Mitchell, M.; Wong, C.-H.; Wilson, I. A. Science 2001, 294, 369.

(62) Rankin, K. N.; Gauld, J. W.; Boyd, R. J. J. Phys. Chem. A 2002, 106, 5155.

(63) Evans, D. A.; Chapman, K. T.; Carreira, E. M. J. Am. Chem. Soc. 1988, 110, 3560.

(64) Grondal, C.; Enders, D. Tetrahedron 2006, 62, 329.

(65) Brown, C. A.; Coleman, R. A. J. Org. Chem. 1979, 44, 2328.

(66) Brown, H. C.; Negishi, E.; Katz, J. J. J. Am. Chem. Soc. 1975, 97, 2791.

(67) Evans, D. A.; Dart, M. J.; Duffy, J. L. Tetrahedron Lett. 1994, 35, 8541.

(68) Ramasastry, S. S. V.; Zhang, H.; Tanaka, F.; Barbas, C. F. J. Am. Chem. Soc. 2006, 129, 288. 
(69) Ramasastry, S. S. V.; Albertshofer, K.; Utsumi, N.; Tanaka, F.; Barbas, C. F. Angew. Chem. Int. Ed. 2007, 46, 5572.

(70) Utsumi, N.; Imai, M.; Tanaka, F.; Ramasastry, S. S. V.; Barbas, C. F. Org. Lett. 2007, 9, 3445 .

(71) Narasaka, K.; Pai, F.-C. Tetrahedron 1984, 40, 2233.

(72) Chen, K.-M.; Hardtmann, G. E.; Prasad, K.; Repia, O.; Shapiro, M. J. Tetrahedron Lett. 1987, 28, 155.

(73) Paterson, I.; Florence, G. J.; Gerlach, K.; Scott, J. P.; Sereinig, N. J. Am. Chem. Soc. 2001, 123, 9535 .

(74) de Napoli, L.; Messere, A.; Palomba, D.; Piccialli, V.; Evidente, A.; Piccialli, G. J. Org. Chem. 2000, 65, 3432.

(75) Blanchette, M. A.; Choy, W.; Davis, J. T.; Essenfeld, A. P.; Masamune, S.; Roush, W. R.; Sakai, T. Tetrahedron Lett. 1984, 25, 2183.

(76) Abushanab, E.; Vemishetti, P.; Leiby, R. W.; Singh, H. K.; Mikkilineni, A. B.; Wu,

D. C. J.; Saibaba, R.; Panzica, R. P. J. Org. Chem. 1988, 53, 2598.

(77) Cren, S.; Wilson, C.; Thomas, N. R. Org. Lett. 2005, 7, 3521.

(78) Murga, J.; Ruiz, P. n.; Falomir, E.; Carda, M.; Peris, G.; Marco, J. A. J. Org. Chem. 2004, 69, 1987.

(79) Paterson, I.; Wallace, D. J.; Velazquez, S. M. Tetrahedron Lett. 1994, 35, 9083.

(80) Goodman, J. M.; Paterson, I. Tetrahedron Lett. 1992, 33, 7223.

(81) Evans, D. A.; Nelson, J. V.; Taber, T. R. In Topics in Stereochemistry; John Wiley \& Sons, Inc.: 2007, p 1.

(82) Carreira, E. M.; Singer, R. A.; Lee, W. J. Am. Chem. Soc. 1994, 116, 8837.

(83) Goodman, J. M.; Paton, R. S. Chem. Commun. 2007, 2124.

(84) Vulpetti, A.; Bernardi, A.; Gennari, C.; Goodman, J. M.; Paterson, I. Tetrahedron 1993, 49, 685 .

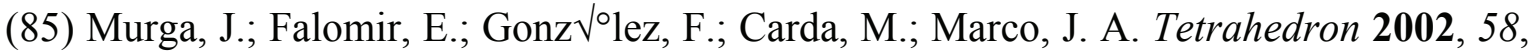
9697.

(86) Rai, A. N.; Basu, A. Tetrahedron Lett. 2003, 44, 2267.

(87) Veitch, G. E.; Boyer, A.; Ley, S. V. Angew. Chem. Int. Ed. 2008, 47, 9402.

(88) Madsen, C. M.; Hansen, M.; Thrane, M. V.; Clausen, M. H. Tetrahedron 2010, 66, 9849.

(89) Evans, D. A.; Cee, V. J.; Siska, S. J. J. Am. Chem. Soc. 2006, 128, 9433. 
(90) Aho, J. E.; Piisola, A.; Syam Krishnan, K.; Pihko, P. M. Eur. J. Org. Chem. 2011, $2011,1682$.

(91) Grubbs, R. H. Handbook of Metathesis: Catalyst Development; WILEY-VCH Verlag GmbH \& Co. KGaA, 2008.

(92) Scholl, M.; Ding, S.; Lee, C. W.; Grubbs, R. H. Org. Lett. 1999, 1, 953.

(93) Crimmins, M. T.; McDougall, P. J. Org. Lett. 2003, 5, 591.

(94) Seto, M.; Roizen, J. L.; Stoltz, B. M. Angew. Chem. Int. Ed. 2008, 47, 6873.

(95) Hoye, T. R.; Eklov, B. M.; Jeon, J.; Khoroosi, M. Org. Lett. 2006, 8, 3383.

(96) Wang, Z. In Comprehensive Organic Name Reactions and Reagents; John Wiley \& Sons, Inc.: 2010.

(97) Cowden, C. J.; Paterson, I. In Organic Reactions; John Wiley \& Sons, Inc.: 2004.

(98) Beemelmanns, C.; Reissig, H.-U. Angew. Chem. Int. Ed. 2010, 49, 8021.

(99) J. J. Li, E. J. C. Name Reactions for Functional Group Transformations; John Wiley \& Sons, Inc., 2007.

(100) Martin, J. C.; Arhart, R. J. J. Am. Chem. Soc. 1971, 93, 2339.

(101) Kocovsky, P. Tetrahedron Lett. 1986, 27, 5521.

(102) Anh, N.; Springer Berlin / Heidelberg: 1980; Vol. 88, p 145.

(103) Cherest, M.; Felkin, H.; Prudent, N. Tetrahedron Lett. 1968, 9, 2199.

(104) Paterson, I.; Burton, P. M.; Cordier, C. J.; Housden, M. P.; Muahlthau, F. A.; Loiseleur, O. Org. Lett. 2009, 11, 693.

(105) Sinisterra, J. V.; Marinas, J. M.; Riquelme, F.; Arias, M. S. Tetrahedron 1988, 44, 1431.

(106) Saito, S.; Shiozawa, M.; Ito, M.; Yamamoto, H. J. Am. Chem. Soc. 1998, 120, 813.

(107) Saito, S.; Shiozawa, M.; Yamamoto, H. Angew. Chem. Int. Ed. 1999, 38, 1769.

(108) Maruoka, K.; Imoto, H.; Saito, S.; Yamamoto, H. J. Am. Chem. Soc. 1994, 116, 4131.

(109) Saito, S.; Nagahara, T.; Shiozawa, M.; Nakadai, M.; Yamamoto, H. J. Am. Chem. Soc. 2003, 125, 6200 .

(110) Frigerio, M.; Santagostino, M.; Sputore, S. J. Org. Chem. 1999, 64, 4537.

(111) Ireland, R. E.; Liu, L. J. Org. Chem. 1993, 58, 2899.

(112) Casiraghi, G.; Battistini, L.; Curti, C.; Rassu, G.; Zanardi, F. Chem. Rev. 2011, 111, 3076.

(113) Denmark, S. E.; Heemstra, J. R.; Beutner, G. L. Angew. Chem. Int. Ed. 2005, 44, 4682 . 
(114) Charest, M.; Felkin, H.; Prudent, N. Tetrahedron Lett. 1968, 9, 2199.

(115) E. Vedejs, S. L. Organic Syntheses 1986, 64, 127.

(116) Julia, M.; Paris, J.-M. Tetrahedron Lett. 1973, 14, 4833.

(117) Baudin, J. B.; Hareau, G.; Julia, S. A.; Ruel, O. Tetrahedron Lett. 1991, 32, 1175.

(118) J. B. Baudin, G. H., S. A. Julia and O. Ruel Bull. Soc. Chim. Fr. 1993, 130, 336.

(119) Blakemore, P. R. J. Chem. Soc., Perkin Trans. 1 2002, 2563.

(120) Blakemore, P. R.; Ho, D. K. H.; Nap, W. M. Org. Bio. Chem. 2005, 3, 1365.

(121) Blakemore, P. R.; Cole, W. J.; Kocienski, P. J.; Morley, A. Synlett 1998, 1998, 26.

(122) Bellingham, R.; Jarowicki, K.; Kocienski, P.; Martin, V. Synthesis 1996, 1996, 285.

(123) Hopf, H.; Plagens, A.; Walsh, R. Liebigs Annalen 1996, 1996, 825.

(124) Evans, D. A.; Fitch, D. M.; Smith, T. E.; Cee, V. J. J. Am. Chem. Soc. 2000, 122, 10033.

(125) Laganis, E. D.; Chenard, B. L. Tetrahedron Lett. 1984, 25, 5831.

(126) Jiang, L.; Job, G. E.; Klapars, A.; Buchwald, S. L. Org. Lett. 2003, 5, 3667.

(127) Strieter, E. R.; Blackmond, D. G.; Buchwald, S. L. J. Am. Chem. Soc. 2005, 127, 4120 .

(128) Gensler, W. J.; Johnson, F.; Sloan, A. D. B. J. Am. Chem. Soc. 1960, 82, 6074.

(129) Crabbe, P.; Garcia, G. A.; Rius, C. J. Chem. Soc., Perkin Trans. 1 1973, 810.

(130) Ho, T.-L.; Fieser, M.; Fieser, L.; Fieser, L. F. In Fieser and Fieser's Reagents for Organic Synthesis; John Wiley \& Sons, Inc.: 2006.

(131) Paterson, I.; Findlay, A. D.; Florence, G. J. Org. Lett. 2006, 8, 2131.

(132) Mathieson, J. E.; Crawford, J. J.; Schmidtmann, M.; Marquez, R. Org. Bio. Chem. 2009, 7, 2170.

(133) Wang, G.; Yin, N.; Negishi, E. Chem. Eur. J. 2011, 17, 4118.

(134) Kumaraswamy, G.; Padmaja, M. J. Org. Chem. 2008, 73, 5198.

(135) Edlin, C. D.; Faulkner, J.; Quayle, P. Tetrahedron Lett. 2006, 47, 1145.

(136) Dolder, X. S. M.; Tamm, C. Helv. Chim. Acta 1990, 73, 483.

(137) Tsimelzon, A.; Braslau, R. J. Org. Chem. 2005, 70, 10854.

(138) Marshall, J. A.; Sabatini, J. J. Org. Lett. 2005, 7, 4819.

(139) Stoller, A.; Mioskowski, C.; Sepulchre, C.; Bellamy, F. Tetrahedron Lett. 1991, 32, 495.

(140) Kumarn, S.; Oelke, A. J.; Shaw, D. M.; Longbottom, D. A.; Ley, S. V. Org. Bio. Chem. 2007, 5, 2678.

(141) Shimada, T.; Yamamoto, Y. Tetrahedron Lett. 1998, 39, 471. 
(142) de Kermadec, D.; Prudhomme, M. Tetrahedron Lett. 1993, 34, 2757.

(143) Thomas, E. J.; Whitehead, J. W. F. J. Chem. Soc., Perkin Trans. 1 1989, 507. 